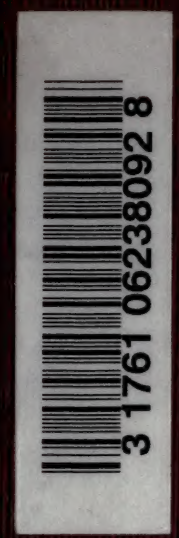


$10 \%$ 
Students Sikrary Che

hedical Buildap

muivect of toronto 



\section{THE BODY AT WORK}


Digitized by the Internet Archive in 2007 with funding from Microsoft Corporation 



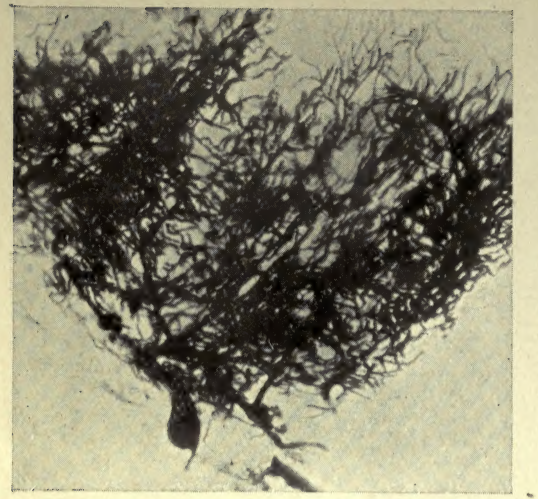

A.

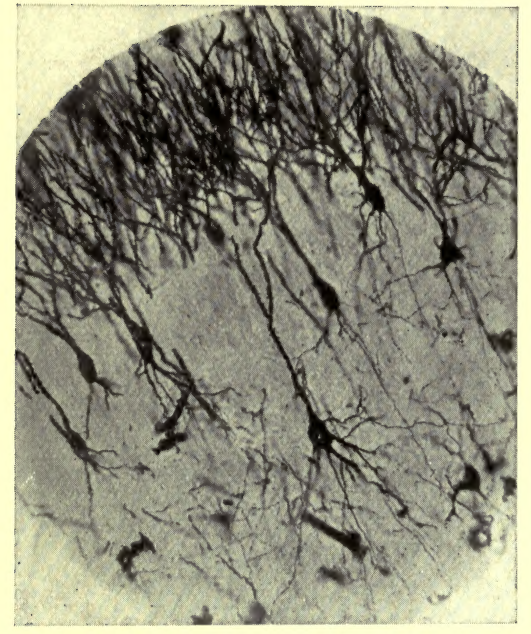

c.

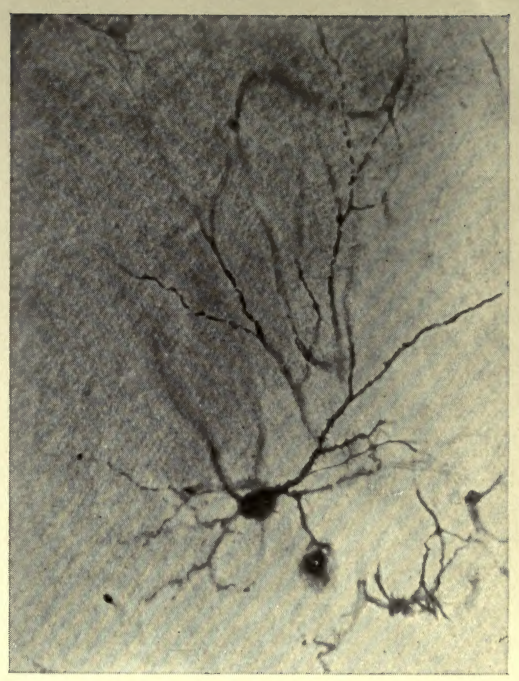

B.

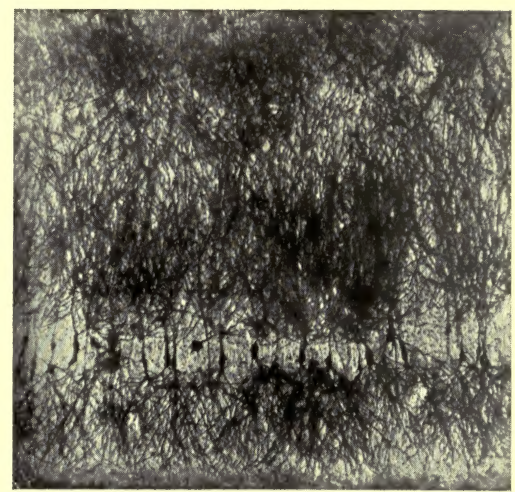

D.

Fig. 1.-Photomicrographs of Cells of the Cortex of the Cerebellum and Cerebrum. 


\title{
THE BODY AT WORK
}

\section{A TREATISE ON THE PRINCIPLES}

\section{OF PHYSIOLOGY}

153

\author{
BY \\ ALEX HILL, M.A., M.D., F.R.C.S.
}

SOMETIME MASTER OF DOWNING COLLEGE, CAMBRIDGE

WITH 46 ILLUSTRATIONS

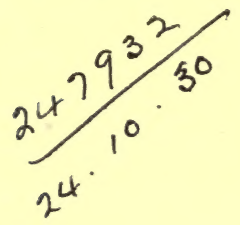

LONDON

EDWARD ARNOLD

1908

[All rights reserved] 



\section{PREFACE}

Few subjects are as well provided with text-books as physiology; yet it may be doubted whether the interests of the amateur of science have been adequately cared for. From his point of view there are certain obvious drawbacks to even the most admirable of text-books. Writing for medical students, their authors assume that their readers have passed through two years of preliminary training in physics, chemistry, and biology; they take for granted that they will have the privilege of supplementing their study of the theory of physiology with practical work in a laboratory ; they treat all parts of the subject with equal thoroughness. In this book I have endeavoured to describe the phenomena of life, and the principal conclusions which have been drawn as to their interdependence and as to their causes, in language which will be understood by persons unacquainted with the sciences upon which physiology is based. I have omitted all reference to experimental methods and to the technique of the science, save when a knowledge of the means by which information has been obtained is essential to a comprehension of its bearing. I have passed over such sections of the subject as are generally considered unsuitable for ordinary discussion. And since this book neither aims at being an introduction to the systematic study of physiology, nor poses as an aid in the preparation for professional examinations, I have treated with some thoroughness the more recondite and the more suggestive results of recent research, and have tried to indicate the trend of modern thought regarding problems as yet unsolved. I have endeavoured to reflect the 
intrinsic interest of the science apart altogether from its medical applications.

An author who attempts the popular exposition of a science must stand sufficiently far away from his subject to lose sight of its details, whilst keeping its outlines clearly in view. The difficulty of finding such a position is probably greater in the case of physiology than in that of any other science. Few of its conclusions are indisputable-even those which seem to be most in accord with the balance of evidence. If my treatment of any vexed questions is unjustifiably dogmatic, this will, I trust, be attributed to the desire to present a definite picture, and not to forgetfulness of considerations which seem to call for qualified statements. All physiologists will agree that a book which recorded every piece of evidence which is difficult to reconcile with the views generally adopted would not only extend to an inordinate length, but would leave a very indefinite impression on the mind of the reader.

In many cases the value of a conclusion depends upon the reputation for insight and accuracy of the physiologist who recorded the observations upon which it is based. It is no want of appreciation of the genius of the workers who have contributed most largely to the advance of the science which has led me to omit, save in a few classical instances, the names of all authorities. It is solely due to a desire to lighten this book of all details not essential to the comprehension of the propositions which it sets forth.

The illustrations are reproductions of blackboard drawings. A few of them have already appeared in my Physiologist's Notebook and Primer of Physiology ; but the large majority are now printed for the first time.

November, 1908.

ALEX HILL. 


\section{CON'TEN'TS}

CRAPTRR
I. PROLEGOMENA
II. THE BASIS OF LIFE -
III. THE UNIT OF STRUCTURE -





\section{LIST OF ILLUSTRATIONS}

FIG.

1. Photomicrographs of Brain-Tissue

2. The Organs of the Chest and Abdomen

PAGR

3. Mucous Gland, Capillaries, and Connective-Tissue Spaces - 38

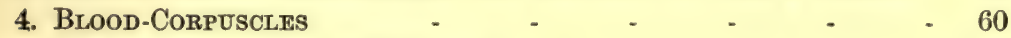

5. Spleen-Pulp, with Phagocytes - - - $\quad$ - $\quad$ - 81

6. Duodenum and Neighbouring Organs - $\quad$ - $\quad$ - $\quad 99$

7. A Lobule of the Liver and Liver-Cells - $\quad$ - $\quad 160$

8. The Diaphragm during Inspiration and Expiration - 172

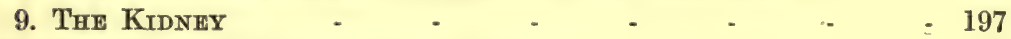

10. The Heart in Longitudinal Section - $\quad-\quad$ - $\quad 218$

11. Horizontal Section of the Heart, showing its Valves - 225

12. Section of the Wall of a Small Artery $\quad-\quad$ - $\quad 233$

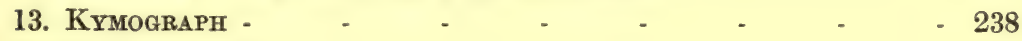

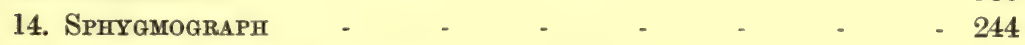

15. Blood-Pressure Tracings $\quad$ - $\quad$ - $\quad$ - $\quad$ - $\quad 245$

16. Minute Structure of Muscle-Fibres - $\quad$ - $\quad$ - 262

17. The Biceps Muscle in Action - $\quad$ - $\quad$ - $\quad$ - $\quad$ - 286

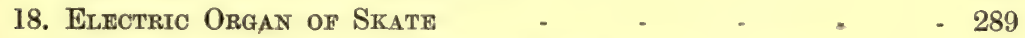

19. Minute Structure of a Nerve-Fibre - $\quad$ - $\quad$ - $\quad 296$

20. Ganglion-Cells with Neuro-Fibrilles of the Leech - - 298

21. The Development of the Granules of the Cerebellum - 304

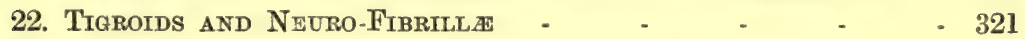

23. Minute Structure of the Cortex of the Cerebellum - 339

24. Minute Structure of the Cortex of the Cerebrum - - 347

25. Functional Areas of the Cortex of the Cerebrum - 352

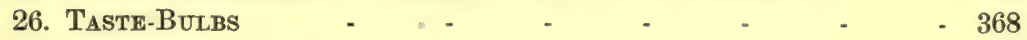

27. Horizontal Section of the Eye $\quad$ - $\quad$ - $\quad$ - $\quad 373$

28. Development of the Crystaline Lens $\quad$ - $\quad$ - $\quad$ - 374

29. Purkinje's Shadows of the Vessels of the Retina - - 375

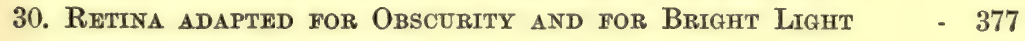

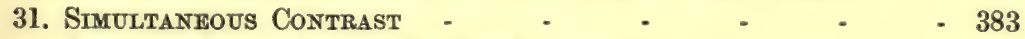

32. Formation of an Image on the Retina $\quad$ - $\quad$ - $\quad 391$

33. The Form of the Eyeball in Short Sight, Normal Sight, and

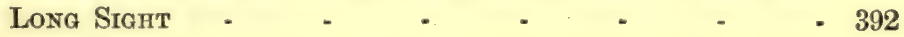


FI.

34. The Bunnd Spot .

35. An Optical Illusion

36. An Optical Illusion

37. An Optical Illusion

38. External, Middle, and Inner Ear

39. The Cochlea

40. Thi Organ of Corti

41. Nerves of the Corneal Epithelium

42. Touoh-Corpuscles -

43. Pactintan Corpuscle

44. The GLotris

45. The Larynx in Longitudinal Section -

46. The Larynx from the Right Side

\section{NOTE ON THE FRONTISPIECE}

Four photomicrographs of cells or parts of cells of brain-tissue, coloured by the chrome-silver method (cf. p. 293).

A. Cell of Purkinje from the cerebellum of a man aged 45. At the bottom of the photograph is seen the rounded cell-body, with the commencement of its axon. The summit of the cell-body bears an elaborately branched system of dendrites, spread out in the plane of the section.

B. A single basket-cell of the cortex of the cerebellum (very highly magnified). The oval cell-body gives origin to four dendritic processes which branch. Thorns are to be seen on the larger process which ascends on the right. From the same process, near its origin, springs a delicate axon which thickens as it proceeds to form a basket at the right-hand lower corner of the photograph. Two other branches of the same axon, which form baskets around other Purkinje-cells, are faintly visible, although out of focus.

C. Seven or eight pyramids from the cortex of the cerebrum of a hedgehog. A little below the centre of the photograph is seen a large pyramid with a single thorny apical process which bifurcates, several basal dendrites and an axon. In the upper part of the photograph are seen the apical processes of a number of pyramids of which the bodies were not included in the section.

D. The margin of the cortex (subiculum cornu Ammonis) from the same specimen. A single row of pyramids extends across the photograph. They are remarkable for the richness of branching of their basal processes, which has earned for the cells which comprise this sheet the name of "double pyramids."

All four sections were cut vertically to the surface. 


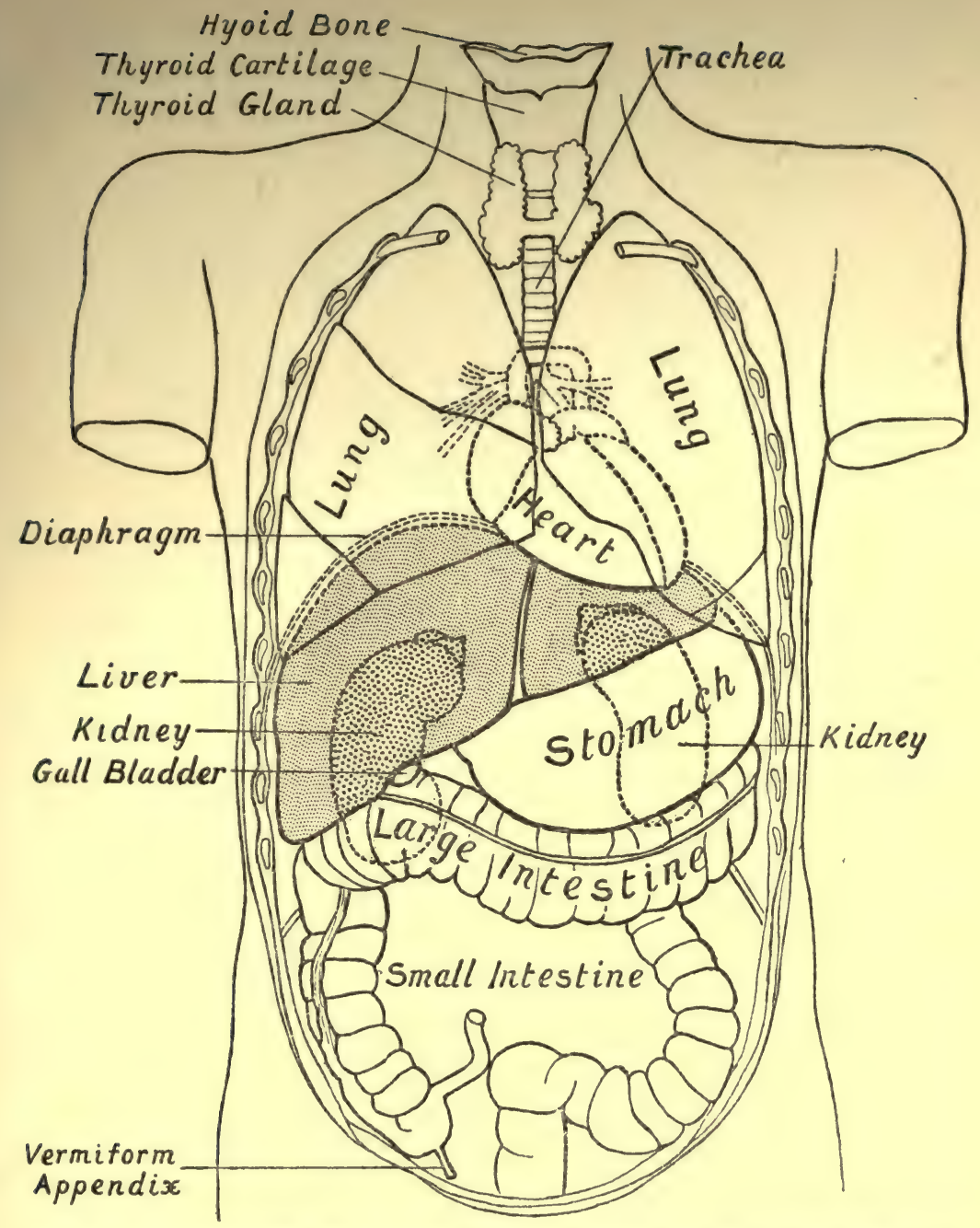

Fig. 2.-Diagram showing the Relative Positions of the Organs of the Chest and ABDOMEN.

The ribs from the first to the tenth have been cut across in the lateral line. The eleventh and twelfth ribs do not reach sufficiently far forwards to be cut. With the exception of a short segment near its junction with the ascending colon, the small intestine has been removed. The trachea is seen to divide into bronchi beneath the arch of the aorta. The right lung has three, the left two lobes. The kidneys are situate behind all the other viscera. On their upper ends rest the two suprarenal capsules. The lower edge of the right lobe of the liver follows closely the line of the ribs and costal cartilages. Below the left lobe of the liver the stomach comes to the anterior abdominal wall. The transverse colon (large intestine) comes to the anterior wall below the stomach. Below the latter the wall is in contact chiefly with coils of small intestine. The vermiform appendix rests on the posterior wall. Spleen and pancreas are not shown in the diagram. 



\section{THE BODY AT WORK}

\section{CHAPTER I}

\section{PROLEGOMENA}

Physiology is the science of the body at work. It is the study of life. Anatomy records how plants and animals are constructed. It maps and measures. Physiology ascertains what they do, endeavours to explain how they do it, and conjectures why.

A knowledge of structure is essential to the right understanding of function; but the physiologist does not contemplate structure with a view to divining possibilities of action. He has no interest in structure as such. To him it is a matter of perfect indifference whether the tendon of a muscle is at its origin or its insertion. He would rather not know which end of the muscle terminates in a tendon. It is waste of his time to notice such a fact, save for the negative, the protective value of the information. If he did not know how the muscle and tendon are related, he might possibly imagine the muscle as doing something of which it is incapable. Observers of living things are often credited with studying structure with a view to determining function. The reverse is the true order of thought and observation. Living things perform certain acts. Having no inherent knowledge of our own microcosm which enables us to say how it works, we cannot, by reflecting upon our own internal operations, explain its various activities. Nor can we make use of the results of introspection when endeavouring to account for the acts of other beings. Our knowledge of how things are done is altogether extrapersonal, objective. It is the result of trial, failure, success in the use of apparatus, our own essays, or those of others. The body is a combination of organs-a term 
used somewhat loosely to designate any piece of the animal mechanism which has a distinct function to perform. The physiologist studies the results of the activity of an organ. He watches it in action, and endeavours to explain the process by which it produces its effects. Then follows the anatomist, who, taking it to pieces, examines it with the utmost thoroughness which scalpel and forceps or microscope allows, with a view to ascertaining whether its structure will support the physiologist's hypothesis as to its mode of action. This in the vast majority of cases has been the history of scientific progress. The physiologist has preceded the anatomist in drawing inferences as to the manner in which things are done. The anatomist, after a further examination of structure, has either admitted the plausibility of his explanation, or has interposed the objection that the part was incapable of working in the way supposed.

This comparison of anatomy and physiology must not be pushed too far. Enough has been said to emphasize the distinction between them. The one treats of form, the other of function. The one looks at structure, the other at action. Anatomy in its limited and logical sense has nothing to do with the uses of a part; its business is to measure it. Physiology has nothing to do with the measurements of parts; its duty is to watch for movement. Every living thing may be contemplated either in its statical or in its dynamical aspect. Physiology looks at it from the latter point of view.

Surveying his province, the physiologist asks himself: "Who are my subjects? What am I to find out about them ? What methods, in addition to direct observation, may I use to obtain this information?" His oversight embraces all living things. It is no longer reasonable to make a distinction between human and animal physiology, or between the physiology of animals and the physiology of plants. No human being can take all science for his field. If he contents himself with scratching its surface, he will assuredly raise but a meagre crop, and that mostly weeds. But he is far behind the spirit of his age if he declines to sow in his own little patch seeds of thought which have blossomed in other localities, however remote. The man whose purpose in studying physiology is to obtain a knowledge of the working of the healthy human 
body, in order that he may know how to set right the accidents, perversions, and premature decay to which human flesh is prone, would remain an empiric of the most rigid type did he not apply to the elucidation of his problems all conclusions reached from the study of other organisms which are likely to prove pertinent. There would be no science of human physiology had observation and experiment been limited to Man. There would be no science of medicine, it may be added, had not the mode of working of the human body, and the influence of drugs upon it, been inferred from the results of experiments upon animals-experiments which could never have been made upon men. Blisters, blood-letting, mercury-poisoning, would still be the physician's remedies for all human ills. "Give the watch a good shaking. It sometimes does good. If that fails, I cannot advise you what to do, as I know nothing about the working of a watch." Even though we open the living human body, as must be done for the purpose of making good such defects as are amenable to surgical treatment, and for a little while observe its wheels go round, we are unable, from fear of damaging the wheels, to introduce the mechanical tests which would tell us how and why they revolve. The man must be allowed to recover with uninjured organs. But, thanks to anæsthetics, there is no test which may not be applied to a live animal with as much propriety as to a dead one. Anæsthetics abolish the distinction, in its ethical applications, between life and death, because we are under no obligation, as in the case of the human being, to allow an animal to recover. Many experiments upon animals will be recorded in this book, and since the book is intended for the general public, who have been singularly misled regarding the nature and methods of vivisection, an opportunity is taken thus early of insisting that anæsthetics have made all things, not only possible, but legitimate. It is unnecessary to commence the description of each experiment with the statement that the animal was first placed in a condition of complete anæsthesia, or to end it with the statement that it was destroyed before it had recovered from the effects of the anæsthetic. The reader may take these facts for granted. In discussing the propriety of operating upon a living but unconscious animal, we are playing a word game as old as Plato's day. What is life? What is 
the relation of the personality to the animal machine which it occupies and operates? For a few minutes a heart removed from the body continues to beat. In a physiological sense it is alive, although the body from which it was removed is dead. Yet the personality does not reside in the heart, as many generations of philosophers believed. It is merely an accident that the body dies when the co-ordinating mechanism, the heart, ceases to pump blood through its vessels. Nor is the personality limited to the brain. Without the sense-organs which place the brain in relation with the body, and owing to the movements of the body-by which the sources of sensations of smell, sight, hearing are ascertained-with the world of which it forms a part, there would be no personality, no Ego. Is it, then, coextensive with the body which exhibits it? A soldier returning crippled from the wars does not finish out his days with his personality curtailed. We are no nearer than was Plato to a definition of life. Such a discussion soon takes us out of the realm of science. Science is limited to the sphere in which the whole is greater than the part. Take away consciousness, and personality ceases. Guarantee that consciousness shall never return. The animal is dead. When considering the propriety of vivisection we must regard life and consciousness as inseparable. There can be no question of right or wrong in regard to experiments on a dead animal, even though a sensitive mind, from association, shrinks from contemplating them. A person who dislikes the idea of dissecting a dead animal is influenced by purely subjective and personal considerations; nor is he prompted by sympathy with an unconscious animal when he recoils from the spectacle of its still moving organs. The term "vivisection" conveys too large a meaning. A negative term is needed, some word which will hold the emotion of pity in check. Pity is misplaced when devoted to the unconscious subjects of physiological experiment; and, happily for animals, as for Man, anæsthetics suspend conscious life. Only a person who has undergone a surgical operation can understand how resolutely the intellect declines to adopt as part of itself things which have not come within its own experience. The nurse's testimony, that a long interval separated the placing of the mask upon the face and the commencement of that dull half-consciousness 
which gradually reawakened into interest in one's surroundings cannot be set aside. The nurse says that during that interval knife, saw, and cautery were busy at their work. Her story is accepted, but it is not believed. All physiological operations are conducted under anæsthetics. In by far the larger number the experiment is continued until life terminates, under anæsthetics. The only ground upon which an objection to vivisection can be based is the ground that it involves the infliction of pain, and it is with regard to this that the greatest misapprehension exists in the public mind. Only in experiments which have for their object the study of the effects of the removal of a certain part, the diversion of a duct, the elimination of the control of a particular nerve, is there any possibility, under existing conditions, that an animal will suffer. In such experiments as these, observations cannot commence until after the animal has recovered. The operation is conducted under anæsthetics, and with the utmost precautions, to prevent any disturbance of the animal's general health. The injury is in almost all cases of a comparatively limited nature, and it is certain that it involves very little pain to the animal when it has recovered from its anæsthesia, since, thanks this time to aseptic surgery, there is no inflammation or other secondary trouble.

The field of physiology embraces the phenomena exhibited by all living things, whether plants or animals. The vegetable physiologist works in one part, the comparative physiologist in another. The work of the human physiologist is more limited in scope. Yet there are few problems relating to Man's mechanism concerning which the physiologist can have direct knowledge. His theories are based upon the results obtained by experimenting upon animals. 


\section{CHAPTER II}

\section{THE BASIS OF LIFE}

Protoplasm was defined by Huxley as " the physical basis of life." It is the material substance which lives. There is no life in anything which does not consist of, or is not supported upon, or permeated by a system of filaments of protoplasm. Huxley's definition indissolubly links in thought protoplasm and life. But it is doubtful whether the definition is in any sense axiomatic. The adjective " physical " has too narrow a range. If the biologist could say to the chemist, "Here is a substance which was alive. If I could restore to it the energy which it has lost, if I could impart to it the movement which I recognize as life, it would again be alive," he would offer the chemist a substance susceptible to the methods of his science, something which he could analyse. If, approaching the physicist with a group of chemical products, he could say, "Into these protoplasm broke up on dying. I cannot assure you that while it was alive they were combined into molecules within your meaning of the term. There may be no such 'substance' as protoplasm in the sense in which you understand the word, but so long as this mass lived these various familiar compounds were bound together in a supermolecular form. Death was their falling apart. If I could cause them to recombine, they would be alive," he would give the physicist a problem within the range of his methods. The physicist could devise a method for measuring these units. The science which can weigh an electron, the thousandth part of an atom, need not fear failure in its attempt to gauge the size of units of structure composed of groups of heavy molecules, albumins, globulins, and other proteins, ${ }^{*}$ with the inclu-

* Proteïn, subs., proteïd, adj., general terms for complex nitrogenous substances, such as albumin (white of egg), the less soluble globulins, fibrin of blood casein of milk, etc. 
sion, perhaps, of fats, sugars, inorganic salts. But herein lies the biologist's dilemma. He cannot assert that there exists a homogeneous substance, protoplasm. He cannot assert that there exists a definite tectonic grouping of heterogeneous substances which, so long as it is maintained, constitutes a physical basis capable, and alone capable, of exhibiting the phenomena of life. Protoplasm is still a hypothetical substance-a name. Truly, in the absence of nitrogen-containing compounds of very complicated chemical constitution there is no life. All living things yield on chemical analysis approximately the same nitrogenous substances. No one can say whether the capacity for living is dependent upon the molecular -that is to say, the chemical-constitution of the basis, or whether it is dependent upon the arrangement of its molecules, its form. It is even open to question whether instability, the capacity for incessant change, both in chemical composition and in form, be not the condition which differentiates living matter from dead. "Physical basis" is too hard a term for this elusive concept of the matter which exhibits life.

If it were possible by a process of elimination to ascertain the substances which must be present in protoplasm, the physiologist might formulate a reasonable hypothesis as to the nature of this " basis." But there is no part of any living thing, or, at any rate, no part which is not microscopic in its dimensions, which can be pointed out as protoplasm and nothing besides. It is impossible to isolate anything which can be described as pure protoplasm. Nor is it possible, by comparing various tissues which are acknowledged to be rich in protoplasm, to ascertain what chemical substances are common to them all.

If it were feasible, by analysing a number of specimens of protoplasm, to make sure that, although $x$ is absent from one, $y$ from another, and $z$ from a third, some one thing, $P$, is always present, then $P$ might be regarded as the physical basis, even though it were evident that $P$ alone was not protoplasm Protoplasm would be $P$ combined with either $x, y$, or $z$. Globulins and albumins and other proteins are always present, but in varying proportions; but it is impossible to make certain that either of these chemical substances is more important than the rest. Nor is it possible to assert of either that it is essential. 
Chemically, protoplasm is a mixture of substances, chiefly proteid, in a condition in which it is capable of manifesting the phenomena of life. But whether it be more complex and of heavier molecule than either globulin, nucleo-protein, albumin, fibrin, or any other of the nitrogenous compounds which take its place when it is dead; or whether it be as simple as eíther of these, but differ from them all in its instability, in the constant flux of its atoms, which causes it at one time to incline towards one of them, at another time to another, are questions which cannot at present be answered.

The uncertainty as to the chemical nature of protoplasm is responsible for an unfortunate irregularity in the use of the term. It is ex hypothesi the most active, the most living part of an animal cell. If the cell has a nucleus and an envelope, the protoplasm must lie in the space between the two. This part of the cell is therefore often termed, without qualification, the "cell-protoplasm." Frequently the abuse of the word is carried still further. Young cells, leucocytes, nerve-cells, etc., which have no envelope, consist of a nucleus embedded in soft cell-substance. The latter is termed its protoplasm. The cell is described as consisting of nucleus and protoplasm, the term assuming an anatomical signification. Not only is such a use of the term bad, because it indicates a confusion of thought, but it brings with it a train of ambiguities. What are the limits of the protoplasm? If the cell-body be firmer towards its exterior than it is within, is the denser substance protoplasm, or is it not? It has not the qualities which are attributed to protoplasm in so marked a degree as has the substance which it surrounds. Hence a distinction is made. The one is "ectoplasm," the other " endoplasm." Within the cell-body are many collections, often in the form of granules, of substances which have not the protoplasmic attributes. They constitute the "deuteroplasm " of certain cytologists. But these enclosed substances may be as far removed from protoplasm as starch grains. It is absurd to use the termination "plasm " for such welldefined products of cell activity as these. The subject is, unfortunately, obscured by conflicting terms. Nomenclatures which were invented with the object of giving definiteness to our ideas have served but to perplex them. The term " proto- 
plasm " should be reserved as a synonym for the substance which is most alive, the substance in which chemical change is most active, the substance which has in the highest degree a potentiality of growth. Anatomical distinctions are better expressed in anatomical terms. We shall treat of such distinctions when considering the organization of the cell.

In the meantime it may be well to consider the attributes which appear to belong to this most-living substance. Its chemical composition can be inferred only from the compounds found on analysis to be present in a mass of organized substance which there is reason for thinking was rich in protoplasm while it was alive. The compounds found vary within certain limits. The quantity of water associated with these compounds is still more variable. Water is essential to the existence of protoplasm. Its power of combining with water in variable quantities is one of its characteristics. Tissue rich in protoplasm yields on an average about 75 per cent. of water. Part of the protoplasm within a cell holds more water associated with it, part less.

Closely associated with its power of holding water is its tendency to assume an architectural form. In large vegetable cells, such as those of the hairs within the flowers of Tradescantia, the protoplasm may be seen, under the microscope, arranged in threads containing granules which are incessantly streaming up and down them. The spaces between the threads are filled with water. Such mobile protoplasm cannot be said to have a structural form. But in the greater number of cells, and especially in animal cells, the protoplasm is disposed in a network, with usually a tendency for the strands of the network to set in lines. In attempting to define these very variable networks, the microscopist is obliged to speak with caution. He finds it very difficult to distinguish between appearances which he is justified in regarding as inherent in the cell-substance, whether alive or dead, and appearances which he may have induced by the action of reagents whilst preparing the tissue for examination. Rarely can he assert that he sees a network in a living cell. When examining a dead cell, he is bound to recognize that the preservatives and hardening reagents which he used may have caused the proteins to coagulate in a particular pattern. If 
he obtains the same pattern with several different methods, he infers that the appearance which he sees is that of a structure existing in the living cell ; but he is never quite sure that it is not an arrangement produced by reagents after death.

The tendency of protoplasm to dispose itself in the form of a network or spongework is of the greatest interest in its bearing upon the theory of its activity in effecting chemical change. The body itself, as we shall find later, is a network of tissues enclosing lymph. The lymph in the tissue-spaces contains foods and waste products in solution. The tissues are constantly taking from it the former, and discharging into it the latter. Every cell is, microscopically, a tissue. The strands of its protoplasm are perpetually sorting foods from its cell-juice, adding to its cell-juice waste products. By diffusion, foods, including oxygen, pass from lymph to cell ; waste products, including carbonic acid, pass from cell to lymph. If water be added to gum, the gum swells. The mixture is homogeneous. Diffusion takes place slowly through the mucilage. When water is taken up by protoplasm, the protoplasm swells; but the mixture is not homogeneous. The protoplasm expands as a wet sponge expands, although the relation of the enclosing reticulum to the water which it encloses is far more complicated. It is, as it were, a sponge made of gum. Some water is combined with the protoplasm ; the remainder fills its spaces. There is an active surface relation between the free water and the protoplasmic threads. As water rises in a capillary tube, as it passes from the inside to the outside of a flannel shirt, so it circulates within the cell.

Irritability is a property commonly attributed to protoplasm, but it is a little doubtful whether there be not again some danger of an illogical use of terms. An amœba, one of the unicellular organisms found in ponds, has the power of moving. If a piece of a water-plant-the stalk of duckweed is a suitable object-be examined with the microscope, these little animals are usually to be found upon its surface. They feed upon algæ more minute than themselves. When they come in contact with something suitable for food, their bodysubstance flows around it. The food is coagulated. So much of it as is digestible is digested; the remainder is extruded. Constantly parts of the body-substance are protruded, other 
parts retracted, in the search for food. Such movement is a response to stimulus. Stimuli received at one part of the body-substance are transmitted to another. The bodysubstance is irritable. It acknowledges stimuli ; it conducts them. But if the amœbæ are watched until, owing to lack of oxygen or other cause, they die, their irritability comes to an end. It is a phenomenon of life. Again the physiologist is in a dilemma. Either protoplasm is not protoplasm when death has supervened, or protoplasm is not irritable as such. It is somewhat paradoxical to ascribe to the physical basis of life a property which depends upon its being alive.

Yet the influence on protoplasm of anæsthetics makes it difficult to understand how it can be either physically or chemically a substance which loses its form or changes its constitution whenever it ceases to display the usual evidences of its existence. Chloroform and similar agents suspend irritability. Yet irritability returns as their influence passes off. They appear to hold it in check without - at any rate visiblychanging the nature of the irritable substance.

All parts of the minute body-substance of an amoba are equally irritable. In higher animals irritability is concentrated in the nervous system. The form of irritability to which consciousness is adjunct is restricted to the cortex of the great brain.

Chloroform and similar agents are termed "anæsthetics" because they abolish the irritability of the cortex of the great brain, before their effects upon other parts of the nervous system are sufficiently pronounced to endanger the working of the animal machine. Pain ceases to be felt before the dose of anæsthetic is sufficient to suspend the irritability of the centres of reflex action. All protoplasm, whether animal or vegetable, is susceptible to the influence of these agents. They cause it to enter into a state which resembles death in all respects save the impossibility of revival. There is a great demand in the Paris flower-market for white lilac in the winter. The plant cannot be forced until after a period of rest. By withholding water and placing the bushes in a cool, shady place, horticulturists endeavour to send them prematurely into their winter sleep. Recently it has been found that from three to four weeks can be gained by placing the 
bushes for a couple of days in an atmosphere charged with the vapour of ether. Some change of state is evidently produced in protoplasm by anæsthetics. It ceases to be capable of rereceiving or transmitting stimuli. But we cannot picture the change as being sufficiently pronounced to justify the hypothesis that so long as it is irritable protoplasm is a complex substance which is resolved, as it loses its irritability, into simpler compounds familiar to the chemist. Perhaps it would be more correct to say, we cannot picture these chemical substances as reuniting into protoplasm when the effect of the anæsthetic passes off. Rather are we driven to think of living matter as a mixture of many substances in a state of molecular interchange, and to suppose that the activity of this interchange is diminished by anæsthetics.

Chemical activity is a property of protoplasm. In its network combinations and decompositions are effected more extensive in range than any which a chemist can cause to occur in his laboratory. From ammonia, carbonic acid, and water, a plant makes albumin. A chemist cannot make albumin, no matter how complex may be the nitrogenous substances which he endeavours to cause to combine. Albumin is resolved by animals into water, carbonic acid, and urea. Cells of the gastric glands set a problem which puzzles the chemist by making hydrochloric acid from sodic chloride without the intervention of a "stronger" acid. Many other illustrations of the same kind might be cited. Although the tissues of animals act chiefly as destroying agents, their protoplasm is not without constructive power. There is apparently no limit to the capacity for synthesis of plants. The chemistry of living things may be divided into two provinces, absolutely antagonistic in the series of reactions which they comprise. The one series is constructive, synthetic; the other destructive, analytical. Construction involves the locking up of energy. It is endothermal. Destruction results in the setting free of energy. It is exothermal. To accomplish synthesis energy must be added. Plants obtain it from the sun's rays. Animals disperse energy, set free by the analysis of substances formed in plants, in maintaining their bodies' warmth and movement.

The chemistry of the laboratory and the chemistry of proto- 
plasm present certain contrasting features. A chemist reaches the compound which he wishes to form by effecting a series of interchanges. For example, he wishes to form uric acid by uniting a nucleus contained in lactic acid with urea. First he introduces chlorine and ammonia into the molecule of lactic acid. He makes trichlorlactamide. Then he heats (supplies energy to) a mixture of trichlorlactamide and urea. Two of the chlorine atoms carry off hydrogen atoms from the urea. A third leaves the trichlorlactamide with its ammonia. Water also breaks away. Uric acid remains.

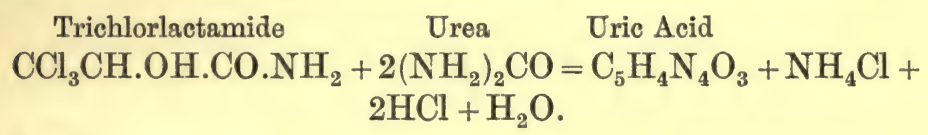

In this example the trichlorlactamide may be said to exchange its chlorine and ammonia for urea. When he planned the reaction, the chemist foresaw what would happen. $\mathrm{He}$ knew that if he weakened the grip of the lact radicle upon them, chlorine and hydrogen, chlorine and ammonia, oxygen and hydrogen, would take the opportunity of getting away together. The lact radicle and urea would be left with dangling arms, which must "satisfy their affinities" by linking up. It would be rash to assert that any reaction is impossible to Nature's chemistry ; but it may safely be said that the reactions which protoplasm effects are, so far as we know them, of a different type from this laboratory example. Uric acid is the chief excrement of birds. It is made in the liver. If the liver is shut off from the circulation, lactate of ammonia is excreted in the place of uric acid. It is therefore, in all probability, lactate of ammonia which the liver transforms into uric acid. We cannot pretend to say how this is done, although an empirical formula for the change might be drafted easily enough.

Lactate of ammonia has the formula $\mathrm{NH}_{4}, \mathrm{C}_{3} \mathrm{H}_{5} \mathrm{O}_{3}$. Uric acid, $\mathrm{C}_{5} \mathrm{H}_{4} \mathrm{~N}_{4} \mathrm{O}_{3}$, contains a much higher percentage of nitrogen. It could be produced from lactate of ammonia by the condensation of the nitrogen-containing nucleus and the addition of a sufficient amount of oxygen to complete the oxidation of the superfluous carbon and hydrogen into carbonic acid and water. It is of little interest to count the number of atoms 
concerned in this process. If a bird be fed upon urea, or even upon various salts of ammonia, its liver will change them into uric acid. Lactate of ammonia is the nitrogen-containing compound with which the liver has normally to deal. It can handle almost any other combination of nitrogen with equal ease. In the protoplasm of the liver the atoms in the molecule of lactate of ammonia are rearranged. The molecules are condensed ; water is set free; oxidation occurs. It seems almost as if molecules, when in contact with protoplasm, lose their individuality. Their atoms fall into new groups. Chains which the chemist finds so difficult to break-chains from which he can remove a link only by insinuating another and a stronger-are, when in contact with protoplasm, groups of isolated links. The links rearrange themselves. They join into new circlets, larger, smaller, more open, closer. As grains of sand on a metal plate group themselves in harmony with the vibrations caused in the plate by drawing a violin bow across it, so the atoms answer to the forces which set protoplasm vibrating. There is no waste of force. The chemist may need to enclose sawdust and lime in a crucible heated in an electric furnace if he wishes to compel them to combine as carbide. He supplies energy enormously in excess of the amount which the new compound will lock up. Under the influence of protoplasm the reactions which occur are exactly proportional to the amount of energy supplied. Or, if it be a reaction by means of which energy is set free, it occurs spontaneously. No energy is absorbed in setting it going. All the energy liberated is effective. The chemist very frequently needs to heat a substance in order to cause it to decompose, even though it be falling from a less stable to a more stable state.

Vital chemistry and mineral chemistry are so widely different in their methods that one is tempted to think of them as different in kind. We find it very difficult to look at both from the same point of view. Men's minds are preoccupied with the things that they have to do for themselves. The chemistry of the laboratory is seen as a science circumscribed by the laboratory walls. If it were possible to stand outside, it would be evident that it is only a part of the science of molecular change. Matter changes its state under the influence of force. Many 
rearrangements are effected by the chemist which do not occur in nature. He has an almost infinite range of action. Yet many of the rearrangements of matter and force which are occurring in the dandelion on his window-sill (if the fumes of sulphuretted hydrogen have not killed it) he is unable to reproduce. It is largely a question of waste. Nature works with greater precision than the chemist ; but the chemist could do all that Nature does if he had but the same control of force.

We have spoken of the reactions which occur in protoplasm as divisible into two great series-the one ascending, constructive, endothermal; the other descending, destructive, exothermal. In the one series energy is locked up ; in the other series it is set free. Synthesis and analysis are names applied to the two series respectively. Synthesis is characteristic of plants, although analysis is also perpetually occurring. Plants fix carbon from the air and liberate oxygen. They also respire, setting free carbonic acid. Analysis is characteristic of animals, although synthesis is not excluded.

Of the chemical processes which occur in plants very little is known. Few halting-places between raw materials and finished products can be marked. The final products are sugars and starches, oils, proteins, and a vast number of other substances-alkaloids, glucosides, etc. Condensation, dehydration, and deoxidation are the methods by which the synthesis of these compounds is accomplished. These methods are adopted simultaneously in varying degree. The large group of bodies known as sugars and starches are, with few exceptions, built on the $\mathrm{C}_{6} \mathrm{H}_{6}$ model; in fruit sugar, $\mathrm{C}_{6} \mathrm{H}_{12} \mathrm{O}_{6}$, six atoms of carbon are linked to one another and to six molecules of water. The formula of starch is $\left(\mathrm{C}_{6} \mathrm{H}_{10} \mathrm{O}_{5}\right)_{\mathrm{n}}$. Not only has water been removed from the molecule, but an unknown number of molecules have been linked together. This condensation and dehydration is effected whenever sugar carried in cell-sap is deposited as starch in seeds or tubers. These compounds are hexatomic. The chemist pictures them as made by the union in the first place of six atoms. As small drops unite to form larger ones, so small molecules, under the direction of the protoplasm of plants, close together.

The reactions which characterize animal protoplasm are of 
a different kind. They belong to the descending series. Close molecules are unfolded. Water is incorporated with them. Hydrogen and carbon are oxidized into water and carbonic acid. The conversion into sugar of glycogen or of starch may be taken as an illustration of expansion. Starch, $\left(\mathrm{C}_{6} \mathrm{H}_{10} \mathrm{O}_{5}\right)_{n}$, becomes maltose, $\mathrm{C}_{12} \mathrm{H}_{22} \mathrm{O}_{11}$, and then dextrose, $\mathrm{C}_{6} \mathrm{H}_{12} \mathrm{O}_{6}$. The grouped molecule of starch opens out. The breaking of the double molecule of maltose into two molecules of dextrose is a further illustration of progress towards simplicity. Hydration, union with $\mathrm{H}_{2} \mathrm{O}$, accompanies this expansion. Hydrolysis is the secret of almost all digestive acts. Starch is hydrolysed into sugar, fat hydrolysed into glycerin and fatty acid, proteins hydrolysed into peptones.

All the chemical transformations which protoplasm is able to accomplish are of the nature of fermentations. The term fermentation was first applied to the effervescence which occurs in grape-juice when its sugar is being converted into alcohol, carbonic acid gas, and certain substances which appear in relatively small quantities. It was discovered later that the yeast which effects this change is a unicellular plant. The term "fermentation" was extended to the production of vinegar from alcohol, and eventually to all such reactions as are carried out by living organisms, or by the secretions or products of living organisms, without the destruction of the agent which is effective in the process. A ferment is an organic body which brings about changes in other bodies without itself undergoing change. At the end of the process, however prolonged, there is as much ferment as there was at the beginning, and its chemical nature is the same. Rennin has been made to curdle nearly a million times its weight of milk, pepsin to digest half a million times its weight of fibrin. As the ferment is not consumed, there is no relation, except one of speed, between the ferment and the quantity of fermentable substance which it is able to transform. We said that a ferment is an organic body. It is necessary to introduce the qualification "organic," because certain reactions termed "catalyses" which occur in mineral chemistry resemble fermentations in respect of the non-destruction of the agent which serves as intermediary. If a solution of cane-sugar containing a very small quantity of sulphuric acid is boiled, the cane-sugar 
is "inverted." It is changed into a mixture of fruit-sugar and levulose. The ferment invertin of the gastric juice and of intestinal juice produces a similar effect; and just as invertin remains unchanged, so also the sulphuric acid is found in the mixture unchanged in nature and in amount after an unlimited inversion of cane-sugar. Great stress was formerly laid upon the similarity between fermentation and catalysis. It has now been shown that catalytic actions are not necessarily of the same nature as fermentation, although the results and, as far as is visible, the means are similar. For example, finely divided platinum (or, better, palladium) causes an indefinite quantity of oxygen and hydrogen to unite. The reaction comes within the category of catalyses. But it is widely different from a fermentation. The metal causes hydrogen to condense, and actually absorbs it into its surface layer. In the liquid form hydrogen cannot resist combination with oxygen. This may be termed a "physical phenomenon," adopting the common distinction between chemistry and physics. There is no reason for thinking that fermentations can be explained in so simple a way. They may, however, be grouped under the designation "catalyses." As the initial conditions and final results are similar, it is inevitable that fermentations and catalyses should obey the same "laws" as to mass action, speed, effect of accumulation of products of action, and the like ; but it does not follow that invertin and sulphuric acid produce their effects in the same way. Fermentations are instances of catalysis, but all catalytic actions are not fermentations.

So far from dwelling upon the resemblance between fermentation and the catalysis of mineral chemistry, chemists nowadays incline to regard fermentation as essentially a reaction of life. It is very difficult, when attempting to present ideas which are new to thought, to adapt, without ambiguity, existing words. It would be absurd to talk of a substance removed from yeast or bacteria or blood-corpuscles by a process which involves cooling with liquid air, grinding with powdered glass, solution in water, precipitation with absolute alcohol, and re-solution in water, as alive. Yet, unlike any known mineral product, it is easily killed. Ferments are not destroyed by cold, but their activity is arrested. They are most active at about the body temperature. Their activity is annihilated 
by heating them, in solution, to the temperature at which albumin coagulates-a little over $50^{\circ} \mathrm{C}$. Although they are not alive, their behaviour very closely resembles that of living matter. They can be obtained only from living things. They produce their effects even though they are present in almost infinitely small quantity. It is impracticable to make a chemical analysis of a ferment, owing, in the first place, to the very small amount available for analysis, and, in the second place, because of the impossibility, with existing methods, of obtaining a ferment pure. The amount of ferment present in even a great mass of yeast, or in many pounds of salivary gland or pancreas, is extremely small. However prepared, it is always accompanied with proteid substances. It is impossible to say whether ferments, like proteins, have heavy nitrogen-containing molecules. The fact that they are not diffusible suggests that they have.

It would be straining language to term fermentation a phenomenon of life ; worse, to define life as a sequence of fermentations. Yet it is safe to say that all the chemical changes carried out by living organisms are fermentations. Fermentation and the chemistry of life are almost synonymous terms.

A very large number of ferments are already known. Each has its own specific work to do: "To every fermentable substance is fitted a ferment, as a key to a lock." It will be understood, from what has been already said regarding our inability to determine the composition of any ferment, that we cannot say whether or not these various ferments differ one from another in chemical constitution. They are classified according to their action, and not according to their nature. Those which

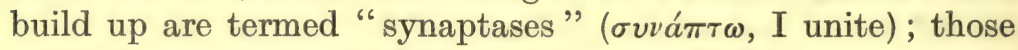
which decompose, or hydrolyse, "diastases" ( $\iota_{\imath a} \sigma \tau a \sigma \iota s$, separation). The termination "ase" is added to the name of the substance upon which the ferment acts, except in cases in which other terms have already become so general as not to be displaceable: amylase, hydrolysing starch; sucrase, inverting canesugar; protease, hydrolysing proteins. Unfortunately, there is little uniformity in this nomenclature ; amylopsin, invertin, pepsin, are terms used as often as those terminating in "ase." As a distinguishing termination, "in " or " $\sin$ " is less desirable than "ase," owing to the fact that it has been appropriated 
already as the termination of the names of albuminoids-e.g., gelatin, chondrin, mucin.

The various ferments are substances which protoplasm sets aside for specific purposes. Primitively, contact with the substance to be fermented determined the nature of the ferment assigned to the task. There are reasons for thinking that protoplasm still retains its power of making a suitable response; cases may be cited in which the lock presented to protoplasm shapes the wards of the key. In such cases the fermentable substance provokes the formation of the ferment. But, for the most part, in situations where particular ferments are regularly needed, protoplasm has acquired the habit of making such ferments and no others. The cells of salivary glands accumulate ptyalin, the cells of gastric glands accumulate pepsin, during the intervals between meals.

The capacity of protoplasm for producing a new ferment when it is needed is shown by such examples as the following: Blood-plasm contains a variety of proteid substances. If a solution of white of egg be added to it, the mixture is clear and uniform. Yet egg-albumin is treated by the blood as a foreign body, a poison. When injected into the veins of a living animal, some of it is excreted by the kidneys, some destroyed in the blood-stream. If several successive doses of egg-albumin are injected into an animal (it is most convenient to inject it into the peritoneal cavity), the power of the blood to destroy the intruder is greatly increased. If now a specimen of blood be taken, and the plasma or serum mixed with egg-albumin, the mixture is no longer clear. The eggalbumin is precipitated. The blood of the animal thus "prepared " has developed a ferment, termed a "precipitin," which throws down egg-albumin. If instead of egg-albumin, which, although a foreign body, is comparatively innocent, a substance which is distinctly poisonous, toxic, be injected into an animal, the first dose, if a large one, will prove fatal. If, however, the first dose be small, and succeeding doses progressively larger, the animal acquires the power of tolerating a quantity of the poison much larger than would have proved fatal in the first instance. A classical example of this, because it afforded an opportunity of directly observing under the microscope the difference between "unprepared" blood and blood from an 
immune animal, is the acquisition by a mammal of the power of tolerating the injection of the blood of an eel. Eel's blood contains a toxin which destroys the red blood-corpuscles of a mammal. The dissolution of the blood-corpuscles may be watched with the microscope. If successively increasing doses of serum of eel's blood be injected into the body of a rabbit, the rabbit acquires the power of resisting the toxin. Further than this, the serum of the immune rabbit injected into a rabbit which has not been prepared confers immunity upon the latter. If the blood of the prepared animal be mixed with the blood of an unprepared rabbit and with eel's serum, and the mixture examined under the microscope, it will be seen that red blood-corpuscles are no longer dissolved. The immune serum is able to save the blood-corpuscles of the unprepared blood from destruction. During its course of preparation the rabbit developed an antitoxin.

If germs of diphtheria are injected into the blood of a horse, the first injections give rise to marked febrile symptoms. After a number of injections the horse becomes completely tolerant of the virus. Not only does its blood develop sufficient antitoxin to protect it against the toxin of diphtheria, however large may be the quantity injected into its system, but the serum of the prepared horse, when injected beneath the skin of a child suffering from diphtheria, carries with it sufficient antitoxin to destroy the toxin which has gained admission to the child's blood.

Many more instances might be cited of this capacity of developing "antibodies" of protoplasm. The leucocytes of the blood are incessantly adapting their chemistry to the needs of the economy. All the tissues, it may be supposed, possess the power of developing resistant ferments; but the leucocytes (Fig. 4) are the undifferentiated cells, the maids-of-all-work. 'They have not specialized as makers of ptyalin or makers of pepsin. They are not completely given up to lifting weights, like muscles, or carrying messages, like nerves.

Bacteria are the world's scavengers. To them ultimately belongs the task of reducing organic matter to the salts which plants reorganize. The cycle of life would be broken if bacteria were suppressed. No sooner has an animal fallen than these little agents commence their beneficent task of resolving 
its carcass into air and soil. Birds and insects may interrupt their work. They may steal portions of the derelict, use them for fuel, or patch them between their own ribs. But they, too, will soon lie breathless on the ground; and the bacteria are always ready to finish their interrupted task. Why should they wait until the slight change occurs, important to us, but of little consequence to them, which marks the transition of living protoplasm into dead proteins? There is nothing in the constitution of protoplasm which makes it harder to break up than protein. There is no quality inherent in living matter which makes it resistant of decay. We resent the officiousness which prompts bacteria to obtain entrance into the ship while it is still under full sail, with a view to commencing the work of demolition. Deep in our minds lies the conviction that it is contrary to the rules of Nature. We are especially annoyed at the many ruses bacteria adopt to disguise their personalities. The bacteria of the soil we can keep at a proper distance. But bacteria of the stream, bacteria of milk, bacteria of the breath that would betray us with a kiss! It is hard to recognize that they are fairly and squarely playing their part. Birds and insects we can beat off with our hands. Our invisible enemies are everywhere. They are constantly insinuating themselves through scratches in the skin, through abrasions in the mouth, through surfaces of the intestine left unprotected owing to the desquamation of its epithelium. But if we are constantly open to attack, we are policed by myriads of zealous leucocytes, ever ready to reduce the invaders to impotence. The germs which have found entrance fire off a toxin. The leucocytes reply with an antitoxin. There is absolutely no limit to the power of protoplasm to protect itself, if only it be not taken by surprise. It can resist any organic poison if it is allowed a sufficient time to produce the antipoison. The ferment of pancreatic juice, trypsin, is a poison which is unlikely to find its way into the blood. When injected it produces disastrous results owing to its immense activity in digesting proteins. An animal "prepared" by the injection of successive doses of trypsin develops an antitrypsin. Injection of pancreatic juice no longer does it any harm. Tapeworms which live in the intestines are bathed in pancreatic juice; they are constantly exposed to its digestive action. They are not digested, 
because they secrete an antibody which prevents the development of the activity of trypsin. It is not in this case, strictly speaking, antitrypsin. It is antikinase, a substance which, if extracted from the bodies of tapeworms and added to pancreatic juice, renders it incapable of digesting albumin. The antikinase does not destroy trypsin, but destroys kinase, the co-operation of which is essential to its activity.

Not only has protoplasm the power of meeting with an antiferment any ferment which might prove prejudicial to its own integrity ; but after it has been once attacked it continues to defend the vulnerable spot. Its tactics are, it must be confessed, somewhat like those of the dusky warrior who, during his first lessons in the art of boxing, made a point of covering with his fist the place where he had just been hit; but even its power of remembering its last injury is of supreme value to the human race. Before the age of sanitary science, and even, in certain backward communities, in these days of its beneficent rule, conditions producing disease were not necessarily set right as soon as the epidemic was over. The close-packed inhabitants of a ghetto were continuously exposed to germs of typhoid fever, small-pox, whooping-cough. But after their protoplasm had once responded to the need for the production of an antigerm, it either continued for many years to keep a stock in hand, or it kept the recipe within easy reach. The memory of protoplasm is amazing. It is commonly said that vaccination is an absolute protection for seven years. There is no doubt but that the immunity from small-pox which it induces, if gradually lessening, lasts for life. The disease, if it attacks a person who has been vaccinated in infancy, is relatively harmless.

Inoculation, vaccination, is the boxing-master's method of utilizing the self-protective instinct of the dusky warrior. Knowing that his pupil will for a long while continue to cover an injured spot, he asks himself : "Where is he most likely, when it comes to a serious contest, to be hit ?" Then he gives him a gentle tap in that particular place. Does he need to know how to defend himself against small-pox? Give him cow-pox. Is he likely to receive a knock-down blow from typhoid fever? Just show him what it feels like to have a gentle shake. Educate his protoplasm to make antityphoid 
ferment, by giving him the typhoid germ in such an attenuated form that it cannot do him any harm.

The chemistry of protoplasm is a science which is growing rapidly, or, to speak less arrogantly and more correctly, our knowledge of the ways of protoplasm, the Chemist, has greatly increased during the last few years. We can but watch protoplasm at work. Our experiments, so called, are but windows which we open in the walls of his laboratory. We cannot take the work out of his hands. The methods of mineral chemistry are useless in this search for knowledge. And, naturally, the longer we watch, the more details do we discover in what seemed at first a generalized procedure. We recognize that several manipulations are required in the carrying out of a reaction which hitherto we believed to take place in a single stage. This is not the place in which to give an account of a subject regarded as belonging, owing to its applications, to the province of pathology. But Nature is one, however many be the companies into which we divide the explorers of her secrets. We have attempted the merest outline of the observations made up to the present, and have submitted the results for the sake of the light which they throw upon the way in which ferments are prepared as they are wanted to meet the needs of normal everyday digestion and metabolism, rather than for the purpose of showing the methods by which protoplasm combats disease.

Amongst the chemical phenomena of life is respiration. Respiration in this very general sense means oxidation. The force which is exhibited in living is obtained from the union of organic materials with oxygen under the direction of protoplasm. This is true of plants as well as of animals. It is true even of the subdivision of bacteria, termed anaerobic, because they cannot live in air. They secrete ferments which enable them to decompose compounds which contain oxygen, in order that they may use the oxygen for respiration. It might have been supposed that green plants which are receiving radiant energy from the sun would convert this energy into the forces which enable protoplasm to display the phenomena of life. But this is not so. The energy which green plants obtain from the sun is used in constructive metabolism, and not in maintaining life. Life-force, if we may use the expression, is derived from the oxidation 
of the substances which the sun's rays enable the plant to make. A plant, equally with an animal, respires. The distinction between the constructive metabolism of a plant and its respiration may be brought out in a striking way by administering to it sufficient anæsthetic to stop the former without stopping the latter. It may be paralyzed without being killed. If a water-weed-potamogeton is the most convenient-enclosed in a bell-glass filled with water and inverted over a dish of water, is placed in sunshine, bubbles of gas rise from the plant. They accumulate at the top of the bell-glass. If the gas be removed and analysed, it is found to be oxygen with a small admixture of carbonic acid. If a second bell-glass containing water-weed be exposed under the same conditions in all respects, save that a small quantity of chloroform is added to the water, the gas that collects at the top of the bell-jar will be much less in amount. It will be found to be carbonic acid without admixture of oxygen. The power which chlorophyll possesses of decomposing carbonic acid with fixation of carbon and liberation of oxgyen is suspended by the anæsthetic ; whereas respiration is not interfered with.

Lastly, we must attribute to protoplasm a capacity of growing. The activity of protoplasm depends upon constant molecular interchange. It incorporates molecules of food. It excorporates molecules of waste. If food is abundant and "vitality" exuberant, it takes in more than it gives out. It grows.

If we attempt to formulate a definition of protoplasm, we find that our ideas are far from clear, owing to want of knowledge. The questions, What is protoplasm? What is life? are equally unanswerable. Their definition is reciprocal. Protoplasm is the substance, the material, which exhibits life. Life is the complex of phenomena exhibited by protoplasm. Al! parts of the body are alive, in their degree. The nucleus of a cell lives, as well as its cell-body. Its capsule may be less alive-that is to say, less vibrant-than the soft cell-substance which it encloses; but it lives. So-called intercellular substance, or matrix, is alive. In growing cartilage the matrix does not behave as a dead substance. It does not crack and gape under the pressure of the dividing and multiplying cell- 
bodies which it contains. If the windows of a house were endowed with the power of spontaneously enlarging, the walls would be crushed. They would bulge, break, tumble. The matrix of cartilage offers as little resistance to the enlargement of the cells which it encloses as the plasma of blood to the multiplication of blood-corpuscles. It grows with the cellbodies, and must be considered as divisible into areas, each of which is the periphery of a cell. Muscle is alive. So, too, are bone, teeth, hair, nails. But as we proceed outwards we find the quality of aliveness growing less and less apparent, until at last we acknowledge that it is unrecognizable. Vibrations diminish in amplitude and in rapidity, until the material of which the body is made appears to be at rest.

Biologists apply the term "protoplasm " to the most living substance of which plants and animals are composed. It may be that there is an entity, protoplasm. It may be that in certain situations this exists in an unmixed state. It may be that the degree of aliveness of a tissue or constituent part of a tissue varies as the quantity of protoplasm which it contains. The tendency of protoplasm to dispose itself in a reticulum in the meshes of which other substances accumulate favours such a view. The cells of the deeper layers of the skin are rich in it. The superficial layers are composed chiefly of keratin. It is possible that the network opens out, and its strands grow thinner and thinner, as keratin accumulates. But it cannot be demonstrated that this is the case. There is no completely satisfactory reason for concluding that the life of a cell of the skin resides in its protoplasmic network, while its keratin is inert.

Many attempts have been made to prove that living cells contain something which dead cells do not contain; but no evidence which will bear sifting has, as yet, been adduced in support of this thesis. 


\section{CHAPTER III}

\section{THE UNIT OF STRUCTURE}

Immediately after its discovery in the seventeenth century, the compound microscope was applied to the study of minute plants and animals, their organs and tissues. In this connection and for this purpose the microscope has steadily improved, until perfection has almost been attained. Calculations based upon the physical properties of refracting media show that the limits of the assistance which it can give to the eye have been very nearly reached. One of the first results of the application of the microscope to the study of parts of plants was the discovery of their cellular structure. Robert Brown, looking at slices of cork, saw that its tissue is divided into compartments. It is difficult to ascertain who it was that first used the word "cell." The resemblance of a slice of vegetable tissue or the surface view of a petal of a flower to honeycomb is so striking that the same comparison probably occurred to the mind of everyone who saw it. Further study with better instruments showed that the cells are not empty. Each cell contains cell-juice, or cell-substance, and in the centre of the cell-substance a miniature cell, the nucleus. Naturalists therefore extended the connotation of the term. A cell was no longer a space with enclosing walls; it had contents. A nucleus was invariably a constituent of the cell. The cell was regarded as an anatomical unit, consisting of a wall, cell-contents, and nucleus. In 1839 Theodor Schwann, using his microscope in the study of animal tissues, recognized the similarity between animals and plants. Animals also, he discovered, are aggregations of cells. He enunciated the Cell Theory. Philosophers are always ready to generalize. It is their business. Seeing that vast numbers of organisms are single cells, that they feed, breathe, divide, and reproduce 
their kind-in fact, carry out all the functions of life-as isolated cells, they conceived the idea that a visible plant or animal is a community of cells, each an organism in itself. As bees are units of a swarm, as men and women are units of a state, cells are units which for the sake of mutual protection remain associated in a multicellular body. The physiological or sociological aspects of this conception we shall consider shortly; but the anatomical basis of the cell theory was laid without a sufficient testing of the facts upon which it rests ; or, rather, one ought to say that, although the axiom, enunciated by Virchow when he applied the cell theory to tumours and other morbid growths, Omnis cellula a cellulâ, holds good, the applications of the theory which certain of its later exponents have made are not necessary sequents.

Every plant, every animal, commences its existence as a single cell. An organism which is permanently unicellular divides. Each of the separate cells into which it divides is a new individual. Higher plants set aside certain cells as ovules, which in due course, after conjugation with pollen grains, grow into plants. In the same way the ova of animals, by repeated cell division, reproduce the species. The individual commences as a single cell. Its complicated body, composed of various organs and various tissues, is formed by the multiplication of cells. Each of the innumerable cells of which it is composed has the structure, and may therefore be presumed capable of performing all the various functions; of a unicellular organism. But it does not follow that the cells retain their individuality. Even unicellular plants (e.g., the extraordinary vinegar and tan fungi, myxomycetes) may for a time merge their individuality in a common mass formed by the aggregation of many cells.

The cells of higher plants are not always, or even generally, anatomically distinct. Their protoplasm, the essential part of every cell, is united with the protoplasm of neighbouring cells by threads which traverse the cell-walls. The cells of the connective tissues of animals are united into a web, or syncytium. This is especially noticeable during early stages of growth. Nerve-cells are connected together by conducting filaments (neurofibrillæ). It is possible that nerve-cells and the muscle-fibres which they innervate are from the beginning 
united by nerve filaments-that the nerve-cell and muscle-cell grow apart without severing this thread-like connection. Certain anatomists regard the nerve strand which connects a cell in the central nervous system with a number of musclefibres, placed, it may be, at a great distance from the nervecell, as the bridge which has never been broken in the process of cell division and displacement, which made one primitive cell into a nerve-cell and a group of muscle-cells. Muscle-fibres are not separate cells, but cell complexes. Each muscle-fibre contains scores, in some cases hundreds, of nuclei (Fig. 16). It is a cylinder, perhaps 2 inches long, in which cell division is incomplete. Tendons are bundles of exceedingly slender fibres which lie side by side, like silk threads in a skein. The row of cells which gives rise to a tendon undergoes incomplete cell division. Their nuclei divide, and a small quantity of soft body-substance is set apart for each nucleus. The rest of the mass consists of fused cells. It constitutes a continuous rod, which becomes fibrillated as it grows. Vegetable cells are separated by cell-walls. Animal cells tend to develop intermediate partitions; but the partitions are so thick that they can no longer be described as walls. In cartilage the cellbodies are embedded in a great mass of intercellular substance, or matrix. In this intercellular substance elaborate developments may take place. Elastic fibres may make their appearance in it to form elastic cartilage, as in the case of the epiglottis. In these various instances, although it is perfectly true that tissues are formed by cell division, the cells are not, strictly speaking, separate units. They are not completely divided one from another. It is impossible to recognize their anatomical boundaries.

But there is a much more serious difficulty in applying the cell theory-the difficulty of deciding what are the essential parts of a cell. Long ago it was recognized that many animal cells-white blood-corpuscles, for example-have no cell-wall. It was therefore decided that cell-body and nucleus are the only essential parts. But what is to be said of the red bloodcorpuscles of mammals? (Fig. 4). Are they cells? They have neither cell-walls nor nucleus; nor does their substance present the structure which is usually associated with the "bodysubstance" of cells. They are not produced, if the view held 
by many histologists be sound, by cell division, in the ordinary sense of the term, but appear as spots, gradually growing into discs inside the body of a blood-forming cell. The discs are extruded when they reach their full dimensions. Yet the tissue, blood, is composed of these blood-discs and the intermediate substance blood-plasm. Mammalian blood might be dismissed as a non-cellular fluid secretion containing formed elements, if it were not for its history. In all animals below mammals the red corpuscles are cells with nuclei and cellbodies. The absence of nuclei in mammals is due to the recognition by Nature of the fact that, as the blood-cells will never be called upon to divide, it is a waste of material to provide each of them with a nucleus. Not only would the nucleus be useless, but it would take up space, diminishing the capacity of the corpuscle for carrying hæmoglobin. The process of cell division is in consequence curtailed. There are, it is true, other ways of looking at this problem. The cells which line the bloodvessels stand in some sort of nutritive relation with the blood. When the lining cells of the bloodvessels are injured or inflamed, the blood clots. But here again it is somewhat straining a point to say that these lining cells are the cells of the blood, and the blood a kind of intercellular substance; especially as a distinction would have to be made between mammals with non-nucleated blood-corpuscles and birds with complete blood-cells.

The physiologist, if he is to feel sure of his ground, needs to know the minute anatomy as well as the naked-eye anatomy of the body. But what is there that he does not need to know? He must be chemist, physicist, biologist, pathologist, and expert in various other branches of science. Microscopic anatomy, or histology, as it is commonly termed, will be called upon in this book only when it has evidence to give which bears directly on physiological problems. We have dwelt at some length upon the cell theory because the physiologist needs starting-points. He needs to have in his mind a conception of the fundamental structure of the body. Protoplasm is the material which lives. We begin with protoplasm albeit our conception of protoplasm is so difficult to formulate that we are obliged to admit that in using the term we are almost guilty of playing with words. Protoplasm 
is the most living substance. The substance which is most alive always presents itself to us as an imperfectly transparent, viscous material, which proves on analysis to contain a large quantity of certain proteins mixed with various organic and inorganic compounds. Protoplasm is organized into, or distributed amongst, cells, which in any given tissue present a fairly uniform size. What determines the size of cells? Speaking generally, cells are small - say about 0.01 millimetre in diameter. In early stages of growth, cell division occurs as soon as the cell attains to something like this size. It would seem that when nutriment is abundant cells add to their protoplasm more than they lose. Having attained certain dimensions at which the conditions most satisfactory for cell life reach their limit, cell division occurs. The big drop falls into two smaller drops, each of which grows more rapidly than the big one was growing at the time when it began to divide. But if there be an optimum size for nutritive purposes, this limit is suspended in many cases, and for various reasons. Take the ovum itself as an example. It is vastly bigger than the cells into which it divides. The yolk of a hen's egg is, when first formed, a single cell. By the time the egg is laid cell division has already set in. In the embryo there are cells which surpass the average dimensions-the unexplained "giant cells" which appear in the liver as soon as it can be recognized as such (c.f.p. 65). These disappear from the liver, but are for a time evident in the spleen. The large cells found in the marrow of bone, some with a great single nucleus, others containing a bunch of separate nuclei, also show that there is no fixed limit of size. It is generally considered that the giant cells of marrow-or, at any rate, those which are multinucleatedare leucocytes which are engaged in scooping out the bone; consuming the hard tissue on the inner surface of the hollow cylinder in order that, by deposition of new material on the outside of the cylinder, the size of the whole bone may be increased-leucocytes battening on bone which, owing to interference with its blood-supply, is breaking down. They have not time to divide. Nourishment is superabundant. Although much too large for a vigorous standard of cell life, they continue to grow, putting off the duty of cell division until the supply of nutritious food begins to run short. 
The most remarkable variations in size are to be found amongst the cells of the nervous system. It may be given as one of the most distinctive characters of nervous tissue that its cells have no fixed or standard dimensions. A nerve-cell enters into connection with other nerve-cells and with musclefibres by means of branches, or cell-processes, as they are termed. The cells may be globular, as in the sympathetic system, or star-shaped. Each cell gives off a certain number of processes, which divide like the branches of a tree, and one process which may run for a very long distance without dividing. This latter thread-like process places it in communication either with a distant part of the central nervous system or with the muscle-fibres which it controls. By means of such a thread a cell in the spinal cord may be connected with muscle-fibres of the hand or of the foot. The thread is really a bundle of filaments (neurofibrillæ) which separate to supply a number of muscle-fibres. It is, in its whole length, a part of the cell in which it originates. The size of the cell varies as the number of filaments in this bundle (termed the "axon"), and possibly also as their length. Hence it comes about that nerve-cells may be amongst the smallest, or they may be the very largest, in the body. The so-called "granules" of the cortex of the cerebellum and of the cerebrum are almost as small as red blood-corpuscles (Fig. 23). Each of them has five or six minute branched processes and an exceedingly delicate axon. The large cells of the cerebral cortex, which send their axons far down the spinal cord, and the large cells of the spinal cord which supply the muscles of the body, have a diameter ten or twelve times as great as that of a granule. But larger still are the nerve-cells which supply the electric organs of the torpedo and other electric fishes (p. 295); and largest of all are the cells which innervate the curious " fishingrods" of the strange angler fish (Lophius piscatorius). It is difficult, owing to their irregular shape, to say how large these cells are; but they are visible to the naked eye.

The anatomical unit of structure is the cell. Cells are the bricks of which the body is built. Some are large, others small, as befits the part which they take in the construction of the body. If the tissue be merely a supporting tissue, connective tissue, cartilage, bone, its cells are uniform in size 
and small. If it have functions to perform which in some cases are carried out best by small cells, in other cases by large ones, the cells are adapted in size to the work that they have to do. Of the various kinds of wandering cells, somethe bone-forming cells (osteoblasts), for example-are small ; others-the bone-eating cells (osteoclasts)-relatively large. Nerve-cells, like telephone exchanges, are large or small according to the size of the area which each supplies.

All animals of complex organization, from starfishes and seaurchins to Man, are inhabited by motile cells. In addition to the bricks which enter into the construction of its fabric, each fixed in its place and definitely united to its neighbours, the animal contains leucocytes which wander through its tissuespaces or float down the streams of lymph or blood. We are disposed to speak of these wanderers as inhabitants of the body, to distinguish them from the elements which enter into the construction of their habitation. It is difficult to avoid the temptation of describing the body as a habitation. Allegorical as Aristotle's distinction between body and soul-between the habitation and that which inhabits-may seem, when contrasted with the exact language of modern science, it would save many a periphrasis if we might still use the monosyllable "soul." The fixed tissues constitute a unity, bound together by nerves. The work done by glands and muscles is done in response to directions conveyed by nerves. It is impossible to say where the control of the nerves ceases - to point out any fixed tissue which is not co-ordinated with other tissues, nor susceptible to the influence of the environment as impressed upon the central nervous system, through the medium of sense-organs. The fixed tissues constitute a habitation for the "soul." They share in a common life. The wandering cells are as independent of control as the parasites which occasionally find entrance into the body. Each must have a soul of its own in Aristotle's sense. Like parasites, they carry on all the business of nutrition, respiration, cell division, without reference to the needs of the fixed tissues. They take what they require from the lymph as it leaves the intestines loaded with the products of digestion ; they take it from the lymph in the tissue-spaces ; they take it from the blood. When nutriment or oxygen runs short, they do not share the privations of the fixed tissues. 
Only indirectly is their well-being affected by that of the body as a whole; only accidentally is the death of the body the occasion of their death. The same might be said of such parasites as the "blood-worms" of Egypt, or the trypanosomes (the cause of "sleeping sickness") of Equatorial Africa. Occasionally, in the rare disease lymphocythæmia leucocytes multiply exceedingly, not, apparently, in response to a call for their presence in large numbers, but in defiance of the needs of the economy, and with baneful results. To the indispensable services which wandering cells render, frequent reference will be made. In the present connection, and while we are searching for the principles of construction of the animal body, it would be desirable, if we could do so, to define the status of wandering cells. If they entered the body from without, they would be parasites of commensal type, intruders who share in the food. and shelter of the body in return for service. But they do not enter from without. They are cells of the growing body which, detaching themselves from the cells which are forming tissues, assume a wandering life. They are not to be recognized in the embryo until development is considerably advanced. Their origin is far from clear, but histologists believe that, although they are not recognizable as wandering cells in the earliest stages of growth, they, or rather their parent cells, are set apart at a very early date. Probably they are not formed in the embryo proper, but in the "extra-embryonic area," from which they emigrate into the embryo. In this sense they come in from outside. But, after all, the extraembryonic area equally with the embryo is a product of the ovum. Looking at the body as a whole, we recognize a common life, a soul in Aristotle's sense, which inhabits the framework of fixed tissues; and at the same time we see a multitude of independent cells, each an organism in itself, produced, like amobæ, from similar independent cells by cell division, absorbing the body fluids, consuming invading germs and fragments of decaying tissues, dying, disintegrating, in their turn absorbed. Wandering cells are autonomous in the largest sense.

All multicellular plants and animals are formed by division of a primitively single cell, the segments remaining in contact. As the scale of life is ascended, the cells which are massed 
together in the body, whether of a plant or of an animal -we are still unable to find any word other than body for the thing as a whole-tend more and more to differ in appearance. Some are large, others small. Some have cellwalls; others have none. Some remain "protoplasmic"; others are largely composed of "metaplasm." Better terms are wanted to connote "most living substance" and "less living substance" respectively. It would be easy to coin suitable words, but, alas ! the nomenclature of physiology is alri rdy hopelessly encumbered, and there is little prospect that a bad word will die when a good one is available in its stead. Differences in structure indicate differences in function. A division of labour has set in. The cell starts with capacities for every function. Its particular situation renders it desirable that it should cultivate one capacity at the expense of the rest. It specializes in a particular direction. If it happens to be placed in the centre of the body on the course of the bloodvessels which bring to the embryo food and oxygen from its mother, it develops a great capacity for taking up food. It accumulates in its substance a vast quantity of nutriment which it cannot consume, holds it, and passes it on into the blood-stream as it is required. Thus the liver is formed. In the embryo it attains to a great size, equal to about one-half the whole body-weight; but whether storing food be its chief function at this stage, or whether the other special functions for which it is responsible are equally important, remains a question for further research. In subsequent life its main work is to store food. After birth, when the child prepares its own food by processes of digestion in its stomach and intestines, the blood-supply of the liver is so modified that the blood from the digestive organs is passed through it. Now and for the rest of life the liver is the storehouse of food, the larder of the body. It is a reservoir from which supplies are distributed as required. A liver-cell retains many primitive characters. It is soft and destitute of envelope. But under the microscope it appears, unless it be taken from a starving animal, unlike any other cell (Fig. 7). It is always loaded with masses of glycogen. Sometimes it contains fat globules also. This is perhaps the simplest of all instances of specialization of function. An amœba can take up food. 
Presumably it always absorbs as much as it can get, the simple law of growth with cell division making it impossible for it ever to get too much. The cells which in the liver are so fortunate as to be placed on the route along which food is carried into the body retain the appetite of an amœba, but lose its capacity for growth and cell division. They return to the blood-stream, when it is deficient in food, the stores which they took up when food was in excess.

The specialization of a gland-cell is opposite in kind to that of a liver-cell. It takes up no more food than it requires, but it has developed a great capacity of producing from the food a substance which would no doubt be needed for its own purposes were it an isolated cell, but which the gland-cell places at the service of the body as a whole. An amoba can digest proteid substances. A cell of the pancreas produces the ferment necessary for the digestion of proteins, and secretes it into the alimentary canal.

To take another instance of specialization. An amœba responds to stimulation by changing its shape. It contracts in one direction, expands in another. A muscle-fibre has developed the capacity of contraction at the expense of all other functions. During the course of its growth it changes from a round cell into one that is elongated. The elongation is in the direction in which it acts with greatest efficiency. Its cell-substance is very highly specialized in order that it may have the maximum capacity of contraction in this direction.

Sensory cells develop to a maximum the capacity of responding to external force; nerve-cells, the capacity of conducting the impulses generated in sensory cells. The body is a republic in which every citizen develops to the highest degree the capacity of doing the thing which his situation makes it desirable for him to do.

The possibility of isolated cell life, and the necessity within certain limits of cell division, have led biologists to dwell too much upon the independence of the separate cells of which the body is composed. Protoplasm organizes itself into cells, but cells are not necessarily anatomically distinct. They may be the partially separate elements of a syncytium, or there may be but the faintest traces of cell separation. The objection to looking upon cells as isolated, self-complete 
units does not hold good to the same extent when they are viewed from a physiological standpoint. A cell is an administrative area. For purposes of nutrition, respiration, and cell division it is autonomous. It is responsible for its own local affairs. If a part is cut off from it, this part loses its vitality; this, at least, is the conclusion drawn from the atrophy of the axons of nerves when they are cut off from the cells of which they are outgrowths. Apparently we must understand by "the cell," when speaking of the cutting off of a part, the portion of the cell which retains the nucleus; although we must be careful not to lay too much stress upon the nucleus as the centre of cell life. Red blood-corpuscles, as already pointed out, have no nuclei, and yet they live. Cell growth, estimated by mere increase in size, does not depend upon the nucleus. Many cells of the skin and its appendages increase considerably after the nucleus shows changes which clearly indicate that it is far advanced towards decay. But increase in protoplasm, cell growth in a legitimate sense, and especially cell division, are dependent upon the presence of an active nucleus. While, therefore, histologists no longer formulate the cell theory in the restricted terms in which it was enunciated some years ago, they still regard the cell as the unit of structure and unit of function. The body is built of cells, and whatever is done by the body as a whole is done by its individual cells. 


\section{CHAPTER IV}

\section{THE FLUIDS OF THE BODY}

From one-fourth to one-third of the whole body is fluid. If the skin be regarded as a watertight bag, three-fourths or rather less of its contents are solid, one-fourth liquid ; and even its apparently solid contents, the tissues, contain much water. Water is an essential constituent of protoplasm. It is also present in cell-juice. The estimate given above does not include the fluid within the cells, but only the fluid with which the cells are bathed. In a general sense this extracellular fluid, excluding blood, is termed lymph. It occupies the spaces of a gauzy "connective tissue," which connects, or separates - the terms are equally appropriate-muscles, nerves, glands, and other tissues of specialized function. Nowhere, except, in a fashion, in the spleen, does blood come in contact with a cell. The lymph which more or less surrounds them is the bath from which cells receive their food and oxygen, into which they excrete carbonic acid and tissue-waste. The network of lymph-spaces is traversed by capillary bloodvessels with walls composed of flattened connective-tissue cells. Such cells are usually spoken of as elements of an "endothelium." As the epithelium covers the surface of the body, so endothelium lines its cavities. Endothelial cells are thin scales or tiles with sinuous borders dovetailed one into another. That the tiles which form the walls of capillary vessels are not cemented together in any proper sense is shown by the facility with which white blood-corpuscles, leucocytes, by their amœboid movements, push them asunder when making their way from the blood-stream into the tissue-spaces, or vice versa. They offer no more resistance to a leucocyte than a pair of curtains hanging in front of a door offers to a child. Yet so 
long as the endothelial cells are alive they keep their edges in such close apposition as to constitute a continuous membrane which shuts off blood from lymph. They are always close enough together to prevent red blood-corpuscles from escaping from the capillary vessels ; but their resistance to the passage of the different constituents of plasma varies greatly. The membrane which they compose is more complete and less pervious, or less complete and more pervious, in accordance

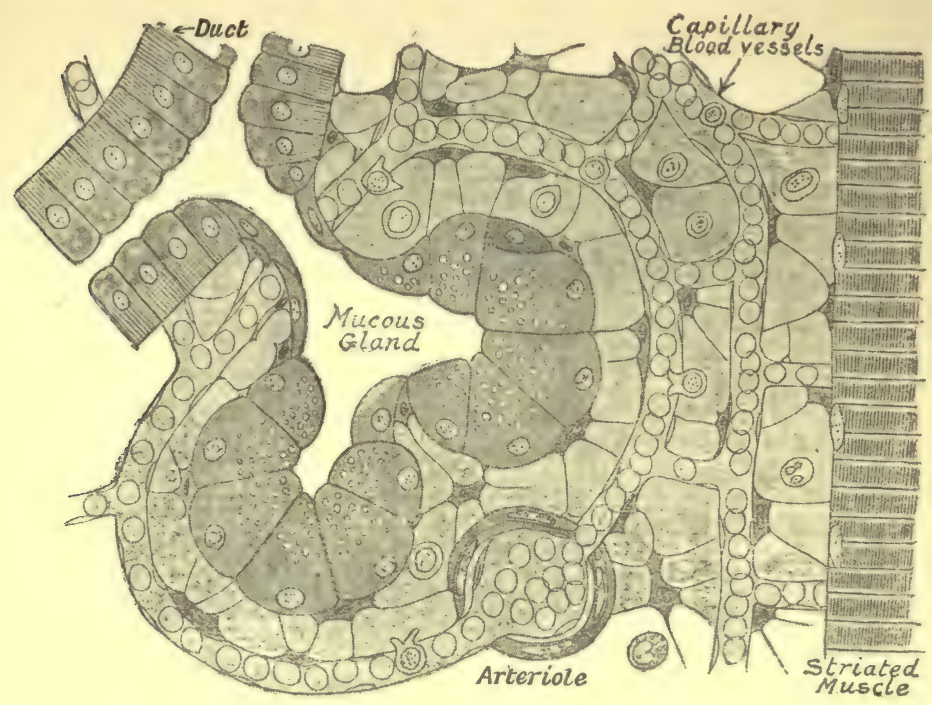

Fig. 3.-A Duotule and Two Amini of a Mucous gland of the Mouth, with a MuscleFibre OUT LoNGITUdinally; CAPILIARY BloOdVESSELS AND CoNNECTIVE TISSUE.

Stellate connective-tissue cells form a labyrinth of intercommunicating lymph-spaces which separate the gland-cells and the muscle-fibre from the walls of the capillary bloodvessels. The capillaries contain circular red blood-corpuscles and nucleated leucocytes. Some of the leucocytes are squeezing their way either out of a capillary into a lymph-space or vice versa. A granular leucocyte is to be seen in a lymph-space at the bottom of the picture.

with the nature of the tissues which surround it, and their varying needs. The blood-passages of the liver may be described as filters. The escape of red blood-corpuscles into lymphatic vessels is prevented, but they offer practically no resistance to the plasma. Plasma- "lymph," as it is termed as soon as it is outside bloodvessels-passes through the walls of the capillaries of the liver unchanged in constitution. Where they traverse glands (other than the liver), muscles, skin, and 
various other structures, the walls of capillary vessels, while offering practically no resistance to water and diffusible salts which can pass through membranes, prevent proteid substances from passing from blood to lymph, except in extremely small quantities. In this way an exquisite balance is automatically maintained. Water and salts pass out as they are needed. But they never pass out in excess, because the protein-containing blood-stream tends to keep them in, in virtue of the same attractive force which enables it to suck in the oxidized products thrown into the lymph by the tissues. Whatever a tissue needs it takes from the lymph. Suppose that bone is being formed. Large quantities of lime and phosphates are needed for the calcification of the cartilage in which it is modelled. The cartilage absorbs lime and phosphates from the lymph which bathes it. Lime salts and phosphates immediately begin to diffuse from blood into lymph. The hurrying blood-stream brings up further supplies from the walls of the intestine, products of digested milk and other foods. Lymph contains (although not in the same proportions) everything which blood contains. Many an analogy may be found in the world of economics, although no illustration would be sufficiently complete. From the lymph tissues take the fuel that they need, the oxygen with which to burn it, the foods for their own repair, the raw materials for their arts. Into it they throw their smoke, their drainage, the slag and refuse of their factories. The blood replaces the supplies as they disappear. It absorbs all waste. Lymph occupies streets, market-place, passages, corridors. The blood-stream is a closed system, rolling down the streets and through the market-place, on its never-ceasing circuit from port and mine to open air and open sea. From the alimentary canal it picks up food and fuel ; the lungs give it oxygen, and disperse its carbonic acid; the kidneys purge it of non-gaseous waste.

The facility with which the constituents of blood pass out to the lymph, and the constituents of lymph pass into the blood, depends upon the condition of the walls of the capillary vessels. Water and substances dissolved in water might pass through the wall of a capillary vessel in either of three waysby filtration, by osmosis, or by secretion. A filter is a porous barrier, which allows water and all substances dissolved in 
water to traverse it. The solution passes through unchanged in composition. Only solid particles are kept back. The rapidity with which fluid passes through a filter varies as the difference between the pressure on the one side and the pressure on the other. A membrane does not allow of filtration. Water and things dissolved in water pass through it by osmosis. Some things it will not allow to pass ; such, for example, as gum, mucin, white of egg. To others it offers resistance in varying degrees. Most of the things that can diffuse through a membrane are capable of crystallization; but the membrane exercises some control over the passage of even crystallizable substances when in solution. If a membranous tube containing water in which proteins, sugar, and various salts are dissolved is hung in a basin of pure water, the proteins remain in the tube; the sugar and the salts pass through its wall into the surrounding water. But they pass at different rates. Those of small molecular weight pass more quickly than those whose molecule is heavy. After a time a condition of equilibrium is established. No more salts pass out of the tube. If now the contents of the tube and the contents of the basin are analysed, it will be found that the tube contains all the proteins, some of the sugar, and some of each of the salts, although not in the proportions in which they were present at the commencement of the experiment. The water in the basin contains some sugar and some of each of the salts, but not in the same proportions in which they are found in the tube. As a matter of fact, the same number of molecules would be present, per unit volume, on each side of the membrane-in the tube and in the basin. In this respect the percentage composition of the two solutions would be the same. But some of the molecules being beavy, others light, the weight of salts which unit volume of the solution in the tube would contain would not be the same as the weight of salts in unit volume of the solution in the basin. A membrane exerts a discriminating action on the substances which pass through it. Secretion is osmosis in disguise. It may be even filtration in disguise. A glandcell (like an amcob) takes things up and passes them out without regard to their osmotic equivalent. It seems to exercise a choice. It seems to act in disregard of the laws 
both of filtration and of osmosis. So, at least, it appears to us when we are looking at the result in ignorance of what has happened inside the living cell. The passage from blood to lymph and vice versa through the wall of a capillary vessel is in certain situations or at certain times a mere process of filtration; at others a process of restricted filtration. If the wall is behaving as a perfect membrane, it is a process of diffusion, or osmosis. It seems unnecessary to regard it, in any case, as a process of secretion. The more widely the capillaries are dilated, the less resistance do they offer to exudation. The narrower their calibre, the greater is the restraint which they place on the escape or entrance of fluid. When the skin of the palm of the hand is not sufficiently thick to protect the soft tissues beneath it from the injurious effects of the prolonged pressure of an oar or an axe, the capillary vessels of the under-skin dilate; more lymph transudes; the skin is raised up as a blister. The same thing happens when the capillaries are dilated and paralyzed by scalding water. The fluid of a blister has much the same constitution as blood-plasm, except that it contains less proteid substance. These results might be regarded as purely mechanical-the direct effects of pressure or heat upon the membranous capillary wall. But the "vital" element is more important. The capacity of endothelium to act as a barrier depends upon its nutritive condition-its vital integrity, as it might be termed; which no doubt in the last resort means its chemical relation to the fluids which bathe it. Now and again blebs, like blisters, are formed on the skin - the herpes which appears about the mouth; urticaria, which is more generally distributed; and various other cutaneous disorders. Frequently a connection can be traced between these eruptions and the consumption of a particular food. An attack of urticaria results not uncommonly from eating lobster, mussels, rook-pie, or some few other articles of diet. Various things-bad fish, for example-may produce the same effect; but shell-fish have an especially evil reputation. If extract of lobster or of mussels be injected into the blood of an animal, the amount of lymph which leaves the blood is markedly increased. The extract acts as a poison upon the endothelium of the capillary walls. It increases its permeability in all conditions in which 
lymph escapes in undue quantity from the blood-stream, or escapes more rapidly than it is absorbed ; the nutritive condition of the endothelium is disturbed. Its unusual permeability is due in part, no doubt, to the dilatation of the capillary tube, the stretching of its membranous wall; but it is due also to the diminished vigour of the endothelial cells. They have lost to a certain extent their capacity for holding their edges in perfect apposition.

When the circulation is sluggish, owing to the inefficiency of the heart, the tissues become odematous. In other words, lymph accumulates in the tissue-spaces. When the skin of a healthy person is pressed, it returns to its natural position as soon as the pressure is removed. If there is a tendency to dropsy-for ages the term "hydropsia" has been thus familiarly clipped-the finger leaves a pit behind it when pressed upon the skin. It is some little time before the lymph in the connective-tissue sponge readjusts the surface. Excessive escape of lymph from the blood, or its insufficient return into the blood, may also be the result of obstruction to the flow in the great veins. When the veins of the leg are varicose, the weight of the column of blood in the distended vessels impedes its circulation. After standing, the tissues about the ankle become œdematous. The œdema disappears on lying down. A hardening (cirrhosis) of the liver impedes the circulation of the blood which comes to it through the portal vein from the walls of the alimentary canal. The capillaries of the stomach and intestine are distended. Lymph accumulates in the abdominal cavity, producing ascites, another form of dropsy.

It is almost hopeless to attempt to disentangle the various factors which disturb the balance between blood and lymphexcessive outflow from blood, deficient inflow from lymph, stretching of the endothelium of the capillary tubes, imperfect nutrition and consequent imperfect apposition of the endothelial scales, increased permeability of the scales. The exudation which accompanies inflammation would seem to be due to the diminished vitality of the endothelium rather than to a mechanical factor, such as increased blood-pressure in the capillaries, and their consequent distention. Ascites is, apparently, a purely mechanical result of the resistance offered 
to the passage of blood through the liver; but pleurisy, the accumulation of lymph in the space between the lungs and the chest wall, cannot be explained in the same way. There is no undue pressure on the vessels in which the blood circulates through the inflamed pleura (the investing membrane of the lungs and lining membrane of the chest), yet the walls of the capillaries fail to maintain a proper balance between blood and lymph.

Hitherto we have spoken of the lymphatic system as a labyrinth of communicating spaces containing stagnant fluid, which is kept in a fitting state by egress and ingress out of and into blood. Such a mental picture is substantially correct. But the system is complicated by the presence of lymphatic ressels. Cells of the connective-tissue sponge-work arrange themselves side by side. They flatten into endothelial scales. The borders of the scales close up. They form lymphatic channels, wider than blood-capillaries, but strictly comparable in every other respect. The lymph capillaries unite into larger vessels. The larger vessels are connected by crossbranches; they form plexuses. Their walls are strengthened with fibrous tissue. Like the veins, they are abundantly provided with valves, which check any tendency to a backward flow on the part of the fluid which they contain. Lymphatic plexuses surround and accompany the larger bloodvessels. They are disposed on the surface of muscles and glandular tissues. They are abundant beneath the skin. Nearly three centuries ago the lymphatic vessels of the mesentery, which collect products of digestion, especially fat, from the walls of the alimentary canal, were recognized owing to the milkiness of their contents after a meal. They were, on this account, termed "lacteals." Other lymphatic vessels, owing to their transparent walls and colourless contents, are not easily seen ; but they are readily injected with mercury or other fluids which render them conspicuous. In the upper part of the thigh, in the armpit, or in the neck, they are about large enough to admit a crow-quill. Those from the lower limbs, from the viscera, and from the walls of the abdomen converge to a receptacle which lies in front of the spinal column. The receptaculum chyli is continued upwards as the thoracic duct, which pours the lymph into the great veins of the 
left side of the neck and of the left arm just where they join together.

The thoracic duct provides for the overflow of lymph from the spaces of the body. There is no circulation of lymph. Lymph from the liver and from the intestines is constantly draining into the thoracic duct, and thus returning to the blood-stream by a short direct route, entering it without the necessity for reabsorption through the walls of capillary vessels. By no means all of this fluid has exuded from the blood-stream. Much of it is water which was poured into the stomach as gastric juice, and into the intestines as the secretions of the pancreas and other glands, or imbibed through the mouth and absorbed by the lymphatics of the alimentary canal. The remainder of the water taken up from the alimentary canal enters its bloodvessels. The diluted blood flows to the liver, loaded with digested products which the liver will store. As the blood parts with them the additional water which has served for their transport exudes from the capillaries of the liver into lymphatics, which empty it into the thoracic duct. Large quantities of water are used in washing out digested products. Secreted into the alimentary canal by the digestive glands, it passes out through its wall as the vehicle of digested products. Collected by lymphatic vessels, it is either carried directly into the thoracic duct, or passed from lymph into blood, carried by blood to the liver, again transferred from blood to lymph, and borne by the lymphatic vessels of the liver to the thoracic duct.

Water exuded from blood into lymph may be reabsorbed into the blood near the place where it was poured out, or it may reach the blood via the thoracic duct. It would seem that the former is the natural, the latter the emergency route; the former the course taken when an organ is tranquil, the latter a necessity when the organ is active. If the large lymphatic vessels of a limb are cut, no lymph escapes from them so long as the limb is at rest. When the muscles contract lymph begins to flow. If the limb is flexed and extended by hand, lymph flows. If the muscles are squeezed or massaged, lymph flows. As the flow is set up both by active contraction of the muscles and by passive movements in which the muscles do not take part, it clearly must be due to external pressure 
on the lymphatic vessels. As they are provided with valves, squeezing them converts them into pumps. The fluid which they contain is bound to go forwards. Additional fluid is squeezed into them from the tissue-spaces. To a large extent, therefore, the outflow of lymph from contracting muscles is to be explained as the result of the pressure which the swelling muscles exert upon the lymphatic vessels within their sheaths. But there is another factor which must not be overlooked, although it cannot readily be estimated. When a muscle is actively contracting its bloodvessels dilate. There is a greater exudation of lymph; and reabsorption by blood is not equal to the exudation. The surplus leaves the limb by the lymphatic vessels. A gland is never at rest. In the intervals between the ejection of its secretion its cells are preparing materials for the next outflow. Lymph is always flowing from a gland; its amount increases as the activity of the gland increases. More lymph leaves the blood when the gland is exceptionally active than when it is relatively quiet. Some of it is not reabsorbed into the blood. A certain proportion of the waste products of the active gland are hurried away by the overflow system in the direction of the thoracic duct.

Lymph is the reservoir of nutriment upon which every cell in the body draws. It is improbable that in health and under normal conditions the activity of any organ is ever restricted for want of sufficient food. As food is removed from lymph, it is instantly replaced by fresh food from the blood. There is some evidence-not very clear-that the removal of waste products offers greater difficulty than the renewal of supplies of food. When the activity of muscles has been excessively prolonged they ache. It has been supposed that their unwillingness to do more work is due, not to the exhaustion of the food which they use up when contracting, but to the inadequacy of the lymph and blood to carry off all refuse. This, at least, is the explanation of fatigue which is usually offered, although it is difficult to understand why the arrangements for removing waste products which have worked to perfection for eight hours should during the ninth hour become rapidly ineffective.

If a frog's muscle, cut out of the body, has been made to 
contract until it refuses to work any longer, it again responds to stimulation after a solution of salt has been passed through its bloodvessels. The salt solution brings no food; the only thing it can do is to wash away waste products. But this experiment upon a tired, isolated muscle does not necessarily throw light upon the nature of fatigue in muscles under normal conditions. The isolated muscle is using up, in contracting, food which it has stored. Cut off from the circulation, it has no means of getting rid of the lactic acid and other products into which food is changed. They may well have accumulated to a poisonous extent long before all the food has been used up. Hardly more cogent is the argument based upon the benefit which a tired man experiences from hot baths, massage, and the like. They take away the feeling of tiredness, but it does not follow that this result is due to the removal of waste products. Quickening the circulation of blood brings about renewal of the lymph. Renewal of lymph means fresh supplies of food as well as removal of waste products. Even human muscles are not perfect as machines. They will not work for an unlimited spell. There comes a time when they must have rest. Something goes wrong in the admirable adjustment which has hitherto provided exactly the right amount of food and exactly the necessary freedom from the products of action. A feeling of fatigue is the signal that the apparatus is not in a condition to work longer; but whether this feeling is due to a dislocation of the balance of supply and loss, or to some deterioration of the apparatus which calls for rest and renovation, it is at present impossible to say. It is not due to the exhaustion of muscle food. A more powerful stimulus, the urgency of fright or some other strong emotion, or an electric current applied directly to the muscle or its nerve, will still induce vigorous contraction. The muscles of a hare that has been coursed until it can run no farther still contain glycogen, muscle-food.

Glycogen is stored in the liver. Fat, if it is assimilated in excess of the needs of the body, accumulates in the connective tissues. Proteins, if in excess, are either destroyed by oxidation, or partly destroyed and partly converted into fat. Increasing the amount and richness of the food does not, if nutrition is already at its best, improve the quality of the 
blood. The surplus of food is either stored or burnt. The composition of lymph is unaffected. Its quality is not improved by taking more food than enough. A perfect balance is maintained. Every cell is able, when conditions are normal, to obtain as much nutriment as it needs. It cannot get more. It cannot lay by food and shirk work. If it did it would grow. Reaching its optimum size, it would divide. Additional tissue would be formed. But when it does more work it needs more food ; and it is a matter of common experience that the system is so adjusted that food is supplied to the tissues, not reluctantly, but with a slight tendency towards generosity. Working harder than usual, they find the lymph by which they are bathed somewhat richer in the materials that they need than the necessities of the case demand. They are able not merely to obtain all they want, but a little more. Activity favours growth.

Many attempts have been made to show that if a part of the body has more than its share of food it grows to an excessive size. John Hunter grafted a cock's spur into its comb. It grew to monstrous dimensions. Such a result favours the view, but it is not quite conclusive. Undoubtedly the comb was richly supplied with blood, but it does not follow that the cells of the spur were able in their new situation to take advantage of this supply. Besides, the spur when projecting from the head was not subject to the accidents to which it was exposed whilst on the leg. Its size was not kept down by friction. Nor was it as hard and compact as it would have been in its normal situation. It is scarcely possible to devise any experiment that would be satisfactory now that the relations between blood and lymph and lymph and tissues are understood. In certain pathological conditions, however, hypertrophy is the result of the hyperæmia of chronic inflammation; and there is little doubt that, if we could arrange for a certain group of cells to receive lymph richer in food and freer from waste products than the perfect adjustment of supply to needs normally allows, the cells would grow.

Under perfectly healthy normal conditions growth can be induced only by use. Nature supplies the fuel which is used during activity, and a balance of food available for the construction of additional machinery. The muscle which is 
called upon to do work develops a greater capacity for work.

When nutrition is not at its best, the growth of muscle may be favoured by external pressure which squeezes lymph out of its tissue-spaces, and therefore leads to increased exudation from the blood. It is not improbable that in badly nourished tissues the circulation of blood is somewhat torpid and the lymph stagnant. A feeble circulation usually results in some œdema. 'The muscles, or rather the connective tissue which envelops and penetrates them, feels doughy, instead of being, as it should be, firm and elastic. Under these conditions massage is undoubtedly of service. Squeezing the muscles displaces lymph, and, if the pressure is properly directed, drives it along the lymphatic vessels. Fresh lymph exudes from the capillary bloodvessels, and the muscle-fibres, surrounded with a more abundant supply of nutriment, benefit, as, in a vigorous person, they benefit from use.

Lymph is an exudate from blood. Its composition therefore depends upon that of blood-plasm, but it tends to differ from it owing to the influence of two causes. In the first place, the walls of the capillary bloodvessels restrict exudation. Red blood-corpuscles cannot pass through them. Proteins which are non-diffusible are, according to the circumstances of the tissues, held back to a greater or to a less extent. The pseudocapillaries of the liver let them pass, as has already been said. The capillaries of the limbs restrict their passage to such proportions as, it may be supposed, are absolutely necessary for the nutrition of the tissues. In the second place, tissues remove food from lymph and add to it waste products. Hence the lymph issuing from a limb, after full contact with the tissues, contains less of the former and more of the latter-less sugar, for example, and rather more oxidized nitrogenous substances, lecithin and other things termed collectively "extractives," because they can be extracted from dried blood or lymph by ether. The reaction of lymph is alkaline. After a time it coagulates, but coagulation is slower, and the clot less firm than in the case of blood.

As the composition of lymph depends upon the source from which, and the conditions under which, it has been obtained, it is unnecessary to state the results of a chemical analysis. 
It suffices to say that lymph contains all the substances which are present in the plasma of blood, but not necessarily in the same total amount or in the same relative proportions. Speaking generally, leucocytes are present in about the same numbers as in blood-6,000 to 8,000 to the cubic centimetre; but leucocytes are everywhere present: in blood, in the lymph, in lymph-vessels, in the tissue-spaces. As they are not passively floating bodies like red blood-corpuscles, but active migratory organisms, they tend to accumulate in one situation and withdraw from another, in accordance with the opportunities which the different localities afford. They desert effused lymph, blisters, ascitic fluid, and the like. They are not found in the lymph in the pericardium. There are fewer in the lymph coming from the intestines after a meal than in the same lymph during the intervals between meals. Their departure from effused lymph might easily be explained. It is not so easy to account for their comparative absence from the lymph in the lacteals when it is heavily charged with fat and other products of digestion. Such leucocytes as are present at this time are loaded with fat granules which they have stolen from the chyle, as the lymph in the lacteals is usually termed. One would need to be very intimate with a leucocyte before one ventured to give reasons for all its movements. Lymph contains the same proteid substances as blood, and in the same relative proportions, but usually in smaller quantity.

Incidental reference has been made to the great lymph-spaces - peritoneal, pleural, and pericardial. The brain and spinal cord are separated from their outer membranes by a lymphspace. There are also spaces within the brain-the ventricles -and a central canal in the spinal cord. The aqueous and vitreous humours of the eye are also lymph-spaces, although the latter contains some remnants of tissue. The joint cavities are lymph-spaces. So also are the bursæ which surround tendons or separate them from bones. It is not, however, justifiable to include all these cavities in a single category, either from the point of view of their purpose, their mode of formation, or the nature of their contents. The peritoneal, pleural, and pericardial spaces are parts of the great primitive body-cavity, or colom. The two first are potential rather than actual. Normally they contain just sufficient fluid to moisten 
the apposed surfaces of the endothelium which lines their walls and covers the organs which they contain. There is no fluid in them which can be collected and labelled "peritoneal" or "pleural" fluid. The purpose of the spaces is to allow of movement without friction-in the one case of the intestines, in the other of the lungs. It is possible to take a spoonful or so of fluid out of the space which surrounds the heart. It has the usual composition of lymph. It contains proteins, but is not spontaneously coagulable. Leucocytes are absent, a fact which probably accounts for its not clotting. The fluid inside the cerebro-spinal system is extremely dilute. Its principal salt-its principal constituent, indeed-is sodic chloride. It contains hardly a trace of proteins, and these in a modified condition-proteoses. It also contains pyrocatechin, a benzoic alcohol. This substance has long been recognized as a constituent of cerebro-spinal fluid, owing to the fact that, like sugar, it reduces copper salts when heated with them in an alkaline solution. It appears to be one of the products of proteid decomposition. Although exuded as lymph from the bloodvessels of the chorioid plexuses, the composition of cerebro-spinal fluid has been profoundly changed by the activity-it might almost be called the digestive activityof the epithelium which lines the cerebro-spinal canal. There is a theory that the ancestors of all vertebrate animals were organized on a very different plan from that of their distant descendants. Our cerebro-spinal canal was their stomach and intestine. It would appear that the lining epithelium of these organs, although disused for millions of years, cannot resist the temptation to digest the lymph which they contain! The fluid in joints contains mucin (the essential constituent of mucus), or a substance resembling mucin. In this case the joint-membrane has added something to lymph without removing or destroying any of its other constituents.

Other illustrations might be given showing how the plasma of blood is altered in composition while it is passing out of, or after it has passed out of, capillary bloodvessels. Perhaps it would be more logical to start on the outer side of the walls of the capillaries; since blood may, very properly, be regarded as a tissue, dependent, like all other tissues, upon diffusion from lymph for the nutrient materials that it needs. 
In the wall of the alimentary canal it receives supplies via the lymph. It drops them in the liver, its garde-manger, to pick them up again as they are wanted. The torrent of lymph which the thoracic duct discharges into the veins of the neck conveys the fat which could not traverse the walls of the capillary bloodvessels, and much of the reserve of food which the blood had deposited in the liver. Only about one-quarter of the fluid of the body (one-thirteenth of the body-weight) is included within the blood-system; but this enclosed fluid, owing to the fact that it is kept in circulation by the heart, replenishes and purifies the much larger quantity which does not circulate. The unenclosed lymph has in particular situations a chemical composition which varies widely from that of the blood. Imagine a marsh through which a river flowsthe vast plains of water-plants on the Nile above Fashoda, for example. There is a constant interchange between the flowing water of the river and the stagnant water of the marsh. In any given part of the marsh the quality of the water will depend upon what it has been able to take from, and what it has given back to, the river; upon what the water-plants have taken from it, and what they have added to it. Boats which cannot penetrate the walls of reed keep to the open channel of the Nile. Fish swim, now in the river, now in the narrow passages and open pools of the marsh. So it is, in a way, with the fluid in the spaces and cavities of the lymphatic system and in the bloodvessels which traverse them, and with its migratory inhabitants. In our extravagant analogy read leucocytes for fish. Fish have two reasons for wandering from river to marsh. Amongst the water-weeds they hunt for food ; they seek quiet places in which to breed. In this matter the analogy holds good. A leucocyte may be overtaken with cell division anywhere-in the blood-stream or in a lymphvessel. But cell division very rarely occurs except in certain favoured spots. The breeding-places chosen by leucocytes are sheltered situations in connective tissue where the bloodsupply is abundant, and the eligibility of such a spot is much increased by its being near to a field where their services are likely to be called for. The nests of connective tissue made by the leucocytes are of three kinds, termed respectively diffuse adenoid tissue, lymph-follicles, and lymphatic glands. The 
connective tissue beneath the mucous membrane of the whole of the respiratory tract-trachea, bronchi, and bronchiolesis diffuse adenoid tissue. It presents no special structure, but its spaces are packed with leucocytes in various stages of cell division, and young leucocytes, or lymphocytes, as they are usually named. Some of the lymphocytes make their way into the blood or into the lymph. Others, acquiring their full dimensions, scour the epithelium which lines the respiratory tract for germs and other foreign bodies which are drawn into the tract with inspired air. They may be seen pushing aside the cells of the lower strata of the epithelium, on their way to the surface, or returning to the subepithelial connective tissue with germs, or particles of soot, or débris of epithelial cells which they have taken into their substance (Fig. 4, B).

The tonsils are examples of follicular lymphoid structures. They lie one on either side of the entrance to the gullet, between the two folds (the anterior and posterior pillars of the fauces) by which the soft palate is continued to the side of the tongue. Normally the tonsil is not visible, but when inflamed it may project sufficiently to be seen ; and its surface may then be covered with mucus and pus. It is liable to become enlarged in childhood, owing to chronic inflammation. A section of the tonsil shows it to consist of clusters of lymphfollicles lying beneath the mucous membrane. The term "follicle" is unfortunate. It conveys no idea of the form or structure of one of these masses of lymph-cells ; and it is, besides, applied to things of an entirely different characterfor example, the pits of mucous membrane which sink down between the masses of lymphoid tissue in the tonsil. The expression "follicular tonsillitis" does not refer to the lymphfollicles, but to the epithelial pits. It is a condition in which a drop of pus is to be seen in the mouth of each of the pits. A lymph-follicle is a small rounded clump of connective tissue, denser on its periphery than in its centre. Its bloodvessels are disposed chiefly on the periphery. Lymphatic streamlets arise in the centre. Its outer portion is closely packed with dividing lymph-cells and young leucocytes, which as fast as they are formed migrate towards the centre, and eventually escape from the follicle by the lymphatic vessels. The connective tissue which invests and separates the follicles is full 
of leucocytes. Removal of the tonsils is followed by no ill effects. They are not essential to our well-being. Nevertheless, they have important functions to perform. They are barracks crowded with leucocytes, which guard the pass into the alimentary canal. Their leucocytes incessantly patrol the mucous membrane, capturing germs, removing fragments of injured epithelium, striving to make good the mischief to which this part of the alimentary canal is peculiarly liable. The enlargement of the tonsil which results from frequent sore throat is a response to the demand for an increase in the supply of these little scavengers, in order that they may cope, not only with objectionable things outside the walls, but with the still more pernicious germs which during an attack of sore throat succeed in breaking through the epithelium. It is the invaders which elude the vigilance of the leucocytes that cause fever and other general symptoms. Other notable groups of lymph-follicles are found in the middle portion of the small intestine, where they form oval patches, about three-quarters of an inch long by half an inch broad-Peyer's patches. The leucocytes which are developed in them search the walls of the intestine for germs. During an attack of enteric fever the patches become inflamed, and one of the greatest risks which the patient runs is the risk of ulceration of a patch and the perforation of the intestinal wall.

The abundant provision for the multiplication of leucocytes shows that the destruction of these cells must occur on an equally large scale. Every day large numbers die. Where this occurs, and how their dead bodies are removed, is not certainly known. Doubtless they are eaten by their fellows, their substance oxidized, and the products-carbonic acid, water, and nitrogenous waste-thrown into the lymph. There is some reason for thinking that a part of the nitrogenous waste is excreted in the form of uric acid (cf. p. 216). The daily production, and consequent destruction, of leucocytes shows that their metabolism is a factor which cannot be overlooked when we are making up the body's accounts.

The fixed tissues receive their nutriment in a digested condition. Leucocytes digest it for themselves. In many cases, although not in all, the cells of fixed tissues last throughout life, so far as their outer form is concerned, although their 
molecules are oxidized and replaced by new material. It is not improbable, therefore, that there is a difference between the metabolism of the fixed tissues and the metabolism of leucocytes. The whole of a wandering cell, its nucleus included, breaks down and has to be removed. We do not know that this occurs in the case of a fixed cell. On the strength of evidence which points, apparently, to a chemical relationship between nuclear substances and uric acid, it has been inferred that the two chief nitrogenous products which are excreted by the kidney are divisible into the one which in the main represents the oxidation of fixed cells, urea, and the other, uric acid, largely derived from the oxidation of wandering cells.

The valiant leucocytes do their best to cope with all the rubbish, whether living or dead, that needs removal. They flock to any situation in which germs are numerous or tissue has been destroyed. If all goes well they take the foreign matter into their substance-dead tissue is matter foreign to the body-and either digest it in the course of their ordinary progress, or retreat with it, if they cannot digest it, to the nearest lymphatic gland. But in their efforts to reach objectionable matter they are apt to wander too far from the healthy lymph from which they obtain oxygen for their own respiration. Unable to breathe, they die. They lose the power of extruding pseudopodia. Their extensible, prehensile processes are drawn in. Assuming a globular form, they float helplessly in what once was lymph. Their body-proteins are largely changed to fat. As " pus cells," they are thrown off in the discharge from an ulcer, or accumulate in the cavity of an abscess. A pus cell is a dead and fattily degenerated leucocyte.

The third kind of breeding-place of leucocytes, a lymphatic gland, has a more elaborate structure than the tissues with which we have already dealt. Lymphatic glands are about the size of beans, and of the same shape. They are found in the course of lymphatic vessels in situations where they are not exposed to pressure, such as the back of the knee, the groin, the front of the elbow, the armpit, in the neck above the collar-bone, and on either side of the sterno-mastoid muscle, behind the angle of the jaw. There are a number in the 
abdomen and in the thorax. Each lymphatic gland is invested by a strong fibrous capsule. Its artery enters, and its vein and efferent lymphatics leave, the concave side (the hilus) of the gland. The lymphatic vessels which bring lymph to it pierce the capsule on its convex side. It is divisible into two parts: (1) The adenoid tissue which surrounds the artery and its branches; (2) the open network of "lymph-ways" which invest this adenoid tissue. Leucocytes divide in the adenoid tissue. The young lymphocytes drop out into the lymph-ways. As a stream of lymph, brought by the afferent vessels, is always flowing into the lymph-ways, and out by the efferent vessel or vessels, the lymphocytes are carried with it towards the thoracic duct. A lymphatic gland is therefore an organ for adding leucocytes to lymph in the course of the lymph-stream. It has, however, another and equally important function. Leucocytes which have picked up germs or other foreign matter pass on with the lymph to a lymphatic gland. After entering its lymph-ways they leave the lymph-stream, squeeze into the adenoid tissue of the gland, and there come to rest with their burden. They remain in the gland until the foreign matter is digested, or, if it be indigestible, until they undergo dissolution, when the particles of soot or pigment are deposited from their débris in a harmless state. When the skin is tattooed, much of the Indian ink and other pigment remains where it was inserted with the needle, but some of it is picked up by leucocytes and carried to the nearest lymphatic gland.

Lymphatic glands are barriers which stop the spread of infection. They are the stations to which our police carry captured germs. The skin of the heel is abraded. Germs from the soil, or elsewhere, which have accumulated in a dirty stocking-owing to the warm moisture enclosed by an impervious boot, the woollen covering of the foot is a peculiarly healthy place for germs-enter the opened lymph-spaces of the subcutaneous tissues. Leucocytes hasten to the spot. They seize the invaders with their pseudopodia, engulf them in their body-substance, enter lymphatic vessels, and are rolled away by the lymph-stream. The instinct which brings them in ever-increasing numbers to the breach in the protecting skin can be explained only in terms of force. From our own conscious action to the causes which determine the movements 
of a leucocyte, or of an amœba, is so deep a drop that we prefer to recognize in the latter a merely chemical attractive force. "Chemiotaxis" we term the influence which draws leucocytes to the place where food is abundant; although it is also the place, one must admit, where in the interests of the body as a whole they run great risk of asphyxiation. It is appetite which draws a schoolboy to a bun-shop; a sense of duty prompts a fireman to risk his life in a chamber filled with smoke. We have no desire to humanize a leucocyte; but it is difficult to emphasize too strongly its independence. It would be absurd to use terms which imply that a leucocyte has a self-directive power; yet it is equally misleading to describe its migration to the seat of injury, its retreat with ingested germs to a lymphatic gland, its wriggling from the lymph-ways of the gland into the shelter of its adenoid tissue, in terms which imply that the forces which direct it are known, and their mode of action understood. The success which attends the inroads of germs is due to their amazing capacity for multiplication when they reach lymph or blood. It is useless to attempt to form an idea of the rapidity with which they divide, since we have no data upon which to base calculations. If the leucocytes fail to deal with the first few that enter, germs soon swarm within the lymph-vessels. This leads to an inflammation of the walls of the vessels, which may then be seen as red lines beneath the skin. These red lines lead upwards towards the nearest lymphatic gland. The glands in the space behind the knee are not usually affected when the focus of infection is in the foot. The red lines can be traced up the inner side of the knee and the front and inner side of the thigh to the groin. The glands in this situation swell until they can be easily felt. If the mischief is in the hand, the gland at the elbow may be affected, but most of the lymphatics pass by it on their course to the glands in the armpit. If a sore throat is the source of infection, the glands beneath the angle of the jaw enlarge. Thus various glands block the further progress of infection. In doing this their resources may be strained to the uttermost ; they may enlarge, become tender, grow soft, fill with pus, break down and discharge the pus without the aid of a surgeon's knife, although as soon as pus is recognizable within them it is wise to let it 
out. If germs pass through these first stations into the lymphvessels beyond them, abscesses are formed in other situations. A condition of " blood-poisoning," so called, is set up.

The readiness with which leucocytes sacrifice themselves in their efforts to remove germs and decaying tissue is a matter of almost every-day experience. The fatty matter produced in the sebaceous glands of the skin normally overflows on to the surface. It serves to render the skin supple and impervious to water. Germs get into one of the sebaceous glands of the face or of the eyelid. The contents of the gland begin to decompose. Leucocytes enter it for the purpose of removing the putrescent substance. They lose their vitality and turn into pus corpuscles. The pimple or the stye bursts, and pus and fatty matter are discharged together.

That the conversion of leucocytes into pus cells is due to want of oxygen has been shown by the following experiment: A minute piece of phosphorus is placed beneath the skin. Leucocytes gather round the spot with a view to removing the tissue which the phosphorus has destroyed. But phosphorus has so strong an affinity for oxygen that it exhausts the supply in the area of tissue which surrounds it. The leucocytes die before reaching the tissue immediately adjacent to the piece of phosphorus. Their dead bodies form round it a raised ring of pus cells. We can explain this readiness of leucocytes to sacrifice themselves in their efforts to reach foreign matter which needs to be removed, only by saying that the attraction of the food is greater than the repulsion of lymph destitute of oxygen. An amœba placed in comparable circumstances gives up the quest of food, however strongly chemiotaxic, and retreats towards water which contains oxygen sufficient to provide for its respiratory needs.

Blood.-A portion of the body fluid is enclosed within vessels and kept in circulation by the heart. The heart pumps blood into the aorta. This trunk gives off large arteries, which in turn divide until the finest capillary vessels are reached. The capillary tubes reunite to form veins, which, with the exception of those which collect food from the digestive organs, convey the blood right back to the heart. The veins which drain the stomach and intestines (the organs in which food is prepared for absorption) and the spleen (the organ in which 
worn-out red blood-corpuscles are in a sort digested) break up in the liver into a second set of small vessels. The pseudocapillary vessels of the liver reunite to form the hepatic veins, which add the blood that has passed through that organ to the rest of the blood which is passing up the inferior vena cava to the heart. A second capillary circulation is found in the kidney also.

The heart is four-chambered (Fig. 10). Its left ventricle drives the blood round the systemic or greater circulation, the blood returning to the right auricle. The right ventricle drives the blood through the lesser or pulmonary circulation, from which it returns to the left auricle. The walls of all bloodvessels, except capillary tubes, are sufficiently thick to prevent the escape of any of the constituents of blood. To support the pressure of the blood which they contain, the arteries and the larger veins need walls of considerable thickness. The walls of the capillaries allow an interchange between blood and lymph in the manner already described (cf. p. 39).

Blood fresh from the lungs, whether still in the pulmonary veins or in the systemic arteries, is scarlet in colour. Venous blood is darker and purple-red, the depth of its tint varying with the extent to which it has parted with its oxygen. It looks less opaque than arterial blood. With this exception, the physical properties and chemical composition of blood are remarkably constant in all parts of the body. Arterial blood contains more oxygen, venous blood more carbonic acid. Other chemical differences can be recognized, but they are relatively very small. The constancy in the constitution of blood is its most notable character. Bleeding, unless excessive, does not greatly affect it. The number of corpuscles is of course diminished, but even these are replaced with great rapidity. The plasma, after bleeding, soon recovers its proteins and salts. A similar readjustment occurs if normal saline solution (water containing 0.9 per cent. sodic chloride), or even a strong solution of salt, is injected into the blood. Within certain limits it is very difficult to disturb the balance of its constituents. It gets rid of substances added in excess, or replaces substances removed, with remarkable facility. If sugar (glucose) be injected into a vein, it escapes through the capillary walls into the lymph. After a short interval the 
lymph contains more sugar than the blood. If an excess of protein, whether of a kind foreign to the blood or its own serum-albumin, be injected, it is removed by the kidneys. The blood has various sources from which it can draw out reserves of anything that is lacking, and various ways of getting rid of anything that is in excess. It draws upon the lymph in the tissue-spaces for water. It discharges salts into the lymph. It also takes salts from the lymph. It draws upon the liver for sugar, and probably for proteins also. In a starving animal the blood still contains sugar long after fresh supplies have ceased to reach it from the intestines. The lungs remove its carbonic acid. The kidneys free it from everything which cannot be otherwise removed. It is essential to the well-being of the organism as a whole that a uniform standard of composition should be maintained by the blood.

Composition.-The structural composition of the blood, and the relation of its several constituents to each other, is best studied under the microscope. A thin transparent membrane in which blood is circulating through small vessels-the web between the toes of a frog's foot, the mesentery, the membrane of a bat's ear-affords an opportunity of observing blood in circulation. In any of the smaller vessels, whether artery or vein, a column of red corpuscles is seen moving in the axis of the stream. This column is surrounded by a layer of clear plasma. Amongst the red corpuscles a few leucocytes may be detected floating placidly down the current. Others are seen in the peripheral layer of plasma, tending to creep along the wall of the vessel rather than submit to be moved forward, as passive objects, by the current. If an irritant be applied to the membrane, the vessels dilate; yet, notwithstanding their wider calibre, the current becomes slower. The red corpuscles mass together. Apparently their constitution is slightly altered by this commencing inflammation, in such a manner that they cease to be clean, independent dises which slide past each other like small boats on a river; they exhibit a tendency to stick one to another. In the capillary vessels leucocytes may now be observed, not merely ereeping along the inner surface of the endothelium, but squeezing themselves between its scales ; making their way out of the vessel into the tissuespaces through which the vessel passes. Such an observation 
gives the clue to the functions of the several constituents of the blood. The red corpuscles carry oxygen in chemical combination with their colouring matter. From them it passes into solution in the plasma; from the plasma through the walls of the capillary vessels into lymph; the tissues take it from the lymph as they require it. As fast as it is removed from lymph it is renewed from plasma. Carbonic acid excreted by tissue-cells is dissolved in lymph. From lymph it is trans-

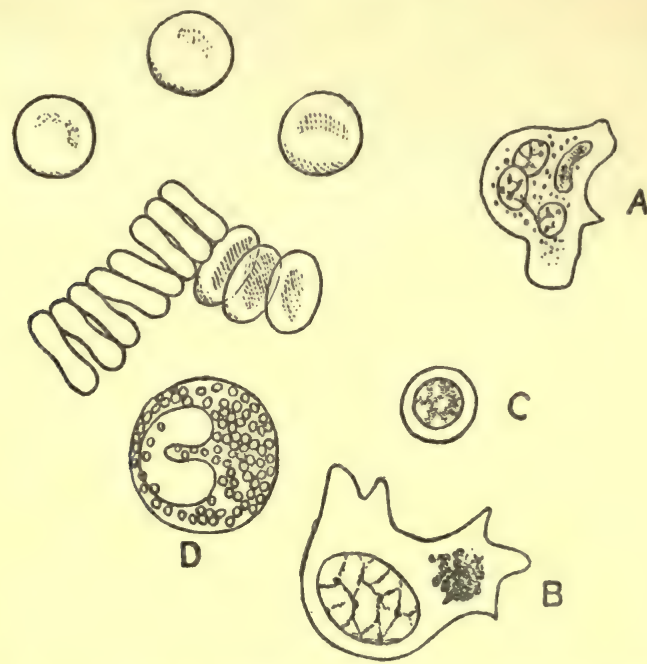

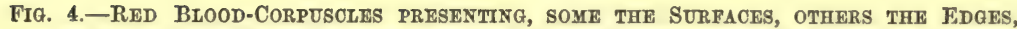
of their Disos, together with Single Representatives of Four Types of LedCoOYTE.

A, the most common type, highly amxeboid and phagocytic. Its protoplasm is finely granular, its nucleus multipartite. B, a leucocyte closely similar to the last, but larger, and containing an undivided nucleus. It is shown with a cluster of particles of soot in its bodysubstance. C, a young leucocyte, or "lymphocyte." D, a coarsely granular leucocyte. Its granules stain brightly with acid dyes-e.g., eosin or acid fuchsin.

ferred to plasma. The reception of carbonic acid by these fluids is not quite so simple as the transference of oxygen from blood to lymph. It is aided by the presence of alkaline carbonates which are always ready to form "acid" salts : not acid to litmus-paper-the blood is always alkaline-but containing more than one unit of acid to one of base. Sodic carbonate has the formula $\mathrm{Na}_{2} \mathrm{CO}_{3}$. With an additional molecule of carbonic acid it becomes $\mathrm{Na}_{2} \mathrm{CO}_{3} \mathrm{CO}_{2}(\mathrm{HO})$-bicarbonate. When in solution it can hold still more carbonic acid. If carbonic acid 
were merely dissolved in lymph and plasma, it would be impossible for the blood to carry it away with sufficient rapidity ; just as it would be impossible for blood to bring sufficient oxygen were it not for the colouring matter (hæmoglobin) which forms a temporary, easily divorced union with it. But from a physical point of view it comes to the same thing. As the tension of oxygen in plasma falls, it dissolves more from the hæmoglobin. When the tension of oxygen in lymph is less than its tension in plasma, the former borrows from the latter. If the tension of carbonic acid in lymph is higher than in blood, it passes to the blood. The rapidly circulating blood at frequent intervals traverses the lungs. The whole blood of the body is exposed to air in the lungs once every minute. Oxygen tension being higher in pulmonary air than in venous blood, this gas is taken up. Carbonic acid tension being higher in venous blood than in pulmonary air, this gas escapes. The plasma in the capillary vessels which traverse the tissues exchanges gases with the lymph with very great rapidity.

The specific gravity of blood varies from 1.056 to 1.059. The corpuscles are heavier than the plasma. Its reaction to test-paper is alkaline, owing to the presence of bicarbonate of soda and disodic phosphate. The alkalinity is greatest when the body is at rest ; it is diminished by severe muscular exercise. Blood contains about 5,000,000 red corpuscles, and 7,000 or 8,000 leucocytes, to a cubic millimetre. Red bloodcorpuscles are biconcave discs destitute of nucleus, and, so far as can be seen, devoid of any investing membrane. Seen in profile they appear biscuit-shaped, because the centre is hollowed out. Their largest diameter is 7.5 micromillimetres $\left(\frac{1}{3200}\right.$ inch $)-a$ measurement of great importance to anyone who works with a microscope, because it serves as a standard by which to estimate the size of other objects. They are soft, but fairly tough and highly elastic. In circulating blood a corpuscle may occasionally be seen to catch on the point where two capillary vessels unite. It bends almost double under the pressure of the column of corpuscles behind it, and then springs forward.

A red corpuscle is a vehicle for hæmoglobin. If blood is diluted with water, or if it is alternately frozen and thawed, the hæmoglobin separates from the corpuscles, which can then 
be seen as colourless discs. Hæmoglobin constitutes 40 per cent. of the weight of a moist corpuscle, or 95 per cent. of its weight after it has been dried. This is an enormous charge for a corpuscle to carry, and the question of how it carries it has been much discussed. It is not in a crystalline state. A corpuscle examined by polarized light is not doubly refractive. Microscopists know that if there were any crystals in the corpuscle it would appear bright on a dark ground when the Nicholl prisms are crossed. It cannot be in solution, since the water which the corpuscle contains would not suffice to dissolve it. It must be combined with some constituent of the corpuscle. But whether it is uniformly distributed throughout the disc, or in a semifluid form enclosed in spaces in a sponge-work; or whether the corpuscle is a hollow vesicle enclosing fluid hæmoglobin-a view which was long ago maintained, and has recently been revived-are questions which still await further evidence.

Red blood-corpuscles, properly so called, are found only in vertebrate animals, although invertebrate animals, from worms upwards, possess genuine blood, and in some of them it contains hæmoglobin, or a similar pigment in the form of globules. These might be likened to the non-nucleated corpuscles of mammals, but it must be remembered that the non-nucleated cells of mammals have been evolved from the nucleated bloodcorpuscles of birds, reptiles, amphibians, and fishes. Below fishes red blood-cells are not found. Hæmoglobin is usually dissolved in the blood of invertebrate animals. It is impossible to trace any relationship between the coloured globules of invertebrates and the blood-cells of fishes. The coloured globules must be regarded as deposits or accretions of hæmoglobin held together by a proteid substance.

The nucleated red corpuscles of submammalian vertebrates multiply by cell division while circulating in the blood-stream. $\mathrm{A}^{*}$ good subject in which to look for dividing corpuscles is the blood of a newt in spring-time, when rapidly increasing activity calls for an additional supply. There is nothing to distinguish the method of division of a nucleated blood-corpuscle from that of any other cell.

The life-story of the red blood-corpuscles of mammals is one of the most fascinating that the histologist has to tell. He 
wishes that he could tell it with assurance; but, unfortunately, there are many uncertainties, due to conflicting testimony, in its earlier chapters. It is unlikely that a blood-corpuscle lives for long. A month or six weeks is probably the term of its existence. The rapidity with which the stock is replenished after bleeding shows that there must be ample provision in the body for making blood-corpuscles. The rate at which they disappear after they have been added in excess shows that there is an equally effective mechanism for destroying them. If half as many again as the animal already possesses be injected into its veins, the number is reduced to its normal limit in about ten days. It is clear that they can be made and can be destroyed with great facility, and it seems a legitimate inference that production and destruction are constantly taking place. Regarding the way in which they are destroyed there is no uncertainty. We shall refer to this subject when describing the functions of the spleen. But how are they made? We can sketch their history in outline, but the evidence is conflicting with regard to all matters of detail.

In early stages of embryonic life all red blood-corpuscles are nucleated, as they are permanently in birds and the other classes of vertebrates below mammals. In embryonic mammals they multiply by division whilst circulating in the blood, just as in the newt. But it is generally believed that this is not the most important source of new ones. During the earliest stages of growth they are being formed in enormous numbers. Such instances of division as can be seen in circulating blood appear to be all too infrequent to account for their rapid multiplication, and there can be no doubt but that a more complicated method of production is more important. Their formation is described as taking place "endogenously." Certain cells termed "vaso-formative," or "vaso-sanguiformative," reach a considerable size, and become stellate in form, or branched. Their nuclei divide without the cell dividing. Each nucleus accumulates a little hæmoglobin round it. A space filled with fluid appears inside the cell. The nuclei project into this space. Then they drop off with their envelopes of hæmoglobin. The outer shell of the big vaso-formative cell becomes the wall of a capillary bloodvessel. By its branches it links up with other vaso-formative cells, making a network of vessels. 
The fluid inside it is the plasma of the blood. The nuclei and their envelopes are blood-corpuscles. This, if it be a true story, is a comprehensive way of making bloodvessels and blood at the same time. Doubts have been thrown upon its accuracy, but many leading histologists strenuously maintain that this description is correct.

At a certain period all nucleated red corpuscles disappear from mammalian blood. Non-nucleated corpuscles take their place. How are the latter formed? For a short stage of embryonic life nucleated cells containing blood-pigment are seen, or are supposed to be seen, in the liver-there is, unfortunately, great difficulty in distinguishing them with certainty from young liver-cells; later they are seen in the spleen; throughout the whole of life they are to be seen in the marrow of bone. The nucleated cells give origin to the non-nucleated corpuscles. It is hardly legitimate to call these cells persistent embryonic corpuscles. Yet the chain which connects the cells which in the embryo are capable of dividing into pairs of nucleated red blood-corpuscles, and the cells which, assuming the rôle of parent cells, do not accumulate hæmoglobin for their own purposes, but for the benefit of the red corpuscles which split off from them, is probably unbroken. In this sense they are persistent embryonic corpuscles which have deserted the blood-stream, and have taken shelter in certain tissues which are particularly favourable for cell division. The situations in which they hide themselves are singularly suggestive. In the liver there is an abundant supply of nutriment, more abundant than in any other part of the body of the embryo. Later, in the spleen, red blood-corpuscles are being destroyed. Materials available for making new ones must therefore be set free. The inside of a hollow bone is a peculiarly sheltered situation. The fat cells of marrow accumulate there after a time; but within some bones the marrow develops very little fat; hence it shows the red colour, which is due to its abundant bloodvessels. This "red marrow" is the most important seat of the manufacture of red blood-corpuscles in adult life. Unfortunately, when we try to answer the question, How are they formed? we are obliged to speak with caution. Some histologists assert that the nucleated cells divide, and that one of the two daughter cells accumulates hæmoglobin, and 
loses-that is to say, extrudes-its nucleus. Others maintain that the nucleated cells become irregular in form ; that hæmoglobin accumulates in the projecting portion of the cell ; that this projecting portion breaks off as a non-nucleated corpuscle. It would be indiscreet at the present time to pronounce in favour of either of these reports, although the decision is of theoretical importance. If the former account be true, red blood-corpuscles are nucleated blood-cells which have lost their nuclei. If the latter account be in accordance with fact, it is hardly justifiable to regard them as cells. They are parts of cells which finish their existence independently of the cell body and nucleus to which they belong. As circumstantial evidence, favouring the theory that cell division is normal and the nucleus subsequently lost, may be pleaded the existence in marrow, and also in the embryonic liver and spleen, of certain very peculiar cells. These cells have long been known as giant cells, and all attempts at accounting for them have broken down. They are relatively of immense size: their diameter may be twenty times as great as that of a red blood-corpuscle. Each contains a huge irregular, bulging nucleus. Hence the cells are termed "megacaryocytes" (bignucleus cells). They must not be confounded with the polycaryocytes (cells with several nuclei), which eat up degrading bone, although it must be confessed that megacaryocytes and polycaryocytes appear to be genetically connected. It is supposed that megacaryocytes consume the nuclei which red corpuscles extrude during the process of their conversion from nucleated cells. Traces of nuclei, or things which often look like nuclei, are found in their body-substance. Their own overgrown misformed nuclei appear to be the result of an excess of nuclear food. It is certainly remarkable that megacaryocytes are not found below mammals. They do not occur in any animal in which red blood-corpuscles retain their nuclei. Polycaryocytes are found in numbers in the bones of growing birds. They are evidently scooping out bone from situations in which it has to be displaced in order that the shape of the bone as a whole may be changed. But there are no megacaryocytes in birds. On the other hand, megacaryocytes are present in the liver, and later in the spleen, of mammals at the periods when blood-formation is occurring most actively in 
these organs. From the liver they disappear early. In most mammals they disappear from the spleen about the time of birth; but in some-the hedgehog, for example-they are found in the spleen throughout the whole of life.

-Hæmoglobin is a substance which has the property of uniting with oxygen to form oxyhæmoglobin-a compound from which the oxygen is, again, very readily withdrawn. It is extremely soluble, but may be made to crystallize by adding alcohol to blood, after setting the hæmoglobin free from the corpuscles by freezing and thawing. From the blood of Man and most other animals it crystallizes in the form of rhombic prisms, whether in the oxidized (oxyhæmoglobin) or non-oxidized condition. The addition of oxygen does not affect its crystalline form ; although crystalline, it is absolutely non-diffusible. This is due to the great size of its molecule, which is probably larger than that of any other substance which is capable of crystallizing.

The percentage composition of hæmoglobin conforms closely with that of albumin and other proteins, with this most important difference : it contains a definite proportion of iron0.336 per cent. That the percentage of carbon, hydrogen, nitrogen, sulphur, and oxygen should agree with that commonly found in proteins is inevitable, since it may be split into a part which contains all the iron, hæmatin, and a proteid part resembling albumin; and the latter constitutes 96 per cent. of its weight.

There is no doubt but that its value as a vehicle of oxygen depends upon the presence of iron. In the matter of taking up and dropping oxygen, hæmatin behaves somewhat in the same manner as hæmoglobin; whereas if iron be removed from hæmatin the "iron-free hæmatin" loses its respiratory value. It is almost certain that a molecule of hæmoglobin contains a single atom of iron. On this supposition its molecular formula may be calculated. It is not quite the same for all animals, although the variations are slight. For the blood of the horse it is as follows :

$$
\mathrm{C}_{712} \mathrm{H}_{1130} \mathrm{~N}_{214} \mathrm{~S}_{2} \mathrm{FeO}_{245} \text {. }
$$

This means a molecular weight of 16708 . We give the figures, because the properties of hæmoglobin will be better understood 
if its prodigious molecular weight is borne in mind. In a sense, the reason for the great size of its molecule is not far to seek. The atomic weight of iron $(\mathrm{Fe}=56)$ is much greater than that of either of the other elements contained in hæmoglobin. The molecule needs to be very great to float an atom of iron. As it is, the corpuscles are heavier than the plasma which surrounds them, in the proportion of about 13 to 12 . Although hæmoglobin is a crystallizable substance, its immense molecule is absolutely non-diffusible. It cannot pass through a membrane. This is of no consequence as regards the relation of hæmoglobin to the walls of the capillary bloodvessels, since it is contained in corpuscles; but it is of great importance as regards its relation to the discs which carry it. A very small quantity of enveloping substance suffices to prevent it from diffusing into the plasma of the blood. The great molecules are held together and isolated from the fluid in which they float by a minimal amount of insoluble globin.

The iron needed for the making of hæmoglobin is obtained both from meat and vegetables. The constituents of an ordinary diet provide from 2 to 3 centigrammes of iron a day. The whole of the blood contains about 4.5 grammes. When corpuscles are being destroyed in the spleen, the iron which their pigment contains is largely reabsorbed and rendered available for further use. The iron in a mixed diet is more than sufficient to counterbalance any loss. Milk contains extremely little iron. Before birth the liver and spleen accumulate a store of iron which lasts until the end of the nursing period, unless this be unduly prolonged. If it be prolonged, the child is apt to become anæmic. Iron has been administered in the treatment of anæmia ever since its presence in the red clot of blood was recognized a hundred and fifty years ago. Physicians are agreed that in the anæmia of young people it is of value ; but observations made with a view to obtaining definite data as to the increase in number of blood-corpuscles which results from the administration of iron, without any other alteration in the diet or the habits of the patient, have not given accordant results. Some observers have obtained an increase with organic compounds of iron, others with inorganic compounds; some are in favour of small doses, others of very large ones. As in the treatment by drugs of other abnormal condi- 
tions, it is difficult to isolate the effect of the drug from the effects of improvements in the general regimen. Yet physicians agree that iron accentuates the beneficial effects of fresh air and improved diet.

1. 'When the surface of the body is struck, the effect of the blow is marked at first by redness. There is nothing to show that small bloodvessels have been ruptured and blood effused beneath the skin. Next day the injured area is reddish-purple. The bruise turns blue, green, yellow, and eventually disappears. In the process of absorption, oxyhæmoglobin undergoes decomposition. First its proteid constituent is removed, leaving a coloured pigment containing iron, termed "hæmatin "; soon reduced by loss of oxygen to hæmochromogen. When Sir George Stokes first described the spectrum of blood (cf.p. 185), he showed that as hæmoglobin may exist in an oxidized and in a non-oxidized condition, distinguished by their spectra, so also may the coloured residue which is left after the proteid constituent has been removed from hæmoglobin. This coloured residue he termed, when oxidized, "hæmatin" ; when not oxidized, "reduced hæmatin." Stokes's reduced hæmatin is now termed "hæmochromogen." Hæmochromogen stands for the coloured nucleus of hæmoglobin. Although it is not present in hæmoglobin as hæmochromogen-hence we must not speak of hæmoglobin as made of a protein, $x$, plus hæmochromogen, $y$-it is to its coloured residue that hæmoglobin owes its value as a carrier of oxygen. Later, iron is removed from hæmochromogen, leaving hæmatoidin, a substance often found at the seat of old hæmorrhages, where it may remain unchanged for a very long time. Hæmatoidin is apparently identical with the yellow pigment of bile, bilirubin. The green colour which shows itself in the bruise seems to indicate that the more oxidized bile-pigment, biliverdin, is formed in the first instance. Red corpuscles, when destroyed in the spleen, pass through transformations similar to those which blood undergoes when effused beneath the skin. Their protein is used by the phagocytes which eat them. Their iron is reserved for the use of the blood-forming cells of the red marrow of bone. The pigment which remains as the residue of hæmoglobin is carried by the splenic vein to the liver, which secretes it as bile-pigment. So much of the bile-pigment as is 
reabsorbed by the wall of the alimentary canal is eventually excreted as the pigment of urine.

Such is the history of the changes which blood-pigment undergoes within the living body. To a certain extent its chemistry can be followed in the laboratory; but it must be remembered, when we are treating of the chemistry of a substance as complex as hæmoglobin, that the products which can be obtained from it in the laboratory are not necessarily those into which it is transformed in the body. In the laboratory oxyhæmoglobin is easily changed into methæmoglobin, a substance of the same percentage composition, but with its oxygen more firmly fixed. Methæmoglobin can be decomposed into a proteid substance and hæmatin. Hæmatin, when acted on by reducing agents, becomes hæmochromogen. Hæmochromogen, when subjected to such a reducing agent as a mixture of tin and hydrochloric acid, gives rise to coloured bodies closely resembling bile-pigments - not as they are secreted by the bile, but as they appear in the urine. It is impossible to prove that the changing colours of a bruise indicate a sequence of chemical transformations from hæmoglobin to bile-pigment, but it is not improbable that such a description is correct. The test commonly used to ascertain the presence of bile-pigment, i.e., bilirubin, is the play of colours which it exhibits when oxidized by fuming nitric acid. From yellow it turns to green, to blue, and then to purple, more or less reversing the colours of the bruise. It is fairly certain that effused blood undergoes changes along lines which, if not identical with those through which blood passes on its road to bile-pigment, are at any rate very similar.

Coagulation of Lymph and Blood.-Two or three minutes after blood has been shed it begins to clot. In ten minutes the vessel into which it has been received may be inverted without spilling the blood. After a time the jelly, holding all the corpuscles, shrinks from the sides of the jar. It squeezes out a transparent, straw-coloured fluid-serum. The clot continues to contract until, in a few hours, about one-half of the weight of the blood is clot, the other half serum. Lymph coagulates like blood, but most specimens clot more slowly, and the product is less firm.

When the process is watched through the microscope-a 
fow drops of the almost colourless, transparent blood of a lobster afford an excellent opportunity of studying the formation of the clot-innumerable filaments of the most delicate description are seen to shoot out from many centres. They multiply until they constitute a felt-work. In the case of blood obtained from a vertebrate animal, this felt-work holds the corpuscles in its meshes. Its filaments exhibit a remarkable tendency to contract. They shorten as much as the enclosed corpuscles allow.

The filaments may be prevented from entangling the corpuscles by whipping the blood, from the instant that it is shed, with a bundle of twigs or wires. The fibrin collects on the wires, while the corpuscles remain in the serum. If this fibrin is washed in running water until all adherent serum and corpuscles are removed, it appears as a soft white stringy substance which, when dried, resembles isinglass.

Clotting is a protection against hæmorrhage. As it oozes from a scratch or tiny wound, blood clots, forming a natural plaster which prevents continued bleeding. It has little if any influence in resisting a strongly flowing stream of blood. But a clean cut through a large vessel is an accident which rarely happens as the result of natural causes. It is not the kind of injury to which animals are liable. When an artery is severed by a blunt instrument, the muscle-fibres of its wall contract. They occlude the vessel. The blood clots at the place where the vessel is injured, and plugs it. This happens also when a surgeon ties an artery. He is careful to pull the ligature sufficiently tight to crush its wall. His sensitive fingers feel it give. He stops before the thread has cut it through. As will be explained later, the clotting of blood is promoted by contact with injured tissue. If in tying an artery its wall be not crushed, the blood in it may remain liquid. When it is skilfully tied, the blood clots, forming a firm plug which is practically a part of the artery, by the time that the silk thread used in tying it is thrown out, owing to the death of the ring of tissue which it compressed. After a tooth has been extracted, the cavity is closed and further bleeding stopped by clotted blood.

When large vessels have been severed, the copious hæmorrhage which follows induces fainting. For a short time the heart stops, or beats very feebly. The blood-pressure falls. 
The bloodvessels contract. A clot has time to form. An emotional tendency to faint at the sight of blood is a provision for giving the various causes which stop bleeding an opportunity of coming into play. It is a useful reflex action, always supposing that the person who is liable to it faints at the sight of his own blood. Amongst other reasons for the greater fortitude of women-they are far less subject to this emotional reflex than men-might be alleged the circumstances of life of primitive people. It was the part of their women-folk to dress wounds, not to receive them.

The phenomenon of coagulation has attracted attention from the earliest times. It was a phenomenon that needed explanation, and culinary experience suggested analogies close at hand. Hippocrates attributed the clotting of blood to its coming to rest and growing cold. The blood which gushed from a warrior's wound formed a still pool by his side. It set into a jelly as it cooled. Until the second quarter of the nineteenth century this theory was deemed sufficient. It then occurred to two men of inquiring mind to institute control experiments. John Davy placed a dish of blood upon the hob. William Hunter kept one shaking. In both experiments the blood clotted more quickly than it did in vessels of the same size, containing the same amount of the same blood, left upon the table.

Even before this date an observation had been made regarding the circumstances in which clotting occurs, which has thrown much light upon the causes of the phenomenon. In 1772 Hewson gently tied a vein in two places. At the end of a couple of hours he opened the vein. The blood was still liquid, but clotted in a normal manner after it was shed. Scudamore showed that blood clots more slowly in a closed than in an open flask. A new theory, as little trustworthy as Hippocrates', was based upon these observations. Blood clotted because it was exposed to air. A record of all observations of the circumstances of coagulation, and of all the theories to which they have given rise, would make an exceptionally interesting chapter in the history of human thought. It would bring into singular prominence stages in the development of what is now known as the "scientific method." Not that Science has a method of her own. Philosophers of all classes would follow 
the same method if their data allowed of its application. The peculiarity of the data with which Science deals is that they can be brought to a test of which the data of historical, or political, or economic theory are not susceptible. They can be confronted with control experiments. The control experiment is the alphabet and the syntax of the scientific method. No hypothesis is admissible into the pyramid of theory until it has passed this test. A natural phenomenon is observed. Every measurement which is applicable is taken and recorded-time, weight, temperature, colour. Scientific observation implies the tabulation of all particulars which are capable of statistical expression. Reflecting upon the relation of the phenomenon to other phenomena of a like nature, the philosopher-it is the philosophy of physiologists which interests us-formulates an hypothesis as to its cause. At this point the real difficulty of applying the scientific method begins. It is easy to formulate hypotheses. It is very difficult to devise control experiments. An experiment must be arranged which will provide that, while all other conditions in which the phenomenon has been observed to occur are reproduced, the condition which was ex hypothesi its cause shall be omitted. This digression into the philosophy of science may seem to be somewhat remote from our line of march, but it may perhaps hasten our progress in the comprehension of the story of physiology. There is no other science in which the control experiment plays an equally important part. Unless this is realized, the whole trend of experimental work will be misunderstood. Scudamore explained coagulation as due to contact with air. Based on the observations we have cited, no hypothesis could have seemed more reasonable. With a view to checking this hypothesis, blood was received into a tube of mercury. It coagulated in the Torricellian vacuum. Scudamore's hypothesis, like many earlier and later, when confronted with a control experiment, was turned away, ashamed.

Clotting is a property of plasma. Red corpuscles play no part in the process. Coagulation does not occur in a living healthy vessel. It occurs when the vessel, and especially when its inner coat, is injured. It is hastened by contact with wounded tissues, especially with wounded skin. Contact with a foreign body also starts coagulation. If a silk thread is 
drawn through a bloodvessel, from side to side, fibrin filaments shoot out from the thread, as well as from the wound inflicted on the vessel by the needle which was used to draw it through.

Plasma contains a substance which sets into fibrin. It has been termed "fibrinogen." It is present in lymph, and in almost all forms of exuded lymph. If sodium chloride (common salt) is added to plasma until it is half saturated-until it has dissolved half as much as the maximum quantity which it can dissolve-fibrinogen is thrown down as a flocculent precipitate. It can be redissolved and reprecipitated until it is pure. When fibrinogen was separated from plasma a step was taken towards the explanation of coagulation. Under certain conditions fibrinogen sets into fibrin. The question which then presented itself for solution was as follows: What is the substance which, by acting upon or combining with fibrinogen, converts it into fibrin? The clue to the solution of this question was obtained from the consideration of certain observations made by Andrew Buchanan in 1830, but long neglected, because their significance was not understood. Buchanan had observed that some specimens of lymph exuded into a lymph-space-the peritoneal cavity, for example-will clot; others will not. He noticed that they clot when, owing to puncture of a small bloodvessel during the process of drawing them off, they are tinged with blood. Determined to ascertain which of the constituents of blood is effective in rendering noncoagulable effusions capable of clotting, he added to them in turn red blood-corpuscles, serum, and the washings of bloodclot. Either of the two latter was found to contain the clotprovoking substance. Thirty years later a German physiologist prepared fibrinogen from effused lymph by precipitating it with salt. He also treated serum in a similar way, precipitating a protein which he termed fibrinoplastin. When these two substances were dissolved and the solutions mixed, he obtained a clot, which he regarded as a compound of fibrinogen and fibrinoplastin. Subsequently he found that the mixture did not always clot, but he discovered that if he coagulated blood with alcohol, and washed this residue, the washings added to the mixed solution just referred to invariably produced a clot. Thinking that the substance which he obtained from his 
alcohol-coagulated blood could not be proteid, he termed it "fibrin-ferment." He neglected the control experiment. $\mathrm{He}$ failed to ascertain whether or not all three substances were needed. Had he tried adding fibrin-ferment to fibrinogen, he would havediscovered that the further addition of fibrinoplastin was unnecessary. He did not ascertain, as he might have done, that the weight of fibrin formed is somewhat less, not greater, than the weight of fibrinogen used. (Fibrinogen gives off a certain quantity of globulin when it changes into fibrin.) He was also wrong in supposing that the water which he added to alcohol-coagulated blood dissolved no protein. His "fibrinferment" is always associated with a protein. Since it may also be obtained from lymphatic glands, thymus gland, and other tissues which contain lymphocytes, it has been inferred that it is itself a protein, of the class known as nucleo-proteins. The fact that it is destroyed at so low a temperature as $55^{\circ} \mathrm{C}$. has been supposed to confirm the theory that it is a protein. But with regard to the chemical nature of fibrin-ferment, as of all other ferments, we are at present in the dark. Under ordinary circumstances, when blood clots, the fibrin-ferment, or plasmase, or thrombin-it has received various names-is set free by leucocytes. Fluids which contain fibrinogen clot on the addition of a "ferment" which is either secreted by leucocytes or set free from leucocytes when they break up-as they are very apt to do, as soon as the conditions upon which their health depends are interfered with.

Freshly shed blood contains minute particles, termed "platelets," in diameter measuring about a quarter that of a red bloodcorpuscle. When the inner coat of a vessel is injured, platelets accumulate at the injured spot. They form a little white heap, from which coagulation starts. Evidently they supply the ferment, or a precursor of the ferment. As yet their origin has not been traced. They are too large to be the unchanged granules of granular leucocytes, but that they are in some way derived from leucocytes seems probable.

The further study of coagulation has shown that the conditions under which it occurs are more complicated than the simple explanation just given would seem to imply. This explanation holds good, so far as it goes, but facts connected with the details of the process have recently been brought to 
light which warn the physiologist that as yet his theory of coagulation is incomplete.

The presence of salts of lime has an important relation to coagulation. If blood is received into a vessel in which has been placed some powdered oxalate of potash, or soap, or any other chemical which fixes lime, the blood does not coagulate. All other conditions are as usual, but lime is withdrawn from the plasma. The non-coagulation of oxalated plasma was interpreted as indicating that lime, under the influence of fibrin-ferment, combines with fibrinogen to form fibrin; that fibrinogen altered by fibrin-ferment combines with lime. This hypothesis was based upon the analogy of the curdling of milk. Milk cannot curdle if lime be absent. If rennin (milkferment), prepared from milk from which lime has been removed, be added to a solution of caseinogen (the coagulable protein of milk), also prepared from lime-free milk, no curd is produced. The addition of a few drops of a solution of chloride of lime results in the immediate curdling of the mixture. Evidently rennin so alters caseinogen as to bring it into a condition to combine with lime. But the analogy does not hold good for blood. In the case of plasma, lime acts, not upon fibrinogen, but upon the fibrin-ferment-or rather upon a precursor of fibrin-ferment-in such a way as to render it effective. Leucocytes produce a prothrombin, which in contact with lime-salts is converted into thrombin, which coagulates fibrinogen.

Fibrinogen is the substance which fibrin-ferment combined with salts of lime changes into fibrin. Yet even now the story is not complete, if the theory of coagulation is to be brought up to date. A perfectly clean cannula is passed into an artery of a bird. If it be thrust well beyond the place where the vessel has been cut, if the vessel be tied so gently as to avoid injury to its inner coat, and if the blood which first passes through the cannula be allowed to escape, the blood subsequently collected will not clot. It contains fibrinogen, lime salts, and fibrin-ferment, ordinarily so called; but the ferment is ineffective. The addition to the blood of a fragment of injured tissue, or of a watery extract of almost any tissue, immediately sets up coagulation. This observation brings fibrin-ferment into line with other ferments. Digestive 
ferments are secreted as zymogens, which require to be influenced by a kinase before they acquire fermentative activity. So, too, must thrombogen be changed into thrombin, under the influence of thrombokinase, before it can act upon fibrinogen. Almost all tissues yield the kinase which actuates fibrin-ferment. The utility of this provision is manifest. A bird's blood contains everything necessary to form a clot with the exception of thrombokinase. The injury which brings the blood into contact with a broken surface supplies this ferment of the ferment. Fibrin-ferment, rendered active, at once changes fibrinogen into fibrin. The same interaction is necessary before the blood of a mammal is susceptible of clotting. But a mammal's blood is even readier to clot than is the blood of a bird; for not only will a broken surface provide it with thrombokinase, but the leucocytes contained within the blood, when injured, also yield it. And the leucocytes are exceedingly sensitive of any change of circumstance ; on the slightest indication that conditions are not normal they set free, perhaps owing to their own disintegration, the kinase which turns thrombogen into thrombin.

There is a constitutional condition, fortunately rare, in which blood does not coagulate. A person subject to this abnormality is said to suffer from hæmophilia. It is alleged that this condition is due to deficiency of lime in the blood; and the deficiency of lime is said to be due to excess of phosphates. The subject suffers from phosphaturia. His kidneys get rid of the superabundance of phosphates by excreting them in combination with lime. If this explanation be correct, there is a chronic insufficiency of lime in the blood, because it is being constantly withdrawn in the process of removing phosphates.

The difficulty in the way of establishing a complete theory of the coagulation of blood increases when the phenomena of incoagulability are considered. Blood may be rendered incapable of clotting in a variety of ways. Leeches and other animals which suck blood have the capacity of rendering it incoagulable. If the heads are removed from a score of leeches, thrown into absolute alcohol, dried, ground in a pepper mill, extracted with normal saline solution, a dark turbid liquor is obtained. This liquor, after filtration and 
sterilization at a temperature of $120^{\circ} \mathrm{C}$., injected into the veins of an animal, renders its blood incoagulable.

The preparation sold by druggists under the name "peptone," when injected into the veins of a dog, renders its blood incoagulable. Commercial "peptone" is a mixture of many substances. Its anticoagulation-effect is not due to the peptone which it contains. It has been supposed to be due to imperfectly digested albumin and gelatin (proteoses), but products of bacteric fermentation (toxins and ptomaines) are more probably the active bodies. Not only is the peptonized blood of a dog incoagulable, but if this blood be injected into the veins of a rabbit (an animal upon which the direct injection of peptone has no effect), it diminishes the coagulability of the rabbit's blood. If peptonized blood be mixed in a beaker with non-peptonized blood, it prevents the coagulation of the latter. There is little doubt but that the poison, whatever it may be, acts upon the leucocytes; and there are some reasons for thinking that the poison is not contained in the "peptone," but is secreted by the liver of the animal into which the "peptone" has been injected.

A still more remarkable property in relation to coagulation must be assigned to leucocytes. The blood of a dog which has been rendered incoagulable by injection of peptone recovers its coagulability after a time. If a further injection of "peptone" be made, the animal is found to be immune. Injection of "peptone" no longer renders its blood incoagulable. In a similar manner the blood develops a power of resisting the action of agents which induce its coagulation whilst circulating in the vascular system. Nucleo-proteins contained in extracts of lymphatic glands and other organs when injected into the veins of living animals cause their blood to clot, provided they are injected in sufficient quantity. If they are injected in quantity less than sufficient to induce coagulation, they render the animal immune to their influence. A larger quantity given to an animal thus prepared fails to take effect. This brings the phenomena of coagulation and resistance to coagulation to the verge of chemistry. They extend into the domain in which pathology reigns. Tempting though it be to record other facts with regard to these phenomena which recent investigation has brought to light, it is 
probably judicious to leave the problem at the frontier. Across the frontier lies a fascinating land, rich with unimaginable possibilities for the human race. Settlement is rapidly proceeding in this country, which is charted, like other borderlands, with barbarous names: "antibodies," "haptors," "amboceptors," "toxins," "antitoxins," and the likefinger-posts to hypotheses which show every sign of hasty and provisional construction. But certain facts stand out, in whatever way theory may, in the future, link them up. The virus of hydrophobia, modified by passing through a rabbit, develops in human beings, even when injected after they have been infected, the power of resisting hydrophobia. The serum of a horse which has acquired immunity to diphtheria aids the blood of a child, which has not had time to become immune, in destroying the germs of this disease. It is a contest between the blood and offensive bodies of all kinds which find entrance to it, whether living germs or poisons in solution; with victory always, in the long-run, on the side of the blood, provided its owner does not die in the meantime. And not only is the blood victorious in the struggle with any given invader, but having repulsed him, it retains for a long while a property which neutralizes all further attempts at aggression on his part. In the past, physicians have fought disease with such clumsy weapons as mercury, arsenic, and quinine. Now they anticipate disease. In mimic warfare with an attenuated virus the blood is trained to combat. Smallpox which has been passed through the body of a cow is suppressed by the blood's native strength. The exercise develops skill to deal with the most virulent germs of the same kind. In cases in which physicians cannot anticipate disease in human beings, they train the blood of animals to meet it ; and, keeping their serum in stock, they can, when the critical moment arrives, reinforce the fighting strength of the patient with this mercenary aid.

The Spleen.-The spleen is placed on the left side of the body, and rather towards the back. It rests between the stomach and the inner surface of the eighth, ninth, tenth, and eleventh ribs. It is quickly distinguished from other organs by its brown-purple colour, a sombre hue to which it owed its evil reputation with the humoralists. The liver's yellow bile 
tinged man's mental outlook, preventing him from seeing objects in their natural brightness; but the spleen made black bile, which, mounting to the brain, displayed its malign influence upon the action of that organ, as, or in, the worst of humours.

The spleen is invested with a capsule of no great toughness. Inside the capsule is "spleen pulp." When the fresh organ is cut across, it is seen that, although most of the pulp is of the colour of dark venous blood, it is mottled with light patches. In some animals-the cat, for example-these whitish patches are small round spots, regularly arranged at a certain distance from the capsule. The distinction into "red pulp" and "white pulp" marks a division into two kinds of tissue with entirely different functions. The white pulp is lymphoid tissue, lymph-follicles developed in the outer or connective-tissue coat of the branches of the splenic artery. Its function is to make lymphocytes, of which, for reasons which will shortly appear, the spleen needs an abundant supply. The constitution of the red pulp is entirely different, and peculiar to the spleen. The branches of the splenic artery divide in the usual way into smaller and still smaller twigs until the finest arterioles are reached; but these arterioles do not give rise to capillary vessels. At the point at which in any other organ their branches would attain the calibre of capillaries, the connective-tissue cells which make their walls scatter into a reticulum. They are no longer tiles with closely fitting, sinuous, dovetailed borders, but stellate cells with long delicate processes uniting to constitute a network. The blood which the arterioles bring to the pulp is not conducted by closed capillary vessels across the pulp to the commencing splenic veins. It falls into the general spongework. The venules commence exactly in the same way as the arterioles end. Stellate connective-tissue cells become flat tiles placed edge to edge. The endothelium of an arteriole might be likened to a column of men marching shoulder to shoulder, three or four abreast; the connective tissue of the pulp, to a crowd in an open place. The column breaks up into a crowd. On the other side the crowd falls into rank as the endothelium of veins. The capsule and the red pulp are largely composed of muscle-fibres. These relax and contract 
about once a minute. By their contraction the blood is squeezed out of the sponge.

If the spleen be enclosed in an air-tight box (an oncometer), from which a tube leads to a pressure-gauge-a drum covered with thin membrane on which the end of a lever rests, or a bent column of mercury on which it floats-the pressuregauge shows the changes in volume of the spleen. The long end of the lever, which records the variations of pressure in the gauge, may be made to scratch a line on a soot-blackened surface of travelling paper. A record of the variations in volume of the organ, which can be studied at leisure, is thus obtained. It shows that the spleen is sensitive to every change of pressure in the splenic artery. Small notches on the tracing correspond to the beats of the heart. Larger curves record the changes of blood-pressure due to respiration. A long slow rise and fall marks the rhythmic dilation and contraction of the spleen itself.

One of the three large arteries into which the cœliac axis divides delivers blood to the spleen direct from the aorta. The splenic vein joins the portal vein shortly before it enters the liver. Thus the spleen is placed on a big vascular loop which directs blood, not long after it has left the heart, from the aorta, through the spleen, to the liver.

The peculiar construction of the splenic pulp which brings the blood more or less to rest within its sponge-work, and the transmission to the liver of the blood which leaves the spleen, indicate that it is an organ in which blood itself receives some kind of treatment. It is not passed through it, as it is through all other parts of the body, in closed pipes. The spleen is a reservoir, or a filter-bed, into which blood is received.

The red blood-corpuscles of mammals are cells without nuclei, and with little, if any, body-protoplasm. They are merely vehicles for carrying hæmoglobin. We should deny to them the status of cell, if it were possible to prescribe the limit at which a structural unit ceases to be entitled to rank as a cell. They are helpless creatures, incapable of renewing their substance or of making good any of the damage to which the vicissitudes of their ceaseless circulation render them peculiarly liable. It is impossible to say with any approach 
to accuracy how long they last, but probably their average duration is comparatively short. The spleen is a labyrinth of tissue-spaces through which at frequent intervals all red corpuscles float. If they are clean, firm, resilient, they pass through without interference. If obsolete they are broken up. In the recesses of the spleen-pulp, leucocytes overtake the laggards of the blood-fleet, attach their pseudopodia to

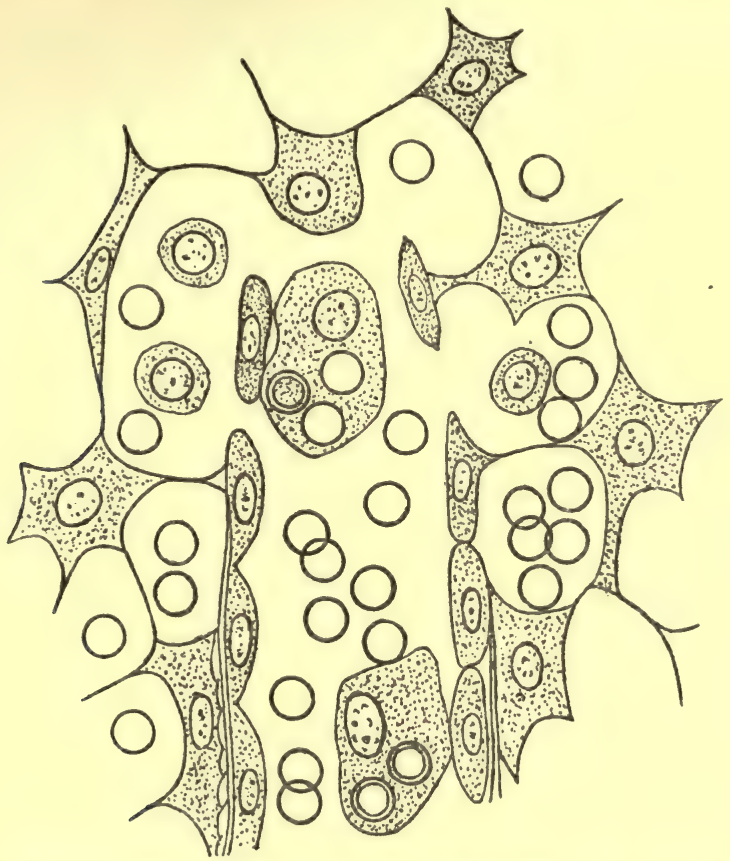

Fig. 5.-A Mindte Portion of the Pulp of the Spleen, very highly magnified.

Stellate connective-tissue cells form spaces containing red blood-corpuscles and leucocytes. In the centre of the diagram is shown the mode of origin of a venule. It contains two phagocytes-the upper with a nucleus, two blood-corpuscles just ingested, and one partially digested in its body-substance; the lower with two blood-corpuscles.

them, draw them into their body-substance, digest them. The albuminous constituent of hæmoglobin they use, presumably, for their own nutrition. The iron-containing colouring matter they decompose, and excrete in two parts; the iron (perhaps combined with protein); the colouring matter, without iron, as the pigment, or an antecedent of the pigment, which the liver will excrete in bile. Hæmoglobin is un- 
doubtedly the source of bilirubin, and general considerations lead to the conclusion that it is split into protein, iron, and iron-free pigment in the spleen; but the details of this process have never been checked by chemical analysis. Neither bilepigment nor an iron compound can be detected in the blood of the splenic vein. The only evidence of the setting free of iron in the spleen is to be found in the fact that the spleen yields on analysis an exceptionally large quantity of this metal (the liver also yields iron), and that the quantity is greatest when red corpuscles are being rapidly destroyed.

As a rule, it is very difficult to detect leucocytes in the act of eating red corpuscles; but under various circumstances their activity in this respect may be stimulated to such a degree as to show them, in a microscopic preparation, busily engaged in this operation. The writer had the good fortune to prepare a spleen which proved to be peculiarly suitable for this observation (Fig. 5). His method was an example of the way in which a physiological experiment ought not to be conducted. Having placed a cannula in the aorta of a rabbit, just killed with chloroform, he was proceeding to wash the blood out of its bloodvessels with a stream of warm normal saline solution, when the bottle from which the salt-solution was flowing overturned. Fearing lest an air-bubble should enter the cannula, he hastily poured warm water into the pressure bottle, and threw in some salt, in the hope that it would make a solution of about 0.9 per cent. The salt-solution was allowed to run through the bloodvessel for rather more than an hour. When sections of the spleen were cut, after suitable hardening, every section was found to be packed with leucocytes gorged with red corpuscles. Some of the corpuscles had just been ingested; from others the hæmoglobin had already been removed. It may be that, for some unknown reason, the destruction of red corpuscles was occurring in this particular rabbit with unusual rapidity at the time when it was killed; but it seems more probable that the animal's leucocytes were provoked to excessive activity by changes in the red corpuscles brought about by salt-solution which was either more or less than "tonic." As a score of attempts to reproduce the experiment, with solutions of different strengths, have failed, it is impossible to be sure that this is a valid explanation. 
There must be something in the condition of worn-out red corpuscles which either makes them peculiarly attractive to predatory leucocytes or renders them an exceptionally easy prey. It does not require much imagination to picture the drama which is enacted in the spleen. Slow-moving leucocytes are feeling for their food. The majority of red corpuscles pass by them; a few are held back. The leucocytes, like children in a cake-shop, cannot consume all the buns. A selection must be made, and preference is given to the sticky, sugary ones. Red corpuscles when out of order show a tendency to stick together. When blood is stagnating in a vein, or lying on a glass slide in a layer thin enough for microscopic examination, its red discs are seen after a time to adhere together in rouleaux. The parable of a child in a cakeshop is not so fanciful as it may appear.

The differentiation of function of organs is not as sharp as was formerly supposed. Evidence of their interdependence is rapidly accumulating. The activity of various organs is known to result in the formation of by-products termed "internal secretions," which influence the activity of other organs, or even of the body as a whole. The spleen enlarges after meals. This may be merely connected with the engorgement of the abdominal viscera which occurs during active digestion, or it may indicate, as some physiologists hold, that an internal secretion of the spleen aids the pancreas in preparing its ferments. The spleen enlarges greatly in ague and in some other diseases of microbial origin. This has been regarded as evidence that it takes some part in protecting the body against microbes. But whatever may be the accessory functions which it exercises, they are not of material importance to the organism as a whole, seeing that removal of the spleen causes no permanent inconvenience either to men or animals. Its blood-destroying functions are taken on by accessory spleens, if there be any, and by lymphatic glands. The marrow of bone also becomes redder and more active. Under certain circumstances, red corpuscles, or fragments of red corpuscles, are to be seen within liver-cells; but it is uncertain whether blood-destruction is a standing function of the liver. 


\section{CHAPTER V}

\section{INTERNAL SECRETIONS}

Thyroid Gland.-On either side of the windpipe, rather below the thyroid cartilage (Adam's apple), lies a somewhat conical mass of tissue. The two masses are connected by an isthmus ; lobes and isthmus make up the thyroid gland. The whole weighs about an ounce. In health it is so soft that only the finger of an anatomist could detect it through the skin and the thin flat muscles which connect the hyoid bone and the thyroid cartilage with the breast-bone. It makes no visible prominence on the front of the neck. The thyroid gland is, however, liable to enlargement, especially amongst the people who live in certain districts. In the Valais, "goitre," as it is termed, is so frequent that anyone walking up the Rhone Valley is sure to meet a number of persons - for the most part womenwhose swollen necks overhang their collar-bones, like halffilled sacks. Goitre is even more common in the Valle d'Aosta, on the Italian side of the Alps. In England this condition, comparatively rare, is known as "Derbyshire" or "Huntingdonshire " neck.

In the majority of cases the tumour in the neck develops slowly, and does not reach its full dimensions until after middle life. Goitre in this form, although inconvenient, causes no serious discomfort. But when it appears in early life, it is associated with an extraordinary complex of malformations and ill-performed functions. The condition into which a goitrous child sinks is known as cretinism. With the exception of the skull-case, its skeleton does not attain to its proper proportions; and, since the soft parts do not equally submit to arrest of growth, the dwarf is heavy and ungainly, with large jowl and protuberant abdomen. The appearance 
of distortion is extraordinarily heightened by hypertrophy of the skin and the subcutaneous connective tissue. Ears, eyelids, nose, lips, fingers, are thick and heavy. The hair and nails are coarse. The skin is folded, wrinkled, rough.

The bodily ungainliness of a cretin has its counterpart in the deformity of his mind. He is an idiot whose deficiency is chiefly marked by apathy.

Cretinism exhibits itself in varying degrees. The description that we have just given would not be accurate for all. For the sake of brevity, we have chosen a case which might be that of a goitrous cretin of a certain type, or that of a cretin whose thyroid gland, in lieu of showing what looks like overgrowth, has failed to properly develop. Nothing is more remarkable with regard to this organ than the fact that the condition associated with its overgrowth and the effects of its atrophy, or inadequate growth, are the same. A consideration of the function of the gland will suggest an explanation of this seeming paradox.

The inconvenience caused by goitre induced surgeons, about twenty-five years ago, to remove the tumour in simple uncomplicated cases. Owing to the accessibility of the gland, the operation is both safe and easy; but its removal was found to be followed by symptoms of a very serious nature, especially overgrowth and œdema of subcutaneous tissue, muscular twitchings and convulsions, mental dulness. About the same date, physicians recognized that the disease myxœdema-so called because the odema is not watery, as in dropsy, but firm and jelly-like-is due to deficiency of the thyroid gland.

No other organ of the body has so weird an influence upon the well-being of the whole. No other organ has an equally mysterious ancestral history. Assuredly the thyroid gland was not always such as we see it now. In prevertebrate animals it must have been quite different, both in structure and in function. From fishes upwards, however, its structure is always the same. It is composed of spherical vesicles or globes. Every globe is lined by a single layer of cubical epithelial cells. Its cavity is filled with a homogeneous semisolid substance known as "colloid." The globes are associated into groups or lobules. They are in contact with large 
wide lymphatic vessels. The organ has a lavish supply of blood. It is also well supplied with nerves. Colloid is the secretion of the epithelial cells which line the globes. As these globes have no openings, the secretion must be passed by osmosis into the lymphatic vessels. There is abundant reason for believing that by this route the products of the gland reach the blood, and are distributed by the blood to all the tissues of the body. And here it is important to notice that associated with the thyroid gland are certain very' small masses of tissue termed "parathyroids." There may be four of these-two on the course of the large arteries which supply the thyroid gland from above, two related with the almost equally large arteries which supply it from below ; but the number varies. The parathyroids do not contain vesicles. They are solid masses of epithelial cells, traversed by bloodvessels and lymphatics. Yet, like the epithelial cells of the vesicles, they secrete colloid. Granules of this substance are to be seen within their cells. We cannot pass over the parathyroids without this reference, since, small though they are, they seem to be quite as important as the thyroid gland itself, judging from the effects which follow their removal.

In all vertebrate animals the thyroid gland has the characters which we have described. What was it like in the ancestors of the vertebrate races? Its microscopic appearance in vertebrates, the only animals in which we know it, is so anomalous as to convince an histologist that it is a makeshift; it looks like an organ which, at a period no longer visible through the mists of time, had a quite different function to perform. This function it has lost-some other organ has taken it on-yet it must do something which is useful to the organism. Otherwise it would not have been preserved. It has been retained for the sake of its by-function, for the sake of the internal secretion which it produces. This is now the only work it has to do.

What was its prime function? It is an axiom of biology that an animal in its individual development recapitulates, albeit with many omissions and abbreviations, the ancestral history of its race. The thyroid gland appears in the embryo as a diverticulum of the anterior wall of the pharynx. It is remarkable in being a single, median, unpaired diverticulum, 
whereas almost all other organs are bilaterally symmetrical. The parathyroids are formed on the two sides in connection with certain of the branchial pouches. In its earliest development the thyroid gland resembles any other gland-a salivary gland, for example. Until a late stage it retains its connection with the back of the mouth. Occasionally indications of this primitive connection persist throughout life. In most cases the place where the duct of the thyroid gland used to open is clearly marked. At the back of the tongue-too far back to be seen without the aid of a dentist's mirror-there is a Vshaped row of large papillæ (papillæ circumvallatæ). Just behind the meeting-point of the two limbs of the $\mathbf{V}$ a pit is to be seen-foramen cæcum. This pit is the vestige of the mouth of the duct of the thyroid gland which opened into the pharynx in the ancestors of fishes. It is an inconceivably long time since fishes diverged from other races of animals. We do not know which of the various orders of invertebrate animals now existent most nearly resembles our prepiscine ancestor. The organ which has developed into the thyroid body of mammals may possibly have disappeared from all the other descendants of the common stock from which vertebrates and their nearest relatives in the invertebrate sub-kingdom were evolved; but it is much more likely that it has been preserved, and is still performing its prime function in the higher invertebrate animals. Probably it is a functional organ in a cuttle-fish or a scorpion or a worm, but so unlike the thyroid gland of vertebrates that we fail to recognize its homology. There are other instances in the body of the persistence of an organ long after it has fallen into such ruin that not even archæologically-disposed biologists can guess what it was like, or what purpose it served in the days when it was at the height of its efficiency; but perhaps there is none other which so pregnantly illustrates the physiological doctrine of functional interdependence. Nature shows herself amazingly conservative in retaining primal organs - the pituitary body, the thymus gland, the thyroid gland, the suprarenal capsules-organs which millions of years ago forgot the very rudiments of their craft ; but her conservatism is not mere force of habit. Although she no longer has any use for the wares which she created these pieces of apparatus to make, she cannot do without their 
refuse. Evon the vermiform appendix may have its use. Dr. Gaskell's theory of the thyroid gland involves a transformation so fantastic that it would provoke a smile of incredulity were we to set it forth without a prologue far more lengthy than our space permits. Yet Dr. Gaskell may be right. We can but guess as to the nature of the prime functions of the thyroid and parathyroids. For many geological epochs they have not been exercised. But whatever else they did when they were indispensable constituents of the organism, their activity was accompanied by the secretion of colloid. Colloid is not made by other organs; therefore the otherwise obsolete thyroids are retained. It is of course not impossible that, in a certain degree, Nature, like a thrifty housewife, finds a new use for superseded apparatus; but we are probably justified in believing that the use is never really new. Not wanting the organ for its original specific purpose, Nature relegates to it alone work which hitherto it had shared with other of her tools.

A comparatively short while ago the attention of physiologists was wholly concentrated upon the obvious or prime functions of organs. Muscles contracted. The stomach digested. The pancreas secreted pancreatic juice. The brain made thought. Now they understand, to put it somewhat metaphorically, that gastric juice is made in the calves of the legs; the ferment of pancreatic juice in the small intestine; thought of a certain emotional quality in the large intestine. The chemistry of the laboratory is far behind the body's chemistry. We cannot detect in the blood coming from contracting muscles the stimulant-possibly a precursor of pepsin - to which the stomach reacts, although the magical benefit of exercise seems to prove that there is a chemical connection between the activity of the muscles and the activity of the glands of the alimentary canal. It has been proved by experiment that a substance produced in the epithelium of the small intestine is the messenger upon whose call depends the potency of pancreatic juice. The clearing of the brain effected by a judicious pill shows that poisons of some kind are absorbed into the blood from an overloaded large intestine. None of the organs lives altogether for itself. The chemical products which it throws off, absorbed by the blood, regulate the 
activity of other organs. Formerly the several parts of the body were looked upon as independent. Their activity was regarded as a direct response to the commands of the nervous system. If it varied in kind, the variations were supposed to depend upon the quality of the nervous impulses which reached the organ. Evidence is rapidly accumulating that many exhibitions of function are evoked by the calls of "hormones," or chemical messengers, not by command of the nerves.

Internal secretions, using the expression in its general sense, are necessary for the co-ordination of the work of the various parts of the animal mechanism. Colloid is the internal secretion of the thyroid gland and of the parathyroids. Unlike most other internal secretions, it is a substance easily analysed, and startlingly definite in its chemical characteristics. It is composed mainly of a protein which contains iodine. From this protein a substance termed "iodothyrin" may be obtained, of which no less than 10 per cent. is iodine; but it is uncertain whether iodothyrin is preformed in the gland. The exact nature of the active substance of the internal secretion of the thyroid gland matters little. Whether it be iodothyrin or a protein, its activity depends upon the fact that it contains iodine in large quantity. Iodine amounts to from 0.3 per cent. to 0.9 per cent. of the weight of the whole thyroid gland in Man.

Iodine is very widely distributed in Nature. It is present in the air, in rain-water, in herbage. It is also present in all parts of the body, although in quantities which are infinitely minute. It is found in sea-water, and is relatively abundant in marine plants. There is no reason for supposing that it is deficient in districts in which goitre is common. It would appear more likely that the soil has properties which result in the fixing of iodine in a form in which it is not available for plant-food, and that in consequence animals are unable to obtain a sufficient supply. Careful analyses have shown that the thyroid glands of sheep bred in mountainous districts where goitre is common contain but one-thirtieth part as much iodine as the thyroid glands of sheep bred in places where goitre is rare. In ancient times burnt sponge and seaweed were esteemed useful in the treatment of goitre. Later, iodide 
of potassium given internally, and tincture of iodine as an outward application, were the approved remedies. It is now known that myxœdema and certain forms of goitre may be checked, and even cured, by administering uncooked thyroid gland or even tabloids of dried extract. Fortunately, it is not necessary to inject it subcutaneously; the iodine-containing compound is so stable as to resist the action of gastric juice.

Iodine stored in the thyroid and parathyroid glands is distributed to all the tissues. The remarkable symptoms which indicate that the tissues are not receiving an adequate supply may occur under either of two conditions. Iodine may be deficient in the food, or the thyroid gland may be incompetent; the former is the commoner cause. And here we see the explanation of the formation of a goitre. By increasing the size of the organ which selects iodine, Nature attempts to obtain and store an adequate supply for distribution to the tissues.

Cretinism has been observed in animals. If attention were directed to this inquiry, it might be found that it is not so exceedingly rare as would be judged from the few observations that have been recorded. A cretin, if a wild animal, falls an easy prey. If a domesticated animal, little trouble is taken to insure its survival. A myxœdematous pig is a dwarf with coarse, sparse hair, thick, warty hoofs, large jowl, heavy ears. It is apathetic. A piglet presenting these characteristics is not altogether uncommon in a litter. Among chickens and pigeons, also, individuals appear which might, judging from their uncouth appearance and mental dulness, be suffering from cretinism. The only way of proving that this is the case is to feed them on thyroid glands; it does not matter from what animal the gland is obtained. Operative cretins, produced by removing the thyroid gland soon after birth, recover their natural characters on a diet containing a daily allowance of thyroid gland. The coarse hairs, or wiry towsled feathers, fall off, and are replaced by a smooth, supple growth. The thickened skin becomes soft and pliant. Mental apathy gives place to alertness. They make up for lost time by growing more rapidly than other animals of the same age, which have not been operated upon, although they never surpass the normal stature. 
Suprarenal Capsules.-Each of the kidneys is capped by a pyramidal body weighing about $\frac{1}{6}$ ounce. Small though it be, this organ is essential to life. As Dr. Addison was the first to discover, in 1855, its disease results in a cycle of symptoms which invariably has a fatal termination. A college friend of the writer suffered from "slackness." Before he had finished a set of tennis, he abandoned the game, and spent the rest of the afternoon lying on the grass, wrapped in a rug. After hall, although he earnestly desired to conquer the subtleties of the Greek grammar, he fell asleep over his books. As his countenance was not ruddy merely, but bronzed like that of a man who has just returned from a yachting cruise, he was the butt of many a joke. Although already a qualified medical man, who had been in practice-he had come to the University with a view to adding the degree of M.D. to his M.R.C.S. - he had no suspicion that he was ill. Thought he wanted "freshening up." Took a trip across the Atlantic. Stumbled over a rope on landing; broke his thigh. Spent two months in a New York Hospital, but the bone did not mend. At last, the surgeons, growing anxious, sent him back to London. He was seen by a leading physician, who told him that he was suffering from Addison's disease. Two months later he died of failure of the heart. Disease of the suprarenal capsules is usually of tuberculous origin. Its symptoms : muscular weakness and excessive liability to fatigue; abnormal pigmentation of the skin ; lowered blood-pressure, and consequent sensitiveness to cold; cardiac weakness. As the pigmentation of the skin and mucous membrane is not invariable, and since it may occur without disease of the capsules, it is not improbable that it is due to disease of the abdominal sympathetic ganglia, which are usually affected at the same time as the capsules.

The suprarenal capsules are composed of columns of epithelial cells, which radiate from a large vein in their centre. They are abundantly supplied with blood and with nerves. The cells near the vein are much larger than those in the peripheral portions of the columns. Amongst them are nerve-cells resembling those of the sympathetic system.

The history of the suprarenal capsules is almost as obscure as that of the thyroid gland. In the embryo they are relatively 
very large-larger at one period than the kidney. At this period bloodvessels are formed in them with great rapidity by a curious process of boring through and channelling out of their cells. There are other facts connected with their development in the individual and their varying form in different classes of vertebrate animals which point to a "previous existence," but there is nothing to indicate that they were ever open glands. In all vertebrates they are closed masses of cells, the only function of which, so far as we know, is to produce an internal secretion; but the importance of this chemical messenger in bringing about the proper working of other organs is almost startlingly evidenced by the collapse which follows disease, or removal of the organ which produces it.

The suprarenal capsules yield a substance which has been termed "adrenalin." It contains nitrogen, is crystallizable and dialysable; but its chemical relationships have not been made out as yet. It is not destroyed by boiling, nor by digestion with gastric juice. Injected into a vein, it causes, amongst other effects, an immense rise in blood-pressure, even though the amount injected be extraordinarily small. Applied locally as a wash or spray, a solution of 1 part in 10,000 produces marked blanching of the surface; and it is useful, in consequence, as a means of checking bleeding in small operations, especially those on the eye or the nose. It is a most energetic poison. Even $\frac{1}{4}$ milligramme is sufficient to kill a rabbit. In short, adrenalin acts like the most powerful drugs known to physicians; and this drug, manufactured by the suprarenal capsules, is constantly added to the blood. Disastrous consequences follow a failure in the regular supply.

The tone of the vascular system is maintained by adrenalin. The nature of its influence upon muscles is not known, but probably the complete loss of muscular strength, which is one of the most noticeable symptoms of disease of the suprarenal capsules, is an indirect result of the lowering of blood-pressure. The muscles, it must be remembered, make up about one-third of the weight of the body of a muscular man. For the exchange of their waste products for food, they are dependent upon an efficient circulation. They are unable to display their normal vigour when the vascular system is not up to its work.

The Pituitary Body is another ductless gland of dubious 
history. It is a round body, the size of a small marble, which occupies a deep recess in the floor of the skull, beneath the centre of the brain. It is composed of epithelial cells collected into irregular groups. No homologue of the pituitary body can be found in the invertebrate sub-kingdom. Its strange mode of development in vertebrate animals-it is present in them all, from fishes to mammals-and the mystery in which its prevertebral existence is hidden, provoke to speculation. We must be content to state that it is undoubtedly masquerading under an assumed name. "Pituitary body" is reminiscent of a long-abandoned theory that it secretes fluid into the upper chamber of the nose.

Disease of the pituitary body is associated with a perversion of growth even stranger than that due to disease of the thyroid gland. The condition has been termed "acromegaly," to indicate that all extremities-toes, fingers, nose, lips, tongueundergo enlargement.

With these three organs - the thyroid gland, the suprarenal capsules, and the pituitary body-we must leave the subject of internal secretions. Each of these organs is a ductless gland. Each has a history which the zoologist is unable to transcribe. The document is a palimpsest, the earlier script so faint as to be illegible beneath the dark letters which a new era has written over it. Even the modern script is smudged and blotted. The laws which it sets forth seem, as a rule, to be destitute of sense, but a sinister meaning is evident at times. We are tempted to regard these codes as obsolete, until the mischief which follows their suppression calls our startled attention to the fact that they are, in the most lively sense, extant. Myxœdema, Addison's disease, acromegaly, are ominous warnings that the three ductless glands are no mere monuments of a past epoch, which owe their survival to Nature's indolence. They teach us that we must not attribute the persistence of such organs to a conservatism which resists innovation, or suppose that they would long ago have been wiped off the statute-book if her inertia could have been overcome. Undoubtedly Nature gives us many excuses for adopting this attitude of mind. The "chestnuts" on a horse's legs, the "dew-claws" of a dog's foot, are vestiges which would have disappeared if every part of 
the body had to establish its claim to be regarded as useful before it became entitled to share in the common supply of food; so, at least, we are disposed to think. But, tempting though it be to attribute to sheer conservatism the retention of an organ which has been superseded in its original functions, and for which we cannot recognize any new use, it is a temptation which must be severely checked. It is safer to suppose that the fact that it has been retained is prima-facie evidence that the body has need of it.

There can be no doubt as to the importance of the internal secretions of the three chief ductless glands. What about other organs-the glands which make external secretions, for example? Does each of them make also an internal secretion which influences the activity of other organs? It is very difficult to prove the production of internal secretions by such organs as the salivary glands, the pancreas, the kidneys, because all the effects which result from their removal may be due to the suppression of their external secretions. It is almost impossible to distinguish the consequences which might be due to the abolition of an internal secretion from those which ought to be attributed to the loss to the body of the chief functions of the organ. Certain physiologists are inclined to think that all organs-not only the glands, but the liver, spleen, muscles, etc.--produce chemical messengers which are discharged into the blood ; and recent discoveries tend to justify this view. As the time approaches when milk will be wanted for the nourishment of offspring, it begins to appear in the breast. Hitherto this has been attributed to nervous contrcl. It is now known that the secretion is provoked by a chemical messenger. If this messenger, extracted from the organ in which it is formed, be injected into the veins of an animal which has no call to secrete milk, it sets up a condition of activity in its mammary glands. Such an illustration of the possibilities of chemical, as distinguished from nervous, control inclines us to attribute the harmonious working of the body in large measure to the mutual influence of its several parts, instead of invoking in every case, as used to be the custom, the directing power of a somewhat bureaucratic nervous system.

It is curious to note that an internal secretion is essentially 
a drug. Faith in drugs has suffered eclipse in latter days, and with good reason. The medicines of fifty years ago so little resembled Nature's pharmacy that there is cause enough for astonishment at the credulity of a generation that believed them to be charms by the exhibition of which they could direct the working of the body. To be quite just, our forebears did not exactly adopt this view. They still believed in remedies. Docks grew in the same hedgerow as nettles. Therefore the juice of the dock was an antidote to nettlestings. Washerwomen found wasps vexatious, but, fortunately, "blue-ball" cured the pain of their stings, and prevented the swelling which otherwise would have occurred.

A new pharmacology is rapidly developing. The physiological action of every substance likely to be of service as a drug is put to the proof. Having ascertained what is wrong, and knowing exactly what effects his drugs are capable of producing, the physician devises the adjustment which he may attempt without risk of making matters worse. He then seeks, if possible, a chemical messenger near akin to the messenger whom Nature herself would send; at least, this is the ambition of the modern pharmacologist. 

little beside salt and a weak ferment. It serves to moisten the food as it is being crushed by the molar teeth. The submaxillary and sublingual secretions contain, in addition to the ferment, ptyalin, mucus which the tongue mixes with the masticated food as it forms it into a bolus suitable for swallowing. The dorsal surface of the tongue is covered by papillæ, which rasp the food against the palate. Of these the greater number are pointed, or filiform. The remainder are flat-topped, or fungiform. The two varieties may be distinguished with a lens, especially on the sides of the tongue. Usually the fungiform papillæ are the redder. In fever, when the tongue is densely furred, they stand out as bright red spots. The back of the tongue is crossed by a $\mathbf{V}$-shaped row of papillæ of larger size, each surrounded by a slight fossa and a vallum, and hence termed "circumvallate." Very minute organs of sensetaste-bulbs-stud the mucous membrane which lines the fosse.

The hard palate ends in a muscular curtain-the soft palatethe central portion of which-the uvula-depends lower than the rest. On either side the soft palate splits into two folds; the anterior, continued to the side of the tongue; the posterior, to the pharynx. "These folds, since they bound the gateway into the pharynx, which is known as the "fauces," are termed the "pillars of the fauces." The tonsil lies between the anterior and posterior pillars of the fauces, but does not appear as a prominence unless inflamed or enlarged.

The pharynx hangs as a bag from the base of the skull. It, like all the rest of the alimentary tract, is lined by mucous membrane. "Mucous membrane" is not a happy term. It does not denote that the epithelium secretes mucus. It may or may not possess this property. Nor does it imply that it has a different origin from the skin-that it arises from hypoblast, the inner layer of the rudiment from which the embryo grows. The term is applied to all internal, and therefore moist, surfaces, whether they arise from hypoblast, as in the case of the lining of the greater part of the alimentary tract, or whether they are involutions of epiblast as in the case of the mouth and also of the extreme lower end of the alimentary tract. Almost the whole of the alimentary canal is, in the first instance, a tubular cavity in the interior of the embryo, lined by hypoblast. This cavity communicates with the 
yolk-sac, but has no openings on the exterior until it joins up with two epiblastic pits-one the stomodrum, or mouthcavity, at the anterior end; and the other the proctodæum, at the posterior end of the body. The distinction between the middle closed portion of the alimentary canal and its two secondary openings suggests morphological speculations, into which we have not space to enter, as to the ancestry of the vertebrates. The majority of anatomists believe that the primitive canal is represented in the middle portion, and that, in prevertebrate animals, it opened to the exterior in a different way. The pharynx is $4 \frac{1}{2}$ inches long. It is enclosed by three thin muscles, which overlap from below upwards-the constrictors of the pharynx. The anterior attachment of the superior constrictor is to the jaw; of the middle constrictor to the hyoid bone; of the inferior constrictor to the thyroid cartilage. Above the soft palate the nasal chambers communicate with the pharynx by the posterior nares. Below the hyoid bone, which is easily felt in the neck as a bony arch just above the thyroid cartilage (Adam's apple), the windpipe, or trachea, joins the pharynx by a single pear-shaped orifice-the rima glottidis. When we consider the mechanism of swallowing, we shall study the arrangements which prevent food, passed through the fauces, from entering either the nasal chambers above or the windpipe below and in front. At the level of the lower border of the thyroid cartilage the pharynx becomes the relatively narrow œesophagus. This tube, which lies behind the trachea, and slightly to its left side, passes with a straight course to the abdomen. It traverses the chest, lying behind the heart, pierces the diaphragm, and just beneath it joins the stomach. Its length is about 9 inches. The stomach is a sickle-shaped bag. It has two apertures-the cardiac orifice, or junction with the cesophagus; and the pyloric orifice, or junction with the small intestine. It is so folded on itself that these two apertures are not more than 4 inches apart. Its outline may be drawn on the body-wall with a piece of charcoal from a point an inch below and an inch to the left side of the lower end of the breast-bone, the position of the cardiac orifice, to a point about 4 inches below the end of the breast-bone, and an inch or two to the right side of the midline of the body, the position of the pyloric orifice, with a slight curvature to 
represent the upper border; to represent the lower border the same two points are joined by a bold curve, bulging upwards to the nipple, outwards to the side of the body, and downwards some distance on the abdomen (cf. Fig. 2).

Such an outline represents the form and position of the stomach when distended; but it is to be understood that its dimensions depend upon the amount of its contents. It is capable of holding about 7 pints. The junction of

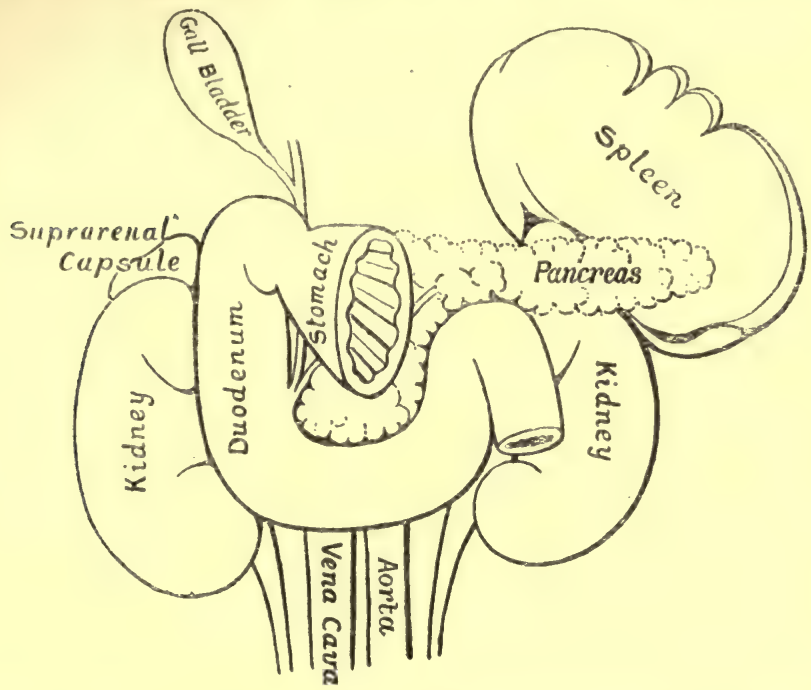

FIG. 6.

The stomach has been cut across a short distance from the pyloric valve, and removed, to show the viscera which lie behind it. The descending aorta and the vena cava rest upon the vertebral column. They are crossed by the pancreas and the transverse portion of the duodenum. The head of the pancreas is enclosed by the curvatures of the duodenum. The ducts of the liver and pancreas are seen entering the descending duodenum side by side.

œsophagus and stomach is closed by a muscular ring, or sphincter muscle - the cardiac sphincter; the junction of stomach and intestine is guarded by a much stronger pyloric sphincter The average diameter of the small intestine is about $1 \frac{1}{2}$ inches. It is wide enough, therefore, to admit two fingers. The length of the tube is about 22 feet. Its first part is termed the "duodenum," because its length equals the breadth of twelve fingers-i.e., about 9 inches. The remainder is divided arbitrarily into jejunum and ileum. The 
duodenum makes three sharp curves. First it inclines upwards and to the right, then vertically downwards, then horizontally to the left, and finally forwards. The ducts of the liver and pancreas open by a common orifice into the descending portion. Its horizontal portion is bound firmly to the vertebral column. After this the whole of the small intestine is supported by the mesentery, a double fold of peritoneum which allows it to hang freely in the abdominal cavity. The mesentery is attached to the back of the body-wall. Commencing on the left side of the second lumbar vertebra, its line of attachment inclines obliquely downwards and to the right, across the vertebral column, for about 6 inches. Measured from its attached edge to the edge which bears the intestine, it has a width of about 8 inches. Its free border has, as already said, a length of 22 feet. Its measurements being as just stated, it is clear that it must be folded backwards and forwards upon itself, like a goffered frill. In the right groin the small intestine joins the large intestine, or colon. It does not, as might have been expected, simply dilate into the large intestine, but enters it on its mesial side, its orifice being guarded by the ileocolic valve. In other words, the large intestine projects downwards beyond this orifice, as the cæcum coli. In many animals the creum is of great length and capacity. In the human embryo it begins to assume a similar form ; but a very small portion only (the so-called "cæcum" of human anatomy) dilates to the calibre of the colon. The real cæcum retains throughout life its embryonic calibre. It has a length of about $3 \frac{1}{2}$ inches, and a diameter of not more than $\frac{1}{4}$ inch. This is the "vermiform appendix," of ill fame, which must be looked upon as one of Nature's misfits. Its great liability to become inflamed is commonly explained as due to the tendency of such articles of food as pips, the fibre of ginger, flakes from the inside of enamelled saucepans, etc., to become lodged in its cavity. But whether this explanation be correct or no-and there are reasons for thinking it somewhat fanciful-it is much to be wished that the process of evolution would hasten the disappearance of this functionless vestige of a cæcum. As there is no tendency towards the inheritance of characters due to mutilation, and since the surgeon's knife now prevents this death-trap from claiming its toll of possible parents, we must 
look upon the rudimentary cæcum, with its liability to inflam. mation, as a permanent burden on the human race. In justice to the appendix, however, it must be pointed out that it has acquired its criminal reputation during the past twenty years. The frequency of appendicitis has increased so enormously during this period that it ought to be possible to correlate its prevalence with the introduction of the cause upon which it chiefly depends.

The colon has a length of about 5 feet. Its greatest width, about 3 inches, is at its commencement, but it is everywhere much wider than the small intestine. Whereas the wall of the small intestine is smooth externally, the wall of the colon is sacculated. Three muscular bands constrict it longitudinally ; circular bands at intervals of about 1 inch or $1 \frac{1}{2}$ inch throw it into pouches. It ascends on the right side, lying far back against the body-wall, to which it is bound by peritoneum, which in this part of its course covers only its anterior surface. Having touched the under side of the liver, it loops forwards and to the left side, crossing the middle line just above the umbilicus. On the extreme left side it touches the spleen, getting very near to the back of the abdominal cavity. It then descends on the left side, again bound to the body-wall by peritoneum, although not so closely as on the right side, until it reaches the inner lip of the crest of the hip-bone. From here onwards the fold of peritoneum which attaches it allows it a free movement. This portion of the large intestine, the sigmoid flexure, may even fall over into the right groin. Lastly it curls backwards into the pelvis, as the rectum.

Movement of the contents of the alimentary canal may be favoured by judicious pressure, or massage. From the description of the situation of its several parts given above, it will be understood that if the right hand be placed on the abdomen immediately beneath the ribs, with the fingers well round to the left side, the stomach will be covered. Pressure from left to right will tend to drive its contents towards the pyloric valve. The small intestine is so irregular in its course as to preclude the possibility of following it with the hand. Pressure first on one side and then on the other, with a general tendency to work from above downwards, tends to press forward its contents ; but, owing to its circular form and strong muscular 
walls, it is not in much need of help. Very different is the position of the large intestine in this respect. Its calibre is much greater, its wall is sacculated, its contents comparatively firm. If the palm of the hand be placed above the right groin and pressure directed upwards, the cæcum coli and ascending colon are emptied. If pressure be directed from the extreme right side just below the ribs, across the middle line to the left side, the transverse colon is emptied. The descending colon needs pressure from above downwards on the left side; the sigmoid flexure, pressure above the left groin, downwards, and towards the middle line.

The inner wall of the œsophagus is smooth, save for the wrinkles into which it is thrown when not distended; but from the cardiac orifice of the stomach onwards the mucous membrane of the alimentary canal exhibits folds and other projections which serve many purposes. They serve to delay the food, keeping it longer in contact with the secreting surface. They increase the area pitted with tubular glands ; they increase also the area through which absorption of the products of digestion occurs. On the inner surface of the stomach the folds produce a reticulated pattern. In the upper portion of the small intestine, especially the duodenum, there are prominent transverse shelves (valvulæ conniventes). No definite folds occur below the upper three-fourths of the small intestine, with the exception of the constrictions of the transverse colon already referred to, which affect the whole thickness of its wall. Throughout the whole of the small intestine the mucous membrane projects in finger-like processes, or villi, which give it a characteristic velvety appearance. The villi are longest in the duodenum.

Lymph-follicles occur at intervals in the intestine. In the ileum they are collected into patches (Peyer's patches), on the side opposite to the line of attachment of the mesentery. They serve both for the supply of phagocytes, which hunt any germs that have penetrated the mucous membrane, and also as stations to which germ-laden phagocytes retreat.

The wall of the intestine is composed of mucous membrane, submucous tissue, and muscle. The mucous membrane is everywhere pitted with tubular glands, termed in the stomach "gastric glands," and in the intestines, both small and large, 
"crypts of Lieberkühn." Their relation to the wall might be exemplified by taking a block of dough about 6 inches thick and pushing a pencil vertically into it almost down to the table on which it rests. The holes should be made as close together as possible, since, especially in the stomach, extremely little tissue intervenes between the tubes of gland-cells. If the piece of dough were placed upon a folded cloth, the cloth would represent the muscularis mucosæ, a layer properly regarded as a constituent of the mucous membrane. The fibres of this coat are disposed in two or three sheets, the fibres of one sheet crossing those of the next. By their contractions they squeeze the ends of the crypts, and probably wobble them about, expelling their secretion. Beneath the muscularis mucosæ is a layer of connective tissue, the submucosa, which contains abundant lymphatic channels, bloodvessels, and nerves. At the pyloric end of the stomach, the tubes of gland-cells tend to pierce the muscularis mucosæ. In the first part of the duodenum, certain tubes, having pierced this layer, branch in the submucosa. A layer of racemose glands is thus formed-the glands of Brunner. Outside the submucosa is the muscular coat proper, composed of plain musclefibres, except in the upper part of the osophagus, where the fibres are striated. It consists of an inner and an outer sheet, the fibres being disposed circularly in the inner, longitudinally in the outer sheet, with a slight departure from this regular arrangement in the wall of the stomach. On its outside the canal is invested by peritoneum, a layer of flattened epithelial cells supported by connective tissue. The abdominal wall also is lined with peritoneum. The smooth moist surface of the peritoneum covering the intestines glides on the peritoneum lining the abdominal wall. Between the two is a "potential" space. In dropsy, fluid accumulates within this space. In a healthy condition the apposed surfaces are merely moist.

The movements of the intestines are of two kinds. At all times they exhibit swaying movements, in the production of which the longitudinal fibres play the chief part, although the circular fibres also contract. The object of this undulation is to thoroughly mix the contents of the gut with its secretions. If pills of subnitrate of bismuth are administered, and their progress observed by the aid of Röntgen rays, they are seen to 
oscillate backwards and forwards on their way down the canal. The slower vermicular movement which squeezes the contents forwards is called "peristalsis." It resembles the progressive contraction of an elastic tube which may be effected by drawing it through a ring, but is rather more complicated. At the point at which it is occurring the circular coat is sharply contracted. Above this it is also somewhat contracted; below it is relaxed. The longitudinal fibres, using the constricted portion as a point d'appui, pull up the segment of the intestine which lies immediately below it, drawing it off the contents of the tube as a glove from a finger.

When food is swallowed, it falls down the osophagus, aided by slight peristalsis. As soon as sufficient has accumulated on the upper surface of the cardiac valve of the stomach, the valve relaxes; at the same time a stronger peristalsis of the lower portion of the œsophagus squeezes its contents into the stomach. Food remains in the stomach until it has reached a certain stage of digestion, the chief object of which is its subdivision into small particles. Until this stage is reached, the pyloric valve is firmly closed. The contractions of the wa!l of the stomach drive its contents round and round-down the greater and up the lesser curvature-mixing them thoroughly with the gastric juice (cf. p. 124). As the acidity of the mixture increases, the peristaltic contractions of the stomach become more vigorous, until, the pyloric valve relaxing, the food is little by little driven into the duodenum.

The alimentary canal has an abundant supply of nerves from the vagus and the sympathetic systems. It contains also within its own wall an enormous quantity of nerve-fibres and nervecells. They are disposed as two plexuses, one in the submucosa, the other between the circular and longitudinal muscular coats. In a specimen successfully stained with methylene blue, they are so abundant as to give the impression that every plain muscle-cell may have its own separate nervetwig. Nevertheless, the contraction of the muscle-cells may take place independently of all nerve-influence-independently, even, of the local mechanism, the plexus referred to above. Nicotin applied to the wall of the intestine paralyses the local nerves; yet rhythmic contractions still occur. They are, however, no longer progressive. They do not drive the contents 
of the intestine forwards. Co-ordinated contraction is observed so long as the local mechanism is intact, even though all external nerves have been cut. The intestines have their own nerve cells and fibres, which, acting as a linked system of reflex centres, provide for the harmonious contraction of their walls. External nerves, sympathetic and splanchnic, convey impulses which either intensify the movements or inhibit them, as need may be.

In the matter of its nerve-supply, the alimentary canal stands apart from the other organs of the body. It may be supposed that it presents a more primitive condition. Its muscular fibres have the power of contracting spontaneously. The pressure of the contents of the tube acts as a stimulus. When the fibres are stretched, they contract. When the tube is dilated, its muscles endeavour to restore it to its normal calibre. Such direct action would not, however, provide for the forward passage of its contents. To bring about peristalsis, a nervous mechanism is needed, as abundant and complicated as that which ensures the progress of a slug or a worm. To deal satisfactorily with the various contents of the tube-liquid, solid, gaseous-the mechanism must be capable of complicated adjustments. The dilated portions of the tube-stomach, cæcum coli, rectum-require special arrangements of muscle and nerve. Nor is the canal altogether independent of the rest of the body. To a large extent its work is carried on without regard to the activities of other organs, yet it is not wholly free from the control of the central nervous system. It is regulated by means of both afferent and efferent nerves of the vagus and sympathetic. Even the brain has something to say with regard to the way in which it shall contract. It is a matter of common experience that emotional influences may affect the movements of the stomach and intestines-" His bowels yearned."

Normally, vomiting is due to irritation of the endings of the vagus nerve in the stomach, although the afferent impulses may have other sources. Touching the upper surface of the epiglottis with the finger will provoke the reflex. So also will stimulation of the olfactory nerves by a foul smell. In this latter case the emotion of disgust to which the odour gives rise brings about the reflex action. A flow of saliva precedes the act of vomiting. A deep inspiration is then taken, in order 
that for a time the lungs may be independent of a fresh supply of air. The glottis is closed, the diaphragm fixed. Contraction of the abdominal wall presses the stomach against the diaphragm; its cardiac sphincter relaxes, and its contents are squirted into the osophagus, which undergoes a forcible retrogressive peristalsis.

It is interesting to note the difference between carnivora and herbivora in regard to vomiting. Carnivora swallow fur and other indigestible materials, as well as many unwholesome things which they need to be able to return. A dog can, apparently, vomit at will. Never, while in a state of nature, do herbivora need to return the contents of the stomach. No provision is made for vomiting. A heifer which has strayed into a dewy clover-field is not unlikely to die from the effects of distension of its paunch, if relief be not given by opening it with a knife. In a horse the cardiac sphincter is strong, the pyloric weak. Pressure on the stomach tends to drive its contents through the pyloric valve into the duodenum, not backwards into the œsophagus. The stomach is not so placed as to allow of its being compressed between the wall of the abdomen and the diaphragm. Horses cannot vomit. It is a mistake to suppose that they suffer from sea-sickness. In rough weather they sweat, their limbs tremble, they go off their feed; but these symptoms are probably due to the fatigue which results from excessive anxiety to maintain their balance, and to fear. We can never know their feelings, but there is no reason for supposing that they experience the sensation of nausea.

Vomiting is a frequent symptom of cerebral disturbance. The fluctuations of pressure which the brain experiences as it rocks about on its "water-bed " within the skull is the cause of seasickness. Yet the motion of a ship may produce violent headache without nausea, the brain only, not the stomach, appearing to be troubled by the motion. Not that headache is a pain "inside the head." Nor is it properly described as a pain in the scalp, although the messages which are felt in consciousness as headache originate in the endings of the nerves of the skin which covers the skull. The excessive sensitiveness of these nerves is due to vaso-motor conditions, usually the dilation, occasionally the constriction, of the 
bloodvessels of the scalp. But the vaso-motor condition is sympathetic with the disturbance of the brain ; and the special urgency or efficiency of the messages from the skin results from their being delivered into excited brain-tissue. Nausea and headache are equally symptoms of the irritability of the brain caused by the motion of the ship. In one case messages from the stomach, in the other case messages from the scalp, acquire undue importance, owing to the agitated condition of the brain-tissue through which they pass. Not uncommonly the voyager, who wakes in the morning reconciled to the changes of pressure which he has experienced while recumbent, finds, when he stands upright, that the base of his brain is as sensitive as ever. Visual sensations also contribute to the brain-disturbance. So, too, do the movements of endolymph in the semicircular canals (cf. p. 335). It is, indeed, possible that this last factor is more important than the variations in pressure on the surface of the brain. Probably it accounts for the after-image of rolling which almost everyone experiences for at least a day after leaving the ship. Its cause being cerebral, the tendency to sea-sickness can be controlled by drugs which, like the bromides, chloral, alcohol, etc., deaden the brain.

Salivary Glands.-The secretion which accumulates in the mouth is the combined product of the sublingual, submaxillary, and parotid glands. It is a very thin, watery solution containing not more than 0.5 per cent. of solid substance. If red litmus-paper is moistened with saliva, it becomes blue, showing that the secretion is alkaline. It contains a ferment, ptyalin, which digests starch. The action of this ferment can be demonstrated by holding in the mouth for half a minute some warm starch mucilage-boiled arrowroot, for example. It quickly loses its viscidity owing to the conversion of starch into sugar. Chemically this change may be demonstrated by adding iodine-water to a specimen of the starch before and after action. Before the starch is taken into the mouth the iodine turns it blue (a characteristic reaction for starch). After it has been exposed to the digestive action of the saliva, iodine fails to colour the mixture, which now contains no starch. All the starch has been converted into dextrin and sugar. If unboiled arrowroot is placed in the mouth, 
some sugar is produced, but the process of conversion is very slow. It is almost impossible to digest raw starch in the mouth sufficiently to render it insusceptible to the colouring action of iodine. The sugar produced by the action of ptyalin is of the same nature as that which appears during the malting of barley. It is therefore termed "maltose." It closely resembles grape-sugar, but is not identical with it.

The Secretion of Saliva.-The accessibility of the salivary glands, and especially of the submaxillary, has led to their being used for a very large number of experiments. They have been studied with the aim of coming to an understanding of the mechanism of secretion in general. The glands consist of tubes of gland-cells, each tube suspended in a basket of connective tissue, in a bath of lymph (cf. Fig. 3). Innumerable capillary bloodvessels traverse the lymph-bath. The arteries which carry blood to the gland are supplied with nerves, which regulate their calibre, and therefore determine the amount of blood which passes through the capillaries into which they break up. The glands also are supplied with nerves which influence their functional activity. Nutrient substances and oxygen pass out of the blood into the lymph. Carbonic acid passes into the blood from the lymph. Waste products are either carried away in the lymph-stream, or make their way through the walls of the capillaries into the blood. Many problems present themselves for solution. How does the amount of work done by the gland affect its supply of blood? Does the quantity of saliva secreted vary directly with the pressure of lymph in the spaces by which the gland is surrounded? Is this pressure wholly dependent upon the pressure of the blood? Are the substances secreted by the gland supplied as such by the blood, or does the gland make the ptyalin and mucus which it secretes? If it makes its secernable products, what materials does it abstract from the blood for the purpose of their manufacture? Does it use the whole of these materials, whatever they may be, or does it use part only and return the residue to the lymph? Does it make its products only when it is actively secreting, or is it always making them, and storing them in its cells in order that it may have a supply to discharge when called upon by the stimulation which results from the presence of food in the mouth? Is their discharge merely a washing-out 
due to the rush of fluid which occurs when the bloodvessels are dilated, or can the gland-cells expel their products in response to nervous action? In what way do the nerves of the gland influence secretion? Do they call for increased production, or increased output, or both? These are some of the problems which the exposed situation of the submaxillary gland allows physiologists to tackle.

By means of a very simple operation, the ducts of one or both parotid or submaxillary glands can be brought to the skin, and made to pour their secretions on to the surface instead of into the mouth. The flow under various circumstances can be watched. The saliva can be collected and measured.

The nerves of the submaxillary gland are easily isolated. A nerve leaves the seventh (or facial), crosses the drum of the ear, comes out through a minute crevice in the skull, and runs for some little distance as a separate nerve before it applies itself to the lingual branch of the fifth; which runs along the side of the tongue. Owing to its passage across the tympanic cavity (drum of the ear), it is termed "chorda tympani." As its fibres are very small, they can be recognized wherever they form a part of the lingual nerve. They leave the lingual to go to a ganglion, the submaxillary ganglion, from which the gland is supplied. The gland also receives branches from the sympathetic nerve which ascends the neck. The lastnamed branches accompany the facial artery. Stimulation of either of these nerves causes the gland to secrete. The flow of saliva which follows stimulation of the chorda tympani is much more copious than that which follows stimulation of the sympathetic, and as a rule it contains far less organic matter, although about the same amount of mineral salts. Under normal conditions the activity of the chorda tympani is brought into play in a reflex manner by impulses which travel up the nerves of taste (the lingual and glosso-pharyngeal) to the cerebro-spinal axis ; but almost any other nerve will serve as an afferent path. The gland may also, as we shall presently explain, be called into activity by the cortex of the brain.

It is certain that in the case of the submaxillary gland secretion is not the direct result of increased blood-pressure. It is not a case of filtration from the blood through certain membranes and cells into the salivary duct. Atropin (bella- 
donna) dilates the bloodvessels, increasing blood-pressure, but it stops secretion. After belladonna-poisoning, the mouth, like the skin, is hot and dry. Other drugs there are which provoke a certain amount of secretion, even after the bloodvessels going to the gland have been tied. It is possible, by stimulating the chorda tympani, to obtain a pressure in the fluid in the duct very much greater than that in the bloodvessels which supply the gland. Here we have clear proof that secretion is not filtration. Filtration is the passage of fluid through a filter-bed from a higher to a lower pressure. In filtration, moreover, soluble diffusible salts accompany the water. The saliva contains only half as much of these diffusible salts as the blood. Therefore the gland tissue stops half the salts. Secretion is an active process carried out by the gland-cells, under the influence of nerves, in opposition to the laws of filtration. The gland-cells determine how much water shall pass through them and what percentage of salts shall accompany the water.

How does a gland-cell make the substance which it secretes? There is no reason for supposing that the ptyalin or the mucus which the salivary glands secrete is present in the blood, either ready formed, or, as it were, half formed, in combinations which can be easily broken up. All the evidence obtainable points to the conclusion that the gland-cells take out of the lymph proteid materials from which they manufacture the peculiar substances which they secrete. During rest, granules accumulate in the cells. During activity they disappear. It has been shown in the case of the gastric glands that these granules consist of the special ferment which the gland secretes, in an inactive form. It may be that it is combined with a substance which prevents it from exerting its digestive action on the cells within which it is made; damped, as gunpowder is damped during transit. Or it may be that it is not a finished ferment ; it may need a further addition to its molecule. During activity, while the granules disappear, proteins accumulate at the bases of the cells, giving to a tube of gland-cells the appearance of a peripheral non-granular zone. This proteid substance must have come from the lymph, and the inference seems inevitable that the cells have taken into their protoplasm a supply of material which will serve for the manufacture of additional granules. Each gland-cell is therefore an indepen- 
dent unit. By its own activity it takes up materials from the lymph, out of which it manufactures its own special products. It stores its products until they are wanted. Then by its own activity it extrudes them into the lumen of the gland-tube. It has, indeed, been shown that, when the nerve going to a salivary gland is stimulated, the gland shrinks, notwithstanding the great dilation of its bloodvessels. Under the influence of the stimulation the granules in the gland-cells imbibe water, swell up, and escape from the cells. The cells discharge their accumulated stores, in the first instance, more rapidly than they take up materials (even fluid) from the blood. For its knowledge (if the term may pass) of what is wanted the gland-cell is dependent upon messages which reach it through the nervous system. These messages take origin in the endings of the sensory nerves of the mouth, pass up to the brain, and are reflected down the nerves to the gland. So accurate is the information conveyed to the glands, that when a horse transfers the work of mastication from one side of its mouth to the other, as it is in the habit of doing about every quarter of an hour, the flow of saliva from the parotid gland on the masticating side is increased; on the other side it is diminished. Two or three times as much saliva is poured out on the one side as on the other.

Not only is the amount of saliva poured out in response to stimulation proportional to the needs of mastication, but the kind of saliva is adapted to the nature of the food. In a dogand this is an observation which can be made only on an animal which lives on a mixed diet-it is possible to determine the amount of the two kinds of saliva secreted and the relation of flow to food. When meat is given to the animal, the submaxillary gland yields its secretion; when it is fed on biscuit, abundance of the watery parotid saliva is poured forth. A mouthful of sand also causes the parotid saliva to flow, in order that the sand may be washed out of the mouth.

More remarkable than the response to direct stimulation is the effect produced by the sight and smell of food. When meat is shown to a dog, submaxillary saliva begins to flow; when it is offered bread, parotid saliva is secreted. And the activity of the glands is not morely a nervous reflex independent of the animal's mind. The moment the dog realizes that it is being played with-that there is no intention of giving it the coveted 
food-the flow of saliva ceases. An emotion may check secretion when every physiological condition is demanding it. This is the explanation of the Rice Ordeal. Dry rice provokes a flow of saliva in the mouth of all save the guilty man. Response to mental impressions is a matter of the greatest consequence in the physiology of digestion. It holds good in the case of the secretion of gastric juice equally with that of saliva. The sight and smell of food sets the juice flowing into the stomach, and the more desirable the food, the more attractive its appearance, the more stimulating its smell, the more rapidly does the secretion flow. Here we touch upon a theme which hardly needs exhaustive treatment. It is not the stoutest people who eat the most, although an impartial survey of one's wellnourished friends will show them to be persons who "take kindly to their victuals." A small quantity of food perfectly digested is more nourishing than much food which the digestive organs do not efficiently prepare for assimilation. Good digestion waits on appetite ; and appetite, in civilized man, is something more than a mere physical need of food. The hunger which leads to the bolting of food without pleasurable anticipation, without mastication, without any consideration of the quality of the viands, is a harmful craving which ends in imperfect assimilation. It is more profitable to toy with a hors d'suvre than to engulf, unthinking, a plateful of beef. But we have said enough to suggest reflections to those who take no thought as to what they shall eat or what they shall drink; and few who take thought need to be convinced.

The Stomach.-The sight and smell of food, its presence in the mouth, and the performance of mastication, which induces a secretion of saliva, gives rise at the same time to a flow of gastric juice. It is psychic stimulation and the act of eating which cause gastric juice to ooze from the gland-tubes of the stomach at the commencement of digestion, not the stimulation of nerve-endings by food which has passed down the esophagus. As a consequence of gunshot wounds, or as the result of operations performed for the purpose of relieving patients whose œesophagus has become blocked, numerous cases have been recorded in which a fistulous opening into the stomach has made it possible to study the interior of this organ. Such cases present an opportunity of 
watching the digestion of various foods introduced through the opening, and of collecting gastric juice for purposes of analysis. A similar condition has been established in animals by operative means. The oesophagus having been cut, and the cut end sutured to the margins of an aperture in the skin, food taken by the mouth escaped by this opening instead of passing into the stomach. A similar opening was made into the stomach for the insertion of food, and for the purpose of studying the effects of reflex stimulation of the gastric glands. As soon as food was introduced into the mouth, gastric juice began to flow. The advantage of this experimental method lies in the fact that the juice secreted was a pure juice-not mixed with food, as in all the earlier experiments in which, the stomach being opened without diversion of the œesophagus, the presence of food within it was the stimulus which led to secretion. No juice flowed in the absence of stimulation; nor was the secretion normal in composition when provoked by a mechanical stimulus, such as the tickling of the gastric mucous membrane by a feather.

My lord the stomach! He is not the only, nor is he the chief, agent in digestion; but with him rests the decision as to whether the food offered to the alimentary tract is suitable in quality and quantity. He is offended if it be not offered with all the circumstance and ceremony which becomes his rank. As an intimation that he is about to receive food, he accepts the news from the mouth that its nerve-endings are subject to mechanical stimulation. But the chewing of indiarubber would produce a like effect. The stomach, therefore, confers with the organs of taste and smell. If their report is favourable, he argues that the substance which the teeth are crushing will justify an outflow of gastric juice. He responds most generously when prolonged mastication assures him that he may trust to receiving the food in a sufficiently subdivided state. At our peril we neglect to propitiate my lord. Not always debonair when treated with consideration, he is morose or petulant when slighted. Never content with lipservice, he exacts the labour of teeth and tongue and palate. The tribute we offer may be of the best-savoury, wholesome, well cooked, well chewed-but if it be not tendered with some degree of love, if thoughts are concentrated on other things, 
if no attention is devoted to the meal, if no sense of liking accompanies our offering, my lord the stomach on his part affords the viands an indifferent reception. In consulting our own tastes we are to a large extent consulting the needs of the stomach. Ravenous and excessive feeding is not an exhibition of taste ; it is a return to the instinct of the savage, who was never sure that he would get his full share, and was afraid to trust that another meal would be obtainable when nature declared it due. Some degree of epicureanism is favourable to digestion. The flow of gastric juice in the stomach occurs reflexly in response to the emotion of appetite, to stimulation of the nerves of taste and smell, to the obscure sensations which accompany the activity of the muscles of mastication.

The gastric juice secreted in a day amounts probably to about 8 or 9 pints. To this we must add, when considering the quantity of fluid which passes through the stomach, the saliva, which certainly reaches as much as 2 pints, and the beverages taken with food.

Gastric juice collected in the manner described above is a clear, colourless, inodorous fluid. It is very acid, and so powerfully peptic as to digest its own weight of coagulated white of egg. Its solid constituents amount to 0.5 per cent. They consist of the two ferments pepsin and rennin, with traces of proteins and mucin, and various inorganic salts. Its acidity is due to free hydrochloric acid to the amount of 0.2 per cent. This acid is more or less in combination with the pepsin. In pure gastric juice hydrochloric acid is the only acid present; but when mixed with food the juice contains other acids also, especially lactic.

When food first reaches the stomach, the alkaline saliva which accompanies it neutralizes the acidity of the gastric juice. For some time, probably about half an hour, the conversion of starch into sugar is still carried on by the ptyalin of the saliva, owing chiefly to the difficulty which the gastric juice encounters in permeating the masses of masticated food. The Bacillus acidi lactici is always present in the stomach. It converts some of the sugar into lactic acid; of this a small quantity is further changed into butyric and acetic acids, with the formation of carbonic acid and hydrogen gas. After a while the lactic acid is absorbed, and hydrochloric acid alone remains. 
The secretion by the gastric glands of so powerful a mineral acid as hydrochloric has always aroused interest. How is it possible for the gland-cells to produce it without injury to themselves, or for the stomach to contain it without self-digestion? Many chemical and physical theories have been advanced in the belief that they rendered the process of its production less difficult to understand. All such theories are, however, inadequate to explain the secretion as a discontinuous process, which occurs only as a response to demand. That the source of the acid is the sodic chloride which the gland-cells take from the blood does not need assertion, but we cannot picture the process by which this exceedingly stable compound is decomposed otherwise than on the assumption that weaker acids, or, rather, acid salts, are also absorbed by the cells, and that, in accordance with the laws which govern the composition of salts in solution, an exchange of acids occurs. If sodic chloride and any acid salt-acid phosphate of sodium, for exampleare in solution in water, the salts do not retain their form as we know them when isolated by crystallization. The mixture contains "free" hydrochloric as well as "free" phosphoric acid. It may be assumed that within secreting cells a similar exchange of acids takes place. By a process which we term "vital," the acids are kept apart, and the hydrochloric acid is extruded by the cells. In the present state of knowledge this vital action is mysterious; but it is no more mysterious than the isolation of pepsin, or any other metabolic event which occurs within a cell.

The proteolytic ferment pepsin is active only in an acid medium. Yet apart from its digestive function as an ally of pepsin, hydrochloric acid by itself also exerts a valuable disintegrating action on certain constituents of the food. Possibly the most important results of the presence of free hydrochloric acid in the great chamber into which food is first received are due to its disinfective property. It destroys all the putrefactive germs which accompany the food, and many germs which, if introduced into the blood, would give rise to disease. It also destroys the germs which multiply in the stomach towards the end of each interval between two meals. When withdrawn from the body, gastric juice will keep an indefinite time, if evaporation of the acid be prevented. 
Pancreas.-In structure the pancreas presents a marked resemblance to the salivary glands. Probably this resemblance is merely superficial. Minute examination reveals points, apparently of great morphological importance, in which the $\dot{y}$ differ. In the gland-tubes of the salivary glands, and, indeed, in all glands with the exception of the pancreas, secreting cells project into the lumen. The secreting cells of the pancreas are invested internally by a layer of flattened scales. (intra-acinar cells). They lie, therefore, between the basement membrane which invests them externally and this second layer of flattened cells which separates them from the lumen of the tube. At a very early date in embryonic life the gland-cells of the pancreas are filled with highly refracting granules. As this occurs long before any digestive action is called for, it may be taken as indicating that the pancreas has functions which other glands-the salivary, for example-do not possess. These granules do not, however, appear in all parts of the tubes. Certain portions of the tubes remain undeveloped-fail, that is to say, to acquire a secreting function-even in adult life. Such patches of cells, not disposed in gland-tubes, are known as islands of Langerhans. When the pancreas is over-stimulated by artificial means, leading to its extreme exhaustion, large portions of its glandular substance return to this primitive condition. The gland-cells not only discharge their stores of granules, but they lose the greater part of their cell-protoplasm. It would seem that, in their effort to meet the demand for ferments, they use up their own cell-substance in their manufacture. Having exhausted their coal, they stoke the furnace with the looms and furniture of the mill. It may be that other glands would do the same if it were possible to stimulate them as strongly as the pancreas can be stimulated. The result is probably due to the extreme susceptibility of the pancreas to the action of secretin, a substance made in the intestine. Secretin can be isolated and injected into the blood. We shall refer again to this chemical stimulation of the pancreas when tracing the progress of food through the alimentary canal.

The secretion of the pancreas is a clear, colourless, alkaline liquid of syrupy consistence. The quantity of juice secreted is relatively small, but the organic substances which it contains 
are in a concentrated form. They constitute as much as 10 per cent. of the pancreatic juice. Proteins are present, if the juice be fresh. If it has stood for any length of time, they are found as peptones. The digestive ferments of pancreatic juice are the most powerful which are secreted into the alimentary canal.

Bile. - In its most important functions the liver has no relation to digestion. It is a storehouse of absorbed food. This organ will therefore be treated in a separate chapter. The bile which the liver secretes into the alimentary canal has no chemical action on any of the constituents of food, with the exception of a feeble tendency to digest starch. Yet it is in some degree accessory to digestion. Poured into the second portion of the duodenum through an orifice common to the liver and the pancreas, it mingles with the semi-digested food, or " chyme," which, about two hours after a meal, passes through the pyloric valve. Gastric digestion has converted the greater part of the proteid constituents of the food into peptones or intermediate stages. The proteoses or propeptones-a name is needed for the intermediate products of proteid digestion which does not commit us to any theory as to their chemical constitution-are quickly peptonized by the pancreatic juice. But portions of the proteins have escaped the action of gastric juice, or have at most been affected by its acid only; these are precipitated by the bile-salts on the mucous membrane of the small intestine, which is raised into projecting flanges for the purpose of delaying the passage of the chyme, in order that it may be thoroughly submitted to the digestive action of pancreatic juice. Bile-salts also favour the digestion of fat, and its passage through the intestinal wall. The action of bilesalts in spreading fats is well known to artists. Ox-gall is smeared upon glass when it is desired to apply oil-paints to its surface. When mixed with oil, it causes its emulsification, or breaking up into microscopic globules. In the absence of bile, but little fat passes into the lymph-vessels which convey digested food from the intestine to the thoracic duct, and so to the great veins of the neck. Its action is mechanical. It favours the digestion of fats by rendering them easily amenable to hydrolysis by pancreatic juice.

Bile as secreted by the liver is a clear, limpid fluid of low 
specific gravity; but during its stay in the gall-bladder it is concentrated by absorption of water, and mucin is added to it. It contains " bile-salts" of complex constitution. These salts favour the solution of certain by-products of cell-metabolism, cholesterin and lecithin; substances which are formed in many cells, both in animals and plants. Cholesterin occurs most abundantly in nerve-tissue and in blood-corpuscles. Lecithin also is a by-product of the metabolism of nerve-tissue. Protoplasm appears to be incapable of oxidizing these substances, as it does other products of metabolism. Other substances of equally complex constitution are reduced to urea if they contain nitrogen; to water and carbonic acid if nitrogen be absent. Cholesterin and lecithin have to be eliminated without further change. Some of the cholesterin is excreted by the sebaceous glands of the skin. It is the chief constituent of "lanoline" prepared from sheep's wool; an unguent which owes its valuable properties to the resistance which cholesterin offers to cell action, and therefore to the action of living ferments. Bacteria cannot turn it rancid. The sebaceous glands have the power of directing metabolism into a channel in which cholesterin is the chief product, but apparently all cells make it in small quantity. The bile-salts carry cholesterin and lecithin into the alimentary canal, from which they are not reabsorbed. Some of the bile-salts are lost to the body, but the remainder re-enter the circulation, and recommence their work as vehicles for these inoxidizable and insoluble substances. In the gall-bladder cholesterin is apt to separate out from the bile in the form of gall-stones; but whether this is due to an excess of cholesterin in the bile, or to an abnormal, inflammatory condition of the lining membrane of the gallbladder, is still an open question.

Bile also contains bile-pigments. Their colour varies in different animals, and changes according as the bile is exposed to the air, or subject to the action of reducing agents. If oxidized, the colour is green (biliverdin) ; if reduced, brownishyellow (bilirubin). Bile-pigment is formed from hæmoglobin, the colouring matter of the blood, after the removal of its iron. Worn-out red blood-corpuscles are destroyed in the spleen, in the manner already described, but it is uncertain whether the conversion of the hæmoglobin thus set free into bilirubin 
occurs in the spleen, or whether this chemical change is reserved for the liver. Physiologists incline to the view that the liver is the seat of the change.

Intestinal Juice.-The mucous membrane of the alimentary tract, as far down as the middle of the rectum, is, as previously stated (p. 102), studded with tubular glands. They secrete a light-yellow fluid, alkaline in reaction, and opalescent. Its most important property is due to a ferment which converts cane-sugar into a mixture of dextrose and levulose, and changes maltose-the sugar produced by the action on starch of saliva and pancreatic juice-into dextrose. It is in the form of dextrose that sugar is carried about the body and assimilated by the tissues.

Intestinal juice also contains a ferment, erepsin, which shakes to pieces the heavy molecules of peptones and partly formed peptones. Under its influence they break up into comparatively simple bodies containing the radicle of ammonia. Substances containing an $\mathrm{NH}_{2}$ group-one $\mathrm{H}$ of $\mathrm{NH}_{3}$ (ammonia) having been given up, in order that the group may have a "free arm " with which to link on to the other component parts of the molecule-are termed "amides." The amides which are most characteristic of the action of erepsin are leucin, an amidated fatty acid; and tyrosin, an amidated aromatic acid. The tendency of proteins to break up along these two linesthe fatty acid line and the aromatic acid line-is of considerable interest. The one line is represented by acetic acid, $\mathrm{CH}_{3}, \mathrm{COOH}$; the other contains the hexone radicle, $\mathrm{C}_{6} \mathrm{H}_{6}$. Benzoic acid, $\mathrm{C}_{6} \mathrm{H}_{5} \mathrm{COOH}$, is representative of the latter. It used to be thought that proteins which were shaken into simple bodies such as amides were lost to the economy. Their downward career was a foregone conclusion. There could be no arresting it before they brought up at the bottom-as urea, $\mathrm{CO}\left(\mathrm{NH}_{2}\right)_{2}-$ the diamide of carbonic acid. It was even supposed that this disintegration of proteins was a provision for getting rid of the surplus animal food which we consume. Physiological chemists now take quite a different view. They believe that the epithelial wall of the intestine through which these substances are absorbed, or the liver, to which they are carried by the portal blood-stream, has the power of recombining these fragments into the complex protein edifice. It is even 
thought that disintegration is a necessary preliminary to the rearrangement of the sub-groups. A large variety of proteins is ingested as food. Many of them, especially the vegetable proteins, are quite foreign to the body. By the activity of pancreatic juice and erepsin, they are broken into small and relatively stable groups of atoms, which are again fitted together into the particular forms of protein which are of use to the economy.

The Story of a Meal.-The chemistry of digestion will be understood most readily if the constituents of a meal are traced from their entrance into the mouth to their absorption through the wall of the alimentary canal, or abandonment as indigestible.

We may describe as a typical meal one consisting of bread, vegetables, cane-sugar, meat, milk, fat, and cheese. In the mouth the various foods are crushed and mixed with the alkaline secretions of the salivary glands. A certain amount of the cooked starch contained in the bread is changed into maltose. In the stomach the digestion of starch is continued for a time, but a large part even of the cooked starch awaits the action of pancreatic juice. A certain amount of cane-sugar is converted into dextrose and levulose, which are rapidly absorbed into the blood; but this action is due to hydrochloric acid, and probably affects a comparatively small part of the cane-sugar swallowed. Fat is quite unaltered in the stomach. All proteins are attacked by pepsin, but some yield to digestion more readily than others, Gluten of bread, like all vegetable proteins, is comparatively resistant; but since it is presented to the action of pepsin in small quantities and in a spongy form-very suitable for digestion-it is probable that most of it is peptonized in the stomach. Chemists experimenting with gastric juice taken from the stomach, and reproducing the conditions as to temperature, removal of products of action, etc., as closely as it is possible to reproduce them in the laboratory, find that the various foods take different times to digest. The proteins of meat are more quickly peptonized when raw than after coagulation by heat. The same is true of white of egg. Amongst different varieties of cooked flesh, beef is more quickly peptonized than fish. The casein of milk is more quickly peptonized than any other protein ; and it also is no 
exception to the rule that digestibility is diminished by cooking. Similar data may be obtained for all foods. They are no doubt useful indications of the course of action which we may expect to occur within the stomach, but we can never be sure that my lord will obey the ruling of the chemist. Practice with a captive golf-ball is a useful preparation for the game ; but there are conditions on the links which cannot be reproduced on the lawn. In an artificial stomach the clean fibre of raw fish digests more slowly than raw beef. Even when the beef is roasted and the fish fried or boiled in the ordinary way, the beef disappears through the dialyser (the bag of membrane suspended in a vessel of warm water in which experimental digestion is carried out) more quickly than the fish. Nevertheless, the living stomach is better disposed towards a mixed meal containing a certain weight of fish than towards a meal in which, the other constituents remaining the same, beef takes the place of fish. Important conclusions may, no doubt, be drawn from observations of the time occupied in the peptonization of pure food-i.e., fibrin, white of egg, clean meat, etc.under conditions simulating those which are present in the stomach ; but they must be accepted with many reservations. In the stomach it is not pure substances, but mixtures, that the gastric juice has to deal with. And here a most important factor comes into play, to which further reference will be made later on. The amount and quality of the secretion of the gastric glands depends upon the nature of the food. Hence a food, or a combination of foods, which digest readily in the laboratory may take a long time to disappear from the stomach, and vice versâ. Digestibility depends upon the nature of the food. It depends also upon its physical state. To take simple illustrations : Cheese contains coagulated casein, one of the most easily digestible of proteins, but the casein is intimately mixed with fat, upon which gastric juice can make no impression. Even when finely divided, the particles of casein are protected from the action of the juice by fat. In the same way the meat of pork is as digestible as mutton, but the fat of pork is quickly melted and very liquid. In the process of cooking the muscle-fibres become saturated with fat.

It is not the function of the stomach to complete digestion. Its business is to initiate it. Food which reaches the stomach 
in fragments is reduced to a condition in which its digestion will be readily completed by pancreatic juice. Gastric digestion produces a much larger proportion of intermediate products, proteoses or propeptones, than does digestion in the duodenum. Such intermediate products are quickly dealt with by pancreatic juice. Artificial tests of relative digestibility do not, as a rule, take the amount of propeptones formed in a given time into account. When considering the digestion of a typical meal, we must bear in mind that it is not the duty of the stomach to pass as much sugar, peptone, and fat as possible into the blood. In fact, very few of the products of digestion are absorbed by the bloodvessels of the stomach. The impermeability of its mucous membrane is shown by the fact that hardly any of the water swallowed passes through the stomach-wall. Practically all the water ingested leaves the stomach through the pyloric valve. Various salts, some sugar, and peptones are taken up by the vessels of the stomach; but the bulk of all the different kinds of food passes into the duodenum in a semi-digested state. The function of the stomach is to carry digestion through a preliminary stage. The process will be completed in the small intestine. It is to be noted that, although water is not absorbed by the stomachwall, alcohol passes through it with great rapidity. The same is true of the various crystalline nitrogenous bodies found in meat-extracts; and also of the essential principles of tea and coffee, which chemically belong to the same class. All these substances are degradation products of proteins produced by oxidation, far advanced along the road to urea. In this selective absorption we see proof of the activity of the cells of the mucous membrane. They take up the substances which it is desirable to remove from the contents of the stomach. Some may be wanted by the body for its immediate use; others are better out of the way, because they are prejudicial to the progress of digestion.

When contemplating the activity of the cells of the gastric mucous membrane, we feel the need of an adjective which shall express our recognition of the fact that they have a power which we cannot confer upon our clumsy mechanical imitation stomach. They can discriminate. "Vital" is the only term available, though much abused. Using it without 
prejudice, as lawyers say, we speak of the "vital activity" of the cells when we wish to imply that things happen in a living stomach for which we cannot make provision in a model. Of the many substances which make their appearance as digestion proceeds, some are absorbed, others left in the mixture.

The mucous membrane shows its power of controlling digestion in yet another way. In the neighbourhood of the pylorus its structure is unlike that which it presents elsewhere. The gastric glands are short, and tend to branch. Their lining cells are all of the same kind. Over the greater part of the inner wall of the stomach the tubes are long. They do not branch. The cells which line them are of two kinds: small cubical cells (the term refers to their form as seen in section), similar to those of the pyloric glands; large oval cells, placed with their longest axes in the same direction as the axis of the gland-tube. These oval cells do not project into the bore or lumen of the tube, but are displaced from it by the cubical cells. They rest on the investing, or basement, membrane. All parts of the gastric mucous membrane secrete pepsin, although the pyloric portion produces very little; the area which contains oval cells alone secretes hydrochloric acid. If a short time after a meal an extract is made from some of the mucous membrane near the pylorus, by pounding it with salt-solution and sand to break up its cells, this extract, when filtered and injected into the blood, stimulates the glands of the cardiac end of the stomach. Under its influence they pour out both pepsin and hydrochloric acid. The extract contains a substance which acts as a chemical messenger. It is a representative of a class of bodies which play a most important part in co-ordinating the activities of the various organs. Hitherto physiologists have concerned themselves with the visible or "external" secretions of glands. They have shown how the production of these secretions is controlled by the nervous system. Recently they have discovered that another set of influences has to be taken into consideration. Glands, and possibly all other tissues, take from the blood the materials out of which they make their characteristic secretions, or, if they do not discharge secretions, the substances which they require for the building of their own structures, and return to the blood "internal secretions" which act as stimuli to other 
tissues with which they are linked in harmonious co-operation. The active principles of internal secretions have been termed "hormones"-from óp $\mu a ́ \omega$, I announce. The glands of the pyloric mucous membrane secrete a hormone which ealls upon the rest of the membrane to pour out gastric juice (cf. p. 89).

What induces the cells of the pyloric mucous membrane to produce the gastric hormone? Their activity in this respect evidently depends upon the presence in the stomach of partially digested proteid substances. The cells judge, as it were, when these substances come into contact with them, that there is more work for the great bag of the stomach to do. They call upon the part which is most active in secreting gastric juice to pour it out quickly and get the business of digestion over. Meat-extracts, which contain the products of protein disintegration, have a similar influence in promoting the formation of the hormone. Hence, no doubt, the general custom, found from experience to be beneficial, of commencing dinner with soup; although it must be remembered that the rapid absorption of meat-extracts makes them peculiarly valuable as restoratives. They afford very little energy, but what they have to give is quickly placed at the disposal of the economy. Persons whose stomachs are unduly irritable are advised to avoid soup. It leads to undesirable activity on the part of the gastric glands, and especially of the acid-secreting cells. Well-chewed bread also encourages the production of the hormone.

Here it may be well to call attention to the evident division of the stomach into two parts-the large bag, or cardiac portion, which hangs down; and the smaller, funnel-shaped pyloric end, which is almost vertical. The distinction between these two parts is faintly visible in the resting stomach, but even opening the abdomen tends to obliterate it. That it is much more evident during active digestion has been shown by adding subnitrate of bismuth to the food, and throwing the shadow of the stomach on a screen with Röntgen rays. When this is done, it is seen that the two parts work in different ways. Food is churned round and round in the cardiac portion, and pressed towards the pylorus. Its fluid products, mixed with the abundant secretion of the gastric mucous membrane, are wrung out of it by the pyloric funnel. 
They are squeezed towards the pylorus, which opens at intervals to let them through. If lumps of solid matter reach it, the pyloric valve closes tightly, until the undigested food has fallen back into the dependent bag. Dyspeptics are sometimes unpleasantly conscious of the contractions of the pyloric funnel. In fact, putting aside pain due to gastritis, all the discomfort of dyspepsia is felt on the right side. Flatus accumulates beneath the pyloric valve. The valve will not open to let it pass. The pyloric portion of the stomach contracts strongly. Notwithstanding the general trend of movement in the opposite direction, the gases are squeezed back into the larger bag, and escape through the cardiac orifice.

Tables have been prepared showing the length of time which various articles of food take to digest. They are based in part upon observations made upon the living stomach in cases in which it has been possible to examine its contents through a fistulous opening; in part upon the results of artificial digestions carried out in the laboratory. It is hardly too much to say that such observations are absolutely without value as tests of the relative digestibility of the several articles of diet consumed as parts of an ordinary meal. The fact that the commencement of the flow of gastric juice depends upon mental stimuli, and its continuance upon hormones, shows how difficult it must be to reproduce the conditions which obtain in a healthy living body. The most wholesome of foods taken by itself may be longer in digesting, or may produce more irritation, than many less desirable things taken in judicious combination. Crushed chicken, hastily swallowed, sometimes proves more difficult of digestion than meat so cooked and served as to stimulate appetite and to demand mastication.

Returning to the story of a meal, vegetables pass almost unaltered through the stomach. Some of the scanty proteins which they contain are peptonized, but unless they are very well masticated or cooked until they are soft, and therefore easily pulped by the churning action of the stomach, the gastric juice has to reach the proteins through cell-walls. None of the digestive juices are able to dissolve the cellulose of vegetable cell-walls. Blocks of vegetable tissue pass down the whole length of the alimentary canal in the form in which they were left by the teeth. Hence the extreme indigestibility of 
ill-chewed cucumber or apple. The pyloric valve of the stomach is forbidden to allow any lumps of food to pass until the very last stage of gastric digestion. Pieces of ill-masticated vegetable tissue lie for a long time in the stomach, irritating the ends of the gastric nerves, until at last the time comes for them to be shot through the pylorus into the duodenum. Many salts which vegetables contain, especially the earthy carbonates and phosphates, are dissolved by the acid of the gastric juice. Meat consists of muscle-fibres supported by connective tissue. In the stomach the gelatiniferous connective tissue is dissolved, setting the fibres free. Further, the fibres being surrounded by a membrane of the same nature-sarcolemmathis is removed; and although it may be hardly justifiable to speak of "Krause's membranes" (cf. Fig. 10) as gelatiniferous septa, the fibres are certainly composed of segments-Bowman's discs, sarcous elements-into which they break up under the action of gastric juice. As a result, meat-fibre is reduced to a finely divided granular condition. The capacity of gastric juice for dissolving collagen (the substance of which connective tissue is composed) may be regarded as its most characteristic, as it is one of its most valuable, properties. Collagen, when boiled or acted on-by acids, takes water into its molecule, becoming gelatin. Under the influence of gastric juice gelatin is rapidly hydrolysed into diffusible gelatin-peptone. Pancreatic juice is unable to act upon collagen, unless it has been previously boiled, or swollen by the action of dilute acids.

Fat is composed of vesicles of oil supported by connective tissue. Gastric juice, by dissolving the connective tissue and the collagenous walls of the vesicles, sets the oil free. The oil, even though it be as firm as suet when cold, is liquid, or almost liquid, at the temperature of the body.

Thus, with the exception of raw vegetables, the hard fibre of cooked vegetables, elastic tissue of meat, and a few other indigestible substances, the meal is reduced in the stomach to a cream-coloured, fatty, strongly acid "chyme." In this condition it enters the duodenum, where it at once comes into contact with an alkaline secretion. The passage of acid chyme down this portion of the canal provokes the discharge of gushes of bile and pancreatic juice. By precipitating partialiy digested proteins and "acid-albumin " bile renders 
the mixture thicker and sticky. It colours it yellowish-brown. Under the influence of pancreatic juice the remaining proteins and proteoses are rapidly converted into peptones, some of which are shaken down by the violent action of erepsin into simpler bodies, such as leucin and tyrosin, etc. The chyme becomes alkaline, grey, and thin. All undigested starch is changed into maltose, and this into dextrose. Cane-sugar is converted into dextrose and levulose. These sugars are absorbed into the blood. Milk-sugar, if not converted into lactic acid, remains as lactose $\left(\mathrm{C}_{12} \mathrm{H}_{22} \mathrm{O}_{11}\right)$, in which condition it is absorbed without "inversion." Fats are split by a ferment of the pancreatic juice into fatty acid and glycerin ; some of the fatty acid combines with alkali to form soap, but of this we shall have more to say later on.

The duct common to the liver and the pancreas opens into the second part of the duodenum. The organs which produce bile and pancreatic juice are comparatively remote from the place where their secretions come into contact with the food. By what mechanism are they thrown into activity when the assistance of their secretions is required? As in the case of the stomach, the agent is a hormone, a chemical messenger. The hormone, termed "secretin," is formed by the cells of the mucous membrane of the duodenum when acid comes in contact with them. It is absorbed by the blood, which carries it to the pancreas and the liver. When it reaches the pancreas, it acts as a most powerful stimulant to the discharge of accumulated ferments, and to the production of an additional supply. It stimulates the liver to pour forth bile. At present we are in ignorance as to the chemical nature of this hormone. It is not a proteid substance, nor is it a ferment. If scrapings from the mucous membrane of the duodenum be crushed with sand and hydrochloric acid, the mixture boiled, neutralized with carbonate of soda, and filtered, the clear, colourless liquid which results has a powerful effect upon the pancreas, when injected, in even small quantities, into the blood. Apparently, the cells of the duodenal mucous membrane are constantly producing and accumulating a substance which is converted into secretin when acted on by acid. It is not necessary for the acid to stimulate the living cells. If the mucous membrane is ground up with sand and salt-solution, the filtrate is inactive: 
but an active extract is obtained by treating the crushed cells with $\mathrm{HCl}$. It changes some substance which they contain (provisionally termed " prosecretin ") into the efficient hormone.

In the lower portion of the small intestine any maltose that remains is converted into diffusible dextrose. A very large amount of water has been poured into the canal in the various digestive juices. This, together with water drunk, is absorbed in the large intestine. At the lower end of the alimentary canal nothing remains but indigestible substances taken with food, chiefly cellulose, and the pigments and other bodies which, as already said, are eliminated in bile.

The absorption of water is checked by the ingestion of extremely soluble salts, such as sulphate of magnesia, the heavy molecule of which diffuses with difficulty. We attribute the fact that sulphate of magnesia remains in the intestine, and prevents water from diffusing out of it, to its slowness in passing through a membrane, because this is what would happen in dialysis ;* but we must remember that the living wall

* Notice the distinction between filtration and dialysis. If water containing soluble and insoluble substances is placed in a porous jar, the water and the soluble substances pass through the pores of the jar. The rate of flow varies as the pressure. If water containing soluble substances is placed in a bladder, and the bladder is suspended in a vessel of water, some of the substances which it may contain - white of egg, for example - are non-diffusible ; others will pass from the water inside the bladder to the water which surrounds it. But every diffusible substance has its own osmotic value. Some pass through the membrane rapidly, soon establishing a condition of equilibrium in the two fluids; others take a long time. Further, if the water on one side of the membrane contains a certain percentage weight of a salt, the molecules of which are large-say sulphate of magnesia - and the water on the other side the same percentage weight of a salt of smaller molecule-say chloride of sodium-water containing the salt of smaller molecule will pass into the water containing the salt of heavier molecule with a certain force. If, to start with, the two solutions are at the same level, the level of the solution containing the less diffusible salt, sulphate of magnesia, will at the commencement of the experiment rise. It is therefore said to exert a greater osmotic pressure than the more diffusible salt-chloride of sodium. Equilibrium will not be established until the fluid on one side of the membrane contains the same number of molecules per unit volume as the fluid on the other side. If the molecules of magnesic sulphate are pictured as oranges, and the molecules of sodic chloride as nuts, it will be understood that equilibrium is not established until the oranges and nuts to the pint on one side equal in number the oranges and nuts to the pint on the other. When these principles are applied to the passage of water containing products of digestion through the wall of the alimentary canal, it is evident that, if we understand all the conditions, the process cannot be explained as merely an exhibition of osmosis. Take the simplest illustration. When blood-serum is placed in the intestine it is absorbed. If it were in a dialyser, there would be equilibrium between the 
of the intestine is not a membrane. The cells which line the intestine take up substances far less easily diffusible than the sulphate of magnesia which they refuse. Nevertheless, speaking generally, it is the less diffusible salts which act as aperients, the more diffusible which are absorbed. The forward passage of the contents of the alimentary canal is hastened by castor-oil. The peristalsis of the intestines is stimulated by certain drugs, such as jalap or the burnt products of tobacco. Another class of drugs, of which aloes is an example, increases the secretion of the intestines, small or large. Certain purgatives, such as calomel, podophyllin, etc., used to be regarded as cholagogues. It was supposed that they increased the flow of bile. This is an error. Their action is complicated, but it affects chiefly the peristalsis of the intestine. The poor misunderstood liver still suffers from the libels of primitive medical science. It is the most innocent of organs, in no way responsible for derangements of digestion. It carries out its functions without haste and without delay. With the possible exception of salicylate of soda, no drug is known which can stimulate it to a more rapid output of bile.

Absorption.-All the cells which line the alimentary canal are capable of absorbing food, if it is presented to them in a suitable form. In a suitable form means, speaking generally, in a diffusible condition, although it must not be supposed that the epithelial cells are incapable, under certain circumstances, of taking up non-diffusible substances, just as a unicellular organism - an amceba-can take in food. If soluble proteins, such as white of egg or acid-albumin, are injected into the large

serum inside the intestine and the lymph on the outside. There would be no osmosis. Or, again, supposing water containing $2 \%$ of common salt is placed in the intestine, we find that both salt and water pass through into the lymph. In a dialyser water would pass from the lymph (which contains salts equal to about $0.9 \%$ of sodic chloride) through the membrane into the stronger solution. A salt-solution needs to be very concentrated to cause water to take the reverse course through the intestinal wall, and so to act as a purgative. When we study absorption from the alimentary canal, we find that its wall, if it wants a salt or any other substance, sets the laws of osmosis at defiance. If the salt is not wanted, the ordinary phenomena of osmosis are exhibited. Sulphate of magnesia (Epsom salt) would be deleterious if absorbed. The intestinal wall behaves towards it like a dead membrane. The salt retains the water in which it is dissolved: possibly water passes out of the lymph into the solution of the salt. The contents of the intestines are in consequence unduly liquid. The salt acts as a purge. 
intestine, a very considerable proportion of the substance so injected is absorbed. It is possible, indeed, to supply in this way the whole of the nitrogenous food needed by the system, none entering by the mouth. If milk is injected, a certain amount of the fat also is retained. It can be shown that such absorption takes place when no digestion of the food occurs in the colon. The food is taken up by the epithelial cells in the form in which it is injected.

The organs specially devoted to absorption are the villi, which project into the contents of the small intestine. Each is a conical process about $\mathbf{0 . 5}$ millimetre long. The villi are longest in the upper half of the small intestine. Below this level they decrease in number and size. A villus is completely covered with epithelial cells of short, columnar form. The free border of each cell is slightly hardened, forming a disc or cap which appears striated in optical section-an indication, as some think, that it is traversed by pores. Others hold that the appearance of striation is due to minute cilia-like projections which beset the free border of each cell. In worms and other invertebrates the cells carry motile projections of not inconsiderable size, which no doubt free their surfaces from the unassimilable matter which tends to accumulate upon them. Possibly they help to fix particles which are suitable for absorption. In mammals the presence of cilia has not been demonstrated. The extreme minuteness of the striæ seems to point to their being merely indications that the border is permeable to fluids, including droplets of fat.

The so-called basement membrane upon which the epithelial cells rest must not be regarded as a membrane in the physical sense. Rather is it a basket-work which supports the cells, without in any degree limiting their power of disgorging into the lymph-spaces of the villi the substances which they have absorbed. Within the villus, connective tissue forms a spongework, the spaces of which are filled with lymph, in which a considerable number of leucocytes roam, on the look-out, no doubt, for any germs which may make their way between the epithelial cells. In the centre of the villus is a lymphatic radicle-i.e., a fusiform cul-de-sac-which is the dilated end of a lymph-vessel. It, like all other lymph-vessels, is walled by flattened endothelial scales. It communicates with the 
lymph-plexus beneath the mucous membrane, which, again, communicates with a coarser plexus outside the muscular coat. From the peri-intestinal plexus vessels lying in the mesentery converge to the receptaculum chyli, the bulbous commencement of the thoracic duct, which lies at the back of the abdomen in front of the bodies of the vertebræ. The thoracic duct runs up the front of the vertebral column, through the thorax, and then hooks over to pour the fluid which it conveys into the great veins shortly before they join the heart. After a meal containing fat the fluid in the lymphatic vessels of the mesentery, the lacteals, has, as already stated (p. 43), the appearance of milk. The fat absorbed by the epithelium covering a villus is passed on into its lymph-space. From this into the central lacteal receptacle, thence to the submucous and periintestinal plexuses, the lacteal vessels of the mesentery, the thoracic duct. Absorbed fat does not pass through the liver, but is carried into the heart; thence through the lungs, and back to the heart, which pumps it to all parts of the body. In addition to the lacteal radicle, the villus contains long capillary bloodvessels, and the arteriole and venule in which they commence and end. These traverse the lymph-spaces of the connective tissue, which contains, not only the fat which the epithelial cells have passed into it, but the other products of digestion also. None of the fat traverses the walls of the bloodvessels; but the other products diffuse from the lymph, through the walls of the vessels, into the blood. Many nervefibres are found in the core of the villus on their way to epithelial cells, or to one or two plain muscle-fibres which are disposed in the direction of its long axis. For each villus is a little pump. By the contraction of the muscle-fibres it is shortened, and the fluid in its lacteal radicle is forced into the submucous vessels.

Two problems have to be considered : First, in what form and by what mechanism are the several kinds of food absorbed? Secondly, what becomes of them after they have been absorbed?

Clearly, the epithelial cell is the absorbing mechanism. It is not a membrane governed by the laws which regulate diffusion of fluids through membranes, but a living cell. There is hardly any limit to its power of selecting the food which it 
ingests. It could, and very possibly it does, ingest albumin and fats as such. Still, the elaborate provision which is made for converting albumin into diffusible peptone, and cane-sugar and maltose into easily diffusible dextrose, suggests that substances which will pass through membranes are more readily absorbed than substances which will not. We are justified in looking upon absorption as a physical problem up to a certain point. But we must not dwell too much on the physical aspects of the problem. If the absorption of food were merely a process of diffusion, an enormous quantity of water would be required to carry the diffusible products of digestion into the villi. The passage of the foods is aided by the selective activity of the epithelial cells. Peptonization greatly facilitates the work of the epithelial cells, but it is not a condition essential to absorption, so far as soluble proteins are concerned. It is, however, essential that the proteins should be presented to the epithelial cells in a soluble form. They could do nothing with the solid fibres of meat, however much they might have been disintegrated by mastication and by the action of hydrochloric acid. It is only after digestion by pepsin and by trypsin that all the proteins of food are brought into solution. Digestion is needed to reduce them to a condition in which the epithelial cells can take them up.

Much thought has been devoted to the question of the form in which fat is absorbed. Fat in the chemical sense-a pure fat, that is to say - is a compound of a fatty acid and glycerin. Suet, lard, butter, vegetable oils, etc., are mixtures of several fats. All consist of glycerin united with fatty acids. The acids are stearic acid, palmitic acid, oleic acid, and others of less importance. Fats are insoluble in water; so also are the fatty acids. A fatty acid combined with an alkali (in place of glycerin) is a soap. Soaps are soluble in water. If milk is examined under the microscope, it is found to contain droplets of fat, varying in size, but all minute. The larger droplets tend to rise to the surface as cream, but the smaller droplets do not run together. If milk from which the cream has been skimmed is sterilized, it retains its normal appearance for an indefinite time. Its fat remains in droplets. In technical language, milk is an emulsion. Theoretically oil and water would make an emulsion, if the droplets of oil were 
rendered sufficiently minute. Such a condition has been almost obtained by agitating oil and water with powdered glass. But the more viscous the medium through which oil globules are distributed, the greater is the resistance to their fusion. If oil which has become rancid-in which a certain quantity of fatty acid has been liberated from the glycerin with which, in a neutral fat, it is combined-is shaken with water containing carbonate of soda, an emulsion is easily formed. The carbonate of soda and the fatty acids form soaps. A solution of soap is sufficiently viscous to keep the droplets of oil apart. Emulsification of fats occurs in the intestine. It might be assumed that the epithelial cells ingest fat in this finely divided state. But it must be remembered that, however minute the droplets, they are enormously large as compared with the molecules of peptones and sugar which the epithelial cells absorb. It is unlikely that fat is absorbed in a manner so widely different from that in which other foods enter the epithelial cells. Nor is it necessary to make any such assumption. Pancreatic juice contains a ferment which rapidly splits fats into their constituent fatty acids and glycerin. In the presence of an alkali the fatty acids are converted into soaps. In this soluble condition of soap and glycerin the fats are probably absorbed. As soon as they have entered the cell, the fatty acids and glycerin reunite to form fats, setting the alkali free. The alkali is returned to the intestine, where it is available as a solvent of further droplets of fat. The droplets of fat accumulate in the epithelial cells. During active digestion they are also to be seen in the connectivetissue cells, in the leucocytes, and in the lymph inside the lacteal vessel. The epithelial cells extrude the oil droplets, backwards, much in the same way as the cells of the mammary glands extrude globules of milk. In herbivora, and in Man also so far as we can judge, the contents of the small intestine are alkaline. Conditions are therefore favourable for the formation of soap. But in carnivora the contents are acid throughout the greater part of the canal. Acid, it need hardly be stated, prevents saponification. Yet carnivorous animals have an immense capacity for absorbing fat. Fatty acids are soluble to a moderate extent in bile. It is possible that, fats having been split into fatty acids and glycerin, the 
fatty acids are carried into the cells in solution in bile. But if in carnivora bile actively participates in the absorption of fat, there is no reason opposed to its having the same function in Man; and, indeed, all observations which have been made upon patients in whom the bile was, for some reason, diverted from the intestine, and in animals in which a fistula of the gall-bladder has been artificially produced, show that in the absence of bile the absorption of fat is considerably decreased. Yet there is no reason for thinking that bile is secreted for the purpose of facilitating the absorption of fat. Just as much bile is poured into the intestine of a cow which is feeding upon grass as into the intestine of a pig or a dog when the animal is consuming a very large quantity of fat. Nevertheless, it appears to be certain that, not in carnivorous animals only, but also in herbivorous animals, the assistance of bile is necessary for the satisfactory absorption of fat. Doubtless the co-operation of bile and pancreatic juice is more important to carnivora than it is to herbivorous animals, in which, owing to the alkalinity of the contents of the intestine, all fatty acids liberated by the action of pancreatic juice might be converted into soluble soaps.

The problem of the form in which foods enter the absorbing cells is intimately associated with the further problem of the form in which they leave them. In the villus, and even within the epithelial cells, fat appears abundantly as such. If, as we have reason for believing to be the case, it enters in the form of soap and glycerin, the reformation of fat is an illustration of the synthetic power of the tissues. For the purposes of the economy it is needed as fat, and not as the constituents of fat. There is no reason for thinking that at any stage in its future progress it is again split into fatty acid and glycerin.

We cannot see absorbed proteins with the microscope, as we can see fat, nor can we apply chemical tests which will distinguish between the proteins which the cells contained before digestion commenced, and the proteins which they have received as its result. Nevertheless, it is certain that peptones are reconverted into proteins as soon as they are absorbed. They are not to be found in blood or lymph. If the peptones absorbed after a proteid meal remained as such 
after they passed through the wall of the alimentary canal, they would produce various undesirable results.

There is some difficulty in following droplets of fat across the space which intervenes between the epithelium of a villus and its lacteal radicle. It has been asserted that leucocytes act as carriers, catching the droplets as they are extruded by the epithelial cells, and bearing them into the radicle, where they set them free. Undoubtedly, many leucocytes are present in the lymph-spaces of a villus. After a meal they are found charged with fat. But it is hardly in accord with what we know of the character of a leucocyte to suppose that it will let go fat which it has once ingested into its own body-substance. A leucocyte is not a disinterested organism. If fat droplets are floating across from the epithelium to the lacteal, leucocytes are pretty certain to steal some of them. But we know of no other case in which they give up what they have stolen, unless it be something which is injurious to their own health. Even then they usually cling to it, whether it be a germ or a particle of soot, until their own dissolution sets it free.

Neither proteins nor sugar reach the lacteal radicle. Both these substance are absorbed from the lymph in the tissuespaces of the villus by the blood-capillaries and venules which traverse them. The veins of the intestine unite to form the portal vein, up which proteins and sugar are carried to the liver, where they are stored, to be doled out into the bloodstream as the tissues need them.

Bacteria of the Alimentary Canal.-The enzymes (ferments) of the several digestive juices are not the only agents which modify the constitution of the foods within the alimentary canal. Throughout the whole of the tract conditions are in many respects favourable for the growth of putrefactive organisms. Mouth, stomach, small and large intestine, has each its special bacterial flora. It is doubtful whether any of these organisms, with the single exception of the bacteria which in herbivorous animals break up cellulose, are favourable to digestion. That they are not necessary has been shown by an ingenious experiment on new-born animals. Guineapigs born in an aseptic chamber, through which filtered air was drawn, and fed every hour on sterilized milk, throve and put on weight. When killed at the end of eight days, no germs 
were present in their alimentary tracts. Yet in all animals under ordinary conditions bacteria are present in great numbers, at any rate, after the nursing period, and, for good or ill, produce important fermentations. Only a single bacillus (B. bifidus), and that a friendly germ, is, it is asserted, present in the intestines of an infant at the breast ; whereas a bottlefed baby houses a variety of parasites.

In the stomach, sugars are changed by the Bacterium acidi lactici into lactic acid, which is further split into butyric acid, carbonic acid gas, and hydrogen. Succinic acid and other substances are also formed. This occurs in the first stage of gastric digestion. When a considerable quantity of hydrochloric acid has been poured out, lactic fermentation is stopped. The small amount of gaseous products formed normally is of little consequence; but flatulence is a most annoying symptom of indigestion. "Put your trust in Providence, and you will feel more cheerful after luncheon," Dr. Jowett is alleged to have remarked to a despondent friend. The presence of food stimulates the stomach to contraction. Accumulated gases are expelled. Hydrochloric acid is secreted, and puts a stop to fermentation for a time. But if the meal be too heavy or the mucous membrane in an irritable condition, the contents of the stomach become unduly acid in the later stages of digestion. Other bacteria then develop, leading to fresh trouble; more gases accumulate, and the dyspeptic's distress is greater than it was before. Unfortunately, antiseptics, such as creosote, and carminatives, such as oil of lavender, oil of peppermint, or alcohol, which for the moment give relief, increase irritability, and consequently in the long-run make matters worse. It is the fermentation of the later stages of digestion which causes most annoyance. Admirable as was the Master of Balliol's advice, it hardly took account of the fact that bacteria which cause flatulence, with its resultant feeling of oppression, are derived for the most part from the imperfectly digested, and therefore actively fermenting, remnants of food which were present in the stomach when the meal was taken. It would be far beyond the scope of this book to consider the pathology of dyspepsia; but the study of normal conditions reveals the fact that some amount of fermentation invariably occurs. The Bacterium acidi lactici is always present in the stomach. 
Normally its activity is arrested by the hydrochloric acid of the gastric juice about twenty minutes after a meal. After this no further multiplication of bacteria should occur. The presence of bacteria which grow in a strongly acid medium usually indicates that the stomach was not completely emptied before fresh food reached it. It may be that the last meal was too large or the interval too short. If the mucous membrane is in an unhealthy condition, its own secretions afford material on which bacteria thrive. Nothing short of washing it out with a stomach-pump will clean it up. The presence, at the time of feeding, of food left over from the previous meal is likely to perpetuate the unsatisfactory state of affairs. All the glands of the alimentary tract exhibit a tendency to periodicity. Their efficiency is greatest when activity follows a period of rest. If the stomach is not able to expel its contents, it has not the opportunity of preparing for fresh duties. Fat undergoes a certain amount of rancid fermentation in the stomach. Proteins are not attacked by bacteria in the stomach unless the condition of the organ is very unsatisfactory. The odour of the products of their decomposition is then recognizable in the breath.

Bacteric fermentations in the small intestine are unimportant under normal conditions, with the exception of the fermentation of cellulose. Cellulose has the same empirical formula as starch. It is completely insoluble, and is not affected by any of the digestive juices. The greater part of the cellulose consumed by herbivora is, however, broken up by bacteria into acetic and butyric acids, carbonic acid, and marsh-gas. In Man also a small quantity is similarly destroyed.

In the large intestine the bacteric fermentations are not unlike those which occur in the stomach, with, in addition, the destruction of proteins, or of products of proteid digestion. The greater the quantity of undigested food which reaches the large intestine, the greater is the development of bacteria. When the stomach is dilated, the ascending colon, and especially its cæcum, is usually dilated also. Bacteric fermentation in the large intestine, with resulting flatulence, is evidence of imperfect digestion, due either to an excess of food or to weakness of the alimentary organs, or, as is more commonly 
the case, to the combination of these two factors. The relation of fermentation to alimentation can be shown by counting the microbes in a specimen of the contents of the large intestine. In a particular case it fell from 65,000 per milligramme upon a mixed diet to 2,000 per milligramme upon a diet of milk.

In the world at large bacteria perform many offices of the utmost usefulness to other living things. They fix nitrogen in the soil, sweeten polluted rivers, reduce animal and vegetable matter to a condition in which it is available as plant-food. Their presence within the alimentary canal is inevitable; but it is somewhat doubtful whether, with the exception of the fermentation of cellulose, they do the economy any service with which it could not dispense. As parasites of the alimentary canal, some kinds are less desirable than others. Recently a method of limiting their variety has been introduced and advocated with much enthusiasm, as favourable to the hygiene of the digestive tract. In countries in which the cows are driven, in summer, to mountain pastures, the peasants of the plains live during their absence largely upon milk brought down at intervals, and allowed to turn sour. Sour milk, in Bulgaria, develops a bacterium of extraordinary vigour. It can live in a medium containing as much as 10 per cent. of lactic acid, a concentration fatal to other forms of Bacterium acidi lactici. It is easily cultivated, and when ingested continues to multiply in the alimentary canal. So peculiarly lusty is this bacterium that it makes life impossible for other germs. As it dies out after two or three months, it seems unlikely that a man who swallows the Bulgarian milk-germ runs a risk of inviting a repetition of the tragedy which followed the acclimatization of the mongoose in Jamaica. Its supremacy has been attributed to its capacity of developing a concentration of lactic acid too high for the well-being of other bacteria; but it is improbable that it has the opportunity of doing this in the alimentary canal of a person living on a mixed diet. The extinction of other bacteria (if they are extinguished) is more likely to be due to an antagonism of a more subtle kind, at present inexplicable, but not without parallel. The purifying influence of the water of the Ganges has for ages been an article of faith. Pilgrims from fever-stricken districts bathe in it, foul it, drink it, with the corpses of their fellows floating down the stream. 
Recently it has been shown that this belief is not without foundation. The water of the Ganges at Benares contains bacteria which are as tigers among lesser vermin. The germs of cholera and typhoid fever disappear from cultures into which these overbearing microbes are introduced.

Conditions Requisite for Normal Digestion.-When M. Chevreul, Professor of Chemistry at the Jardins des Plantes of Paris, attained his hundredth year, an interviewer very naturally inquired of him, "Have you always had a good digestion?" To this the still vigorous Professor answered: "I really cannot say, for I have never noticed." So long as it is well used, the stomach is an unobtrusive organ. It is tyrannical when it deems itself the victim of inconsiderate treatment. A study of its physiology serves to show that it will work contentedly only upon certain clearly defined terms, of which the following are perhaps the most important: The stomach exacts due warning that its services are wanted. The nerves of smell and taste must announce the approach of food and guarantee its quality. "What may I eat?" asked a large-framed, strenuous, eager, over-worked barrister of a great physician. "Eat, sir? You may eat whatever you like. But be quite sure that you do like it." Wise advice. The human race would not have developed its strong preferences for certain kinds of food if all foods were equally suitable to satisfy its needs. Taste is not a matter of fashion. It is the expression of the experience of mankind. Fanciful as civilization has made us, and easily as appetite is perverted, if we are sure that we really like, and want, a food, we may trust that our liking will guide us as safely as it guides a buffalo or a deer. "Eat what you like." Eating with liking carries with it the idea of obtaining the maximum of satisfaction from the exercise of this necessary function. Most things which are reckoned unwholesome are full in flavour or rich in consistency. They satisfy the palate when spread out very thin. It is poor economy to help oneself to caviare with a table-spoon. In the second place, the stomach must be assured that the teeth are doing their proper share of work. Among the many halftruths which every year are exalted to the level of a revelation or a rule of conduct is the doctrine of the "chewers"persons who take no meals, but industriously and almost 
continuously masticate nuts and biscuits. Thirdly, the meal must not be so large that the stomach cannot deal with it " at a sitting." In from two to three hours the last of the food should have passed through the pylorus, allowing the stomach to rest before it is called into activity again. As proteins are practically the only foods which are digested in the stomach, the work required of this organ depends upon the quantity of proteins present amongst the constituents of a meal. Meat is the food richest in proteins, although bread, vegetables, milk, cheese also yield them. Some people can digest three meat meals every day; but others, probably the majority, find that it is unwise to take any considerable quantity of meat more than once in twenty-four hours. It is only when the cells of the gastric glands have accumulated a store of pepsinogen-granules that proteid digestion is vigorously carried on. Fourthly, the food must be in a form in which it does not irritate the stomach, provoking an outflow of acid out of proportion to the pepsin which accompanies it. Experience alone can teach the foods which are to be avoided on this account. But speaking generally, it may be said that the stomach resents the presence of substances which cannot be amalgamated into chyme. Its task is the reduction of the mixture of foods which compose a meal to the consistence of a smooth cream. Hot buttered toast or pie-crust are made of wholesome constituents enough, but, fat being melted into the starch, the fragments are impermeable to the gastric juice. They act mechanically as irritants of the mucous membrane. Again, it may be said that "pure" foods are apt to provoke acidity. Nothing could be more wholesome than eggs or pounded meat or custard pudding; but taken by themselves these articles of diet over-stimulate the mucous membrane. They need to be diluted with starch-foods, or even with cellulose.

And this calls attention to the dietetic value of vegetables. Vegetables, which consist chiefly of innutritious cellulose, distribute the digestible constituents of a meal and increase its bulk, greatly favouring its progress through the alimentary canal. Especially in herbivora is it important that the bulk and looseness of the food should be well maintained. Rabbits thrive on sugar, starch, and albumin, mixed with such an 
absolutely indigestible substance as horn-shavings. If the inert substance be omitted, they die of intestinal inflammation, although fed on the same mixture of pure foods. Other rules which govern digestion might be mentioned; and it is needless to point out that, when the mechanism is deranged, steps adapted to the particular malady must be taken to bring it back to a normal condition. There is, however, one precaution upon which, in a certain number of cases, it is impossible to lay too much stress. The digestion of proteins is seldom carried out satisfactorily when much sugar, and especially much cane-sugar, has been eaten at the same meal. Excessive lactic fermentation prevents the proper peptonization of meat. The chemistry of digestion is not sufficiently well understood to enable the physiologist to say what is amiss; but probably by-products of peptic digestion are produced. To many people this is of little consequence; but to those who exhibit a gouty tendency it is, unfortunately, a most serious matter. Civilized races are particularly subject to the uric acid diathesis. In the course of nitrogenous metabolism uric acid is formed in place of fully oxidized and easily soluble neutral urea. Although the chemical sequence has not been discovered as yet, there is no question but that imperfect gastric digestion means the formation of uric acid, with all its lugubrious results : malaise, neckache, emotional depression. Birds and reptiles form uric acid as the end-product of nitrogenous metabolism, not urea. So also do city-fathers, butchers, and others whose diet consists too largely of meat. Many nervous, ill-nourished men and women tend to do the same, however abstemious their meals. It is useless to tell such persons to reduce the amount of proteins in their diet. Their attempts at increasing the starch, sugar, and fat at the expense of nitrogenous foods lead to dyspepsia, which makes matters worse. They often find, however, that if they are careful to restrict to the narrowest limits the amount of carbohydrates (especially sugar) which they take in conjunction with meat, fish, eggs, or other proteid foods, the formation of uric acid ceases. Sugar, bread, fruit, and other carbohydrates, may be taken in abundance, and with great advantage, at breakfast and lunch, without proteid food, if dinner consists of broth, fish, meat, cheese, vegetables, with a minimum of bread. 
The History of the Foods after Absorption.-All foods, with the exception of inorganic salts and salts of various vegetable acids, fall into three classes: (1) Proteins-substances of complex chemical constitution, containing nitrogen; (2) carbohydrates-so called because hydrogen and oxygen, in the proportions in which they enter into the formation of water, are united with carbon ; (3) fats. Proteins of various kinds are consumed as food. The peptones produced from them by digestion also vary. Yet very little is known as to the differences in physiological value which distinguish the various kinds of protein when absorbed into the fluids of the body (cf.p. 134). All carbohydrates after digestion and absorption appear as dextrose. The various fats preserve their individuality until they are taken up by the tissues. When fixed in the tissues, they assume, except under somewhat abnormal conditions, the composition characteristic of the fat of the animal which has eaten them. If a dog which has been severely starved is fed upon mutton-fat, it puts on in the first instance fat which resembles that of a sheep rather than the normal fat of a dog. As soon, however, as it is well nourished (which would never occur unless some protein and carbohydrate were added to the mutton-fat), its fat assumes the usual form.

For practical purposes we are obliged to speak of the three classes of food-proteid, carbohydrate, and fatty-as if there were but one member in each class. And we have abundant evidence that such a simple classification is fully justified. The body has so large a power of altering chemically the nature of the food which it absorbs that it makes little difference in the further history of the food whether the protein supplied to it be an albumin or a globulin; the fat, stearin, palmitin, or olein ; the carbohydrate, starch or sugar.

In earlier days it was customary to regard the body as the receiver of a variety of foods which it could break down into simpler substances by oxidation, but could not reconstruct. Plants were regarded as the manufacturers of organic compounds, animals as the destroyers of the complex substances made by plants. The union of molecules, synthesis, was looked upon as the function of the vegetable kingdom. Animals built into their tissues the products elaborated by plants; some of these products they shook to pieces for the 
purpose of setting their energy free; others slowly disintegrated as the result of tissue "wear and tear." Gradually it was realized that many chemical changes occur in the body which cannot be viewed as merely exhibitions of its analytical capacity. The tissues were recognized as laboratories in which reactions occur which consist in something more than the splitting of complex into simpler molecules. The instances earliest understood were connected with the history of carbohydrates and fats. In the disease diabetes an enormous quantity of sugar is excreted, amounting in extreme cases to between 1 and 2 pounds per diem. When carbohydrates are present in the food, the amount of sugar excreted in diabetes is greater than it is when they are withheld; on an almost exclusively proteid diet the amount of sugar excreted far exceeds the amount of carbohydrates in the food. Another illustration of the power of making sugar possessed by the animal economy is afforded by a dog fed upon lean meat, and nothing else. Sugar is found in its blood, and a store of carbohydrate (glycogen) in its liver. The formation of fat is an instance of constructive metabolism. There is abundant evidence that the quantity of fat produced may greatly exceed the quantity contained in the food. Animals are fattened for the market on a diet which contains less fat than that which accumulates in their bodies. When nursing her young, an animal may secrete in her milk much more fat than she obtains as such in food. It was a great mistake to suppose that the body is dependent upon its tradesmen for fat and sugar. It can make either of these substances out of a mixed. diet in which it is relatively deficient. It must, however, be a mixed diet. An animal cannot live exclusively on fat or exclusively on carbohydrate. It is impossible, therefore, for us to determine whether, if given the one alone, it can turn it into the other. Chemists were very unwilling to credit the body with the power of performing even the simpler of these transformations-the conversion of carbohydrate into fat. Proteins are essential constituents of a fattening diet. Their immensely complex molecule has always afforded a tempting field for arithmetical ingenuity. It is easy to remove from it the atoms needed for the composition of fat, and yet to leave such groups of atoms as might reasonably be supposed to con- 
stitute its " nitrogenous moiety." The hypothesis that the metabolic capacity of the body is limited to analytical processes justified the supposition that, when more fat is laid on than the food contains, the balance comes from proteid substances, which split into nitrogenous and fatty moieties. It has been shown, however, that an animal during fattening may put on more fat than is contained as such in the food, or obtainable from its diet, even though all the atoms of carbon and hydrogen in its proteid food were devoted to its formation. The balance must come from carbohydrates. Perhaps a still more striking illustration of constructive capacity is the power of making glycerin. If a dog receive fatty acids in its diet, it accumulates normal fats. The glycerin which, united with fatty acids, constitutes the fat, was not contained in its food. Starch and sugar are sources of fat. As yet there is no evidence that fat can be converted into sugar.

The chemistry of $\{$ he nitrogen-containing compounds appears to present more difficult problems. Plants build up proteins. Is the animal's relation to these substances limited to their disintegration? Do proteins inevitably descend from step to step until they reach urea? There are reasons for thinking that, even when dealing with nitrogenous substances, the metabolic power of the body is not exclusively analytical. The liver can make urea from ammonia-salts, such as lactate, or even carbonate, of ammonia-substances more stable, and therefore in the chemical sense simpler, than urea. This is an indication, though a faint one, that the body has a constructive capacity, a power of producing more complex from simpler substances, even in the case of nitrogenous compounds. Beef-tea, mutton broth, meat-extracts have long been regarded as foods of value when the power of assimilation is low. Chemists point out that the nitrogenous substances which these decoctions contain are so near the bottom of the ladder that the energy set free by their further oxidation to urea is scarcely worth consideration. They admit that their ready availability renders them useful as restoratives, but they deny them the status of foods, on the assumption that their further progress must be downward. As was stated when the conversion of peptones into leucin and tyrosin was described, evidence is beginning to accumulate which shows that within certain limits, at 
present impossible to define, the system can reconstruct its proteins from amides and other simple products of their degradation.

The animal economy receives, and after due digestive preparation absorbs, three classes of food-nitrogenous, fatty, and carbohydrate. If either of the two latter kinds be deficient in the diet, the body can to a certain extent produce it from the other two. What is the special value of each kind of food ? What use is made of it? Before attempting to answer these questions, we must endeavour to trace the further history of the foods after they have traversed the wall of the alimentary canal.

After leaving the stomach and intestines, the foods follow two different routes. Proteins and carbohydrates are carried by the portal vein to the liver. Fats are carried by the thoracic duct to the general circulation. An excess of fat is found in the blood in all parts of the body after a meal rich in fat. The eventual destination and fate of fatty foods is unknown. Under certain circumstances they are added to the fatty deposits in connective tissue ; but if no additional fat is being laid down, they go to other tissues, in which they are oxidized into carbonic acid and water. When the amount absorbed is excessive, a certain quantity of fat may be stored in the liver. In the cells of this organ it is housed for a time, in order that it may be distributed to the tissues after they have used up the supplies which first reach them through the general blood-stream.

Proteins are completely lost to sight after they are absorbed into the blood. They take part, of course, in the formation of growing tissue, blood-corpuscles, skin, hair, nails. It is also common to speak of them as making good the wear and tear of active tissues, although it is very doubtful whether we can legitimately speak of the wear and tear of tissues. The protoplasm which does the work of the body is not worn out in the same way as the materials of which a machine is made. There is no friction to rub it down. Proteins, like other foods, are used up as sources of muscular energy and heat. Eventually they are reduced to urea, carbonic acid, and water. Chemists naturally seek for substances intermediate in constitution between proteins and urea. They 
assume that the degradation of proteins will occur in regular steps; complex, partially oxidized, nitrogenous compounds being formed first-in the muscles, for example-to be further oxidized in the glands. The existence in all organs of nitrogenous "extractives," which can be separated out when the organ is subjected to chemical analysis, seems to justify the search for stages; but hitherto this search has been singularly unsuccessful. Urea is the final product. It is not found in muscle, nor, indeed, in any tissue other than the liver, which, as already said, has the power of making it, even from salts of ammonia. It is therefore clear that if proteins are destroyed in muscle and other tissues, and if all urea is made by the liver, the antecedents of urea must be carried from the muscles to this organ. The substance which is most characteristic of muscular metabolism is lactic acid. It is not impossible that all the nitrogenous portion of the complex proteid molecule is reduced to ammonia $\left(\mathrm{NH}_{3}\right)$, which may be regarded as the simplest of all nitrogenous compounds, and that this, combined with lactic acid $\left(\mathrm{C}_{3} \mathrm{H}_{6} \mathrm{O}_{3}\right)$ as lactate of ammonia $\left(\mathrm{NH}_{4} \mathrm{C}_{3} \mathrm{H}_{5} \mathrm{O}_{3}\right)$, is carried by the general circulation to the liver, where it is converted into urea. A considerable amount of lactate of ammonia may be injected into a vein without any of it overflowing through the kidneys. It is all reduced to the condition of urea, water, and carbonic acid. If the liver is so diseased as to be functionless, or if by operative measures it is thrown out of action, salts of ammonia are excreted by the kidneys instead of urea. In birds and reptiles uric acid takes the place of urea. Their livers yield uric acid on analysis. If lactate of ammonia be injected into their blood, it is converted into uric acid, so long as the liver is intact.

We know nothing of the forms assumed by the proteins absorbed into the blood, of the organs in which they are stored, or of the higher terms of the series of substances through which they pass before they are finally excreted as urea, water, and carbonic acid. No nitrogenous compounds are found in lymph or blood which can be pointed out with confidence as the products of tissue wear and tear. When considering the sources of muscular energy, we shall have something more to sayregarding the part that proteins play in the economy.

If there is great difficulty in following fats and proteins 
after their absorption, it is quite otherwise when we come to deal with sugar. Carbohydrates are the great sources of energy. Muscular work may be generated by the oxidation of either of the three classes of foods, but undoubtedly the carbohydrate glycogen is its most constant source. Provision is therefore made for the storing of glycogen in the liver, and the distribution to the muscles of a regular supply. After a meal the portal blood, on its way from the intestines to the liver, contains a higher percentage of sugar than the blood in the hepatic vein or in any other vessel. If sections of liver be examined after feeding, and compared with those obtained after a period of starvation, it is found that the cells of the well-fed liver contain glancing masses of a substance which takes a port-wine colour with iodine. This is glycogen, or animal starch. It has the same empirical formula as starch $\left(\mathrm{C}_{6} \mathrm{H}_{10} \mathrm{O}_{5}\right)_{\mathrm{n}}$. In the dry state it is a greyish powder, which, unlike starch, forms an opalescent solution in cold water. Like starch, it is non-diffusible. In the animal kingdom it stands to sugar in the same relation as starch to sugar in plants. If a sheep be killed while it is feeding in the paddock, and its liver removed and weighed, it will be found that it is from one-third to one-half heavier than the liver of a sheep of the same weight obtained from a butcher; for butchers have the stupid practice of starving animals before they kill them. It was long ago discovered that it is unnecessary to feed an animal for a day or two before it is killed, and this option has been elevated into a prohibition. A tradition has grown up that it is undesirable to give food for some time before killing. Not only will the liver of a sheep killed during active digestion be found to be heavier than that of a starved sheep, but it will also prove more succulent; for it is loaded with sugar (into which glycogen is rapidly converted after death), as well as with proteins and fats, which are withdrawn from it when the animal fasts. It appears that the liver cannot secure the whole of the sugar which is absorbed after a full meal. Some of it passes into the general circulation, and is stored in the muscles; but the liver always maintains a considerable reserve. Even after prolonged deprivation of food, it holds on to a certain quantity, especially in carnivora. Glycogen is found in the liver of a dog after a 
long period of starvation. The muscles lose during activity the glycogen which they contain when at rest.

It has already been pointed out that the body is not entirely dependent upon external agencies for the production of the sugar which it needs. When the supply is inadequate, it manufactures glycogen for itself out of the other constituents of the diet. It can, indeed, make it at the expense of its own proteins. If a dog which has been caused to do muscular work, without a sufficiency of carbohydrate food, until (as judged from a control experiment) all glycogen has disappeared from its liver, be placed under the influence of a narcotic drug, which arrests the activity of its muscles, glycogen reappears.

Dietetics.-Even those who are most ignorant of the science of physiology flatter themselves that they have one piece of information: "The whole of the body is renewed once in every seven years." I cannot trace the origin of this sapient apothegm, which for generations has passed current. If seven weeks or seventy years were the period allowed for the renewal of the tissues, the statement would be equally near the truth. Judging from the rate at which they are destroyed, it is unlikely that blood-corpuscles live for more than five or six weeks. Hairs are shed about two years after they first appear above the surface. On attaining this age a hair drops off and a new one takes its place. The superficial cells of the skin are shed in great numbers every day, and their place taken by younger cells which come up from the deeper layers. The cells of many glands would seem to have a comparatively short term of life. On the other hand, some tissue-elements are far more permanent. By the time a child is a year old all its nerve-cells are in position. They last as long as the individual lives. When the statement with regard to the renewal of the tissues is understood as meaning, not that the cells are destroyed and replaced by new ones, but that within a period of seven years all the molecules which enter into their protoplasm are extruded from the body and replaced by molecules received as food, the assertion verges on the transcendental. It is unlikely that we shall ever obtain data against which it can be checked.

The essential part of every living cell is its spongework of protoplasm. "Bioplasm" is perhaps a better term to use when we are speaking of protoplasm as a structure, since it does not 
suggest any prejudice with regard to its chemical constitution. Within the meshes of the bioplasm are nutrient materials, as yet unused, and worked-up products in various stages. It has always been taken for granted that when treating of nutrition, we have to consider the repair of the bioplasm, as well as the provision of raw material which it can convert into the specific products of the cell. Suppose that the cell belongs to the class of supporting tissues; let it be a cell of cartilage, for example. The bioplasm manufactures a collagenous substance which remains in and around its meshwork. If it be an epidermal cell, it forms horny substance. If a secreting cell, it accumulates secernable products. If a muscle-cell, it develops a large quantity of material, which by a change in form produces movement. In this last case we suppose that the energy set free as muscular force is due to oxidation. More stable bodies take the place of a less stable substance. After contraction the relatively complex contractile material is renewed from the foods stored in the muscle-cell ; or if it be not, in the ordinary sense of the word, destroyed, if it has merely parted with certain oxidizable constituents, it obtains a fresh supply of such constituents from the foods which the muscle-cell contains. Even in the case of cartilage or epidermis, we imagine that, since the matrix is "alive," it is always undergoing molecular change, and consequently always requiring food. The fact that every tissue, however inert, dies when, owing to the blocking of the bloodvessels which irrigate the part, its supply of nutriment is cut off, justifies this belief that all living tissue is undergoing change.

When we make up a balance-sheet of the body as a whole, placing to the debit side the food which it receives, and to its credit side the work done in external movement and in the production of heat, we again find reason for believing that every part of every cell is constantly undergoing change.

The balance-sheet of the body can be drawn out in either of two ways. We can estimate the quantities of nitrogen, carbon, hydrogen, and oxygen supplied to it in the several foods, and compare them with the amounts of each of these four elements given off in urea, carbonic acid, and water, making, of course, a note of the body's balance in hand at 
the beginning and at the end of the period of observation. Or, we may estimate the amount of potential energy contained in the food, and ascertain the use to which this energy is put in doing external work, in maintaining the temperature of the body, and in warming the breath and other excreta.

If we are making up the balance-sheet of a fully-grown man, we may take for granted that he is not making fresh tissue. During the period throughout which he is under observation, care is taken to avoid altering the conditions of his life in such a manner as to lead him to develop additional muscle. If he gains in weight while under observation, he is putting on fat. If he loses in weight, he is sacrificing fat.

The whole of the nitrogen taken in leaves the body in urea, unless, as we have said, growth of tissue is taking place. The body has not the same temptation to store nitrogen as it has to store carbon. Consequently, it is very sensitive to any deficiency of nitrogen in the diet. If food does not contain as much protein as is needed, the deficit is made up at the expense of the tissues. It does not necessarily follow that under these circumstances a man loses in weight. He may be putting on fat, although losing in strength owing to waste of muscle. For observations upon the income and expenditure of the body to be of any value, a condition of " nitrogenous equilibrium" must be established. The nitrogen taken in must equal in amount the nitrogen given out.

Very exact determinations of income and expenditure may be made by placing an animal, or even a man, in a box through which air is drawn. A record is made of the volume of air drawn through the box. The percentages of water vapour and carbonic acid which the air contains are estimated before it enters and after it leaves. The solid food consumed and the urea excreted are also measured.

If it is desired to measure the amount of heat given off, an animal may be placed in a calorimeter.

Even when most passive, the subject under examination, whether an animal or a man, is expending energy in keeping the body warm, in movements of respiration, and in shifting position. If it is desired to ascertain the relation of oxidation to external work, it is easy to devise a form of resistance, such 
as the turning of a wheel, or the lifting of a weight which can be measured.

In testing diets, it suffices to make sure that nitrogenous equilibrium is maintained, and then to estimate the gain or loss in weight and the output of energy in external work.

The Relative Value of Foods.-Dried proteins contain about 15 per cent. nitrogen, 54 per cent. carbon, 7 per cent. hydrogen, 22 per cent. oxygen, a little sulphur, and frequently some phosphorus. A large proportion of their carbon and hydrogen is available for combustion. Fats contain 75 per cent. of carbon, and a considerable quantity of hydrogen available for combustion; carbohydrates, 40 per cent. of carbon, with hydrogen and oxygen in the proportions in which they occur in water. If 1 gramme of protein is oxidized to the condition of urea, carbonic acid, and water, sufficient heat is liberated to raise the temperature of 4,100 grammes of water 1 degree centigrade. Its calorific value is therefore expressed as 4,100 calories, the unit of measurement-a caloriebeing the amount of heat needed to raise 1 gramme of water $1^{\circ}$. The calorific value of 1 gramme of fat is 9,300 calories ; of 1 gramme of starch, 4,100 calories. Thus, the energy potential in protein and in starch is the same; that in fat more than twice as great as that in either of the other foods.

A Normal Diet.-Nitrogenous equilibrium and body-weight can be maintained and work done on diets which vary widely in percentage composition. This is a question which we shall consider at greater length later on. In the meantime, for the sake of illustration, it is necessary to formulate a diet which is fairly representative of the selection of foods made by a man of average weight-say 70 kilogrammes (145 pounds)-who desires to do a moderate day's work in comfort. It has been found to amount to about 100 grammes of protein, 100 grammes of fat, 240 grammes of carbohydrate, all measured dry and as pure foods. If the several elements of such a diet be multiplied by the figures which represent their calorific value, it will be found that the man is supplied with $2,324,000$ calories. The illustration that we have chosen is the diet of a professional man who is not engaged in hard physical work. The pure foods would be found to the amounts stated in 17 ounces lean meat, 4 ounces butter, and 17 ounces bread. The day's 
diet would, of course, be much more varied than this, but it is simpler to express it in these terms.

Such a diet would hardly answer the requirements of a man doing hard muscular work. Experience shows that he would expect to receive a more liberal supply of energy, and that to obtain it hewould increase slightly his allowance of proteins, and very considerably increase the quantity of carbohydrates that he consumed. The diet of European workmen is remarkably constant in the relative amounts of its several constituents, no matter what their nationality or the exact form of their work may be: Proteins, about 135 grammes; fats, 80 grammes; carbohydrates, 500 to 700 grammes-giving a supply of energy equal to 3,500 to 4,000 kilo-calories.

Speaking generally, carbohydrates are the source of muscular force, and fats of heat. In warm climates men work on carbohydrates. The 'rickshaw men of Japan are said to eat only rice on working days, and to reserve fish for days of leisure. The Japanese, as is well known, consume extremely little fat. The Esquimaux and other inhabitants of high latitudes eat immense quantities of fat. Proteins constitute the luxurious element of a diet. Not only are they more attractive to most palates, and therefore preferred by persons whose dietary is not severely regulated by price, but the body prefers them. It works with greater alacrity when supplied with more protein than, in a strictly physiological sense, it needs.

The supply of food must exceed the apparent demand. The most efficient of motors cannot convert more than 15 per cent. of the energy potential in its fuel into work. If a man endeavours to obtain a better result than this from his muscular system, if he tries to make his machine do more than 15 units of work for every 100 units of energy with which he supplies it, he does it at the expense of his own tissues. First he loses in weight, owing to the consumption of fat; then the excess of nitrogen discharged over nitrogen consumed shows that he is burning up the proteins of his own tissues. It is needless to add that the weakness which results puts a stop to excessive work. Muscles, as we shall find when we consider the relation of their output of work to the energy supplied to them, can produce a much better result than the best of engines; but we are speaking of the body as a whole, which wastes energy in the 
movements of respiration, masticating food, shifting position, maintaining the body-temperature, etc.

Health may be maintained and work done on diets which depart widely from the one which we have selected as a standard. Darwin found the Guanchos of South America living exclusively on meat. Nansen and Johannsen, when seeking the North Pole, lived for months on meat and blubber. Millions of the inhabitants of India abstain from meat and meat-fat, their diet consisting of rice, buttermilk, and a little fruit. In the case of all persons with whom the price of food is an important consideration, carbohydrates are preferred to proteins and fats. Oatmeal is very much cheaper per unit of energy than meat. A man may be a meat-eater or a vegetarian, although he is probably unwise in overlooking the obvious teaching of his teeth and digestive organs, which are those of an omnivorous animal. His prehistoric human ancestors lived chiefly on the harvest of their spears and tomahawks. If we insist upon looking back still farther, we discern a cleavage of the race into the arboreal fruit-eaters, which still retain pre-human characters, and the more enterprising and energetic troglodyte hunters from whom the human race was evolved.

A man may vary his diet within wide limits. Innumerable considerations lead certain individuals to desire to depart from the diet which we have termed "normal "-i.e., typical of inhabitants of the temperate zone. One man rebels against the expense of living; he would fain reduce the quantity and the cost of food. Another, having to traverse regions in which food is scarce, wishes to ascertain the lightest, and therefore the most portable, combination of its essential elements. A third-and he belongs to a much larger classtormented with indigestion or harassed by gout, asks, "Why must I consume things which give the stomach trouble, or produce disagreeable and incapacitating after-effects?" Many circumstances prompt to experiments in diet. Much latitude is undoubtedly allowed. But there are limits within which alone health can be maintained and work done. It is of great interest to ascertain exactly how wide these limits are; and especially important is it to find out the lower limit, the minimum of food, and the minimum of each particular kind of food, which will enable the human machine to work. The problems 
involved are somewhat complicated. If it were possible to live on a single food, it would be as easy to ascertain the irreducible minimum as it is to find out with how much coal or with how much petrol an engine can be made to turn a wheel. But to support the body several different kinds of food are indispensable. It is therefore necessary to determine, not only the minimum quantity of the combined foods, but also the minimum amount of each kind of food, and the effect upon the total of variations in the relative amount of each of its several factors. The problem is complicated, but certain limits are impassably defined. In the first place, with regard to the total amount, the work which the body does cannot under any circumstances be reduced below a certain level. The food consumed must provide a supply of energy equal, at the least, to the performance of the minimum of work. The body must receive each day food of due caloric value. Then with regard to the amount of each several constituent. Many considerations lead us to wish to increase one of them or to diminish another. Some food is cheap, and other food is dear. Economic reasons are in favour of the cheaper food. Even ethical considerations are not without weight. We have, perhaps, a prejudice against sacrificing life to supply the pot. We have doubts as to whether our system can properly digest, metabolize, and excrete meat. We need an unambiguous answer to the question, To what extent can nitrogen-foods be replaced by carbon-foods, and vice versa? A cell, as already said, consists of a framework of bioplasm bathed in cell-juice which contains nutrient substances and manufactured products. The bioplasm is alive; the proteins, carbohydrates, and fats of the cell-juice are the materials with which it is nourished, and upon which it works. Some physiologists incline to the view that non-living substances must enter into the bioplasm before they undergo metabolism. They consider that the molecules of the non-living substance must at the time when they undergo a chemical change be physically and chemically a part of the living substance. Others take the opposite view: that the living substance does not undergo change, but brings about changes in the non-living substance which is in contact with it, enclosed within its meshes. This is a problem which is not likely to be solved, nor is its solution 
of great importance in relation to the question which we are discussing. Whichever of the two views be justified, we have to distinguish between the bioplasm of the cell-the machineand its raw materials and manufactured products. The question to which we want an answer is the following: Must the bioplasm undergo change? There seems to be no reason in the nature of things why it should. It is not, as we have already pointed out, subject to wear and tear. A perfect machine would in the absence of friction, which rubs down its steel and brass, continue to turn out its products so long as it was supplied with raw materials and the energy needed to manufacture them. We could imagine the bioplasm as indestructible, receiving energy from a portion of the foods, and expending this energy in the production of chemical change in the remainder. We could imagine that when once the tissues had attained their full growth they would require no more protein for their own nutrition; they would be occupied in producing heat and motion from the non-nitrogenous foods. But observation shows clearly that this is not the case. The force which energizes the bioplasm, enabling it to evoke metabolism in non-living substance, is obtained at the cost of its own destruction. The bioplasm wastes unless constantly supplied with proteid food.

Under ordinary circumstances the amount of urea excreted varies directly as the quantity of nitrogen contained in the food. Since urea contains 45 per cent. of nitrogen, and protein 15 per cent., every gramme of urea excreted represents 3 grammes of dry protein consumed; or, in terms of nitrogen, every gramme of nitrogen excreted represents 6.25 grammes of protein consumed. If all food is withheld, the excretion of nitrogen falls, but it never reaches zero. Many observations have been made on fasting men. On the second day of fasting the nitrogen excreted falls to about 13 grammes, representing 80 grammes of protein used up. It is generally thought that by the second day all "floating proteins" are exhausted, and that therefore nitrogenous metabolism is reduced, as it were, to a business basis. So long as the supply of food is abundant, the body has a luxurious habit of using proteins in preference to non-nitrogenous food. But after a day's starvation there is no longer any fancy metabolism, 
no consumption of proteins as fuel when cheaper fats and sugar would answer equally well. In the case of Succi, who fasted for thirty days, the nitrogen excreted fell to $6 \cdot 7$ grammes on the tenth day, to $4 \cdot 3$ grammes on the twentieth, and to $3 \cdot 2$ grammes on the last day. Clearly, we have to make a distinction, when all food is cut off, between the oxidation of the protein which, failing all other material, is withdrawn from the tissues for the purpose of supplying the force absolutely necessary to maintain respiration and such other movements as are inevitable, and to keep up the temperature of the body-force which under other circumstances might be supplied by non-nitrogenous food-and the oxidation to which bioplasm is inevitably subject, so long as it is alive. The oxidation of bioplasm under ordinary circumstances of course supplies force; but it does not follow that this is sufficient to maintain the respiratory movements and the contraction of the heart. When a herbivorous animal is starved, it not infrequently excretes more urea at the commencement of the starvation period than it was excreting when well fed. Its activities did not come to a standstill when carbohydrate food was cut off. For a time they were maintained at the expense of its own tissues. On the other hand, the results obtained from the observation of the man who went without food for thirty days show that Nature is able to economize force by reducing the metabolism of living substance below the normal. It might be supposed that the irreducible metabolism could be ascertained by giving a nitrogen-starved animal non-nitrogenous food, but it is found that this scarcely affects the tissue-waste. Becoming more active, the tissues, while saved from the necessity of supplying fuel for the production of heat and motion, suffer more waste. Again, it might be expected that if to an animal which had been starved for a few days, until its urea had fallen to the starvation limit, exactly sufficient protein were given to supply this amount, the tissues would be saved. It is found, on the contrary, that nearly twice as much urea is excreted as before. If the quantity of protein be steadily increased, equilibrium is at last established, but not until the amount of nitrogen in the protein given is two and a half times as great as the amount excreted during the starvation period. Additional food at once gives rise to additional 
waste. The tissues which during the period of scarcity had reduced their oxidation to a minimum become more active at the first hint of returning plenty.

This last experiment illustrates a general law. An increase of proteid food within certain limits increases the metabolic activity of the tissues-provokes them to extravagance. It is possible, by adding protein to a mixed diet which sufficed for the maintenance of body-weight and nitrogenous equilibrium, to bring about a nitrogen deficit and to reduce the body-weight. Or, if the body is gaining in weight, owing to the accumulation of fat, the substitution of protein for carbohydrate (weight for weight, since their caloric values are the same) will lead to its reduction. It is difficult to avoid the use of fanciful language in accounting for these results. The animal economy is like an over-careful housekeeper, who, when meat is scarce, doles out porridge also with a thrifty hand. When meat is plentiful she is prodigal with every article of diet. Protein is the most costly of foods. Any indication that it is scarce leads to a shutting-down of activity. On the other hand, no other food is so readily absorbed (unless the digestive organs be protein-sick); none is so quickly incorporated in the bioplasm ; none is so easy to decompose. When fed with protein the machinery hums. The insatiable appetite for beef and eggs which overtakes a man of sedentary habits after a long morning in a boat or on a bicycle does not indicate that his muscular tissue is suffering from wear and tear. It does not prove that he is setting free energy by oxidizing proteid food. It shows that he is asking certain tissues which are accustomed to a quiet life to exhibit prodigious energy. They will not shake off their customary sloth unless he stimulates them with sumptuous fare. At the end of a week he finds that proteins are not the best fuel for steady work. If he consumes sufficient to supply all the energy needed by his muscles, he is hampered' by a quantity of nitrogenous residues which have to be reduced to urea and eliminated by the kidneys. He goes back approximately to his old regimen, so far as proteins are concerned, and consumes more carbohydrates for the supply of the force which his increased muscular activity demands.

It is possible to live on meat alone, but the quantity required 
is very great, involving the digestive organs, the liver, and the kidneys in an excessive amount of work. On the other hand, it is possible to reduce the consumption of proteins to a minimum by substituting for them fats and carbohydrates. But, again, after the proper balance is disturbed, the substitution ceases to be a simple problem in arithmetic. The carbonfood has to be increased out of all proportion to the protein which it replaces. If a dog which is being fed on a diet natural to it-chiefly meat-is in a condition of nitrogenous equilibrium, carbohydrate may be substituted for some of the meat. But from the very beginning it is found that, if nitrogenous equilibrium is not to be disturbed (if the dog is not to be induced to consume its own tissues), a weight of carbohydrate must be given considerably greater than the weight of the protein withdrawn. The disproportion increases as the experiment proceeds, until perhaps 12 to 15 grammes of carbohydrate have to be substituted for every gramme of protein. The proteid food has now come down to $\mathbf{1 . 5}$ gramme per kilogramme of the animal's weight. Owing to the increase of carbohydrate, the caloric value of the total food, nitrogenous and non-nitrogenous, is several times as great as the animal requires. The surplus is oxidized without any equivalent in work. At about this point the experiment is brought to an end, owing to the failure of the digestive organs to deal with so large a mass of food.

The value of gelatin as an article of diet is of interest in this connection. Gelatin is not, strictly speaking, a protein, and it cannot be built up into the tissues. It does not prevent, nor even delay, starvation. Yet up to a certain point it can be used as a substitute for proteid food. In the observation just referred to, protein might be withdrawn at any stage, without disturbing nitrogenous equilibrium, by substituting about 2 grammes of gelatin for every gramme of protein withdrawn. It spares protein, although it does not take its place. It is said that the minimum of protein necessary for the maintenance of nitrogenous equilibrium may be reduced to about one-half by the substitution of gelatin. This has been interpreted as indicating that when we have reduced the oxidation of nitrogenous substance to its smallest amount the nitrogen comes from two sources in about equal propor- 
tions-(a) the bioplasm ; (b) the food-proteins in contact with it. It is inferred that gelatin, although it cannot be built up into bioplasm, may take the place of proteins present in the cell-juice. It appears to be impossible to starve the cell until it consists of a bioplasm framework bathed in nitrogenfree cell-juice. As the non-living proteins of cell-juice are removed, they are, if no nitrogenous food be given, renewed by the breaking down of bioplasm. When gelatin is absorbed, it takes its place in the cell-juice, and the breaking down of bioplasm is no longer necessary. When digestion is impaired, or vitality lowered, decoctions of meat which contain extractives of low calorific value, useless, without synthesis (cf. p. 144), for the purposes of tissue-repair, may to a certain extent save tissue-waste. In the same way, gelatin, which is very rapidly digested in the stomach, may cover the consumption of proteins, although it cannot take their place.

To sum up: The requisite daily income of energy must come from both nitrogenous and non-nitrogenous food. It is impossible to reduce the nitrogenous factor below a certain minimum. From this minimum upwards, until a certain level is reached, every additional unit of nitrogenous food enables the system to dispense with more than its equivalent of nonnitrogenous food. When the proper balance of foods is attained, there is no waste either of labour involved in digestion, or of labour involved in metabolism and excretion.

The Liver.- The liver weighs from 3 to $3 \frac{1}{2}$ pounds. It lies beneath the diaphragm, more on the right side than on the left. Its posterior border, which rests against the last three ribs (separated from them by the diaphragm), is about 3 inches thick. Its anterior border is thin, and keeps close along the line of the ribs. If the organ is neither unduly enlarged nor squeezed out of its place owing to the use of a tight corset, it does not project below the ribs, save where it crosses the space between the rib-cartilages below the end of the breastbone.

The liver is supplied with blood by the hepatic artery. This vessel is small for so large an organ. Although responsible for the nutrition of the liver, it does not bring it the materials which are stored in its cells. A much larger supply of blood is derived from the portal vein, which breaks 
into capillaries, or, to speak more accurately, into sinuses, or pseudo-capillaries, in the liver. The blood, whether conveyed to the organ by the hepatic artery or by the portal vein, is
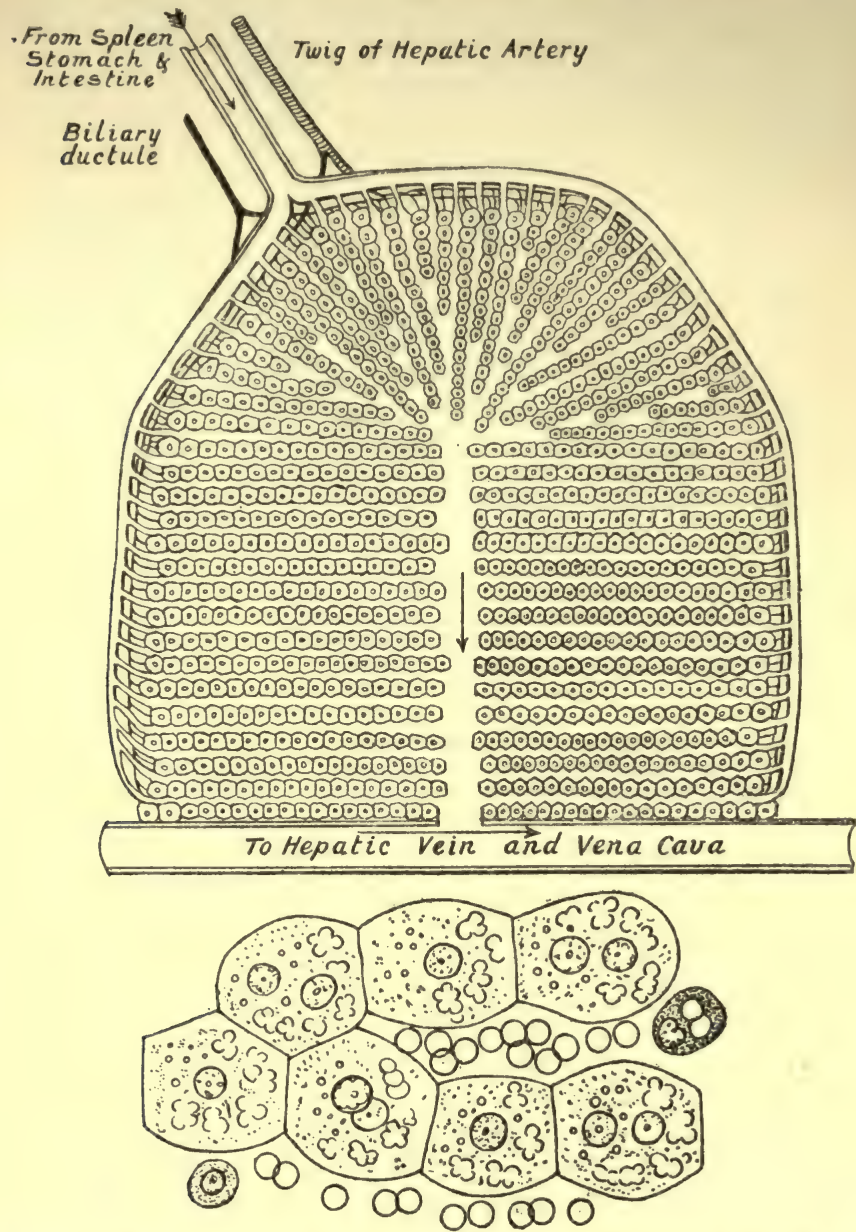

Fig. 7.-Diagram of a LobUle of the LIVER DIVIded VERTíally throdgh its Axis.

In its centre is a space, the intralobular vein, through which the blood falls into a branch of the hepatic vein, on its way to the heart. An interlobular branch of the portal vein, which brings the blood from the digestive organs, pours it by many smaller vessels over the surface of the lobule. It filters into the lobule through innumerable pseudo-capillary vessels, or spaces, between the radiating columns of liver-cells. Arterial blood is brought to the lobule by a twig of the hepatic artery. Bile is drained away from it by an affluent of the hepatic duct. In the lower part of the diagram seven liver-cells are shown, forming a divided column, magnified about 300 diameters. The cells are loaded with glycogen, and contain minute globules of fat. Red blood-corpuscles and two leucocytes are seen between the columns of liver-cells. One of the leucocytes has ingested two blood-corpuscles. 
drained away by the hepatic veins. The plan of structure of the liver is best understood when viewed with reference to the hepatic veins. These, if traced backwards, are found to break up into fairly straight vessels, each of which has a large number of lateral branches. Each of the lateral branches is in the centre of a mass of cells, which are packed round it in radiating columns. These masses, which have a diameter of from 1 to 2 millimetres, are termed "lobules." By mutual pressure the lobules are squeezed into a pentagonal or hexagonal form. The vein in the centre of the lobule is the intralobular vein. Turn now to the portal vein; this is seen to break up into branches which run between the lobules, and are therefore termed "interlobular veins." The branches of the hepatic artery also run between the lobules, as do the radicles of the bile-duct. Each lobule is a liver in miniature. The blood of the portal vein, which has come from the spleen, in which red blood-corpuscles are destroyed, and from the stomach and intestines, from which it has absorbed the products of digestion, is poured over the surface of the lobule, to be filtered through into its central intralobular vein. In its passage from the interlobular veins (and branches of the hepatic artery) to the intralobular vein the blood is confined to radiating capillary channels; but since these merely prevent the escape of red blood-corpuscles without imposing any restrictions upon the exudation of blood-plasma, the portal blood is to all intents and purposes filtered through the columns of liver-cells. The body-substance of the liver-cells is soft, destitute of envelope, and capable, when free on the (warmed) stage of a microscope, of changing in form, somewhat after the manner of a leucocyte. Such cells have a great capacity for taking up the products of digestion. Possibly they take up and store fats and proteins, but undoubtedly it is their chief business to absorb sugar which accumulates as glycogen in their substance. The glycogen is handed out to the hepatic blood as required. The pigment which results from the disintegration of red blood-corpuscles in the spleen is secreted, along with the bilesalts, into minute channels, or canaliculi, which groove the flat surfaces of adjacent liver-cells. These canaliculi converge to the bile-ducts. The liver is therefore at the same time the storehouse of sugar which it takes up from the blood when 
it is in excess, and passes out to the blood when it is deficient, and an excretory organ which eliminates the refuse of hæmoglobin. The iron derived from hæmoglobin it stores, and returns to the blood.

Another function of the liver has been referred to already. It is the organ, and, as far as we know, the only organ, in which urea is made in mammals, and uric acid in birds. If the liver of a freshly killed animal be excised and a stream of blood passed through it, the blood which leaves the organ contains urea. If a salt of ammonia, even the carbonate, be added to the blood, it is converted by the liver into urea. When a bird's liver is made the subject of the same experiment, uric acid appears instead of urea. The liver can convert many nitrogenous substances into urea, but it seems probable that, normally, the salt with which it has chiefly to deal is lactate of ammonia (cf. p. 146).

A few words must be added with regard to the functions of the liver during prenatal life, obscure though these functions are. The liver develops very early, and attains a relatively enormous size. At the third month it weighs as much as the whole of the rest of the body (cf.p. 34). Yet it cannot, one must suppose, have to do much of the work which falls to its share in postnatal life. Food is reaching the embryo in a constant stream, and not as the result of intermittent meals. The embryo has no need to store glycogen; nor does its liver, on analysis, yield much of this substance. In the embryo glycogen is widely distributed throughout the tissues, not specially accumulated in the liver. No digestion is occurring in the alimentary canal. Bile is not needed to aid the hydrolysis and absorption of fats. A small quantity of cholesterin and less lecithin is being eliminated, but not much bile is needed to facilitate this process.

A process which is proceeding at a great rate in the embryo, in various situations, is the formation of red blood-corpuscles. In this the liver takes part. But its duty in regard to bloodformation is not sufficiently onerous to account for its size. The formation of blood-corpuscles in the liver is observed with difficulty in microscopic sections. It is therefore impossible to speak with certainty as to the extent to which it is going on, but it may be safely asserted that this function by itself cannot 
be held to account for the great size of the organ in embryonic life. What other office it fills at this period is a question which still awaits an answer.

There is no more curious chapter in medical history than the story of the views held at various periods with regard to the functions of the liver. From being a mere mass of "parenchyma" serving as packing for the abdominal viscera, it was elevated to the rank of Grand Purifier of the "humours" of the body. Next, its excessive activity became the cause of that form of dyspepsia known as "biliousness." Still later its want of activity was its chief vice. A "sluggish" liver was held responsible for mental perversity and moral dulness. Calomel, podophyllin, and other drugs were used as whips to stir it up ; and the increased secretions of the alimentary canal were mistaken for bile. Poor patient organ! It is the stillroom of the body, in which the day's supplies are stored, and from which they are served out, without haste and without delay. And it makes urea. What else it does we have yet to find out; and it is not impossible that when physiologists have quite shaken themselves free from the explanations based upon conjecture, which their predecessors have handed down, they may discover that it has other duties which are not obvious, but of great importance. 


\section{CHAPTER VII}

\section{RESPIRATION}

LiFe means change. We cannot imagine its continuance without liberation of energy. Arrest of molecular activity is death. There is no possibility of its revival. A watch that has stopped may be started by shaking. On the cessation of molecular activity an animate being becomes inanimate. Dead, it is liable to further chemical changes. Bacteria invade it. They shake down its complex unstable compounds into simple, stable, so-called "inorganic groups"; but the ordered combination with oxygen, which constitutes living, can never recommence. Putrefaction may be prevented by the exclusion of germs. The inanimate mass of organic material may remain unchanged. Its return to life would be a miracle. From time to time a frog is found enclosed in old red sandstone, or some other rock which for countless ages has lain beneath the surface. The cleft through which the frog entered a few hours or days before it was discovered is overlooked. It is supposed to have lived "in a state of suspended animation" for millions of years. The fact that no frogs are to be found among the fossils of the old red sandstone is an objection too casuistical to be seriously entertained. The physiologist's demand to know what has become of the mountains of solid carbonic acid, water, and urea which the frog must have produced during its unimaginable term of incarceration is regarded as the natural expression of his prejudice-that life cannot continue without molecular change. And he is bound to admit his inability to prove that it cannot. Nevertheless, his experience that, whenever and however he may, by experimental methods, arrest change, he loses the power of causing it to recommence justifies him in his conviction that life is change. Even a living seed is to his mind an organism whose complex constituents are slowly- 
however slowly - setting free energy by settling down the steps which lead to stability and ultimate, inanimate rest; and the only source of this energy is combination with oxygen. In the case of a seed the oxygen need not come from without. Seeds retain their power of germination after long occlusion in nitrogen or other neutral gases. But all the time some change is occurring, some internal oxidation which resolves their less stable into more stable compounds. Otherwise they would not be alive. A physiologist is willing to believe that this may continue for ten years, fifteen years-for any period that the botanist tells him that he has, under verifiable conditions, observed that it does occur; but when he is told that peas taken from the hand of an Egyptian mummy, or seeds set free by the spades of navvies after a far longer burial, have been found to retain their vitality, his credulity is stretched beyond breaking-point. He cannot imagine a change so slow as to be spread over a geological period, still without exhaustion of all changeable compounds.

The term "respiration" has been extended until it is synonymous with "oxidation." At one time it was supposed that the combination of oxygen with oxidizable substances occurred in the lungs. The lungs were the hearth of the body, to which the blood brought fuel which burned in the air drawn into them. When it was understood that the actual combination of combustible material with oxygen occurs, not in the lungs, but in the tissues, a somewhat illogical distinction was made between "external respiration" - the combination of oxygen and blood in the lungs-and "internal respiration" - the combination of oxygen and tissue-substances. The terms are not comparable. The taking up of oxygen by the hæmoglobin of blood is a different process to the union of oxygen, after the hæmoglobin has parted with it, with the carbon, hydrogen, and nitrogen of the tissue-substances.

The blood-stream carries both fuel and oxygen to the tissues, but the fuel while in the blood is not in an oxidizable condition. The foods are taken up by the tissues. They enter into combination with their protoplasm. Oxygen also combines with tissue-substances. In proportion as the tissues are active oxidized compounds are split off. They fall into the lymph, whence they are absorbed by the blood. If they are 
nitrogenous compounds, they are carried to the liver, formed into urea, and passed to the kidneys for elimination. If carbonic acid, it is carried to the lungs for exhalation. The water formed by combination of hydrogen and oxygen may escape from the lungs, the kidneys, or the skin.

Two or three pounds of mixed foods are consumed every day. By the blood they are carried to the tissues, whence an equivalent quantity of waste - that is to say, oxidized-material is removed. About $1 \frac{1}{2}$ pounds of oxygen is required to burn the day's fuel.

The problems of respiration are twofold. In the first place we have to consider the physics and chemistry of the combination of hrmoglobin with oxygen, and of the elimination of carbonic acid from the blood in the lungs; secondly we have to explain the transference of oxygen from hæmoglobin to the tissues, and the reception in the blood of carbonic acid produced by the tissues.

The apparatus by which air is brought into relation with the blood consists of lungs and windpipe. At its upper end, where it joins the portion of the alimentary tract common to deglutition and respiration, the special respiratory tube is protected by the larynx. The nasal chambers belong to the respiratory tract; the gullet, or pharynx, is common to the two functions.

The mucous membrane which lines the nose and windpipe is kept moist in order that it may catch particles of dust drawn in with the air. At the same time the nasal chambers serve to warm the air, and to add moisture to it if it be too dry; for the lining epithelium of the lungs would suffer if dry air came in contact with it. The wall-surface of the nasal chambers is increased by the projection of folded and chambered "turbinate bones." The importance of warming the air before it is admitted to the lungs is remarkably illustrated in the case of certain sea-birds. The nasal chambers of the frigate-bird, and of some other birds which resemble it, are exceptionally complicated. Since the animal is devoid of any sense of smell, and the air which it breathes must be nearly saturated with moisture, the only function which can be assigned to these convoluted passages is that of warming inspired air.

The larynx will be more minutely described when it is con- 
sidered as the organ of voice. In connection with respiration, it must be regarded as primarily a valve which closes the entrance to the windpipe during swallowing. It is overhung by a leaf-like appendage-the epiglottis - formed of exceedingly elastic tissue. It was thought until lately that the epiglottis drops over the aperture of the larynx when food is passing down the gullet, and springs up again as soon as the act of deglutition is over; but recent observations have shown that during deglutition the epiglottis is pressed against the back of the tongue, and that the closure of the larynx is effected by its own sphincter muscles. The mucous membrane of the larynx is extremely sensitive to stimulation by anything which would be prejudicial to the tissue of the lungs. When its sensory nerve-the superior laryngeal-is stimulated, the larynx closes. It is the agent in carrying out many reflex actions, in which not the larynx only, but also the muscles of the chest and diaphragm, take part. For example, it immediately stops inspiration if an irritating vapour is present in the air. It stops respiration if any foreign body, such as a crumb of bread or a drop of water, touches the mucous membrane. When the trunk of the nerve is stimulated by an electric current, respiration is inhibited. Further, under suitable stimulation the nerve brings about respiratory movements in which inspiration is gentle and expiration sudden, violent, convulsive. Rib-muscles and diaphragm combine to produce a cough, which ejects the noxious body. Again, its stimulation in a different way probably helps to produce constriction of the smaller bronchi which regulate the amount of air supplied to the air-cells of the lungs; although this constriction may be largely due to a reflex which starts in the aircells. The epithelium of the air-cells has an immensely rich supply of sensory nerves. In some persons this protective mechanism is very prone to overact its part. A little dust or foul gas in the air leads to such marked contraction of the bronchi that respiration becomes very difficult. Such an exaggerated tendency to reflex action constitutes the neurosis, asthma. In this malady the mechanism is unduly sensitive. Very slight stimulation leads to a maximum discharge of impulses to the muscular tissue of the bronchi.

The trachea has a length of about 4 inches. It extends from 
the lower edge of the cricoid cartilage, which is easily felt in the neck beneath the thyroid cartilage (Adam's apple), to the under side of the arch of the aorta, where it divides into the right and left bronchi. The epithelium which lines the trachea and bronchi is ciliated. The cilia propel the secretion which accumulates on its surface upwards towards the larynx. The wall of the windpipe is kept open by rings of cartilage which are incomplete behind, where the trachea and œsophagus are in contact. Rings and plates of cartilage also support the bronchi. The bronchi divide and subdivide until their diameter is reduced to about $\mathbf{0 . 2}$ millimetre. Each bronchiole then breaks up into a bunch of very thin-walled, elongated infundibula, club-shaped, and with a diameter about five times that of the bronchiole with which they are connected. They may be three or four times as long as they are broad. The wall of an infundibulum is pitted like a piece of honeycomb into shallow chambers-the air-cells or alveoli.

The walls of the air-chambers, or alveoli, are formed of a membrane upon which is spread a network of capillary bloodvessels. The air-chambers are so closely packed together that a common wall separates one chamber from the next adjoining. Minute bloodvessels pierce the partitions which separate the chambers, appearing now on one side of the wall, now on the other. The air-chambers are lined by thin epithelial scales or tiles. The blood in the capillary vessels is separated from the air in the air-chambers by the wall of the capillary; by a lymph-space, probably rather potential than actual; and by the epithelial tiles. This covering suffices to prevent the escape both of red corpuscles and of plasma, yet offers very little resistance to the passage of gases from the blood into the air, and from the air into the blood.

Leucocytes make their way between the tiles, and creep over their internal surfaces, searching for cell débris or foreign matter. Anything that they find they carry to the clumps of lymphoid tissue which occur in the outer wall of the bronclii. In a town-dweller, leucocytes are found in these lymphthickets, charged with particles of soot. They show droplets of fat and other evidences of degeneration. At other spots are to be seen little collections of soot which have been left behind after the dissolution of the leucocytes which brought them there. 
It is not possible to make anything like an accurate estimate of the number of alveoli in the lungs ; 725,000,000 is a figure arrived at by measuring the average cubic capacity of an alveolus, and comparing it with the total cubic capacity of the lungs. Each alveolus supports some forty or fifty capillary vessels. The superficial area of vascular membrane exposed is placed at 90 square metres, or about 100 times the area of the skin. Figures such as these convey very little meaning, but they help one to realize the magnitude of the provision made for the aeration of the blood.

Pneumonia is a condition in which the lining of the airchambers is inflamed, usually, possibly always, owing to the entrance of bacteria. Lymph exudes through the walls of the alveoli. Epithelial scales flake off. Pus-cells (dead leucocytes) accumulate in the air-chambers. Respiration is curtailed, and dyspnœa results. After a time, if the case progresses favourably, "resolution," as it is technically termed, begins to occur. The exuded substances are either expectorated or absorbed, and the lung-tissue returns to a normal condition.

Here a few words may be devoted to respiratory sounds. Spirare means to sigh. Breathing received the name by which it is known in physiology from the sound which accompanies the exit of air from the nostrils. Since the introduction of auscultation as a means of ascertaining the condition of the lungs, other sounds, not heard until the ear or a stethoscope is placed against the chest, have acquired great importance. These sounds, termed " murmurs," may be divided into two classes. (a) When the ear is placed against the windpipe, or in the middle of the back between the shoulder-blades, a murmur is audible, due to the movement of air through the larynx. If the larynx, the trachea, or the bronchi contain mucus, it is a harsh, rough, bubbling, or crackling sound. It accompanies both inspiration and expiration. (b) A softer, more delicate murmur is heard when the ear is placed against the front or the side of the chest. This is the vesicular or pulmonary murmur. It is heard during inspiration, and is due to the passage of air out of the smallest bronchi into the more spacious infundibula in which they end. These two kinds of murmur must be rigidly distinguished-the laryngeal murmur, heard in situations in which no lung-tissue interveres between the 
ear and the great tracheal or bronchial tubes; and the pulmonary murmur, heard over all regions where the bronchi are buried in lung. Healthy lung is as bad a conductor of sound as a sponge or a wad of cotton-wool. The laryngeal murmur is inaudible in regions in which lung lies beneath the chest-wall. It would be far beyond the scope of this book to attempt to describe the very varied alterations in the chestsounds which may be produced by disease. The student would do well to familiarize himself with the nature of the sounds which are heard in health, and the situations in which they are heard, in order that he may be able, in abnormal conditions, to recognize that something is wrong.

The chief departures from the normal may be grouped under the following heads : (1) The pulmonary murmur may lose its soft, smooth, sighing character owing to inflammation of the alveoli and infundibula. It may be as loud in expiration as in inspiration. Only a practised ear can estimate the significance of these changes. (2) The laryngeal murmur may be reinforced by "râles "- a convenient term for supplementary sounds. The source of such râles may be a cold in the chest, laryngitis, or bronchitis of various degrees. (3) The laryngeal murmur may be heard in situations in which lung intervenes between the ear and the larger bronchial tubes. This can be due only to the lung being in an abnormal condition as a conductor of sound. Instead of being as spongy as well-made Vienna bread, its air-spaces are filled with solid or fluid deposit. It is as firm as dough. To such a condition it attains at the height of pneumonia - a stage termed "hepatization" because in section it looks like liver rather than lung.

Breathing is the enlargement and diminution of the chest, which causes air to be drawn into and expressed from the lungs. The windpipe being open, the air inside the lungs is, of course, at the same pressure as the atmosphere. Expansion of the chest results in the equal expansion of the lungs. Since there is no air-space between the outer surface of the lungs and the inner surface of the chest-wall, the lungs cannot separate from the chest-wall when it expands. But the lungs contain elastic tissue always slightly on the stretch. If the chest be punctured, and air admitted between the chest-wall and the lungs, the lungs collapse. The expiratory movement, 
the contraction of the chest, is due to the elasticity of the lungs. This tendency on the part of the lungs to contract is sufficient in quiet respiration to restore the chest to its usual size after inspiration, and thus to expel air. The lungs are held open owing to the negative pressure in the space which separates them from the chest-wall. This negative pressure has a most important relation to their permeability by air. Imagine the condition reversed. Picture a lung into which air is forced by a muscular pump. After each stroke of the pump the lung would collapse. Its finest tubes and their dilated terminations could be maintained as open spaces, between the strokes of the pump, only by giving a considerable thickness and firmness to their walls. Such a substantial structure would be unfavourable to an interchange of gases between the blood and the air. The reverse of this condition is found in Nature. The lung is stretched from without. Its tissue, delicate as crêpe, cannot collapse even at the end of the deepest expiration.

The ribs are united by intercostal muscles, disposed in two sheets. The fibres of the external intercostals are directed downwards and forwards, those of the internal intercostals downwards and backwards. In tranquil respiration the chest is enlarged by the external intercostal muscles, which raise the ribs, and the diaphragmatic muscle, which renders peripheral portions of the diaphragm flat. The rôle of the internal intercostal muscles is a subject still under discussion. For the most part, physiologists regard them as accessory to expiration, but some hold that they combine with the external intercostals in raising the ribs and twisting them outwards during inspiration. The diaphragm is a partition which separates the thoracic from the abdominal cavity. It is in the form of a vault. The central portion of the dome is membranous, its margins muscular. Its membranous centre is in contact with the pericardium, which encloses the heart. The level of this part is therefore fixed, except in forced inspiration, when it descends slightly. It constitutes a fixed plane for the muscles of the diaphragm, which are attached below to the vertebral column and the ribs. When the muscles contract in inspiration, the curvature of the marginal portions of the diaphragm is diminished, and the chest-cavity consequently enlarged. 
During expiration the space between the muscle of the diaphragm and the chest-wall closes up, and the lower border of the lung slips out of it.

There is a marked difference in the relative extent of the costal and diaphragmatic movements in men and women. In women respiration is chiefly costal; in men it is chiefly diaphragmatic. In men the abdomen moves forwards, as the diaphragm descends in tranquil breathing ; in women the chest rises. Men who wish, for the purposes of athletics, or singing,

A

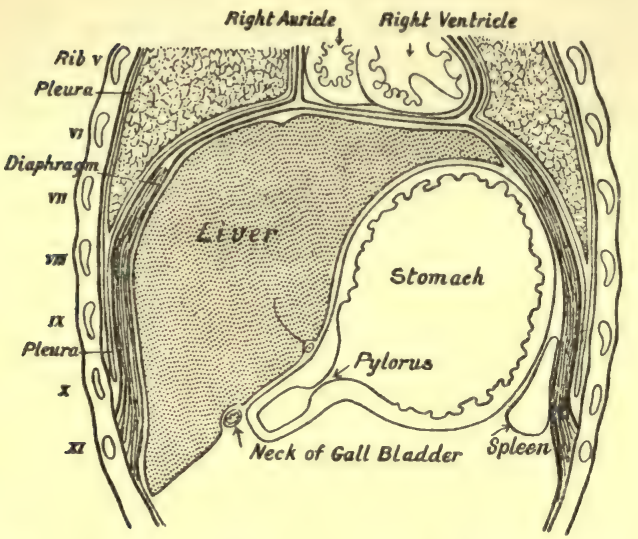

B

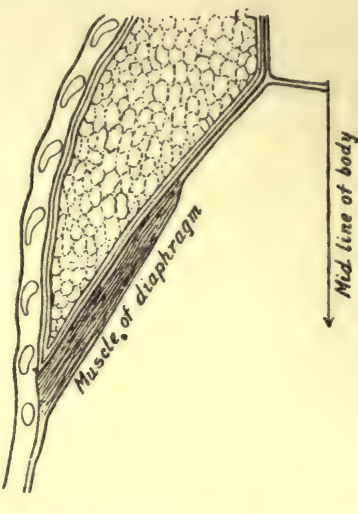

Fig. 8.-The Diaphragm and ORgans in Contaot with it-A, in Expiration ; B, at the End of a Deep inspiration. Transverse Vertioal Sections in the line of the ARMPIT.

A, At the end of an ordinary expiration the lung does not extend below the upper border of the eighth rib. From this level to the middle or lower border of the tenth rib the two layers of the pleura covering respectively the inner wall of the chest and the upper surface of the diaphragm are in contact. B, When the lung is distended with air it occupies the whole of the pleural cavity.

or public speaking, to retain the power of making the most of their chest-capacity are wise in not allowing themselves to fall into the habit of lazy, abdominal breathing.

When additional pressure is required, when respiration is forced, various external muscles attached to the spinal column, the shoulder-blades, and the clavicles, as well as the muscles of the abdomen, come into play.

The chest is lined and the lungs covered by a serous membrane-the pleura. Normally there is only just sufficient lymph in the space between the visceral layer of the pleura 
which invests the lungs and the parietal layer which lines the chest-wall to prevent friction during respiration. When the pleura is inflamed, one layer of the membrane rubs against the other. In the early or "dry" stage of pleurisy, the physician recognizes this condition by the friction-sound which he hears on placing his stethoscope against the chest. In a later stage lymph (pleuritic fluid) is poured out. It accumulates in the lower part of the chest, and is recognized by the absence of the resonant note which, under normal conditions, is given out by the chest when percussed.

The lungs are not compressed during expiration; they are not squeezed, as a pair of bellows or a sponge may be squeezed, emptying it of its contents. At the end of tranquil expiration the lungs still contain about $3 \frac{1}{2}$ litres of air. At the top of tranquil inspiration the volume of their contents does not exceed 4 litres. It is evident, therefore, that air is not drawn into and driven out from the air-chambers by the movements of respiration. The tide of air does not extend far beyond the ends of the bronchi. The gases in the air-chambers are exchanged with the fresh air drawn into the infundibula by diffusion. The composition of the air which is in contact with the bloodvessels is constant. It is about 4 per cent. poorer in oxygen and 3 per cent. richer in carbonic acid than the outside air.

Of the air drawn into the windpipe during an inspiration, about one-third returns to the open with the following expiration; two-thirds remains in the lungs. If, therefore, the air taken in at each tide equals one-seventh of the quantity already in the lungs, and if of this one-seventh two-thirds remains, each alveolus renews about one-tenth of its air. Its contents are completely changed in ten respirations.

Fresh air is composed of 21 per cent. oxygen, 79 per cent. nitrogen, and a trace ( 0.04 per cent.) of carbonic acid. Forced by a syringe through lime-water, fresh air does not produce any appreciable milkiness, whereas air breathed through a tube into lime-water renders it turbid owing to the formation of carbonate of lime. Carbonic acid $\left(\mathrm{CO}_{2}\right)$ occupies the same volume as its oxygen $\left(\mathrm{O}_{2}\right)$ would occupy if free. The oxygen which breathed air has lost slightly exceeds in amount the carbonic acid which it has gained in exchange. The differ- 
ence is due to the retention of some of the oxygen for the purpose of uniting with hydrogen to form water, and of forming urea. The proportion between carbonic acid gained and oxygen lost, $\frac{\mathrm{CO}_{2}}{\mathrm{O}_{2}}$, is termed the " respiratory quotient." Its value varies, of course, with diet. In a herbivorous animal, whose food consists of carbohydrates, it departs but little from unity; in a carnivore, which eats fat and nitrogencontaining food, it is about $\mathbf{0 \cdot 8}$.

The respiratory exchange is very much smaller in coldblooded animals than in animals which maintain the temperature of the body at a fixed level. In warm-blooded animals it rises as the temperature falls, falls as it rises, the increased oxidation warming the body, the diminished oxidation allowing it to cool ; whereas in cold-blooded animals it increases as the temperature rises, owing to the greater activity induced by warmth, and falls as the temperature falls.

The respiratory exchange is increased by muscular activity. If the amounts of oxygen absorbed and carbonic acid given out are measured while a man is at rest, and again while he is doing hard physical work, it is found that during work the respiratory exchange is twice as great as during rest. During periods of starvation the respiratory exchange remains unaltered, since heat has to be constantly produced if the temperature of the body is to be kept from falling.

Since the purpose of respiration is to give to the blood the opportunity of renewing its supply of oxygen, and of getting rid of the carbonic acid with which it is charged, it might be supposed that the respiratory exchange would be increased, so far as the intake of oxygen is concerned, by breathing oxygen gas instead of air ; but it appears that under normal conditions nothing is gained. When an animal is breathing air, its blood takes up all the oxygen that it wants-all the oxygen, that is to say, for which its tissues are asking. Offering it pure oxygen in place of mixed oxygen and nitrogen does not induce it to take up more. The hæmoglobin is almost saturated with oxygen when the blood leaves the lungs under ordinary conditions. In certain diseases of the lungs, however, in which the blood becomes unduly venous, the respiration of oxygen may be beneficial ; but even in these cases the results are dis- 
appointing, because the system is suffering much less from deficiency of oxygen than from accumulation of carbonic acid. Substituting oxygen for air does not facilitate the escape of carbonic acid.

The nervous mechanism of respiration has been the subject of much investigation and of many experiments, without, it must be confessed, the development of a quite complete or satisfactory theory. Rospiration is a rhythmic process. About seventeen times in a minute the intercostal and diaphragmatic muscles contract. Inspiration is immediately followed by expiration, the falling movement being due, as already explained, to the elasticity of "the lungs, which are stretched during inspiration. A slight pause intervenes between the end of expiration and the commencement of the next inspiratory movement. Tranquil respiration is a succession of reflex inspiratory movements, the depth of which varies according to the needs of the body-that is to say, according to the condition of the blood. If the need for aeration of the blood becomes urgent, the depth of inspiration is increased, and expiration also becomes an active movement, certain muscles, especially those of the abdomen, being called into play. In this condition two sets of reflex actions alternate. A large number of nerves are concerned even in tranquil respiration. If a man in falling "breaks his back" at the junction of the cervical and thoracic regions, costal respiration ceases. The series of intercostal nerves which arises from the dorsal spinal cord below the level at which it is injured are thrown out of action. Diaphragmatic respiration still continues, because the nerve of the diaphragm, the phrenic, arises from cervical roots. The lungs are supplied by the vagus nerve. This nerve joins the medulla oblongata as one of a group of three-glossopharyngeal, vagus, and spinal accessory-which by a large number of roots enter the groove between the olive and the restiform body. The vagus is the channel along which afferent impulses from the lungs enter the medulla. Such impulses call for respiratory movements. Cutting both vagi, however, does not put an end to respiration. Inspiratory movements continue, but they are much deeper and separated by much longer pauses. Such a form of respiration is inefficient. The blood is not properly aerated. The animal falls into a condi- 
tion of dyspnœa, which ends in death. When the central end of the cut vagus is stimulated, the movements become more natural. Clearly, the respiratory reflex is not dependent upon the vagus, since it continues after the nerve is cut, although the impulses which pass up this nerve regulate its rhythm. They govern the length of the inspiratory movements, cut them short at the right moment, and secure their succession at proper intervals.

The transfer of afferent impulses into efferent channels occurs in the medulla oblongata. Long ago it was found that if the brain above this level be removed, part by part, respiration is not interfered with until the medulla oblongata is injured. When a cut is made into the floor of the fourth ventricle not far to one side of the middle line, the respiratory movements on that side of the body cease. If the injury be bilateral, even though very limited in extent, respiration stops. This spot was therefore spoken of as the " respiratory centre." Flourens, who first discovered it, believed that it was a mere spot. He gave to it the fanciful name of noud vital. It is the place at which the afferent nerves which call for respiration are brought into connection with all the various motor nerves which bring about the respiratory movements of nostrils, larynx, chest, and diaphragm. Possibly the knife in Flourens' incision divides the tract of fibres which distributes afferent impulses, but whether the junction be a defined tract or no, injury to this region of the medulla throws the nervous mechanism of respiration out of gear. At this particular spot lies the "centre" for respiration-the one part of the nervous system which must be intact if the movements of respiration are to be carried out. There is no reason for thinking that respiratory impulses are generated at this spot. It is a centre in the same sense in which Crewe is a centre for distributing the goods of Lancashire and other parts of England to North Wales. The use of the term "nerve-centre " has been very much abused. Centres were supposed to be collections of cells, each group of which had some prerogative of initiation. Reasoning from the analogy of human institutions, it was thought necessary that the nervous system should be organized into departments severally responsible for the administration of the activities of certain sets of muscles : one centre controlled 
respiration, another the beat of the heart, another deglutition. The centres were dependent one on another; each regulated lower centres, and was governed by those above it, in this bureaucratic scheme. We know nothing of any function of nerve-cells other than that of transmitting impulses. All that we know about nerve-cells is that ihey place afferent and efferent routes in communication, and interpose resistance into nerve-circuits. Every nerve-cell of the grey matter of the brain and spinal cord gives off processes which ramify. The ultimate twigs into which a branch divides are in connection with other sets of twigs derived from the end-branchings of nerve-fibres or processes of other nerve-cells. A nerve-fibre is but the axis-cylinder process of a nerve-cell. Impulses encounter resistance in passing along the neuro-fibrillæ (cf. Fig. 22) contained in the twig-connections of the ramifying processes of nerve-cells. There is no reason for supposing that anything like the same resistance is offered to the passage of impulses along the fibrillæ where they lie within the stout branches of the cellprocesses or within the body of the cell. It is easy to make a pictorial representation of such a mechanism. Imagine a model of the stem of a tree made by binding together a large number of wires; its branches as containing small groups of wires ; the ultimate twigs as separate wires. Carry wires from the roots of one tree to the branches of another. Trees so constructed might be taken as representing nerve-cells. We have not as yet succeeded in demonstrating the isolated neurofibrillæ as they pass over from the end-twigs of a nerve-fibre to the end-twigs of a nerve-cell branch, but we have abundant reason for believing that they do so pass, and that the resistance to the passage of a nerve-impulse is interposed in this neutral or junctional zone. This resistance has to be overcome. It is overcome by the summation of impulses. All nerveimpulses are vibratory. The first vibrations fail may to get through ; but if the vibrations continue, they exert a cumulative effect. After a time they overcome the resistance; sensory impulses flow through the centre into motor channels. In this way we endeavour to explain the rhythmic discharge through the respiratory and other centres. It has not been found possible to determine the source of all the afferent impulses which reach the centre. Respiration continues after 
all accessible nerves have been cut, including even the posterior roots of the cervical nerves. Probably it is a mistake to look for definite afferent channels in the medulla and the rest of the brain. All parts of the body need aerated blood. From all parts, including nerve-tissue itself, arises the demand for respiration. Possibly nerve-centres have the power, as it were, of storing impulses, and discharging them after the stream of fresh arrivals has ceased to flow. They may acquire a habit.

The resistance in the centre is profoundly affected by the condition of the blood. As the blood becomes more venous, impulses pass across the nerve connections with ever-increasing force. Kept in the first instance to definite channels, they spread as the centre becomes more excitable farther and farther afield, reaching one group of muscles after another, and pressing them into the service of respiration. When, in dyspnœa, every muscle which can in any way help the movements of the chest is doing its best, others which are useless for this purpose receive the reflected impulses and join in, producing general convulsions. The increased activity of the respiratory centre which is produced by slight venosity of the blood is shown in the rapid and deep inspirations which are caused by violent exercise. Perhaps it is justifiable to go a step farther, and to assert that there is something in blood which has been rendered venous by muscular activity which is specially exciting to the respiratory centre. If the blood from a limb be prevented from returning to the general circulation, by compressing or tying its great veins, and if the muscles of the limb be strongly stimulated by an electric current, their activity, so long as the passage through the veins is blocked, has no influence upon respiration. But, on relaxation of the pressure on the veins, respiration may become twice as deep and twice as frequent as it was before the muscles were stimulated, although the limb is now in a condition of perfect rest.

What is the special action of the vagus nerve? Its superior larnygeal branch checks inspiration and induces expiration, as already said. The impulses which pass up its main trunk bring about ordered movements. They are not dependent for their generation upon the condition of the blood in the lungs. When the chest is filled with nitrogen, inspiration and expira- 
tion alternate in the usual way, although the blood is growing steadily more venous. The failure of inspiration io bring about aeration of the blood does not lead to a prolongation of the inspiratory effort. Inspiration is cut off and expiration established in regular sequence. In performing " artificial respiration" (cf.p. 184) for the purpose of saving life, in cases in which respiration has ceased owing to the lungs being filled with water, or for other reasons, the chest is enlarged by raising the arms above the head, and diminished by pressing the elbows against the sides. Enlargement promotes a tendency to expiration, compression a tendency to a natural inspiratory effort. Evidently there is a connection between the movements of the chest and the stimulation of the respiratory centre. If respiration is being carried on artificially, by forcing air from a bellows into the trachea, the nostrils dilate as the chest is distended, and contract as it is emptied, so long as the vagus nerve is intact, just as they do in normal respiration. This shows that, when the chest is emptied, a message is sent through to the nucleus of origin of the nerve which supplies the dilator muscles of the nostril. When the lungs are full, a message calls upon the nostrils to contract. The only factor which is common to pressing in and pulling out the ribs, and filling and exhausting the lungs with a bellows, is the alteration in the form of the lungs which is produced by the two methods. It is impossible to resist the conclusion that the stretching of the tissue of the lungs stimulates the nerve-endings of the vagus. The impulses thus induced automatically stop inspiration, and lead to an expiratory effort.

There are many indications that the nervous mechanism of respiration is a double one, certain stimuli inducing expiration, with inhibition of inspiration, others inhibiting expiration and inducing inspiration. There are, however, many difficulties in the way of formulating a satisfactory theory of the relation of these antagonistic actions. We may frequently observe indications of such an antagonism between the two phases of the respiratory mechanism. Cold water dashed on the back of the head (when the head is being shampooed) induces a long inspiration with inhibition of expiration. A blow in the pit of the stomach " knocks all the wind out of a man." Expiration is prolonged until the lungs are unusually empty, and yet the 
victim of the blow feels as if he would never again be able to draw breath.

Modified Respiratory Movements.-The object of coughing is to expel foreign matter from the windpipe or larynx; of sneezing, to clear the nose. The former action consists of a long deep inspiration; the closure of the glottis; a forcible expiration. The blast of air encountering a closed glottis acquires considerable pressure. When the resistance of the glottis is overcome, the blast rushes through, carrying with it mucus or bread-crumb, or whatever the substance may be which irritated the endings of the superior laryngeal nerve. In sneezing, the back of the tongue is thrust against the palate, closing the aperture of the fauces. Inspiration is prolonged. A strong expiration follows. The blast rushes through the nasal cavities. This reflex is usually provoked by a tickling of the endings of the fifth nerve in the nasal mucous membrane. It is also caused in many persons, through the optic nerve, by a bright light ; an apparently purposeless reflex about which we shall have something more to say in a subsequent chapter. Laughing and crying are modified respiratory movements as useless, so far as any immediate purpose is accomplished, as sneezing in response to a bright light. As means of expressing emotions they have been cultivated by the human race. Possibly a case for crying might be made out on physiological grounds. Under certain circumstances it relieves a feeling of distress which, while it lasts, is detrimental to the proper functions of the body. Laughing undoubtedly is beneficial. The rapid movements of the chest quicken the circulation. The shaking of the midriff favours the discharge of digestive secretions, accelerates the movements of the alimentary canal, and generally is beneficial to digestion. But "laugh and grow fat" is not necessarily the order of cause and effect. An efficient digestion and a good capacity for assimilation lead to a sense of bien-être which predisposes to a merry view of life.

Yawning is a deep inspiration with open mouth and larynx. It commences usually at the end of a normal inspiration, a slight pause being followed by further inspiration, deep and prolonged. Its commencement seems to be due to impulses generated by the relaxation of the tone of the muscle which 
holds up the lower jaw. The masseter goes off duty for a moment, allowing the jaw to fall. A reflex contraction of the muscles which open the mouth immediately follows. Muscles of the neck and head also come into play. Not improbably the yawn ends in a general stretch. If the origin of this reflex is obscure, its usefulness is marked. The circulation is quickened, the blood is changed, nervous system and muscles again become alert.

"Apnœa " is the condition of arrested respiration. If a man about to dive into the water breathe deeply and rapidly half a dozen times, he abolishes for a while the desire to breathe. One is naturally inclined to explain this as due to a surplus of oxygen taken into the blood, but a moment's reflection shows that this cannot be the cause. In the first place, as we have already pointed out, the blood which leaves the lungs in tranquil respiration is very nearly saturated with oxygen. It can take up but little more. Again, the deep inspirations do not change the air in the air-chambers; time is required for the renewal by diffusion of their gaseous contents. It is improbable that the constitution of the air in the alveoli is sensibly altered by a few deep breaths. Probably the explanation is to be found in the effect upon the nerve-centre of distention of the chest. Stretching the nerve-endings of the vagus in the lungs inhibits inspiration. If the stimulation be excessive, inspiration is inhibited for a considerable time. That this is the right theory of apnoea is proved by repeatedly inflating the lungs of an anæsthetized animal with a pair of bellows. The same arrest of inspiration is induced whether the lungs are inflated with air or with a neutral gas, such as nitrogen, so long as the vagus nerve is intact. If this be cut, inflation with a neutral gas no longer produces apnœa.

"Dyspnoa " is the term applied to the complex conditions and movements which result from deficient aeration of the blood, or, rather, from the distribution of insufficiently aerated blood to the centres in the medulla oblongata. The blood of the rest of the body may be in a satisfactory condition, but if, owing to ligature of the carotid and vertebral arteries or other causes, the blood supplied to the brain be inadequate to its proper nutrition, the phenomena of dyspnoea are as marked as they are when air is prevented from entering the lungs. 
That the excitability of the nerve-centres in the brain is greatly increased when this organ is supplied with venous blood, and that their tendency to transmit impulses which call for respiration is consequently exaggerated, is remarkably shown by the following experiment: Two rabbits-A. and B.-are placed under the influence of chloroform. Their carotid arteries are cut, and a crossed circulation established by connecting the proximal ends of A.'s arteries with the distal ends of B.'s, and vice versa. The head of each rabbit is now supplied with blood from the heart of the other, the rest of its body by blood from its own heart. A.'s chest is now opened, so that its lungs collapse and cease to take part in respiration. The animal continues to make the movements of respiration in a tranquil manner, whereas $B$. is thrown into violent dyspncea. The animal whose brain is receiving aerated blood remains normal, notwithstanding the fact that its lungs and the rest of its body are poisoned with venous blood. The animal whose brain is supplied with venous blood becomes dyspnœic, although its lungs and body are receiving pure arterial blood.

There is a regular sequence in the phenomena of dyspnœa leading up to the final stage termed " asphyxia." If the trachea be suddenly blocked, so that no air can pass, the respiratory movements at once become deeper and more rapid. This condition is termed "hyperpnœa." In a comparatively few seconds the system appears, as it were, to find out that inspiration is not needed. Expiratory efforts begin to preponderate. They increase in violence. All accessory muscles are brought into play. The cry for air is heard even by muscles which cannot help. Muscles of the limbs contract, although their contraction has no effect upon the capacity of the chest. Every expiratory effort is accompanied by convulsions of a flexor type. At the end of two minutes there is usually a sudden change. Attempts at expiration cease. Slow, deep, infrequent inspirations take their place, accompanied by convulsions of extensor muscles. Pupils are widely dilated, mouth open, head thrown back. The subject is absolutely insensitive to every kind of stimulus. The pulse shows a high arterial tension. The beating of the heart is slow and strong. In about four minutes from the time at which the windpipe was blocked respiratory movements cease. The arterial tension falls. The 
heart's action grows rapidly weaker, although for two or three minutes longer it may still continue to flicker. Recovery is possible until it finally gives up. After death the right side of the heart is found gorged with blood, the left side empty, showing that the heart had been unable to force the blood through the capillaries of the lungs.

Under all ordinary conditions the sequence of phenomena of asphyxia is the same-a stage of exaggerated breathing (hyperpnœa), a stage marked by the co-operation of muscles which are not called into action in tranquil breathing (dyspnœa), followed by the condition of asphyxia properly so termed. An animal whose supply of fresh air is cut off passes through these three stages, whether it be enclosed in a small space or in a very large one. It must, however, be noted that in asphyxia several factors combine in varying degrees. Carbonic acid is in excess in the blood, oxygen deficient. The nervous mechanism which regulates respiratory movements is thrown out of gear. Motor and inhibitory impulses are in conflict. It is important, if these complex phenomena are to be analysed, that one factor only should be altered at any given time. For example, carbonic acid may be allowed to increase in the air while a constant oxygen-tension is maintained. Under these circumstances the dyspnœic contractions are much less marked. No convulsions follow. The paralysing action of carbonic acid predominates. Anæsthesia passes into complete unconsciousness. Death is tranquil. And this, speaking generally, is what happens in disease of the lungs. Asphyxia comes on slowly. The supply of oxygen is undiminished, but carbonic acid accumulates in the blood, acting as a narcotic poison which lowers the excitability of the nervous system, suspends consciousness, and slowly brings the vital activities to a standstill.

In cases of drowning, when the lungs are filled with water, the resistance to the passage of blood through their capillary vessels is greater than it is when they are still filled with air. The heart is sooner beaten in its effort to drive the blood through them. Usually it stops in about four minutes. Yet it is difficult to say for how long after a person has been immersed in water it may be still possible to resuscitate him. Reports vary, owing in large measure to uncertainty as to the exact time at which the immersed person sank and his lungs 
filled with water. It is a wise precept that artificial respiration should be tried in every case, without waiting a single instant to ascertain whether the heart still beats. The first thing to do is to empty the chest of water. Then place the subject on his back. Kneel on the ground behind his head. Grasp an arm just below the elbow, in each hand. Draw the arms up above the patient's head, so that the pectoral and other muscles drag on the ribs, enlarging the chest; then lower them, and press them into the sides. This must be done with the natural rhythm of respiration, and not more frequently than twenty times in a minute. It is well if an assistant draws the tongue forward, to give free admission to air. Presumably the slight exchange of air brought about by mechanical expansion and compression of the chest favours the passage of blood through the capillaries of the lungs; but the real object of artificial respiration is to stretch the endings of the vagus nerve, and in this way to originate impulses which will call the respiratory centre into action. Perhaps it may not be superfluous to point out that the failure of the pulse must not be taken as an indication that the heart has ceased to beat. Owing to the obstruction to the circulation through the lungs, the left side of the heart is almost empty. Very little blood is pumped into the aorta. None reaches the wrist.

Exchange of Gases in the Lungs.-In the lungs each red corpuscle takes from the air a charge of oxygen which it carries to the tissues. In the tissues the plasma of the blood receives carbonic acid, which escapes from it when it reaches the lungs. Water dissolves oxygen and carbonic acid. Towards animals and plants which live in it, water plays the same rôle as the atmosphere towards dwellers on land. The quantity of a gas which will dissolve in water is proportional to the pressure to which it is subjected. If water were the circulating fluid, some oxygen would enter it in the lungs; some carbonic acid would be taken up in the tissues and liberated in the lungs. But it is clear that the small quantity of fluid which the vascular system will hold would be incapable of serving as an efficient medium of exchange between the tissues and the lungs. When a given quantity of venous blood is agitated with air, five times as much oxygen is taken up as the blood could carry if the gas were simply dissolved. Both oxygen and 
carbonic acid are held by the blood in chemical combination.

The condition in which oxygen is carried was discovered in 1864 (cf. p. 68). From all time it had been noticed that the blood which flows from a vein is darker and of a more purple tint than the blood which spurts out of a cut artery. Shortly before the date mentioned above, the spectroscope had begun to be used to distinguish more accurately than the eye can do the groups of rays which a coloured solution transmits. The colour of a ray of light depends upon its wave-length. The light of the sun, when its rays are sorted by a prism, according to their wave-lengths, shows all colours from the long waves of red to the short rays of violet, with certain gaps. At intervals where rays are missing, the spectrum exhibits dark bands-Fraunhofer's lines. The colour of a solution is measured by placing a flat-sided vessel containing it in the course of a beam of the sun's light, on its way to a prism. When the rays are spread out, it is observed that certain groups have been absorbed by the coloured fluid. The colour of the solution is due to the rays which it transmits. It had been pointed out in 1862 that blood diluted with water absorbs parts of each end of the spectrum, and also two groups of rays lying between the fixed bands of Fraunhofer which spectroscopists had labelled D and E. Stokes observed that this is true only of arterial blood. Venous blood absorbs a broad band in this part of the spectrum in place of the two narrow bands. He showed that, "like indigo, it is capable of existing in two states of oxidation, distinguishable by a difference of colour and a fundamental difference in the action on the spectrum. It may be made to pass from the more to the less oxidized condition by the action of suitable reducing agents, and recovers its oxygen by absorption from the air." The reducing agents of which Stokes made use were alkaline solutions of ferrous sulphate or of stannous chloride containing some citric or tartaric acid. These subsalts of iron and tin very rapidly absorb oxygen from the air or from any chemical substance which parts with it readily. With thes esolutions Stokes replaced the tissues. He abstracted the oxygen from the oxyhæmoglobin; then, shaking the solution of reduced hæmoglobin with air, he reproduced the action which occurs in the lungs. 
If the hand be held between a spectroscope and the source of light, in such a position that the beam passes through the thin tissue of two fingers where they are in contact, the spectrum of oxyhæmoglobin is obtained. If now the circulation through the fingers be impeded by putting strong indiarubber bands round them, the blood becomes venous, and the two narrow bands of oxyhæmoglobin give place to the broad band of reduced hæmoglobin.

Although very soluble, hæmoglobin may be obtained in crystals, the form of which varies in different animals. When obtained from human blood, the crystals are rhombic prisms ; from the guinea-pig, tetrahedra ; from the squirrel, hexagonal plates. Yet it is unlikely that the hæmoglobin of one animal differs chemically from that of another in any proper sense of the term. Probably the form of the crystals depends upon the amount of water of crystallization. The apparent polymorphism of hæmoglobin may be associated with the great size of its molecules (cf. p. 66).

Even when in the crystalline form, hæmoglobin can take up oxygen ; but the difficulties which attend its purification and crystallization render somewhat uncertain the amount of oxygen which a gramme of crystallized hæmoglobin can absorb. In solutior, 1 gramme can take up 1.34 cubic centimetres. The whole of the hæmoglobin of the body would, therefore, if it were all in the oxidized condition, hold about 4 grammes of oxygen.

It is not with oxygen alone that hæmoglobin can combine. It can absorb the same volume of carbonic oxide or of nitric oxide gas. Both of these gases it holds more firmly than oxygen. Neither carbonic oxide-hæmoglobin nor nitric oxidehæmoglobin is of any use to the tissues. If the blood becomes charged with the fumes of carbonic oxide (CO) given off by a coke-fire, this gas proves extremely poisonous. The blood does not lose it in its circuit through the body, nor is it exchanged for oxygen in the lungs.

The instability of the compound of hæmoglobin and oxygen is shown under the air-pump. The pressure of air in the open equals 760 millimetres of mercury. When the pressure falls to about 250 millimetres, the oxygen is rapidly given off. This is a matter of considerable interest in its bearing upon the ques- 
tion of the height to which it is possible for a human being to ascend. An animal placed in a chamber from which the air is pumped dies when the pressure falls to 250 millimetres of mercury. It has been ascertained that a man under the same circumstances can bear with impunity a reduction to 300 millimetres. How much lower must the pressure fall before it proves fatal ? Of three aeronauts who ascended in the balloon $Z$ enith to a height of 8,600 metres (26,500 feet), two died. The third, Tissandier, became unconscious, but recovered during the descent. The pressure of the atmosphere at such a height is 260 millimetres. The greatest mountain heights yet attained are 23,100 feet (Aconcagua, in the Southern Andes), reached by Fitzgerald, and 23,400 feet (Trisul, in the Garhwal Himalayas), reached by Dr. Longstaff and his companions. The pressure at this height was 320 millimetres. From these facts it is clear that mountaineers have just about reached the limit; but since they have not as yet mounted to a height at which the barometric pressure is less than 300 millimetres, it is possible that slightly higher mountains are still waiting to be conquered. At 23,000 feet the oxygen contained in arterial blood does not exceed 10 volumes per cent. (cf. p. 190). It is therefore about half the normal amount. Hence the breathlessness and sense of feebleness experienced by climbers. The least exertion leads to the consumption of all the circulating oxygen. But since the effects of want of oxygen are felt at altitudes much lower than those to which reference has been made, it is clear that the question cannot be regarded as simply ore of physics. The nervous system suffers when an attempt is made to do work with a deficient oxygen-supply. Violent headache and nausea attack most persons long before a level is reached at which the combination of hæmoglobin with oxygen ceases to be possible. The occurrence of this " mountain sickness" reminds us that we must not take for granted that the nervous system will continue to do its work right up to the altitude at which oxy-hæmoglobin is dissociated. Still, the figures show that, apart from these nervous symptoms, which disappear after a time, no serious disturbance occurs even though the atmospheric pressure be but little higher than the absolute minimum at which hæmoglobin combines with oxygen.

The eapacity of the blood for rapidly absorbing oxygen in 
the lungs and readily parting with it to the tissues is easily and completely explained by the property which hæmoglobin possesses of forming an unstable compound with this gas.

It is quite otherwise with regard to the liberation of carbonic acid. The problems presented by the solution of this gas in blood and its elimination in the lungs are difficult to solve. Less than one-tenth of the volume of carbonic acid which can be extracted from blood by the air-pump is simply in solution. The remainder is in loose chemical combination, the chief agents in holding it being the alkaline carbonates which the plasma contains. With an excess of carbonic acid they form acid carbonates, which give up carbonic acid and again become normal carbonates in the lungs. About one-third of the carbonic acid is, however, held by the blood-corpusclespartly in virtue of their alkaline carbonates and phosphates, partly in combination with their globulin. The affinity of these several vehicles for carbonic acid is sufficient to enable them to take it from the lymph, and to hold it while the blood is in the veins. When they reach the capillaries of the lungs, they part with their burden of carbonic acid to the air. It is in connection with this renunciation that certain difficulties remain to be explained. The carbonic acid is given up with greater readiness than our knowledge of the chemistry of the compounds into which it enters in the blood would lead us to expect.

Why does oxygen enter blood as it circulates through the lungs, and carbonic acid leave it? We have referred to the immense surface which the lungs expose to air. If a soapbubble be filled with a mixture of oxygen, nitrogen, and carbonic acid, and if the oxygen be in smaller proportion, and the carbonic acid be in greater proportion, than in the air of the room, oxygen will enter the bubble, and carbonic acid will leave it, by diffusion. If, instead of filling a bubble with gas, we fill a bladder with water charged with carbonic acid, but destitute of dissolved oxygen, a similar exchange with the gases of the air will take place. It is merely a question of "gaseous tension." The tension of the gases in the lungs is measured by passing a small tube down the trachea, and along one of the two chief bronchi until it becomes blocked in a bronchus just large enough to admit it. Respiration is carried on under normal conditions in the remainder of the lung ; but in the lobe 
which the catheter blocks diffusion from stationary air to tidal is no longer allowed. At the same time, since the circulation is not interfered with, the gases in the blood of the occluded lobe of the lung are not in markedly different proportions from those in the air-chambers of other parts. If at the end of a sufficient interval the air of the occluded lobe is drawn off and its gases measured, their tensions can be compared with the tensions of gases in specimens of arterial and of venous blood. If from 10 c.c. of fluid 1 c.c. of gas can be removed by the air-pump, the volume of gas dissolved is 10 per cent. of the volume of the fluid which dissolved it. Commonly this is written " 10 volumes per cent." To ascertain experimentally the tension of a particular gas in a particular fluid when dissolved to the amount of 10 volumes per cent. at the ordinary pressure of the atmosphere and at the temperature of the body, it would be necessary to place it in an open vessel in air containing a sufficient admixture of the gas to prevent its escape from the fluid. Suppose that it were found that, when the fluid containing the dissolved gas was placed in air mixed with the same gas to the extent of one-tenth of its volume, the fluid neither gave up gas nor absorbed more gas, the tension of the gas would be equal to one-tenth of an atmosphere. Since the pressure of the atmosphere equals 760 millimetres of mercury, the tension of the dissolved gas would be 76 millimetres. If more gas were added to the air, more would dissolve in the fluid; if some of the gas were removed from the air, gas would escape from the fluid. Gas passes from the medium in which its tension is high to the medium in which its tension is low. The tension of carbonic acid in tissues, particularly in muscles and glands, is higher than in lymph; in lymph higher than in blood; in blood higher than in air. Hence it passes by these several stages from the tissues in which it is formed to the air in the lungs. Much ingenuity has been devoted to perfecting methods for the determination of the tension of carbonic acid in lymph and in venous blood. Frequently results have been obtained which seemed opposed to the doctrine that carbonic acid progresses from one medium to another in accordance with the law of pressures ; but such perplexing results were probably due either to imperfections in method or to the establishment of abnormal physiological conditions during the course of the 
observations. When, for example, it was found that the tension in lymph was less than the tension in blood, the specimen of lymph examined was probably not in the same condition as the lymph in the tissue-spaces where the exchange occurs. The experimenter in such a case was in error in supposing that the specimen of lymph which he examined contained as much carbonic acid as did the lymph in the tissue-spaces from which the blood which he compared with it received its supply of this gas.

We have already given the figures for the composition of the air in the air-chambers of the lungs. The figures commonly accepted as correct for the percentages of the several gases in the blood are, at $0^{\circ}$ C. and 760 millimetres of mercury pressure :

$\begin{array}{lccc} & \text { Oxygen. } & \begin{array}{c}\text { Carbonic } \\ \text { anhydride. }\end{array} & \text { Nitrogen. } \\ \text { In } 100 \text { vol. of arterial blood . . } & 20 & 39 & 1-2 \\ \text { In } 100 \text { vol. of venous blood . . } & 8-12 & 46 & 1-2\end{array}$

This table shows the gain in oxygen and the loss in carbonic acid which results from the passage of blood through the capillaries of the lungs. The aerated blood returned to the heart by the pulmonary veins contains 8 to 12 volumes per cent. more oxygen, and about 7 volumes per cent. less carbonic acid, than the blood which the pulmonary artery carries to the lungs.

As to the physics of this exchange, the air in the recesses of the lungs contains about 16.36 per cent. of oxygen, and an amount of carbonic acid variously estimated at from 2.57 per cent. to 3.84 per cent. Of the 760 millimetres of mercury which the atmosphere holds up in a barometric tube, the oxygen in the alveoli of the lungs supports $\frac{760 \times 16.36}{100}=124.33$ millimetres ; the carbonic acid, at the lower figure quoted $(2.57$ per cent.), $19 \cdot 5$ millimetres.

The tension of gases in arterial blood is ascertained by opening an artery into a closed vessel which contains nitrogen mixed with oxygen and carbonic acid at about the tensions which it is computed that they have in the blood. If the amounts of these gases are exactly right, no exchange occurs between the blood and the mixture of gases. The mean of many observations 
made in this way by various physiologists is, for oxygen in the blood 72.2 millimetres mercury pressure, for carbonic acid 20.5 millimetres mercury pressure. At a glance it is seen that, since the tension of oxygen in the blood never exceeds 72 millimetres, whereas its tension in pulmonary air never falls beneath 124 millimetres, there is no difficulty in accounting for its passage from air to blood. The position is somewhat otherwise with regard to carbonic acid. Aeration continues in the lungs until the tension of this gas in the blood returning to the heart does not exceed 20.5 millimetres; whereas the tension in pulmonary air, even accepting the lowest figure obtained by experimental means, is as high as $\mathbf{1 9 . 5}$ millimetres. 'This leaves a very small margin of pressure to account for the escape-and it is undoubtedly a rapid escape-of carbonic acid from blood as it circulates through the lungs. As was said regarding the fixation of carbonic acid in the blood, it is somewhat doubtful whether the problem has been completely solved.

The carbonic acid exhaled contains all the carbon of the digestible food, with the exception of a comparatively small quantity given off in urea. It amounts to about 900 grammes per diem.

How are we to determine the quantity of air which an individual requires? We can but make the general statement that it must be sufficient to dilute the carbonic acid exhaled to an extent which precludes poisoning. It is impossible to fix a limit. Breathing becomes embarrassed, and frontal headache and other symptoms make themselves felt when 10 per cent. of pure carbonic acid is mixed with air. Even in so large a proportion as this, carbonic acid is not fatal to life. Yet an atmosphere in which there is present a hundredth part of this amount of carbonic acid, produced by respiration, is extremely injurious to health under the ordinary conditions in which people live. It may be asserted, therefore, that under ordinary conditions 0.1 per cent. is the extreme limit for wholesome living. But again we are obliged to add that air contaminated to this extent is not under all circumstances injurious to health. The explorers on the recent Antarctic Expedition were obliged at times to sleep three men in one sleeping-bag, with the aperture of the bag tightly closed. The atmosphere must have been heavily laden with carbonic acid. Dr. Wilson assures us that it was 
impossible to keep a pipe alight inside the bag. Not that any man so placed would desire, one would imagine, to add the combustion-products of tobacco to those given off from the lungs! The survival of the explorers proves that it is impossible to fix a limit of safety even for the carbonic acid in air vitiated by respiration. It is, however, a matter of common observation that air which is moist and warm, owing to respiration, and tainted with the odours of humanity, is extremely prejudicial to those who live in it. Such an atmosphere is a favourable medium for the conveyance of germs, whether of the common cold or of a more virulent type. At one time it was supposed that the volatile emanations which can be condensed, along with water, by hanging a vessel of ice to the ceiling of a crowded room, were actively poisonous; but this statement has not been confirmed by recent research. It is unnecessary to call any such evidence in support of the thesis that human beings thrive better in fresh air than in foul. The admirable results achieved by the "fresh-air cure" show that there is no degree of vitiation which can be pronounced innocuous. Nevertheless, public opinion demands that sanitarians should give some figure as a guide. Commonly they fix the maximum of carbonic acid compatible with health at 0.06 per cent., the quantity of carbonic acid being taken as the measure of all impurities present. An adult exhales about 0.6 cubic foot of $\mathrm{CO}_{2}$ per hour. Fresh air already contains about 0.04 per cent. If, therefore, the percentage is not to rise higher than 0.06 per cent., each adult must be supplied with 3,000 cubic feet of air per hour. With good ventilation air may be changed four times an hour, and therefore 800 cubic feet is regarded as sufficient space for each occupant of a room. The figure may pass. It is a reasonable basis from which to calculate the packing capacity of a dormitory. So long as a man has 800 cubic feet of air to himself, he may safely feel that he has room to stretch his lungs. Dwelling on this figure may make him feel uncomfortable when he finds himself in a railway carriage, seated five on a side, with the windows closed. In the theatre or in church he may doubt whether he has all the fresh air to which his humanity entitles him. But, as a philosopher rather than as a physiologist, he reflects that, whether on the Antarctic icecap in a sleeping-bag or standing on a summit in 
the Alps, he takes all that he can get, for fresh air is one of the few good things of which one can never have enough.

Tissue Respiration.-A frog will live for seventeen hours in an atmosphere of nitrogen. Under these circumstances it is clearly impossible for it to take up oxygen, yet for several hours it gives off as much carbonic acid as it would do if it were living in air. Such an observation as this proves that oxidation does not occur in the lungs, but deeper in the body. At one time the blood was regarded as the seat of oxidation; the products formed by the splitting up of proteins in the tissues were supposed to be passed into the blood, where they came in contact with the oxygen carried by hæmoglobin. A certain amount of oxidation does take place in the blood, as in all other tissues, for blood is a living tissue and needs to respire. But the oxidation whioh occurs in the blood is small in amount as compared with that in the organs which the vessels traverse. Muscle and other tissues detached from the body and free from blood give off carbonic acid. It is possible to wash the blood out of the vessels of a frog and to replace it with a solution of salt. In an atmosphere of oxygen such a "saline frog" lives for a day or two, taking in the same quantity of oxygen and giving off the same quantity of carbonic acid as a normal frog. The oxygen is chiefly absorbed through the skin, the carbonic acid discharged from the lung. This experiment shows that blood is not essential for oxidation. Oxidations do not occur in the salt solution with which blood is replaced. Taking all the evidence together, it seems to be safe to conclude that the tissues absorb the oxygen which the oxyhæmoglobin brings into their neighbourhood, and that they have some capacity of storing it. A piece of detached muscle which gives off carbonic acid in an atmosphere of nitrogen would appear to be holding a store of oxygen, much as hæmoglobin holds it. The proof is not quite so definite as might be desired; but we are probably justified in holding the belief that the main part of the respiratory exchange occurs in the tissues. Lymph dissolves oxygen which it obtains from the blood. The tissues take it from lymph. Tissues set free carbonic acid which lymph dissolves. Its tension being higher than in blood, carbonic acid diffuses from lymph, through the walls of the capillary vessels, into blood, from which it passes into the air in the lungs. 


\section{CHAPTER VIII}

\section{EXCRETION}

MaNy things enter into the alimentary canal. If an analysis were made of a day's food and drink, from the cup of tea on waking to the cocoa or other potion which is regarded as a necessary preliminary to settling for the night, it would be found that a great variety of substances were included in the food or taken as adjuvants to food. All these things, differing widely in chemical constitution, must leave the body. Some are not digested. They do not, properly speaking, enter into the diet. Such are the cellulose of vegetables, especially skins, husks, woody fibres ; elastic fibres of meat; horny substances, etc. The quantity varies greatly, according to the nature of the diet. About 2 ounces (weighed dry) is the average. With this indigestible refuse is included undigested food, if the diet be excessive, and a variety of substances secreted by the liver, such as cholesterin and bile-pigment, some residues of the secretions of the alimentary canal, and products of bacteric fermentations. All food which is digested and absorbed is oxidized. It leaves the body by the lungs, the kidneys, or the skin. Foods, as already stated, are classified as proteins, carbohydrates, and fats. The chief excreta are carbonic acid, water, and urea. Carbonic acid makes its exit from the lungs ; water from the lungs, the kidneys, and the skin ; urea from the kidneys. The three great groups of foods and the three great groups of excreta overshadow in amount all the other substances which pass through the system. A balance-sheet in which proteins, carbohydrates, and fats appear on one side, carbonic acid, water, and urea on the other, is substantially correct. The energy which is set free by burning in a calorimeter the items entered on the debit side, after deducting that yielded by burning the urea (carbonic acid and water are in- 
capable of further oxidation), gives a day's income. Other constituents of the diet are so small in quantity as to be negligible in making up the body's accounts. The chemical changes which they undergo add practically nothing to its eapacity for work. Yet some of them are essential to the maintenance of health. Of such are common salt (sodic chloride), alkaline and earthy carbonates, sulphur, phosphorus, etc. These things, together with some products of action of the bacteria in the alimentary canal, the final stage of hæmoglobin, imperfectly oxidized nitrogenous substances, and other soluble substances which enter with, or are formed from the food, are removed by the kidneys. We speak of the elimination of waste products, as excretion. Not that there is any physiological distinction between exoretion and secretion. Both terms refer to the selection or production and the discharge of materials by cells. If the product discharged has a useful function to perform-if it be a digestive ferment, for example-it is said to be secreted. If it is of no further use to the economy, we say that it is excreted-got rid of. In some cases either term is equally appropriate. The sebum prepared by the sebaceous glands is useful as a lubricant of the skin. It is thrown off. We may speak of the glands as either secreting or as excreting this fatty substance.

The Kidney.-From worms upwards, all animals possess organs for the removal of waste products in solution. This statement might, indeed, be widened so as to include animals even lower than worms. All animals which have a cœlomic cavity - a space between the alimentary canal and the bodywall-have organs for the removal of soluble waste. The segmental organs of worms are obviously the same organs as the kidneys of manmals; the latter are distinguished from their prototypes by greater concentration of structure and specialization of function. The kidney is the oldest of organs, if its antiquity be estimated as the length of time during which it has had a form practically identical with that which it now presents. The lungs are of late appearance in the animal scale. Alimentary canal, heart, brain, have passed through many transformations. The kidney assumed its permanent form very far back in the history of the animal kingdom. The most primitive animal which has a digestive cavity, and 
vessels in which the products of digestion circulate, needs an organ which provides for the overflow from the body-fluids of all substances which are injurious or effete.

The kidney is an aggregation of long urinary tubules. The head of each tubule is dilated into a globular capsule, into which a tuft of bloodvessels depends. This is the sink into which the waste-water of the blood drips. The long urinary tubules are lined with cells well qualified by form and constitution to search the blood in the capillaries which border them, for substances which, not being easily diffusible, have to be forcibly dragged from it and added to the water trickling down the pipe which connects the rain-water head with the sewer. The hydrostatic conditions of this apparatusthe provision for greater or less flow of blood through the tufts (glomeruli) which hang in the capsules, and for longer or shorter exposure of the blood to the purifying activity of the epithelium of the renal tubules-will be described after a very brief account has been given of the structure of the organ.

The outer border of the kidney is convex, its inner border concave. The concavity is termed the "hilus." The central depression of the hilus is embraced by the expanded end of the ureter-the tube which carries the secretion of the kidney to the bladder. The renal artery and the renal nerves enter, and the renal vein leaves, the kidney at the hilus.

If a kidney be split longitudinally, it will be noticed that its outer part, the cortex, is darker in colour than its inner part, the medulla (Fig. 9). The glomeruli already referred to occur in the cortex. The medulla is occupied by radiating tubules, collected into groups. Those of each group converge towards a common duct. From twelve to eighteen ducts open into the expanded end of the ureter, each at the apex of a pyramid. If the section of the kidney be examined with a lens, it will be seen that narrow rays from the medulla extend into the cortex. The cortex is therefore made up of interdigitating pyramids of dark substance, consisting of glomeruli and the contorted tubules, about to be described, and of lighter substance, consisting of straight tubules continuous with those of the medulla.

The urinary tubules are the separate pieces of apparatus of which the kidney consists. The problems connected with 
a single tubule are therefore the problems of the kidney as a whole. These structures are all exactly alike. The descrip-

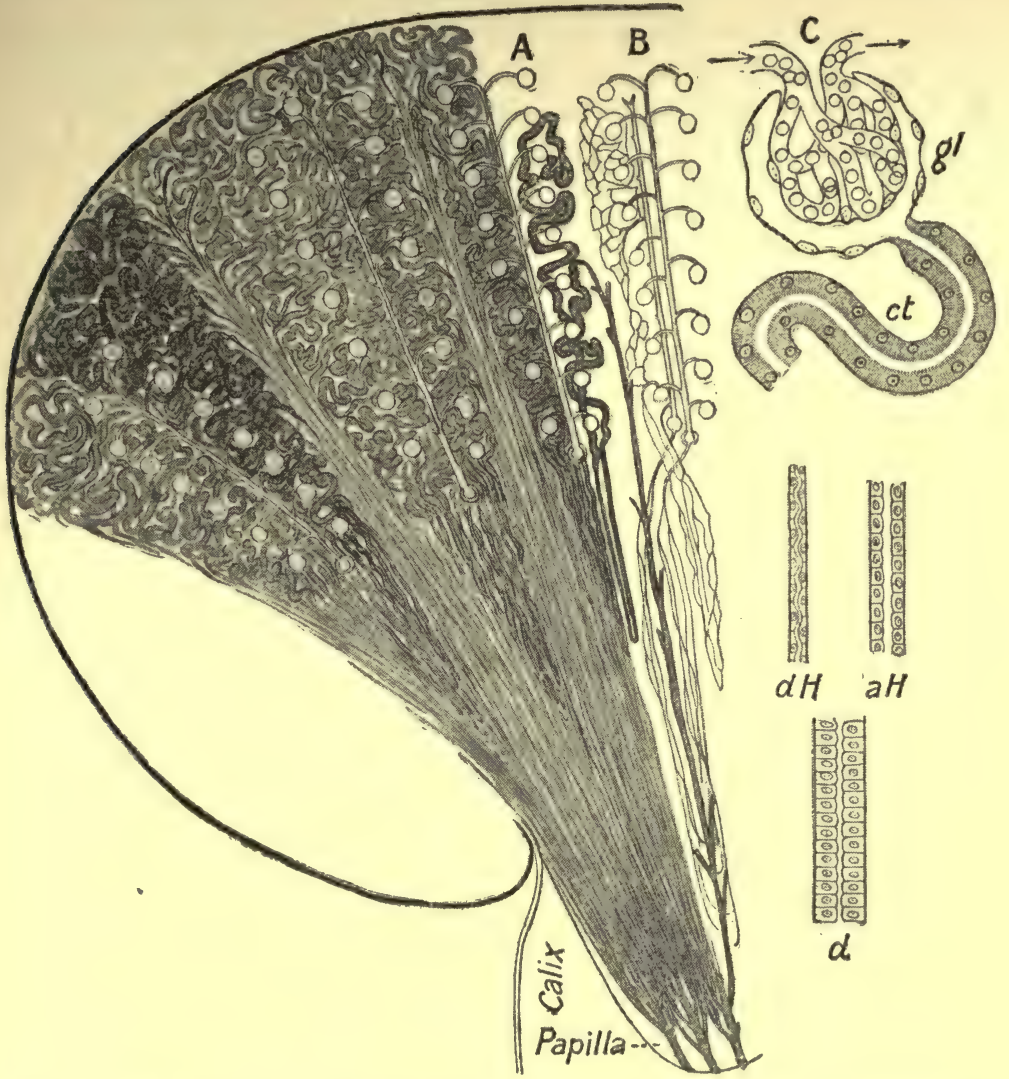

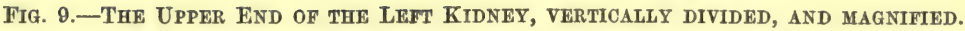

It is invested by a capsule with which, at the hilus, the dilated end of the ureter blends. A portion of a papilla (the end of a pyramid) is shown projecting into one of the calices into which the ureter dilates. The peripheral portion of the kidney containing glomeruli and contorted tubes is termed its cortex, the central portion medulla. At $\mathbf{A}$ is shown a single urinary tubule. Commencing at the third glomerulus, it winds in the cortex, descends into the medulla, turns in a loop of Henle, again winds in the cortex, and ends in a collecting tube, which joins a duct. The arrangement of the bloodvessels is shown at B. A straight artery and a straight vein lie side by side. The artery gives branches to the glomeruli. The venules from the glomeruli again divide into capillaries, which supply the contorted tubes and loops of Henle. The ducts are supplied by long arterial capillaries. C shows the structure (magnifled) of a glomerular tuft of capillary vessels, invested by a capsule which closes into a contorted tube, $c t ; d H$, a descending limb; $a H$, an ascending limb of a loop of Henle; $\boldsymbol{d}$, a duct.

tion of any one of them applies to all. Each begins as a capsule containing a glomerulus. The wall of the bulb-which 
is merely a thin basement membrane covered by epithelial scales-is involuted by the tuft of bloodvessels. The vessels do not penetrate its capsule. Between the tessellated epithelium which covers the tuft and the similar epithelium which lines the capsule there is a space communicating by a narrow aperture with the next portion of the tubule-termed its "contorted" part, because it is twisted about like a tangled thread in the cortex of the kidney. The contorted tubule is of relatively large calibre. The cells which line it are irregular in form and indistinct in outline. The basal half of each cell, between its nucleus and the basement membrane, is vertically striated, or "rodded," as it is usually termed. Such an arrangement of the protoplasm of a cell is commonly associated with a habit of absorbing fluid. It would seem to indicate in this case that the cells take water and various substances dissolved in water from the direction of the basement membrane. After a time the contorted portion of the tubule, although still sinuous, becomes more nearly straight-the "spiral portion" - and assumes a radial direction. In the zone between the cortex and the medulla, the spiral portion tapers into an exceedingly slender tubule which, after running some distance in the direction of the hilus, turns back again towards the cortex, making a loop, known as the "loop of Henle." The ascending limb of this loop is of larger calibre than the descending limb. The descending limb is lined by flattened epithelium, each cell so thin that (in microscopic sections as ordinarily prepared) its nucleus bulges into the lumen of the tube. The cells of the ascending limb are more nearly cubical in form. On reaching the cortex, the tubule again becomes contorted. The second contorted portion narrows into a "collecting portion," which joins a ductule. The ductules unite together, until at last a single duct is formed which opens at the apex of a pyramid. The cells of the ductules are cubical or columnar. Their cell-substance is clear, whereas that of the cells lining other parts of the tubule is cloudy in appearance.

Such a tubule, viewed as a hydrostatic mechanism, presents three portions, evidently fitted for different functions : (1) The glomerulus is an apparatus which allows of the rapid exudation of water from blood. (2) The contorted portions of the 
tubule present the appearance of a secreting mechanism. The large soft, cloudy cells which line them are eminently fitted to take from the blood, or rather from the lymph which fills the tissue-spaces which intervene between the walls of the capillary bloodvessels and tubules, the various substances which they excrete. (3) The loop of Henle is a remarkable piece of apparatus, the purpose of which has been a subject of much controversy. Looking at it from the point of view of hydrostatics, it seems safe to conclude, from its extremely narrow bore, that it raises the pressure of the fluid in the glomerulus and first contorted portion; but it may have other functions also.

A consideration of the arrangement of the bloodvessels of the kidney bears out the conclusion that the secreting apparatus is divisible into at least two separate portions, possibly into three. The glomeruli are supplied by short and relatively wide arterioles. Each arteriole breaks up, as soon as it enters the capsule, into a bunch of capillary vessels, which, in the same abrupt manner, reunite to form a venule. On leaving the capsule, this little vein behaves in a fashion for which the only parallel is to be found in the portal system of the liver. Instead of uniting with a larger vein, it again breaks up into capillary vessels, which supply the contorted tubules and loops of Henle. The medulla of the kidney is supplied by long arterial capillaries of the usual type. The short arterioles of the glomeruli are controlled by nerves which, constricting them, or allowing them to dilate-possibly by actively causing them to dilate-rapidly diminish or increase the amount of blood passing through their tufts of capillary vessels. Here, therefore, is a mechanism by which the glomeruli can be suddenly flushed with blood-a condition favourable to exudation into the urinary tubules. The interposition of a second set of capillaries prevents this sudden flushing from unduly disturbing the pressure in the vascular system as a whole. In the renal-portal capillaries of the kidney the blood-pressure is fairly constant and, presumably, low. The use of the term "renal-portal" is justifiable, not only on the ground that the vessels of the kidney behave like those of the portal system of the liver, but also owing to the very significant fact that in fishes and amphibia the kidney actually has a double bloodsupply. In such an animal as the frog the glomeruli are 
supplied with arterial, the tubules with venous, blood. The glomeruli receive branches from the renal artery, the tubules from a portal system derived from veins of the abdomen and hind-legs.

Sir William Bowman, who in 1842 gave the first detailed description of the microscopic structure of the kidney, concluded that, whereas " the tubes and their plexus of capillaries are probably the parts concerned in the secretion of that portion of the urine to which its characteristic properties are due (the urea, lithic acid, etc.), the Malpighian bodies [i.e., the glomeruli] may be an apparatus destined to separate from the blood the watery portion."

All physiologists are in accord in regarding the glomeruli as the principal seat of exudation. There is great diversity of view as to the function of the tubules. In 1844 Ludwig advanced the opinion that all the constituents of the urine pass through the glomeruli in a large excess of water, and that in the course of the tubules this excess of water is reabsorbed. This theory was based, among other considerations, upon the extreme thinness of the epithelium which covers the glomerular tufts; he judged that water would filter through it very readily. A large amount of experimental work has been directed to the solution of these two problems-viz., (1) Do urea and other similar substances pass through the glomeruli ? (2) Is water returned from the tubules to the venous system? Our views as to the functions of the kidney as a whole will not be greatly influenced by the answers that may eventually be given to these questions; yet their discussion is of very great interest, owing to the nature of the evidence which may be marshalled on either side.

There is, perhaps, no other organ in the body the problems with regard to which seem to be so nearly plain questions of hydrostatics. It is easy to make a model of a urinary tubule and its blood-supply. If such a model were shown to a sanitary engineer, and he were asked to explain the working of the drainage system of the body, and especially to answer the two questions which we have propounded, he would say that there could be no doubt as to the part of it through which most water enters the tube, the glomerulus. He could give no opinion as to whether urea, uric acid, and other substances of a like 
nature, accompany the water until he had tried the experiment of separating blood from water containing the inorganic salts of urine by a permeable membrane-the blood. being at such a pressure as the physiologist told him he might expect it to have in renal arterioles, the water at such a pressure as he might expect it to have at the upper end of a urinary tubule. $\mathrm{He}$ would find that urea, and still more uric acid, is very reluctant to pass through the membrane. Again, when asked whether water, in which urea and other things were dissolved, would leave the tubule-say from the loop of Henle-to pass back into the blood, he would repeat his experiment with a membrane. This time he would allow the urine and the blood to be at the same pressure (or, possibly, would assign a higher pressure to the former), and he would dilute the urine to make the conditions agree with those which Ludwig supposed to exist; but his experiment would prove to him that, unless the urine were very dilute indeed, water would still tend to pass into it from the blood, and not vice versa. And here it may be remarked that the results of these experiments might have been predicted by calculation. When Ludwig advanced his theory, osmosis was a mysterious phenomenon. Its laws have since been accurately ascertained. Given the molecular weights of bodies in solution and their degree of concentration, the direction in which they will pass through a membrane can be predicted. The force with which water will tend to pass from one solution to another can be calculated. Urine as secreted contains far more urea, sodic chloride, and other salts than blood. It has a much higher degree of concentration. The concentration of blood is 0.55 ; that of urine, 1.85. Water passes from a less concentrated to a more concentrated solution, not vice versa. As a solution of a problem in hydrostaties Ludwig's hypothesis is untenable.

Osmosis.-Cells of all kinds, both vegetable and animal, are limited, or surrounded by a layer of cell-substance which is firmer than, and probably different in constitution from, the substance in the interior of the cell. This outer layer is a living membrane. The nutrition and growth of the cell are dependent upon the capacity of its limiting membrane for regulating the ingress and egress of water and of substances dissolved in water. The phenomena of osmosis-that is to 
say, of the passage of water and of solutions through membranes-are of such high importance in relation to the life of the tissues that it may be permissible to make a further digression for the purpose of describing them (cf.pp.40, 128). A very simple apparatus will suffice to exhibit a phenomenon which will give an idea of the meaning of osmosis. If the top of a glass funnel, covered with a piece of bladder, so fastened to its edge as to make it water-tight, be fixed in an inverted position in a glass vessel, the glass vessel filled with water, and the funnel filled to the same level with a solution of sugar, it will soon be evident that water is passing through the membrane into the funnel. The level of the sugar-solution will rise in the tube of the funnel. If, instead of water outside the funnel and sugar-solution inside it, a strong solution of sugar be placed in the funnel and a weaker solution outside it, water will leave the weaker for the stronger solution, and sugar the stronger solution for the weaker. If some of the solution in the funnel be removed from time to time so that the pressure in it is kept down to the same level as that outside it, water will continue to enter through the membrane and sugar to leave the contents of the funnel until the concentration of sugar is the same on the two sides. The fluids will then be of identical composition, and therefore isosmotic. In the further consideration of the phenomena of osmosis, a distinction must be made between permeable and hemipermeable membranes. Suppose in the first instance that a permeable membrane is used. Let it be so placed as to separate two watery solutions of different constitution, yet of the same osmotic pressure. By their being of the same osmotic pressure is meant that they are of the same molecular concentration. The liquid A contains certain salts in solution; but the liquid B may contain the same salts in quite different proportions. It so happens, however, that the salts are so balanced that the total tension of the salts in $\mathrm{A}$ is equal to the total tension of the salts in B. At first there may be some change in level in the two liquids, owing to differences in rates of diffusion through the membrane of the various salts which they contain ; but after a time the levels of the two liquids will be the same. To outward appearance, nothing will have happened. Nevertheless, if the experiment has been continued for a sufficient length of time, 
it will be found that great changes have occurred in the constitution of the two liquids. At the commencement, although their total tensions were equal, the proportions in which the various salts were distributed in $\mathrm{A}$, and therefore their partial tensions, were very different to their proportions and partial tensions in B. At the end of the experiment each of the several salts is equally divided between $\mathrm{A}$ and B, supposing the volume of A to equal that of B. This experiment shows that the molecules of substances in solution are free to move. They behave like gases. Gases diffuse through a membrane until their partial tensions are the same in the two spaces which the membrane separates. The æther in which physicists picture gases as dissolved offers no resistance to the migration of their molecules; neither does the solvent-water, for example-prevent the movement of salts which are distributed through it.

One other illustration of the phenomena of osmosis will suffice to give an idea of the laws by which they are governed. In the case just cited the membrane was permeable to all the salts in solution. When the phenomena of osmosis were first investigated, a distinction was drawn between substances which will pass through membranes-crystalloids-and substances which cannot pass-colloids. We have already had occasion to note that, whereas albumin is a colloid which does not diffuse, its hydrate, peptone, is a crystalloid which does. The term "crystalloid" indicates that substances which can be crystallized are diffusible. Substances which are diffusible are therefore allied to those which crystallize. The nature of the membrane used to test diffusibility was not at first taken into account. Now a distinction is drawn between membranes which are permeable to all diffusible substances, and membranes which are permeable to the solvent, but impermeable to the substances which it dissolves. The latter are termed "hemipermeable." Imagine now that water is separated from a solution of sugar by a membrane which stops sugar, but is permeable to water. Water will pass through the membrane into the solution of sugar. The level of the solution will rise. Pressure will be needed, and a very considerable pressure, to prevent its rising-to prevent endosmosis, that is to say. The force needed to resist osmosis is 
directly proportional to the degree of concentration of the solution. If the solution contain 1 per cent. of sugar, a pressure of 500 millimetres of mercury is needed; if it contain 2 per cent., a pressure of 1,000 millimetres; if 6 per cent., of 3,000 millimetres.

In the next experiment separate two solutions, A and B, by a hemipermeable membrane. Let A contain one salt only- $X$; let $B$ contain several salts-X, Y, Z. Water will pass from A to $\mathrm{B}$, or vice versa, unless the osmotic pressure of the salts which the solutions contain is the same, The osmotic pressure will be found to be the same if the total number of molecules dissolved in A equals the total number of molecules dissolved in B. If in A there be $\mathrm{N}$ molecules of $\mathrm{X}$ (per unit volume), and if in $\mathrm{B}$ there be $n X, n^{\prime} Y, n^{\prime \prime} Z$, the osmotic pressure will be the same provided $n+n^{\prime}+n^{\prime \prime}=N$. This, it will be seen, is a very different matter from equality of percentage composition. Some molecules are light; others are heavy. The percentage weight of $\mathrm{X}+\mathrm{Y}+\mathrm{Z}$ in $\mathrm{B}$ may be very different from the percentage weight of $\mathrm{X}$ in $\mathrm{A}$. To estimate the osmotic pressure of a mixed solution, it is not sufficient to add together the percentages of the various salts which it contains. "Concentration," in the sense in which it was used in regard to blood and urine, refers to the number of molecules of dissolved substances in a given volume, not to their weight.

It would be undesirable to attempt in this place to enter upon the theory of osmosis. Enough has been said to suggest to the reader that he should, when endeavouring to apply its laws to the explanation of physiological phenomena, bear the following facts in mind: Some membranes are permeable to water and to the crystalloids which it dissolves; others, although permeable to water, are impermeable to substances in solution. Some substances are diffusible through permeable membranes; others are not. Osmosis of water occurs from the solution of lower to the solution of higher concentration. Diffusion of crystalloids is their escape, owing to their own molecular movements, from a situation in which they are denser to a situation in which they are less dense. It must be added, however, that various circumstances prevent the reduction of the laws of osmosis to simple terms - the tendency of salts to dissociate when in solution, their bases and acids acting 
as independent "ions," is an example of the complications which produce apparent departures from these laws. It must further be added, and with emphasis, that, important though it be that anyone who attempts to explain the interchanges which occur between the various fluids of the body should be conversant with the laws of osmosis, it is impracticable, and in some cases misleading, to rigidly apply them. Living membranes and dead membranes do not necessarily control diffusion in the same manner. Still less do the laws which govern diffusion through dead membranes hold good, without qualification, to living cells.

To return to the sanitary engineer whose opinion we asked regarding the mode of working of the drainage system of the kidney. Probably he would deny that the problems came within his province. "They are not physical, but vital," he would say. "I know nothing about the vital action of the cells which line the tubule." Objection may be taken to the form of expression, albeit he was fully justified in declining to discuss the question any further. He does not know enough about the internal structure of a cell to be able to predict the phenomena of osmosis which will occur within it. No one can say what capacity living cells may have of taking substances from the blood, returning some of them, and excreting others. This unknown capacity leads to results which, when they do not appear to be in accordance with the laws of physics, are commonly termed "vital." The term is a stumblingblock which has tripped up generations of physiologists. The expressions "vital action" and "physical phenomena" have been used as if they were antithetical, whereas all vital actions are physical phenomena. "Vital" in this sense connotes "as yet unknown." Yet, in truth, there is abundant excuse for the use of a term which covers ignorance, so long as its connotation is not extended until it assumes a positive, antiphysical sense. "Physical" and "vital" are expressions which point a contrast constantly present to a physiologist's mind. He knows perfectly well that the passage of water and salts through a membrane, and their passage into and out of a living cell, are equally phenomena of osmosis. But the former process he can test and measure in his laboratory ; the latter he can but observe in much obscurity in the living body. $\mathrm{He}$ 
cannot make a model of a living cell. In the case of the salivary gland, as we have already seen, living cells take water from lymph, and discharge it as saliva in apparent opposition to osmotic force. They reverse the direction of the flow which would occur were lymph and saliva separated by a membrane. But a cell is not a membrane. It is an extremely complicated structure with an elaborate architecture of its own. As well might we compare the distribution of water by a County Council water-cart and its passage through a brewery. According to all the laws of hydrostatics, the water which flows into a brewery should leave it through its drains. Its exit in barrels on drays is antiphysical. When the physiologist can explore the living cell, he will discover that the imbibition and extrusion of water, the selection, retention, and discharge of salts, are phenomena as strictly physical as their passage through a dialyser in his laboratory. In the meantime he can but contemplate the cell with a certain degree of awe. His best devised model of a urinary tubule may lead him into error, for the simple reason that he cannot line it with living cells. A living cell has a power which upsets all calculations, falsifies all experimental findings. Its protoplasm can isolate and place out of action any of the substances which enter it. If observations eventually prove to us that water passes from the urinary tubules into the blood, "in the face of osmotic force," we shall be constrained to explain this antiphysical phenomenon as due to the action of living cells. The cells, we shall say, take up fluid from the urinary tubules, fix its urea and other salts in their protoplasm, discharge its water into the venous blood, return the urea and other salts to the urine. Given this property of protoplasm, such a process is strictly in accordance with physical laws.

Enough has been said regarding the theory, or want of theory, of the action of the kidney. Turning now to matters of observation, it can easily be shown that the epithelium of the tubules has the power of excreting into the urine highly complex materials which diffuse with difficulty. If a substance soluble in blood, but insoluble in urine, an alkaline salt of indigo, for example, be injected into the vascular system, it is rapidly excreted by the kidney. The indigo is precipitated even before 
it comes in contact with the acid urine. If the animal be killed a short time after the administration of the indigo, the contorted portions of its tubules and the ascending limbs of the loops of Henle are strongly coloured blue. An ammoniacal solution of carmine may be used for a similar experiment; but the results are not nearly so sharply limited to the large-celled portions of the tubules. Even the glomerulus is coloured red, a fact which has been interpreted as showing that, although the greater part of the carmine is excreted into the tubules, some of it accompanies the water which exudes from the blood through the glomerular tufts.

The practical identity in structure of the kidney in birds and reptiles and mammals would seem to have an important bearing on this controversy. The urinary excretion of birds consists almost exclusively of uric acid. As seen under the microscope, it is a semisolid white deposit, made up of crystals, supposing no special precautions have been taken to obtain it fresh. The water, pigment, and salts which are essential elements of the excretion of mammals are practically absent. Yet the kidney of a bird presents the same arrangement of glomeruli and tubules as the kidney of a mammal, although the glomeruli are relatively smaller. Uric acid diffuses with great difficulty. If it is, so to speak, washed through the glomeruli, and the water which dissolved it reabsorbed by the tubules, an enormous quantity of water must pass through the kidney in order that it may carry the uric acid in its stream. If uric acid be excreted by the epithelium of the tubules, it is difficult to account for the presence of glomeruli, since no water leaves the kidney. Crystals of uric acid are to be seen in a section of the kidney, not only in the cells of the tubules, but also in the glomeruli ; but it may well be that in both situations crystallization has been induced during the preparation of the section. It jars an histologist's conception of the constitution of a secreting cell to contemplate the formation within its network of protoplasm, and the extrusion from it, of sharp-angled crystals. As a matter of fact, it is not in its crystalline form that uric acid is excreted by birds, but as quadri-urates-i.e., salts containing only one-fourth of their "normal" complement of base; crystalline spheres or amorphous deposit, not angular crystals. These quadri-urates decompose very quickly, setting free 
crystals of uric acid. It must be confessed that, in whatever way one attempts to account for the excretion of uric acid by birds, the similarity of structure of their kidneys and those of mammals is difficult to reconcile with the wide difference in consistency and in chemical composition of the excrement.

Reflecting upon all the evidence bearing upon the mechanism of the mammalian kidney, the majority of physiologists come to the following conclusions: The greatest outflow of water occurs in the glomeruli. The water is accompanied by salts, including a small quantity of urea. The contorted and spiral portions of the tubule and the ascending limbs of Henle's loops add to the urine the remainder of the urea, together with various bodies still less readily diffusible.

It may be that the chief function of the loops of Henle is to oppose resistance to the passage of fluids, thus heading up the secretion, and favouring the osmosis of water into it from the blood of the glomerular capillaries. It is possible that the calibre of the slender descending limbs is influenced by external pressure, their partial occlusion being increased, and the pressure in them raised, when the organ is very active and its intermediate zone turgid with blood.

Various drugs influence the secretion of the kidney. In some cases their action seems to be mainly hydrostatic. They change the rate of flow by altering blood-pressure. Digitalis increases the force of the heart. The heart beating more strongly, blood-pressure rises. Higher blood-pressure is accompanied by a more copious secretion. This action of digitalis is far more marked when the heart is out of order than when it is healthy. In heart-disease the blood-pressure is unduly low, and the tissues become water-logged in consequence. When the bloodpressure is restored and a brisker capillary circulation established, water and waste-products, which have accumulated in lymph, pass, as they ought to do, into the veins. Carried into the general circulation, they overflow from the kidney.

It is a little difficult to realize the abundance of the bodyfluids. From one-quarter to one-third of the whole bodyweight is due to lymph, using this term in its most general sense. The waste-products of tissues collect in the lymph. The blood circulating through capillary vessels which traverse lymph-spaces takes up water and waste-products. Its just 
composition is maintained by the eliminating activity of the kidneys.

Even in the diuretic action of digitalis we see indications of something more than an alteration of the hydrostatics of the blood-supply of the kidney. The brisker circulation carries waste-products to the liver; the liver transforms nitrogenous refuse into urea; urea stimulates the renal epithelium. It would be a mistake to lay too much stress upon the direct effect of the drug upon the blood-pressure in the kidney. Other illustrations throw the mere hydrostatics of the problem into the background. Adrenalin (extract of suprarenal capsule) causes a severe contraction of the small arteries, which raises the general blood-pressure considerably; but the increased bloodpressure is not accompanied by diuresis, because the glomerular arterioles share to a full extent, perhaps to a disproportionate extent, in the general constriction. In migraine and certain other disorders it frequently happens that the blood-pressure in the aorta is unduly high, yet very little fluid enters the renal tubules. If a "saline diuretic," potassic nitrate, sodic acetate, or some other drug of the same kind, be administered, a copious flow is established, the blood-pressure is relieved, the distressing symptoms disappear. Then, again, certain diuretics, such as "sweet spirits of nitre," tea, gin, etc., may bring about a flow out of all proportion to the alteration they produce in the hydrostatics of the circulation. The diuretic action of these various drugs is clearly due to increase in permeability of the renal epithelium. And, of all stimulants to secretion, urea, the natural stimulant, is the most effective. If a kidney be removed from the body, a cannula inserted into its artery, and defibrinated blood caused to circulate under pressure through the organ, water may or may not drip from the ureter. On addition of urea to the blood, a copious excretion is set up. In explaining the mode of working of the kidney, as, indeed, in explaining that of every other organ of the body, the mechanical aspects of the problem must be kept in the background. When we are contemplating the plan of construction of the kidney, the hydrostatics of the circulation attract attention; but alterations in hydrostatic conditions are not the initiating cause of a greater or less flow of urine. The chemical condition of the blood circulating through the kidney is the initiating cause. 
When the presence in it of urea demands a more copious flow, the hydrostatic conditions are adjusted to this need. In the case just cited of the isolated kidney, it might be urged that the flow caused by urea is a mechanical effect. The cells of the contorted portions of the urinary tubules remove urea from the blood. They secrete it into the tubules. The solution of urea, being headed up towards the glomeruli, owing to the resistance offered to its passage down the tubules by the narrow, descending limbs of Henle's loops, surrounds the capillary tuft. Urea rapidly attracts water from the blood. A copious flow is the result. But it is just this contrast between the capacity of removing urea possessed by living cells, and the passage of urea in solution from one side to the other of a membrane, which justifies the retention of the expression "vital." Mechanical conditions are those which we can imitate in a model ; vital conditions, those which at present we are unable to reproduce.

Nitrogenous Waste.-Meat, fish, eggs, milk, vegetable albumins, are the sources of nitrogen. The kidney is the organ which eliminates it from the body. Since all nitrogenous food which is digested is eventually reduced to simple, soluble compounds which appear in the urine (the quantity thrown off in perspiration is so small as to be negligible), the proportion which the nitrogen of the urine bears to the nitrogen in the food is a measure of the efficiency of digestion. A certain quantity of the nitrogen eliminated is in the form of uric acid, creatinin, and other compounds of a like order; but these less oxidized substances, though always present in some degree, are not, in Man and other mammals, the normal end-products of nitrogenous metabolism. Urea is the final and simplest product. It is therefore sufficient to estimate the quantity of urea excreted, and to compare the nitrogen which it contains with the nitrogen ingested in the form of "animal food." About nine-tenths of the nitrogen ingested should be accounted for by urea. When alimentation is excessive or digestion imperfect, the proportion is less than this; some nitrogenous food is not absorbed ; some that is absorbed is imperfectly oxidized.

Urea is characteristically an animal product. Inorganic chemistry deals with stable, organic chemistry with unstable, compounds. Not that there is any boundary between inorganic 
and organic chemistry. They are merely terms which it is convenient to use to indicate the groups of atoms which occupy the chemist's attention at the time. Nor is stability an attribute of certain groups, instability an attribute of others. Stability is relative, not absolute. But admitting these terms as convenient indications of degree, it may be said that inorganic chemistry has to do with such substances as carbonates, nitrates, ammonia ; organic chemistry, with compounds in which carbon is not satisfied with oxygen, as it is in carbonic acid; nitrogen not satisfied with oxygen, as in nitric acid, or with hydrogen, as in ammonia. Carbonic acid (anhye)drid has the formula $\mathrm{CO}_{2}$; ammonia, the formula $\mathrm{NH}_{3}$. Urea is a combination of the two compounds. It is carbonic acid in which one (divalent) atom of oxygen is replaced by two (monovalent) atoms of ammonia. It is ammonia in which two (monovalent) atoms of hydrogen are replaced by one (divalent) atom of carbonic acid.

$\begin{array}{ccc}\text { Carbonic anhydride } & \text { Urea } & \text { Ammonia } \\ \mathrm{CO}_{2} & \mathrm{CO} & \\ & \mathrm{NH}_{2} & \mathrm{NH}_{3}\end{array}$

Urea is an amide-carbonic diamide. It very readily takes water into its molecule, changing into carbonate of ammonia. $\mathrm{N}_{2} \mathrm{H}_{4} \mathrm{CO}+2 \mathrm{H}_{2} \mathrm{O}=\left(\mathrm{NH}_{4}\right)_{2} \mathrm{CO}_{3}$. This change is rapidly brought about by the influence of bacteria in urine exposed to the air.

In thinking of the transformations which proteid substances undergo in the system, it is legitimate to regard their nitrogen as from the first united with hydrogen in the form of ammonia. Not that the grouping is so simple as this. An albumin is not an amide. But in the dance of atoms of its great molecule as it progresses through the system-forming part of the blood, taken up by the cells as floating protein, incorporated in the protoplasm of the cells, shaken into smaller aggregates in the muscles-nitrogen and hydrogen are partners. They leave the body hand in hand. Gusts of oxygen atoms enter through the lungs; use blood-corpuscles as carriages; dismounting, they traverse lymph, forcing their way into the interior of the cells ; they join in the dance. With their strong arms they detach carbon atoms and hydrogen atoms from the huge albumin chain. 
As carbonic acid and water they bear them to the lungs. But nitrogen clings to hydrogen. Oxygen cannot detach its grasp. Out of the molecule of albumin this firmly united couple slips, without contributing anything to the energy which moves the body and keeps it warm. Nitrogen is not a source of energy. It even saves a portion of the hydrogen of albumin from combustion. Urea burnt in a calorimeter has a balance of energy to give up.

Many attempts have been made to ascertain the stages through which proteins pass on their road to urea. The search for intermediate compounds is probably futile, since there is no sufficient reason for supposing that proteins disintegrate in stages, each a step less complex than the food and a step nearer to urea. Every nitrogenous extractive found in the tissues is, of course, on its road to urea. It will be removed as urea, unless indeed, like uric acid or creatinin, it has to be excreted without further change. But it appears to be impossible to discover in the tissues any nitrogenous compounds which occur in sufficient quantity to justify us in regarding them as inevitable halting-places on the downward road (cf. p. 146).

The metabolism of albuminous substances, like other oxidations, takes place chiefly in muscles. Very little is known regarding the nature of the products. Urea is not amongst them. Whatever they may be (cf. p. 267), they are carried to the liver, in which they are turned into urea.

The metabolism of the body is not normally derived from the oxidation of nitrogenous foods. Failing a sufficient supply of other kinds of food, they may be used as sources of energy; but we must picture them as splitting into carbonaceous and nitrogenous portions. If, after the reserve of glycogen in the liver has been brought low by abstention from carbohydrates and fats, nitrogenous food is consumed, and the muscles are then called upon to do severe work, the amount of carbonic acid and water given off rises at once. The excess of urea derived from the nitrogenous food which was destroyed for the purpose of liberating the energy which the muscles expended makes its appearance some time later. If the diet contains a sufficiency of carbohydrates, muscular work does not increase urea. The output of urea is exceedingly steady. It is not increased by muscular work, nor diminished, beyond 
a certain limit, by absence of food. The tissues are constantly throwing off nitrogen-containing molecules, which, if the body is not to waste, must be as constantly renewed.

Uric Acid.-When nitrogenous metabolism has reached the bottom, when albuminous substances have been shaken into the simplest and most stable compound or compounds which the muscles are capable of making (we know not whether the end-products be one or many), they are carried to the liver by the blood. The mammalian liver converts them into urea; the liver of birds and reptiles changes them into uric acid. Uric acid is not, however, completely absent from the urine of carnivorous animals. In Man the amount excreted is about 0.8 gramme per diem, but subject, even in perfect health, to considerable variations $(0.2$ gramme to 1.4 gramme). There is no reason for thinking that uric acid is made in the liver of mammals. On the contrary, it seems to be either an endproduct of the disintegration and oxidation of leucocytes (cf. p. 53), or, like certain other more complex nitrogenous compounds which appear in very small quantities in the urine, the relic of albuminous food which has missed the broad downpath, via muscles and liver, to the kidney. It is a troublesome burden for lymph and blood, and, unfortunately, the kidney finds difficulty in throwing it out. Uric acid has a pernicious way of accumulating in tissue-spaces, producing all the malevolent symptoms of gout. During an acute attack of gout the quantity of uric acid in the system may be largely increased. It may be so abundant in the blood that, when a sample is allowed to cool, uric acid begins almost immediately to crystallize out. Speaking generally, it is right to ascribe gout to an over-production of uric acid ; but it must be remembered that the balance between elimination and production is very delicately adjusted. During an attack of gout the amount excreted in the urine is not increased; frequently it is less than usual. The clearing up of the attack is accompanied by abundant excretion of urates, or lithates ( $\lambda i \theta_{o s}$, stone), as they used to be called, because the "stones" which are found in the bladder consist largely of uric acid. From this it appears that faulty distribution and inadequate excretion have more to do with the development of the symptoms of gout than over-production. In a previous chapter (p. 140) 
we gave as the predominant cause of gout acid fermentations in the stomach. It does not, by any means, follow, however, that we were right in correlating imperfect digestion with an excessive formation of uric acid. It may well be that the gouty symptoms to which hampered peptic digestion gives rise are due in larger measure to a disturbance of the composition of the body-fluids which renders them unfit to carry uric acid to the kidneys in such a form, or in such relation to the fluid in which it is dissolved, as will insure its escape into the urinary tubules. The interference with the efficient working of the system caused by accumulation in it of uric acid gives a particular interest to all that is known regarding the nature and origin of this substance.

Uric acid has the formula $\mathrm{C}_{5} \mathrm{H}_{4} \mathrm{~N}_{4} \mathrm{O}_{3}$. It is a more complicated and a more stable body than urea. The deposits of guano in Peru contain uric acid (the excrement of birds) which has remained practically unchanged for years-for centuries, perhaps. Its chemical nature is not completely understood. It can be readily made to yield urea; and it can be formed by conjugating urea with a nucleus derived from lactic acid (cf. p. 13). Its formula is therefore commonly represented as that of a diureide-a substance containing two urea radicles :

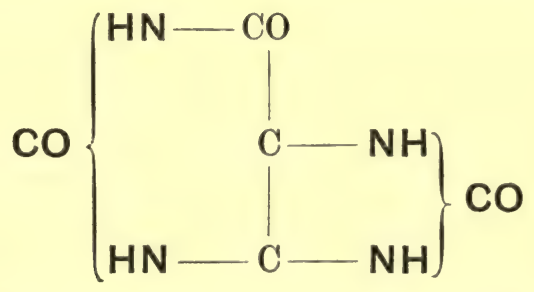

But notwithstanding this inclusion in its molecule of two radicles of urea, it is safe, when one thinks of the contrast between urea and uric acid, to lay stress, in the case of the former, on the binding of nitrogen to hydrogen ; in the case of the latter, on the binding of nitrogen to carbon.

Uric acid is soluble with difficulty; it crystallizes in rhombs. It forms salts, normal and acid. Those which appear in the urine are always acid salts. As a treatment for "stone," lithia water has long had a reputation which it probably deserves, the acid urate of lithium being the most soluble salt 
of uric acid which the kidney can secrete. When uric acid is in excess in urine, brown crystals of uric acid are deposited as "gravel" soon after it is passed. Even when not in excess, uric acid crystals appear after a sufficient time. In other cases uric acid, when in excess, is thrown down in the form of a cloud of acid urates of sodium and other bases, which renders the urine turbid. These urates are redissolved when the water is warmed.

The more fortunate of human beings need never concern themselves with the chemical history of uric acid. It is always present in their body-fluids. It is excreted by the kidney. Its formation is of no greater interest than that of creatinin and other nitrogenous compounds which escape the almost universal reduction to urea. Persons who have a uric acid diathesis are in a very different plight. Every scrap of evidence bearing upon its origin is of supreme importance. Unfortunately, the evidence collected as yet is scanty, and its application for remedial purposes impracticable.

The only disease in which uric acid is invariably in excess is leucocythæmia. This is a condition or habit marked by the presence in the blood of a very great number of white blood-corpuscles and a paucity of red ones. The connection between this disease and the production of uric acid is made plain by certain experiments in diet. If flesh which contains relatively a large proportion of cell-nuclei is eaten, the uric acid excreted is markedly increased. Sweetbread, especially " neck sweetbread "-i.e., thymus gland-is a mass of comparatively small cells with large nuclei. If thymus gland be substituted for all other meaty foods, the quantity of uric acid appearing in the urine is doubled. A large increase in the quantity of ordinary meat or fish consumed also increases uric acid, because all meat-fibres contain nuclei. If egg-albumin be taken instead of meat, uric acid is not increased. A sudden excess of muscular work leads to an increase in uric acid, owing presumably to the unusual activity of the tissues. This used to be very noticeable in the case of young men during the first few days of "training" under the old system ; but it may have been due to the generous consumption of chops and steaks, rather than to the increase in physical work, and consequent destruction of tissue. Nuclei contain nucleo-proteins, which split into 
proteins and nuclein. Chemically, it is reasonable to attribute to nuclein the parentage of uric acid ; a plausible line of descent can be traced. The association of leucocythæmia with the production of uric acid is probably due to the destruction of leucocytes which are present in abnormal numbers (cf. p. 53).

Such is the evidence at present in the hands of physiologists. Naturally, physicians have endeavoured to turn it to account. Patients have been recommended to avoid animal foods which contain nucleo-proteins-to take, instead of meat and fish, eggs, milk, cheese, vegetable-albumins. Certain physicians contend that such a diet is followed by the happiest results; others, equally competent, and perhaps less biassed by "medical theory" - the most dangerous of handicaps for anyone who practises an art which must ever remain empirical-are satisfied that equally good results are obtained by excluding from the diet eggs, milk, and cheese. Physiological discoveries suggest treatment. Modern medicine is in the fullest sense applied physiology. But treatment based upon theory must be controlled by unprejudiced observation. It is possible that the gouty diathesis may be held in check in certain cases by the exclusion from the diet of certain kinds of nitrogenous food. The experience of generations has taught us that the injudicious use of such articles of diet as fruit, pastry, sugar, which do not contain nitrogen, is the main factor in inducing an attack of gout ; that imperfect digestion, sluggish circulation, insufficient activity on the part of the kidneys, lead to the accumulation in tissue-spaces of the fons et origo malorum. Even sweetbread, which with the precision of a chemical experiment increases the production of uric acid by a healthy person, is not necessarily found unwholesome by those who are inclined to gout. It is amongst the most digestible of all meat foods, and easy digestion covers a multitude of metabolic sins. 


\section{CHAPTER IX}

\section{THE CIRCULATION}

THE blood circulates in a closed system of tubes, continuous from the heart back to the heart. The walls of these vessels separate the blood from the tissues. Nowhere, except in the spleen, does it come into contact with any cells other than the lining cells of the vessels in which it flows, and the exception made by the spleen is more apparent than real. The spleen (p. 79) is a kind of sponge invested with a firm capsule. Small arteries discharge their blood into its spaces; small veins collect it. But the organ is essentially a part of the vascular system. Its spaces take the place of the capillary vessels which connect arteries with veins in other situations.

The blood makes a double circuit. From the right heart it passes through the vessels of the lungs. Returning to the left heart, it is driven through the body. Although the heart consists of two separate pumps, it makes but a single organ. Its division into right auricle and ventricle and left auricle and ventricle is but slightly indicated on the surface. In most invertebrate animals the two pumps are distinct. In some the lung-heart and the body-heart are on opposite aspects of the body. But one must not, when thinking of the morphology of the vertebrate heart, picture it as formed by the juxtaposition of two, originally separate, pumps. Truly, in its very earliest stage of growth, it is represented by two tubes which lie, in the embryo, far apart. But these, before we can speak of the existence of a heart, fuse into a single tube, with four contractile bulbs in series. As the heart develops, the dilatation at its hinder or venous end and the dilatation at its anterior or arterial end disappear. A partition is formed which divides the two middle bulbs into right and left auricle and right and left ventricle respectively. Immediately after birth the lungs are, 
for the first time, distended with air. Up to that particular minute they have had no functional use. Nothing would be gained by compelling all the blood of the body to traverse the vessels of the embryo's lungs. Until birth, therefore, the interauricular septum is perforate. The blood takes a short-cut, through the foramen ovale, from right auricle to left. But by birth-time a curtain has grown down on the left side of the

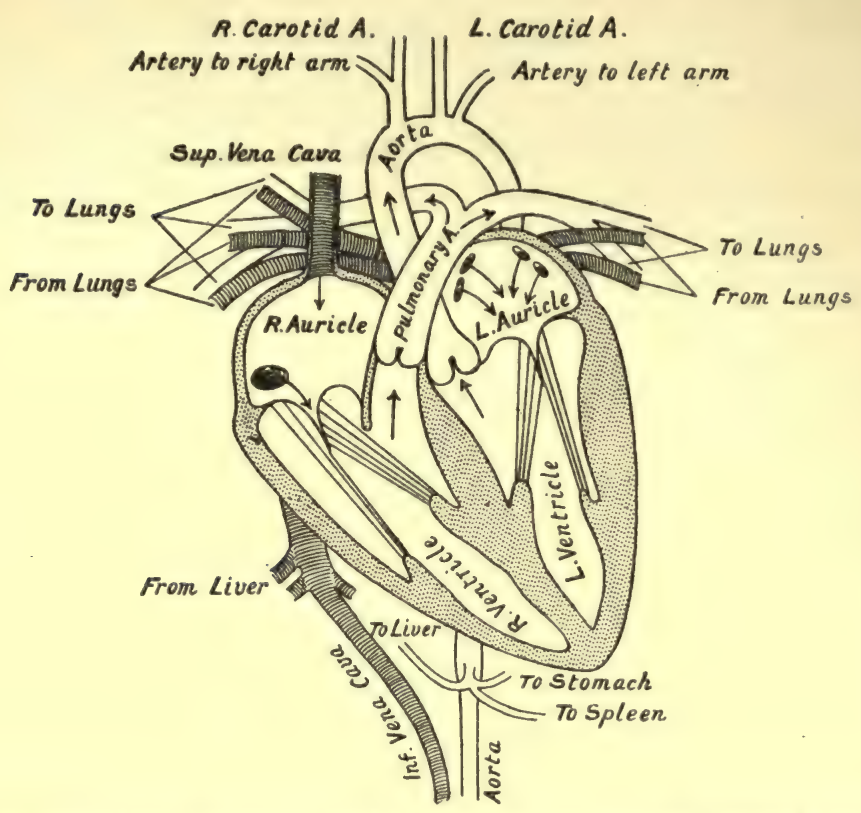

Fig. 10.-The Heart out in the Plane of its Long Axis, and the Vessels whioh open INTO AND OUT OF IT.

Chordæ tendineæ attach the margins of the auriculo-ventricular valves to musculi papillares which project from the inner aspect of each ventricle.

foramen. When the lungs are expanded by the forcible enlargement of the chest-cavity which contains them, their bloodvessels are distended by the same extensile force. Blood is sucked into them from the right side of the heart. A difference in pressure on the two sides is established. A condition is set up which is favourable to what may almost be termed the adherence of the flap which hangs down on the left side of the foramen ovale. The growth of its margin very rapidly obliterates the hole. Occasionally the closure of the foramen 
is not complete. A child grows up with a perforate interauricular septum. If the aperture be very small it causes little inconvenience. Shortness of breath and blueness of lips indicate its existence if it be large enough to lead to deficient aeration of the blood.

The two sides of the heart being quite separate, it is clear that all blood ejected by the right ventricle into the lungs must return to the left auricle, to be driven by it round the body. Yet it does not follow that the heart must at each stroke drive exactly the same quantity of blood into the pulmonary artery and into the aorta. On the average, each of the two sides ejects the same amount-about 3 ounces. Nor does it follow that as much blood is lodged in the lungs as in the whole of the rest of the body. The amount varies, but on the average the lungs contain not more than one-fifteenth of the whole blood. The heart may be likened to two cogwheels; the blood-stream to a chain, folded into a figure eight, against which the cogwheels work. Synchronously each cogwheel lifts a link, the right one of the smaller, the left one of the larger loop. Any given link returns to its starting-place in half a minute. Such an illustration gives an idea of the arrangement of the circulation as a whole, although the motion of a fluid is widely different from the motion of a chain.

If, the jugular vein of the neck being cut, a colouring mattersuch, for example, as ferrocyanide of sodium or methylene blue -is injected into its central end towards the heart, it begins to appear in the blood which issues from its distal end in half a minute. In this short space of time it has passed through the right heart, 'through the lungs, through the left heart, and through the vessels, arteries, capillaries, and veins, of the head. Half a minute is therefore the "circulation time." Not that all the blood-corpuscles of the body make the circuit as rapidly as this. The time taken depends upon the particular route they follow in the greater or systemic circulation. Some traverse the vessels which supply the walls of the heart itselfa short journey; others go down to the foot and up again. But the average circulation time does not exceed a minute or a minute and a half. It is particularly in the veins of the liver and other abdominal viscera that blood tends to linger. Usually half the blood of the body, or even more, is lodged in these 
capacious reservoirs. It is thanks to their capacity for storing blood that a supply is provided adequate to meet any special demand. If a man runs a few hundred yards, two-thirds of the whole blood of the body is transferred to his limbs. It is quickly withdrawn from the abdominal vessels when it is wanted elsewhere; but, failing an efficient cause for removing it, its accumulation induces lethargy. Even tight-lacing has been defended by an eminent physiologist on the ground that it prevents accumulation of blood in the abdomen. But tightlacing diminishes the capacity of the chest, hampers the action of the heart, checks the circulation, distorts the abdominal viscera, and generally deforms and jams the domestic machinery, even though the professor be right in his view that visceral compression may favour alertness of mind. More by token, it interferes with this admirable adjustment by which blood is distributed to the various parts of the body in proportion to their needs. The brain is the only organ which has any difficulty in securing all it wants, and its claim to so much blood might be disputed. Nature has not provided for long-continued passivity of the body associated with strained activity of mind. When the stimulus to mental activity is not unreasonable, most "nervous" people are apt to discover that their brains are better supplied with blood than is good for their health.

The effect upon the distribution of blood throughout the body of squeezing the viscera is experienced after taking a deep breath and contracting the muscles of the abdomen. The contents of the abdomen are compressed between the depressed diaphragm and its muscular wall.

Certain other forces co-operate with the beat of the heart in causing blood to circulate. Two such factors are especially deserving of attention. In the first place, the movement of blood in veins is largely dependent upon external pressure. The veins are valved at frequent intervals, the folds in their interior being of course directed towards the heart. Any external force which empties a section of a vein drives blood forward. A "good stretch" brings the lateral pressure of contracting muscles to bear upon the walls of the veins which lie between them, or beneath. More blood is delivered to the heart. The exercise of standing erect in the attitude of attention, and then slowly raising the arms until the thumbs meet 
above the head, and slowly lowering them again, has a remarkable effect in quickening the circulation-increasing the bloodsupply of the brain. Changes of posture, by relieving pressure on subcutaneous veins, removes an impediment to the flow of blood.

The second of the factors to which we have referred as adjuvant to the heart's action is the negative pressure of inspiration. In explaining the effect of this force upon the circulation, the relation of the lungs to the thorax must be taken into account. The box in which the lungs are enclosed is too big for them; nevertheless, being extensible and elastic, they always fill it. They follow its movements when in inspiration the muscles between the ribs enlarge it, and when in expiration it diminishes again. No air or fluid, save the moisture which lubricates the surface of the pleura, reducing friction, occupies the (potential) space between the lungs and the chest. But the moment the chest is punctured the lungs collapse. Air is sucked into the pleural cavity. The lungs fill the chest only so long as there is neither air nor fluid between it and them. Lung-tissue is extremely delicate. Each air-cell is a cup of thin membrane holding together a basketwork of capillary vessels. So long as the chest-wall is stationary the negative pressure in the pleural cavity has no effect upon these slender tubes. But when the chest expands, the capillaries are between two minus pressures, the pull of the chestwall and the resistance offered to the entrance of air into the lungs by the passages through which it has to pass. The calibre of the lung-capillaries is increased, just as it would be increased were they hanging in an air-pump while the piston was drawn out. More blood passes to the left heart through the wider capillaries. Ejected into the aorta, it raises the pressure in the arterial system. A record of the pressure in an artery shows a rhythmic rise for each heart-beat. It shows also a rise with inspiration and a fall with expiration. These larger undulations correspond with the movements of the chest, although they are necessarily somewhat late on respiration, for the first effect of the dilatation of the capillaries is to cause them to hold more blood and to deliver less. The first effect of expiration, on the other hand, is to urge on the blood which the dilated vessels contain. In any case a single beat is 
needed to throw into the aorta the blood which has been received by the right auricle.

The expansion of the chest influences the flow of blood in yet another way. The heart and the great vessels which join and leave it are themselves enclosed within the chest, subject to the negative pressure produced within that cavity by the elasticity of the lungs. The lungs pull upon the pericardium, the membranous covering of the heart. When this pull is increased owing to the forcible expansion of the chest, blood is sucked into the great veins, just as air is sucked into the windpipe. The thick-walled aorta, containing blood at high pressure, does not feel the effect of slight variations in the pressure round it. The soft-walled veins are expanded during inspiration to a not inconsiderable degree. What relief a deep yawn gives by hastening a languid circulation! Leaning over an account-book late in the afternoon, every condition is unfavourable to the flow of blood. It accumulates in the legs and in the abdomen. The head is thrown back and the mouth opened wide, while the chest expands in a long deep inspiration. Down on the liver, stomach, and intestines presses the flattened diaphragm, squeezing their blood towards the heart. The negative pressure within the chest sucks this up, and draws down the blood contained in the great veins of the neck. The capillaries of the lungs are widened, allowing blood to pass more quickly from the right side to the left side of the heart. The heart responds to the call upon it, throwing all that it receives into the aorta. Only a great effort of the will had kept the pale brain at work ; in the attic it suffers more than organs on the lower storeys from insufficient pressure. For a short time after the yawn it finds itself nourished with an adequate supply of blood.

The negative pressure in the thorax is considerable at all times. If a manometer-a $\mathbf{U}$-shaped tube with mercury in its loop-be connected with a cannula passed through the wall of the chest, the difference of level of the mercury in the two limbs of the $U$ is a measure of the force with which the lungs are endeavouring to shrink away from the chest-wall. Even at the end of expiration the mercury in the limb next the chest stands about 6 millimetres higher than the mercury in the outer limb. During a deep inspiration the pressure in the chest falls 30 millimetres below the atmospheric pressure. Hence a 
problem is presented of which no completely satisfactory solution has yet been given. How comes it that lymph is not sucked into the pleural cavity? In health there is no more pleural fluid than just suffices to keep the membrane moist. The endothelial cells which cover the surface of the pleura resist further exudation. Valves in the lymphatic vessels prevent backward flow. Yet in disease, when the pleura is inflamed, lymph pours out quickly, often to be reabsorbed with equal rapidity when the pleurisy subsides. This flow uphill, from a lower to a higher pressure, can be explained only as a phenomenon due to the "secretory" capacity of endothelium. As an answer to the hydrostatic problem this is hardly satisfactory.

The circulation of the blood is the result of the difference between the pressure in the vessels through which it leaves the heart, and that in the vessels through which it is returned. The pressure in the aorta amounts to about 200 millimetres of mercury. In the venæ cavæ it is nil, or, owing to the aspiration of the thorax, less than nil.

The Heart.-Inspection of the liver, the spleen, or the kidney helps but little to the comprehension of the mechanism of these organs. It is quite otherwise in the case of the heart. Its mechanics being comparatively simple, physiology is concerned with measurements, with the conditions under which it can and cannot work, and with the action upon it of the nervous system and of drugs. The heart of any mammal will suffice for anatomical study. A sheep's heart is about the same size as that of a man, and exactly similar, save in minute particulars, which do not appreciably affect its mode of working.

The heart is a hollow muscle, composed of minute contractile cells. Each cell is a cylinder, about twice as long as it is broad, with an oval nucleus in its centre. There is no impropriety in speaking of the heart as a single muscle. Muscles which we can move at will, " voluntary muscles," consist of fibres, each from 1 inch to 2 inches long, and of about the thickness of a piece of thread (Fig. 16). Every fibre is surrounded by a membranous sheath, its sarcolemma, which completely isolates it from the others. Each has its separate nerve-supply. A voluntary musclefibre is a cell-complex. The single embryonic cell which grew into the fibre underwent nuclear division until hundreds of nuclei were formed, but its cell-substance was not divided into 
territories appertaining to the several nuclei. In heart-muscle, on the other hand, nuclear division has been followed by celldivision; but minute protoplasmic bridges are left between the cells. The whole of the heart-substance is thus in structural continuity. The cells are not invested with sarcolemma. As the result of this arrangement, an impulse started in one part of the heart spreads over the whole, with certain limitations as to the directions in which it is able to travel, whereas in voluntary muscle a separate impulse must be delivered to each fibre. The wave of contraction commences in the great veins, the venæ cavæ and pulmonary veins, near their junction with the heart, spreads from cell to cell throughout the auricles, and onwards down the ventricles to the apex of the heart. The substance of the heart has not, however, a homogeneous appearance. Its cells are collected into fascicles, which lie in various planes and cross the axis of the heart at various angles. In a boiled sheep's heart it is easy to separate one fascicle from another, and to distinguish the sheets into which the fascicles are collected. The four valves of the heart lie in almost the same plane. They are supported by a fibrous plate divided into four rings (Fig. 11). Most of the fascicles are attached to this plate, though some which encircle the auricles are independent of it. With one or with both ends attached to the plate, fascicles loop over the auricles. They run down the ventricles with a twist from right to left. Those on the surface turn in at the apex of the heart, and run up the inner surface of the ventricles. Some of them go to form the free columns which are found on the inner surface of the ventricles, pointing towards the valves-musculi papillares. The fibrous plate which supports the valves cuts off almost all of the muscle which makes the walls of the auricles from that which constitutes the ventricular walls; but a thin sheet is continued from the inner surface of the auricles down the interventricular septum. To a considerable extent the walls of the two auricles and of the two ventricles are respectively continuous, insuring synchronous contraction.

The arrangement of the fascicles accounts for the changes in form which the heart undergoes when it contracts. Systole commences in the cardiac ends of the venæ cavæ and pulmonary veins. They empty the last of their blood into the auricles, and close to prevent regurgitation, their mouths not being 
valved. Then the auricles quickly shrink in all dimensions, and as soon as their contraction is at its height the ventricles contract, while the auricles relax. The ventricular wave runs from base to apex too rapidly to be followed with the eye, and ends, owing to the involution of the fascicles, in the musculi papillares. As soon as ventricular systole has commenced, the auricles relax. After emptying their contents into the aorta and pulmonary artery, the ventricles relax, their contraction giving way first at the apex, and being longest held at the base. Then follows a pause (diastole), during which both auricles and ventricles are flaccid. If the pericardium is open, the heart is

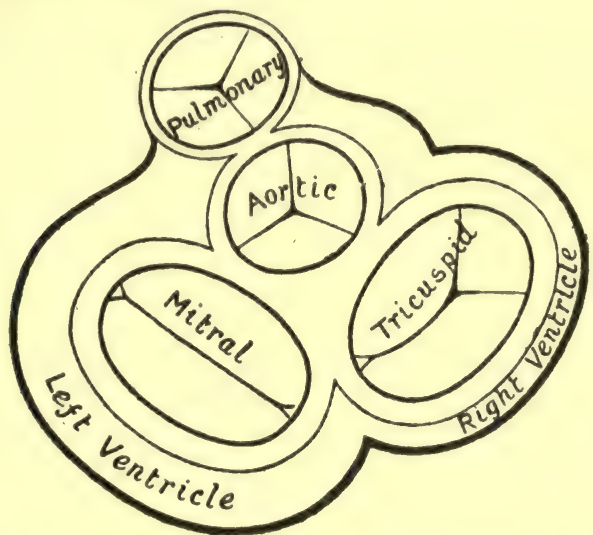

Fig. 11.-A Seotion approximately at Right Angles to the long axis of the Heart, eXPosing the Four Valves whioh lie very nearly in the SAMe Plane.

The semilunar valve which guards the aperture of the pulmonary artery is the nearest to the breast-bone.

seen to become round instead of oval in transverse outline during systole. It shortens. Its apex twists a little to the right, and projects forward. But if it is within its pericardium the shortening is not accompanied with any displacement of the apex. Instead of the apex mounting, the base descends. The front of the right ventricle, at some little distance from the apex, presses the chest-wall forwards in the fifth intercostal space, about an inch to the inner side of a line falling vertically through the nipple. This pressing forwards is felt as the "impulse of the heart."

The contraction of the heart is not a see-saw of auricles and ventricles. During diastole blood is falling from the veins 
through the auricles into the ventricles. In a sense, the auricles are not necessary parts of the double pump. They collect blood while the ventricle is contracting, thus preventing it from heading up in the veins. They save time. Their contraction completes the filling of the ventricle, so that the instant the ventricular contraction begins blood enters the aorta and pulmonary artery.

The Valves.-If ever expressions of admiration were appropriate in a treatise on the animal body, such preface might be permitted to a description of the cardiac valves. Which means no more than this: Men make pumps. Therefore they are in a position to appreciate the mechanism of the heart. We cannot admire what we do not understand. If we made secreting organs or self-contracting springs, glands and muscles would evoke our commendation. We should recognize that Nature's apparatus is even better adapted to its work than any that men can make. This is the admission which is forced from us when we study the heart.

The apertures connecting auricles and ventricles are extremely wide, allowing the contents of the former to be emptied into the latter almost instantaneously. If we attempted to make a pump fulfilling this condition, we should find that it failed in several respects. In the first place, the rush of fluid from the one chamber into the other would press the flaps of the valves back against the wall of the second chamber. They would cling to the wall, and would not float up quickly into place when the second chamber was squeezed. Let us call the two chambers $A$ and $V$ for brevity's sake. When V contracted, some of the fluid would be thrown back into $A$, because, the resistance in that direction being lower than the resistance offered by the column of fluid above the pump (the resistance in the aorta is very high), the contents of $\mathrm{V}$ would rush past the margins of the A-V valve. This would happen even though its flaps were not pressed back against the wall. Further, at the height of contraction the membranous valve would bulge backwards into A, making a cup towards $\mathrm{V}$ which $\mathrm{V}$ could not empty. In the heart these difficulties have been overcome.

The tricuspid valve, which separates the right auricle from the right ventricle, has three flaps. The mitral valve, on the left side of the heart, has but two. The flaps are composed of 
tough membrane, but are comparatively thin. The following direction for deciding at an autopsy whether or not they were healthy at the time of death was given many years ago by a surgeon of repute: "You ought to be able to see the dirt under your thumbnail when you place it beneath one of the flaps." Surgery has improved in cleanliness as well as in other ways; indeed, the possibility of advance has been due to the recognition of the need for transcendental cleanliness. But this is a digression. The margins of the flaps are crenulated. Threadschordæ tendineæ-are attached to them like the stay-ropes of a tent. At their other end these tendons are attached to the musculi papillares already mentioned. The bunch of tendons from each papillary muscle spreads, to be inserted into the contiguous margins of two flaps. We have mentioned some of the difficulties which have been overcome in the construction of the pump. (1) The flaps do not flatten back against the wall of the ventricle during systole of the auricle. It must be remembered that during diastole of both chambers blood is flowing through the auricle into the ventricle. The latter being partly filled before systole of the auricle commences, the flaps are floated up. This is greatly favoured by the form of the inner wall of the ventricle. It is not flat, but raised in pillars - columnæ carneæ. The spaces between these pillars cause backwash currents, which lift the flaps and help to bring them into apposition as soon as systole of the ventricle commences. (2) No blood which has entered the ventricle is thrown back into the auricle. The valve "balloons" over the blood in the ventricle before the contraction of the auricle has ceased. The thin margins of its flaps come together with great rapidity. The tendinous cords holding their edges on the ventricular side, they meet, not edge to edge, but folded flap to folded flap. (3) The valve does not bulge into the auricle. On the contrary, at the height of systole it is pulled into the ventricle by the contracting musculi papillares. As the ring to which the valve is attached is diminished in size, by the contraction of the base of the heart, which continues, it will be remembered, until after the apex has begun to relax, the edges of the flaps are folded farther and still farther over by the pull of the musculi papillares, and the blood is squeezed out from between the wall of the ventricle and the indrawn valve. 
The "semilunar valves," which close the apertures into the aorta and pulmonary artery, have each three flaps. The aortic semilunar valve, which has the higher pressure to bear, shows its characteristic features in a rather more marked degree than the other. Each of its three flaps is a half-cup. At the centre of the margin of the half-cup is a small fibrous nodule. The edge of the cup on either side of this is very thin. Fine elastic fibres radiate from the nodule to all parts of the flap. The wall of the aorta shows three bays, or "sinuses," one behind each flap. Hence, when the valve is forced by the rise of pressure in the ventricle, the flap is not flattened back against the wall of the aorta. There is always a certain amount of backwash in the pocket behind it. The instant the pressure in the ventricle begins to fall, the three flaps come together with a click, so smart as to be plainly audible over most of the front of the chest. The click is the "second sound" of the heart. The auriculo-ventricular valves also make a sound when they close ; but this "first sound of the heart" has a different character. It is prolonged, soft, low-pitched. It is customary to represent the sounds by the syllables "lūbb dŭp-lūbb dŭp," the pause during diastole being of about the same length as the sounds when the heart is beating with its normal rhythm. The duration of systole is little affected by variations in the rate of beat. It is diastole that is shortened or prolonged. The second sound is due entirely to the closure of the semilunar valves. It is heard most clearly when the stethoscope is placed over the region where the aorta comes nearest to the wall of the chest-at the second rib cartilage on the right side of the breast-bone. The first sound is loudest near the apex of the heart. It is generally agreed that it is not wholly due to the closure of the auriculo-ventricular valves, but possesses a second constituent. Some persons assert that they can with the ear distinguish the clearer valvular sound at the commencement from the general rumble which overtakes it. The main part of the sound, if it have two constituents, or the whole sound, if there be no distinguishable valvular constituent-observers differ-is just the noise of a distant cab (bruit du cab) or the waves on a faroff beach; it is the sound which the ear picks up from any irregular mixture of tones which it cannot analyse. It is 
the resonance-tone of the ear. That the membranous valves play the leading part in producing the first sound cannot be doubted, whether by their first closure or by their subsequent vibration. We should be inclined to attribute to them the whole performance, were it not that the first sound, or at any rate a sound, is heard during the beating of a bloodless heart. If an animal be killed and the heart removed from its thorax with the utmost despatch, it will beat for about a minute while lying in the palm of one's hand. When a stethoscope is applied to the ventricle, a "first sound" is heard. This was described as a muscular sound, owing to a misconception. It is similar to the sound which is heard when a stethoscope rests upon a contracting biceps. Until recently the voluntary contraction of a muscle was believed to be vibratory-a tetanus. The sound corresponds to a rate of about thirty-six vibrations to the second. There being reasons for thinking that muscle contracting naturally does not vibrate as fast as this, the sound was interpreted as the first overtone of the musclenote. Muscle was said to vibrate eighteen times a second. The similarity of the first sound of the heart and the ordinary muscle-sound led physiologists to infer that the contraction of the heart also was a tetanus. But this was a mistake. Neither voluntary muscular action nor the contraction of the heart is an interrupted contraction in this sense. In the case of the musculature of the heart especially, contraction is a steady shrinking, followed by a steady relaxation. The sound produced by the bloodless heart is due to the various displacements which occur when it contracts. Its interior is very irregular, with its columns, papillary muscles, tendinous cords, valves. The displacement of these various structures is responsible for the noise.

The sounds of the heart afford to the physician a means of ascertaining with the utmost nicety the condition of the valves. If the sounds are altered from the normal in the least degree, the valves are not healthy. Alteration of the structure of a valve is in ordinary parlance heart-disease. It is usually indicated by an addition to the normal sound. Such addition is termed a " murmur "; in French, un bruit de souffe. Either term is somewhat misleading to the tyro. We remember a fellow-student to whom our chief had in vain expounded the 
nature of a murmur. "Surely, Mr. S., you can hear the murmur in this case." We others could hear it as we stood around the bed. After listening for a minute, S. replied: "I think I could hear it, sir, if the heart wasn't making such a thundering noise." The thundering noise was the murmur. It is the business of the physician to recognize that there is a departure from the normal, to analyse its character, to determine the time at which it is heard in relation to the cardiac cycle, and to locate the place on the chest where it is heard most loudly. He is then in a position to state which of the valves is affected and what is the nature of its lesion. Is it a lesion obstructing an orifice, or is it causing regurgitation of blood? Or is one of the valves, as is commonly the case in heart-disease, imperfect in both respects ?

A murmur, in the strictest sense, is a sound added to a heartsound. It is due in all cases to vibration of a fluid column ("fluid vein" is the term in physics). When fluid passing under pressure along a tube of a certain calibre enters a tube of smaller calibre, no vibration occurs. When it passes from a tube of smaller calibre into a larger tube or space, it is thrown into vibration. Under normal conditions no vibration occurs in the heart. The auriculo-ventricular orifices are so large that auricle and ventricle form a single cavity when the valve is open. The ventricles drive the blood into tubes of smaller dimensions than themselves. These are not the conditions which set up vibration in a fluid column. But if one of the orifices is constricted, owing to thickening or partial adhesion of its valve, the fluid column vibrates on entering the space beyond it. The sound is propagated forwards, beyond the constriction, not behind it, and transmitted to the wall of the ventricle, aorta, or pulmonary artery, as the case may be. When either of the auriculo-ventricular orifices is constricted, the vibration of the fluid column can be felt as well as heard. The finger placed against the chest-wall at the spot where the impulse of the heart occurs is sensible of a thrill. The vibration may occur whilst blood is flowing through an auricle into a ventricle, before the auricle contracts. In time, it is presystolic. The murmur produced by regurgitation into an auricleis synchronous with systole. The murmur due to regurgitation into a ventricle past an incompetent semilunar valve is postsystolic. 
We have said that the heart is so formed that no vibrating fluid vein is produced when it is functioning normally. Murmurs are due to alterations in the valves which are visible after death. This statement needs modification. Not infrequently functional murmurs are heard, which disappear again after a time-in a few weeks, or even days, perhaps. The explanation of murmurs of this class is very difficult. They are heard most frequently in anæmic persons, and appear in these cases to be due to the heart having shrunk, owing to the blood in circulation being deficient in quantity, until the cavities of the ventricles have a smaller diameter than that of the great arteries into which they expel their contents.

Such is the explanation of the physical cause of murmurs given by Chauveau and Marey, the physiologists who have paid most attention to this subject. But it must be remembered that the valves which, when diseased, are the sources of the murmurs are membranous structures. It may be that fluid veins would be produced by them if they were rigid ledges which jutted into the blood-stream ; but, being membranous, they are capable of vibration. Certain physicists are of opinion that a murmur is caused, not by the vibration of a fluid vein, as such, but by the vibration of the membranous structure which impedes the passage of the fluid. The physics of the problem is of little consequence to the physician. The murmur is produced at the spot where a diseased valve is situated, and is propagated forwards. It enables him to ascertain with accuracy what is amiss with the heart.

Bloodvessels.-The greater circulation occurs through a closed system of vessels which unite the left ventricle with the right auricle. The aorta gives off lateral branches. Its branches branch. Subdivision continues until the vessels are just wide enough to allow blood-corpuscles to pass in single file, or but little wider. When a bough of a tree divides, the united cross-sections of its twigs, their soft bark being stripped off, may be a little larger than the cross-section of the bough ; but the disparity is usually small. The united cross-sections of the smaller arteries is considerably greater than that of the trunks which give origin to them. By the time the capillaries are reached, their total bed-their united cross-section-is about 640 times as great as that of the aorta. This estimate is based 
upon the diminution in the rate at which blood flows through the vessels. The velocity with which a stream flows through a channel varies as the cross-section of the channel. In a capillary vessel the blood flows at the rate of from 0.5 millimetre to 1 millimetre per second. In the aorta the velocity is about 320 millimetres per second. In the re-formation of the venous system a converse process of reduction occurs, but not with anything like the same rapidity. The united calibre of the two venæ cavæ, in which the reduction is complete, is about twice that of the aorta. From this it follows that the veins hold much more blood than the arteries; and since veins are more easily distended, the amount that they can hold varies within wide limits. They constitute to some extent a reservoir for blood.

The capillary vessels are the tubes of the circulatory system in which blood comes into use. On the average they are about 0.5 millimetre long. Through them the blood flows slowly. Through their walls alone is there any exchange worth mentioning between the blood within the vascular system and the lymph by which it is surrounded. Interest therefore centres in these vessels. Their walls are formed of endothelial tiles. In the centre of each thin transparent tile is a boss, where its lens-shaped nucleus is situate. The outline of the tile is sinuous. Its margin dovetails with the margins of those adjacent to it. Oxygen and carbonic acid, nutrient substances and waste-products, pass rapidly through the endothelial cells. Leucocytes have the power of pushing the cells aside, in order that they may make their way out of the blood into the lymph which fills the tissue-spaces. With the exception of the lens and cornea of the eye, cartilage, and the various epidermal structures, all tissues are traversed by capillary vessels. It is not difficult to calculate the number of such vessels in the body exclusive of the liver and the lungs. The diameter of the aorta is 28 millimetres, that of a capillary about 0.008 millimetre. The cross-section of all the capillaries added together is 640 times that of the aorta, as already stated.

Many schemata have been devised to illustrate the vascular system ; but all are misleading, inasmuch as they fail to give any idea of the extent to which the subdivision of its vessels is carried. If the water-pipes supplying a town branched 
until the original conduit was represented by five to six thousand million little pipes, the friction which the pumping-station would have to overcome would be very great. But little force would remain in the water when it reached the smallest pipe. Still greater is the resistance to the flow of blood, which is slightly viscous, and contains solid corpuscles, which increase friction. Two thousand miles of capillary tubing in the body of a man, without reckoning the vessels of his liver and lungs !

Water is supplied to houses in rigid tubes. Arteries are elastic, and their elasticity is self-regulating. The cause of this will be apparent if a section of an artery is examined. It

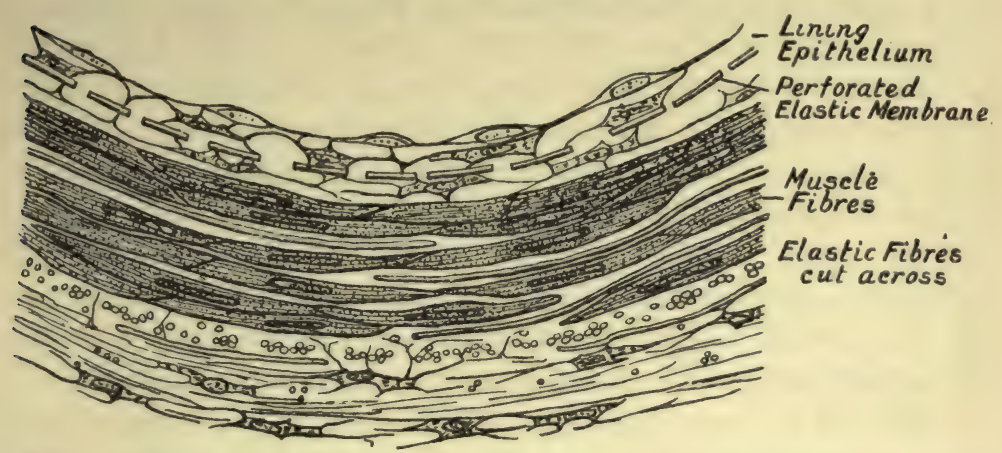

Fig. 12.-A Portion of the WaLl of a Small ARtery odt transversely aNd highly MAGNIFIED.

Its inner coat consists of a lining sheet of epithelial scales supported by connective tissue and a strong elastic membrane. This membrane is perforated with holes which place the lymph-spaces on its two sides in continuity. The middle coat is composed of plain muscle fibres and patches of elastic membrane ; the outer coat of elastic fibres, mostly longitudinal, and connective tissue.

contains much elastic tissue. It also contains plain musclefibres. The smaller the artery, the greater is the amount of muscle relatively to the other constituents of its wall. The wall of a vein contains very little muscle, and not much elastic tissue. The muscle of all arterial walls is in a chronic state of tone. To some extent the degree of tone is varied automatically. Pressure within an artery acts as a stimulus to the musclefibres of its wall. Any increase leads the fibres to contract more strongly. Any diminution induces them to relax. The arteries resist distension; they do not narrow to any great extent when pressure falls. But more important than this automatic mechanism for maintaining a uniform pressure in 
the capillaries in general are the changes of pressure in particular localities, brought about by the mediation of vasoconstrictor and vaso-dilator nerves. In almost all organs and parts of the body the automatic tone of arteries is enhanced by impulses which flow continuously down vaso-constrictor nerves. These impulses start from, or, to speak more accurately, pass through, the vaso-motor centre in the medulla oblongata. From every part of the body impulses ascend to this centre, urging it to keep up the blood-pressure by universal constriction. Yet no separate organ would be interested in sending such a message if it were not open to it to ask at the same time that the constriction of its own vessels might be relaxed. Hence it may be said that every individual in the community is crying out for universal economy, with more generous treatment of himself. The response made by the State to the latter part of his demand is in proportion to the vehemence with which it is presented.

If the spinal cord of an animal be cut across near the medulla oblongata, respiration being maintained by pumping air into and out of the lungs, the heart continues to beat with undiminished force, but the pressure in the large arteries falls to one-third of its normal height. Constricting impulses no longer pass down the spinal cord from the vaso-motor centre. This experiment also illustrates the truth of the statement that models of the vascular system-arrangements of pumps and indiarubber tubes-are more likely to mislead than to inform. In an artificial schema the relaxation of the constriction of the small tubes on the proximal side of the capillary vessels would reduce friction. Fluid would reach the capillaries in larger quantity, and pass through them more quickly. The pressure in the tubes which represented veins would consequently approach more nearly to that on the arterial side. But when the spinal cord is divided the pressure falls in the veins, as well as in the arteries. This is due to another factor, and one of very great importance in the regulation of the circulation. The blood from the digestive organs is collected by the "portal system " of veins. These do not join the inferior vena cava ; they go to the liver, where they again break up into capillaries. It is not until after this second distribution through minute vessels that the blood is re-collected by the hepatic veins and 
forwarded to the heart. As in the case of the arteries, the portal system of vessels is controlled by the nervous system. When the spinal cord is divided they also dilate. The whole vascular system becoming more capacious, blood-pressure falls in veins as well as in arteries.

When the digestive organs are active, other parts of the body are kept short of blood. It chanced to the writer, in his student days, to spend the early summer in Paris, with a big healthy Yorkshireman as companion. We dined together each night at one of the restaurants of the Palais Royal à prix fixe. After dinner, with British regularity, my friend called for the Times. Then followed a short period of placid reading, interrupted by the remark: "How cold it is!" Half an hour later, giving himself a shake: "Suppose we go and dine somewhere else?" His well-ordered digestive organs had made short work of the two-frane dinner. They had been ably supported by the vasomotor system of nerves which provided them with the bulk of the blood, while limbs and skin ran short.

Vaso-constrictor nerves leave the spinal cord by the roots (called "rami communicantes") of sympathetic ganglia. Beyond the ganglia they apply themselves to the large arteries whose course they follow. The constrictor nerves for the face and neck leave the spinal cord within the chest by the roots of the first four thoracic nerves. They do not at once apply themselves to the great artery of the head. Until the upper part of the neck is reached, they traverse the ganglionated sympathetic cord, which lies behind the carotid artery and internal jugular vein. If in a rabbit this cord be cut, the vessels of its ear dilate, as evidenced by the rosy blush which is observed when a light is held behind it. If the upper part of the sympathetic cord be stimulated, the ear grows pale. The redness of the ear remains for many days after section of the nerve; but gradually the engorgement diminishes, and the vessels acquire the power of automatically regulating the flow.

The classical experiment with the rabbit's ear suffices to show the relation of bloodvessels and nerves which holds good for all areas of the skin. The condition of the skin is the chief factor in regulating the temperature of the body. In a cold atmosphere its vessels are severely constricted to limit loss of heat. When one passes into a warm room the constriction is 
relaxed. The skin is flushed; heat is thrown off by radiation. The sweat-glands secrete water, which is evaporated by the heat of the skin. Constriction and remission of constriction are the processes which diminish or increase loss of heat.

This mechanism is different in the case of glands and some other structures which, when active, require an abundant supply of blood. Such organs are provided with vaso-dilator in addition to vaso-constrictor nerves. The most conspicuous example of this is to be seen in the case of the submaxillary gland. The nerve to this gland runs for some distance as an isolated thread-the chorda tympani. Stimulation of the chorda tympani has the double effect of dilating the arteries of the gland and of causing it to secrete. But the administration of atropin prevents secretion. Vaso-dilation is then the only visible effect. Stimulation may increase sixfold the outflow of blood from the veins of the gland. It rushes through with such rapidity that it retains its bright arterial hue. The gland also receives a twig from the sympathetic cord in the neck, which, as already stated, controls the vessels of the face. By stimulating the one nerve or the other the physiologist can at will increase or diminish the amount of blood flowing through the submaxillary gland. Stimulating any sensory nerve causes in a reflex manner an increased outflow of constrictor impulses from the centre in the medulla oblongata to all parts of the body, with the exception of the part to which the sensory nerve appertains. Its own constituency receives an increased supply of blood. It is not difficult to appreciate the importance of this double action. A part is injured. The restrictions placed upon its supply of blood are suspended. Lest its increased consumption should lead to a general fall in pressure, all other parts have their supply curtailed. The effect is even more pronounced than this. The whole blood-pressure is raised above its ordinary level. The flow of blood to the injured part is therefore greater than it would be were relaxation of its arteries the only change.

The most important of all constrictor nerves are the splanchnics which control the supply to the stomach and intestines. When these nerves are cui, the digestive organs become engorged to such an extent that a pronounced fall of the general blood-pressure is the result. Their stimulation renders the digestive organs anæmic. We have already shown 
that the relaxation of vaso-constriction occurs in a reflex manner. The reflex relaxation of the splanchnic area is a matter of great importance, because it can be brought about by stimulation of one of the sensory nerves of the heart. The higher the blood-pressure, the harder the heart would work if left to itself. It is an impetuous organ, always trying to quicken its pace and to increase the force of its beat. Excessive zeal would get it into trouble if severe precautions were not taken to hold it in check. True, it is encouraged by certain "accelerator nerves" - sympathetic filaments which leave the spinal cord by the anterior roots of the second and third thoracic nerves; but the influence which the accelerators exert under normal conditions is not, it would seem, very pronounced. The nerves which restrain the heart are much more in evidence than those which urge it on. The arrangements for diminishing the work of the heart are of two kinds. In the first place, branches derived from the vagus act as a continuous check. From a certain spot in the medulla oblongata, the cardio-inhibitory centre, impulses are always descending to slow the heart. They are of reflex origin, but a high blood-pressure in the centre increases the facility with which they are transmitted. Some of these stimuli originate in the heart itself, ascending and descending the vagus nerve. The remainder come from various sources. A severe injury to any part of the body slows the heart. Injury to the intestines, such as occurs in peritonitis, is particularly effective in increasing vagus inhibition. Slowing of the heart lowers blood-pressure. When both vagi are cut, the heart begins to gallop whatever may be the pressure against which it has to work.

A sensory nerve of the heart, termed the " depressor," is the chief agent in lowering blood-pressure. Its course is not the same in all animals, but it runs more or less in conjunction with the vagus. Usually it joins its superior laryngeal branch. Impulses which ascend this nerve inhibit the constriction of the splanchnic vessels. They open a floodgate which brings down the general pressure. The severe pain and extreme distress of angina pectoris are the cry of the heart when blood-pressure is too high-when it feels unable to work against it. This was recognized by physiologists long before a remedy was known. A systematic search was instituted for a drug which could be 
used with safety to lower blood-pressure. The discovery that the inhalation of amyl nitrite answers this purpose and fulfils this condition was the result.

When we consider the hydrostatics of the circulation, it becomes evident that changes in the force with which the heart beats, and changes in the calibre of the bloodvessels, work together in determining blood-pressure. Both vessels and

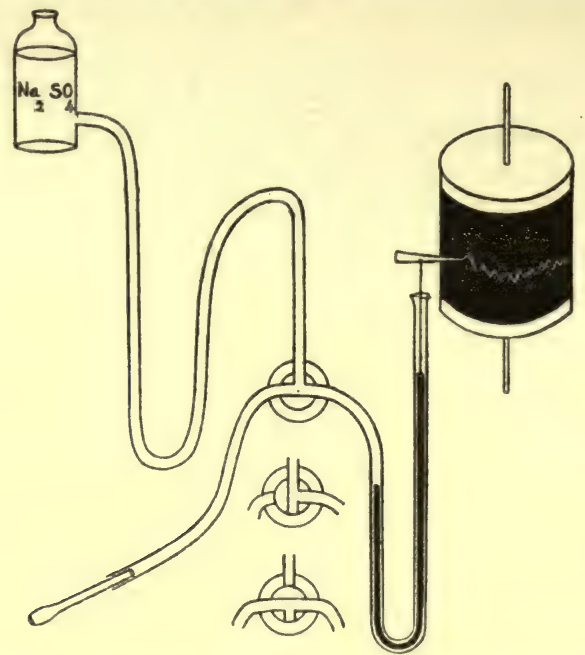

Fig. 13.-Manometer for measuring BLOOD-PREssure.

A U-tube contains mercury, on which floats a rod supporting a scratching point, which makes a "tracing " on blackened paper wrapped round a revolving drum. Between the manometer and the cannula which is introduced into the central end of a cut artery is a threeway cock, which leads to a pressure-bottle containing a half-saturated solution of sodic sulphate. This solution prevents blood from clotting. Before it is connected with the artery the apparatus is filled from the pressure-bottle. The cock is then turned into the second position, and the bottle raised until the mercury in the manometer stands at a level somewhat higher than that which it may be expected to attain under the influence of blood-pressure. The cannula being then inserted into an artery, the cock is turned into the third position, which places the manometer in connection with the blood, and excludes the pressure-bottle. As the mercury is a little higher than blood-pressure, some of the sodic sulphate solution enters the artery, but no blood enters the cannula. The scratchingpoint, rising and falling with every variation in blood-pressure, makes a record on the sootblackened paper, which is subsequently removed from the drum, and varnished.

heart contract automatically-the former continuously, the latter rhythmically. The heart of a frog, if it is enclosed in a moist chamber, beats for a long time after its removal from the animal. Even when cut in pieces, in certain ways, the separate pieces beat. A strip from the ventricle of a tortoise's heart, kept gently stretched by the weight of a light lever attached to one of its ends, continued to contract rhythmically 
for forty-eight hours. When the heart has come to a pause, it cannot be started again by stimulating any nerve. It has in the most marked degree its own views as to the rapidity and force with which it ought to beat. But within certain limits it is under nervous control. The accelerators hasten it, to its own detriment. They belong to the division of katabolic nerves-a name given them to indicate that they waste the tissues, impoverishing their condition. The vagus nerve slows the heart. It protects it from itself. Its action is anabolic. The condition of the heart is improved under its influence. If it has been kept in check for a time by stimulation of the vagus, the heart beats more strongly when this nerve ceases to act than it did before it was induced to rest.

The arteries also are under the influence of two antagonistic sets of nerves. Those which increase their tonic contraction are almost universal in their distribution. It may be that those which actively check it are equally widespread, but the evidence is not altogether free from ambiguity. On certain organs - such as the salivary glands, already instancedwhich require great variations in the amount of blood supplied to them, the influence of dilator nerves is very marked. The simplest hypothesis as to the mode of action of vaso-constrictor and vaso-dilator nerves leaves the initiative with the musclefibres of the vessel-wall. The distending internal pressure of blood is the stimulus which induces the muscle to contract. In some invertebrate animals-the snail, for example-if blood be prevented from entering the heart, so that there is no distending pressure, the heart stops. In higher animals the heart has acquired a habit of contracting, which keeps it going in the absence of its proper stimulus. The two classes of nerves exercise opposing influences on the muscle. Vasoconstrictor nerves increase the excitability of its fibres ; vasodilator nerves diminish it. Only thus can we explain their action on a common basis. A good deal might be said as to the reasonableness of such an explanation. Our views as to the relation of nerve-influence and muscle-contraction are apt to go astray, owing to the fact that generations of physiologists have observed the phenomenon of a spasm of a muscle following on a sudden stimulus to a nerve. The two events are evidently related. The stimulus appears to set up a new condition in 
the nerve-to initiate a process which was not occurring before the electric current was passed through it. The muscular spasm equally appears to be an isolated event. As usual, we are misled by the analogy of human inventions. We compare the nerve-impulse to the fall of a hammer, the muscle-spasm to the explosion of gunpowder. We forget that nerve and muscle are in permanent connection; that the impulse is a sudden exaggeration of an influence which the nerve is continuously exerting, the contraction an exaggeration of metabolic changes which are constantly occurring in muscle. (See in this connection the explanation of muscle-tone, p. 273.) In the case of plain muscle, nerve stimuli do not cause contraction ; they merely increase the excitability of the muscle. It may be more difficult for us to figure to ourselves the way in which dilator nerves diminish excitability; but the existence of such an anabolic influence is beyond the reach of doubt. Heart and bloodvessels are part of the same system. The heart has its accelerator and inhibitory nerves, the bloodvessels their constrictor and dilator nerves. For both vessel-wall and heart the stimulus to contraction is the distending pressure of blood - although it is not altogether necessary that this stimulus should be acting at the time. Sympathetic and vagus nerves can to a certain extent control the beating of a bloodless heart. The heart-tissue has acquired the habit of beating, and the habit of listening to advice conveyed to it through these nerves.

The self-adjustment of the blood-tubes to the pressure to which they are exposed is exhibited in the adaptation of their degree of contraction to the position of the body-to the weight, that is to say, of the column of fluid which they have to support. Everyone has played the game of "right hand or left." When the hand is held above the head the blood leaves it, and the hand becomes cold; but if there be need for adjustment, and time is given for the mechanism to come into play, it works to perfection. When we are standing erect, there is neither too much blood in the feet nor too little in the head. But after a fortnight in bed a convalescent finds, the first time that he stands upright, that his legs are quickly engorged-his slippers after a few minutes feel too tight for him-whereas the brain becomes so anæmic that he turns giddy, or even faints. Numberless illustrations of vaso-motor action are met with 
in daily experience. It is a curious fact that the nerves which control the calibre of the bloodvessels tend to overact their part. When an organ demands more blood, it is supplied at the expense of the rest of the body, and especially of the parts most nearly adjacent. This is partly a mechanical effect. If all the houses in a terrace are supplied with water from a common main, the bursting of a water-pipe in one of them will reduce the supply of its neighbours more than it will reduce the supply of houses in distant parts of the town. But vaso-motor nerves, in their compensating adjustment, go farther than this. A thimbleful of blood removed by a leech produces an effect upon an underlying engorged organ altogether out of proportion to the hydrostatic requirements of the case. "Cupping" the loins diminishes the congestion of the kidneys. This is the explanation of the curative efficacy of various agents which, with improvements in surgery and the introduction of more reliable drugs, have almost disappeared from the surgeon's armamentarium - scarification, blisters, setons, and the like. Such methods have been relegated to veterinary practice.

There is a marked tendency to see-saw between the skin and the mucous membrane of the alimentary canal. During active digestion, when the "splanchnic area" is full of blood, the skin is cold. Hot fomentations, by dilating the vessels of the skin, diminish congestion of the alimentary tract. An inflamed throat is relieved by a compress round the neck. Conversely, it must be admitted that, in certain persons, slight constriction of the vessels of the skininduces inflammation of the mucous membrane. This is one reason for the almost universal dread of draughts. A draught cools a limited area of the skin. Some of us cultivate a love of draughts. They are the sensible evidence of the entrance of fresh air. Yet we admit reluctantly that certain fragile mortals are not altogether fanciful in supposing that a draught may give them a catarrh or a toothache. If asked why they object to draughts, many persons answer that they "are afraid of catching a chill "-carrying us back to the time before the clinical thermometer was invented; to days when the shivering fit, or "rigor," which first calls attention to the fact that the temperature is already two or three degrees above the normal, was supposed to be the com- 
mencement of the illness. The patient imagined that the " chill" caused him to shiver, and that if he had not "caught" it he would not have been ill. The substitution of the term "cold" for " rheum," naming the malady after one of its prominent symptoms, has done much to perpetuate this superstition. "Chill" is a word we scarcely dare to mention. When doctors could no longer attribute to witchcraft the occurrence of disorders for which they had no other explanation, they invented the luminous theory that inflammatory diseases-especially those of the stomach, liver, and lungswere produced by "a chill." At one time all diseases which were not evidently infectious were caused by chill. The discovery of germs and the recognition of their maleficent activity has stripped this cloak of ignorance off almost every case of abnormal tissue-metabolism. It is recognized now that the germ is the disease, not the effects which the germ produces. Pneumonia is impossible in the absence of the pneumococcus, however severe the chill to which the patient was exposed when out in the cold and wet. Consumption is the effect produced by the tubercle bacillus. If there are no bacilli, there can be no consumption. Yet these two diseases illustrate the possibility of the use of the term "chill" without impropriety. The coccus of pneumonia may frequently be found in the mouth of a healthy person. If everyone with whom the tubercle bacillus has at some time come in contact were inevitably its victim, no human being would be free from phthisis, if any still survived. There are conditions of health, or rather of unhealth, in which the economy is less resistant than usual to the germs. Apparently the vaso-motor disturbances of internal organs caused by the cooling of the surface of the body, if it occur when health is otherwise depressed, contributes to the production of such a state.

The vaso-motor system is influenced by emotions. It is a little difficult to express accurately the relation between emotion and vaso-motor change. Some psychologists regard the vaso-motor change as the emotion. "All emotions," says a prominent exponent of this view, " are wholly due to excitation of a particular kind of the vaso-motor centre." The person about to be subject to an emotion of shame, anger, fear, disgust, recognizes a fact or circumstance, or conjunction of circum- 
stances, which justifies the emotion. (We are assuming that emotions may be justified; that the intellectual appreciation of a situation and reasoned decision regarding the action which it demands is not sufficient.) This recognition as an intellectual act of the higher brain is accompanied by certain forms of enhanced activity or inhibition of activity of the vaso-motor centre in tho medulla oblongata which cause changes in the degree of contraction of the bloodvessels of certain organs. The vascular changes produce an alteration in the state of the organ which is reflected in nerve-currents sent back to the brain, providing the background of feeling which constitutes emotional tone. We are not prepared to endorse this extreme view of the nature of an emotion. A maiden's blush is not an emotion of embarrassment or shame. It is its harmony. Her mind plays the air. The sensations which originate in the flushed skin of the face sustain it with their accompaniment. The emotional tone keeps attention fixed on the fact or circumstance which led her to conclude, by the exercise of her reason, that she was placed in an awkward situation. This fixing of attention is frequently so pronounced as to inhibit all other intellectual action. The maiden is less quick than she would have been, had the emotion not glued her thoughts together, in recognizing the readiest means of extricating herself from embarrassment. All nerves found within the chest and abdomen were in very early times termed "sympathetic." The cord in the neck was the "little sympathetic." The name explains itself; but it will be understood that it implied much more in the days when the liver, spleen and heart were supposed to pour out emotions than it does now. The vagus nerve was termed the " middle sympathetic." Shame inhibits the activity of the vaso-constrictor nerves of the face; dilation of the vessels which they supply is accompanied with constriction of other cutaneous nerves. Kipling must, we think, have embellished Nature when he represents the very unimpressionable hero of Lungtungpen as admitting "I niver blushed before or since; but I blushed all over my carkiss thin." Usually the carmine of the face contrasts with the pallor and coldness of the hands. Still, we are not prepared to assert that it is impossible, under circumstances as trying as those in which Private Mulvaney and his companions were placed, for all the 
cutaneous constrictor nerves to let go their grip at the same time. Terror heightens the control of the vaso-motor centre over the vessels of the skin; it increases vagus inhibition of the heart. Even disgust evoked by a revolting sight or a foul smell may call the vagus so forcibly into action as to bring the heart to a standstill.

The Pulse.-The arterial system is always distended. The pressure in the largest arteries amounts to about 140 millimetres of mercury. The source of pressure is the beat of the heart pushing the blood forward against the resistance offered to its flow by the smallest vessels. At every stroke another 3 ounces is added to the already overfull vessels. In the aorta,

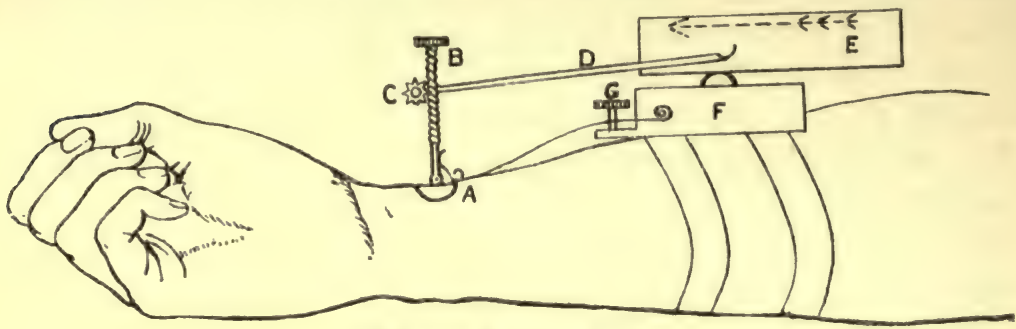

FIG. 14.-SPHYGMOGRAPH.

A, An ivory button which is pressed on the skin over the radial artery by a metal spring. B, A continuous screw which works against the cog-wheel $\mathbf{C}$. By rotating $\mathbf{B}$, the lever $\mathbf{D}$ is raised to a position in which its point scratches the travelling-plate $\mathbf{E}$ (covered with blackened paper). F, A box containing clockwork which moves E. G, A screw by means of which the pressure of the spring is adjusted to the force of the pulse.

therefore, the blood moves forward with jerks, but by the time it reaches the capillaries the intermittent accessions of force have been taken up by the elastic walls of the vessels and returned to the stream in the form of constant pressure. In the very smallest arteries the blood flows in a steady stream. If the corpuscles in a capillary vessel are watched under the microscope, they show no variations in rapidity synchronous with the beat of the heart. The "pulse" in the larger arteries is the push given to the column of blood by the sudden contraction of the left ventricle. Its propagation along the arteries will be understood if it is remembered that the blood is contained within elastic tubes. The first effect of the ejection into the aorta of an additional quantity of blood is the distension of its wall. The wave of distension travels down all the arteries of the body with gradually decreasing force. 


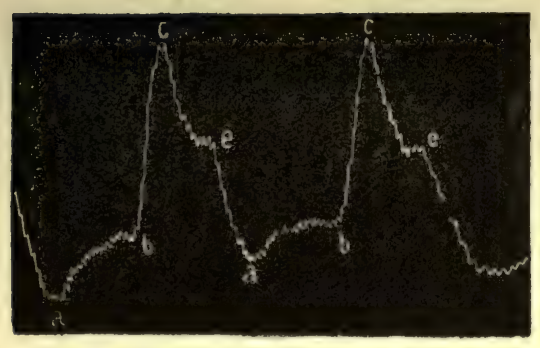

A

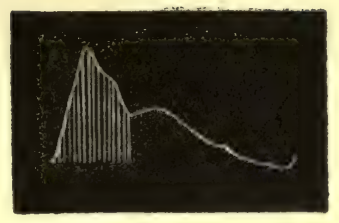

B

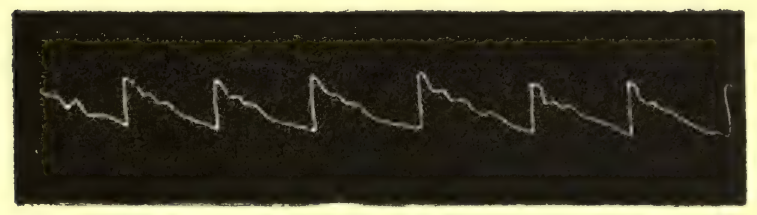

C

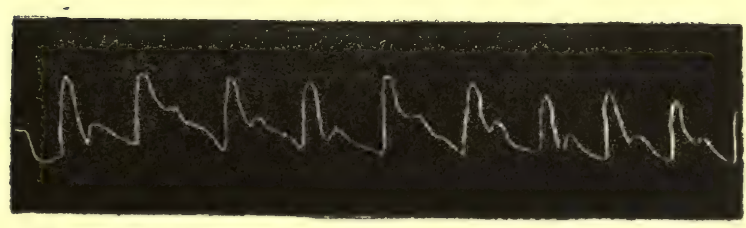

$\mathrm{D}$

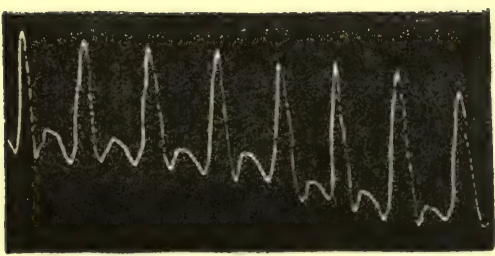

E

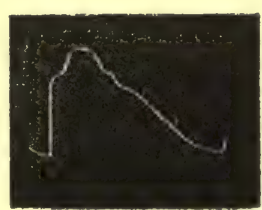

F

FIG. 15.

A, A cardiogram, or tracing of the impulse of the heart, recorded on a blackened plate borne on the end of a vibrating tuning-fork; $a-b$, the systole of the auricles; $b-e$, the ventricular systole. From $c-e$ the heart is shrinking as blood leaves it by the aorta and pulmonary artery. B, C, D, E, F, Sphygmograms. B is shaded to show the portion of the pulsewave which corresponds to the systole of the heart. C, High-tension pulse of vigorous health. D, Low-tension pulse. E, Dicrotic pulse of fever. F, Hog-backed pulse of hardened (atheromatous) arteries. 
Much may be learned from the pulse with regard to the condition of the vascular system, although it is impossible to balance the effects of the several factors which go to the production of its various modifications. The character of the pulse depends upon the vigour with which the heart is beating, the efficiency or otherwise of the cardiac valves, the quantity of blood in circulation, the suppleness of the arterial walls, the degree to which they are contracted, the resistance offered by the smaller vessels. Departures from the normal may take the direction of unduly high tension or of unduly low tension. In place of the sudden rise and more or less gradual fall, with the slightest possible roughness due to secondary waves, which constitutes a healthy pulse, the rise may be shorter, its subsidence prolonged. This is a high-tension or hard pulse. The pressure in the arteries is unduly high, or the walls of the vessels are not as elastic as they should be. Considerable pressure is needed to obliterate such a pulse-i.e., to prevent it from passing on beneath the finger. As the converse of this condition, the difference between the beginning of the pulse and its end may be very marked, the vessel suddenly dilating and as suddenly collapsing. But little pressure is needed to stop such a low-tension pulse from passing beneath the finger. Usually it has a distinct secondary or dicrotic wave. Some tactile education is needed by the finger that aspires to read the pulse. It was hoped that the personal equation would be of less importance if mechanical records were substituted for statements as to the impression produced upon the observer. Various forms of sphygmograph ( $\sigma \phi v \gamma \mu \rho^{\prime}$, pulse) have been invented for this purpose. The form commonly used (Fig. 14) consists of a metal spring which is adjusted so that a button beneath its free end presses on the radial artery at the wrist. The force with which it presses is regulated by a screw. At each pulsation its free end is lifted by the distension and rounding of the artery. Its movement is transmitted by means of a continuous screw, attached to it vertically, to a cogged wheel, which in its turn raises a lever. The end of the lever scratches blackened paper fastened on a plate moved by clockwork. Records made in this way are useful for future reference. They are not, however, so valuable as it was anticipated that they would be. The form of the tracing 
depends to so large an extent upon the amount of pressure exerted by the spring, and the amount of pressure must be adapted to the vascular tone in every case. Some of the most interesting tracings are obtained from old people affected with atheroma of the arteries. This is a condition in which, owing to old-standing inflammation of the subepithelial coat of the vessels, the arteries have lost their suppleness. They are hard and inelastic. Instead of showing the normal steep face of the pulse-wave rising abruptly to its highest point, the tracing rises vertically for a short distance, and then slopes upwards. The wave is flat-topped or hog-backed.

All pulses are dicrotic, although the dicrotism may not be sufficiently pronounced to be felt with the finger. The notch which divides the primary from the secondary wave is produced by the closure - that is to say, by the falling down of the aortic valve. The wave from the commencement of its ascent to the dicrotic notch corresponds to the period during which blood is passing from the heart into the aorta. This part of the tracing represents systole of the ventricle after the semilunar valve has been forced. It is the push given to the bottom of the column by the additional 3 ounces of blood thrust into the aorta. The effort of the ventricle then comes to an end. The pressure beneath the semilunar valve is less than that above it. The valve closes. If the blood were contained in an open tube, the wave would now end, save for secondary oscillations, due to inertia of the fluid. But the arterial system is practically closed owing to the fineness of the tubes into which it ultimately divides. Its walls are elastic. They distend, taking up the pressure and returning it again in the second half of the wave. In fever, after the consumption of alcohol, and in other conditions in which the finest bloodvessels are dilated, the division between the two parts of the wave is very marked. Dicrotism is plainly felt. We have used the expression " finest vessels " rather than " capillaries," because the ascription to the capillary vessels of all peripheral resistance has led to misunderstanding. Resistance is offered throughout the whole vascular system, with the exception of the largest veins. It is greatest in the small arteries, capillaries, and small veins. It is so adjusted as to fall to zero just before the blood reaches the heart. 


\section{CHAPTER $\mathrm{X}$}

\section{MUSCLE}

Living matter, protoplasm, is irritable. It responds to influences impressed upon it by its environment. An effective influence, termed a "stimulus," produces a change in protoplasm at the spot at which it acts. From this spot the change spreads outwards as an "impulse." Protoplasm is said to "conduct." A stimulus may be likened to a blow given to a fixed but elastic mass; an impulse to the vibration which travels outwards from the spot struck. Unfortunately, the term "stimulus" is used both for the stick that strikes, the stimulator, or stimulant, and for the blow that is struck; but breaches of logic seldom lead to confusion in an experimental science. The context indicates the particular application of the term. The manifestation of stimulation is a physical or chemical change-most obvious when it is one of form. This change of form may occur at the spot stimulated, or may be deferred to a distant part to which the impulse is conducted.

In opening the study of muscle and nerve we need to form a conception of the nature of these three functions-irritability, conductivity, and changeableness of form. Not that the functions are as distinct as the ideas to which the three terms give rise. They are three aspects of a common function; although this is a reflection which will carry more weight when the ways in which protoplasm reacts to external forces have been considered.

A stimulus may be mechanical, something in the nature of a blow which displaces the particles of protoplasm ; or it may be chemical or thermal, disintegrating a portion of its substance ; or electrical, divorcing the ions of its molecules. Only the last in any way resembles a natural stimulus; since elec- 
trical stimulation alone can be repeated without the substance stimulated showing any evidence of injury in the process. Mechanioal, thermal, chemical stimuli destroy a portion of the protoplasm upon which they act. Yet even the weakest of electric currents is a gross disturbance as compared with natural stimuli, such as touch, warmth, sound, light. The essential and most distinguishing quality of living matter is its return to its original state immediately after stimulation. It does not even wait until the stimulator has ceased to act. An effective influence is a sudden change in the environment. It is answered by a sudden response, followed by a return of protoplasm to the state in which it was before the impact of the external force. The change progresses through the protoplasm as a transitory alteration of state, the perticles concerned in conducting it returning to their original condition the moment it has passed. No non-living matter responds to force in this way. If a stone is dropped into a pond, a wave circles outwards from the spot it strikes; but this is a wave of displacement, not a change of state. Suppose the pond contained a solution of sugar which the impact of the stone changed into vinegar, and that the zone of vinegar spread outwards, the liquor returning to the condition of sugar and water as it passed. Here we should see some analogy to the progress of an impulse. But no non-living matter behaves like this. A product of the laboratory may be so unstable as to explode when shaken, passing on the slightest provocation into a more stable state. It does not return after the explosion to its previous strained condition. Having thrown away its energy, it continues on a lower plane. Protoplasm parts with energy to recover it again. It returns to instability after assuming a more stable form.

If we are to form a conception of the cause of the irritability of living matter, we must have a mental picture of the physical conditions which distinguish life from death. All matter is in a state of motion. It consists of separate molecules, each moving in its orbit with vast rapidity. A molecule is a cluster of atoms. The dimensions of its orbit depend upon the number and weight of the atoms in its cluster. If we could watch the dance of the molecules of proteins and other substances into which protoplasm breaks up on dying, we should see each 
separate cluster executing the figure appropriate to its mass, indifferent to the movements of neighbouring groups. But if living protoplasm were of the company, the scene would be one of vastly greater animation; for now it is the ambition of our dancers to form a single group. To this they can never attain. There is a physical limit to the number of dancers who can hold together while the music carries them in wide sweeps backwards and forwards across the floor. At every gust of wind which bursts through an open doorway a group breaks, to clasp hands again as the wind subsides. Protoplasm is always on the verge of instability; always snatching at additional atoms which it draws within its ring; always shaking off other groups of atoms because the ring is too large to hold together. Touch it, and it falls into simpler combinations. Kill it, and it becomes a mixture of organic and inorganic compounds which we know and can name. But as long as it is alive-as long as it is protoplasm, that is to sayintegration and disintegration are occurring. Simultaneous complication and simplification is life. The protoplasmmolecule, if we dare to think of it as a molecule, in the sense in which a chemist uses the term, is always changing. It is its variability which makes stimulation possible. Irritability is a tendency to dissociation under the influence of an external force, with reassociation when the force ceases to act.

The molecules which protoplasm gathers into itself may be classified under the headings oxygen, foods, water, and inorganic salts. It is the two latter which most affect its state, conferring upon it the capacity of exhibiting the phenomena of life. Water and the ions of salts dissolved in water, electrolytcs, are linked to the other elements of its groups. Striving to find room for more molecules of water and more ions, protoplasm expands. It becomes more mobile and more irritable; for irritability and mobility vary as the number of these extraneous groups of atoms which protoplasm is in a position to let drop. As an impulse travels through it they lose their hold, recovering it as they pass the impulse on. This progress towards expansion is the lifeward tendency; the quickening of activity which leads also to the incorporation of additional atoms of nitrogen-containing substances, and consequent growth. 
The opposite tendency is deathwards. Protoplasm drops extraneous groups of atoms; retires into itself; loses irritability; settles down to rest.

The molecules of proteins exhibit a property which appears to pertain in some degree to living matter also. When their relation to the water in which they are dissolved and the electrolytes which it contains is disturbed, they appear to go out of solution, they coagulate. This disturbance is brought about in all proteins by heat ; in some it is the result of altering the amount of salt in the water in which they are dissolved. Coagulation in protoplasm is the prelude to death; but it would appear that a step in this downward path is taken whenever an impulse is conducted. Coagulation is due to the clustering of the molecules of a protein. When protoplasm drops electrolytes and water its molecules cluster in some degree, regaining their independence and reattaching their accessory groups of atoms as the cause which drove them to make for safety passes by.

Our conception-not of life, but of "the physical basis of life," may be very wide of the mark. The account given above is intended as little more than a hint of the lines along which thought is travelling at the present time. The reader must not regard it as a serious attempt to present in detail the views of any of the workers who are endeavouring to apply the results of recent discoveries in molecular physies to the solution of problems in the chemistry of living matter. There can, however, be little doubt but that we are on the eve of further advances which will secure data upon which it will be legitimate to construct hypotheses. At present it would be unreasonable to do more than indicate the direction from which it may be hoped that light will shine.

A stimulus is a change of circumstance rather than a transient disturbance. When an electric current is thrown into it, protoplasm dissociates-parts with something. It instantly re- . associates. The continued passage of the electric current does not maintain it in a dissociated condition. When the current is cut off, the sudden change again acts as a stimulus. Within limits, the efficiency of an electric stimulus varies as its suddenness. Similarly with all other stimuli to which protoplasm responds: crushing, burning, chemical decomposition, are 
effective at the moment of their occurrence. When they generate a succession of responses, it is because they continue to produce changes in the protoplasm. Their continued action does not, under ordinary circumstances, prolong the response.

Response to stimulation travels as an impulse through protoplasm. An impulse is commonly likened to a wave, but enough has been said already to prove that the simile is misleading. It is not of the same nature as the wave which a stone starts on the surface of a pond, a pulsation of sound through air or water, an undulation of light or heat in the æther. These various kinds of waves are waves of displacement, a swing first to one side and then to the other. An impulse traverses protoplasm, whether it be the apparently diffuse protoplasm of a leucocyte or the severely oriented protoplasm of a nerve or muscle, as a change which may be described as chemical, with reservations as to the meaning allowed to this term. We may without impropriety represent the fall (dissociation) and subsequent rise (association) graphically as a wave; but even then it is but a half-wave, and inverted. It is a very different thing to the onward progression of an accession of force, with which it is not infrequently confused.

All protoplasm is not equally susceptible of stimulation. Probably it is safer to put this in a different form. Protoplasm is not everywhere equally exposed to stimulation, nor is it when especially exposed to stimulation in one way equally accessible to all other effective forces. A sense-organ is a collection of cells in which protoplasm is so disposed as to be susceptible to a certain kind of stimulus. It is a "receptor" for a particular force. At the same time it is essential to its efficiency that it should be insusceptible to other forces. The protoplasm in certain of the sense-organs of the skin dissociates when compressed, in others when warmed. The cells of these receptors have a certain structure which exposes their protoplasm in such a manner that it cannot escape dissociation when, in the one case, the cells are squeezed, or when, in the other case, they are heated. The ear contains sensory cells so constructed that the protoplasm which they contain dissociates when affected by pulsations of sound. In the receptors of the tongue and the nose protoplasm is exposed to the influence of chemical 
stimuli ; in the eye it is exposed to the dissociating action of light.

Protoplasm is responsive to external force. It conducts the impulses to which stimulation gives rise. Eventually the impulses, which travel along strands of tissue highly specialized for the purpose of conduction-nerves-reach collections of protoplasm which are so disposed that when they dissociate energy is set free. A comprehensive term is much needed for the connotation of this third essential property of protoplasm, the capacity of liberating energy which characterizes "effectors." An external force, so small in intensity as to be negligible when we are dcaling with the body's accounts, acts upon the protoplasm of a receptor. A change in state results. The change is conducted to an energy-liberating organ. This organ is supplied with blood which brings it food. Food is its store of energy, the raw material from which it manufactures its ammunition. When an impulse reaches an energyliberating organ its protoplasm dissociates. But here the protoplasm is so disposed-the cells which contain it have such a form - that when it dissociates a change in the cell follows; it alters in shape, or it discharges into its environment heat, or electricity, or light. The dissociation and reassociation of the protoplasm of an effector involves chemical change. Molecules of water and of carbonic acid are cast off. The energy sacrificed in letting matter fall into these very stable forms is the energy made visible, as it were, in lifting a weight or dispersing heat. It must be replaced if the organ is to retain its power of acting when next an impulse reaches it. To replace it, protoplasm takes up food and oxygen from the blood.

The liberation of energy which occurs when a muscle contracts is not a special phenomenon-something which does not occur when the muscle is at rest. It is an intensification of a process which is always taking place. The substance of muscle, like that of nerve and every other tissue, is always combining with oxygen and giving off water and carbonic acid. When we are auditing the body's accounts, we enter so and so much food and oxygen on the debit side, we credit it with the same weight of water and carbonic acid; or we debit it with the energy potential in the food, and enter to its credit the 
mechanical work done and the heat set free by the oxidation of this food. Food is the petrol the combustion of which causes the movement of the car. The external force which stimulates a receptor is too insignificant in amount to be carried to account. Physiologists neglect it, just as engineers neglect the energy liberated by the sparking-plug which ignites the petrol, when they are estimating the efficiency of a motor.

Compared with the amount of energy actually received from the environment when a sensory cell of the eye or ear is excited, the energy needed to start an artificial impulse in a nerve is relatively enormous; yet a well-known comparison of the energy conveyed to the nerve in a certain experiment with a nerve-muscle preparation from a frog, and the energy expended by the muscle in contracting, brings home to our minds the fact that it is impossible to carry even this item to account. The energy furnished to the nerve from an electric condenser measured $0.001 \mathrm{erg}$; the energy expended by the muscle reached 100,000 ergs.

It is easy to determine the amount of mechanical work which results from a given expenditure of energy. By alternately flexing and extending the joints of his legs, a man lifts his own weight up a hill of a certain height. The work can be measured in foot-pounds or in kilogrammetres. But this by no means accounts for all the energy potential in his food. A still larger amount is expended for the purpose of keeping the body warm, or, not improbably, making it too warm ; in either case generating heat which is dissipated into the atmosphere. When a machine is being planned, attention is concentrated upon the problem of how to get the largest result in work for a given quantity of fuel. Fuel costs money. All energy dissipated as heat is wasted. Every ounce saved makes for economy. Engineers therefore speak of the "efficiency" of an engine as the relation between the work actually done and the work which would have been done if no energy had been wasted. In the best steam-engines it stands at about 1 to 10 . Since the chief function of muscle is to do mechanical work, physiologists are apt to adopt the engineer's point of view. But in the case of muscle this is justifiable only in a limited degree. The body of a warm-blooded animal is maintained at a temperature higher than that of the surrounding air. Muscies are the 
chief producers of heat. If they turned all the energy which they receive into work, they would be inefficient as regards this very important function. Yet even from the engineer's point of view muscles are more efficient than the best of engines.

It is almost impossible to determine with accuracy, in regard to isolated muscles, the amount of food taken up from the blood, and the return in work by the muscles of the energy potential in the food. Calculations have to be based upon observations of food consumed, gain or loss of bodyweight, work done by a man or an animal during a period lasting for several days. We shall consider the evidence obtained in this way in a subsequent section (p. 149). But whether we study isolated muscles or the body as a whole, the relation between work and heat varies within wide limits. So wide, indeed, are the variations as to justify the conclusion that there is no necessary relation between the two phenomena. Muscles develop heat when they are quiescent. Activity is accompanied with an increased evolution of heat; but, if it be desirable, the evolution of heat is reduced until it is, relatively to the output of work, much smaller than in the case of any engine which has yet been made. It is sufficient in this connection to state that, under certain conditions, the return in work may amount to about one-half. The comparison with an artificial motor, of whatever kind, breaks down. In an engine combustion develops heat, heat causes steam or gas to expand, the expanding gas pushes a piston. In muscle certain of the carbon, hydrogen, and oxygen atoms contained in protoplasm combine to form water and carbonic acid-compounds too stable to be reassociated with the remaining atoms of the protoplasm-molecule. They are replaced by complex, energy-yielding substances-foods - and by oxygen, carried in the blood. Their displacement brings about a change in the form of the molecules which involves, owing to their peculiar orientation, a change in shape of the muscle as a whole. Such an explanation is, perhaps, more exact than our knowledge at present warrants; or rather let us say, since we do not know what the expression "the form of a molecule" means, it has an appearance of an exactitude which does not characterize it. It is merely intended to help the reader to realize the hopelessness of attempting to compare muscle with 
any mechanical contrivance. In the boiler of a steam-engine heat is applied to water until its molecules cannot remain in so close a state of aggregation. Their orbits are greatly increased. The cause of the thrust given to the piston of an engine is the increased amplitude of movement of the molecules of steam behind it. In a combustion engine a mixture of petrol and air is ignited. Energy is set free by the resolution of unstable petrol into stable water and carbonic acid. This energy heats the gases, causing them to expand. Waste of energy as heat is inevitable in a machine which depends for its motive-power upon the translation of molecules. The source of muscular force (if it be not intramolecular change) is certainly not, directly, increased amplitude of molecular swing.

But we must not conclude, as we are tempted to do, that muscle is capable of liberating as mechanical work the whole of the energy supplied to it in food, seeing that its activity is always accompanied by evolution of more heat than can be attributed to friction. If the bulb of a thermometer be inserted into a group of muscles, the instrument shows a marked rise of temperature when the muscles contract. Even though the temperature of the chamber in which an animal is placed is equal to its own, the animal makes more heat if compelled to work, notwithstanding the fact that the consequent rise of its body-temperature may prove fatal.

Nor can muscles dispense unlimited heat without doing mechanical work. If I am too cold, the obvious means of getting warm is jumping about. There appears to be a level of heat-production which cannot be exceeded without movement. When more heat is called for than quiescent muscles can produce, they exhibit flickering contractions, shivering, without moving the limbs. The signal for increased production is given by the skin. The skin is sensible of the amount of heat which is being lost. Exposure to cold air makes one shiver, by suddenly withdrawing heat. But an increase of temperature in the blood behind the skin has an exactly similar effect. In the first stage of fever, when the temperature of the body has risen two or three degrees, and before the system has become accustomed to this state of affairs, the skin announces to the muscles that heat is being rapidly lost. A 
severe shiver, termed a "rigor," is the result. At the same time loss of heat by evaporation is checked, just as it is when the skin is cold. The sweat-glands are rendered inactive. A phenomenon which marks the nightly fall of temperature in consumptive patients is the sudden return of activity in these glands.

Muscle when most highly developed has an extraordinarily definite structure. It is minutely subdivided into units which appear, looked at separately, simple in design. We are tempted to believe that the explanation of the way in which each of these units works is not far to seek. It is disappointing to be obliged to admit that, notwithstanding all the thought which has been devoted to the problem, we are as far as ever from a definitive solution. We understand the principles on which steam-engines, combustion-engines, electric motors are planned. We compare muscle with each of these mechanical contrivances in turn, expecting to discover the principle of its construction. Many ingenious hypotheses have been formulated; but the fact that some of these are mutually destructive shows clearly enough that as yet no approach to certainty has been made. Probably the fundamental error lies in attempting to compare muscle with a mechanical contrivance. The apparent simplicity and regularity of structure of "striped muscle" misleads us. We ought to have commenced our investigations at the other end of the scale of mobile tissue-to have begun with semifluid and apparently homogeneous animal matter, working upwards to the tissue which, being limited to the one function of movement, and movement in one direction only, has, as it were, crystallized along the lines of force.

All protoplasm is mobile. Its particles move one on another. Hence follows either circulation of the living matter within the cell or change in shape of the cell. The two phenomena are identical in nature. Circulation is best studied in a largecelled, transparent part of a plant. A filamentous water-weed is suitable for the purpose. If this be examined. with a microscope while still alive, its cells are seen to contain a watery juice enclosed in spaces of denser cell-substance. Bridges of cell-substance span the spaces. The particles of which these bridges consist are in a state of constant streaming motion, 
which has, it is needless to say, no effect upon the shape of the cell (cf. p. 9).

The unicellular animal amœba, leucocytes, and certain spores of plants, are devoid of cell-wall (cf. p. 28). Their soft protoplasm is not limited by a rigid case. When it streams, the form of the cell is changed. True, we must not think of the body-substance of an amœba as homogeneous. It exhibits an internal structure. Yet its architecture is not, so far as we can see, sufficiently fixed to restrict the directions in which it can stream. Any change of shape is possible. We cannot find in Nature an isolated clump of living protoplasm; nor do we suppose that, if we found it, it would prove to be homogeneous. It appears to be necessary that protoplasm and metaplasm-the terms have no chemical significance ; "primary" and "secondary," or "chief" and "subsidiary" would be equally distinctive-should be intermixed. Streaming is apparently due to alterations in the surface relations of the two substances.

In multicellular animals certain elongated cells are arranged in groups, with their long axes all pointing in the same direction. They can change in shape, diminishing in length, with equivalent increase in breadth. Since all the cells of a group undergo this change of form at the same time, the result is an alteration in the shape of the animal of which they are a part. Applying the experience which we have gained in studying the movements of unicellular organisms, we conclude that these elongated cells are composed of two substances-protoplasm and metaplasm. The restriction of their capacity for altering their shape to one direction indicates that their protoplasms and metaplasm are not indifferently mixed. The two substances set in lines in the direction of the long axis of the cell. Hence, when streaming occurs-when the force which keeps the molecules of protoplasm and of metaplasm in their respective rows is relaxed-the lines thicken. The cell broadens, with an equivalent diminution of length.

Muscle-fibres exhibit all degrees of specialization. The simplest, "plain muscle-fibres," are found in the wall of the alimentary canal, of bloodvessels, of ducts, in the tissue of the spleen, in the skin, and elsewhere. Each fibre is a fusiform 
cell. Save for its central nucleus and a little granular protoplasm in which the nucleus is embedded, the cell may show no architectural features. But in most varieties of plain muscle, and especially in that of the alimentary canal, the substance of the fibres is striated longitudinally. This is visible evidence of the orientation of the molecules of protoplasm and metaplasm in the direction of the long axis of the fibre. It shows that the streaming of particles occurs along these lines. It is, as it were, a diagram of the lines of force.

Heart-muscle has been described already (p. 224). Its striation, which is both transverse and longitudinal, is so delicate as almost to defy microscopical analysis. The transverse striæ are the darker and more distinct. But close examination shows that the transverse striæ do not indicate the direction in which the particles of cell-subs'ance are oriented. They are oriented longitudinally. The cell is a bundle of rods of substance A, embedded in substance B. The transverse markings are very thin lines which cross the bundles at right angles.

The third variety of muscle is the kind by which locomotion is effected. It is present in large masses-all the red tissue to which the term " meat" is commonly applied. It accounts for about 35 per cent. of the body-weight. This kind of muscle is not composed of single cells, but of compound cells, or cellcomplexes, termed "fibres." A fibre may attain a length of upwards of 2 inches, with a breadth of about $\frac{1}{500}$ inch. In most cases the fibres are attached by one end to a bone, by the other to a tendon; and since they are shorter than the muscle as a whole, the tendon commences as a membrane which covers the surface of the muscle, sloping to it from the bone to which by their other ends the fibres are attached. A fibre is developed from a single cell. The cell elongates, its nucleus divides, and the daughter-nuclei divide until several hundred have been formed; but cell-division does not follow. The result is a cylindrical mass enclosed within a delicate membranous sheath, the sarcolemma. In the early stages of its development its nuclei are in the axis of the fibre, but subsequently they are displaced outwards. In the most highly specialized muscle, known as the "white" variety, they lie just beneath the sarcolemma (cf. Fig. 16, B). 
The feature of this type of muscle is its transverse striation, almost mathematically regular. Commonly striated muscle is spoken of as "voluntary," because, for the most part, it is under the control of the Will; but the term, in so far as it implies a connection between structure and mode of actuation, is misleading. Transverse striation is evidence of capacity for rapid action. The muscles which the Will directs exhibit promptitude; but striated muscle, which is not under the direction of the Will, is found in certain situations-e.g., the upper part of the osophagus. Conversely, many animals can voluntarily call into action muscle which is not striped. A turkey erects its feathers by setting in motion little groups of "plain" fibres, which pull on elastic tendons attached to the tips of the buried ends of their shafts. Plain muscle contracts less promptly and relaxes more slowly than the striped variety. Cardiac muscle is quicker in acting than plain, but does not hold the contraction so long.

All striped muscle is not equally rapid. Two varieties are distinguishable: "white fibres," which respond suddenly to a single stimulus and quickly relax ;" red fibres," which respond in a more leisurely way, but remain contracted longer. In some muscles these two types of fibre are intermixed. Others are wholly red or wholly white. Everyone is familiar with the contrast which the white flesh of a turkey or of the domestic fowl presents to the red flesh of game-birds and birds of prey. In the breast of a blackcock a sheet of white muscle overlies a mass of red. When the bird is cooked the difference in colour is strongly marked. Of the two muscles which, in a rabbit, correspond to our muscles of the calf, the superficial, gastrocnemius, is white; the deeper, soleus, red. The former acts over both knee and ankle joints; the latter over the ankle only. The muscle which, acting over a longer range, has to contract more quickly is white ; the shorter, more slowly acting muscle is red. Experiment shows that red and white muscles are distinguished by a difference in the promptitude with which they respond to an electric current. It shows, too, that the white muscle is exhausted sooner than the red. It cannot give so many successive responses to stimulation without a rest. We shall find, when we are considering the minute structure of striped muscle, a difference between its two varieties which 
we can correlate with their different modes of action. All human muscles belong to the red kind.

The most efficient muscle-fibres in the animal kingdom are found in insects. This will not surprise anyone who thinks of an insect's power of movement. If a man could jump as many times his own height as a flea can, he would clear the dome of St. Paul's. An ant can drag an object sixty times as heavy as itself, with no wheels beneath it to diminish friction. Under the same conditions a horse cannot drag much more than its own weight. A dragon-fly, it is asserted-although we have not met a man who guarantees that he has made the observationwill support its heavy body in the air by the rapid vibration of its wings for four-and-twenty hours without alighting. The chirp of a cricket is produced by the rubbing together of its hind-legs. A mosquito sounds its war-cry much in the same way. The pitch of the note proves that the insect's muscles are contracting and relaxing at least 300 times a second. None of these figures must be applied without qualifications in estimating the relative strength of insect and human muscle. Weight for weight, the muscle of a flea is not so much stronger than ours as the figures might lead one to infer. To ascertain the numerical relation, it is necessary to compare the total cross-section of the two chief segments of a flea's leg with the cross-section of the extensor muscles of a man's thigh and calf, and a man's weight with the weight of a flea. Nevertheless, after all deductions have been made, a considerable balance of superiority lies with the insect as regards the strength of its muscles, their rapidity of contraction, and power of repeating contraction without fatigue. An insect's muscle is the most suitable that can be obtained for microscopic examination. Its pattern is larger and more distinct than that of other animals. That the pattern should be larger is not quite what might have been expected. It would not have surprised us had we found the pattern finer in the more effective type.

Nothing is easier than to mount a specimen of insect-muscle. The large water-beetle (Dytiscus marginalis) is an excellent subject. It is so easily handled. Having cut off the animal's head, a leg is pulled out from the thorax. It is split open with a penknife, and a little of the muscle is dug out from within its 
hard case, placed on a clean slide, and covered with a coverslip. If the preparation has been made quickly and cleanly, the muscle remains alive for five or ten minutes. Not only can it be studied, with the microscope, unaltered by reagents, but under the most favourable circumstances the progress along its fibres of waves of contraction can be watched. The structure of the fibres is more easily made out if a little saltsolution or white of egg is added to the preparation.

Striped muscle is crossed by bands, dim, bright, and dark. The sequence is as follows: Starting with the very thin dark line, which often appears as a row of dots, the next band is bright ; then comes a dim band about twice as broad as the bright one ;

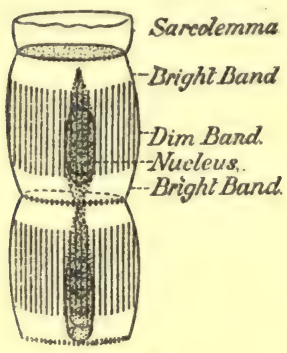

A

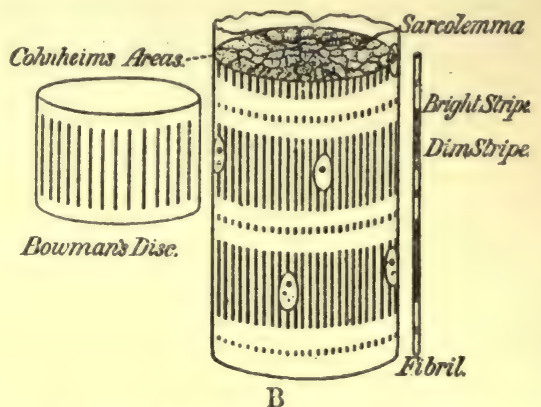

Fig. 16.-A, a Minute Portion of an Insect's Muscle-Fibre, mighly magnified.

B, White Fibre of mammalian Muscle.

A, The nuclei are in the core of the flbre. B, The nuclei lie immediately beneath the sarcolemma. The disc on the left of this flbre, and the fibril on its right, show the two ways in which striated muscle-fibres tend to cleave. The dark line, or row of dots, is known as Dobie's line, or Krause's membrane. The figures are severely diagrammatic.

then another bright band. This sequence is repeated with extreme regularity from end to end of the fibre. Usually the bands cross the whole breadth of the fibre, although occasionally it is divided by longitudinal lines into parts in which the stratification is shifted a little backwards or forwards. A segment of a fibre comprises the substance between two dark lines-i.e., two bright bands with a dim one between them. If the muscle has been hardened in one of the fluids commonly used for the purpose of preparing tissues for the microscope, with its two ends fixed, say, by binding them to a piece of a match, so that it could not shrink, a thin clear line appears crossing the middle of the dim band. This seems to show that the fibre is not made up of single dim dises between two bright 
discs, but of couples, comprising half a dim disc and a bright disc. The thin dark lines indicate that the fibre is divided into compartments by transverse septa, which are probably reticulated. The appearance of a transverse line of dots, in place of a continuous line, is due to the existence of very fine longitudinal markings (it is unsafe to give them a name which connotes structure). Where the longitudinal lines cross the transverse lines, the optical effect is the appearance of a dot.

If pieces of muscle are placed in a solution of osmic acid, they become hard and brittle, and their markings are accentuated. Muscle from the claw of a crab or a lobster is very suitable for this purpose, owing to its exceptional freedom from connective tissue. After this hardening the fibres are easily separated with the aid of needles into fibrils immeasurably slender. An isolated fibril shows with extreme distinctness the alternation of dark, bright, dim, bright, dark markings already described. The appearance of a cross-section of a fibre also proves that it is a bundle of fibrils. The cut ends of the fibrils appear as dots surrounded by homogeneous substance. In this respect there is an important difference between red muscle and white. In the red fibres the fibrils are fewer and thicker than they are in white, and the embedding substance is more abundant. It is generally assumed that the homogeneous substance, sarcoplasm, is the nutrient protoplasm of the fibre, the fibrils the contractile elements. The more complete the differentiation of the fibre into fibrils, the more rapid is its action; the more abundant the sarcoplasm, the greater its capacity for continued work.

If a living muscle-fibre is observed while a wave of contraction is passing down it, the ends of the fibre being free, so that its shortening is not prevented, it is noticed that the widening of the fibre is accompanied by the thinning, even to obliteration, of the bright bands. The dim dises extend laterally, without any noticeable diminution of their thickness. It looks as if the bright discs, or something contained in the bright discs, were absorbed into the dim discs. The fibre is, as we have already pointed out, striated longitudinally. The striation is more clearly visible in the dim dises than it is in the bright ones. That the dim disc has an architectural structure absent from the bright disc is placed beyond doubt when a muscle- 
fibre is illuminated with polarized light. The dim dise is then found to be doubly refracting; the bright disc is not. When the prism in the tube of the microscope is placed with its axis at right angles to the axis of the prism which intervenes between the source of light and the stage of the microscope, a succession of bright bands is seen corresponding to the dim bands seen with unpolarized light. The rest of the fibre is invisible, because it has not the property of twisting the undulations of light which the lower prism has set all in the same plane. Various hypotheses as to the cause of contraction, or, to speak more correctly, as to what happens during contraction, have been based upon the thinning of the bright dises. It is assumed that the dim dises have a definiteness of structure which the bright discs do not possess. They are thought of as being traversed by pores, or as consisting of short rods. Microscopists who take the latter view believe that during contraction the more fluid substance, sarcoplasm, which occupies the bright bands is drawn into the dim bands between the short rods, or sarcostyles, which are consequently separated more widely.

No tissue could be more unsuitable than muscle for microscopic examination; for none other offers the same optical difficulties. This will be evident to anyone who considers the description already given of the markings which it exhibits. Whatever may be the true interpretation of these markings, it is clear that they point to an almost infinite multiplication of minute elements adjusted with absolute accuracy side by side and end to end. A cylinder filled with these transparent objects has to be viewed by transmitted light. The elements, whatever may be their nature, refract light in different degrees. It is impossible to eliminate the effects of internal reflection, refraction, and interference of waves of light. The most alluring hypothesis must be accepted with a considerable amount of reserve. Any fact which seems to militate against it must be taken into consideration. The view set forth above, in general terms, is very attractive to everyone who wishes to bring muscle within the category of machines. Suppose we accept the hypothesis that the dim band is a plate made of sarcostyles surrounded by sarcoplasm then the impulse which reaches a fibre causes an alteration in 
the surface relations of the rods to the substance in which they are embedded. Molecules of fluid from the bright bands are drawn in amongst them; the rods are pushed farther apart; the fibre broadens with a corresponding diminution in length. This brings muscular contraction into the category of the phenomena which play the most important rôle in bringing about the varied activities of the animal mechanism. Contraction is due to osmosis.

The separation of muscle into fibrils after hardening does not seem to bear out either the rod or the pore hypothesis of the structure of the dim disc. It must be remembered, however, that before the fibrils are teased apart the substance of the fibre has been coagulated. The fluid in the bright disc may thus have become as much a part of the fibril as the rod in the dim dise. The longitudinal striation of plain muscle and the appearance of continuous fibrillation in heart-muscle is more difficult to reconcile with the hypothesis that striped muscle is composed of interrupted rods.

Muscle transforms the energy supplied to it by the blood into mechanical work. It is doubtful whether any hypothesis as to structure will help us to an understanding of the way in which this transformation is effected. Explanations are seductive, but all attempts at explaining the connection between molecular change and change in shape must be viewed with suspicion. It is quite clear that muscle as a motor is not to be compared with any form of motor with which we are acquainted. It is also clear that the theory of muscle must be applicable to all its varieties-striped, cardiac, and plain. It must cover the alterations in form of an amoba and the streaming movements of protoplasm within a vegetable cell. Probably it must extend farther, and cover the discharge of electricity by an electric organ and the emission of light by the lamp of a firefly. We are on ground so treacherous that we are not sure whether, in crossing it, we may lean with confidence on the laws of thermodynamics; and doubt as to the applicability of these laws to living tissue almost upsets one's mental balance. Until we have evidence to the contrary, we are bound to exclude such a misgiving from our minds. If we allow it to influence us at all, it is merely to the extent of causing us to hesitate to assume that the explanation of muscular con- 
traction can be based upon an analogy between muscle and any known mechanical contrivances for generating power, not even excluding apparatus designed for the purpose of measuring osmotic force.

If living muscle is frozen, pounded with snow containing 0.6 per cent. of sodic chloride, and placed upon a filter, a fluid plasma passes through the filter as the mixture thaws. Like blood-plasm, it clots spontaneously-without, however, so far as is known the intervention of a ferment.

All muscles become rigid after death, owing to the coagulation of their plasma. It used to be thought that contraction was a stage towards rigidity - a stage from which muscle, so long as it is alive, recovers. This view was based upon the fact that exhausted muscle-such, for example, as that of a hare which has been coursed-becomes rigid much sooner than rested muscle. But this phenomenon has a different explanation. The setting of muscle in rigor mortis is due to the development of lactic acid (one of the waste-products of active muscle). The more there is of this ready formed at the time of death, the more quickly does coagulation of muscle-plasma occur. The formation of lactic acid is due to deficiency of oxygen. So long as muscle obtains as much oxygen as it wants, its metabolism is complete. The oxidized products which it loses are water and carbonic acid. This is true also of the changes which occur after death. If a strip of muscle is hung in an atmosphere of oxygen, it forms no lactic acid, and it does not become rigid. If, on the other hand, the supply of oxygen has run short before death occurred, rigor mortis sets in very quickly. A dead frog takes a long while in becoming rigid, and its rigidity is transient. Until the moment of death the frog is taking up oxygen through its lungs, and even after death it probably takes it, as it does when it is alive, through the skin. A fish becomes rigid very quickly. For some time after it is caught it continues to live; but, being unable to breathe in air, every molecule of oxygen which was in its body when it left the water is used up before it dies.

In the human body rigor mortis usually sets in from two to four hours after death, and lasts about two days; but both the rapidity of its aprearance and its duration depend upon various circumstances. As muscles become rigid they con- 
tract, moving the limbs, and the shortening is more extensive than mere coagulation of muscle-plasm would account for. It is evident that a process similar to functional contraction precedes coagulation. Many a watcher in the chamber of death has been startled by the shaking of the bed. Even a sound resembling a sigh may be caused by contraction of the muscles of the chest. Placing his hand over the region of the heart, the attendant finds the body warmer than it was when life became extinct, for much oxidation has since taken place.

What chemical changes occur in muscle when it contracts ? What is the chemical source of its power? Carbonic acid is given off. This is the only product which we can collect and measure ; but it is taken for granted that hydrogen atoms also combine with oxygen, forming water. There is no reason for thinking that nitrogen is removed from the molecules of jts protoplasm with any greater rapidity during the activity of muscle than when it is quiescent (cf. p. 212). Is the oxidation immediate and complete, or does it occur in stages? For many years attention has been directed to lactic acid, partly because this substance is found in muscle which has been made to contract under experimental conditions, partly because, on theoretical grounds, glycogen (animal starch) is looked upon as the most important of muscle-foods. Lactic acid- $\mathrm{C}_{3} \mathrm{H}_{6} \mathrm{O}_{3}$-has the same percentage composition as glycogen $-\mathrm{C}_{6} \mathrm{H}_{12} \mathrm{O}_{6}$. Its formation from glycogen merely involves a rearrangement of atoms. It has been supposed that lactic acid is formed in the first instance, and then, if the supply of oxygen be sufficient, oxidized to carbonic acid and water. But this hypothesis may be resisted on various grounds. Undoubtedly, lactic acid appears when oxygen is deficient. Under all circumstances and in all tissues a certain amount of it is formed. There are reasons for thinking that it carries away the nitrogen which is wasted, as lactamide. But it does not follow that under normal conditions, when muscle is abundantly supplied with blood, lactic acid appears in any greater quantity during activity than during rest. The hypothesis is due to the misconception which we have already endeavoured to correct. It is difficult to get away from the steam-engine analogy. A steam-engine is made of 
iron and brass. These materials are subject to wear and tear ; but they are not the source of its power. Its power is due to the combustion of fuel. Muscle, physiologists formerly said, is made of protoplasm. This wears down when it works, setting free creatin and other nitrogenous débris. Its fuel is glycogen. This is not the way, however, in which the matter is now regarded. Protoplasm is not the machine only, but also the source of power. Glycogen is not burnt in a framework of protoplasm. When muscle contracts, protoplasm casts out $\mathrm{CO}_{2}$ and $\mathrm{H}_{2} \mathrm{O}$. Glycogen is the food readiest to restore to it the atoms which it has lost.

Another consideration opposed to the hypothesis of the conversion of glycogen into lactic acid is the uselessness of such a transformation from a physical point of view. The stability of the atoms of $\mathrm{C}_{3} \mathrm{H}_{6} \mathrm{O}_{3}$ is so little greater than that of the atoms of $\mathrm{C}_{6} \mathrm{H}_{12} \mathrm{O}_{6}$ that practically no energy is set free when the one substance changes into the other. We cannot, however, overlook the fact that the formation of acid may be a means of profoundly altering the state of the colloid substances dissolved in cell-juice. The casein of milk coagulates when milk turns sour. The neutralization of a faintly alkaline solution of a protein (and muscle is faintly alkaline) will throw it out of solution. The appearance of lactic acid may be intimately associated with movement of protoplasm, and yet the change of glycogen into lactic acid not be the source of the energy which muscle expends.

Fatigue.-For its continued activity muscle needs an adequate supply of food and oxygen. If the blood which distributes food is circulating properly, and the liver, the great depot of food, is well stored, fresh supplies are brought to the muscles as they are needed. There are muscles-those of the eye and of the heart, for example-which never become exhausted. However continuous their activity, they take food from the blood as rapidly as they waste it; a statement which, perhaps, needs qualifying by the addition, "so long as the work exacted of them is such as may be reasonably expected." If, in a picture-gallery, one keeps the eyes elevated for an hour or more a headache follows. Our eye-muscles have taken over their duties on the understanding that we look down or straight forwards far more often than we look up. If a long-sighted 
child is required to focus his eyes upon a printed page without the aid of spectacles, not headache merely, but actual disease of the brain, may be the result. The ciliary muscle within the eyeball, which effects accommodation of the eye for near objects, is unduly strained. Even the use of our modern type, with its vertical height greater than its breadth, which has taken the place of square Roman letters, is probably related to the development of astigmatism of the lens, and thus indirectly a cause of headache. It is asserted on high authority that vertical astigmatism, the commonest form, is not present in the eyes of children before they learn to read. Headache is an exaggeration of the feeling of fatigue. It may be interpreted as the brain's expression of unwillingness to be made to work; a protest always to be listened to, notwithstanding that it does not necessarily follow that unwillingness to work is the result of overwork. Constipation, irritation of the sensory nerves of the stomach, overdosing of the brain with alcohol, and many other causes, may, through the vaso-motor system, set up the conditions which normally result from activity unduly prolonged. The fact that a central disturbance, headache, results from undue muscular work calls our attention to the double nature of the mechanism concerned in movement. Muscles are set in motion through the intervention of the nervous system. After they have worked to an unusual extent the nerve-centres connected with them grow tired. This, at least, is a legitimate inference from the fact that headache occurs when certain muscles of the eyeball have been subjected to an improper strain. But it must be remembered that the muscles of the eyeball never tire. They do not, like other voluntary muscles, give notice that they are in need of rest. It is not so clear that the central mechanism is in any way involved in the fatigue which is produced by excessive use of arms or legs. The muscles of the limbs (and the central nervous system) are protected by the sensations which originate in muscles when they are overworked. The fact that a weary man can, if a great emergency demands activity, use his muscles with as much vigour as if he were fresh from bed, has been cited as an argument in favour of the view that fatigue is of central origin; but it is an argument which works both ways. A strong emotion causes a fervent response from the 
nervous system. Tired muscles contract energetically when the impulses which reach them are sufficiently urgent.

Nothing so definitely removes muscle from the category of machines as its liability to fatigue. To speak of a muscle as tired is, of course, to transfer to an object a term which is applicable only to a phenomenon of consciousness; but it is necessary, unless a cumbrous expression is to be used, to designate thus the effect upon the muscle of prolonged activity. The petrol may be low in the tank, but the quantity burnt in the cylinder at each stroke is not reduced. If an isolated muscle is repeatedly stimulated by an electric current of a certain strength, the response which it makes improves for the first two or three induction shocks; then it begins to weaken. At each succeeding spasm the muscle shortens a trifle less than before. More remarkable than the diminution in the amount of work done by a muscle which is growing tired is the prolongation of the time taken both in contracting and in relaxing. Further, it has been shown that the fatigue which accompanies the contraction of an isolated muscle is not a condition dependent upon the shrinking of the store of energy which it possessed when it was first thrown into activity. Muscles undisturbed as to blood-supply, and contracting under the direction of the Will, also exhibit it. Speaking generally, it may be said that the tiring of muscle is not so much due to the exhaustion of its store of food as to accumulation of products of action. Vigour is restored to a tired muscle by passing through its bloodvessels a stream of salt-solution, which brings it no food, but washes away some of its waste. But the problem is far more complex than this. The machinery is not simply clogged with the products of its own activity. If the blood of a tired animal is injected into the vessels of one that is rested, the muscles of the latter exhibit the phenomena of fatigue. Evidently muscle is self-protective. During activity it prepares a "fatigue-substance" which poisons its own nerve-endings, making them worse conductors from nerve to muscle of the commands which descend from the brain. Not only does the fatigue-substance dull the nerve-endings in the particular muscle which has contracted, but, being distributed by the blood to the whole body, it produces a general effect. If the legs have been severely worked, they exhibit 
fatigue in the highest degree; but after a long walk the arms also are less ready and less capable than the state of their nutrition warrants.

The condition of stiffness experienced for a day or two after excessive exercise is due to various causes in combination. The fact that it may be remedied by encouraging the circulation through the muscles most affected, as by hot baths and massage, tempts us to assign it also in large measure to accumulation of products of action; but the means taken to reduce stiffness favour the nutrition of the muscles both by giving them more food and by carrying off their waste.

Equally remarkable with the self-protective disposition of muscle, which forbids it to give, except at the instance of increasingly urgent messages from the central nervous system, more than a part of the work of which it is capable, is its preparation for meeting an increased demand. It grows with use. Running increases the girth of the leg by developing especially the muscles of the calf. Raising weights enlarges the muscles of the shoulder and arm. Use-growth may reach inconvenient proportions. Nothing is more noticeable during the training of young athletes, whose nutritive responsiveness is at its height, than their liability to pass through a stage in which they are " muscle-bound." Their legs grow bigger, but their pace falls off.

The development by means of exercises of a strong muscular system has received much attention during recent years. Our ancestors cultivated strength and agility in certain movements without paying much attention to the muscles by which the movements were performed. It is fashionable nowadays to lay stress upon the importance of maintaining an abundant musculature, because of its relation to general "fitness." The balance between muscular activity and the organic functions which is observed by everyone who takes an active holiday proves beyond doubt that the nutritive condition of the various glands and of the heart and bloodvessels is in some degree dependent upon the condition of the muscles. Possibly they secrete into the blood other "messengers" in addition to fatigue-substance-messengers whose call wakes up the organs of digestion. The man who is so fortunate as to be able to use his muscles in the open air has no need of 
exercises in his bathroom. Failing out-of-door opportunities, much can be done by the systematic use of the various muscles working against resistance. It is alleged, and we are not disposed to dispute the justice of the contention, that movements made with the fullest degree of mental concurrence have a more rapid effect upon the growth of muscle than actions more or less unconscious. Muscle and nerve are parts of a single mechanism. It may be that fixing the attention on an exercise, and watching its performance in a looking-glass, aids the nutrition of muscles by increasing the influence of their nerves, possibly by improving the nutrition of their nerve-centre. Unfortunately, this is one of many theories which hardly come within the reach of a control-experiment. Could one concentrate attention on the movements of the right arm, then absentmindedly repeat them with equal vigour with the left, it might be possible to ascertain whether there is anything in this idea. Two other contentions with regard to the best way of performing movements, with a view to the promotion of muscular growth, appear to be justified by their results. Working against a moderate or light load is said to be more effective than putting muscles to a severe strain. A small number of maximal contractions, it is said, induce more rapid growth than many partial shortenings. According to this scheme, when a particular muscle needs strengthening, because in a certain action it is to be the chief performer, it is made to bring its two ends as near together as the plan of its attachments allows. Maximal shortening is apparently favourable to blood-supply and otherwise promotes nutrition.

Tone.-Hitherto we have spoken of quiescence and activity, as if muscle were doing nothing when not visibly contracting. A wrong impression may be engendered by these terms. Muscle is never idle. During sleep, and still more when a person is under the influence of anæsthetics, the muscles approach the condition of machines at rest. But again the language of the workshop is inapplicable. When a headless frog is hanging from a hook its legs are slightly bent. All its muscles are weakly contracted, if we understand by contraction a condition in which the length of muscle is less than it would be were it not alive. But the flexors are tenser than the extensors, hence the crooking of hip, knee, and ankle. If the sensory 
roots of the sciatic nerve are cut, the leg straightens out. So long as the nerve was intact the weight of the limbs acted as a stimulus to sensory nerve-endings, causing a reflex "tone" of the flexor muscles via the spinal cord. The tone of the extensor muscles was less because they were not stretched by the weight of the limbs. Every joint is under the influence of antagonistic muscles which are perpetually watching one another. When the limb is extended the flexors become anxious. When it is flexed the extensors get ready for a spring. Only when it is half flexed is there anything approaching to a truce. And this in most cases is the position of greatest comfort. But even when most at rest, muscles still possess a certain degree of tone. The tendency to shortening in one set causes it to pull against, and thereby increases the tone of, its opponents. When a muscle contracts it does not lift a loose bone. It has to overcome the tone of the muscles which would cause a movement in the opposite direction. And here another adjustment comes into play. The same gross stimulus which leads to the contraction of A starts impulses of a finer kind for B, directing it to relax its tone. We have seen how the heart and bloodvessels are under the influence of two sets of nerves of opposite sign-anabolic, diminishing irritability; katabolic, increasing it. All muscles are under similar management; but we can rarely detect the influence of the anabolic, inhibitory nerves, the brakes, because the katabolic display is overwhelmingly conspicuous. We must be content with two experimental demonstrations. An animal's hamstrings have been cut; the flexor muscles of its thigh are therefore severed from their attachments below the knee. The tone of the extensor muscle keeps this joint extended. If now the pad of the foot be tickled, the flexor muscles contract, just as they would do if they were still able to carry out the reflex action of raising the foot. They cannot do this, because their tendons are divided; nevertheless, the knee bends owing to reflex relaxation of the extensor muscles. Still more striking evidence of reciprocal contraction and relaxation is afforded by the claw-muscles of a crayfish. A weak stimulus to its nerve causes the claw to set open; astronger stimulus causes it to close. Both these movements are due, not to " contraction," but to change of tone. Under certain con- 
ditions, a current passed through its abductor muscle, the claw being open at the time, causes closure by inhibiting the tone of this muscle. In this case the stimulus acts directly on the muscle, producing an effect which is opposite to the one we are accustomed to associate with stimulation; in place of contraction, relaxation.

Contraction of muscle, moving something, impresses one as a positive phenomenon. Relaxation seems negative-the undoing of contraction-and to a very large extent this attitude of mind is justified. Return of a muscle to its full length is due either to stretching by the weight it has lifted, or to the antagonism of other muscles. An isolated muscle lying on a pool of quicksilver does not return to its full length after it has contracted. But it is necessary to banish the machine idea. A machine gives out all the energy it has in store. Muscle is extremely parsimonious. No stimulus can induce it to part with more than a fraction of its energy. Recovery is as definite a function as disturbance. A machine starts when a crank is moved, stops when it is replaced. Muscle has a certain degree of automatism, although its tendency to act on its own account has been almost completely transferred to the governing nervous system. Muscle and nerve work together, and the efficiency of muscle depends upon the maintenance of its relations with its nerve. If the nerve is cut, the muscle atrophies. We will not stop to consider whether wasting may be properly attributed to disuse, or to vasomotor changes. In its lowest form nervous influence shows itself in the regulation of the nutrition of muscle. A somewhat more forcible exhibition of control is seen in the regulation of tone. The maximum is reached when a wave of undoing which has passed down a nerve infects the protoplasm of muscle with the same tendency to disintegration. The musclesubstance explodes. The muscle shortens.

Remarkable evidence of the existence of muscle-tone is afforded by the knee-jerk. Place a person on an upright chair, with his legs crossed, muscles lax, foot hanging free. With a paper-knife or the end of a stethoscope, or even the hand used edgewise, tap the ligament which connects his kneecap with his shin. The tap is instantly followed by a jerking forward of the foot. The deep muscles of the thigh, vastus, 
and crureus, have contracted. This phenomenon is easy to account for. When we are standing upright, the trunk is supported on three joints, of which one-the hip-is a perfect ball and socket, and the other two-knee and ankle-are of the same order so far as the absence of any provision for locking them is concerned. If the muscles on the front and the back of the leg did not constantly adjust our balance, by swaying the trunk forward when it falls back, and pulling it back when it sways forward, the joints of the leg would double up beneath us. A photographer knows how little confidence is to be placed in a man's assertion that he is able to stand still. This see-saw of alternate contraction and relaxation is kept up by means of nerve-impulses which ascend from the nerve-endings surrounding the separate bundles of tendnos, or from the Pacinian bodies which are found in abundance in the neighbourhood of tendons and ligaments, or from the elaborately twisted nerve-fibres found in musclespindles, or possibly from all three classes. Muscle and tendon are richly supplied with sense-organs susceptible to pressure and stretching. There is an abundance of nerve-endings to choose from. The slightest change in their tension, whether due to the muscle's own contraction or to the action upon it of other muscles or weights, is recorded not only in the spinal cord, but also in the cortex of the cerebellum, and, if the contraction is an act of volition, in the cortex of the great brain. Although it was skin which was tapped, skin-nerves have nothing to do with the jerk. It was the result of the slight sudden stretching. In short, the tone-mechanism has been fooled. Notice the position of the leg. The knee is semiflexed ; the foot is hanging free. There is nothing for the extensor muscles of the thigh to do. Now, if ever, they are justified in dozing. It is not to be wondered at that the sudden stretching of the ligament takes them off their guard, or that on waking they give a quite unreasonable start. The phenomenon is, as we asserted, easy to account for. It would also be easy to explain, if it were not for the extreme rapidity with which the jerk follows the tap. The interval is about one-hundredth of a second. This is thought to be too short to allow an impulse to ascend a sensory nerve, pass through the cord, and descend a motor nerve. It is true that these reflexes of adjustment 
must stand on a different level to other reflexes. The toneimpulses which cause them are incessantly patrolling to and fro from sense-organs to nerve-endings. The paths they follow must be the most open in the nervous system. Receptors and effectors must, in an electrician's phrase, be incessantly switched on ; or, to express the analogy more accurately, the flexor and extensor tone mechanisms are incessantly and reciprocally switching each other on and off. It must be confessed that it is very difficult to explain the knee-jerk if it be not a reflex action, but, as has been supposed, a direct response of the thigh muscles to their own stretching. The latter hypothesis does not appear to be reconcilable with its dependence upon the maintenance of the nervous connection of the muscles with the spinal cord. It cannot be elicited unless the "spinal arc" is intact. It ceases after the severance of either sensory or motor roots. Nor will it occur if the supply of blood to the lower end of the spinal cord has been cut off. Still more difficult is it to explain its extraordinary sympathy with everything that happens in the whole nervous system, if the impulses which cause it do not pass through the spinal cord. By a very simple mechanical arrangement it is possible to record the amplitude of the knee-jerk. The foot moves a lever which writes on a travelling surface. The jerk is elicited by the hammer of a clock strapped to the shin. In this way it is possible to extend the period of observation over several consecutive hours, the subject becoming completely oblivious of the movement his foot is making once a second, if it be screened from his view. In deep sleep the jerks stop; but the subject may doze, and still jerk follows tap. And the record made by his foot mirrors all the changes in his nervous system. If he clench his fist, the movement is reinforced, as it is when a child cries, a lamp is lighted, his ear itches. There is music in an adjoining room. His foot is the baton which beats fortissimo to Wagner, and is lulled to piano by the "Lieder ohne Wörte." On a bright day this spinal pulse throbs gaily. It is indolent in dull, depressing weather. The knee-jerk is the physician's guide to the condition of the nervous system.

Elasticity of Muscles.-Muscles are very extensible, and after stretching return to their original length. Their elasticity is a quality of great practical importance. It enables them to 
meet sudden resistance without rupture, as when a man alights from a height. At the moment when the feet touch ground elasticity dissipates the shock. The stretching of the muscles then leads reflexly to the increase of their tone. Here we see an advantage in the short reaction-time of the kneejerk. Tone comes into play long before impulses generated by contact of the sole of the foot with the ground have had time to reach the brain, or even to induce reflex contraction through the spinal cord. The elasticity of the muscles is also of use in the performance of certain sudden actions. A pea is flicked across the room by pressing the thumb-nail against the pad of a finger, or a finger against the thumb, and releasing it with a jerk.

An electrical change accompanies an impulse in its passage down a nerve, and a wave of contraction in its passage along a muscle. In 1788 Galvani observed that the hind-limbs of a frog, suspended by a metal hook to metal railings, twitched when the wind blew them against the bars. The hook passed through the lumbar plexus of nerves. He recognized that the cause of the twitch was the closing of a circuit. The birth of dynamic or galvanic electricity dates from this observation; and ever since this phenomenon was first observed the electric changes in nerve-muscle preparations made from frogs' legs have been favourite subjects of research. Many obvervations with regard to nerve-conduction and muscle-contraction may be made, and many experiments performed, without special apparatus. A frog having been killed by cutting off its head, or by placing it beneath a tumbler with a wad of cotton-wool soaked in chloroform, the skin of the leg is removed, displaying the khaki-coloured muscles, bluish tendons, and bright white threads of nerve. A stretch of the largest nerve of the back of the thigh, the sciatic, is isolated. All the muscles of the thigh are then cut away and the bone nipped across just above the knee. The bones below the knee are removed, the superficial muscle of the calf, the gastrocnemius, being allowed to hang free, its bifid end attached to the fragment of thigh-bone. Its lower end terminating in the tendo Achillis, with its insertion into the prominence of the heel, is left intact. The bone is fixed in a clamp. A light lever made from a wooden spill is suspended from the tendo Achillis. The nerve may then be stimulated in various ways : by crushing in a pair of forceps, 
burning with a heated needle, touching with a drop of glycerin or a strong solution of salt. But of all methods of stimulation, the best is the current from an induction coil. Since it does not injure the nerve, it can be applied as often as may be desired. The amateur provided with an induction coil is in a position to study the relation between stimulus and response. He can vary the strength of the stimulus and vary the weight which the muscle has to lift. He can observe the progressive onset of fatigue, and otherwise gain much information regarding the behaviour of muscle as an isolated piece of apparatus.

It is the ambition of the expert to obtain absolutely correct records of the time-phases and of the changes in electric potential of nerve and muscle under varied experimental conditions. For this purpose he needs the finest apparatus which instrument-makers can furnish, and the knowledge and dexterity requisite for its employment. Consider, for example, the record of the change of form. A nerve-muscle preparation, - obtained by the method already described, is arranged so that the point of the lever scratches on a rapidly travelling blackened surface. As the muscle contracts it makes a "tracing." A tuning-fork vibrating at the rate of, say, 400 times a second also scratches a tracing on the same travelling plate. It is easy to time the several phases of contraction and relaxation by comparing them with the undulations made by the tuning-fork. By means of an induction shock a single impulse is generated in the nerve and a single spasm evoked in the muscle. Our tracing shows that the spasm lasts about onetenth of a second, and that about half this time is occupied by contraction, and half by relaxation. But the ascending curve is usually a little steeper than the descending curve, and the apex a little nearer to the commencement of ascent than to the termination of descent. An electric signal marked the instant at which the current was sent into the nerve. The time taken by the impulse in travelling from the spot where the electric current entered the nerve to its junction with the muscle can therefore be estimated. The contraction begins so much sooner or later, according as the shock is delivered nearer to, or farther from, the muscle. By shifting the electrodes up and down the nerve, the rate at which the impulse travels 
is directly measured. After the time that the impulse took in reaching the muscle has been allowed for, there still seems to be an interval before the musele begins to shorten. This was termed the "latent period," under the impression that some time is actually lost in turning the nerve-impulse into a muscle impulse. The impulse was supposed to be latent in the endplates of the nerve. Various hypotheses were formulated as to the nature of the transformation. The progressive improvements in apparatus and methods is testified by the diminution in this latent period as given in the textbooks of successive decades. It is now put at $\frac{1}{400}$ second, and is regarded by most physiologists as a delay due to the inertia of the muscle. Owing to its elasticity, the molecular change in muscle does not immediately affect its shape. When the latent period appears to be longer-say $\frac{1}{100}$ second-the balance is due to the inertia of the recording apparatus. Usually the curve shows a rise lasting $\frac{4}{100}$ second and a fall occupying $\frac{5}{100}$, due to the fact that inertia of muscle and apparatus delays the commencement of the rise, but does not hasten the termination of the fall.

When an impulse is generated artificially by an induction shock, a single spasm or twitch is the result; but in Nature contraction is never limited to a single twitch. Impulses descending from a motor nerve-cell to a muscle are always rhythmic. They follow at the rate of eight or ten a second in human nerves; and since in our muscles contraction and relaxation take longer than in a frog, a second impulse reaches the muscle before the effect of the first has passed away. The muscle has not had time to relax, when it is again called upon to contract. Hence a summation of contractions. The muscle continues to shorten until the maximum of contraction is reached. This condition is termed "tetanus," to distinguish it from a single spasm. In fullest contraction the length of a muscle may be diminished by one-half, or even by two-thirds.

It would be impossible to treat of the electrical phenomena displayed by nerves and muscles without presupposing some acquaintance with the methods and laws of physics. As this is contrary to our understanding with our readers, we must be content with the statement of a few salient facts. At the moment when an impulse is passing along a nerve, or a wave 
of contraction along a muscle, the electric potential of the active part of the structure, whether nerve or muscle, is different from that of the not-acting parts on either side of it. A battery in its commonest form is a glass vessel containing sulphuric acid in which a plate of zinc and a plate of copper are immersed. The zinc is electro-positive as regards the copper. In a muscle the contracted portion is electro-positive as regards the parts uncontracted. The degree of positivity can be measured by connecting the muscle at two spots with the two wires of a galvanometer. When one wire makes contact with the contracted portion, and the other with a part which is not contracted, a current passes through the galvanometer, causing its needle to swing; and since the wave of contraction is not stationary, but passes down the muscle, the current is subsequently reversed. The wave, as it were, first tilts up one end, and then, passing on, tilts up the other, letting down the first. The contracted spot is electro-positive to the spot not contracted, and then the latter, contracting, becomes electropositive to the former, which has relaxed. The needle of the galvanometer swings first to the left, then to the right. The importance of this method of investigation lies in the fact that the electric variation exactly represents, both in time and in intensity, the change which is occurring in nerve and in muscle. By following it, we can ascertain the rate at which an impulse travels down a nerve. We can determine its length and its "form." Represented on paper, it is a wave. This wave travels in warm-blooded animals with the rapidity of 35 metres in a second. When it reaches a muscle, its ratethat is to say, the rate at which the wave of contraction invades the muscle-is 6 metres in a second. The time during which any particular level in the muscle remains contracted in a single spasm, under the influence of an artificial stimulus, is about 0.05 second. The length of the wave is 300 to 400 millimetres. These measurements give us a very clear idea of the events which occur in a nerve-muscle. An impulse picked up by a motor-cell in the spinal cord runs down its axon-termed later a nerve-fibre-with great rapidity. Even the most distant muscle is reached in less than one-thirtieth of a second. From the end-plate of the nerve it travels in both directions along the muscle-fibre-or group of fibres, since each nerve divides into 
branchets for thirty to forty muscle-fibres-with reduced velocity. Every particle of each fibre rises and falls; but, seeing that the wave of contraction is much longer than the fibre, the whole fibre is in a state of contraction at the same time, although not with equal vigour throughout its whole length.

We cannot dismiss the further consideration of the electric phenomena of nerves and muscles without some inquiry into their meaning. It is evident that they are intimately related to the molecular changes which constitute an impulse. But at present the physics of the phenomena are beyond our grasp. We may speak in a general way of dissociation of ions ; but we do not really know what is happening at the spot which is in a state of impulse. We cannot bring the transformation which it is undergoing into line with chemical and physical transformations which we understand. Probably the electrical phenomena which mark it are not peculiar to muscle and nerve. All living changes of state are of the same nature. Cellular activity, or protoplasmic activity, to use a better term, wherever it occurs, is accompanied by electrical change. But it so happens that nerve-substance and muscle-substance have a definite orientation which gives to the electric force a cumulative effect. In a liver-cell it is dispersed in all directions. In a muscle the change of potential at one particle is added to the change at the next, until the sum of all these changes, transmitted along the length of the fibre, is sufficiently large to deflect the needle of a galvanometer. Owing to its summation it attracts our attention.

Although they cannot tell the true significance of the electromotive change which marks the passage of an impulse, physiologists are in a much better position now than formerly to controvert cartain popular misconceptions. There is no such thing as "nerve-force" in the vulgar sense. A nerve does not transmit energy to a muscle. The muscle obtains the energy which it dispenses when contracting from the foods with which the blood supplies it. The nerve transmits an excitation. Over-excitability is not a sign of strength, but of weakness. Nor is an impulse in a nerve an electric current. It may be generated by an electric shock, but a chemical stimulus is equally as effective. The slow rate at which it travels, as compared with electricity, puts it altogether out of comparison 
with an electric current. Its relatively rapid progress, on the other hand, equally excludes the hypothesis that it is a movement of ions, as that phenomenon is observed in solutions of salts.

What is the nature of the process by which energy is conveyed along a nerve? When speaking of the passage of impulses from receptors to the central nervous system, and through this to effectors, we have used the vague expression " molecular change," to avoid the necessity of being more precise. But the problem is of such profound interest that we look with eagerness for any hint of the direction from which light will eventually be thrown upon it. Recent discoveries regarding the nature of electricity, combined with investigations at present in progress as to the physical constitution of proteid substances, give more than a hint. Hitherto the choice has lain between a chemical and a physical explanation; now the border-line between chemistry and physics, always wavering, has disappeared. The hypothesis that an impulse is a progression of chemical change has meant in the past that the "wave" was due to the oxidation of substances contained in nerve, with liberation of $\mathrm{CO}_{2}$ and $\mathrm{H}_{2} \mathrm{O}$. Various considerations render such metabolism of the substance of which nerve-fibres are composed improbable. In the first place, nerve-cell bodies contain a store of material, tigroids (p. 320), which is recognizably drawn upon during nervous activity. It would appear, therefore, to be the tigroids, and not the substance of the nerve-fibre, which supply the energy transmitted along a nerve. Then, again, the axon of a nerve-fibre, enclosed as it is in a tube of fat, is peculiarly ill-placed for the reception of the nourishment which would be needed to make up for waste, if its metabolism be fluctuating and at times excessive. Nor have nerves more than a very meagre blood-supply. Secondly, observation does not give any support to the hypothesis of fluctuating metabolism. A nerve does not give off more $\mathrm{CO}_{2}$ when active than when passive. Nor does it become acid. Thirdly, nerves, or, to be quite accurate, medullated nerves, are indefatigable. Their capacity for conduction is not diminished by previous use, as it would be were it dependent upon their reserve of nutriment. These various considerations rule out a "chemical" expranation of the old-fashioned type. 
It is premature to do more than outline the " physical " theory which seems destined to take its place ; and the reader will perhaps forgive if, for the sake of elearness, the case is put with unjustifiable definiteness and simplicity. Proteid substances are constituted of clusters of molecules. The form of the clusters depends upon the salts (or, more precisely, the ions) with which they are associated, and the associations depend upon the electric charges which the ions carry. In resting nerve-protoplasm the clusters are small, and, since the total surface-area of a number of small spheres is greater than the surface-area of the same weight of matter when condensed into large spheres, there is, so to speak, more surface for the ions to cling to. Conversely, when the ions leave the small clusters, the latter are not protected from the influence of mutual attraction. They fuse into larger clusters. Fusion is carried to its extreme limits when a protein coagulates. A nerve-impulse is a "wave" of partial coagulation. The positive electricity generated in a cell-body by the metabolism of its tigroids repels the positively charged ions which cling to the nearest protoplasm-clusters in the axon. Like acrobats swinging from trapeze to trapeze, each flight of ions dispossesses the ions from the clusters in front of it ; and in this way the disturbance progresses down the axon as an electric wave.

Thus we interpret the shadow cast by a theory of which either of several pioneers who are diligently climbing may at any time obtain a view. The conductivity of protoplasm (and what is true of its conductivity will be found to hold good equally for its irritability and changeableness of form) is due to the readiness with which its molecules enter into unstable associations with electrolytes. The instability of these associations is related to the tendency of the molecules to cluster. An impulse is passed along a nerve as a displacement of ions ; the ions being transferred from one molecule, or group of molecules, to the next. Such an explanation of an impulse involves no chemical breakdown of nerve-substance during its passage along a nerve. It transfers the metabolism which liberates energy (reinforcing thэ impulses which have originated in sense-organs) to the nerve-cell bodies. It is based upon certain experimental data which appear to have been established; but, like all other hypotheses which are intended to 
account for physiological phenomena, this one must be brought to the test by varying the conditions under which impulses pass along nerves, and ascertaining whether the consequent alteration in the force, rate, and other attributes of the phenomena are in accordance with physical laws. In applying these tests to the activities of protoplasm, we are, however, met by an insuperable difficulty. The matter which transmits nerveimpulses is alive. We have no laboratory standards by which to judge whether the changes in conduction which are produced by changes in the conditions of the conductor are, or are not, consonant with physical theory. It is with protoplasm that we are dealing, and not with a mixture of proteins in solution. If we surround a nerve with nitrogen, it loses its conductivity in five hours, to recover it when oxygen replaces the neutral gas. This has been regarded as proving that metabolism of the nerve is necessary for the transmission of impulses. But conductivity is a phenomenon of life. Deprivation of oxygen for five hours must bring the nerve-substance to the verge of death. It might be argued that the retention by the nerve for so long a time of its power of conducting impulses shows that its metabolism is not a cause of the phenomenon. Again, it has been shown that warming the nerves of cold-blooded animals greatly increases the rapidity of conduction. It is more than doubled in the nerves of the "foot" of a slug - and a similar increase has been proved for the nerves of a frog-by a rise of temperature of $10^{\circ} \mathrm{C}$. Reflecting on the results of this experiment, a physicist would exclaim : "Then an impulse is a wave of chemical change. A rise of $10^{\circ} \mathrm{C}$. increases the rate of chemical processes from two to three times; whereas no known physical process is accelerated by more than 5 to 15 per cent." But the physiologist remembers that a rise of temperature of $10^{\circ} \mathrm{C}$. increases all the activities of a frog. He is hardly prepared to say that its greater vivacity may not be the expression of more rapid oxidation; but he sees no fore-ordained balance of vital enterprise and chemical change. He is, or ought to be, extremely suspicious of any explanation which appears to override physical laws ; yet, at the same time, he is aware that until he has more accurate knowledge regarding the constitution of protoplasm he will not be in a position to understand how 
physical laws apply. The protoplasmicity of protoplasm is increased by warmth. What change of molecular constitution does this imply?

The view that in a muscle molecular change gives rise to an electrical change, which in turn produces the change in form, has been very widely held. The hypothesis was based on observations which seemed to show that the electric variation travels a little ahead of the wave of contraction ; but every improvement in recording apparatus has diminished this apparent want of synchronism. There can be little doubt but that the lagging behind of the wave of contraction is due to the inertia of the muscle and of the recording apparatus. Molecular change and electric variation are simultaneous. If this be true, the electric change cannot be regarded as the cause of the molecular change, in the sense, at any rate, in which they used to be considered as cause and effect.

The power of muscle varies as its cross-section. For human muscles the maximum lift amounts to from 7 to 10 kilogrammes for each square centimetre. This is a large figure, but it must be remembered that, owing to the arrangement of the bones as levers, most muscles act at a great mechanical disadvantage. The greater the difference in distance from the fulcrum between the point of application of the force and the point of incidence of the weight, when the force acts nearer to the fulcrum than the weight, the greater is the mechanical disadvantage. The greater also is the rapidity with which the weight is lifted. What is lost in strength is gained in swiftness. Contrast the slow steps of a negro, whose long heel separates the point of application of the power (tendo Achillis) from the fulcrum (the ankle-joint), with the springy movements of a European. A European needs, and as a rule has, a better developed calf, which allows him his more sprightly gait, without sacrificing his carrying power. Our preference for slender wrists and ankles is not purely æsthetic, unless we admit, as may be maintained, that all natural canons of taste rest upon utility. Slimness of joints means nimbleness. A few muscles act directly, without loss of power-as, for example, the masseter, which lifts the lower jaw (hence a grand capacity for cracking nuts) - but most muscles move levers of considerable length. Compare with the masseter the biceps and brachialis 
which lift the forearm. Their tendons are inserted into the radius and the ulna at a distance from the elbow-joint which is about one-tenth as great as the distance from it of a weight held in the hand. Their united cross-section is about 16 square centimetres: $\frac{16 \times 10}{10}=16$. One cannot hold out in the hand, the elbow being pressed against the side, so that these muscles alone are acting, a greater weight than 16 kilogrammes (34 pounds), although the muscles are exerting a traction ten times as great as this. The strength of muscle when pulling straight is well illustrated by the thick white mass in the centre of an oyster. It keeps the shell closed until a force equal to 1,300 times the animal's weight has been applied. This muscle also affords a

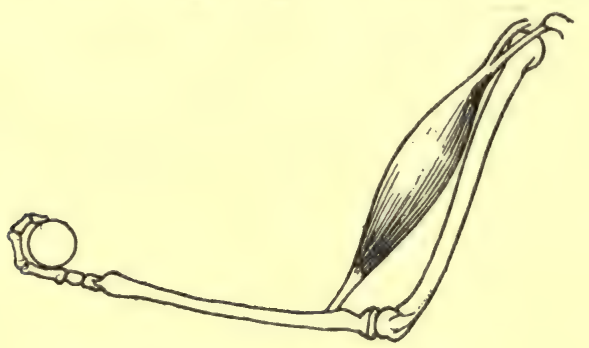

Fig. 17.-Biceps MUSCle in AOTION.

good illustration of the part played by reflex contraction in opposing stretching - the reaction by which tone is maintained. Anyone who inserts an instrument, such as the end of a screwdriver, between the slightly open valves of an oyster lying under water will find that he needs to give it an exceedingly smart twist if he would catch the muscle asleep. Stretching it causes a reaction proportional to the stretching force.

The fact that the output of energy by muscle is proportional, within certain limits, to the work to be done, is brought out even in laboratory experiments. A nerve-muscle preparation teaches that the amount of work is not a function of the stimulus. Within certain limits a stronger stimulus evokes a higher and stronger lift ; but the stimulus remaining the same, the work done by muscle (i.e., the product of weight multiplied by height) is, up to a certain optimum, increased by increasing the weight. Often a very light load is not lifted as high by 
a nerve-muscle preparation as a slightly heavier one. No satisfactory theory of this reaction to load has yet been formulated. Explanations have been put forward, but they merely substitute one unknown for another, a not uncommon drawback to explanations.

Muscles are strongest when at their full physiological length. As he dips an oar into the water a man exerts the greatest force of which he is capable, provided that he is not guilty of "missing the beginning." Hands over the stretcher, body between the knees, ankle, knee, hip, fully flexed, arms straight -all his strongest muscles are at their greatest physiological length. Rowing is an exercise which has no rival. Every muscle in the body, from little toe to little finger, comes into play under the conditions which suit it best. And not less admirable is the effect upon the abdominal muscles during recovery at the end of the stroke ; and the rhythmic movement which encourages deep and measured respiration.

The greatest output of work is obtained when muscles contract against a progressively diminishing load. Towards the end of the lift the load must be small, if contraction is to be carried to its extreme limit. The provision for this is well seen in the case of the muscles of the arm when lifting a weight up to a position above the head. A portmanteau is held in the hand. Its handle is gripped by flexing the fingers. And here it may be noted that, since the range of movement of a muscle varies as its length, the thumb and fingers are not worked only by muscles contained in the palm of the hand. Fingers are bent and wrist flexed by muscles of which the origin is carried up even to the lower end of the humerus. As the portmanteau hangs by the side, biceps and brachialis are at their fullest length. Suppose it to be necessary to place it on a cab. These muscles begin the work under the best conditions. They could not, however, lift the portmanteau far did not the muscles of the shoulder displace the elbow from the side, so that at the end of their pull, the forearm being almost vertical, the muscles of the arm have little more to do than to move the hand inwards towards the head, in preparation for the extensor thrust. The secret of getting the greatest amount of work out of any particular muscle lies in securing for it the due co-operation of other musoles. 


\section{Electric Organs.}

Muscle disperses energy in the forms of mechanical work, heat and electricity. Its structure, as already pointed out, is peculiarly favourable for the display of electromotive force. In certain fishes muscle is so modified as to give an electric discharge without developing mechanical work. The production of an electric change is a by-phenomenon of muscular activity. It becomes the sole function of an electric organ. If the skin be removed from the tail of a skate, a cylindrical column of brawny tissue about the size of a finger will be found embedded amongst the muscles near its root on either side. These are electric organs, although so weak that it is barely possible to feel the shock which they give in a live fish. The nearly allied Torpedo of the Mediterranean has far more powerful batteries. They are situate near its gills, occupying the whole thickness of the fish from skin to skin. When the back of a torpedo is pressed, it discharges a current of 30 volts, or even more. Still more violent are the shocks given by an eelGymnotus-which haunts the tributaries of the Amazon, a terror to all who have to cross their fords on foot; or the African fish, Malapterurus. The current which these animals develop attains an intensity of 200 volts. With the exception of those of Malapterurus, all electric organs are modified muscle, and closely similar in structure. The organs of Malapterurus appear to be modified glands. The skate's electric organ may be taken as typical of the rest. When sliced with a knife, it is seen to be divided by firm connective tissue into minute chambers. These chambers are piled into hexagonal columns, which lie lengthwise in the organ (they are set dorso-ventrally in Torpedo). Each chamber contains a jelly-like substance which embeds an electric disc. The disc divides the chamber into a smaller anterior and a larger posterior compartment. Each chamber is supplied with several nerves which ramify into innumerable twigs on the front surface of the disc. The development of the disc must be considered for a moment if its structure is to be understood. It starts life looking as if it would grow into a voluntary muscle-fibre. A nerve joins it, forming an end-organ in the usual way. Then the end-organ increases its spread unduly, 
while the rest of the fibre fails to grow. The structure becomes toadstool-shaped, with the nerve arborizing on the seat of the stool. The front aspect of the disc, therefore, corresponds to a nerve-ending in a muscle. Its middle layer indicates clearly that the fibre makes an abortive attempt to develop
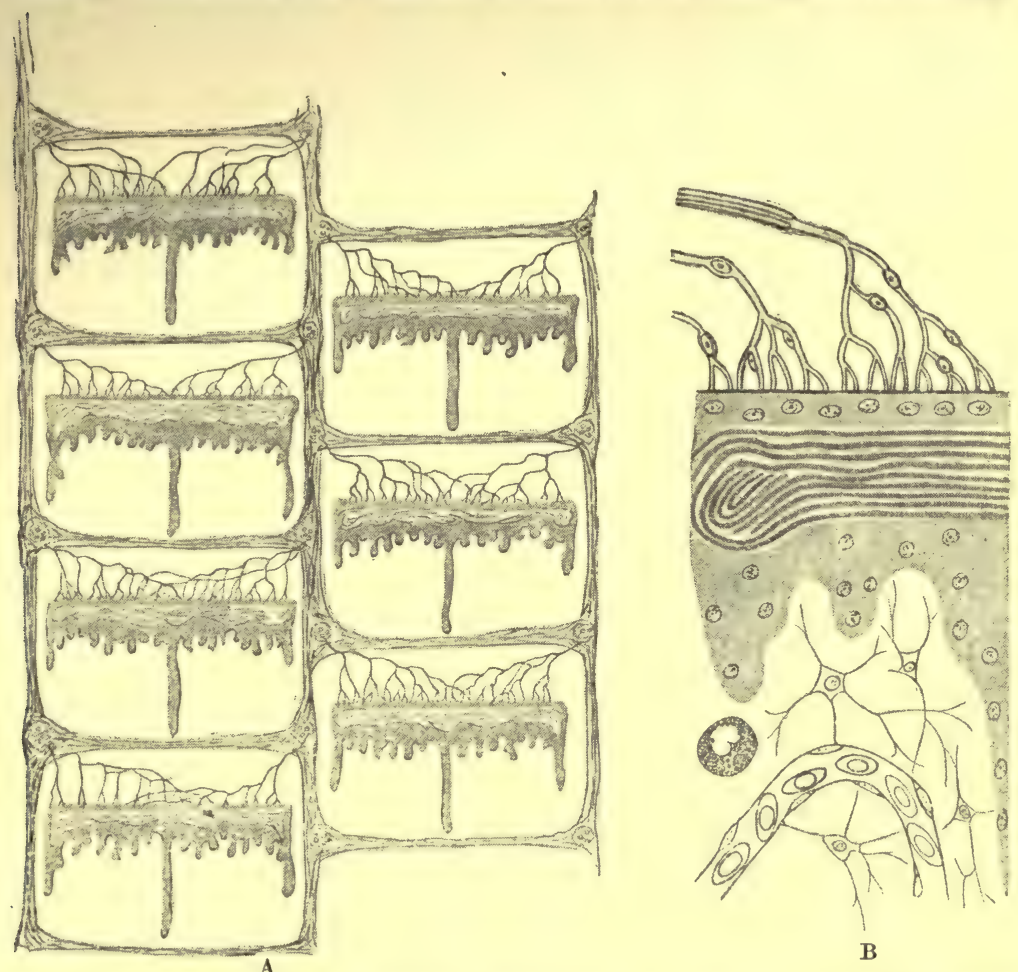

Fig. 18.-Eleotrio Organ of a Skate in Longitudinal Section-A, Slightly, B, Highly MAGNIFIED.

A shows the compartments into which septa of flbrous tissue divide the organ. In the centre of each compartment is a disc formed from a modified muscle-flbre. Nerves ramify in abundance on its anterior surface. B, a minute portion of a disc. At the top are seen nerve-fibres in delicate nucleated sheaths; then follow the nucleated layer with which they come in contact, the contorted laminæ which represent the striations of the muscleflbre, the granular nucleated substance of its posterior layer, some connective tissue, a capillary bloodvessel containing oval nucleated corpuscles. In a tissue space, a single coarsely granular leucocyte is to be seen.

cross-striation. It is laminated, the laminæ strangely contorted ; in section they appear, not as plain lines, but as rows of dots, evidently a suggestion of longitudinal striation. The posterior layer of the disc consists of granular protoplasm drawn out as a number of short backwardly directed tongues, 
and one long process, the stem of the stool. No structure could be more suggestive of the function of the organ; but no one has as yet succeeded in catching the suggestion and pressing it into a definite explanation of the way in which it works. Certain physiologists, laying great stress on the fact that the functional connections between an electric organ and its nerves are not easily interrupted by the administration of curari, atropin, and other drugs, which block the passage of impulses from nerves to muscles, look upon the nerve-layer of the disc as the generator of electricity, and the rest as an accumulator or resonator, which stores, or exaggerates, the electric charge. Others consider that the portion of the disc which is altered muscle-fibre-the middle, or middle and posterior layersgenerates the electromotive force, the nerve simply calling it into activity. All agree that a brief interval (about 0.003 second) elapses between the arrival of the nerve-impulse and the discharge of an electric shock. This "latent period" may be used as an argument in favour of either view. It would be in harmony with the general account which we have already given of protoplasm as a liberator of energy to suppose that a nerve-impulse, having reached a disc, immediately infects the protoplasm of the disc, inducing molecular commotion, and that the ions move in such directions as to disturb the electric equilibrium of the disc, its front surface becoming in relation to the back as zinc to copper in a battery. The current generated in the fish is in the direction from head to tail. It is certain that the change does not occur until an impulse reaches the organ. The organ is not charged by the nervous system during a period of inactivity, and then discharged by a releasing impulse. This is sufficiently evident from the fact that when a piece of the organ, with its nerve, is removed from the fish, although much sooner exhausted, it responds like a nerve-muscle preparation to repeated stimulation.

The usefulness of a torpedo's electric organs is unmistakable. They are powerful enough to paralyse every animal that touches its back, whether foe or little fish suitable for food. But of what service is its feeble battery to a skate? This and the allied question as to the advantages which can have accrued to the ancestors of the torpedo who first began to change innocent muscle into a weapon of offence are 
usually answered by pointing to the liability of flat fish lying on the bottom of the sea to become resting-places of parasites, corallines, and other fixed growths. Very mild shocks would suffice to disturb the peace of would-be settlers In the same way, the electric organs of fresh-water fish may, when rudimentary, have protected the skin from invasion by moulds.

\section{Luminous Glands.}

If it be difficult, when considering the dispersal of energy as mechanical work, heat, or electricity, by living tissues, to bring the phenomena into line with those of which physics takes experimental cognizance, how are we to approach the problems involved in the generation of light? Yet the photogenic property of protoplasm is widely distributed. Protozoans and various other invertebrate animals cause the so-called phosphorescence of the sea. The abysmal depths of ocean are lighted by forests of luminous polyps, and traversed by fishes whose heads are furnished with lamps. By her own light the female glow-worm enables her winged mate to keep his tryst. Fireflies (Lampyrus) flash amongst the orangetrees of Italy, and blaze (Pyrophorus) beneath the mangoes of Ceylon.

Luminous organs vary too widely in structure to allow us to pick out, as in the case of electric organs, the features which are common to them all. In Pyrophorus the organ is a double mass of cylindrical cells near the tip of the abdomen. The cells are set vertically to the surface, and are supported by a tubular membrane. Their substance contains a kind of fat. Beneath them there is a layer of cells, not luminous, but evidently a part of the photogenic apparatus, containing chalky granules. The organ is well supplied with nerves and with respiratory tubes (tracheæ).

More interesting than its structure is the study of the peculiar character of the light which the organ emits. It gives a spectrum which extends from the red (beyond Fraunhofer's line $B$ of the solar spectrum) to the first blue rays (F). It shows no lines. Green rays appear only when the light is bright, and then they are the brightest of all the rays. The light is practically destitute of actinic or chemical rays. A 
photographic plate may be exposed for several minutes, almost without changing, to the light of a firefly bright enough to enable one to read with ease in a dark room ; whereas light of equal brilliance from any other source would change it in the fraction of a second. Nor are heat-rays mixed with the light. Measurements show that the activity of the photogenic organs does not give rise to any greater rise of temperature than would occur in the case of any other gland.

The contrast between the emission of light by an animal and its production in any other manner is very striking when the physical evidence, or want of evidence, of what happens in the protoplasm which produces it is considered. The fact that no heat accompanies the light precludes us from attributing it to oxidation. If a firefly is enclosed in a vessel of oxygen, its lamp burns no brighter-clear evidence that its luminosity has nothing in common with the burning of a match or the glowing of a stick of phosphorus. Nor is the lamp put out when the insect is suddenly exposed to great cold $\left(-100^{\circ} \mathrm{C}\right.$.). It continues to shine until the cold kills it. There is no relation between the luminosity of a firefly and the phenomenon termed "phosphorescence" by physicists. Sulphide of calcium-the substance used for rendering matchboxes visible in the dark-returns light which it has absorbed. A firefly's power of emitting light is in no wise affected by keeping it for a long while in the dark.

Like all other events in vital chemistry, the generation of light by protoplasm is due to a process of fermentation. The luminous organs may be crushed, and the mixture of fermentable substance and ferment extracted with water. The extract is luminous. If an extract is prepared rapidly, and evaporated to dryness in vacuo, the residue glows when moistened with water. That two substances are present in the extract, one (luciferin) fermentable, the other (luciferase) a ferment, is proved by the following experiment: A certain quantity of extract is divided into two portions. One part (A) is allowed to glow until its capacity for emitting light is exhausted. The other portion (B), as soon as it is separated, is heated to $55^{\circ}$ to kill the ferment. B still contains luciferin; A contains luciferase, although all its luciferin has been used up. Recombined, the extract is luminiferous. 


\section{CHAPTER XI}

\section{THE NERVOUS SYSTEM}

TwentY-FIVE years ago a new process was introduced for colouring the elements which by their combination make up the nervous system. With its aid anatomists discovered the inadequacy of their conceptions of nerve-cells. It was already known that a nerve-fibre - that is to say, its essential part, its core-is a part of a cell, the body and other parts of which are situate within the brain or spinal cord, or in one of their dependents, a ganglion. But the new method showed the nerve-cell as more elaborate in form than anything which had been imagined hitherto ; and since the word "cell " was often loosely used when the cell-body alone was referred to, it seemed worth while to give the unit of structure a new name. The term "neurone" was introduced to emphasize its functional individuality. The nervous system is an association of neurones.

By the extremely simple expedient of placing a small block of nerve-tissue in bichromate of potassium, and then transferring it to nitrate of silver, jet-black pictures of nerve-cells are obtained showing with amazing completeness all the details of contour of their bodies and all the intricacies of branching of their limbs. The most surprising feature of the process is the absence of confusion in its results. Dyes were in use which stained one kind of cell better than another, or picked out a particular part-usually the nucleus-of every cell. If the chrome-silver process had acted in the same way, a dense black preparation in which no details could be distinguished would have been the result. But instead of treating all cells alike, the process blackens one cell here and another there, leaving hundreds or thousands untouched. It shows no preference for any particular kind of cell. In one section 
large cells are picked out, in another small ones; in a third no nerve-cells are blackened, but connective tissue is brought into view. When the block of tissue soaked with bichromate of potassium is immersed in a solution of nitrate of silver, the chromate escapes from it into the surrounding liquor much more quickly than the nitrate gets in; and when at last the nitrate of silver enters, it finds that some of the cells have fixed the chromate in their substance. This retained chromate combines with silver. The product is rapidly reduced to a black subchromate. No explanation of the fixing of the chromate by individual cells has yet been offered. It is a remarkable fact that another process which similarly makes choice amongst the elements has since been introduced, giving even more valuable results. Pieces of fresh tissue are placed in a very dilute solution of methylene blue. When staining is satisfactory, nerve-cells alone take up the dye. The selection of individual nerve-cells is not carried so far as it is by the chrome-silver method, but it is exhibited to a certain extent. It is probable that nerve-cells live (in a physiological sense) longer than other tissue elements. Methylene blue contains some easily removable oxygen of which the oxygen-starved nerve-cells take advantage. The reduced methylene blue remains in their substance, so that when the preparation is reoxidized by exposure to air the pattern of the nerve-cells is rendered conspicuous. When a few cells are selected, it is, presumably, because they were the only ones alive at the time when the dye entered the tissue. Preparations made from the wall of the alimentary canal seem to justify this simple explanation. They show patches in which muscle-fibres are stained, patches in which there is no staining, and intermediate zones in which nerve-cells are coloured and muscle-fibres are not. But the hypothesis is inadequate to meet all cases. When first employed, the blue was injected into the animal in successive doses until it killed it. The staining was believed to occur intra vitam. Subsequently it was found that its application to fresh tissue, or, for certain results, to tissue which has been kept for some hours, is equally effective.

Without an understanding of the nature of the two new processes, and of the character of the results which they yield, it would be impossible for the reader to realize the extraordinary 
advance in our knowledge of the finer structure of the nervous system which has marked the period during which they have been employed.

The chrome-silver process is the more useful for the central nervous system. Methylene blue gives better results with tissues containing minute nerve-cells and the branches of nerves. The latter method has revealed such a profusion of nerve-twigs as would never have been suspected but for its use. Consider, for example, the lining epithelium of the lungs (p. 168). Every one of its flattened cells has its own nerve twig or twigs. They lie between the cells. They give branchlets which enter them. A similar statement might be made regarding the richness of the nerve-supply of the muscle-fibres of the alimentary canal, or of the cells of glands, and possibly of other tissues. Each fresh success achieved in the application of the method makes a further revelation of the abundance in which nerves are distributed, increasing our sense of the dependence of all vital processes upon nervous control, and our appreciation of the unifying and integrating importance of the nervous system.

The term " neurone" is used by certain writers with a view to emphasizing their belief, not in the functional individuality alone of the unit of structure, but also in its anatomical isolation. The peculiarity of the methods of coloration which we have described lies, as already pointed out, in their selecting the cells which happen to be in a particular nutritive condition, and ignoring their neighbours. Hence pictures of separate and discrete units are obtained. This proves the nutritive autonomy of the cells, but it does not necessarily follow that $\mathrm{A}$ is not structurally connected with $\mathrm{B}$, and $\mathrm{B}$ with $\mathrm{C}$. Impulses are passed along the chain from A to C. Functionally, therefore, they are linked together; but until the question as to the way in which contact is established is settled, it is as well to think of the neurones as anatomically discrete.

It would be impossible in this book to describe all the varieties of neurone, for nothing is so characteristic of these elements as their enormous range both in size and form. It may be truly described as having no limits. Each of the two electric organs of Malapterurus is governed by a single neurone. Its cell-body is a fifth of a millimetre or more in diameter- 
large enough to be seen with the naked eye-and traversed by capillary bloodvessels. The axon of this nerve-cell-its single nerve-fibre-ramifies to supply a separate branch to each of the 2,000,000 chambers of the electric organ, and each branch breaks up into a bunch of twigs within the chamber. Contrast with such a giant cell as this one of the granules of the retina or cerebellum, the smallest cells to be found in the body, yet each a perfect neurone, exquisitely elaborate in form.

As types for description we may take one of the motor cells of the spinal cord and a granule of the cerebellum. Every nervefibre which supplies a group of voluntary muscle-fibres is a

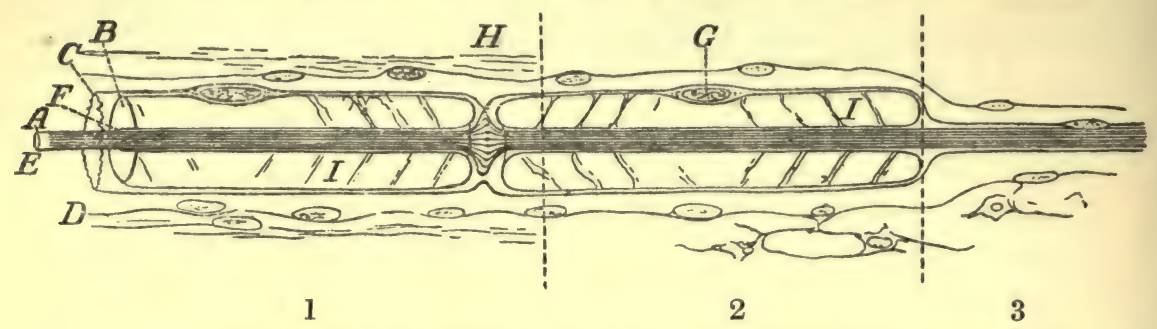

Fig. 19.-A Nerve-Fibre consisting of A, the Undivided, Fibrillated Axon of a NerveCell, with its Various Wrappings.

In segment 1 the wrappings comprise $\mathbf{B}$, a tube of phosphatic fat (myelin), interrupted at $\mathbf{H}$, a node of Ranvier; C, a delicate membrane (sarcolemma) ; D, connective tissue ; E, the rind of the axon; F, a tubular space containing lymph, between the axon and its sheath of myelin; G, nucleus of an enwrapping cell. At $I$ the myelin is seen to be divided into overlapping conical rings. 2, The medullated nerve-fibre, running an isolated course, is merely enclosed in a tube of connective tissue containing lymph. 3, As a " grey " or "non-medullated" fibre, the axon has lost its myelin sheath.

thread drawn out from a large cell-body which lies in the grey matter of the spinal cord or of the axis of the brain. The fibres pass out in the anterior root of a spinal nerve or in a cranial nerve. The cell-body may have a diameter of as much as $100 \mu$ ( $1 \mu=0.001$ millimetre). In shape it is like a very irregular starfish, owing to its being continued into several, usually four or five, thick tapering branching limbs or processes, known as dendrites, in addition to its slender thread-like axon. From its origin in a cell-body to itsdestination in a muscle-it may be a few inches, or it may be a yard away-the axon is an unbroken thread. A short distance from the cell-body it enters a tubular sheath, which protects and insulates it, recalling the covering of gutta-percha in which the wires of a 
telegraph cable are enclosed. The sheath is of a phosphatic fat, invested and held in place by a delicate transparent membrane, neurilemma. Beneath this membrane nuclei occur at regular intervals, and midway between each two nuclei the sheath is cut across by a septum. Such interruptions or nodes show that the sheath is not a part of the nerve, if the term is used in the most restricted sense. Each internode is a cell which has been wrapped round the nerve for its protection. The axon with its sheath is spoken of as a nerve-fibre. A large number of nerve-fibres bound together by connective tissue constitute a nerve. In some cases the axon before it leaves the spinal cord, but after it has entered its myelin sheath, gives off one or two lateral branches ("collaterals"), which return to arborize in the grey matter of the cord. It does not appear that they are always present in the case of the motor neurones of the spinal or cranial nerves-probably they are usually omittedbut collaterals are important features of the large neurones of the cortex of the cerebrum and cerebellum (Figs. 23, 24). Usually two, three, or four such branches start off at right angles from the axon, and after a time turn back towards the surface, dividing into a few extremely slender branches. Their purpose is an enigma. Possibly they bind a group of cells together in functional unison. Such an explanation would seem reasonable in the case of an arrangement of collaterals on the plan we have just described; but in various situations in the brain cells are seen of which the axons, instead of becoming nervefibres, break up completely into collaterals, which branch repeatedly.

By various methods it may be shown that dendrites, cellbody, and axon contain fibrils (Fig. 22). These neuro-fibrillæ lie parallel to one another in the axon. Where it divides they are distributed amongst its branches. Possibly they also branch. In the neurones of Malapterurus, already referred to, this would appear to be inevitable. The discovery of neuro-fibrillæ seemed to carry us a step nearer to a comprehension of the physics of nervous conduction. They clearly indicate that particles of the substance of a nerve-fibre are oriented in the direction in which impulses pass. It is a structural differentiation similar to the fibrillation of muscle, and probably of the same order-a response to the same demand. But when we examine the 
arrangement of the fibrils in a cell-body and its dendrites, the appearances which we discover serve to perplex us. They complicate instead of simplifying our mental picture of the conduction of nervous impulses. The coarsest and most distinct neuro-fibrillæ are to be found in annelids, the ganglioncells of a leech, for example, affording excellent preparations. These cells are pear-shaped, with a single stalk. As is usual in invertebrate animals, they do not exhibit separate dendrites

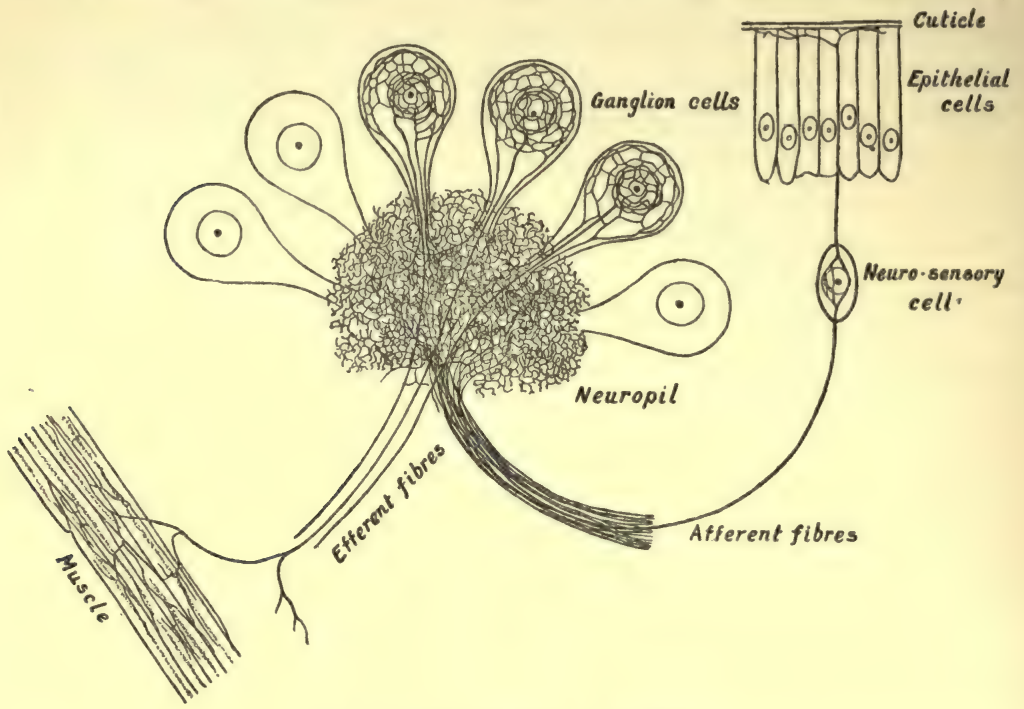

Fig. 20.-A Ganghion of a Leech.

Pear-shaped cells are set round a feltwork of nerve-fibrils (neuropil). A neuro-sensory cell is shown with one fibre directed peripherally, branching on the surface; and one directed centrally, ramifying in the neuropil. Several very slender fibrils from the neuropil pass up the stalk of each ganglion-cell. They join a network near its surface. This net is connected by radiating fibrils with a coarser net which surrounds the nucleus. From the central net a relatively stout flbril passes to muscle-fibres.

and axon, but dendrites and axon pass out from the cell in the common stalk. The bodies of the cells are set round a felted mass of nerve-filaments, into which their stalks break up. Just beneath the surface of the stalk of one of these cells two or three very fine neuro-fibrillæ are to be seen. A single, much coarser fibril occupies its axis. The fine fibrils join a network at the periphery of the cell-body. The thick fibril is connected with a coarser network which surrounds the nucleus. Radiating threads unite the finer with the coarser net. It has been 
suggested that afferent impulses ascend the fine fibrils, pass from the finer to the coarser net, and take their exit by the thick fibril, which can be traced into a motor nerve. Such a transit could not, so far as one can imagine, have any effect upon the distribution of the impulses which pass through the neurone; besides, there are reasons for believing that the course taken by impulses which are delivered to the ganglion by sensory nerves is determined by the feltwork in its centre, the neuropil. It is probable that during their passage through the cell-body impulses acquire the energy requisite to discharge the muscles to which the motor-fibre carries them. In vertebrate animals, sensory nerves are branches of neurones of which the cell-bodies lie in cranial or spinal ganglia. They resemble the ganglion-cells of the leech in as much as they are unipolar; both branches, the one which collects impulses from sense-organs, and the one which distributes them to the spinal cord, come off from the cell in a common trunk which afterwards divides, although the unipolar condition of the cell of the spinal ganglion is not primitive, but acquired. In the earliest stages of its growth the cell is bipolar. Its two ends subsequently grow together for a certain distance, the common portion being the vertical limb of the T (cf. Fig. 21, which shows the growth of a granule of the cerebellum). The body of the cell contains a network not unlike the network of the leech. It is probably related to what may be termed the charge of the neurone, the development of a suitable degree of force in the impulses which pass through it.

The neuro-fibrillæ of a large nerve-cell, such as a motor cell of the spinal cord, are exceedingly slender (Fig. 22). They branch and reunite. A certain number gather towards the axon; but the majority pass through the cell from one dendrite to another, or from one branch of a dendrite to another branch. It is very tempting to suppose that neuro-fibrillæ are connected with conduction. When first discovered they were regarded as conducting strands; but it is evident that they are not comparable with telephone wires or other isolated or separate conductors. There are good reasons for regarding dendrites as collecting processes, taking up impulses from the end-twigs of the nerves which branch in the grey matter around them, passing them through the cell-body into the axon. The continuation of neuro-fibrillæ from dendrite to 
dendrite seems to be irreconcilable with the hypothesis that they are disposed in the lines of conduction.

In common with those of various other types of neurone, the dendrites of spinal motor cells are beset with "thorns." These projections are not rugosities or serrations, but short, delicate threads which stand out at right angles from the dendrites (cf. Fig. 1). About a dozen years ago, the writer made a careful investigation of these structures; at a time when most anatomists regarded them as artifacts. He found that their claim to be regarded as parts of the neurone is as good as that of its axon or its dendrites; although never seen on certain types of cell, the thorns, of cells which carry them, are perfectly definite in arrangement and spacing. In some kinds of cell they are more numerous, in others less. Neuro-fibrillæ, as we now know them, had not been discovered at the date when this investigation was undertaken; but on various grounds the conclusion was arrived at that thorns are the cell-ends of fibrils which pass from the end-twigs of arborizing axons into dendrites. Upon this conclusion was based an hypothesis of conduction which is here submitted, not because there is not much to be said against it-or, at any rate, many a hiatus in knowledge to be filled-but because it happens to be the writer's own. The chrome-silver and methylene-blue methods which reveal the existence of thorns do not stain neurofibrillæ. They colour the soft protoplasm in which fibrils are embedded. By modifying the chrome-silver method in every way which still allows a result to be obtained, it was found that thorns sometimes appear as comparatively long slender filaments, at others as shorter filaments ending in minute knobs, or as filaments bearing two or three dots ; or finally no filaments are visible, but the dots are in the position which they would occupy if fibrils were present, but not stained. From this it was argued that the soft protoplasm which during life surrounds the filament as a continuous film, either falls back towards the cell after death or is made to shrink into the cell by reagents. This accounts for the appearance of rod and knob. What is supposed to happen may bo illustrated by dipping a wire in treacle. At first, when the wiro is withdrawn, it is surrounded with a film. Then the film gathers into droplets. It was suggested that the entrance of impulses into dendrites, their con- 
duction across the space which separates the end-twigs of axons from the dendrites into which their impulses pass, is by means of the thorns, although the thorns are not in themselves conductors. Conduction occurs only when films of cytoplasm surround the thorns. The first effect of impulses is to call out the films, in the same kind of way that a current of electricity converts a row of falling drops into a continuous stream. A succession of impulses, by adding to the number of the filaments which are enveloped in cytoplasm, or by increasing the amount of cytoplasm investing certain groups of filaments, increases the openness of the path. Sleep is a condition in which all paths are open. Hence no impulses are effective. Wakefulness, alertness, depends upon the closing of all paths save those which are actually in use. We may go further. The power of concentrating attention is the power of limiting the spread of nerve-impulses in the brain. Alcohol opens extra paths; the concentrated effort which was making progress with a problem becomes more diffuse. The first effect appears in greater brilliance of thought, gained at some sacrifice of cogency. Unexpected analogies are discovered. Imagination takes a wider range. But as the dose is increased, a condition akin to sleep is set up. Nerve-impulses become ineffective because, many paths being open, they do not attain a sufficient intensity in any set of paths. These few illustrations are given for the sake of showing the need of a theory of the opening and closing of paths. It is not suggested that they favour the particular hypothesis here set forth as to the structural arrangement which provides the paths and regulates their accessibility.

Recent discoveries in the finer structure of the central nervous system have provided many problems which at present appear insoluble. One of the discoveries most difficult to make use of in constructing theory is the existence of extracellular or pericellular nets, which have the appearance of extraordinarily delicate cases of wire-netting immediately surrounding the nerve-cells. It is somewhat remarkable that the spacing of the nets is often very similar to, if not identical with, the spacing of thorns. While some anatomists look upon the nets as nervous, others regard them as pertaining to the connective tissue of the nervous system. At present it is not 
known how impulses get across from the finest visible twigs of arborizing axons to the dendrites of the neurones which they influence. The wealth of structural detail which recent research has revealed is an embarrassment to anyone who tries to devise a scheme. Not improbably, pericellular nets are intermediate factors in the exchange; or, if not the nets, the structures whose existence is indicated by the appearance of the nets. In the case of many of the finer markings which staining methods bring into view, it is impossible to say whether they indicate the presence during life of the structure as it appears to be, or whether the markings are due to coagulation of plasma or to strain caused by shrinkage in coagulating agents. In a sense this is not of much consequence. Coagulation in a uniform pattern would mean the existence of an architectural substructure which determines the pattern. We may be looking at the cake or at the tin the cake was baked in.

There is a danger of seeing too much in a nerve-cell when examining it under the highest powers of the microscope, and of endeavouring to picture in too much detail the arrangements which regulate the flow of impulses. Its markings are so complicated as to suggest to the mind of the observer that it is itself a microcosm-a nervous system in miniature. Neurofibrillæ appear to offer many alternative paths within the cell. It is unlikely that such a way of looking at the unit of structure is the right one. A certain motor cell of the spinal cord is connected by its axon with thirty or forty separate musclefibres; but there is no reason for thinking that the fibres ever contract save as a single group. The axon consists of parallel fibrillæ, but these do not appear to be needed as separate conductors; an impulse travels down the fascicle. It does not appear to be necessary in the case of a motor cell, and presumably the statement holds good for the large cells of the cerebellum and cerebium to picture any arrangement for the simultaneous conduction in its axon of several impulses, or for the conduction of one impulse along one of its fibrillæ and a different one along another. What is necessary is that this particular efferent path $\mathrm{Z}$ should be accessible from every other part of the nervous system-from A to Y. If, merely for the sake of filling the space which would otherwise be blank in the mental picture, we imagine a pericellular net connected 
by thorns with the body and dendrites of the nerve-cell $\mathrm{Z}$, then the net is the meeting-ground of all the routes through which $\mathrm{Z}$ is called into action. A nerve-wave from any of the neurones $\mathrm{A}$ to $\mathrm{Y}$, breaking upon this net, passes along the thorns into the protoplasm of $\mathbf{Z}$.

In size a granule of the cerebellum presents a marked contrast to a motor cell of the spinal cord; yet it is formed on essentially the same plan. From its minute round body (about $8 \mu$ in diameter) four or five slender dendritic processes are drawn out. Each dendrite ends in a little bunch of twigs, resembling fingers curved over the palm. Its single slender axon runs towards the surface of the cortex. As the granules lie at a considerable depth, this course is, for those which distribute to the most superficial layers, a long one. They pass from the granular to the molecular layer between the big cells of Purkinje. When the axon has reached a certain level in the molecular layer, it divides into two threads which run for a great distance, right and left.

The granules of the cerebellum have a curious developmental history. Every neurone in the body has a lifelong existence. Except for the rare accident of its destruction by disease it occupies its station to the hour of death. But at the time of birth many neurones are still immature. Not all the granules of the cerebellum have yet assumed their permanent form or situation. Beneath the pia mater there is still a layer of minute undifferentiated cells. These, as they grow into granules, elongate, in the first instance, into long spindles. Subsequently they sink down through the molecular layer and between the cells of Purkinje, leaving the poles of the spindle as the right and left divisions of the axon (Fig. 21). It is interesting to learn that such a migration is possible. It is also of interest to find that a tiny granule of the cerebellum goes through the same stages in attaining its adult form as one of the large cells of a spinal ganglion.

There are many different types of neurone. Any attempt to describe them, or to give an account of the various details of structure which recent improvements in technique have enabled anatomists to observe, would fill a lengthy treatise ; and would, moreover, be beside our aim, which is limited to obtaining such an idea of the unit of the nervous system as will 
enable us to form a conception, however crude, of the way in which it works. From the brief account that has been given, it will be evident that anatomists are approaching to an understanding of the mechanism. It will also be evident that they have already more information than they can apply. They are cognizant of many details of structure which they cannot interpret in terms of function; and at the same time are aware of wide gaps in their knowledge regarding facts

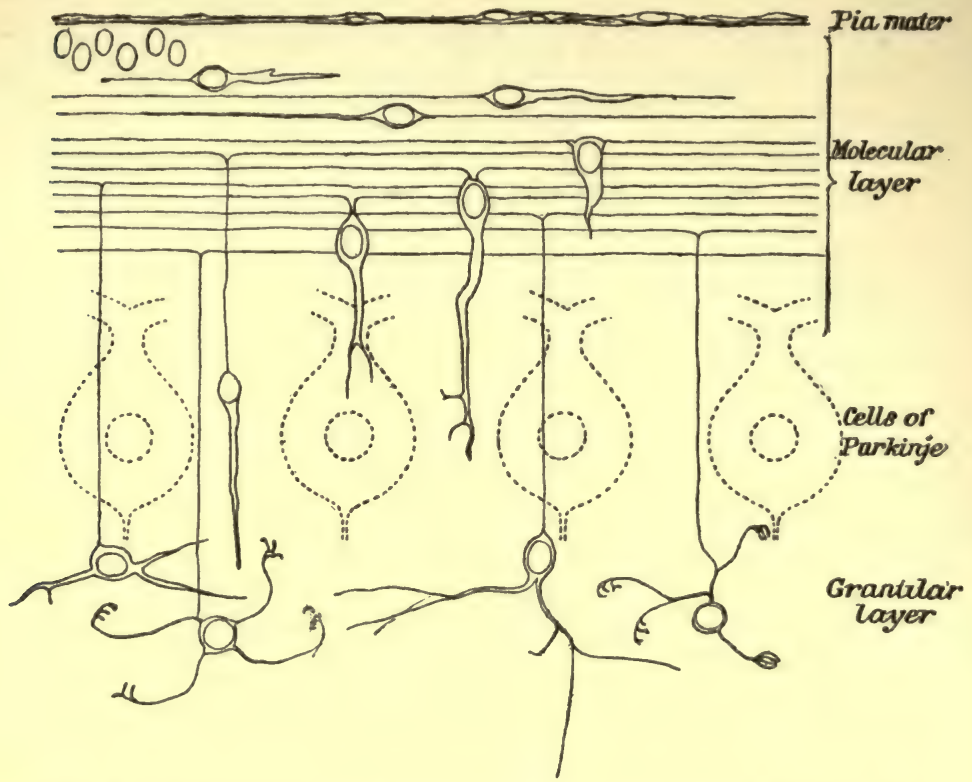

Fig. 21.-The Growth and migration of Grandles of the Cerebelium.

Half a dozen nuclei of as yet undeveloped granules are seen lying beneath the pia mater.

From this level to the bottom of the drawing granules are shown in successive stages of growth. These developing granules, selected from various preparations of the cortex of the cerebellum, were drawn from nature.

which are essential to the construction of any scheme. This much is clear: A sense-cell on the surface or beneath it is touched (probably entered) by the ultimate twig of the outer limb of a neurone whose cell-body lies in a spinal ganglion, while its inner limb, as a fibre of a posterior root, enters the spinal cord. In the spinal cord the root-fibre splits into an ascending and a descending division which rain branches into the grey matter over a considerable area above its point of 
entrance, and a smaller area below it. The finest twigs of these branches are to be seen in the vicinity of the cell-bodies and dendrites of certain other neurones. The axons of these second links arborize in a similar way in the vicinity of large motor cells, whose axons in turn become fibres of anterior roots. (For simplicity's sake no reference is made to hosts of other neurones which link the ganglion-cell and the motor-cell to other cells higher in the cord or brain.) An impulse generated in the sense-cell on the surface of the body runs up the root neurone into the cord, where the ultimate twigs of the posterior root fibre offer it a wide choice of distribution. Following the path of least resistance, it passes into neurone No. 2. Again, the arborization of No. 2 offers it alternative paths. It makes a choice which lands it in No. 3. No. 3 passes the impulse on to the muscle-fibres with which it is connected. Three points are especially worthy of attention: (1) The impulse has a wide (literally, an unlimited) choice of routes. The skin of the finger is touched. Any muscle may respond, although resistance is so graded as to cause the impulse to seek in the. first instance the group of muscles which is most often required to act in consequence of stimulation of the finger. This means, we may suppose, that it follows the chain which, having the smallest number of links, offers least resistance. If it cannot get through to these muscles, owing to the fact that other impulses, acting simultaneously, either increase the resistance in this particular path, blocking its way, or reduce the resistance in an alternative path, it spreads farther afield. (2) Owing to the ramification of the root-fibre which conveys it to the cord, an impulse is not limited to a single line of distribution. It reaches many secondary links. It may therefore influence various effector neurones simultaneously. For example, a stimulus which calls extensor muscles into action, at the same time inhibits their flexor antagonists. (3) The path which it finally takes is accessible to all other impulses. Its root neurone was peculiar to itself. Link No. 2 was more or less a common path. Neurone No. 3 is open to every impulse which traverses the nervous system.

Anatomy justifies the construction of the scheme just outlined. But there are many points regarding structure upon which a physiologist desires information, many details that he 
wants to see filled in. How is the impulse passed from the arborization of axon No. 1 to the dendrites of neurone No. 2 ? By what structural arrangement is resistance introduced, and how is it regulated, if it varies? Supposing the resistance to be higher in one path than in another, or supposing that more force is needed to enable an impulse to invade a wider field, how is additional energy supplied? To the first question no answer can be given at present-the mechanism by which impulses are transferred from one neurone to another is unknown; yet it is convenient to find a name for the junction of axon-endings and dendrites. It is termed a "synapse," on the understanding that the word involves no hypothesis as to its structural nature. It is generally held that resistance is introduced into nerve-circuits at synapses ; although this again is a provisional statement. The phenomena for the explanation of which the idea of synaptic resistance was introduced, may be accounted for on a purely anatomical basis of distribution. The extent to which one neurone influences another may depend upon the size of the brush of fibrils with which its axon touches it. If a certain force is needed to discharge a neurone, a nerve-current must either have a sufficiently high potential when it reaches it, or it must act upon it for a sufficient length of time. There is little to choose between the arguments which place the resistance at the synapse and those which transfer it to the nerve-cell body.

As a mechanism the nervous system is unthinkable, unless we picture its units as independent, yet capable of forming associations; as functionally discrete, yet entering into functional continuity. When acting, they act as chains. Impulses run from link to link, from the end-twigs of an axon of one cell to the dendrites of the next. Neurones are so arranged as to make it impossible for impulses to escape backwards out of dendrites into axon-twigs. In this respect the system is valved. But there is no reason for thinking of the substance of the neurone as polarized in any way. The physical accompaniment of an impulse-the electric variation-travels with equal facility up and down its axon.

There is no evidence of any specificity of neurones; on the contrary, it is clear that impulses of every kind-that is to say, from every source, for we recognize no specificity of im- 
pulses - can travel equally well through neurones of all forms. At every junction, in passing through each synapse, they are delayed. It takes at least 0.01 second (less if the knee-jerk be a true reflex action) for a message delivered to the cord by a sensory root to reach a motor root. This hundredth of a second-the sum of the delays entailed in fording two or three synapses-is regarded as the minimum reflex time. To it must be added, in considering any particular reflex action, the time taken in travelling up sensory and down motor nerves. Delay indicates resistance. If a sensory stimulus be not sufficiently pronounced to provoke a reflex action, the reflex may be obtained on intensifying it. Prolonging or repeating the stimulus-really the same thing, since sensory impulses are rhythmic, not continuous - has a far more potent effect than increasing its force. The resistance of synapses gives way after a number of impulses have bombarded them. The desire of brushing a fly from the skin, if resisted, becomes intolerably urgent after a time. A persistent outflow of impulses produced by the irritation of a spot in the cortex of the brain overwhelms the nerve-muscle system in an epileptic fit. The following is an experiment illustrating the spread of impulses from their customary path to another less often used: A piece of blotting-paper, wet with vinegar, is placed on the inner side of the thigh of a brainless frog. There is no use in trying the experiment on a frog which retains its brain; the substitution of one action for another would be an exhibition of the adaptation of means to end-a demonstration of the animal's right of choice. Besides, the frog might choose not to act, and so the experiment would fail. The brainless frog wipes off the blotting-paper with the foot of the same side. This foot is then fixed so that the action cannot be performed, and the blotting-paper replaced. After a longer interval the frog removes it with its other foot. Evidently it is more difficult for the impulses generated by the irritation which the vinegar causes to get across the cord than it is for them to reach motor neurones on the same side. Evidiently, too, the continued irritation of the vinegar adds to the travelling power of the impulses. They are strengthened until they are capable of overcoming the resistance in the longer path. "Resistance in conductors" and "potential of current" are terms with 
which the study of electricity has rendered us familiar ; but it must be evident from the experiment just described that these terms are not really applicable to nervous phenomena, convenient though they may be for use in an allegorical sense. Holding the foot does not, by any mechanism which we can recognize, switch off the shorter circuit, yet the impulses abandon it for the longer path. There is no evidence of a struggle to free the foot that has been fixed, coincident with the spread of impulses, as they gather sufficient strength to reach the nervous mechanism of the other leg. The right foot not being available, the impulses choose the route to the left foot. Any attempt to explain this in terms of resistance and potential involves the formulation of a number of subsidiary hypotheses; easy to devise, no doubt, but stultifying to the explanation exactly in proportion as they complicate it. Yet the hypothesis of lines of greater and of less resistance (keeping as far away from electrical analogies as possible) is essential to any explanation of nervous phenomena, and is, moreover, justified by the evidence available. There are two causes in chief upon which it depends : (1) The greater the number of neurones in a linear chain, the greater is the number of synapses to be traversed. If $\mathrm{A}, \mathrm{B}, \mathrm{C}$ are in the same circuit, the sum of their resistance has to be overcome. (2) The greater the number of neurones amongst which a nerve-current has to be subdivided, the smaller the charge available for each of them. Imagine $\mathrm{A} \underset{\mathrm{C}}{\mathrm{B}}$ so placed as to divide $\mathrm{B}$ and $\mathrm{C}$, the charge delivered by A between. This arrangement has, probably, an anatomical expression which accounts for the relative ease or difficulty of a path, even on the supposition that impulses do not open out as they advance-do not spread along all the branches into which an axon divides-but keep to a given line. The axon of neurone A divides, to branch about B, C, and D; but its representation in the several pericellular nets (the expression may pass for the sake of the simplicity which it introduces into the picture) is unequal. In the vinegar experiment the impulses delivered to the spinal cord by the root-ganglion neurone $A$ pass to neurone $B$ of the posterior horn. B's axon arborizes more freely about the cell-body of neurone $\mathrm{C}$ in the anterior horn of the same 
side than it does about neurone $\mathrm{D}$ in the anterior horn of the opposite side. Hence the impulses generated by the vinegar stimulate $\mathrm{C}$, sufficiently to discharge it, so long as that road is open, more quickly than they stimulate D. That C should be dischargeable only so long as the foot is free implies that the activity of the neurone is in some way conditioned by its relation with the muscles which it innervates. When the foot is held this relation is interfered with, giving to the impulses generated by the continued action of the vinegar time to overcome the resistance of $\mathrm{D}$.

The simile of the opening up of paths is fairly applicable to the results which follow the use of artificial stimuli. Neurones seem to link up in series under the influence of the impulses which bombard them, popping like fireworks united by a common fuse.

Experimental evidence points to the following conclusions : (1) Resistance is offered at a synapse. This resistance must be overcome before an impulse can get through from neurone 1 to neurone 2. (2) The impulse does not, properly speaking, pass from 1, through 2. It infects 2 , causing it to discharge a fresh impulse. (3) Time is of the essence of this process. Either the impulses head up at the synapse, or, passing through into the neurone, they produce a cumulative effect within it, which provokes it to discharge. (The latter hypothesis, which is the less likely of the two, transfers the resistance from the synapse to the neurone to be infected.) These conclusions are based upon experiments of the following kind: The minimal stimulus which will evoke a reflex action is determined. A stronger stimulus is then applied. The reflex occurs more promptly, and is more pronounced. But on further increasing the stimulus, it is found that the limit of effectiveness is soon reached. The proportional relation of response to stimulus is much less evident than it is when the experiment is tried with a nerve-muscle. Choosing a reflex action easily provoked, the afferent path is stimulated with an electric current interrupted fifty times a second. The impulses which flow down the efferent path to the muscle follow one another at the rate of about ten a second. A column of nerve-fibres within the spinal cord is stimulated fifty times a second. Again, the discharge into anterior roots has the natural rhythm of about ten. The cortex of the "motor area" of the great brain is 
stimulated with a rapidly interrupted current. The muscles which it governs contract with their natural rhythm. The cortex is sliced away, and the stimulus applied to the white matter beneath. A similar result is obtained. Evidence such as this points to an independence of action on the part of the neurones which one can express only in terms of resistance and explosion. But there is another line of thought which leads to the development of a picture of the working nervous system which seems at first sight incompatible with the one that we have sketched. The phenomenon of the knee-jerk (p. 274) reveals a nervous system so intimately linked together, so homogeneous, so mobile, that no event, however trivial, occurs in any part without sending a vibration throughout the rest. Instead of a multitude of batteries enveloped in a labyrinth of wires interrupted by myriads of switches which are crackling on and off, the image of a sheet of water better figures our conception-a material so frictionless that it is a-ripple from side to side and end to end, from the most distant rivulet which feeds it to the farthest trickle in which it drains away. It is a fluid in a state of infinite commotion, the movements of its particles varying in amplitude from tremulous quiverings which scarcely frost the silver of its surface to waves which, breaking on the muscular system, throw it up in heaps. The vinegar experiment seems to demand a scheme of batteries and wires. The knee-jerk points to a continuous conducting medium. Other phenomena suggest the superposition of the two pictures; the conception of a nervous system consisting of a uniform medium conducting, not indifferently in all directions, but with such freedom that from our point of view the paths are infinite in number; and within this conducting medium nerve-cell bodies and their processes which collect and distribute groups of vibrations sufficiently strong in combination to produce visible effects. In order that one of these neurones may be stimulated to discharging-point, the medium by which it is surrounded must be thrown into such a state of agitation as suffices to infect it. The considerations which point to the formulation of this double or superposed scheme are such as follow : The passage of tone-impulses does not appear compatible with the ideas we have formed on other evidence of synaptic resistance and neuronic discharge. They are too 
feeble for such a mechanism. The short "reflex time" of the knee-jerk points to the passage of the agitation up a sensory root to the spinal cord, and through a non-resistant medium to the environment of the motor cells which it discharges, missing the neurone or neurones which intervene in the case of ordinary reflex actions. This is an illustration of the way in which tone-impulses, which we imagine as conducted by the non-resistant medium, pass over into discharges which produce visible effects. Again, the phenomena of inhibition appear to require the supposition of extra-neuronic conduction. Whenever a reflex path is in use, all other paths in its neighbourhood are closed. The passage of impulses leading to a particular reflex action is favoured by the suppression of conduction in its vicinity. When $\mathrm{A}$ is talking to $\mathrm{D}$ through the nerve-telephone, $\mathrm{B}$ and $\mathrm{C}$ are compelled to hold their peace. Inhibition is a phenomenon of universal occurrence. In relation to various actions, it is sufficiently pronounced to be visible in the effects which it produces. A simple experiment will illustrate this. Holding water in the mouth has no effect upon respiration, but during the act of swallowing respiratory movements are suspended. Whilst the swallowing reflex is occurring the respiratory reflex is inhibited. This might be attributed to the volitional control of respiration, and certainly when attention is being directed to the process volition plays a large part. But if a finger is placed on the pulse, it is possible to detect that, during the act of swallowing, the pulse quickens, owing to the suppression of the slowing action of the vagus upon the heart. Here is a case in which inhibition is in no degree a voluntary action. Nor is it of any value as an adjunct to the particular reflex with which it is associated. It is an illustration of the universal rule that activity of any one spot in the nervous system is the cause of the quieting of the surrounding area. Impulses which reflexly check the heart cannot get through the medulla oblongata whilst the swallowing impulses are traversing it. Inhibition has been described as a drainage of nerve-force into the active area. On the structural side it seems to require the conception of an extra-neuronic substance which, agitated in the vicinity of the cells which are to be discharged, is brought to rest around neighbouring cells. The promulgation through the nervous system of the state which, 
when it reaches the centres of consciousness, produces pain also seems to call for an hypothesis of extra-neuronic conduction.

Any reference to pain in a work on physiology needs a few words of preface, since popularly the term "pain" is used in various senses. When I see pink geranium and nasturtiums growing in the same flower-bed, I may exclaim: "It is positively painful." The want of harmony, and at the same time the insufficiency of contrast, of chalky pink and translucent orange, jars my æsthetic sense. Dislikes, however well founded, are ruled out in thinking of the physiology of pain. Further, in defining pain, we must be careful to isolate the real thing, and not to confuse it with sensations which seem to lead up to it. If, putting my finger in a pair of pincers, I touch it as lightly as possible, the first sensation is one of contact ; a little harder, and it becomes a sense of pressure; harder still, and all sense of contact or pressure is lost in pain. It is usual to regard pain as sensation carried to excess. But neither is this physiological. An excessively bright light or an excessively loud sound is disagreeable. It causes a sudden movement for the purpose of avoiding it-just such a movement as one would make if one touched a red-hot poker-but it is not, strictly speaking, painful. Not uncommonly in cases of accident or disease of the spinal cord a sharp distinction is drawn between the sense of touch and the capacity for experiencing pain. Below the injury the patient retains his sense of touch undiminished in acuteness, but no blow, or cut, or burn, causes him any pain. The pain caused by squeezing the finger in a pair of pincers is not, therefore, an excess of touch sensation. Pain begins to be experienced in the skin just when the object applied to it is affecting it to an extent which might do harm. If the point of a needle touches it, it causes pain as soon as the pressure is a trifle less than that needed to pierce its surface. A hot object begins to hurt when the temperature reaches $48^{\circ} \mathrm{C}$. -almost enough to coagulate the tissue fluids. Pain is not a discriminative sensation. If I hold my arm out at right angles, I am conscious for the first few minutes of its weight, and have, besides, some sense of the traction exerted by the muscle of the shoulder. At the end of ten minutes these sensations are merged in pain, and for some time after lowering the arm the shoulder-muscle aches, much as it does in rheu- 
matism. Pain is an effect upon consciousness, which absorbs, engulfs, and therefore obliterates sensation. To use an ancient phrase, "It is less that I feel pain than that I am pain." If we speak of the capacity for pain as a sense, we may call it for the purpose of our present argument the "sense of damage." The nerves of the skin are acutely affected by any agent which is likely to do harm. It is their business to convey to the central nervous system an influence which so affects it as to set up in consciousness the condition of pain. Sensations of damage evoke reflex movements by means of which the part of the body likely to be injured, or the whole body, is removed to a safe distance. It being the duty of the skin to give this warning, a service of nerves sensitive to noxious agents has been developed which scouts in co-operation with the services devoted to the recognition of physical contact and heat and cold (cf. p. 425). If, imagining that the fire has not been lighted, I touch an almost red-hot stove, I acquire quite a considerable amount of information of which I am able to make use. I gain an accurate notion of the situation of the stove, and I put the right part of my finger in my mouth. The skin sends to the brain the ordinary sensations of touch and pressure before the condition of pain is established. In seeking for a definition of pain, we must eliminate the two attributes which have characterized all the forms of stimulation which we have considered up to the present time: (1) The tendency to provoke movement; (2) the supply of information. If I am suffering from a whitlow, the last thing that I am disposed to do is to jerk my finger about. Although it enhances the urgency of skin-reflexes, pain, in general, inhibits movement instead of provoking it. This is well illustrated in pleurisy. So long as a man is healthy he is quite unconscious of the fact that at each respiration the lower part of the lung slides on the lining of the chest-wall ; but commencing inflammation on the surface of one of the lungs causes intense susceptibility to friction, and the pain produces an effect which the man is quite unable to produce by an effort of will; it stops the movements of the chest on the damaged side. Pain is inhibitory, not stimulant. It is not, properly speaking, a sensation. Frequently being mixed with sensational elements, it conveys topographical information; but pure pain approaches 
in quality the nebulous sense of distress of a patient who, when asked where he felt it, replied : "Nowhere ; but there is a deal of it in the room."

Sufferers describe pain in figurative language, as " burning," "stabbing," "throbbing," "aching," and so forth. Two persons afflicted with the same lesion, the same source of pain, use approximately the same terms. Hence we cannot say that pains do not differ in character. But this is not a sufficient reason for assigning any specific quality to pain. It varies in severity, in continuity or intermittence, in suddenness of onset, in the sensations which accompany it, in the emotional tone to which the disturbance of the organ from which it proceeds gives rise, in the tenseness of the part affected and its consequent sensitiveness to a throbbing pulse. All these things make a complex of pain plus sensation, which causes toothache to differ from headache, and both from the pain of burned skin. But they do not give specific qualities to different varieties of pain. This being the case, there is no need to presume the existence of special nerve-endings for the reception of pain, or of a special region of the cortex of the brain for its reception. On the contrary, the evidence is conclusive that the nerve-fibres which serve the more highly specialized senses, which have well-defined connections in the cortex of the brain, do not convey the influence which enters consciousness as pain. It is the innumerable nerves which have no specialized receptors that take up pain. The afferent nerves of the viscera-the vagus and sympathetic-convey no impulses which enter consciousness, so long as the tissues which they supply are healthy. They have no representation in the cortex. The organs with which they are connected (with trivial exceptions, easily accounted for) are absolutely insensitive to injury. Before the virtues of chloroform were known-in the days when, however severe the operation, the patient had to nerve himself to bear it without an anæsthetic-surgeons proved that the liver or the intestines, or practically any other viscus, may be cut or cauterized without the patient being aware that it is being touched. The same is equally true of the brain itself. But if damage in a viscus is set up gradually, its nerves convey to the central system an agitation which has the most pronounced results upon consciousness, and on the way 
profoundly affects the reflex actions which the spinal cord can carry out, and also its capacity as a conductor. Once in his life, perhaps, a man passes a gall-stone; for generations such a thing may not have happened in his family. Yet the man finds that he is provided with a nervous apparatus which conveys to consciousness intensest pain.

It is difficult to think of pain as travelling along nerves in the form of rhythmic impulses, similar to those which produce in consciousness the effects which we have distinguished as sensations. A few lines above we stated that no impulses which affect consciousness normally travel up the vagus or the sympathetic nerve, limiting the term "impulse," perhaps unjustifiably. The vagus conveys an influence which enters our experience, as hunger. Probably other states of feeling for which we have no names, which resemble pain and hunger and their opposites, are set up through the agency of visceral nerves.

Fifty years ago attention was called to the difficulty of finding pain-paths amongst the white tracts (nerve-fibres) of the spinal cord. It is as difficult to point them out now as it was then; but the inference that pain travels up the grey matter has given way to the "neurone theory"; under a misapprehension as the writer holds. Pain travels slowly. If one happens to notice a person who unsuspiciously touches a hot surface, one observes that an interval elapses between contact of his finger with the iron and the exclamation with which he "relieves his feelings." It amounts to more than a second-if the iron is not very hot, to several seconds-whereas the "reaction time" for touch is only one seventh of a second. The slowness of movement of pain through the nervous system can on the neurone theory be explained only on the hypothesis that it travels from link to link along a very long chain of very short neurones. That pain is a state of the grey matter rather than a succession of impulses, and that (within the cerebro-spinal axis) the state is transmitted through an extraneuronic medium, seems a simpler explanation.

The state set up in the segment of the cord in which afferent fibres, conveying pain from viscera, embouch affects its conductivity. It subdues reflex action through the segment, and at the same time facilitates or reinforces the transmission of 
sensory impulses towards the seat of consciousness. This shows itself in the apparent increased sensitiveness of the skin of the area of the surface supplied by the posterior root which joins the segment of the spinal cord into which the pain influence is also being poured. For example, afferent sympathetic nerves from the cardiac end of the stomach join the sixth and seventh thoracic spinal nerves. Other afferent fibres run up the vagus to the medulla oblongata. When the cardiac end of the stomach is diseased, pain is referred to the skin area supplied by the sixth and seventh dorsal roots. The ordinary inevitable stimuli acting upon this area cause pain. Experimental stimuli which elsewhere would be felt as touch or warmth are painful. The impulses to which they give rise pass through pain-agitated segments of the spinal cord. The vagus nerve carries its pain influence to the medulla oblongata. Now, it happens that the sensory nerve of the face-the fifthspreads for a considerable distance up and down the axis of the brain. The fifth nerve in consequence pours its sensory impulses into a region which is pain-agitated by those fibres of the vagus which come from the cardiac end of the stomach. Hence disease of that organ gives rise also to an "illusion" of pain-pains and illusions of pain are philosophically indistinguishable-on the surface of the head. The viscera, having no direct access to consciousness, appear by deputy. When the stomach is distressed, it makes its appeal to the whole body politic for considerate treatment through certain nerves which have the privilege of appearing at Court. The message is misread as coming from the front of the chest- "heart-burn "or from the shoulder, or from the scalp, or from the other skin areas which these nerves serve. When the liver is in trouble, consciousness, having no knowledge of its whereabouts-is it the business of hand and eye to explore another man's liver, or incumbent upon the mind to accept their findings?-infers that the cry comes from the shoulder. Nor have the tissues beneath the root of the nail, or the muscle of the shoulder, or the pulp of a tooth, any direct representation in consciousness; but since the pain-condition in the grey matter converts it into a microphone, messages from neighbouring structures which otherwise would fail to arouse attention, after traversing the pain-segments of the nervous 
system, ring out clearly, and hence the mind locates approximately the "pain" of the whitlow, the muscle-ache, the decayed tooth. Sufferers from toothache are familiar with the phenomenon of the spread of pain from a definite spot to the whole jaw or the whole side of the head, dependent upon the spread of the pain-agitation from the segment of the axis of the brain in which the dental nerve ends to neighbouring segments. Our ability or inability to localize a pain does not depend upon the presence or absence of painnerves, but upon the existence or non-existence of nerves coming from the same organ, or from its neighbourhood, and capable of conveying impulses to the seat of consciousness. In passing through the part of the spinal cord or of the axis of the brain which is disturbed by the influence exercised by a damaged organ, silent impulses acquire force sufficient to render them audible, and combine with the pain to produce a feeling which consoiousness can analyse, to a certain extent. Informed as to its whereabouts by these accentuated sensations, consciousness recognizes a sense of pain limited in its topographical extension.

Sneezing when a bright light falls upon the eye is a curious illustration of the exaggeration of the effectiveness of sensory impulses when they happen to be poured into an agitated segment of grey matter. About one person in every three is affected in this way. A friend of the writer, who was particularly sensitive, rising in the night because he heard his child cry, three times lighted a candle and three times sneezed it out before he could watch the application of match to wick without suffering from a nerve-storm. Some nervous dogsespecially fox-terriers-are very liable to this neurosis. Many persons who do not sneeze feel, when the sunshine stimulates their retinæ, a tickling in the nose. Again the illusion is to be traced to the door of the fifth nerve-the sensory nerve of the whole of the face. The nose is the true tip of the body. Morphologically it is anterior to the eyes. Just as the fifth nerve extends its distribution to the nose, so also its rootfibres extend their connection within the axis of the brain forwards, until they traverse the mid-brain, the primary centre of the optic nerve. A bright light, by stimulating the optic nerve, sets up a commotion in the mid-brain. The ordinary 
every-moment impulses from the nose, carried by the fifth nerve to this region, ought not to appear in consciousness at all ; but owing to the excited condition in which they find the grey matter they assume an importance which does not belong to them, and discharge the reflex action of sneezing, just as they would do had one taken snuff. Several lessons are to be learned from this phenomenon-as, for example, one which cannot be too often impressed, that the impulses which appear in consciousness (or, more accurately, the impulses to which attention is directed) are but a most insignificant fraction of those delivered by sense-organs to the central nervous system. The impulses which give rise to the sensation of tickling in the nose are not exceptional impulses which happened to be started when the light fell on the eye. They were reaching the brain in a steady flow before the agitation of the mid-brain gave to them exceptional force. No consideration regarding the working of the nervous system has a more important bearing than this. We cannot picture to ourselves the activity of the sensory nervous system. Our experience is limited to the scattered sensations which we perceive. Are the sensory nerve-endings incessantly responding to external forces, throwing an almost continuous procession of impulses up each of the millions of nerve-fibres which connect them with the central system? Such a conception is probably nearer to the truth than the conception which we should develop if we trusted to experience. Yet even experienco tells us that an infinity of messages is delivered to the brain, of which consciousness takes no account. Changing trains at a roadside station in France, my attention was attracted by an electric bell on the platform, which was ringing continuously. "Why does the bell ring ?" I asked the stationmaster. "To make known that everything goes well," was the response. "If it stops, something is wrong." "But do you not become so accustomed to it that you cease to hear it ?" "Yes, truly ; it rings day and night. One does not pay attention to it until it has stopped." Sensory impulses generated by the contact of my skin with the chair that I am sitting on are incessantly ringing the bell of consciousness. I should notice them immediately if they stopped. As it is, they do not attract my attention until they ring a little louder than usual, or until 
some particular group, owing to unrelieved pressure, produces a cumulative effect. Another lesson; that the condition of the nervous system, and therefore its conductivity, is determined at any given moment by the sensory impulses which are reaching it. We cannot describe the effect of a bright light as pain, yet it agitates the grey matter, altering its state, in the same way as the nerve-inflow which we recognize as pain. A wet rag on the forehead does not assuage a headache by cooling the brain (cf. p. 106). The headache is "in the scalp." The cool wet rag diminishes the dilation of the bloodvessels of the forehead, and quiets the impulses from the skin which are pouring into a tract of grey matter pain-agitated by the influences ascending a visceral nerve-usually the vagus.

It is necessary to warn the reader that a reversion to the old idea of "conduction through grey matter"-i.e., otherwise than by a chain of neurones-is unorthodox. It is set forth here because it seems to the writer that the various phenomena which have to be accounted for fit in best with the hypothesis of a double path. If evidence of the anatomical possibility of extra-neuronic conduction is asked for, it may be pointed out that the chrome-silver and methylene-blue methods, upon which our knowledge of neurones is based, do not, in the very nature of the case, show that grey matter consists only of neurones and their obvious branches. As they select particular elements of structure, we can never by their use alone know what they fail to show. Attention may also be called to the fact that the same staining process which reveals pericellular nets (p. 301) shows also a structure resembling a network in the substance which intervenes between them. Truly the method is a rough one. It may well be thought that the nitric acid used to fix the tissue may cause strange coagulations with solution of uncoagulated substance ; but, as was remarked with regard to the pericellular nets, regular patterns indicate architectural differentiation. But whether these nets do or do not give hints as to the nature of the conducting medium, there is no difficulty in finding sufficient material, after all the substance entering into the formation of the conducting neurones, as we imagine them, has been accounted for. $E x$ hypothesi, the conducting material is provided by the fibrils of the sensory nerves in their extensions beyond the limits to 
which the deposit of subchromate of silver extends, when the chrome-silver method of displaying neurones has been used. Sensation-impulses enter neuronic chains. The condition which, when it affects the seat of consciousness, is known as pain, progresses up the vertebrate neuropil.

Energy is developed within the nervous system. The force of impulses is adjusted to the resistance which they have to overcome. Stimulation of the millions of twigs of the vagus nerve in the lungs brings about the gentle movements of ribs and diaphragm which constitute peaceful respiration. A crumb of bread touching the mucous membrane of the larynx stimulates a few of the endings of the same vagus nerve. Like an avalanche, the impulses gather head as they advance, causing, not the diaphragm and intercostal muscles alone to do their utmost, but calling into action half a dozen accessory muscles of respiration. It is difficult to account for this reverberation of the messages which clamour for the ejection of the crumb of bread without figuring them as spreading from neurone to neurone, urging each in turn to deliver its maximal discharge.

Neurones are provided with material which serves as a store of energy. In their cell-bodies, including their dendrites, are to be seen coarse granules of nucleo-protein, which, being fitted in between groups of neuro-fibrillæ, assume an angular form. They are known as Nissl's corpuscles, or are termed "tigroids," owing to the spotted appearance which they give to the substance of a cell. If the nerve-cells of birds be examined just after they have alighted from a migratory flight, the granules are found to be few and small. In a bee returning to the hive at evening with its last load of pollen, they are smaller than they were when it commenced its morning's work. They disappear in certain pathological conditions, and under the influence of various drugs; and since their presence is revealed by staining, their disappearance is spoken of as " chromatolysis."

The wasting of tigroids during functional activity proves clearly that nerve-cells do work, in the physical sense. Energy is expended in transmitting messages from receptor to effector, from sensory cell to muscles, from recipient nerve-ending to glands. Have nerve-cells any privileges or duties? Their 
functions, so far as we have considered them hitherto, are automatic, from a mechanician's point of view. Their situation and connections determine the direction in which they conduct, and the degree in which they reinforce stimuli impressed upon the nervous system by the environment, including what may be termed the internal environment, food in the alimentary canal, secretions in ducts, and so forth. Have the

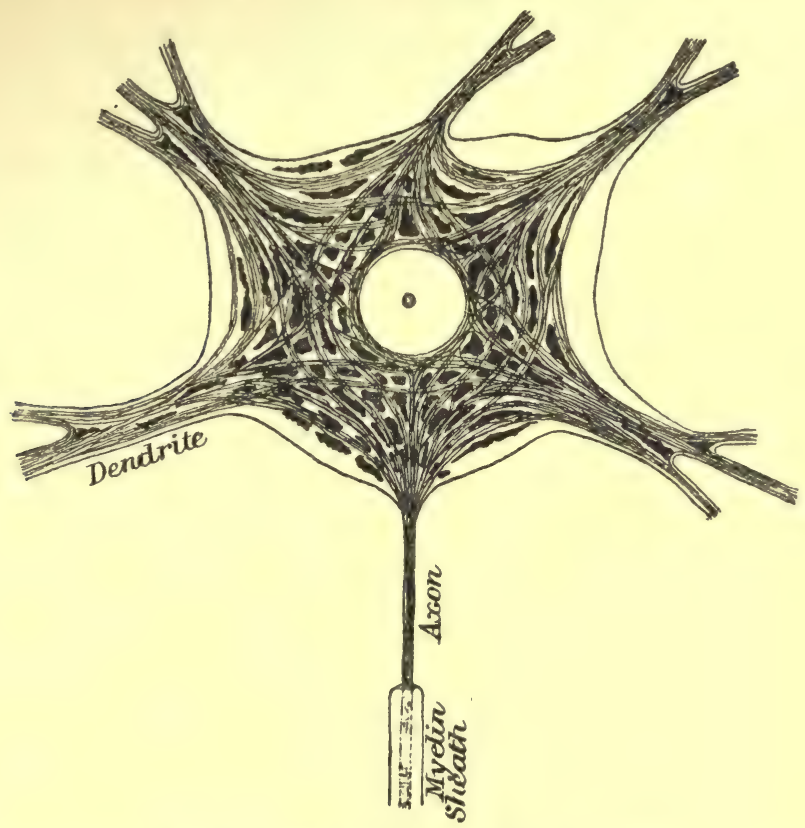

Fig. 22.-The BODY OF A MOTOR NeURONe.

In its centre is a large clear spherical nucleus, with a nucleolus. The body-substance is prolonged into five dendrites and an axon. Neuro-fibrillæ are seen in dendrites and axon. They traverse the body of the cell in all directions, in little bundles which are separated by angular granules of stainable substance (tigroids).

cells any directive or executive functions? There is no evidence that they have; nor, it must be added, is there any line of reasoning which leads inevitably to the conclusion that they have not. Remembering that, until recently, it was the custom to solve all obscure problems and to shelve all difficulties by conferring human attributes upon nerve-cells and collections of nerve-cells, termed "centres," a physiologist admits the negative with reluctance. The unconscious argu- 
ment in the past used to run somewhat thus : "I decide to act or to abstain from action. The nerve-cell is the mechanism by means of which I decide. Therefore the nerve-cell decides.' (In the past a distinction was drawn between the cell-body and its processes, but that, we now see, was absurd.) It is very difficult to relinquish completely this attitude of mind. I feel, I remember, I will. There must be a something which feels, remembers, wills. But a physiologist finds in the nervous system no evidence of a capacity for any function other than that of conduction, with adjustment of the force of current. He can no more discover feeling, memory, or will in a chain of neurones than he can find music in a violin. He hears the strings singing in the breeze. He can twang them with an electric shock. But he has no vision of ghostly performers, no glimpse of the conductor's baton. Yet he knows, as every sane man knows, that the neurones are the instruments played in the orchestra of mind. He knows that, while all are sounding, some are muted, in order that the others may produce a dominant effect. He knows, too, whenever he decides to continue writing or to close his notebook, that the conductor is raising the baton or allowing it to sink by his side.

A neurone or nerve-cell is a transmitting link. It is scarce a thing to wonder at that physiologists, having wrestled successfully with the superstition of the "pontifical nervecell," are unwilling to reinstate it even as doorkeeper in a free church. It may be that it exercises some discretion in admitting impulses, but until its authority as a guardian of the path which stretches behind it has been established, it is better to regard it merely as a door which swings open whenever pressed with sufficient force.

Is it possible to classify neurones according to their function? They can be classified according to size, and, with some degree of completeness, according to form. But if, as we believe to be the case, size and form are governed by purely physical requirements, the divisions into which the cells fall have no physiological significance. The motor cells of the spinal cord and axis of the brain are large and irregular in shape. Their dimensions are clearly dependent upon the size, thickness rather than length, of the nerve-fibres which are drawn out from them. They discharge impulses to groups of voluntary 
muscle-fibres at a considerable distance. Small cells could not do the work. Precisely similar reasons can be given for the large size of the cells of Purkinje in the cerebellum, which transmit the elaborated product, as we may term it, of this organ to the great brain ; and for the dimensions of the large pyramids of the great brain, which convey its decisions to the spinal cord. The small pyramids of the cortex of the great brain distribute the first crude impressions of sensations to neighbouring (association) areas of the cortex. A cell of Purkinje (Fig. 23) has a more complicated, and at the same time a more regular, form than any other nerve-cell. It resembles an exceedingly richly branched espalier pear-tree, set at right angles to the narrow convolutions of the cerebellum ; a disposition easily accounted for, when the structure of the cortex of this organ is considered. Its outer layer in which the espalier processes ramify is traversed longitudinally by an infinity of nerve-threads, the bifurcated axons of granules. These granules are small neurones which take up impulses from afferent ("mossy") fibres, and distribute them to the dendrites of the Purkinje cells-each collecting from a few fibrils only of the sensory channels. (The word "sensory" is used to indicate that sense-organs are their provenance, and not that their messages become sensations.) The numerous spreading branches of a Purkinje cell, disposed in a transverse plane, are obviously arranged to hold up and keep apart these myriads of longitudinal threads. A cerebral pyramid is shaped like a fir-tree. It is placed in a definitely stratified layer. By its branches it collects impulses from the superficial strata, which it transmits through its stem to the white matter beneath the cortex. The various parts of the central nervous system have work of different kinds to do, and we find interposed in the circuits which compose the several parts cells of various types. We speak of the large cells as " motor," the granules as "sensory," the small pyramids as " association" cells-such terms indicating the positions which they occupy in the ares, but not defining their functions. Of specialization of function the physiologist cannot obtain a hint. He cannot classify nerve-cells in groups concerned in reflex action, in feeling, in remembering, in willing, in thought. On the contrary, he can assert with confidence that such distinctions are not to be drawn. 
In various situations in the central nervous system a certain type of cell is found for which, in the present state of knowledge, it is impossible to account. We mention these cells lest it should be inferred, from what has been said above, that all neurones can be fitted into a simple scheme of conducting arcs. In the spinal ganglia there are neurones whose axons divide to form "baskets" around other ganglion-cells. In the cerebellum there are similar cells, the axons of which divide into branches, which break up to encase Purkinje cells. Cells of the same kind are found in a few other situations. In some cases the end-branches which enter into the formation of the baskets are few in number, and thick and clumsy. They grasp the body of the cell which they surround, with gouty fingers, as it were. In other cases the basket is a tangle of fine threads. It is difficult to see what rôle cells of this kind can play in conduction. From the olfactory and optic centres nerve-fibres extend outwards to the olfactory bulb and retina. Here again is an arrangement which does not fit in with any scheme. We might multiply examples. But enough has been said, perhaps, to convey the impression which we wish to leave, that, although experiment abundantly proves that the nervous system consists of an association of sensorimotor conducting arcs, and although anatomical investigation demonstrates the existence of chains of neurones which take part in the formation of such arcs, it is impossible to reduce the system to schemata or to prepare diagrams in which all structural elements are, even hypothetically, fitted into place.

It may be convenient at this point to call attention to the differences which distinguish the sympathetic system - the ganglia and nerves of the viscera and bloodvessels-from the system devoted to bringing sense-organs into connection with the skeletal musculature which we have chiefly considered hitherto. The fibres of the posterior root of a spinal nerve which convey impulses from the skin and muscular senseorgans, and the fibres of its anterior root which convey impulses to skeletal muscles, have a similar diameter of about $15 \mu$. In addition to these, the roots contain fibres which carry impulses from and take them to the viscera. Those which bring impulses from the viscera vary greatly in thickness, some being as large as the other sensory nerves of the posterior 
root. The diameter of the fibres whioh go to the viscera is not more than one-fifth as great as that of the other fibres of an anterior root. Similar slender fibres are found in the vagus nerve. If all organs are removed from an animal's chest and abdomen, a string of small pearl-like ganglia, united by a longitudinal cord, is seen lying on either side of the bodies of the vertebræ, one ganglion for each segment. This string of ganglia is termed the "sympathetic chain" (cf. p. 243). The small medullated fibres of the anterior spinal roots join these ganglia. Some of them arborize about their cells; some pass by them to arborize in ganglia which lie farther afield, on the course of the great bloodvessels and within the viscera. The axons of neurones whose cell-bodies are within a ganglion break up into bunches of non-medullated fibres. In this way the fibres of the sympathetic system are increased in number. Each of its neurones is a multiplying and distributing station. There is no evidence that it in any way serves as a "centre," takes part in reflex action, or otherwise usurps the functions of the grey matter of the spinal cord. Nerve-cells are thickly strewn between the mucous membrane and the muscular coat, and again between the two layers of the muscular coat of the alimentary canal. It is not so certain that this system has no " central " functions. The remarkable degree in which the wall of the intestines retains its capacity for co-ordinated movement, after all nerves which reach it from the ganglia and through the vagus have been cut, suggests that the plexus of nerves within it does act to some extent as a reflex centre. If we leave the case of the intrinsic nervous system of the alimentary canal open, awaiting further proof, there is no reason for looking upon the sympathetic system as in any degree independent of the spinal cord and brain. It does its work on a large seale, and its work is of a low order. Nature does not need to connect up the viscera and bloodvessels with the central nervous system by means of fibres as thick as those used for skeletal muscles. It is more convenient to provide for the multiplication of the nerveswhich must be extremely numerous, owing to the relatively minute size of the muscle-fibres for which they are destinedoutside the central system than it would be to include the necessary distributive cells within it. Again, we find that a nerve-cell, when we see it at close quarters, shows no evidence 
of administrative capacity. Although of a different shape, a ganglion-cell of the sympathetic system is as large and as complex in form and structure as a pyramidal cell of the cortex of the brain; yet the work which it does is of a purely mechanical order. It receives, reinforces, transmits impulses which reach it from the central nervous system.

The often-repeated statement that a nerve-fibre is a drawnout process of a nerve cell-body has prepared the reader to anticipate that it dies when cut off from its central connection. When the axon is dead, the sheath which invests it rapidly loses its tubular character. If the situation of the cell-bodies of a nerve be known, it can be at once foretold on which side of the cut degeneration will occur. Suppose that the median nerve has been severed at the wrist. All nerve-fibres on the distal side of the wound must atrophy, whereas none of the fibres on the proximal side will be affected. The motor fibres have their cell-bodies in the spinal cord, the sensory in the spinal ganglia. Degenerations following lesions in the central nervous system have taught pathologists more about the course of the fibres in the white matter than any other class of observations. Degeneration above the lesion is spoken of as ascending, below as descending-not that it progresses upwards or downwards. It occurs throughout all the stretch of the fibre which has been isolated from its cell-body at the same time, or nearly so. The thought that impulses can no longer ascend or can no longer descend, as the case may be, has given sanction to the expressions " ascending" and "descending" degeneration.

Restoration to functional activity of tracts of fibres which have degenerated in the brain or spinal cord never occurs, but severed peripheral nerves regenerate. Not that fibres join cut end to cut end, however clean the wound. A wound in the wrist which has divided the median nerve may heal in a few days " by first intention," so far as other tissues are concerned; but the patient does not for two or three months recover the power of using the muscles of the hand which the nerve supplied or the sense of touch in the area of skin to which it was distributed. The ends of the axons on the proximal side of the wound have to grow downwards to establish new connections in the muscles and in the skin. 
The interval which elapses between the healing of the wound in the wrist and the restoration of sensation and power of movement is occupied in their downgrowth.

The re-connection of regenerated nerves with their terminal apparatus presents to the mind a curious problem. There is no evidence that as function is re-established the brain has to re-learn the situation of the sensory spots on the skin, or to re-acquire skill in using the muscles which again come under its control. From the moment that the outgrowing nerves have recovered their terminal connections skin and muscles have their right representation in the brain, however much the two cut ends may have been twisted in their relation one to another. It seems inconceivable that each nerve-fibre can find its way to its original station ; but if it does not, our conception of the mode of working of the nervous system still needs much refining from the telephone-exchange analogy by which we naturally help out our explanations. If a telephone cable has been severed, it can be made useful again only in one of two ways. Either the two segments of every wire that has been cut must be reunited, or the subscribers' numbers must be redistributed.

The experiment of uniting the proximal segment of one nerve with the distal segment of another of a quite different function gives results which have an even more disconcerting effect upon our theory of the nervous system. The sympathetic eord of the neck and the vagus nerve lie very close together, alongside the carotid artery. The vagus is both afferent and efferent. The sympathetic is wholly efferent-i.e., it conducts impulses, which enter the sympathetic chain within the thorax, in the direction of the head. If both nerves are cut, and the end of the vagus turned round, so that it is in apposition with the upper end of the sympathetic, its regenerating fibres make their way along the sympathetic cord, headwards, to the superior cervical ganglion. They arborize about the bodies of its ganglioncells, just as the sympathetic fibres used to do. The vagus is a nerve of many functions. Amongst others, it inhibits the contraction of the heart, constricts the bronchi of the lungs, dilates the bloodvessels of the intestines, and helps in regulating the movements of these viscera. After it has taken the place of the upper segment of the sympathetic it dilates 
the pupil, constricts the bloodvessels of the ear, erects the hairs of the head, as if to the manner born. To take another example, in a monkey the two nerves supplying respectively certain flexor and certain extensor muscles of the forearm were cut, and their ends crossed, so that flexor nerve-fibres grew down to extensor muscles, and extensor fibres to flexor muscles. There was no bungling of reflex actions or of voluntary actions when the new roads were first used. The monkey did not jerk its hand open when it tried to scratch or to grasp a nut.

When experimental data first began to accumulate, physiologists drew diagrams and made models of the nervous system in which they represented it as composed of conducting arcs. The arcs were superposed to indicate that they were of various grades-spinal for ordinary reflexes, bulbar for co-ordinated actions, through the grey matter in the centre of the great brain for "ideo-motor" actions, through the cortex of the great brain for voluntary acts. They spoke of authority and responsibility, comparing the nervous system to an army or a club. It is premature to attempt a theory of the nervous system compatible with recent discoveries regarding its structure and mode of working, but it is clear that the diagrams and metaphors to which we have just referred were misleading. In place of attempting to disarticulate the machine, we ought to emphasize its structural unity. The results obtained by uniting heterologous nerves cannot be explained by reference to a model made of wires and pieces of cork. They do not fit in with any organization of human units or with any postal system or telephonic apparatus for transmitting news. Probably the lines of thought which will prove most fruitful are somewhat as follows: (1) An efferent discharge occurs as the result of the opening of a circuit from a muscle back to the muscle. Afferent impulses-call them sensory, on the understanding that this does not imply that they appear in consciousness-are ceaselessly flowing from receptors to effectors in the muscle. A sensation-in the case of skeletal muscles usually a skin sensation-reinforces them to dischargingpoint. If the spinal cord has been severed from the brain, the up-and-down flow does not reach beyond its grey matter. It is short-circuited. If the brain is in normal connection with the spinal cord, sensory impulses travel upwards to its cortex (with- 
out, save in exceptional instances, arousing consciousness, or, as we should prefer to express it in this connection, without attracting attention) to a degree which varies with the several classes of receptor and with the animal. A monkey reduced to the condition of a "spinal animal" -i.e., with its spinal cord severed from its brain-is less competent than a dog, and a man is far less competent than a monkey. In other words, a man habitually uses his brain more than does a monkey, and a monkey more than a dog. The proportion which brain-weight bears to body-weight roughly indicates the part the brain plays in conducting the traffic of the body. (2) Communication within the nervous system is almost unrestricted. If, before the median nerve was divided at the wrist, receptor A usually initiated a current which passed through the circuit to effector $\mathrm{X}$, and receptor $\mathrm{B}$ to effector $\mathrm{Y}$, and if the new fibres which grew downwards lost their way so that the one which used to receive messages from $\mathrm{A}$ attached itself to $\mathrm{B}$, and the one which used to transmit commands to $\mathrm{X}$ attached itself to $\mathrm{Y}, \mathrm{A}$ is not thereby cut off from $\mathrm{X}$, or B from $\mathrm{Y}$. Such a mechanical association is restricted to our diagrams. It does not enter into Nature's plan. The spinal cord is not scored with unchangeable paths. A messenger from A could always reach either $\mathbf{X}$ or $\mathbf{Y}$. It was not the path, but the struggle with competing messengers, which directed him to $\mathrm{X}$.

When we endeavour to picture the mechanism of the nervous system, we find ourselves faced by phenomena which appear irreconcilable. One set of observations leads to the conception of closed paths; another set points to an open conductor. The experimental crossing of nerves to which we have just alluded shows that the nervous system is adaptable, to a degree which seems extraordinary to anyone who attempts to compare it with any of Man's devices for establishing communication. Paths appear to make themselves. On the other hand, the more important, and therefore dominant, reflex actions, such as swallowing, breathing, the maintenance of position, are due to the union of receptors and effectors by lines which are either reserved for their sole use, or, if shared by other currents, it is on the understanding that they have a first and altogether prepotent claim. No competing impulses can divert them or block their way. All reflexes which in the 
history of the race have established their right to dominance not only seize and hold a route through the nervous system, to the exclusion of all competitors, but, as we have already shown in the case of the swallowing impulse, the traffic in neighbouring routes is suspended for their benefit. At the other end of the scale we find reflexes which may be termed "occasional," in that, although of frequent occurrence, they exhibit illimitable variability in form. Occasional reflexes require, as a preliminary to their transmission, that the afferent impulses which give rise to them should secure for a time the exclusive use of the motor neurones by which they are carried out. The receptors bring the motor neurones into tune with themselves, and while in tune they will respond to impulses from no others. But the tuning lasts for a short time only. Either receptor or neurone, or both, soon tire. There is no danger of a particular reflex being prolonged to the detriment of the organism as a whole. As an illustration of an occasional reflex, we may cite the scratching movement of a dog. Its skin is punctured by a flea. It scratches the place. A second flea bites it somewhere in the same neighbourhood. The dog does not shift its hind-foot so as to scratch midway between the two bites. It finishes out one scratch before paying attention to its second tormentor. The exact position to which the hind-foot is raised depends upon the position of the irritant; and since this may be shifted over a very considerable surface, the form of the reflex varies equally widely. Each of the very numerous receptors in the skin tunes a slightly different group of motor neurones; and since a second irritant may reinforce the first, instead of making an alteration in the group of neurones which the reflex is discharging, it is clear that there is no fixed path uniting receptor A with neurones $\mathrm{X}, \mathrm{Y}, \mathrm{Z}$ and receptor $\mathrm{B}$ with neurones $\mathrm{W}, \mathrm{X}, \mathrm{Y}$. If, however, the second irritation occurs at a spot lying at a considerable distance from $\mathbf{A}$, in place of reinforcing the scratching movement which A has set going, it weakens and shortens it. The receptor $\mathrm{C}$, which is calling for the discharge of a markedly different set of motor neurones, tends to inhibit those which are already active. These results are tested with precision upon a "spinal dog" and with the aid of an electric needle, the other pole from the battery being a large flat plate placed in contact with the 
animal's body. The conception of definite paths, to which the contemplation of permanent reflexes gives rise, is inappropriate to occasional reflexes. The latter show so wide a range of variability and adaptability as to prove that a given receptor may bring any of a great variety of groups of motor neurones into connection with itself ; just as a given group of neurones may be played upon by impulses from a great number of different receptors. We have called it a tuning of the motor neurones. One metaphor is as good as another. The physical process which in the brainless frog underlies the preparation for discharging motor neurones in the spinal cord, on the same side as the leg on which vinegar is placed, so long as that leg is free, and on the opposite side, when that leg is fixed, is unknown. We seem to catch a glimpse of a doubleness of action, receptors in the muscles combining with receptors in the skin in determining the paths along which impulses shall be reflected-the efficient muscles sensitizing their own neurones to the tuning influence of impulses from sounding cutaneous nerve-endings. But it is impossible to formulate a working scheme in the present state of knowledge.

Sense-Organs and Nerve-Centres.-A vast amount of labour has been devoted to the study of the external form of the central nervous system and to unravelling its internal structure ; to plotting out its various groups of nerve-cells, to disentangling its innumerable tracts of fibres. The surface of the brain and spinal cord has been mapped and measured. Every millimetre of its substance has been cut into sections on the microtome. Organs which, fifty years ago, appeared too complicated for investigation have been described in the minutest detail. An immense accumulation of data is available for purposes of reference; yet anyone who submits the theory of the nervous system as it is held at the present day to a general review must allow that the results of anatomical research enter but little into its construction. The reason for this is not far to seek. As knowledge has advanced, the apparent, or rather the expected, complication of the system has given place to ideas of unity and simplicity. Its external configuration and the varied arrangement in "nuclei" of its nerve-cells may, without impropriety, be described as accidental. The form of the body and the consequent location of the clients of the nervous 
system determine the disposition and degree of concentration of its various business centres. It shows, when followed throughout the whole animal kingdom, extreme variability of its constituent organs, with absolute uniformity of plan. Indeed, from the physiological point of view the term "organ " is scarce admissible. It implies diversity of function in too high a degree. The several parts into which the central nervous system is obviously divisible co-operate so intimately as to preclude us from thinking of them as separate organs.

If the citadel of the central nervous system is to be captured, all lines of approach must be tried. Its outward form must be studied, its minute structure examined with the microscope, its modifications in various animals compared, its development followed, its reactions to artificial stimuli tested, its pathological deficiencies and vagaries watched. Yet, of all the means which have been made use of in attempting to penetrate its secrets, the study of its history, by the methods of comparative anatomy and embryology, has probably contributed most to the development of sound ideas regarding the manner of its working. The first differentiation visible in the blastoderm-the globe of cells into which the ovum divides and out of which the embryo is built-has relation to the formation of the nervous system. If the earliest stages of its growth are followed, and the different phases through which it passes are compared with the forms which it assumes permanently in lower animals, the plan or type upon which it is constructed shows up distinctly. Looking down the line to the earliest vertebrata, we can discern clearly the form of nervous system possessed by their prototype. Not that this "ideal ancestor" ever existed. Experience teaches that it is unlikely that any animal that ever lived was absolutely regular and symmetrical in all its parts; nevertheless, the type can be presented in a perfectly regular scheme. The ideal ancestor of the vertebrata was segmented, like a caterpillar or a worm. Its mouth was not at the anterior extremity of the body, but two (or more) segments behind it. Every segment bore a sense-organ (at one period two sense-organs) on either side. Beneath each sense-organ there was a clump of " grey matter." Each segment also contained (although not at the earliest epoch) two clumps of nerve-cells and neuropil in 
a more central situation. These "ganglia" were united by longitudinal and transverse commissures. They received the axons of the cells which lay in the clumps beneath the senseorgans. They gave axons to various muscles. Such is the type out of which the modern nervous system has developed: two separate sense-organs and a complete nervous system for each segment, the sense-organs connected with the ganglion of the same side, the ganglia of the two sides bound together across the middle line, and each row of sense-organs and each row of ganglia united by longitudinal commissures into a chain. From the nervous system as we see it now the majority of these segmental sense-organs have disappeared; but the mode of formation of the cerebro-spinal ganglia shows that they are the clumps of nerve-cells which lay beneath the vanished organs. In the nose and the eye the grey matter retains its original situation in the immediate vicinity of the receiving epithelial cells-as the olfactory bulb and the deeper (anterior) layers of the retina. The ganglia of the auditory nerve lie within the bones of the ear. Spinal ganglia are close to the spinal cord. Auditory and spinal ganglia contain only the cell-bodies of the first collecting neurones (sensory nerves) together with certain curious bracketing cells already referred to (p. 324), all the other constituents of the peripheral clumps of grey matter which are found in the olfactory bulb and retina having been withdrawn from the spinal ganglia into the axis of the brain and spinal cord.

The sense-organs in front of the mouth have had from the beginning an immense advantage over the others as observingstations. Whereas the body-organs collected information regarding the things with which the animal came in contact, and consequently specialized in touch, pressure, temperature, and, in the case of fishes, sensitiveness to the chemical constitution of the medium in which the animal lived, the head-organs specialized in responsiveness to forces acting from a distanceparticles suspended in air, vibrations of light, pulsations of sound. Sensitiveness to touch, if it is to be useful, must be widely distributed. The body-organs therefore broke into scattered groups of sense-cells. Touch-spots are scattered all over the surface, although they are set much closer together in the areas of skin which are usually the first to come into 
contact with external objects than they are elsewhere. The efficiency of the sense-organs of the head-nose, eye, and eardepended upon their remaining compact. Progress in animal life, as we understand it - the rise from lower to higher forms -has depended upon increasing integration of the body and co-ordination of its functions. The nervous system is the agent which has accomplished this unification. Each step in advance has depended upon the provision of more nervetissue for the lacing together of the various parts. We have seen already (p. 329) how intimate is the union of receptors and effectors of every kind via the spinal cord and brain. The overwhelming predominance in the direction of action of the nose, the eye, and the ear has led to the accumulation in their vicinity of the ever-increasing grey matter. The cerebral hemispheres, or "great brain," are pouched outgrowths from the first pair of ganglia directed towards the olfactory pits. The original eyes bore a similar relation to the second pair of ganglia-the epithet " original " implying that the eyes which we now use are not the organs with which our prevertebrate ancestors saw. First one of the original eyes disappeared, and then the other. The vestige of the second is still to be seen in the "pineal body" which is found on the dorsal side of the brain of every vertebrate animal-in a mammal deeply hidden in the cleft between the cerebrum and cerebellum. In place of the pineal eyes two other sense-organs have specialized as eyes. They are constructed on a different plan, being, to put it shortly, pineal eyes turned inside out ; for whereas in the pineal eyes, as in most of the eyes of invertebrate animals, the rods and cones, which are the cells of the retina sensitive to light, are directed forwards towards the lens, the rods and cones of our permanent eyes are directed away from the source of light. This change has made it possible to provide more abundantly for their nutrition, and hence a greater power of discriminating separate points in space and of distinguishing colours is conferred upon them. The substitution of other sense-organs for the original eyes has complicated the pictures which are presented to us by a brain in its successive stages of growth ; but it does not prevent us from recognizing the general plan. Probably the secondary eyes, like their predecessors, belonged to a pre-oral segment. The sense-organs of a segment behind the mouth 
developed into ears ; and the ear was in its earliest phases, and still is, something more than an organ of hearing. Its semicircular canals give information of displacements in space. Knowledge of the position of its body is, to a fish, of far more importance than its ability to hear breakers on the rocks. Three looped tunnels, opening at either end into a common chamber, are hollowed in the bone which contains the ear (cf. Fig. 38). Placed at right angles one to the other, they occupy all three dimensions of space. Open a notebook until, one of its covers lying horizontally, the other is vertical, and place a sheet of paper vertically against the bottom of the pages. A curved line drawn on each of these three surfaces will represent the three semicircular canals. Arrange another notebook in the same way, and let the two rest on the table with the two vertical covers inclining one to the other, anteriorly, at an angle of 90 degrees. The six surfaces will be in the planes of the six semicircular canals. Within each bony canal is a membranous tube, to which nerves are distributed, filled with fluid. When the position of the head is changed, the fluid within the membranous tubes slides on their walls. It is left behind at the moment the movement commences. It overtakes its receptacle when the movement stops. The stimulus received by the nerve-endings is recognized as indicating an alteration in the orientation of the head. If the movement of the fluid is violent, as when one waltzes, the loss of the sense of position disconcerts the brain to such an extent that giddiness results. For a time the quiet assurance upon which so much depends, that one knows how the body stands in relation to its surroundings, gives way to a chaos of sensations. From the nature of the case, the information which the semicircular canals afford relates to change. They give no help in ascertaining the position of the head when it is at rest. This must be the reason, although the connection is not very clear, for the waning of the effect in consciousness when stimulation is prolonged, and also for the very marked after-sensation. At the commencement of a voyage attention may be unpleasantly attracted to the rolling of the ship. After a few days it ceases to be noticeable ; yet when the voyager, the night after landing, wakes in the dark, he finds his bed-room as unsteady as his cabin. Rising hurriedly, the attempt to adjust his position to 
the heaving floor (we speak from personal experience) may result in a heavy fall. Although this phenomenon must be classed with other " after-sensations," it is so prolonged as to suggest that consciousness, having become accustomed to a world which causes a backward and forward flow of endolymph, misinterprets the absence of sensation as indicative of change.

Taste is, practically, a special kind of smell. A fish's olfactory membrane, taste-buds, and chemical organs "of the lateral line". serve the same sense, although, no doubt, they are applicable to the analysis of different forms of matter in solution.

Our ideal prevertebrate has now left its primitive undifferentiated condition. In front of its mouth it bears organs with which it searches the world. Close behind the mouth are its auditory and orienting organs. The rest of the surface of the body is endowed with the capacity of recognizing "taste," temperature, and contact. Smell, sight, and orientation determine the development of the brain.

The cerebrum which has eventually become, as the seat of consciousness, and hence the apparatus of mind, the dominant factor in the nervous system, was in the first instance the part of the brain concerned with the distribution to the muscles of impulses generated in olfactory organs. There is scarcely any indication in a fish's brain of the representation in the cerebral hemispheres of any other sense, even that of vision.

A bird's brain presents a striking contrast to the brain of a fish. With the exception of the apteryx and other groundbirds of New Zealand, all birds are apparently destitute of the sense of smell. Vision is the sense upon which their activity depends. It has invaded the cerebrum, converting it into an organ in which sensations of sight are worked up into " mindstuff." The optic lobe connection is restricted to the production of reflex actions in which vision is immediately followed by movement.

All the senses are represented in the great brains of mammals. The cerebrum, which owes its existence to its connection with the favourably-situated sense-organ of the nose, and grew in importance when vision invaded it, has now taken in the senses of hearing, taste, and touch. Only what may be termed in general visceral sense, and the sense of orientation, are excluded.

Looking back to the starting-point, we see a segmented 
animal; its segments of equal value; its nervous reactions unisegmental, although linked in functional sequence. If it starts to walk, owing to stimulation of one of its sense-organs, the impulse to walk spreads from segment to segment. Comparing the latest product of evolution with the earliest, we find that nervous tissue has concentrated at the anterior end of the body. The double chain of ganglia, now condensed into the axis of the brain and the spinal cord, still contain all the effector neurones by which muscles are called into action. Sensory nerves still arborize in the axis, providing the mechanism for actuating motor neurones. But the vast majority of intermediate or intercalated neurones have been attracted to the two huge brain-masses-the cerebellum and cerebrum. In the former all sensations (not conscious) connected with tone, position, orientation and equilibrium are worked into appropriate impulses for the regulation of the muscular system. In the latter all sensations which convey information regarding the relation of the environment, including the body, to the ego-the not-me to the me-are transformed into motor discharges which set a-going the movements (and the thoughts) by means of which the purposes of life are fulfilled; for in the cortex of the great brain alone is the passage of nerve-currents accompanied by consciousness. Concentration of nerve-tissue allows of the combination of sensations. It also facilitates the no less important effect of mutual influence, interference. Sensations are suppressed, and therefore the multitude of reactions to which they would give rise are inhibited, in the interests of restricted and sustained. movement or thought.

The Cerebellum.-Sharks and other swift-swimming fishes have large, deeply fissured cerebella, for the cerebellum is the part of the brain which has gathered into itself most of the grey matter associated with balancing, attitude, posture. The cerebellum is in birds large and deeply folded. Developed. from the ganglia to which the auditory nerve distributes impulses from the semicircular canals, it has established connections with all the other nervous tissues concerned with sensations of position, strain, or pressure, including the eyes, which afford information regarding the position of our limbs relatively to the trunk, and of the whole body relatively to 
external objects. Morphologically it is a median growth. The adverb is one of those qualifying terms, convenient in science, which direct thought without confining it. As used above, it implies that anyone who passes before his mind the cerebella of all animals from fishes to Man, and in all stages of growth, from their earliest appearance in the embryo to their condition in the adult, sees the organ as a median prominence surmounting the medulla oblongata. The bulgings of its sides which, in human anatomy, are termed hemispheres, do not disturb its central, unpaired plan of structure. It has, it is true, a lateral appendage on either side (the combined flocculus and paraflocculus of mammalian anatomy), but this lobe, although of great historic interest, is so small, as compared with the median growth, as not to affect our general conception of the form of the organ. By transverse fissures the cerebellum is divided into a series of lobes.

In appearance the cerebellum varies greatly in the different classes and orders of Vertebrata. Yet underlying this variety there is marked unity of plan. A sagittal section of the organ of a shark, of a bird, of a kangaroo, of a dog, of a whale, of Man, shows that it is divided, from before backwards, into the same number of lobes in animals occupying every position from the bottom to the top of the vertebrate scale. A very little effort to grasp the significance of this mystic number, nine, convinces one of the hopelessness of any attempt to correlate the form of the cerebellum with the muscular development or sensory endowments of vertebrates as a sub-kingdom. It is the same for animals with limbs and animals without; animals with well-developed noses or eyes, and animals destitute of one or other of these sense-organs. This uniformity is extremely significant, when contrasted with the wide differences exhibited by the cerebral hemispheres. It shows that, unlike the great brain which mediates between the several senses and the muscular system, the little brain is concerned in bringing about adjustments to the environment which are equally important to all animals, no matter how far they may depart from the common type. The cerebellum is crossed by deep fissures, dividing it into narrow convolutions or folia. The folia are grouped in nine lobes. If the reader has secured as an illustration the brain of a sheep, he will notice that the 
lateral regions of the cerebellum present a complicated appearance owing to the contortion of the folia, which results from the unequal development on its sides of the several lobes. In its total size the cerebellum keeps step with the cerebrum, the right side of one organ being associated with the left side of the other.

A

B

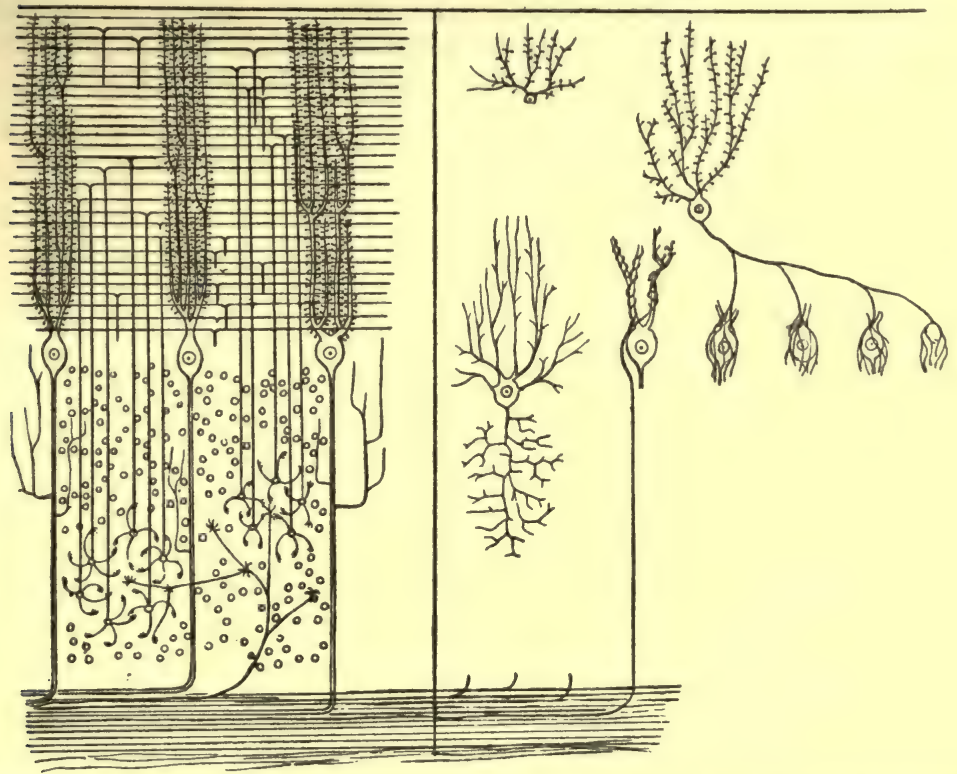

Fig. 23.-Vertical Section of the Cortex of the Cerebellum, cut Parallel with the LONG AXIS OF A FOLIUM.

A shows three cells of Purkinje, their espalier systems of dendrites being seen in profle. A "mossy fibre" enters the granular layer from the white matter. About a dozen of the granules are shown, each with four or five dendrites and a single axon. The axon bifurcates in the molecular layer, its two branches running for a considerable distance to left and right along the folium. B shows the other nervous elements which are found in the cortex : a cell of Golgi with a ramified axon, a climbing fibre, a basket-cell, of which the axon divides into four branches, and a small stellate cell.

The grey matter which covers the surface of the cerebellum, its cortex, is singularly regular in microscopic pattern (Fig. 23). It is divided into three sheets: superficially, the molecular layer in which the dendrites of the cells of Purkinje branch ; beneath this, the thin layer in which are situate the cell-bodies of these neurones ; thirdly, the layer of small cells, or granules. Cells of Purkinje and granules have been already described 
(p. 303). To these must be added the stellate, bracketing cells of the molecular layer, the axons of which divide to form baskets about a number of Purkinje-cells, and the cells of Golgi of the granular layer. These last are comparatively large cells, which have thornless dendrites, and axons which branch repeatedly in the granular layer, without passing into the white matter which underlies the cortex. Two kinds of nerve-fibre bring impulses to the cortex : (1) "Mossy" fibres, which bear rosettes of filaments which distribute impulses to the granules; and (2) "climbing" fibres or "tendril " fibres, which, passing through the granular layer, cling like ivy to the trunk and principal boughs of the dendritic processes of Purkinje-cells. The axons of the cells of Purkinje undoubtedly carry impulses away from the cortex, but their destination is not certainly known.

The uniformity of structure of the cerebellum suggests that it "acts as a whole." Anatomy gives no warrant for the expectation that work of different kinds is done by its several lobes. Its simplicity leads one to hope that its mechanism may some day be understood ; but at present there are so many gaps in our knowledge that it is difficult, perhaps hardly profitable, to attempt to string together the few anatomical facts of which we are sure.

By means of tracts of afferent fibres the cerebellum has a very extensive connection with the grey matter of the cerebrospinal axis (including the optic thalamus) into which sensory impulses of all kinds are poured. Experimental results indicate that the organ distributes impulses to the whole length of the cerebro-spinal axis, from the level of the neurones which govern the muscles which move the eyes to its far hinder end. No nerve-roots enter it. Its afferent fibres are the axons of cell-bodies which lie in the posterior horns of the grey matter of the spinal cord and in the corresponding grey matter of the axis of the brain, especially that part related to the nerve from the semicircular canals. Another set of afferent fibres lies at the periphery of the spinal cord, forming one of the best defined of the spinal tracts. It is also one of the oldest, being found in the same situation in all vertebrate animals. Its fibres, which are exceptionally large, are the axons of cells which form a very definite column-the "vesicular column of 
Clarke" - on the median side of the posterior horn. Further than this we cannot go. We are ignorant of the nature of the sensory impressions collected by the cells of Clarke. The cerebellum also receives through its middle peduncle the axons of cells which lie in the pons Varolii on the opposite side; which cells are discharged by impulses descending from the cortex of the great brain. It is not improbable that it gives to the great brain as many fibres as it receives from it.

If we had no experimental evidence as to the part which the cerebellum plays in the harmonious working of the whole nervous system, we should infer from its structure and connections that it is somewhat mechanical, a co-ordinator of the activities of other parts rather than in itself a functionally independent organ. Pathological and physiological observations very definitely justify this conclusion. They show that the cerebellum is not essential to life. It may be completely destroyed by disease or removed by operation without robbing the individual of any single function or capacity. Disease of the cerebellum does not diminish the patient's sensitiveness to every kind of stimulus, nor does it deprive him of the use of any single muscle; but it reduces him to the condition of a person who in gait, but not in mind, is habitually drunk. When he walks he staggers from side to side; when he stretches out his hand it trembles. His movements are jerky; his head shakes, his eyes oscillate; he suffers from a feeling of giddiness; his speech comes haltingly. Cerebellar ataxia, which is a rare disease, resembles in many respects the much commoner "locomotor ataxia" produced by disease of the spinal ganglia and the parts of the cord connected with posterior roots; but careful analysis of the symptoms shows that they are due, not to want of the sensations which guide movements, but to inability to regulate the force of muscular contractions. A man suffering from locomotor ataxia falls when he closes his eyes, because, not being able to feel with his feet, he is dependent upon vision for information as to his attitude. When the cerebellum is diseased, the patient is no less unsteady with his eyes open than he is with them closed.

The results of cerebellar disease or injury bring home to us the fact that a nice adjustment of movements is needed to maintain equilibrium. A dog from which the cerebellum has been 
removed retains all its natural enterprise, all its instincts, all its emotions ; but every action which requires it to maintain its centre of gravity in an unstable position gives it trouble. Placed in water so that its body is supported,it swims almost as well as a normal dog. It is, however, easy to lay too much stress upon the balancing function of the cerebellum. The disturbance of this function attracts our attention; yet it is probably but the indirect result of the suppression of activities of a more widespread character. No animal ventures such liberties with its centre of gravity as the biped Man accomplishes, without thinking, every time that he descends a flight of stairs. Yet the cerebellum of the limbless whale, that lives in a medium which decentralizes its gravity, so to speak, bears the same proportional relation to the rest of the nervous system as that of Man. Strangely enough, it is the only cerebellum in the animal kingdom which so closely resembles Man's that it might be passed off as belonging to a human giant; another reminder of the difficulty of deducing the functions of the several parts of the organ from a study of their relative development. What have a man and a whale in common which determines the identity in form of their cerebella? How has it come about that two cerebra as widely unlike as a man's and a whale's should be associated with a common form of cerebellum ?

If we apply to grey matter the distinction between sensory and motor nerve-tissue-having no exact terminology, it is difficult to avoid these metaphorical expressions-the cerebellum is essentially a sensory development. It grows from the very margin of the infolding groove, which, when closed, becomes the central canal of the brain and spinal cord, its elements being marshalled in intimate association with sensory root-fibres. Its millions of loops formed by the axons of granules and the collecting processes of Purkinie-cells, are by-paths which tap the conductors of sensory impulses. From some-those, for example, which originate in the muscles and tendons, and in the semicircular canals-more of the impulse is diverted to the cerebellum, from others less. The organ has no motor functions. It does not discharge neurones which control skeletal muscles, or plain muscle, or glands. Yet it influences the passage of impulses through sensori- 
motor chains, and apparently its influence is universal. It regulates tone, reflex action, voluntary action. There is no part of the nervous system over which its control is not felt. By its action on the apparatus which binds the infinity of receptors which the body contains to its muscle-fibres and other effectors, it unifies the body. The cerebrum, as we shall see, is the organ which unifies the personality. In the progress of evolution two functions which were originally combined have, for convenience of concentration, been divorced. The great brain has been set free from the more mechanical part of the work. That it can perform the functions of the cerebellum as well as its own is proved in cases of congenital deficiency of that organ. In several instances malformation, amounting to a very considerable reduction in the size of the cerebellum, was not detected until after death, there being no symptoms of a sufficiently pronounced character to call attention to it during life.

The Cerebrum.-All observations made on the great brain prior to 1870 showed it as absolutely inexcitable. Surgeons and physiologists agreed that cutting, burning, passing electric currents through its substance, neither yielded evidence of sensation nor movement of any part of the body. Concerning its structure little was known beyond the fact that whereas the grey matter, or cortex, which covers its surface contains nerve-cells, only fibres are to be found in the white matter which constitutes the greater part of its bulk. It seemed a hopeless task to attempt to make anything out of a mass of tissue so uniform in constitution and so irresponsive to experiment. Removing portions of it appeared to cause a general dulling of the intellect without loss of any particular mental quality. Physiologists, therefore, spoke of the cerebrum as "functioning as a whole." Phrenologists, having classified the various phases of mental activity as "faculties," discovered "bumps" on the surface of the skull which they correlated with the possession of the several faculties in a marked degree. They parcelled out the brain in organs concerned with different kinds of thought; but their localization of function was anatomically as baseless as their classification of the various aspects of mind, viewed as a system of philosophy, was absurd. In 1870 it was announced that electrical 
stimulation of certain areas of the cortex of the cerebrum of an animal under the influence of an anæsthetic, and therefore incapable of voluntary action, induces definite movements. Although the surgical applications of this discovery have proved immensely important, its physiological value, as affording a method of investigating the functions of the brain, is extremely small. Yet the discovery gave an impetus to the further study of the cortex, which has been rewarded with many exact results. By the discovery of its excitability to electric currents it was proved that the whole cortex has not exactly the same work to do, or-perhaps this is the safer form of statement-does not do its work in exactly the same way. As soon as it was known that it is divisible into areas differing in function, many methods by which the delimitation of the areas might be attempted were devised. The converging efforts made during the past forty years by comparative anatomists, histologists, physiologists, pathologists, and physicians, have resulted in the acquisition of an accurate, if very restricted, understanding of the construction and mode of working of the apparatus of thought. Of some of the new data the psychologist is able to make use; but so far as the physiologist is concerned, it is the vehicle of mind which is the subject of study, not its contents.

A new subject has been created since 1870. There is therefore nothing to be gained, so far as our present purpose is concerned, from the consideration of views which were current before that date; and since, as must always occur when a science is rapidly advancing, observations which logically should have been the first to be made were not thought of until it became necessary to devise methods of checking results obtained in other ways, we will consider the various sources of our information without regard to the chronological order in which they were opened up.

The cerebral hemisphere contains two large central masses of grey matter, the nucleus caudatus and the nucleus lenticularis, often described as a single structure under the name "corpus striatum." Their functions are unknown. The nerve-fibres which connect the cerebral hemispheres with the rest of the central nervous system form two thick limbs or crura on the under side of the brain. Each crus turns upwards 
into its hemisphere, between the nucleus caudatus and optic thalamus (the latter belongs to the "between-brain ") on the inner side, and the nucleus lenticularis on the outer. In this passage the compact crus, which is somewhat flattened, is termed the "internal capsule." Immediately above the three grey masses the internal capsule disperses as a fountain of fibres which go to all parts of the cortex. Mingled with these radiating fibres are vast numbers of others, proper to the hemispheres, which run tangentially. Some, crossing the median plane, as the corpus callosum, bind the two hemispheres together. Others form tracts which can be followed from one end or pole of the hemisphere to the other. Groups of fibres, dipping but little below the cortex, unite nearly adjacent spots or neighbouring convolutions.

The folding of the cortex beneath fissures is due to the necessity of disposing of a certain bulk of grey matter without increasing its thickness beyond the proper limit. Since the superficial area of a sphere varies as the square of its radius, whereas its capacity varies as the cube, it is possible for a fixed relation to be maintained between the amount of cortex and the amount of white matter in the brain, only by the folds increasing in depth as the size of the brain increases. Fissuring is a response to a mechanical need. This does not imply, however, that the lines along which it takes place are devoid of morphological meaning. The similarity in pattern of the convolutions and fissures in various animals, and the regular progress of their development in each individual, prove the contrary. If they are not absolutely trustworthy as boundaries of areas of separate function-and further evidence will be needed before a decision can be pronounced upon this disputed question-they are in the main satisfactory as landmarks.

As the nervous system grows, the axons of its neurones acquire their fatty (myelin) sheaths in the order in which they come into functional activity. The passage through them of impulses is the stimulus which leads to the deposition of fat. The study of the progress of myelination enabled the anatomist Flechsig to ascertain the situation within the brain of the tracts of fibres related to the several senses, and hence the traffic of the areas of the cortex to which they go. Glistening white streaks appear successively in the pulpy yellowish-pink 
substance of the interior of the brain. At the time of birth all the fibres which enter or leave the cerebral hemispheres have acquired their myelin sheaths. In the baby's brain the senseorgans have established all their connections with the cortex. No new fibres will appear in the nerves of the eye, the ear, or the other sense-organs, nor will their end-stations in the cortex be further multiplied. (The use of the expression "endstations" is legitimate so far as sensations are concerned; notwithstanding that all sensory impulses are retransmitted by neurones in the cerebro-spinal axis.) But the cortex is very far from having finished its growth. It contains a large amount of embryonic tissue, which gradually spreads outwards from the developed areas into the surrounding unoccupied zones. The taking up of new territory, and the consequent increase in the size of the brain, is continued into adult life. The study of progressive myelination enabled Flechsig to divide the cortex into "sensory centres," and intervening "association zones"; although, doubtless, the difference in function between the portions which receive sensations direct and the portions in which the products of sensation are worked up is one of degree, and not of kind.

The structure of the cortex is not quite the same in sensory and association areas ; but it is everywhere so far from showing the diagrammatic simplicity which characterizes the cortex of the cerebellum as to make it difficult to summarize the modifications which distinguish its various regions. To a considerable extent its elements shade one into the other, differing in size and in orientation rather than in form. Commonly it is described as divisible into five layers : (1) A thin superficial layer, containing cells of various forms and fibres derived from the cells of the deeper strata. Some of the cells are pluripolar, possessing several axons which run parallel with the surface. Their destination is unknown. They do not appear to form baskets like the cells of the molecular layer of the cerebellum. The dendrites of pyramidal cells extend into this layer. (2) The layer of small pyramids ; cells with a branching apical process, root-like dendrites from the basal angles of the pyramid, and an axon which sinks into the white matter. (3) Granules. Carmine or other nuclear stains show that small cells are present in very large numbers, especially in the sensory areas ; 


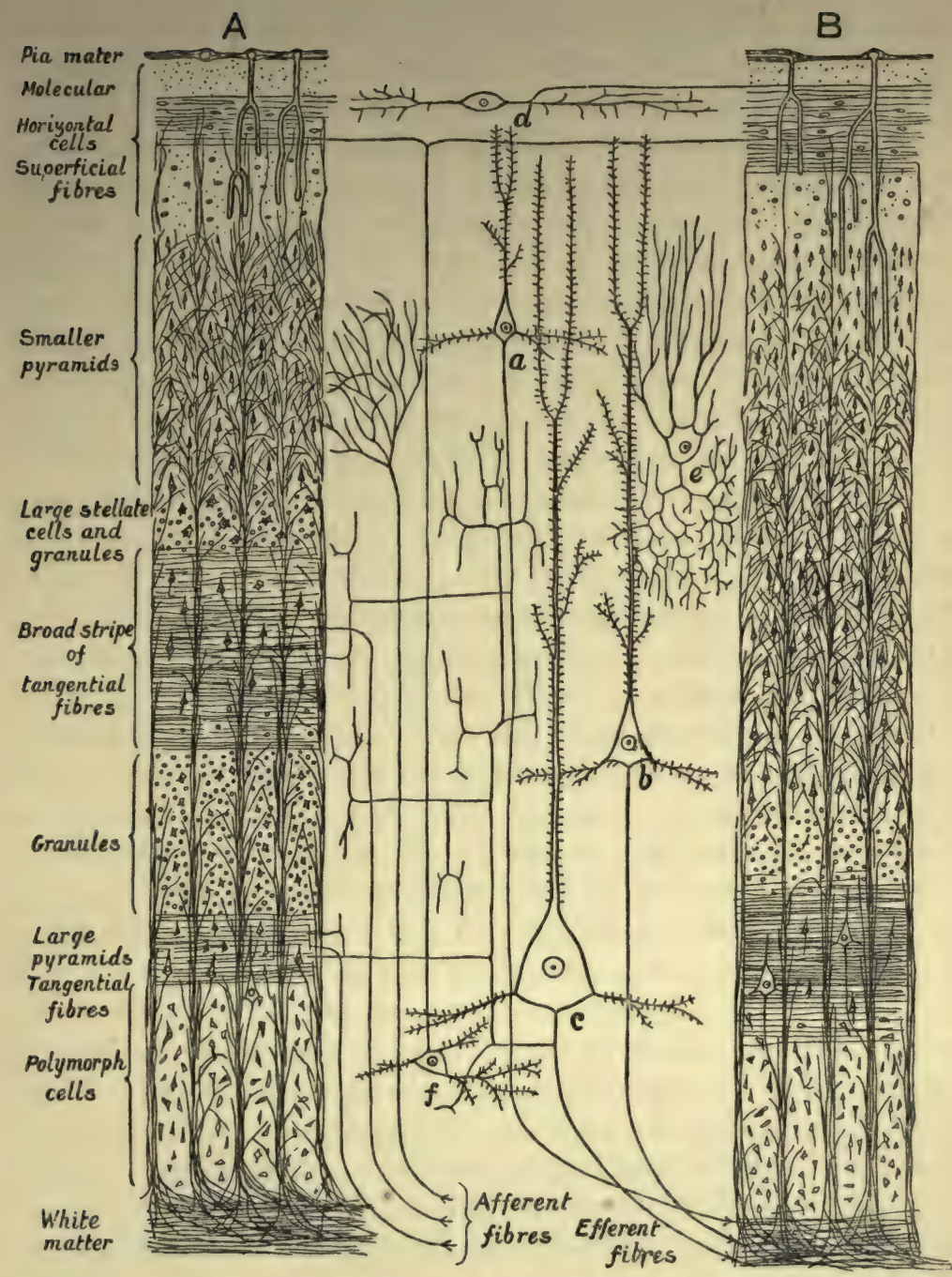

Fig. 24.-Vertioal Seotions of the CoRtex of the Cerebrum-A, of the VisUal Sensory ; B, of the Visual assoctation area.

Between the two sections are shown the principal types of cell, at the levels at which they are severally found : $a$, small pyramid; $b$, medium-sized pyramid; $c$, large pyramid. The size of a pyramid is an indication of the distance to which its axon extends before branching ; the longer its traject, the more widespread, it would seem, is its terminal arborization. The axon of $c$, one of the very large pyramids found in this association area, passes to the front of the cerebrum, where it breaks up in an association area of the tactual sense of the hand, or of sensations concerned with the regulation of gait, or in a centre for movements of the eyeball. $d$, a tangential cell of the surface; $e$, a Golgi cell with ramifled axon; $f$, a polymorph cell, with its axon directed towards the surface. In sensory areas, tangential flbres and granules are more numerous; in association areas, small and medium-sized pyramids. 
but since they are not, like the granules of the cerebellum, coloured by the chrome-silver method, their form and the disposition of their axons are unknown. (4) Large pyramids exactly similar in form to the small ones. Their apical processes are very thorny. Their axons give off several collaterals. Pyramids are the most conspicuous elements in the cortex. Properly speaking, they do not occur in layers, but are scattered throughout its whole thickness, although their cell-bodies are not seen in either its most superficial or its deepest strata. The largest are those of which the axons either descend into the spinal cord or pass to a very distant region of the cortex. They are found singly or in small clusters in the deeper levels. (5) Polymorphous cells, some of them pyramids lying on their sides, or even directing their axons towards the surface ; some fusiform or irregular cells; some Golgi-cells (p. 340). The axons of pyramids enter the white matter, and many fibres from the white matter radiate towards the surface between the pyramids; but the way in which afferent, sensory fibres are connected with the collecting processes, dendrites, of the pyramids is not known. We have already referred to thorns, and to the possible nerve-net (p. 301). Sheets of tangential fibres also occur in the cortex. A particularly distinct sheet divides the granules in the visual cortex into two strata. In sections of this region the sheet of fibres appears as a white line, distinctly visible without a lens.

The limits of the several areas can be determined by examining the structure of the cortex ; but the individual peculiarities of the various regions are not so marked as to indicate that they have different kinds of work to do ; if by kinds of work we wish to imply that one part is "sensory," another "motor," a third concerned with "intellectual processes." On the contrary, its relative uniformity shows unmistakably that all parts are engaged in the same work. Nevertheless, certain broad conclusions can be drawn with regard to the form of the neurones more immediately concerned with sensation, with motion-that is to say, with the discharge to the grey matter of the cerebro-spinal axis of the impulses which call its neurones into activity-and with the secondary processes, called collectively "association," which occur within the cortex. Granules, as everywhere throughout the nervous system, are 
receivers and distributors of sensory impulses; although a study of the cerebral cortex does not justify the conclusion that they are necessary links in its sensori-motor ares. Large pyramids are occupied with the nutrition of fibres which have a long traject through the system. Hence they are " motor." They constitute a marked feature of the area which is susceptible to stimulation. They occur also in the visual area and elsewhere. Small pyramids are associational; that is to say, their axons do not leave the cerebral hemispheres. They distribute impulses from sensory areas to association zones, and from one part of an association zone to another. The layer of polymorphous cells is relatively thicker in animals in which the cortex of the brain exercises less control over action than in animals in which the cortex is supreme-in a rabbit thicker than in a monkey; in a monkey thicker than in Man. This layer is therefore said to be concerned with the lower functions of the cortex, whatever this expression may mean. Since the relative abundance of small pyramids is a test of the supremacy of the cortex, we may speak of them vaguely as concerned with its higher functions. But a surer test of the capacity of the cortex for the elaboration of the raw materials of thought which sensory nerves deliver to it is the relative abundance of the tissue which intervenes between its cells. The number of cell-bodies to be counted in a square millimetre of a section of a given thickness is smaller in Man than in a monkey, in a monkey than in a dog, and in a dog than in a rabbit.

A comparison of the brains of various mammals in which particular sense-organs are either deficient or exceptionally well developed affords the clearest proof of the localization of sensory areas. This, if it were possible to make satisfactory measurements, would be by far the best class of evidence as to the part played by the several senses in an animal's mental life. Unfortunately, measurement appears to be out of the question ; but a glance at a rabbit's brain, placed by the side of a mole's, shows that vision is localized in the occipital region. All marine mammals are destitute of the sense of smell; the brain of a dog, compared with that of a porpoise or a whale, shows that the sphenoidal region (cf. Fig. 25) is associated with this sense. The brain of an otter exhibits very clearly the area into which impulses arising in 
the nerve-endings of the sensory bristles of the cheek are poured.

"Nihil est in intellectu quod non prius in sensu fuerit." The organ of the intellect is the cortex of the great brain, a sheet of grey matter which has developed in connection with the various sense-organs. The cerebral hemisphere of an infant is merely an extension of the nerve-tissue associated with its sense-organs. Such it remains in a microcephalous idiot. In the lower animals its capacity of growth after birth is very small. But in a normal child the inflow of impressions through senseorgans, the experience acquired regarding itself and its surroundings, education, whether accidental or directed, causes the extension of nerve-tissue from the sensory areas into the expansible intervening zones.

There is still some uncertainty as to the nature of the sensations received in the excitable area. They may be termed " kinæsthetic" (sensations connected with movement) without more exact definition. Some physiologists consider that tactile sensations, as well as the obscure sensations, originated in the nerve-endings in muscles, around tendons, or on joint-surfaces, are distributed to the areas, which, when stimulated, are shown to represent fingers, hand, arm, and other parts of the body. Others have sought, though with doubtful success, for a tactile area, independent of the kinæsthetic centres. When first discovered, these centres were termed " motor," and still this term may be retained, on the understanding that it does not imply that the exchanges which occur in the kinæsthetic centres are of a different nature to those which take place elsewhere. The region which they occupy has become the motor area of the cortex because voluntary movement is possible only under the guidance of sensations of movement. A sound or a retinal image may prompt the movement; but the part of the temporal region, or of the occipital region in which the soundmovement exchange or sight-movement exchange occurs must act through the motor area by opening kinæsthetic-movement arcs. Destruction of a part of the kinæsthetic cortex causes in Man and the higher apes permanent paralysis for the movements directed by the spot destroyed. In lower animals the definition of the movement centres is vague, and their removal produces only temporary results. Their mastery 
over the muscles is less complete than in the higher apes and Man.

Practically nothing is known with regard to localization of function in the association-zones, with the exception of the localization of the centres for words; but this exception is so remarkable as to suggest that if there were any other faculties, interference with which caused defects as distinct as those which characterize disorders of speech, it would be found that the association-zones are made up of definite centres. As the evidence stands with regard to the broadest continental divisions, we can merely state that it points, although not very clearly, to the connection of the frontal zone, the region in front of the kinæsthetic area, with ideas of personality, of other zones with ideas of environment. Injury to the frontal region has in certain cases resulted in the victim's losing his knowledge of himself, his name, and his relation to his family. On the other hand, gunshot wounds and other definite injuries have in a large number of cases destroyed portions of the cortex behind the forehead without causing any recognizable intellectual change. It is quite certain that this part of the brain performs no functions which are of a different, or, as it is often called, higher order than those of other association-zones. It has been stated that disease of the zone which intervenes beween the visual and auditory areas is more likely to cause hallucinations, disease of the frontal zone delusions. A patient fancies in the one case that he sees things that are not there, or hears voices when no one is speaking; in the other case he imagines himself a king; but evidence connecting localized disease with mental derangement is very scanty. The functional disturbance which causes lunacy is usually of a general character; or, if local to begin with, it becomes general before the death of the patient makes possible the examination of his brain.

Derangements of speech throw a flood of light upon the organization and manner of working of the association-zones ; and, owing to the accident of the continuation of the line of the carotid artery by the middle cerebral artery, which supplies the speech centre, there is no other spot in the cortex so likely to be thrown out of gear. A little plasma coagulates on one of the cardiac valves, or about an atheromatous spot in the aorta. Detached by the blood-stream, it is shot into one of the branches 
of the middle cerebral artery, which it plugs, causing apoplexy. A larger or smaller number of muscles on the opposite side of the body are paralysed. If the plugging occurs on the left side of the brain, it is accompanied by aphasia; but only if it occurs on the left side, owing to the fact-perhaps the most

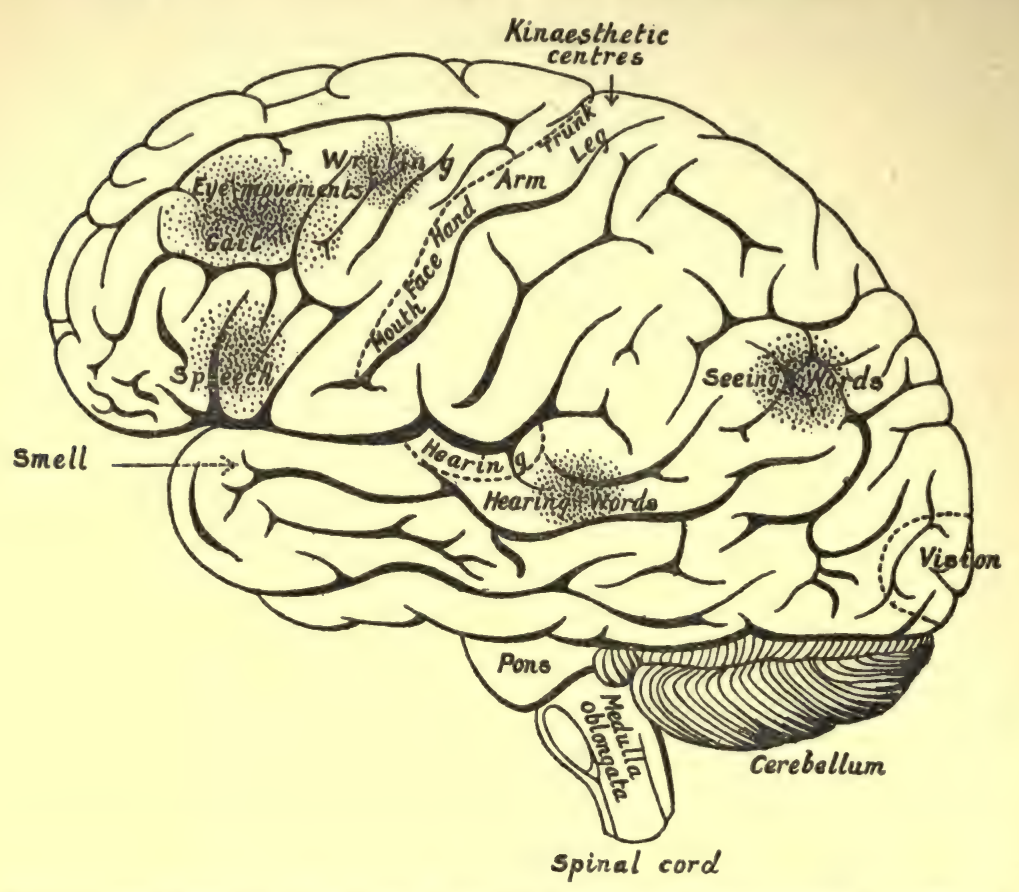

Fig. 25.-The SuRface of the Left Cerebral Hemisphere, Cerebellum, aNd Medulua OBLONGATA.

Sensory areas are enclosed by broken lines ; certain centres in the association-zones are marked by dots. The sensory area of smell is on the inner aspect of the brain; so also is the area of vision which borders the calcarine and retrocalcarine fissures, and only rarely extends on to the external surface, as shown in the diagram. The sensory area of hearing is largely hidden within the fossa of Sylvius, the opening into which is indicated by the dark line above it. The kinæsthetic-sensory areas for the various muscles of the body occupy the territory between the dotted line in front and the bottom of the fissure of Rolando behind. They do not extend on to the posterior wall of this fissure. It is impossible at present to define the boundaries of any of the centres in the association-zones.

remarkable in connection with the localization of speechthat only on the left side is the cortex trained to utter words. In course of time the patient may recover the power of speaking, but not until he has, with almost as much labour as in childhood, educated the right side to do the work. There are four 
speech-centres, quite distinct one from the other. Near the visual area is the centre for seeing words, or rather the centre for seeing the meaning of words. If this centre be diseased, a written word is merely a crooked line. Behind the auditory area is the centre for recognizing the meaning of words heard. If it is interfered with, the most endearing or commanding phrases produce no more impression on the hearer than a bird's song. In front of the hand-area-its localization is less certain than that of the other three-is the centre for writing. In it are associated words heard or seen, with the movements necessary for the making of letters. In the centre first referred to, as being the one most often thrown out of gear, which lies in front of the area for the mouth and throat, words heard or seen are translated into movements of the parts which give them sound. No other actions illustrate so clearly the "law of neural habit." In the infant's brain sounds of words are distinguished from other sounds. They are associated with the objects which they name. Movements of the mouth and throat, made at first ineffectively, blunderingly, succeed after a time in securing the thing of which they sound the name to the child's satisfaction. Thus, two centres are gradually established in his mind. Sounds and ideas of things are associated in the one; words and ideas of the movements necessary to their pronunciation in the other. Either of the four speech-centres may be placed out of action without the others suffering. A man may be able to write without being able to read what he has written. He may read aloud, although apparently deaf to speech. He may be unable to write or unable to speak, although understanding what he reads or hears. Aphasia, when partial, illustrates still further the law of neural habit. The ability to remember nouns, especially proper names, is most easily lost. Few are the people who, as age advances, do not suffer from this failing. Even the names which are most familiar elude the memory. From one point of view this is strange. Nouns-substantive are the words first learned. Of all words they have the most definite objective association. But it is just their definiteness which makes them difficult of approach when the apparatus of mind is working badly. There are so few paths by which they can be reached. Their mental associations are limited. A patient 
who is recovering from the effects of a lesion which has rendered him partially aphasic may be able to recall adjectives when he cannot recall nouns. He may say, "Give me the black," when he wants ink, and "Give me the white," when he needs paper. Or he may retain control of verbs. "Where is the — what I put on-what I think with ?" may be the circumlocution for hat.

Psychologists explain the voluntary production of a movement as the setting flowing of a sensori-motor current. Everyone agrees that it is impossible to think of the impulses which produce movement as originating without sensory antecedents. Hence psychologists picture the nerve-current as originating on the sensory side. Kinæsthetic images of the sensations which will result from the movement are described as being called up in the mind by the agitation of the part of the brain which, by association, is linked with the neurones which discharge impulses to the appropriate spots in the grey matter of the spinal cord. The idea of movement flows over to the muscles. But this conception of the relation of mind to body assumes too much. It postulates an existent mind in which the images of movement-sensations-the memories, that is to say, of the sensations which previously accompanied movement-are stored. The study of the apparatus of mind does not warrant this assumption of an existent mind. It finds nothing in the nervous system but apparatus. There is no mind existent in the brain during sleep. It would appear to be sufficient to describe the origination of a voluntary movement as the opening of the channels which convey the afferent impulses which are ceaselessly pouring into grey matter from nerve-endings in and about muscles into efferent channels. Our conception of the number of sensations which reach the realm of consciousness is ludicrously restricted by our inability to pay attention to more than one sensation at a time-a restriction, it is needless to remark, which is imperative in the interests of consistency of behaviour. Two personalities paying attention to different sequences of sensations would give incompatible orders. One would command the muscles to cause the body to recline; the other would direct them to make it stand up. From myriads of sense-organs impulses are continuously rippling through the cortex of the brain. The term 
"impulse" is too heavily weighted by its association with the idea of currents which are strong enough to prove effective without the intervention of consciousness; but no other is available. They ring the bell of consciousness, however little may be the attention which their summons secures. Attention cannot be directed to two things simultaneously. It moves, as it were, on a succession of points. On some it rests longer than on others. They make an impression which can be recalled; the rest being passed by so rapidly that they are not remembered, it is as if they had never been perceived. They blend, as a succession of moving lights blend, in producing a background to consciousness. Not recognizing their separateness, we interpret them as fused. A good deal of misleading metaphor has been used, as it seems to the writer, in accounting for the effect upon the mind of impressions which make but a weak demand upon attention. They are spoken of as " marginal " perceptions, from the analogy of the ineffectiveness of impulses generated at the periphery of the retina, as compared with those which give rise to direct vision. A "subconscious," or even " unconscious," self is evoked. The self cannot be less than conscious. Self is the passage of attention from sensation to sensation. Its relation to the not-self is temporal, not spatial.

Every sensation which is called up into consciousness, though it occupy attention for the shortest possible time, tends to give rise to movement-is, indeed, in its very nature an impulse flowing through a sensori-motor arc. The circuit for the voluntary execution of a movement is represented as flowing through kinæsthetic-movement ares. This may be necessary for volitional actions, but it is not essential for reflex actions. A spinal frog will remove an irritant from its back with its hindleg, after the roots of all the afferent nerves of the hind-leg have been cut. In this case the reflex is direct, from injured skin to muscles of the leg. It is not double-muscular sensations from the leg, liberated into efferent leg-muscle-nerves by skin-sensations originated simultaneously in a part anterior to the segments in which the roots have been cut.

The unit of sensation to which attention can be directed has yet to be defined. Like sensations-sensations which are correlated in experience, that is to say-seem to fuse in con- 
sciousness. A sequence of similar sensations appeals to attention. Unlike sensations interfere one with another. The apparent fusion is not a composite neural effect which consciousness views as a single unit. Not even identical images simultaneously formed on the two retinæ produce a superimposed effect upon a particular spot in the brain. Different brain-spots receive the two separate images which the mind views as one. This raises a doubt as to whether perceptions are, properly speaking, fused. It suggests that they are separate points upon which attention rests in rapid succession ; but such a hypothesis does not preclude the conception of the production of a composite sensation by impulses coming simultaneously from the same sense-organ-e.g., a unified neural effect as the result of several musical tones.

Every neural agitation which attracts attention has an effect upon the growth of the nerve-strands in which it occurs. Memory is not an existent. It is the repassage of the same strands. There is no such thing as memory. It is the neural apparatus which responds in a similar way to a similar agitation. It is difficult to speak of association and neural habit, the phenomena upon which not only all mental life, but all co-ordinated activities, are based, without using such expressions as " the broadening of the path" or "the thickening of the conductor" by the impulses which pass through it. Apparently these analogies may with safety be pressed curiously far. Chaotic response to stimulation is unknown. Thanks to the nervous system, action exhibits an ordered relation to stimulation. This relation is determined by education, giving the term a connotation wide enough to cover all experience. Nerve-tissue adjusts itself to experience; and since the nerve-matter which takes the pattern is not labile, the process of organization is consecutive and the result permanent. One pattern is not destroyed as another is impressed. Hence temporal associations are formed. What has been thought once will be thought again, if the circumstances in which it was thought recur. What has been done once will be done again under the influence of a similar sequence of stimuli. The conductors are widened every time that they are used. But, so far as concerns the mind, a reversed influence comes into play. The wider the conductor, the less appeal 
to attention is made by the impulses which pass through it. It is as if currents which have to overcome resistance in a narrow path acquire a higher potential than those which find an open road. And since the making of the road depends upon attention, the limit of broadening is reached when a volitional act becomes a habit. The first time that a piece of music is played consciousness is alert. Marks on the page and movements of the fingers are felt intensely. With each repetition the need for attention subsides.

A skilled movement is impossible in the absence of guiding sensations. I decide to button my coat. Sensation-paths from the muscles of the forearm are opened into motor paths extending from the large pyramids in the arm-centres of the kinæsthetic cortex. But it is not sufficient that the action be started : it must be guided by the sensations which movement produces. If my fingers are numb with cold, I cannot button the coat. The muscles which move the fingers are warm enough beneath the sleeve, but my attempts to will them to move are as futile as they would be if the muscles belonged to some other person. The will has no power over the muscles. It is essential that the sensations which accompany the act of buttoning the coat flow through the same paths as hitherto in the cortex of the brain. Flowing through the same paths, they produce the same effect in consciousness, the same perceptions. In ordinary parlance, one cannot perform any act unless one can remember what it felt like to perform it on a previous occasion. It is almost as sound physiology to describe the voluntary action of fastening a button as commencing in the skin of the fingers as to describe is as commencing in the brain. The act is due to the direction of attention to impulses which flow from muscle to muscle, and from skin to muscle.

All skill in the use of muscles is acquired by the method of trial and error. Familiar movements are tried, combined, modified with a view to the production of a new result. A man accustomed to striking with the right hand forwards endeavours to swing a golf-club with the left hand backwards. For a long time the result is anything but a success. At length the head of the club takes the right curve. It not only hits the ball with its centre, but it carries it through in the right line. The ball travels 120 yards or so towards the green. 
In golfing terminology, a successful drive is always " an awful fluke"; but the fluke once accomplished, nothing is easier for the golfer than to drive equally well on all succeeding occasions. $\mathrm{He}$ need merely remember exactly what it felt like to give the club a perfect swing, and exclude all other sensations while he is passing these memories through his sensori-motor arcs!

The fact that we can deliberately improve an action, fitting it to the attainment of the object of desire, by suppressing wrong and emphasizing right sensations, shows how large a part consciousness plays in the affairs of the nervous system. This brings us to the frontier of physiology. At this boundary the authority of the physiologist ends. He cannot define consciousness ; he cannot investigate it. Yet he naturally asks whether the machine which he is investigating is a machine and nothing more. When the possibilities of reflex action were first recognized, thought tended to dethrone feeling and Will in favour of automatism. If the actions of a spinal frog exhibit so distinct a purposive character, why, it was asked, should we assume that the frog with a brain is anything more than a reflex machine? Light, heat, sound are playing upon its senseorgans ; surely these stimuli suffice to set going all the sensorimotor currents which lead to the various movements which in their totality constitute the frog's behaviour! And why assign to a mammal a self-directing authority which we deny to a frog? The increased complexity of its behaviour is more than accounted for by the greater variety of its nervous arcs. All animals, it was argued, including Man, are reflex machines. Their thoughts and actions are the effects of the play upon their nervous systems of forces from the outer world. Each inherits a nervous system of a certain pattern. Its individual development is conditioned by the sensations which pass through it. The sensations are impressed by the environment. Therefore the individual is a puppet, his activities the dance of circumstance. Consciousness is an "epiphenomenon." Few physiologists or students of animal behaviour take this material view of life at the present day. The fact that it leads inevitably to the conclusion that consciousness is an "epiphenomenon" (Huxley's term) is its reductio ad absurdum. It is not in harmony with the economy of Nature that an animal should be endowed with the capacity of feeling pain and pleasure, if such 
endowment is useless to it. It can be useful only by directing activity towards the attainment of pleasure and the avoidance of pain. This admitted, the mechanical theory falls to the ground. There is an "It" which feels, selects feelings, chooses those which have a pleasant tone, wills to perform the acts by which they are attained. It follows that the value of consciousness lies in the prerogative which it confers of adapting action, within certain limits, to circumstance. An animal succeeds in life in proportion as the nervous system which it inherits reacts satisfactorily to its environment. A chick which, after being hatched in an incubator, has been isolated for twelve hours without food, seizes a grain of corn the instant that it sees it. Its brain contains ready-made sensori-motor arcs connecting the spot in its cortex in which the visual impression of the grain is perceived and the motor neurones which control the pecking muscles. A sheep-dog is quickly broken to sheep, because its ancestors have been selected by mankind from amongst dogs that readily adapted themselves to this work. The breeder has selected a pattern of brain with the same success with which, when appearance is the only desideratum, he selects a pattern of coat. Beavers set to work at constructing a dam at the only spot in a valley at which it is possible to create an artificial lake, because for countless ages Nature has ruled out the animals which constructed their dams in unsuitable places. Man also inherits a brain-pattern; but, not being required to shift for himself soon after birth, he goes through a long period of infancy and tutelage, during which, by force of circumstance and his own Will, the pattern is elaborated. His supreme success is due to his capacity for adapting means to ends. He inherits very few instincts. Except as regards organic functions, his spinal cord is subservient in almost all respects to his brain. Most of the actions of an animal are instinctive-a word which has been sadly misapplied. Its connotation is negative rather than positive. Owing to the marked pattern of its brain, an animal finds it difficult to avoid acting in a particular way. As the nights grow longer and its hours for feeding are curtailed, a swallow is impelled by its instinct to go South. It makes the same use of its sensations during its migration, and is as completely dependent upon them for its guidance as a man would be. 
The lower we descend the scale, the more inevitable do an animal's movements become; but there can be no doubt but that consciousness is of value to an animal, as to Man, in that it gives to its individuality the capacity, within such limits as Nature has selected, of resisting or modifying its ancestral instincts when they are not absolutely appropriate to the occasion.

Sentience implies personality. "No system of philosophy can extrude the ego." The difference between the performance of the animal machine as a physiologist studies it, and its behaviour when under the control of its own driver, is the difference between reflex action and choice. The ego interacts with physical forces. It does not come within the province of the physiologist to explain the source of the force which interferes with force. He finds no trace of it on either credit or debit side when making up the body's accounts. He is unable to enter, "Item, to the development of consciousness . . . so much." He can form no conception of this immaterial manifestent which hovers over the infinitely numerous sensorimotor exchanges which are always occurring in the cortex of the brain, giving to a particular group of agitations, now here, now there, a special quality; but the manifestent is needed to account for the potency of the reinforced agitations which enables them to take possession of the nerve-paths by which muscles are reached.

It is for the psychologist to define the application of the terms "consciousness," " attention," "will." He cannot define the attributes of the ego which these terms connote. The moralist must show the way in which they determine, or should determine, conduct. Yet within the plain limits of physiology, attention, using the word in its every-day sense, modifies the responses of the nervous system in a degree which cannot escape observation. It is astonishing to anyone accustomed to hospital surgery (although even in this field singular exceptions are met with) to see the grave operations which a veterinary surgeon may perform, without the animal showing any evidence of pain, provided its apprehension has not been aroused and its attention directed to what is being done. A horse standing in front of a crib of oats, untied, will hardly whisk its tail while the surgeon is making a great wound in its 
flesh, and sawing off a bony excrescence. The knife does not come within the experience of a horse. It has no anticipations, and its skin, intensely sensitive to the tickling of a fly or the smart of a whip, is relatively insensitive to a cut. An eminent surgeon of the last generation (the writer, as a student, "dressed" for him in his old age) was in the habit, having arranged that his patient could not see what he was doing, of performing operations of a very painful nature whilst assuring his patient, "I am merely making a thorough examination, in order that I may be perfectly certain of the cuts that I shall have to make to-morrow in the operating-theatre when you are under chloroform." We are not concerned with the ethics of his method ; but the assurance, "Now that's all over ; you will never need to have that operation performed again," saved many a sufferer from a night of apprehension and a miserable " coming round."

It was stated, during the South African War, that at Ladysmith the bearer of a critical despatch, who was struck in the palm of the hand by a bullet which traversed the whole length of his forearm, did not discover that he was wounded until he saw the dripping blood, after his errand was successfully accomplished. To deliberately cut oneself with a razor is most painful, yet shaving in the morning, with thoughts concentrated on the doings of the day, it is often the sight of blood which directs attention to the fact that the skin is severed. Of all evidences of self, the power of paying attention is the most noteworthy. We can direct attention to certain sensations, which then become perceptions, and we can deliberately ignore others,within certain restricted limits.

The control of the nervous apparatus by the self is a truth which no student of the physiology of human beings can ignore. Isolated from its relation to all other scientific truths, it has been made the basis of a nescience which, although positively merely foolish, is, negatively, harmful-yet a form of folly which answers well to the needs of persons of a certain category.

It may be objected that the picture of the relation of mind to brain which is here presented-the one, activity, motion, the other a labyrinth of conducting paths-makes all mental phenomena entirely dependent upon current sensations. No results could happen if the sensations were not there. It 
affords no ground for the explanation of mental images, hallueinations, dreams. A few lines may be spared to show that this objection does not hold. We cannot attempt to explain the conscious control of thought. It is a part of the impenetrable mystery to which we have just referred. But, granted that it obtains, the direction by the ego of afferent nerve-currents through the same strands which formerly vibrated to sensations which drew a picture, and hence the revival of its image, is no more incomprehensible than the liberation of afferent impulses from musales into efferent channels. Brain-chains are composed of many links. Their interconnection is illimitable. When I recall the appearance of the house in which I lived as a child, I throw into the chain impulses (from somewhere) which traverse the final links, where passage implies consciousness. At the edge of the lace-work of linked threads the impulses light up a pattern which childhood's experience worked into the apparatus of thought.

If we were to admire the perfection of any special aspect of the brain's functioning, the rarity of hallucinations might give us cause for wonder. That impulses so seldom leave their own paths is more astonishing than that occasionally, when the brain is excited and its nutritive conditions deranged, the impulses which the ego can direct into channels where they revive an image should sometimes, and with far greater force, make their own way down well-worn paths, lighting up a picture which deceives the ego. Dreams, by contrast, throw up in a strong light the part played by attention in intelligent life. The capacity for alertness is due to the favouring of one set of impulses by suppressing others. The favoured impulses hold the road. Concentration of attention is keeping thought to one line by resisting all temptation to wander into by-paths. The waking condition is the state in which all nerve-ways are closed, with the exception of those which consciousness is using. The more severe the closure, the more vivid is consciousness. In sleep all paths are open. In none is the potential acquired by impulses in the process of overcoming resistance high enough to evoke consciousness. A burst of impulses ascends from the stomach, set a-flowing by undigested fragments of salmon and cucumber, or mounts from the arm on which the sleeper has been lying until its circulation has been 
arrested. They reverberate through the open corridors of the brain. If they are sufficiently noisy to awaken the sleeper, he, detecting them in this path and in that, supposes them to be on the same errands as the impulses which commonly pass thus. If dreams are analysed, it will be found that, although the combinations of impressions may be uncommon and extremely bizarre, the impressions are selected from the most familiar. The images of which the dream is compounded, which may have lost all normal relations and may have assumed impossible proportions, are those which the mind most frequently conjures up. In the large majority of instances some happening of the day preceding can be recognized as the prompting cause. A remembered dream is the photograph taken by consciousness of the sensations which have bombarded it into activity. Especially if due to impulses originated by visceral discomfort, the dream may have an unpleasant tone. This may take various forms, but the emotion most commonly aroused is fear. The objects visualized may have preposterous dimensions, or they may be not sufficiently distinct for recognition-elusive imps; but most commonly distress is caused by the want of harmony of sensations, due to the absence of kinæsthetic elements. A man is lying on the railway-line; a train is approaching with increasing speed ; he cannot get up. $\mathrm{He}$ is in the pulpit, but cannot speak. Dreams thus confirm the view set forth above as to the cause of volitional action. Ability to perform an act depends upon the flow through the kinæsthetic centres of the brain of impulses generated in the muscles by which the act is to be, or is being, performed. Kinæsthetic sensations do not under any circumstances play the same part in mental life as sensations from the skin, the eye, or the ear ; when the body is passive in bed they are not flowing into the cortex. The dreamphotograph shows elements demanding movement, but affords no evidence that movement is in progress. 


\section{CHAPTER XII}

SMELL AND TASTE

IN Man the chief function of these senses is to guard the entrances to the respiratory and digestive tracts. In this they are not conspicuously efficient, since various poisonous gases, salts, and powders, escape their vigilance. Merely a selection of the substances which occur in air and in food are recognized as having odour or flavour. Smell and taste are only partially distinguished in ordinary parlance. No odorous substance is spoken of as tasteless when taken into the mouth. Its volatile constituents, escaping to the chambers of the nose, are said to afford a certain flavour. On the other hand, it is recognized that substances which stimulate the tongue alone-bitters, acids, sweets, and salts, unmixed with volatile bodies-have no odour.

Biassed as we necessarily are by the paltry rôle assigned to smell in our mental life, it seems a little unworthy of the present functions of the great brain that it should have developed in association with the nose. Yet smell and taste are the oldest of the senses. Their origin goes back to the days of chemiotaxis, when the organism, having no specialized sense-organs, was attracted to its mate or to its food, and repelled from conditions unsuitable for its well-being, by particles in solution acting as chemical stimulants. An amœba is chemiotactically drawn towards its food, one spore of an alga is attracted to another, by the particles of matter which drift across the interval between them.

In the life of many animals smell plays as important a part as that of either of the other senses. One has but to watch a dog "looking" for its master, already full in view, with its nose, to realize that smell is the sense on which a dog chiefly 
relies. We describe it as looking, because in ourselves the eye has so far outdistanced the other senses as a channel of information that we speak of "looking" when we mean seeking, and say that "we see" when we wish to imply that we understand.

The difference between smell and taste is, in fishes, a difference in the quality of the sensation, and not in its " modality" or kind; but in terrestrial animals the olfactory membrane of the nose has become specialized for the recognition of particles suspended in air, the tongue for substances dissolved in water. The olfactory membrane, which lines the upper two of the three chambers of the nose, is covered with elongated cells of two kinds : $(a)$ Columnar cells, fairly thick ; and (b) fusiform cells, each carrying at its free extremity a bunch of exceedingly minute hairs. The fusiform cells are neuro-epithelial cells of the most primitive type. Before nerve-cells, properly so called, appeared, certain favourably-placed epithelial cells were connected by protoplasmic bridges with muscle-fibres, to which they delivered the impulses which were generated in them by external forces. Later some of the neuro-epithelial cells sank beneath the surface, where, as ganglion-cells, they served as intermediaries between groups of sensory cells on the surface and the nerve-net which lay more deeply in the tissues. The olfactory membrane perpetuates the earlier stage; in so far as it consists of elements which are combinations of sense-cells and nerve-fibres. Each of its fusiform cells sends inwards a nerve-filament, which, traversing the submucous tissue of the nose and the bone (cribriform plate) on the base of the skull, between the orbits, enters the olfactory bulb. The olfactory bulb is a part of the local nervous mechanism of smell. It is the ganglion of the nerve of smell plus nerve-elements which in all segments behind the eye have been withdrawn from the neighbourhood of the sense-organ into the central nervous system (cf. p. 333).

The way in which odorous particles in air stimulate the fusiform cells is unknown. The quantity which suffices as a stimulant is so small as to put chemical stimulation out of the question. A few grains of musk will scent a room for years. 0.00000004 milligramme of mercaptan (sulphur-alcohol) is recognizable in a litre of air. This is a dilution to 1 in $50,000,000,000$. 
Probably even such figures as these would be thrown into the shade if we could estimate the minimum amount of human effluvium which will enable a dog to follow his master's trail. Explanations have been sought in alterations in the vibrations of molecules of air caused by the presence amongst them of relatively heavy molecules of volatile substances ; but the difficulty of accounting for the generation of nerve-impulses in the sensory cells remains as great as ever. The hairs borne by olfactory cells are so short that it is impossible that they should project beyond the film of moisture on the surface of the membrane. This seems to preclude an answering vibration. Yet an increase in the thickness of this layer and in its density, due to the presence in it of mucus secreted during a catarrh, renders the sense-cells incapable of responding to odorous particles.

Smell in an animal is not a test of the quality of the air it is breathing, but a source of information as to the direction in which it may seek its prey; or, although far more rarely, as to the direction from which the advance of a foe is to be feared. Hunting animals depend for the most part on the nose. Hunted animals rely chiefly on the eye.

If we attempt to analyse our smell-sensations, we find that we can pick out a number of varieties which appear so unlike as to have nothing in common: Putrid meat, burning indiarubber, sulphuretted hydrogen, ammonia, roses, onions, lemon verbena, methylated spirit. Everyone can make for himself a list of typical odours which seem to have specific qualitiesodours so distinct that he never confuses one with another. He can also class together scents about which he is often uncertain. The type-odours he can distinguish when present in a mixture; whereas odours which are less distinct reinforce or modify one another. It has been found, by careful experiment, that certain type-odours even tend to neutralize each other. Musk and bitter almonds, for example, if present in small quantities and properly proportioned, produce a very dim sensation, whether supplied as a mixture to both nostrils, or the one assertive odour to one nostril and the other to the other. This last observation is of great importance. It proves that their mutual destruction does not occur on the olfactory membrane. It is not due to physical interference. The sensation 
of musk is delivered to one side of the brain, the sensation of bitter almonds to the other; but when attention is directed to these two sensations there is found a quality in the one which is irreconcilable with the quality of the other.

In certain persons and under certain pathological conditions, sensitiveness to particular odours, or groups of odours, is absent, while for the rest the sense is normal. Methylated spirit, prussic acid and mignonette, constitute a group which not infrequently drops out. Instances have also been reported of persons unable to smell vanilla (to which some are hypersensitive), and of others insensitive to violets, although normally sensitive to the scents of other flowers. The notes sounded in consciousness extend over a long gamut; but there are reasons for thinking that the number of keys on the clavier which odoriferous substances strike is limited. Eleven is the number provisionally adopted. The effect in consciousness varies according as one key or another is struck, or several at the same time with varying degrees of force.

Many attempts have been made to associate the sensationqualities of the various odours with the chemical or physical properties of their odorants, with but little success as yet. To excite the sense of smell, a gas must be at least a little heavier than air. No volatile body, it is stated, is so heavy as to be odourless ; on the contrary, speaking generally, heavy molecules are more stimulating than light. The quality of a smell-sensation would therefore appear to depend upon the period of vibration of the molecules of the substance which evokes it; but, as already stated, a consideration of the apparatus which responds to stimulation by odoriferous particles does not help us to an understanding of the way in which the particles act upon it.

Taste is far more limited in its range of sensations than smell. The back of the tongue is sensitive to bitters, the tip to sweets and salts, the sides to acids. Mixtures of these qualities are distinctly analysable by the sense of taste. Our sensations of taste do not fuse. Slight differences in the way in which the organs on the different parts of the tongue react to stimulation enable us to recognize that a sapid substance is a mixture. When, with a great flourish of trumpets, saccharin was introduced as a safe sweetener for gouty people, an attempt was made to provide them with saccharin-sweetened jam. The 
effect of the jam upon the person who consumed it was truly humorous. First a suspicion of tartness, then its adequate suppression, followed by nauseating sweetness. The senseorgans which subserve the sense of taste are clusters of fusiform epithelial cells, collected in "taste-bulbs" (Fig. 26). Each gustatory cell bears a minute bristle, which projects through

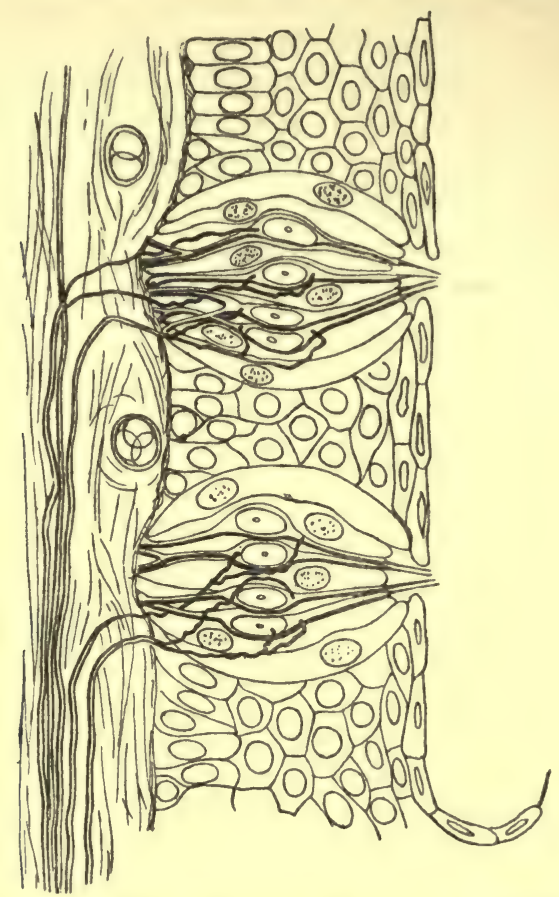

Fig. 26.-Highly Magnified Section throdgh the Wall of a Circunvallate Papilua of the TONGUE, SHOWing Two TASTE-BULBS.

These sense-organs are groups of elongated epithelial cells, set vertically to the surface. Their cells are of two kinds - the one fusiform, slender, bearing each a bristle-like process which projects through a minute pore left between the superficial cells of the general epithelium; the other thicker and wedge-shaped. Nerve-fibres are connected with the fusiform cells.

the pore left by the cells of the surrounding epithelium which constitute a globular case for the bulb. As in the nose, eye, and ear, a second thicker variety of epithelial cell is also present. The nerve-fibres of the taste-bulbs are not, as in the olfactory membrane, processes of their cells, but branches of the fifth nerve which ramify amongst them. On the back of the tongue taste-bulbs are much more numerous than elsewhere. They are 
not as sensitive as the cells of the olfactory membrane ; nevertheless, they enable us to detect 1 part of quinine in 2,000,000 parts of water.

Sensations of taste and smell endure for a long time after stimulation, because the odorous or sapid substance remains in contact with the sense-organs. This accounts for the confusion into which a man is thrown if he sip alternately port and sherry. After a short time he cannot tell the one from the other. The organs are quickly fatigued, using the term loosely. How intolerable patchouli would be to the ladies who use it were it otherwise! If for some time one sniffs the odour of mignonette, it ceases to be recognizable; whereas, turning to a rose, the olfactory membrane is found to be as sensitive as usual. When the sense is fatigued for a particular smell, it is dull for others of the same group, thus affording an opportunity of classifying smell-sensations according to their qualities; but the method is difficult to apply. Taste-organs are greatly affected by temperature. Quinine is not tasted just after drinking ice-cold water. Alcohol, ether, or chloroform paralyses the organs much in the same way. Castor-oil slips down the throat unnoticed if the mouth, just before swallowing it, has been rinsed with brandy or with a strong solution of tincture of chloroform.

Englishmen make but little use of their sense of smell. It might teach them much regarding the various emanations from putrid matter which are produced by bacterial action; but, dreading drains, they decline to cultivate proficiency in the exercise of this sense. The nose is valued for the warning it gives of " nasty smells," but is not allowed to analyse them. Burnt milk, soap-boilings, rancid oils, are taboo, because they are associated with bungling in the kitchen. With moderated ardour, we allow our sense of smell to distinguish foods and beverages, but we are not a race of epicures. The perfumes of flowers are classed as " nice smells." The idea of greediness is not associated with their enjoyment; besides, they remind us of gardens, sunshine, pretty forms and colours. When bottled, musk, orange-blossom, violets, lavender, are valued not so much for their own sweetness, as for their singular efficiency in obscuring nasty smells. Few persons practise the recognition and distinction of even pleasant odours. Very 
few, on first coming across a scented herb or shrub, pay sufficient attention to its perfume to impress it on their memories. They note the shape of its leaves and the colour of its flowers, but they are unable to identify it by its odour when they meet with it again. It is not much to be wondered at, therefore, that this slighted sense tends to leave us after middle life. It has been asserted-and probably the statement is justifiedthat rarely is the olfactory bulb of a man over forty free from signs of atrophy. We have no statistics concerning the brains of Japanese, who regard the sense of smell as one of the chief avenues of pleasure; but it may be that in this respect their brains present a contrast to our own. Yet the deadening of the sense is scarcely noticed, since its results are of little consequence as compared with those which follow loss of sight or loss of hearing. Many a man, as he grows older, declares that the cook of his club has lost his cunning, or frankly asserts that he " no longer cares for kickshaws. Cold beef, beer, and pickles, are good enough for him." He little suspects that his palate has lost its power of distinguishing the flavours of dainty meats and wines. Others continue to be exacting, because their imaginations still endow food with the qualities which they remember, just as people eat preserved asparagus or tinned peas because they look-however little they taste-like the gifts of Spring.

Taste accompanies the reception of food in the mouth. We have no knowledge of the situation of our own olfactory membranes, and therefore we suppose that a flavour, whether it be due to stimulation of taste-bulbs or olfactory membrane, is in the mouth. The odour of a flower we mentally project to a distance, because we associate the sight of a flower with its perfume. A dog, able to judge the freshness or staleness of a scent, must project its sensations of smell in the same way in which we project our sensations of sight. It forms an estimate, of a sort, of the time that it will take in reaching the source of the scent. Its excitement increases as the trail grows fresher.

Taste and smell are heavily laden with affective tone. When disagreeable, the feeling which they evoke is near akin to pain. It may gather head until, like hunger, it causes the discharge of motor neurones; but under its influence 
food is ejected, instead of preparation being made for its reception.

Taste and smell are senses which afford us no information with regard to time or space. They give rise to massive sensations. Such sensations, devoid of detail, produce a frame of mind rather than thought. The smell of tobacco does not distract attention. On the contrary, the steady flow of impulses to which it gives rise helps to inhibit, to subdue, the yapping of more exigent sensations. And since sensations of smell have no features of their own, they form a background to sensations of other kinds, entering with them into memory. No two scenes are exactly alike. One cannot recall another. But the scent of syringa is always the same. Wherever smelled, it opens the pathways in the brain in which were first associated a June evening and syringa, with a scene and a situation upon which memory loves to dwell. 


\section{CHAPTER XIII}

\section{VISION}

THE eye is enclosed in a globe of fibrous tissue, of which the front part, or cornea, being transparent, admits light. The epithelial layer which covers the cornea, conjunctiva, is also transparent. No bloodvessels enter these colourless tissues, unless as the result of inflammation due to infection or to exposure to sunshine or dust. For nutrition they are dependent upon the plasma which, exuding from, and returning to, the vessels which surround them, circulates in their tissuespaces. In advancing years, when the circulation is less brisk, a ring of opaque tissue, arcus senilis, encroaches on the cornea. In the interior of the globe, just behind the cornea, is a projecting shelf, formed of a ring of tissue supported by buttresses, ciliary processes. It is continued inwards as the iris, a muscular curtain. The "hyaloid membrane" lines the back portion of the globe. Continued on the inner side of the ciliary processes, it splits into several layers, which pass, one in front of the lens, others to its edge, to which they are attached, and still another, very thin, behind it. Since it holds the lens in place, the anterior portion of the hyaloid membrane is known as its "suspensory ligament." Thus the eyeball is divided into three chambers. The anterior is filled with watery lymph, aqueous humour. In it, resting on the anterior surface of the suspensory ligament of the lens, is the iris. The middle chamber contains the lens. The posterior chamber is filled with a liquid jelly, vitreous humour.

By the contraction of the circular fibres of the iris, the aperture of the pupil is diminished, limiting the light which enters the globe. This adjustment occurs when the illumination is bright. It is also brought into action for the purpose 
of cutting out divergent rays, which would not be clearly focussed when objects near at hand are looked at. The posterior surface of the iris and the inner surfaces of the ciliary processes are covered with dense black pigment. It is this pigment, showing through the uncoloured connective tissue and plain muscle-fibres of which the iris is composed, that gives their colour to grey and blue eyes. In many eyes the iris contains a brown pigment in its substance.

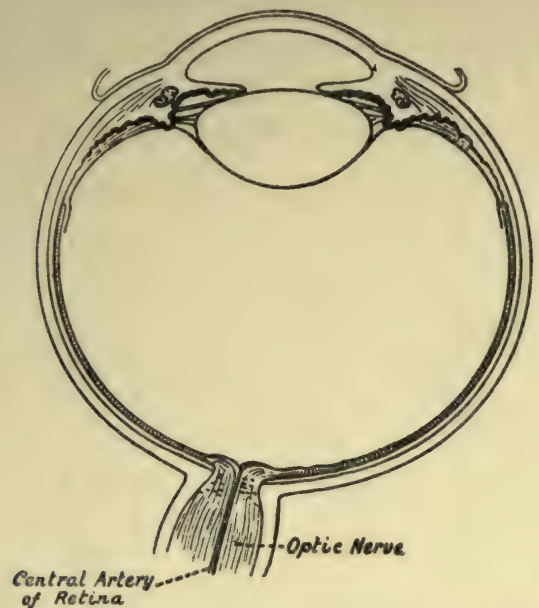

Fig. 27.-Horizontal Section throdgh the Right Eye.

The slight depression in the retina in the axis of the globe is the fovea centralis, or yellow spot the optic nerve pierces the ball to its inner or nasal side. The lens, with its suspensory ligament, separates the aqueous from the vitreous humour. On the front of the lens rests the iris, covered on its posterior surface wtih black pigment. On either side of the lens is seen a ciliary process, with the circular fibres of the ciliary muscle cut transversely, and its radiating flbres disposed as a fan.

The back portion of the globe of the eye is covered with a curtain, the retina, formed by the spreading out of the fibres of the optic nerve in front of various layers of nerve-cells and the sensory cells of the organ of vision, rods and cones. The retina lies between the hyaloid membrane, which encloses the vitreous humour, and a layer of pigment which " backs" it, as a photographer backs a plate when he proposes to use it towards a source of light-to take a photograph of a window from within a room. The serrated margin of the retina is somewhat anterior to the equator of the eyeball. The pigment which backs the retina is contained in a sheet of cells 
which belongs to the pouch of brain that extended outwards towards the eye-pit (p. 334). Properly speaking, therefore, it is a layer of the retina.

Three sets of tissues take part in the development of the eyjeball. (1) The epithelium covering the surface of the head is depressed as a pit, which gradually closes into a hollow sphere. This sphere, when its cavity is filled up, owing to the great elongation of the cells of its posterior half, becomes the lens. It breaks away from the rest of the epithelium of the surface, which clears to transparency as that part of the conjunctiva termed the "corneal epithelium." (2) The retina, as already stated, is a hollow outgrowth from the interbrain.
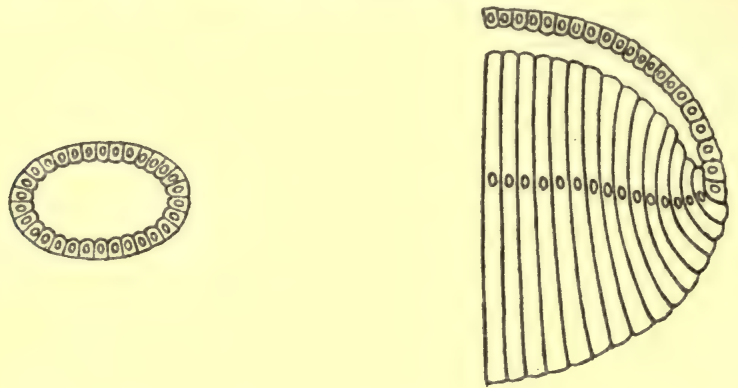

A

B

Fig. 28.-Diagrams showing the Mode of Formation of the Crystalline lens.

A, A pit in the epithelium on the surface of the head has closed into a hollow sphere. B, The cells of the posterior wall of this sphere are growing forward, as the flbres of the lens which traverse its whole thickness, with the exception of the cubical epithelium on its front.

As this pouch approaches the lens, its anterior half is pushed back into the posterior half, forming a cup with a double wall. The anterior, or inner, sheet of the bowl of the cup develops into the nervous layers of the retina, the posterior sheet into its pigmented epithelium. (3) Connective tissues are transformed into the other constituents of the globe-cornea, iris, vitreous humour, etc. The globe is complete, except at a spot on the nasal side of its posterior pole where the optic nerve pierces it.

The bloodvessels of the retina, entering with the optic nerve, ramify on its anterior surface. Under ordinary circumstances we ignore the shadows which they cast, as we ignore the blindspot which coincides with the disc of insensitive tissue presented 
by the end of the optic nerve, and many other imperfections; but it was shown by Purkinje many years ago that by a very simple manœuvre they may be forced upon our notice.

By making use of Purkinje's figures, it can be proved that the level in the retina at which undulations of light give rise to the impulses which evoke visual sensations coincides with the back of its anterior sheet-i.e., with the layer of rods and cones. A person stares fixedly at a white sheet in a dimly lighted room while an assistant, by the help of a lens, focuses

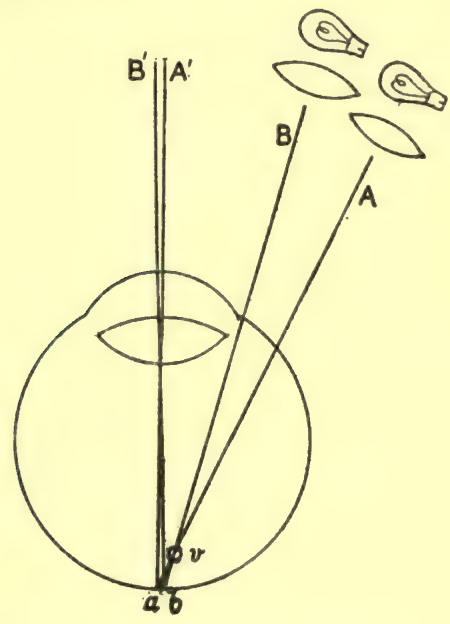

Fig. 29.-PURKINJE'S SHADOWS.

A beam of light traversing the eyeball in the direction $\mathbf{A}$ throws a shadow of the vessel $v$, lying on the front of the retina, upon the sensitive layer at its back. When the light is moved from $\mathbf{A}$ to $\mathbf{B}$ the shadow moves from $a$ to $b$. The mind, supposing the shadow to be a dark mark on the nearest wall or screen, infers that this mark moves from $\mathbf{A}^{\prime}$ to $B^{\prime}$.

a strong light on the front of his eyeball, to the outer side of the cornea. The rays, traversing the white of the eye, throw shadows of the retinal vessels on the layers behind them; but this not being the way in which light normally enters the eyeball, the person experimented upon supposes that he sees the shadows in front of him. He mentally projects them on to the white sheet. The pattern of his retinal vessels appears on the sheet in grey streaks. When the spot of light is moved, the shadow-pattern shifts, and in the same direction; since, 
as the retinal image is reversed, a movement from right to left is interpreted by consciousness as a movement from left to right. Given the angle through which the light is moved, and the apparent displacement of the shadows, it is a simple matter to calculate the distance behind the bloodvessels of the sensitive layer of the eye. So definite are Purkinje's figures that the shadows of individual blood-corpuscles can be followed, and the rate at which they are moving in the capillaries of the retina calculated.

The retina is the organ of vision. Cornea, iris, lens, vitreous humour, are parts of the camera in which this sensitive screen is exposed; and of the retina, the sensitive layer is the layer of rods and cones. Interest therefore centres in these structures. They are disposed with the utmost regularity on the posterior surface of a thin, reticulated membrane-the outer limiting membrane. But rods and cones are only the outer halves of sensory cells, the inner portions of which, reduced to a minimum in thickness, except where they contain their nuclei, lie in the outer nuclear layer. Rods are the larger elements. Each consists of an outer segment, or limb, of relatively firm substance transversely striated, and liable to break into dises; and an inner limb of much softer substance, again divisible into two parts, the outer longitudinally striated, the inner granular. Cones are almost identical in structure with rods, save that their outer limbs are much smaller, their inner limbs rather fuller. In frogs and various other animals, but not in Man, each cone contains at the junction of its two limbs a highly refracting globule of oil, often brightly coloured, red, yellow, or green.

The layers in front of the rods and cones contain nervous elements accessory to them. In the "inner nuclear layer" are the ganglion-cells of the retina, homologous with the cells of the ganglia on the posterior roots of spinal nerves; but, in the retina, bipolar and extremely minute. On either side of the rather thick layer occupied by the nuclei of these ganglioncells (and of cells of other types which, for the sake of clearness, we omit) is a felt-work of nerve-filaments in which their two extremities arborize. The most internal, or anterior, layer consists of a single sheet of rather large collecting cells and of their axons, which stream towards the optic nerve. Each 


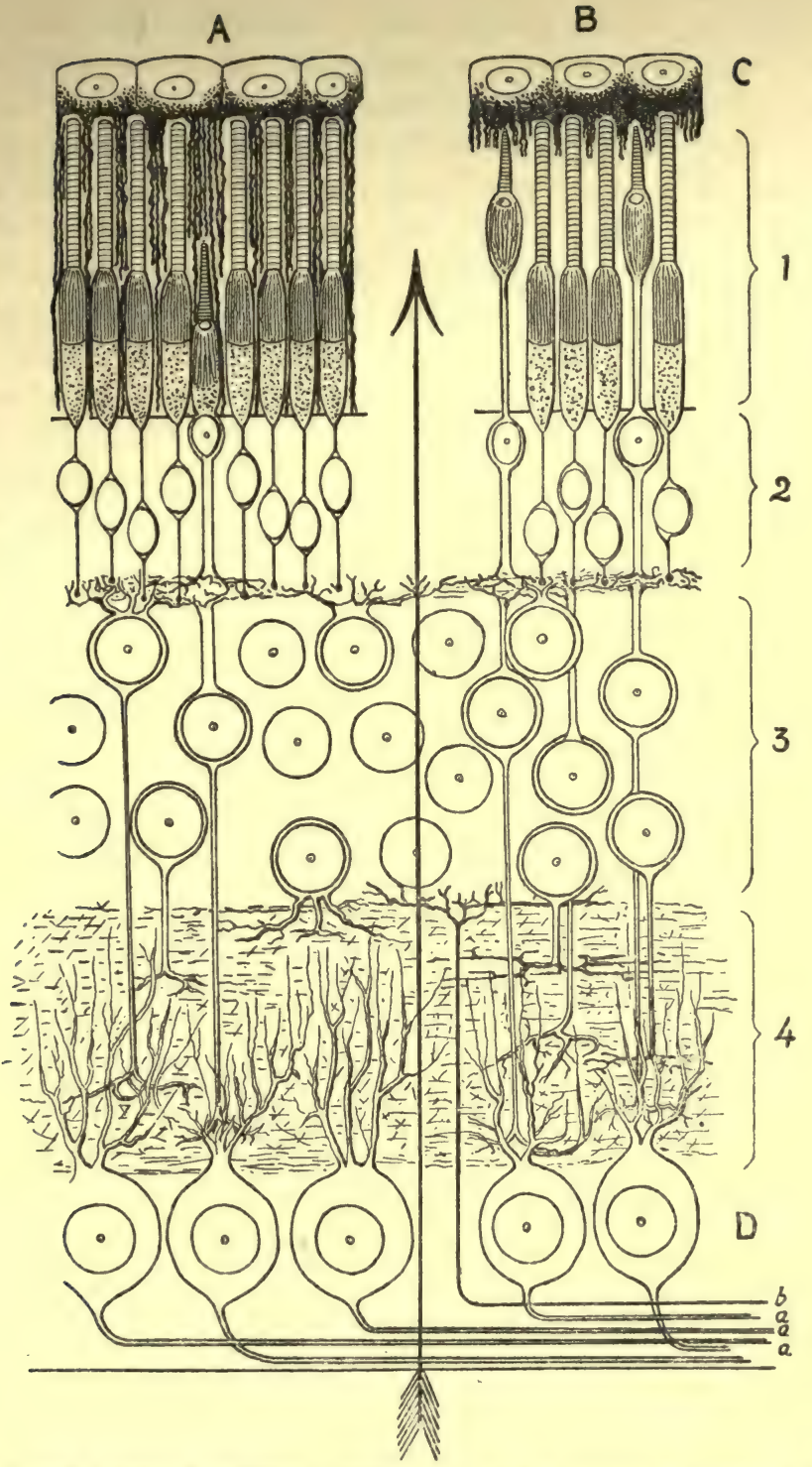

Fig. 30.-The Retina in Vertical Section-A, after Exposure to Bright Light; B, AFTER RESTING IN THE DARK.

The arrow shows the direction in which light traverses the retina. C, Retinal epithelium, with its pigmented fringe. 1, Layer of rods and cones, separated by the external limiting membrane from 2, the layer of the nuclei of the rods and cones. 3, The ganglion cells of the retina, which are homologous with the cells of the afferent root of a spinal nerve. Their peripheral axons ramify beneath the sensory epithelium (rods and cones and their nucleus-bearing segments), their central axons in 4, the inner molecular layer. D, Collecting cells on the front of the retina; $a a b$, their axons which conduct impulses to the brain; $\boldsymbol{b}$, an efferent fibre from the brain. 
cone has its proper ganglion-cell, collecting cell, and efferent fibre. Rods are served in groups by ganglion-cells and collecting cells. From this it may be inferred that a cone is a sensory unit, an inference confirmed, as we shall show presently, by direct evidence. The connections of the rods show that they also are sensory elements, although it may be doubted whether they are sensory units. The optic nerve contains a very large number of fibres-about a million-all small, but some distinctly larger than the rest. The largest very probably belong to the collecting cells of rods. But the retina certainly does not contain a million collecting cells. A considerable residue of fibres is therefore unaccounted for. It is supposed that they are afferent to the retina, but we have no knowledge regarding the nature of the impulses which descend from the brain.

The retinal pigment is not merely a backing for the sensitive screen. It undoubtedly plays an important part in vision. That it is not essential is evident from the fact that albinos, whose eyes appear pink owing to the absence of pigment, and the consequent showing through of the blood in the exceedingly vascular membrane which lies behind the retina, can see; although their visual sense cannot be described as normal. They are exceptionally sensitive to an excess of light. We shall return to this subject after describing the differences in manner of functioning which distinguish rods from cones, differences so marked as to justify us in speaking of two kinds of vision.

During twilight warm tones gradually fade out of the landscape; cold blues and greys predominate. A time arrives when scarlet poppies look black, although yellow and blue flowers and green leaves can still be dimly distinguished. In full daylight colours are seen at their brightest in the high lights ; where the light is dim they tend to appear in different shades of grey. At night, if the sky is star-lit, all colours give place to a slightly bluish grey in the high lights, black in the shade. But a not very uncommon abnormality is nightblindness-inability to see at all when the light is not bright enough for the recognition of colours. In persons so affected the rods do not function; for it is with the rods that we see in weak light. They record differences in intensity between the 
lower limit of their sensitiveness and the higher degree of brightness, at which they are superseded by cones; but they afford no information regarding colour. Their monochrome is interpreted by the mind as a bluish grey, apparently because, since they are insensitive to red rays, the sensations of which they are the source are associated with the blue end of the spectrum. When the cones are stimulated very slightly, the reinforcing grey of the rods enables us to distinguish all other colours, save red, which appears black. In bright light the rods are in a permanent state of exhaustion; they do not contribute to vision. Rods respond to stimulation more slowly than cones. This fact enables us, by a very pretty experiment, to distinguish the two kinds of vision. A disc of green paper about the size of a threepenny-bit is pasted on a red surface. Held at arm's length in a room lighted by a single candle, the disc looks dull green when the gaze is directed at it; but if the gaze be directed 2 or 3 inches to one side of it, it appears brighter than before, but less distinct and almost grey. The explanation of this is to be found in the fact that at the posterior pole of the eye there is a shallow cup-fovea centralis-which carries cones only, without rods. This small depression is the area of direct vision, the only spot at which we see things quite distinctly. At the fovea the nuclei and nerve-cells of the retina are withdrawn from in front of the cones to the margin of the cup, in order that they may not interfere with the passage of light. The pit and the ring round it contain some yellow pigment. Hence it is usually termed the "yellow spot." When we are looking straight at the green disc, it is focussed on the yellow spot. It then excites a sensation of greenness; but since this is not reinforced by any rod-sensations, the green is dull. When it is focussed outside the yellow spot, it stimulates rods and the sparse cones which lie amongst them; and the rods being more sensitive than cones to light of low intensity, the disc looks brighter. If, while the observer is still gazing fixedly at a spot to the side of the dise, the red paper be waved rapidly, but gently, to right and left, a brightish grey cover seems at each movement to slip off the dark green disc, and to regain its position a moment later, with a jump. The grey rod-sensation, developing more slowly than the green cone-sensation, is, as 
it were, left behind. The two are separated at the moment when the paper starts to right or to left.

Astronomers have long recognized that one of the smaller stars which catches the attention when they are not looking directly at it may be invisible when the gaze is directed to the spot where it ought to be. It was visible when focussed on rods, but it is not visible when focussed on cones. In most birds the retina shows cones alone. To anyone who for the first time enters a dovecote at night the experience is very curious. A candle is for him a sufficiently strong illuminant, but it does not give light enough to enable the pigeons to see. Although evidently alarmed by the noise made by the intruder, they allow themselves to be taken down from their perches without making any attempt to escape. If, startled by the touch of a hand, they take to flight, they fly against the wall. Pigeons are night-blind. The retina of an owl bears chiefly rods, the outer limbs of which are exceptionally long.

The outer limbs of the rods are coloured reddish purple. This colour is quickly bleached by light. If a frog which has been kept for a short time in the dark be decapitated, its head fixed for ten minutes in a situation in which a window is in front of it, then carried to a photographic dark-room, where an eye is taken out by red light, opened, and the retina removed, a print of the window will be seen upon it. Such an optogram may be fixed by dipping the retina in alum.

The retina is easily detached from its pigment-layer. If it has been bleached by exposure to light, it regains its "visual purple" when again placed in contact with its pigment. Evidently the visual purple is renewed from the pigment which lies behind (and around) the rods.

From the cells of the pigment-layer a fringe of streaming processes depends amongst the outer limbs of the rods and cones (Fig. 30). In a dull light the processes hang but a short way down ; in a bright iight they react almost to the outer limiting membrane. They supply pigment to the rods, but their relation to cones is not understood. It is clear, however, that the cones, although they are not coloured, are dependent upon the pigment-fringe, since they always remain in contact with it. Their inner limbs elongate in the dark, lifting them to the 
pigment, and shorten in bright light. These movements may merely indicate that the cones require a backing of pigment, but it would seem more probable that, like the rods, they absorb a substance which is sensitive to light, although we cannot recognize it by its colour.

The responsiveness of the rods to light is due to visual purple. As every lady is aware, colours, especially mauves and lilacs, are bleached by light. The chemical change affected by light in the colour of the outer limbs of the rods is the stimulant which originates impulses in the nerve-fibres connected with them, and it is generally believed that cones-the more highly specialized sensory cells-are stimulated in the same way. Visual purple is particularly abundant in all animals that range at night, with the exception of the bat. But its absence in the bat does not militate against the theory that it is the cause of night-vision, for it has been shown that a blind bat flies with almost as much freedom, and avoids obstacles-even threads stretched across the room-with as much skill as one that can see. It is guided by the bristles of its cheek. So, too, is the cat, which has the reputation of being able to see in the dark. Undoubtedly a cat's eye is an exceptionally efficient organ in dim light, just as it is exceptionally sensitive to sunshine-it is provided with an iris which contracts the pupil almost to a pinhole-but the cat trusts to the bristles of its cheek for information regarding the things which block its path.

Most of the peculiarities which distinguish the reactions of the eye from those of other sense-organs can be explained by its mode of stimulation-the initiation of a nerve-current by a chemical change. No stimulus, if sufficiently strong, can be too brief. The retina reacts to an electric spark in the same way as a photographic plate; but, unlike the plate, the retina is restored to its previous condition of sensitiveness in about one-tenth of a second. A visual sensation lasts about one-tenth of a second. This prolongation of the sensation is, however, a mental, not a retinal, effect. The mind continues to see an object which has been illuminated by a flash until the retina is again in a condition to send brainwards a second impulse. Were our sensations coincident in duration with the stimulation of our sense-organs, we should live in a flickering cinemato- 
graph. When one is watching a moving point of light-the glowing end of a match, for example-the prolongation of sensation has its disadvantages; the moving point is interpreted as a streak of light. If the illumination be very brilliant, the object seen may give rise to a prolonged afterimage. A glance at the sun leaves in the mind for seconds, or even for minutes, the image of a glowing disc. Sensations due to stimulation of the yellow spot last longer than those which originate in the peripheral retina. If, in a train, one is being carried at a certain pace, past a fence composed of upright palings, one sees the separate slats until the eyes are directed towards them, when they fuse into a continuous screen.

The phenomena of negative or complementary images are of retinal origin. The bright image of the sun, if the stimulus has not been too violent, gives place to a black disc. If one closes the eyes after staring at a window, a black surface crossed by bright lines is seen in place of a white surface with dark frames to the panes. If, after staring at a red surface, one looks at the ceiling, a green patch is seen; after yellow, blue. Every colour has its complement, which may be determined in this way. There is much uncertainty as to the exact terms in which this phenomenon is to be accounted for, but little doubt as to its being due to the peculiar mode of reaction of the retina to light. Chemical substances which have been used up have to be restored, and during the period in which they are coming back to what may be termed a neutral condition the retina delivers to the brain impulses of the opposite sign.

Contrasts which are experienced simultaneously are more difficult to understand than those which appear successively. In Fig. 31 the half of the grey cross which is surrounded by black appears brighter than the half which lies on white paper. A grey cross on a red background looks green; on a green background, red; on yellow, blue ; on blue, yellow. If green is on red, it looks greener than if it is on white or black. These simultaneous contrasts are seen best when the strength of the colours is reduced by covering them with tissue-paper. It is as if activity of any one part of the retina is accompanied by activity of the opposite sign in the remainder. But it is unsafe, in explaining our various sensations, to lay too much stress on the mode of stimulation. The mind judges sensations in 
the light of previous experience. In anatomical language, the effect of sensations upon the personality depends upon the paths which impulses follow in the brain, and the associations which have been established by previous impulses which have followed the same paths. The retina enables us to distinguish tone and colour. By the variations in tone, the juxtapositions of light and shade, we recognize form. All streams of impulses which do not present tone-variations-do not, that is to say, reproduce the details of a scene-are interpreted in terms of colour. Every child discovers that the tedium of the intervals during which it is proper that his eyes should be closed may be relieved by pressing his knuckles against the lids. Although

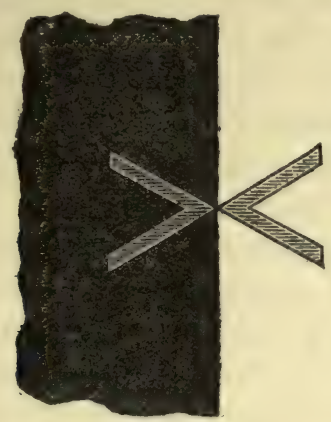

Frg. 31.-Sinultaneous Contrast.

The shading of the two V's is exactly similar; but the figure in half-tone on black appears brighter than the flgure in half-tone on a white ground.

the world is shut out, a phosphene offers itself for his consideration-a yellow or white disc of irregular form with a red margin, changing into lilac bordered with green, and then into yellowish-green with a blue edge. Such, if my recollection can be trusted, were the pictures which I used to see as a boy ; but no adjustment of pressure calls them forth with anything like the same vividness now.

All the senses show a tendency to rebound after activity, exhibiting contrast-phenomena ; but the contrasts of vision are more marked and varied than those of the other senses, as everyone who is curious in the observation of his own sensations is aware. Negative after-images are generally referred to the retina; but various other kinds of after-image and contrastphenomena must be attributed to the judgments passed by the 
mind upon the sensations which it receives ; and not to physical changes in sense-organs. Positive after-images are well-marked appearances, although less common, perhaps, than the phenomena of reversal of sensation of which we have just written. On waking in the morning, one looks at the window ; shifting the gaze to the ceiling, an after-image of the window appears, just as one saw it, with bright panes and dark frame. The "dark adapted eye," being exceptionally sensitive, yields the same persistent positive after-image as the eye in its usual condition yields, after being directed towards the sun at mid-day. Movement-after-images can be explained only by referring them to misdirection of judgment. If the gaze is fixed on a rock close beside a waterfall, then shifted to a bank covered with grass or bushes, the part of the bank which occupies the lateral part of the field of vision appears to rush upwards, reversing the movement of the water. When the gaze has been fixed upon falling water-a narrow stream sparkling in sunlight-a central strip of the field moves upwards, the margins remaining stationary. If one stares at the spot on the surface of a basin of water on which drops are falling from a tap, and then looks at the floor, it is seen to contract towards the spot looked at, reversing the movement of the ripples in the basin. These observations reveal a fact of great importance in the physiology of vision. It is, probably, impossible truly to fix the gaze. The muscles of the eyeball keep the retinal field in constant movement-larger movements with minute oscillations superposed. When, as in watching a waterfall, movement has for a time taken a definite direction, its cessation is judged to mean reversal.

The anatomical unit of sensation is a cone. The forea centralis, the only part of the retina capable of receiving sensations sufficiently discrete for reading, contains cones alone. If the gaze be directed but a very few millimetres on to the white margin of the page, letters lose their form. In the fovea the centre of one cone is $3.6 \mu$ distant from the centre of the next. Two stars are visible as separate stars if they subtend an angle of at least 60 seconds with the eye. Their images on the retina are then $4 \mu$ apart. Parallel white lines ruled on black paper, held at such a distance as causes them to subtend angles of 60 seconds with the eye, appear not straight but 
wavy, showing that their images are taken up, not by a continuous substance, but by the mosaic of cones. So far the explanation of the visual unit is strictly anatomical; but it must be added that trained observers can recognize the separateness of objects which subtend angles of much less than 60 seconds-not more than 5 or 6 seconds. This can be accounted for only on the hypothesis that images far closer together than the width of a cone produce a specific effect in passing across the anatomical unit.

In 1807 Thomas Young, the physicist, formulated a theory to account for colour-vision. He supposed that the retina contains three kinds of apparatus- $a, b$, and $c$-each especially responsive to a particular kind of light, all three slightly stimulated by rays of all colours. (Young imagined three kinds of nerve, but modern supporters of his theory suppose three different substances chemically changed by light.) A prism spreads out the rays which are combined in white light into a band in the order of their wave-lengths-those which have the longest wave-length $(0.8 \mu)$ and the slowest rate of vibration (381 billions to the second) at one end, those which have the shortest wave-length $(0.4 \mu)$ and the most rapid vibration ( 764 billions to the second) at the other : between these two extremes every intermediate grade of length and rapidity. These are a mere fraction-a small group-of the waves which the æther transmits, but they are all that we can see. The long, slow vibrations give rise to sensations which we describe as red; the short, rapid vibrations we describe as violet. Our names for the tints which intervene are singularly old-fashioned and unsatisfactory, but all persons agree that they recognize in the spectrum a certain number of definite colours. Some normal-sighted persons say twelve, others eighteen. It is largely a question of terminology.

Many considerations show that it is quite unnecessary to imagine that the retina is affected in a different kind of way by every kind of light, or by each of several groups of waves. If the red of the spectrum is mixed with yellow, we receive an impression of orange, which is identical with the impression produced by waves of the mean length of red and yellow; orange and green give yellow; yellow and blue, green. Any two complementary colours yield white. By taking three 
colours-say, red, green, and violet-we obtain, when they are duly mixed, not white light only, but light of any other tint, although not of spectral purity, since it is mixed with white. Young considered that all the conditions of colour-vision would be satisfied, all our various sensations provided for, if the retina contain three kinds of apparatus which light, according to its quality, affects in varying degrees; and with this theory of three kinds of apparatus- $a, b$, and $c$-the theory of three elementary or fundamental colour-sensations is indissolubly linked. The colour $x$ produces its intensest effect when $a$ is stimulated, with the least possible stimulation of $b$ and $c ; y$ is the reaction of $b, z$ of $c$. Recent studies of the curves of intensity give us the tints of $x, y$, and $z$ as carmine-red, applegreen, and ultramarine blue.

The blending of sensations is illustrated with the well-known colour-top. But perhaps the most striking proof that three elementary colour-sensations are adequate to produce our visual world is afforded by photographs taken with the threecolour method. Three plates are exposed-(a) behind a red screen, $(b)$ behind a greenish-yellow screen, (c) behind a blue screen. They are fixed in such a way that the portions acted upon by light are rendered insoluble, whereas the rest of the film can be dissolved away; $a$ is then stained red, $b$ greenish yellow, $c$ blue. The three are superposed, and the result appears to the eye as an exact reproduction of the subject of the photograph in all its hues. It shows every shade of orange and green and violet. It is as bright-that is to say, as full of white light-as the original.

Various objections may, however, be brought against Young's theory. Of these, the most weighty are: (1) The retina does not contain three kinds of apparatus, as Young supposed; nor can we find three kinds of photochemical substances, as required by the theory in its modern form. If we could find them, a fresh difficulty would arise; for we have no reasons for supposing that one and the same nerve-ending can receive stimuli of three different kinds. (2) The theory offers no explanation of negative after-images-the complementary colours experienced when the eye is closed after staring at a brightly coloured object. (3) It does not adequately account for the various deficiencies of colour-blindness. 
It is well recognized that there are various degrees of colourblindness, and that the colour-vision of persons considered normal presents different grades of refinement. Nevertheless, the abnormalities of colour-blind persons are so marked that cases fall into definite classes. Those whose cones do not function-which means that their yellow spots are either undeveloped or diseased-see all things grey. They are totally colour-blind. Excluding these, the colour-blind may be grouped in one or other of two divisions- $(a)$ those who confuse red and green, $(b)$ those who confuse yellow and blue. One person out of every thirty-five is red-green blind. The proportion is even higher if males only are considered, showing how very unfortunate is our choice of warning signals. A man who is red-green blind cannot tell the port from the starboard light. Blue-yellow blindness is, on the other hand, extremely rare. According to Young's theory, colour-blindness is due to the absence of one of the three sets of visual apparatus. But cases do not altogether conform to this hypothesis. We knew an amateur water-colourist, since deceased, who derived intense pleasure from the beauties of Nature, and showed no mean skill in reproducing them with his brush, notwithstanding the fact that he was red-green blind. Each night his sister arranged his paint-box for him, and only rarely did he use vermilion to fill in a foreground of lush green grass. But this mistake, when he made it, did not destroy his own satisfaction in the picture. It was clear that red had a value for him, although he confused it with green. It is impossible for a normal person to see through the eye of one who is colour-blind, and there is no other means of comparing his sensations with our own. The mistakes which the colour-blind make in sorting coloured objects and in naming mixtures of light selected from various parts of the spectrum show the range of their deficiency, but give us no information regarding the qualities of the sensations which they retain.

The test of colour-sensitiveness usually employed is the grading of a large number of wools of different tint. The order in which the colours should be arranged is not a matter of opinion. They must be placed in the order in which they occur in the spectrum-i.e., arranged according to their wavelengths. In the cases of colour-blindness which are most fre- 
quently met with the defect may be described as due to an absence of the sense of redness, or as an absence of the sense of greenness. The two conditions can be distinguished. But since the eye is not dark for red (although in certain cases vision is very weak for the red end of the spectrum) or dark for green, the abnormality cannot be adequately accounted for on structural grounds. It is not explicable on the hypothesis that one of three sets of responsive sense-organs (or nerve-fibres) or photochemical substances is absent from the eye. Again, it is generally agreed that the sensations of white, yellow, and blue of the red-green colour-blind are similar to those of normal persons. This is not in harmony with the theory of the omission from their eyes of one of three pieces of colour-apparatus.

Professor Hering, of Leipsic, adopting the generally accepted view that light effects chemical changes in substances contained in the retina, to which changes stimulation of nerve-endings is due, formulated a theory of colour-vision which many physiologists prefer to Young's. He imagines that the retina contains three kinds of pigment, each of which is, as he believes all living substance to be, in a constant state of change. It is at the same time being built up and destroyed. Using the terms which connote the opposite directions of metabolism, the pigment is simultaneously undergoing anabolism and katabolism; the two processes, when the retina is at rest, maintaining equilibrium. When light acts upon either of the substances, it hastens, according to its quality, either the one process or the other; and the chemical change, whether it be constructive or destructive, stimulates the endings of optic nerves. Hering assumes, therefore, that there are six elementary qualities of visual sensation-red, green, yellow, blue, white, black. Red, yellow, white are due to anabolism of the visual substances; green, blue, black are due to their katabolism. The installation of yellow amongst the unanalysable colours is a relief to many minds. It is almost impossible to think of yellow as a compounded colour. White also, we feel, is not a compounded colour, despite our knowledge that a prism scatters from it all the hues of the rainbow. Black, many persons assert, gives them a definite sensation, and not merely a sense of rest. (Parenthetically, it may be observed that the 
feeling that a colour is pure or mixed is not to be trusted. It may be based upon the chromatic aberration of the eye, or it may be reminiscent of the paint-box. We know that we cannot make yellow by mixing red and green pigments, hence we feel that it is pure. Of green we are not by any means sure; gamboge and Prussian blue come into our minds.) Except when the light which falls upon the retina is giving rise to one of the four pure colour-sensations, all three substances are simultaneously affected, although one may be undergoing katabolism while the other two are being built up, or vice versa. Hering accounts for simultaneous contrast by assuming that the activity of any one part of the retina induces an opposite kind of change in the remainder, and especially in the vicinity of the primarily active part. When a certain patch is developing a sensation of red, the rest of the retina develops a sensation of green.

The great merit of the theory is, however, to be found in its offering an explanation of complementary after-images. The green patch seen with closed eyes after one has stared at a red object is due to the rebound of metabolism. In returning to a condition of chemical equilibrium the retinal substance acts as a stimulant which evokes the antagonistic colour. But it is a theory which makes very large assumptions. It assumes, for example, the possibility of the existence of a substance which is built up by light from one end of the spectrum, and decomposed by light from its centre. Not that Hering regards the existence of three retinal substances as essential to his theory. He is prepared to transfer to the brain the seat of the substances, or the substance, which, by their, or its, anabolism and katabolism, produces antagonistic colour-perceptions ; but in this he is abandoning physiology for metaphysics. We have no warrant for imagining that there exists in the brain any substance which, by undergoing physical changes of various kinds, produces various psychical effects. The problem to be solved is physiological. Rays of light of different wavelengths excite the retina to discharge impulses which are variously distributed in the brain. The effects which they produce in conseiousness depend upon their distribution. The impulses to which the longest rays give rise evoke sensations of red, those due to the shortest, sensations of violet. 
And what is true of the retina as a whole is true, apparently, of each individual cone. In what way does light act upon a cone? It is one of the most fascinating problems in physiology. Round it our thoughts revolve whenever we are trying to form conceptions of the nature of stimulation, sensation, and perception. Each of the two theories which we have expounded above helps to group together certain of the more striking phenomena of colour-vision, but neither gives a satisfying explanation of their causation.

The sensitiveness of the retina is in a remarkable degree adjusted to the intensity of the light. When a dark room is entered, the pupil dilates; but one's power of distinguishing objects continues to increase after the pupil has reached its maximum size. At the end of ten minutes the eye may be twenty-five times as sensitive as it was when the room was entered. This adaptation to darkness is due in large degree to the substitution of rods for cones as the organs on which vision chiefly depends. But it cannot be wholly due to this, since it occurs when one is working with a red light. Probably the red used in a "dark room " is not sufficiently near the end of the spectrum to be completely without influence upon visual purple, but it is a colour to which rods are comparatively insensitive. Other evidence also points to an adaptation of cones as well as of rods.

Accommodation of the eye for distance is brought about by a mechanism which allows the lens to change in shape. It becomes more convex when a near object is looked at than it was when adjusted for an unlimited distance, which is its condition when the eye is at rest. Adjustment for near objects involves muscular action, and is accompanied by a sense of effort, however slight. Whilst the eye is at rest the lens is mechanically compressed against the anterior layer of its suspensory ligament. Accommodation for near vision is effected by the ciliary muscle, which is placed in the shelf of tissue which projects into the interior of the eyeball. This muscle is made up of a ring of circular fibres, and to the outer side of this, of fibres which radiate backwards and outwards. The longitudinal, or radiating, fibres obtain their purchase by attachment to the firm wall of the globe just beyond the cornea. They spread into the front of the loose chorioid membrane 
which lines the eye behind the retina. By the joint action of these two sets of plain muscle-fibres the suspensory ligament is slackened, and the extremely elastic lens, previously compressed, bulges forwards. The radius of curvature of its anterior surface changes from $10 \cdot 3$ millimetres for distance to 6 millimetres for vision at the "near point." It was stated, in connection with the development of the lens (p. 374), that the cells of the posterior half of the hollow sphere out of which it is formed grow forwards into extremely long fibres, which traverse its whole thickness. These fibres are bent like the segments of a carriage-spring. Their anterior ends rest against the flattened ligament of the lens; the vitreous humour,

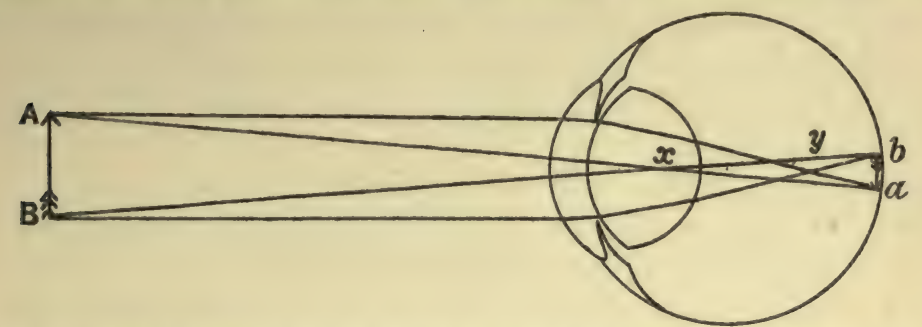

Fig. 32.-The Formation of an Image by the Refracting Media of the Exe.

$x$, The common centre of curvature (nodal point of the several media). Rays which pass through this point are not deflected. $y$, The principal focus of the system. All rays which are parallel to the optic axis converge to this point. The image of the point $\mathbf{A}$ is formed at $a$, the spot at which a ray parallel with the optic axis meets an unbent ray-the image of $B$ at $b$.

which is always under tension, compresses their posterior ends. When removed from the eye, the lens becomes rounder than it is in situ, even when accommodated for near objects. But in later life it grows stiff. It ceases to bulge forwards when its ligament is slackened. Hence it becomes necessary to aid the presbyopic eye with convex glasses when it is used for near objects, although for distant vision it remains as effective as ever. If the ciliary muscle is constantly and completely relieved of the labour of accommodation, it grows lazy, or rather wastes from want of use. A person who relies on spectacles loses his power of accommodation ; but ophthalmologists agree that self-focussing, if it give rise to a sensation of strain, is bad for the eyes. In myopic persons the eyeball is too deep; objects are focussed in front of the retina. In hypermetropia ("long sight") the eyeball is too shallow; 
objects are focussed behind the retina. Concave glasses correct the one condition, convex glasses correct the other. Glasses are also very commonly called for to neutralize another defect-regular astigmatism-which may be present by itself,

A

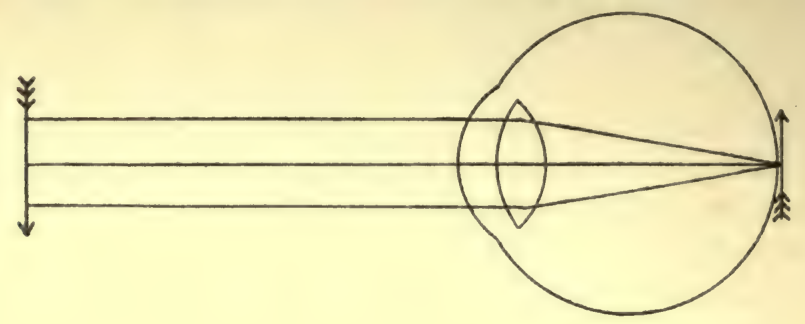

B
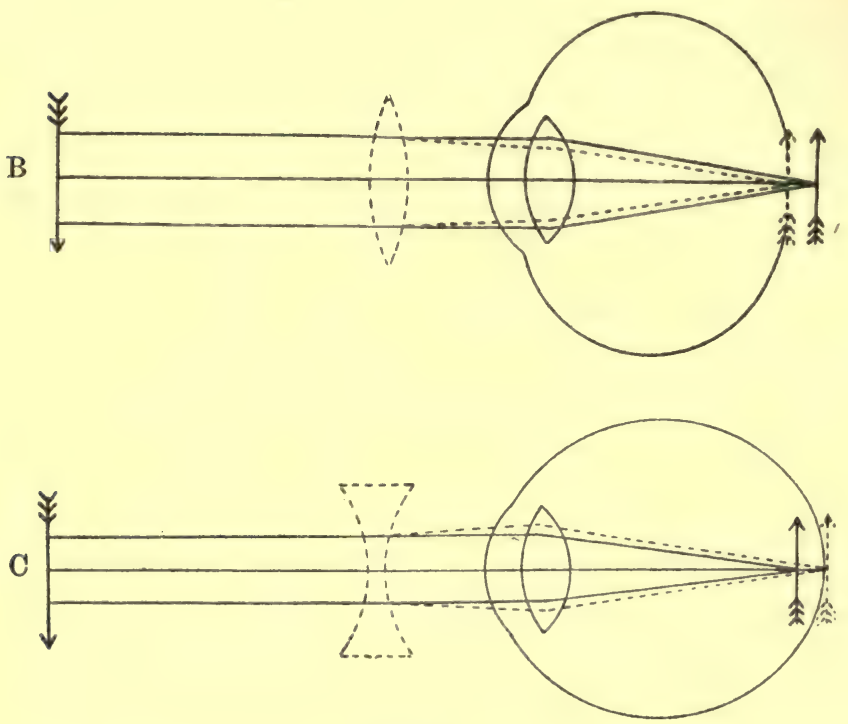

FIG. 33.

A The normal eyeball, in which, when the ciliary muscle is relaxed, parallel rays are brought to a focus on the retina. B, A hypermetropic eyeball. Its depth being less than normal, parallel rays are not brought to a focus on the retina when the eye is adjusted for distant vision without the aid of a convex glass. C, A myopic eyeball. Its depth being more than normal, a concave lens is needed to diminish the convergence of parallel rays.

or may accompany insufficient length or too great length of the optic axis. It is due to unequal curvature of the cornea. Usually the curvature is sharper in the vertical than in the horizontal meridian (cf. p. 269); as a consequence, points in a vertical line are focussed in front of points in a horizontal line. 
Cylindrical glasses, not lenses, are required to correct this defect. And here it may be well to call attention to the fact that rays of light are more sharply refracted by the surface of the cornea than they are by the crystalline lens. The lens has a high index of refraction (1.45), but it does not lie in air (the index of refraction of which is 1 ), but between two humours which have about the same index as water-namely, 1.336. The bending by the combined action of the cornea and the lens of rays of light which come from a source so distant that they may be considered as parallel brings them to a focus on the retina, when the lens is at its flattest. When the lens is at its roundest, rays which diverge from a point only 5 inches in front of the eye are focussed on the retina. The lens is therefore essential for accommodation, but, after its removal for cataract, vision, even for near objects, is rendered possible by the use of convex glasses.

A star or a distant gas-lamp is seen as a point of light with rays. Usually this figure, which has given origin to the expression " star-shaped," shows three greater rays alternating with three lesser rays. Such an image is not produced by a point of light near to the eye, since it is due to the puckering of the lens when flattened against its ligament. It brings into evidence the three axes on the front of the lens and the three axes which alternate with them on the back, with regard to which the lens-fibres are disposed.

As an adaptation of living tissues to optical purposes the eye is above admiration, yet it presents many defects, which an optician corrects in the instruments which he manufactures. A remarkable fact in the physiology of - vision is our unconsciousness of the imperfections of its organ. An unusual experiment is needed to bring them to our notice. If we look through a common glass lens uncorrected for unequal refraction of rays of different wave-lengths, we recognize that a bright object is shown with a colour-fringe, yet we take no cognizance of the colour-fringes which surround the images of all bright objects focussed upon our retinæ. If we think about the matter, we recognize a feeling that blue in a window of stained glass appears farther away than red; but this might well be due to association. Blue glass is chiefly used for the sky. If we look at a bright object through purple glass, we 
see it either red with a blue fringe or blue with a red fringe, according as the eye is focussed for red or for blue. The purple glass having absorbed all intermediate rays, we become aware that we cannot focus the two extreme ends of the spectrum at the same place. Since a greater effort of accommodation is needed to focus red, we judge that the bright object is nearer to us when it appears red than when it appears blue.

Spherical aberration is another fault of the lens. The rays which enter its margin are brought to a focus sooner than those which pass through its centre. This is due to the fact that its surfaces are regularly curved, whereas a glass lens is corrected by grinding it flatter towards the margin. This defect is partly corrected by the cornea, which has an ellipsoidal surface, and partly by the greater density of the centre of the lens. Yet it is still necessary for the eye to be "stopped down " by the iris when a near object is looked at, although less light is entering the eye than when it is directed to the horizon-a condition which would lead a photographer to open his iris-diaphragm.

Of all the imperfections of the eye which the mind ignores, the most remarkable is the gap in the field of vision, due to the gap in the sensitive layers of the retina, which occurs where the optic nerve enters it-the blind spot. Hold this page of the book 10 inches from the face, keeping the lines of print horizontal. Close the left eye and look at $\mathrm{X}$ with the right eye. The black disc disappears, because its image is focussed
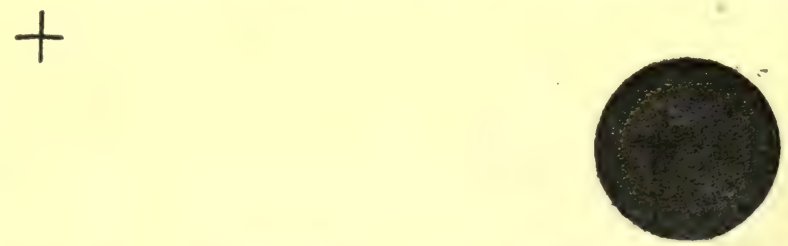

on the blind spot. Since the picture on the retina is reversed, it is clear that the optic nerve enters the globe to its inner side, and slightly above its horizontal meridian. But, unless we employ an unusual test, we are quite unconscious of the fact that a definite hole is punched in the picture. The mind fills it in, and the way in which it does so is extremely suggestive. 
It lies about it-in a downright ingenuous fashion if it is confident of credence, in a more subtle way if a simple falsehood is likely to be challenged. In place of the black disc make nine conspicuous crosses :

\section{$\mathrm{X} \quad \mathrm{X} \quad \mathrm{X}$ \\ X $\quad X \quad \mathrm{X}$ \\ $\mathrm{X} \quad \mathrm{X} \quad \mathrm{x}$}

Hold the paper in such a position that $X$ falls upon the blind spot. It ought to disappear, but the mind assures you that there is a cross at that spot. The mind completes the field. In place of the crosses use noughts and crosses, thus :

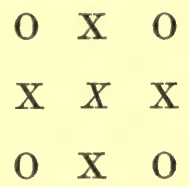

Now let $X$ fall on the blind spot, and allow the eye to go just a little out of focus. The four marginal crosses draw inwards :

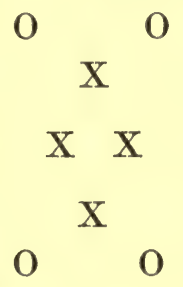

The mind contracts the field. Still denying the gap, but not having sufficient data from which to invent an object, the fraudulent nature of which would not be found out the instant that the gaze is shifted, the mind lies regarding the position on the paper occupied by surrounding objects.

Is it quite fair to the mind to say that it lies about the blind spot? The mind judges sensations in the light of experience. An association of previous sensations teaches me that the wall of the room is not pierced by a round hole a foot in diameter opening into outer darkness. Many sensations 
have discovered to me the fact that the designs on a wallpaper succeed one another with unbroken regularity. Fixing my gaze on one of them, I cannot by any effort of attention efface the pattern which happens to be focussed on the blind spot. I know that I shall see it the instant that I move the eye. If I let my eye roam until the face of my wife falls on the blind spot, its image disappears. I know its lineaments far better than I know the pattern on the wall-paper, but I cannot fill it into the picture. Her hands are visible, and the work which is resting in her lap, but in a mysterious way the background draws together where the face should be. My mind refuses to pass a false judgment; but it also refuses to see that there is a gap.

This exceedingly instructive observation teaches the relativity of sensations. It shows that a sensation has no objective value until judgment has been passed upon it by the mind. The meaning of this we express in figurative language, none other being available. We speak of a new sensation as being compared with sensations previously received-taken into the picture-gallery of the mind, and placed in its due position amongst the infinitely numerous records which are stored there. If we try to make a nearer approach to correlating physical with psychical activity, we say that sensation has no value save that which it acquires from its temporal relation in the sequence of sensations to which attention is directed, and that this value depends upon the relation which similar sensations have possessed in former sequences. There is no gap in binocular vision. An object focussed on the inner (nasal) side of the right eye, where the blind spot is situate, is focussed on the outer (temporal) side of the left eye. The left eye sees the object to which the right eye is blind. Since we have almost invariably used two eyes in the past, experience teaches that there is no gap in the field of vision. Hence the new group of sensations which alleges that there is a gap must be corrected. The field must be filled up in the way which experience shows to be most likely. The retina is a sheet of rods and cones, each of which has a nervous connection with the brain proper to itself. The retinal field is associated with the brain-field. But this does not imply that we may think of the mind as having a spatial distribution 
in the brain. Pressure on button A or button B in the retina causes bell $\mathrm{A}^{\prime}$ or bell $\mathrm{B}^{\prime}$ to ring in the brain, but it does not follow that perception $A^{\prime \prime}$ or perception $B^{\prime \prime}$ will be heard in the mind. It will be heard if this is the association established by custom, since mind is the product of experience. But the new sensation is creating precedent as well as being judged by it.

Point $\mathrm{A}$ in the right retina is associated by experience with point $a$ in the left, and point B with $b$. These are termed corresponding points, because they are similarly stimulated in binocular vision. The mind, therefore, judges that it receives the same information from each pair of corresponding points. The position of corresponding points will be understood if the right retina is imagined as put inside the left, precautions being taken to make the yellow spots coincide, and to avoid twisting the retinal cups in taking them out of the eyeballs. Great care is taken to maintain the points in correspondence during the various movements of the two eyeballs. In addition to the four recti muscles which move the eyeball upwards, downwards, to right and left, two oblique muscles give it the requisite amount of rotation. We have learned to give the same value to the impulses from two corresponding points. But under changed conditions the correspondence changes. When a squint develops in childhood, it follows one of two courses; either the obliquity of one of the eyeballs increases until it looks towards the nose, and its images cease to interfere with the images in the dominant eye-they are ignored by the mind-or a fresh correspondence is established between points in the oblique eye and points in the eye which looks straight forward. If we are severely critical, we find, from a study of the form of the eyeball, that it is impossible that the same rods and cones should occupy corresponding points in different positions of focus and with different degrees of convergence of the eyeballs. To permit of this the retinal cups would need to change in shape. But again mechanical correspondence is of little consequence. In the light of experience the mind judges that points correspond. When we are gazing at a flat surface, the mind judges that corresponding points are giving it similar information. It does not see a flower on a wall-paper twice as bright or twice as red with two eyes as 
with one. If the eyes are normal, the impression received through the two is precisely the same as the impression received through either singly. But when we are looking at solid objects, the image on one retina is not the same as the image on the other. One eye sees farther round the object on the one side, the other on the other; and it is just this disparity in the pictures, aided by the feeling that the eyes are converging, that gives the impression of solidity. Correspondence of points, on the other hand, is not necessarily sufficient by itself to convince the mind that the pictures presented by the two eyes are identical. When a flat triangle such as this

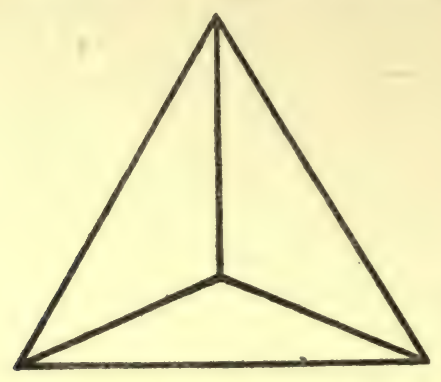

is regarded with the two eyes, its black lines fall on corresponding points ; but the figure is associated in the mind with other sensations-sensations of movement and touch. Notwithstanding the identity of the retinal images, the mind tries to see them as disparate. The figure troubles the eyes. At one moment the meeting-point of the three central lines projects forwards, at the next it recedes. That similarity of retinal images counts for something is shown by closing one eye. The uncertainty of shape of the figure is rendered more troublesome. It changes still more rapidly from convex to concave. When the point seems to be in front of the page, the accommodation of the eyes is adjusted for nearness; when behind the page, for greater distance. But the illusion that the object occupies three dimensions is not dependent upon the sense of contraction of the ciliary muscle. When the paper is moved towards the eye, its centre recedes; it is left behind until the ciliary muscle has had time to contract. When it is moved away from the eye, it projects until the ciliary muscle has had time to relax. Accommodation follows judgment, not judg- 
ment accommodation. The mind is extremely suspicious of the veracity of its newsagents. Disparateness of images, convergence of the eyeballs, shifting of accommodation for the various levels of an object in space, should be indisputable evidence of solidity or of hollowness. Conversely, the absence of either factor should be conclusive proof of flatness. But the mind does not trust to isolated sensations; it looks for associations of sensations. When the finger hints, "I could touch that sharp point," it is useless for the eye to aver that there is no point to be touched.

If two exactly similar photographs are placed in a stereoscope, the fact that the eyes are not converged gives to the common picture an appearance of depth, notwithstanding the fact that corresponding points on the two retinæ are stimulated. If the two photographs have been taken, as they should be taken for this purpose, with a double camera, the disparity of the retinal images immensely enhances the impression of solidity.

It is impossible to exaggerate the dependence of sensation on judgment. At birth a child commences the long process of education which enables it to associate the sensations derived from its retinal images with the movements which place it in contact with things. It discovers that, when it is necessary to make the eyes converge, the object is near at hand. It also associates the voluntary action of contracting its ciliary muscle with nearness. Unconverged and unaccommodated eyes come to mean distance. So, too, do indistinctness due to absorption by the atmosphere, blueness due to the same cause, a small image on the retina. But there are obvious limits to its power of ascertaining the distance of an object, and therefore, conversely, of its power of estimating size. We have no idea of the size of the retinal image of the sun. Very few people would be prepared to believe that the angle which the sun subtends with the eye barely exceeds half a degree. (The first finger, viewed in profile, at arm's length, covers one degree of arc.) A disc of paper of the right size, placed at the right distance, looks far too small to represent the sun. The most brilliant of orbs bulks larger than this in our minds. Everyone who for the first time looks at the sun through well-smoked glass, or, better, through a flat-sided 
vessel filled with ink and water, is astonished that it looks so small. Nor are we prepared to accept the evidence of a camera that the sun at the zenith does not produce a smaller image on the retina than the sun when rising above the horizon. Yet if a photographic plate is exposed to the rising sun, and again, without changing its focus, to the sun at the zenith, the two images are practically equal. There is a slight difference due to the greater refraction of rays passing tangentially through the atmosphere, but it is so slight as to bear no relation to the difference between our two judgments of size. When the sun is rising behind trees and houses, we compare it with objects which we know to be large and distant; yet it looks almost as large when rising out of the sea. One of the causes of the illusion is our conviction that the sky is flattened; and this, again, is due partly to its paler tint-its less substantial blueness-near the horizon, and partly to our impression that it is spread out over a flat earth. When the sun is in what we deem to be the more distant part of the vault of heaven, we judge it to be farther from us, and therefore larger than when it is above us. Yet the last word has not been said in explanation of a phenomenon which has been studied by mankind since the dawn of science. Helmholtz attributed the apparent greater distance, and consequent greater size, of the sun and moon when near the horizon to the indistinctness of their discs. When its image is so reflected from the zenith as to cause the moon to appear to rest upon the horizon, it does not, he said, increase in size. In answer to Helmholtz's explanation, it may be objected that, when at midnight he brought the full moon down from the zenith, he did not bring with her the conditions of light and colour by which she is customarily surrounded when floating on the horizon. If, when watching the moon which has just risen, vast in diameter, out of the sea, one interposes between it and the eye a sheet of paper in which a small hole has been made, and looks at the moon with one eye through the hole, it instantly shrinks to the size which it appears to have at the zenith. It is not even necessary to blot out the whole of its trail of light on the sea. At the same time, it appears to retreat to a great distance. This shows how complicated are the associations upon which judgments of size and distance are based, and to how small an extent 
they are determined by the size of the image on the retina. This observation is most surprising if made one or two nights after full moon, when twilight is already dim at moon-rise.

Our estimate of the distance away from us of an object on the horizon is based upon the time and effort which experience tells us we should need to spend in reaching it. The untried appears shorter than the tried. Anyone who compares his feeling of the number of yards he would have to climb up a pole reaching to the zenith with his feeling of the number of steps he would need to take to reach the horizon will recognize that the horizon appears to him to be the farther away.

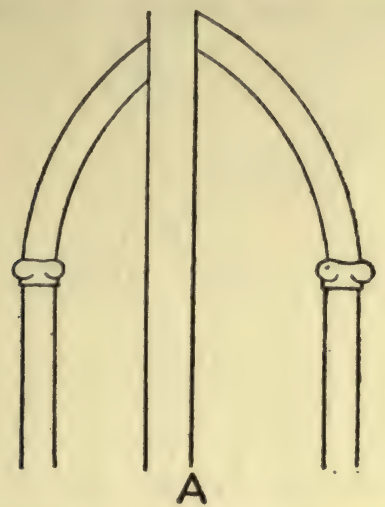

Fig. 36. A Symmetrical ARoh, divided by a Vertical Line, A, which passes throdgh ITS APEX.

In representing a solid object an artist conveys the idea that light is falling obliquely upon it. One side of the object, therefore, is more strongly illuminated than the other. By depth and gradation of shade he indicates the extent to which the thing projects forwards, if solid, or falls back, if hollow, He makes the margin of a ball hazy, in the expectation that the spectator will look at the spot nearest to him-an artifice which he may easily press too far, since the eyes wander restlessly over a flat surface. In representing distance he is dependent upon giving to the various objects in his picture sizes equivalent to the sizes of their images on the retina, making them brighter or paler and more or less distinct. Yet he cannot hope to simulate the convincing evidence of distance which is afforded by our sense of the degree of convergence of 
our eyes. Hence, as Francis Bacon pointed out, a picture appears more real when one eye is closed than when both are open. Its middle distance at once falls back.

Innumerable are the illustrations which may be given of errors of sensory judgment, but none are more striking than the various figures which may be drawn with converging or diverging lines. The mind under-estimates acute and overestimates obtuse angles. It is impossible to convince oneself that in Fig. 36 the line A bisects a symmetrical arch. Equally difficult is it to believe that in Fig. 37 the line with diverging terminal segments and the line with converging terminal segments are of exactly equal length. In the Ruskin Museum

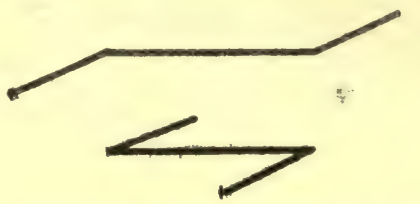

Fig. 37.-Two Horizontal Lines of Equal Length-The One with Divkrging, the Other with Converging, Terminal Lines.

at Sheffield there is a sketch by the master of the façade of a church which shows a vertical tower to one side of a triangular pediment, or, rather, this is what the sketch was meant to show, and does show, when measured on an architect's table. In effect the tower appears to be leaning towards the pediment. Errors of judgment of this type have been attributed to the curvature of the lines of a rectilinear image on the retina, the mind judging the distance between two points by the length of the chord, and not the length of the are which joins them. This is very simply illustrated by the example of the apparently greater length of a filled space than of a vacant one.

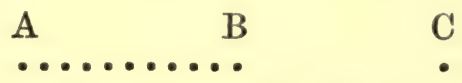

A B looks longer than B C. If A B C be represented as a curved line, the arc A B will, of course, be longer than the chord B C. But it is not safe to suppose that the mind compares the length of an arc with the length of a chord. Judgment is based upon experience, and probably the illusion is due to more subtle causes than the curvature of the retina. 
The mind does not look at the retina. If it did, it would find the reversal of the picture the least of the inaccuracies which it had to correct. It would find it very difficult, for example, to superpose in its stereoscope the photographs of a vertical tower taken simultaneously by the right eye and the left. The curved images on the retina of the vertical lines which define the angles of the tower, as seen with one eye, could not be made to correspond with the images focussed by the other eye. The Greeks felt this when they settled the form of a column. The canon of the swelling entasis and increasing taper above it did not destroy the appearance of uniform thickness which the shaft presented. It gave to the eye just the slight help which it needs to enable it to picture the shaft as of the same thickness from base to capital. 


\section{CHAPTER XIV}

\section{HEARING}

THE ear, like the eye, records amplitude of vibration; loudness. It also records rapidity of vibration, musical pitch, which corresponds with colour. But it seems to have a more difficult task than the eye, since it has to analyse, or at any rate has to transmit information regarding the form of compound vibrations. The meanings of these distinctions may be illustrated by reference to a tracing on the cylinder of a phonograph. A needle attached to the posterior surface of the thin metal plate against which one speaks scratches the surface of a rotating cylinder of hardened wax. Examined with a lens, the record is seen to be an irregularly changing line. The depth of the marks is a measure of loudness. Their varying number in a given time indicates the changing pitch of the voice which produced them. Their form is a record of the quality of its tone. The work of the ear, so far as it consists in the estimation of the amplitude and rapidity of pulsations of sound, is easy to describe, but the acoustics of form are complicated.

Light is transmitted as vibrations of æther. They are transverse to the direction in which the light is travelling. Sound cannot travel through a vacuum, since it is dependent upon displacements of material particles. The particles move forwards and backwards in the direction in which sound is progressing. Sound is a sequence of pulsations, alternate condensations and rarefactions of the media which conduct it. Their particles are first pressed together, and then rebound to positions farther apart. A sequence of to-and-fro movements, each smoothly continuous throughout the whole duration of a pulsation, would produce a pure musical tone. Tuning-forks carefully bowed settle down after a few seconds into unbroken oscillations, which convey to the air the to-and-fro movements of pure tones. Such tones vary in nothing but loudness and pitch. If their pulsations are slow, we speak of the pitch as "low"; if they are rapid, we say that their pitch is high. 
But if the sound produced by tuning-forks (and low-toned stopped organ-pipes) be omitted from the list, no pure tones reach our ears. The notes of flutes, fiddles, trumpets, pianos, have each a certain "quality" characteristic of the instrument. Even in a violin the G string has not the same timbre as the D string. Owing to the elasticity of the substances which originate and of the substances which transmit sound. its pulsations are not simple to-and-fro movements, uninterrupted from beginning to end. Each pulsation is partially broken at intervals ; and the quality of the sound depends upon the number and relative accentuation of these partial interruptions. Sound travels through air at the rate of 1,100 feet per second. This figure, divided by the number of vibrations per second of a tone, gives the wave-length in air of a tone of that particular pitch. For example, the middle $\mathrm{C}$ has a vibratory rate of 256 . Its wave-length is, therefore, somewhat over 4 feet. The lowest tone of an organ has a wave-length of 37 feet ; its highest of $3 \frac{1}{2}$ inches. These figures give no information, however, regarding the movement of the particles which pass on the sound. When air is transmitting a notesay the middle $\mathrm{C}$-its separate molecules do not move through a distance of 4 feet. Each molecule moves but a short distance, varying with the loudness of the tone; but the "wave" of crowding runs straight forward from the piano-string to the ear, the molecules at the end of each stage of 4 feet taking on a backward movement, so that the crowding, so far as the molecules of that particular section are concerned, returns to its starting-point. Between the piano-string and the ear there is a crowding and forward movement at $0,4,8,12 \ldots$ feet ; a spreading and backward movement at $2,6,10,14 \ldots$ feet. Most illustrations which are intended to aid the mind in forming a definite picture of the transmission of sound are liable to be misinterpreted, because they translate rectilinear movements into waves. They represent the movements of the string, and not the movements of the molecules of air between the string and the ear; but with the aid of the imagination one may picture the positions of the particles in this path. The pulse, we will suppose, has just reached the limit of 12 feet. Half-way from its 8 -foot halting place the molecules are again crowded, althoungh not so densely. One-third of the distance from the same point there again appears a tendency to 
crowd. This latter point marks an interval of one-third of this wave plus the wave which led up to it. At the end of the ninth foot there is a crowding, though less marked-this wave plus the two preceding waves, divided into fourths. Within these intervals are other points at which the molecules have closed together, the distances from a nodal point depending upon the number of waves involved, and, speaking generally, growing less marked as the number increases. Such are the very complex pulsatile movements which reach the ear.

Every musical sound produced by a piano, a violin, or other instrument, is compounded of a fundamental or prime tone, and overtones, partial tones, or harmonics. The following table shows the more important partial tones which accompany the prime tone when the middle $\mathrm{C}$ on a pianoforte is struck :

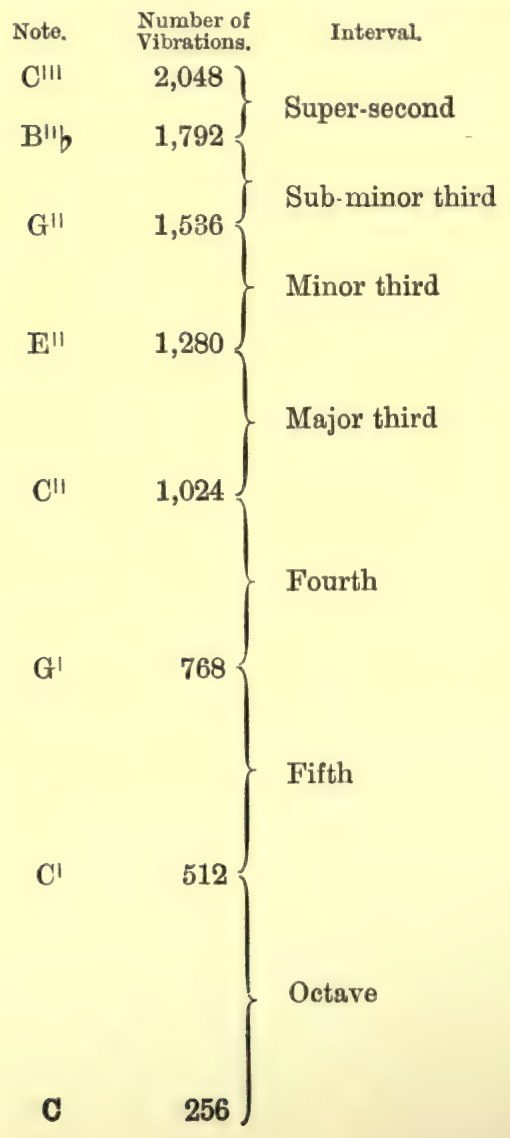

Ratio. Number of

$8 \quad 7$ th

$\frac{8}{7} \quad 6$ th

$\frac{7}{6}$

5th

$\frac{6}{5}$

4th

$\frac{5}{4}$

3rd

$\frac{4}{3}$

2nd

$\frac{3}{2}$

1st

$\frac{2}{1}$ 
The quality of a musical note depends upon the number and relative loudness of its overtones. When several notes are sounded simultaneously, they blend into a chord or harmony, provided the intervals which separate them are equal to the intervals which separate the simpler overtones. Each of the notes yields overtones. The tones blend into a concord. Their partials are in unison. The variations in air-pressure of the compound tone are strictly periodic. If the ratios of the frequencies of its constituent notes are simple the product is a rich, full sound, such as a common chord.

At least one other character of the pulsations of sound must be taken into consideration if we wish to picture the nature of the force to which the ear responds. Tones which reach it from several instruments simultaneously are not necessarily in unison, or even in harmony. The overtones of a single note sounded on a piano or violin-the statement does not hold good for bells, nor is it strictly true of flutes or horns-must necessarily bear a simple proportional relation to their prime tone. They divide the grand pulsation into fractions "without a remainder." But the vibrations of two tuning-forks which are slightly out of unison interfere one with the other at regular intervals. They produce "beats." Everyone is familiar with the curious effect which is produced upon the eye when one row of railings is seen through another, or one expanse of wire-netting behind another. Sets of lines which occupy nearly the same positions in the line of sight combine to make a large pattern, which overlies the smaller pattern of the rails or netting. The same thing happens with sounds which coincide at considerable intervals, although in the case of sounds interference is as marked as reinforcement. If whilst a tuningfork yielding 101 vibrations per second is singing another of 100 vibrations is brought into play, the vibrations of the second fork are superposed on those of the first. At a certain moment the forward movement of molecules of air induced by the first fork is reinforced by a forward push from the second. But half a second after this coincidence of phase an opposite result is produced-50 $\frac{1}{2}$ vibrations of No. 1 have passed, but only 50 of No. 2. No. 2 is going backwards (inwards), whilst No. 1 is moving forwards (outwards). The same molecules are impelled backwards by No. 2 and forwards by No. 1. The result 
is a pause. The compound sound produced by the two forks reaches the ear in throbs. If the forks were vibrating at the rates of 101 and 99, there would be two pauses and two beats in every second; if at the rate of 202 and 198, four. The number of beats per second equals the difference in frequency of vibration of the tones. A pianoforte tuner does his work best if he has a musical ear, yet he may discharge his duties with competence without one. Having struck a note, he sounds its octave, holding both keys down, and listens for the beat. If the first note gave no beat with his tuning-fork, the second is in tune when it likewise gives no beat with the first. We have met a tuner who did his work in this way ; but it must be admitted that his tempering of the intervals of the octave with which he commenced, and consequently of the other octaves above and below it, left something to be desired. The result might have been satisfactory had he been provided with twelve tuning-forks.

The question as to whether beats, when sufficiently rapid, blend into a tone has been much discussed, without a decision. Probably they do not. The complementary question as to the cause of dissonance is also not completely closed. Two notes harmonize, as we have seen, when the ratio of their frequencies is a simple fraction. Musicians are not quite agreed as to the level of numerical complexity at which a compound tone first produces a feeling of discomfort. A good deal depends upon its position in the scale and the instruments which are combining to produce it. A minor third $\left(\frac{6}{5}\right)$ is on the safe side. This is the first chord in our list of intervals in which a beat can be detected. Slow beats, however, do not distress us. It is the rapid beats of conflicting overtones which give a harsh, rough character to a compound note. The level at which a line is drawn between harmony and dissonance seems to depend to a considerable extent upon musical education, using the term in its widest sense. In primitive musicHungarian, Scotch, Welsh-intricate minor chords predominate. The minute subdivision of the octave in Indian music is quite incomprehensible to a European ear. Musical cultivation tends to eliminate complex fractions. It is, however, to be noted that the history of Western music also shows the influence of an opposite tendency. Later generations have 
admitted as harmonies combinations which earlier generations could not tolerate.

Pitch, quality, harmony, and dissonance are distinguished by the human ear. These are the attributes of musical or periodic sounds. In a separate class must be included noises of all kinds, termed in acoustics "aperiodic," because the vibrations which cause them are not rhythmic. The teeth of a policeman's rattle may click a hundred times a second, but it does not make music. Even with a rapidity of interruption greater than this (at least 500 times per second) a succession of noises fails to blend into a smooth, continuous sound. The ear recognizes the loudness, duration, and even to a very high frequency the repetition of unmusical sounds.

The ear as a sense-organ can be followed down the zoological scale to jelly-fish. In its primitive form it is a chamber lined with epithelial cells bearing hairs, containing an otolith, or ear-stone. Otoliths are rounded calcareous masses which play an important part in the ears of all animals up to fishes. Even in man they are found in the more subdivided form of otoconia. Contact of the otoliths with the sensory hairs originates impulses in the nerves with which primitive ears are abundantly provided. Advisedly we use the word "ear" in place of "auditory organ." In all animals this organ affords information of a double nature-movement of the external medium in which the animal lives, and movements of the animal in the medium. When the animal moves, its sensory hairs are displaced with regard to the otolith; when the water in which it is swimming pulsates, its otoliths are shaken against the sensory hairs. Displacements of the animal and agitations of the water produce similar effects. The ear in this stage is an organ of touch. It might well be questioned whether an animal fitted with a piece of sensory apparatus of this kind is endowed with a sense which we may properly, after reflecting upon our own sensations, term "hearing." It is, however, stated that certain transparent crustaceans, in which the functioning of the ear-organs may be watched through a lens, show in these organs hairs of varying length which vibrate to tones of different frequency. This observation apart, it might be doubted whether fishes hear, if we mean by the word "hearing" the recognition and 
discrimination of tones of high frequency-musical tones. Their ears serve equally to inform them of the changes in position of their heads and of the tremblings of the sea. The shocks transmitted through the sea are near akin to the slower vibrations of sound, if the fishermen of the Mediterranean are justified in their practice of beating a wooden clapper which rests upon the seat of the boat as they row backwards and forwards in front of a curved net. They believe that the fish are frightened by the noise ; but it matters little whether we describe the fish as hearing a noise, or as feeling the percussions of the clapper conducted through the water. To the more rapid vibrations of the clapper, the fish are probably insensitive. The cochlea, which we have every reason for regarding as the organ by which sound is analysed, is not possessed by fishes. It makes its first appearance in reptiles. Birds, it is evident, are able to distinguish musical tones. Their cochleæ are very short, and are destitute of "rods of Corti." For a moment this appears surprising, but it must be remembered that the range of tones which any bird discriminates is very short, however nicely it may value the notes within its range. In mammals the ear is clearly divided into three parts, to which the three functions which have grown out of the specialization of the sense of touch are allocated. (1) The semicircular canals are concerned with the sense of orientation. (2) The utricle and saccule reverberate to noise-the rumbling of trains, the boom of guns, the beats of dissonant musical tones. We do not know how to classify the agitations of the atmosphere which surrounds us and of the earth on which we stand, nor can we point with any certainty to the groups of stimuli which for us have taken the place of the grinding of stones on the beach and slapping of rocks by waves. (3) The organ of Corti in the cochlea discriminates and analyses musical sounds. To these three sense-organs, which are situate in the inner ear, certain structures are accessory.

The concha, which enables a horse or a cat to collect sound and to localize its source, is in ourselves merely an ornament to the side of the head.

The external meatus is a curved tube, about an inch long. Frequently a tuft of hairs guards its entrance. The wax 
secreted by its wall serves to attach particles of dust, and to deter insects from entering the tube. The air at the end of it is at a uniform temperature. It is closed by the membrana tympani, or drum. This membrane receives the vibrations of

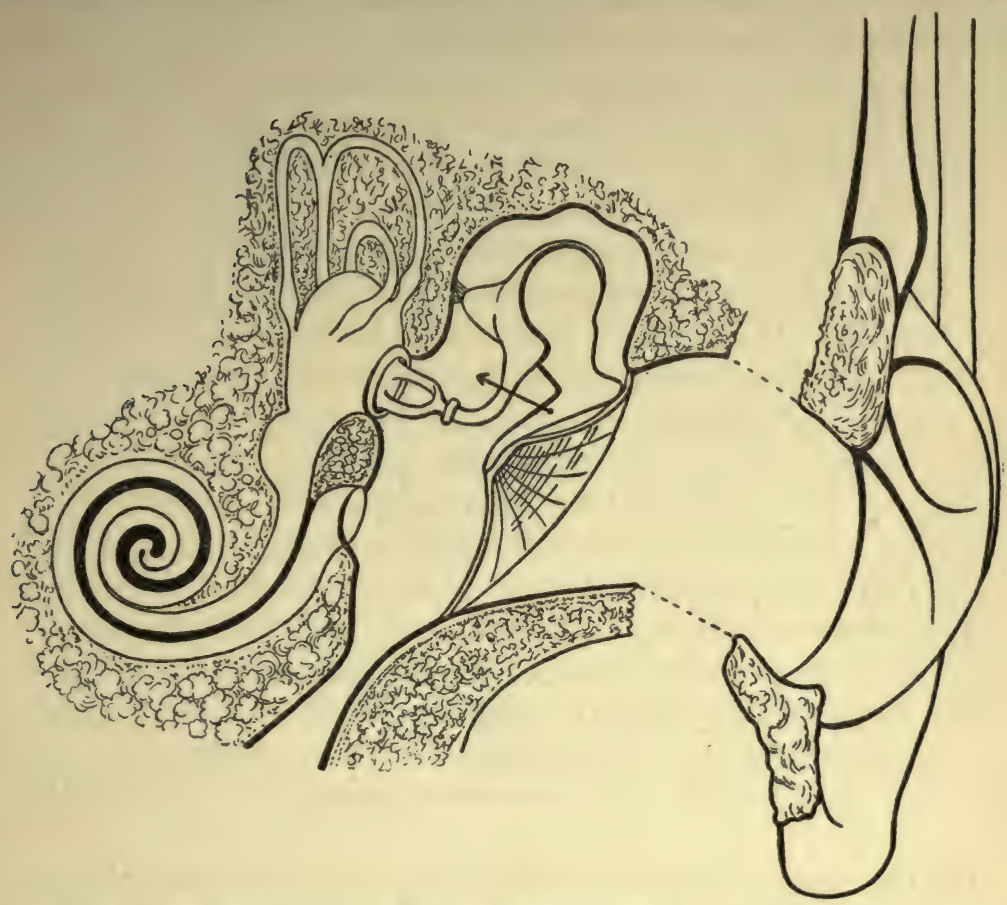

Fig. 38.-The Extrenat, Middie, aNd Internat Ear of the LifT Side.

From right to left, the figure shows the concha andlobule of the ear in profle; the external meatus (abbreviated) ; the drum, divided vertically, its posterior half visible; the hammerbone, with the tip of its long arm attached to the drum, an arrow indicating the point of attachment and line of action of the tensor tympani muscle; the anvil attached by a ligament to the bony wall of the middle ear; the stirrup, with its foot-plate almost flling the oval window; the labyrinth, with the three semicircular canals above, and the scala vestibuli below. The curled black line shows the situation of the scala media, or ductus cochleæ (which contains the organ of Corti). Pulsations of sound which move the membrana tympani are transmitted by the three bones to the oval window. They shake the perilymph, producing waves which travel along the scala vestibuli to the apex of the cochlea, whence they return by the scala tympani to the round window (if they do not take a shorter course through the ductus cochles). The Eustachian tube opens out of the lower part of the middle ear.

sound; and, in order that it may collect them with absolute impartiality, it is in every respect the opposite in shape and structure to the top of a drum. The stretched parchment which covers a drum is flat. Its tension is uniform in all its 
parts. Movements have the greatest amplitude at the centre. Every precaution is taken to insure its emitting, with as little confusion as may be, the particular note to which it is tuned. The drum of the ear is shaped like the mouth of a trumpet, depressed to a point, but convex from this point outwards. Its elastic fibres, which are partly radial, partly circular, are at many different tensions. Its deepest part, to which the long arm of the hammer-bone is attached, is not its centre.

The " middle ear" is an irregular cavity communicating with the pharynx by the Eustachian tube. It is filled with air at the same pressure as the atmosphere. Except during the act of swallowing, when it is at first shut tightly and then opened, the pharyngeal end of the Eustachian tube is gently closed. When one is dropped in a lift rapidly down the shaft of a mine, the difference in pressure between the external air and the air in the middle ear stretches the drum to such an extent that deafness to low tones is produced. Conversation becomes inaudible. The deafness is remedied by swallowing saliva, and thus opening the end of the Eustachian tube. The commonestcause of permanent deafness is inflammation followed by thickening of the mucous membrane of the lower end of the Eustachian tube, with its consequent closure, due to frequent sore-throats. The air in the middle ear is slowly absorbed. It needs to be constantly renewed through the Eustachian tube.

On the inner wall of the middle ear are two small apertures - the oval window and the round window. Both are closed with membrane. Into the oval window is fitted the sole-plate of the stirrup-bone. Three bones-hammer, anvil, and stirrup - combine in transferring the movements of the membrana tympani to the oval window. They constitute a jointed lever, which swings about an axis passing through the ligament of the anvil (Fig. 38), the excursions of the long arm of the hammer being reduced in amplitude by one-third at the stirrup-plate. As the oval window has only one-twentieth of the area of the drum, the movements of the latter are transmitted with concentrated force. Two points in the mechanism of these bones may be specially noticed: (1) The head of the hammer is free to rotate in the cavity of the anvil, checked by a cog. Every inward movement of the drum is faithfully trans- 
mitted to the oval window; but when the drum moves outwards, the hammer does not necessarily carry the anvil with it. (2) A muscle-tensor tympani-is inserted near the elbow of the long arm of the hammer. When high notes are listened to its contraction tightens the drum, rendering it more responsive to rapid vibrations. It has a tonic action, but it does not make any special contraction for low notes.

Behind the two windows, within the solid bone, is the inner ear, which our ancestors very aptly termed a "labyrinth." It is filled with fluid-perilymph-which is shaken by every movement of the stirrup-plate. Since water is incompressible, no waves could be raised in the perilymph were there no second aperture. Every vibration conveyed by the stirrupplate after passing through the labyrinth ends as a vibration of the membrane which closes the round window.

Nowhere does perilymph come in contact with auditory cells. All the endings of the nerve of hearing are contained within a membranous labyrinth which lies within the bony cavities. The way in which the waves of the perilymph are dispersed over the surface of this closed sac can be inferred from the diagram (Fig. 38). They sweep round the utricle and saccule, are lost in the narrow spaces which surround the semicircular canals, run up the scala vestibuli of the cochlea. The course of the waves which traverse the cochlea is of especial interest in connection with the physiology of hearing.

The cochlea - snail-shell-is a spiral tunnel of three turns, in hard bone, about an inch in length. A shelf of bonelamina spiralis-projects into the tunnel on its convex side. From the free margin of this spiral lamina two membranes extend to the outer wall of the tunnel-one firm, containing straight, stiff, and probably elastic fibres which radiate outwards (the basilar membrane) ; the other an extremely delicate film of connective tissue. The tunnel is thus divided into three compartments, known as the scala vestibuli, scala media, scala tympani. The scala media belongs to the membranous labyrinth. Waves transmitted through perilymph pass, as we have already explained, up the scala vestibuli. At the apex of the cochlea the two scalæ are in communication; but the aperture is small, and it is unlikely that waves reach the lower passage from the upper through this opening. They pass 
through the thin membrane which roofs the scala media, shake its endolymph, and reach the lower passage through the basilar membrane. It is noteworthy that, since the round window at the lower end of the scala tympani is, with the exception of the oval window, the only opening of the bony labyrinth, all waves transmitted through the oval window must travel part of the way or all the way up and down the cochlea.

The organ of Corti is spread out on the basilar membrane. It is an epithelial structure of extreme regularity and uni-

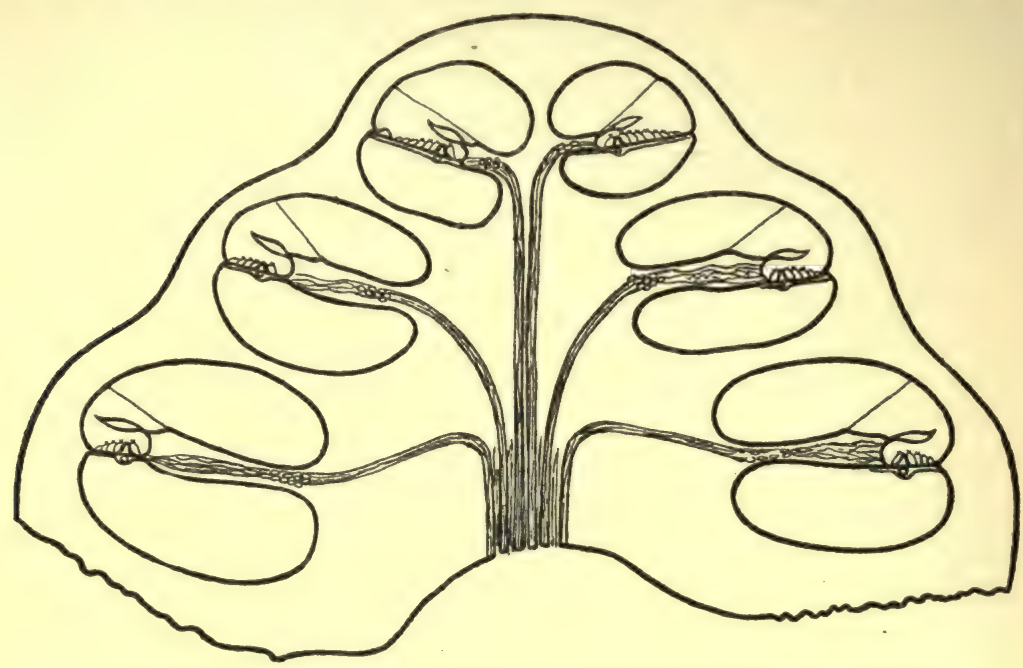

Fig. 39.-A Section throdgh the Axis of the Coldm of the Coohlea.

The spiral sheet of nerve-fibres which supplies the organ of Corti is cut in eight places. If the bundle to the lowest coil of the shell (on the left side of the diagram) is followed, it will be seen to bear ganglion cells where it enters the bony spiral lamina. This lamina divides the tube into two canals $\rightarrow$ scala vestibuli above, scala tympani below. From the edge of the lamina the membrane of Corti stretches to the outer wall. Above the organ of Corti is the membrana tectoria, and above this a very thin membrane which cuts off the ductus cochleæ from the scala vestibuli.

formity. Near to the edge by which the basilar membrane is attached to the spiral lamina rests a double row of rods of Corti, stiff pillars which lean one towards the other, over the tunnel of Corti, the convex head of the outer rod fitting into a concavity in the head of the inner one; in some places one outer rod fits against two inner rods, as the latter are rather the more numerous. On the inner side of the inner rod is seen, in transverse sections a single plump cell filled with cloudy protoplasm, and bearing on its free surface a tuft of very short 
hairs. On the outer side of the outer rod are three or four hair-cells, each with a cloudy outer segment containing the nucleus, a granular middle segment, and a stiffish stalk, which attaches it to the basilar membrane. Between the hair-cells are supporting cells, thicker below, tapering above, containing in their substance a firm fibre. Still farther to the outer side are epithelial cells, of no special interest. The purpose of the rods of Corti and the supporting cells is to give attachment and support to a reticulated membrane of exquisite delicacy, through the oblong apertures of which the hairs of the haircells project into the endolymph. The spiral lamina is

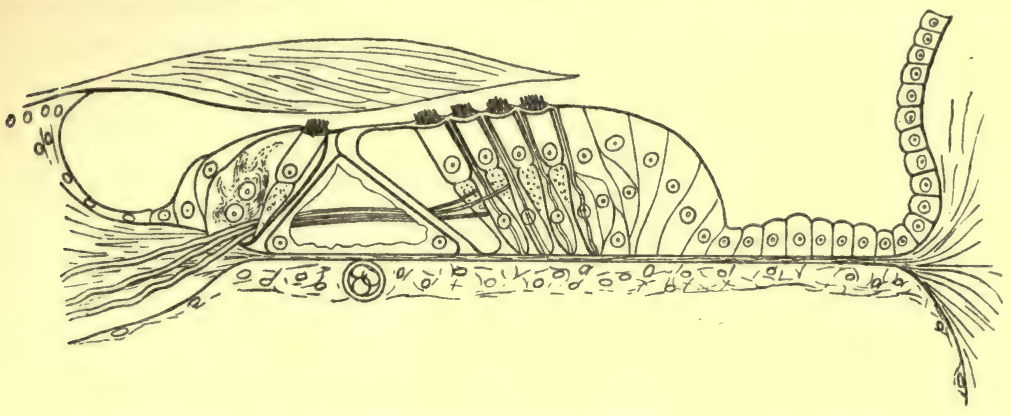

Fig. 40.-ORGaN of CoRTi.

The spiral lamina, on the left of the drawing, gives attachment to the membrane of Corti, which stretches to the opposite wall. Below the membrane is a bloodvessel which runs its whole length beneath the tunnel of Corti. The tunnel is formed by pillars-the inner on the left, the outer on the right-which meet above it. On the left of the inner pillar is a hair-cell ; to the left of this a nerve-cell with two nuclei. To the right of the outer pillar is a space ; to the right of this four hair-cells alternating with four supporting cells, which hold up the reticulated membrane through apertures in which the tufts of hairs project. Three nerve-fibres are seen in the spiral lamina; they cross the tunnel to ramify between the rows of outer hair-cells. The lamina tectoria rests upon the tufts of hairs.

traversed by a vast number of fibres of the auditory nerve, which, losing their medullary sheaths, pass across the tunnel of Corti as naked axons, to end amongst the hair-cells. Above the organ of Corti, attached by its edge to the spiral lamina, is a thick, gelatinous, fibrillated structure-membrana tectoriawhich rests as a coverlet on the surface of the organ. It has been supposed that it serves to damp the vibrations of the hairs after they have been set in motion by the waves passing across the scala media; but it not impossibly plays a more active part in hearing than this.

The organ of Corti is, beyond doubt, the apparatus which analyses sounds; but the problem of the way in which it 
responds to tones of different pitch, or analyses compound tones, is not as yet even approximately solved. To escape the acoustic difficulties which have to be faced by anyone who endeavours to expound the theory of the cochlea as a piece of analytical apparatus, various suggestions as to the possibility of an action en masse have been advanced. For example, the basilar membrane has been compared to a telephone-plate which takes up vibrations and transmits them through the auditory nerve to the brain. But if the organ of Corti be the transmitter, there is no ear in the brain to analyse the vibrations given out by a receiving telephone-plate; and without a receiving plate and a listening ear a telephone is purposeless. According to this hypothesis, the basilar membrane vibrates as a whole, moving the hair-cells in various "patterns"; the pressure of the hairs against the tectorial membrane causing irritation of the cells which bear them, and hence producing stimulation of various groups of nerves. Other pattern theories are somewhat similar. But it is obvious that all hypotheses of the vibration of the whole of the basilar membrane, or of large parts of it, simultaneously, leave to the mind the responsibility of reading the pattern which the impulses generated in the organ of Corti make in the brain. It is conceivable that every fraction of a semitone which a musician can discriminate, and every combination of tones which he can analyse, is transmitted to the brain by a large number of co-operating nerveimpulses; but such a theory involves a complexity of mental associations difficult to contemplate.

According to the general principles enunciated in this book, analysis of stimuli is the function of sense-organs. It cannot in all cases be compared with the analysis effected in a physical laboratory ; nor is this necessary ; but it must be carried so far that nerve-impulses which have no specific qualities apart from their source shall give rise to effects in consciousness which have no basis other than the topographical distribution of the said impulses in the brain. There may be sensory impulses of different orders; there may be in the brain psychophysical substances which react to impulses of various orders in various ways; but until we have some hint of the existence of specific impulses and specific psycho-physical substances, we are not justified in postulating their existence 
simply in order that we may escape from physiological embarrassments.

The organ of Corti has in the highest degree the appearance of a piece of apparatus for the analysis of sound. If the basilar membrane, with the cells which rest upon it, be cut out and laid flat, the suggestion of some kind of instrument is very strong. It is a long narrow ribbon, narrowest at the bottom of the spiral, increasing to about twice the width at the apex. It is crossed by radiating fibres, presumably elastic. The cells which rest upon it carry vibrating hairs, and are supplied with nerves. The rods of Corti hold up the reticulated membrane, which keeps the hair-cells in place. It is not to be wondered at that when its structure was first discovered it was thought that the problem of the analysis of musical tones was solved. If two pianos in perfect tune are in the same room, when one is played the corresponding wires of the other twang. Anyone who sings into a piano, whilst the loud pedal raises the dampers, feels an increased fulness in his voice. This is the familiar phenomenon of resonance. Why should not the fibres of the basilar membrane resonate to the tones conveyed to the ear-the shorter ones at the base of the cochlea to high tones, the longer ones at the apex to low tones? This is the order in which we should expect the pulsations of sound which ascend the scala vestibuli to be taken up-the more rapid near its commencement, the less rapid farther up it. But an explanation of the physics of the selection of vibrations of different frequencies by different sets of the elements which make up the organ of Corti, if such selection occurs, is still to seek. In the first place, the fibres of the basilar membrane are so exceedingly short. What could a fibre less than 0.5 millimetre in length make of the vibrations of a 36-foot organ-pipe? Even if this objection be waived, as certain eminent physicists hold that it may be, there is not a sufficient difference in length between the longest and the shortest fibres to account for the great range of tones which we are able to discriminate; nor is there any evidence that some fibres are more tightly stretched than others.

A further consideration which tempts physiologists to look upon the organ of Corti (including the basilar membrane) as a series of resonators is the somewhat remarkable agreement 
between the number of separate pieces of apparatus of which it appears to be composed and the number of different musical sounds which, if it were a series of resonators, it might be called upon to discriminate.

The squeak given by a bat at each turn in its flight has a pitch of about 11,000 vibrations to the second-the sixth $\mathrm{E}$ above the middle $\mathrm{C}$ (Tyndall). In a group of persons listening for the squeak there are usually some who cannot hear it. Above this the range of hearing is very variable. The suddenness of transition from perfect hearing to total want of perception makes experiments with small pipes or with a siren somewhat amusing, when a number of persons are tested at the same time. One complains that the note is intolerably loud and shrill, whilst others assert that there is perfect silence. Thirtythree thousand vibrations is usually regarded as the upper limit for the human ear, but certain physiologists place it at 40,000 , or even higher. The upper limit is of little consequence, since there is very little power of discriminating rapidities above the highest note used in music-the piccolo stop of the organ, with a pitch of 4,096 . It is possible that a sound with a lower frequence than 27 (the contra-bassoon) may be heard as a tone-16 according to certain writers; but again our power of discriminating very low notes is small. Over a certain range a skilled musician can tell that a note is out of tune when it is one sixty-fourth of a semitone higher or lower than it ought to be. If we assume that by allowing equal sensitiveness for a range of seven octaves, the excess of the allowance over the actual sensitiveness towards either end of this stretch would compensate for the comparatively few distinctions which the ear can make either below or above it$64 \times 12 \times 7=5,376$. A much higher estimate, based upon observations which seem to show that the ear can distinguish sounds less than one sixty-fourth of a semitone apart, places the total number at 11,000 .

On the assumption that one piece of apparatus is tuned to resonate for every distinguishable sound, between 5,000 and 11,000 pieces of apparatus would be required. Taking one of Corti's arches as the centre-piece of the resonator, although the rods are certainly not vibratile structures, we find the number to be 3,848 (the number of the outer rods); 
if either rod with a hair-cell, or hair-cells, is the analytical element, 9,438. Counting gives 3,487 inner, 11,700 outer, hair-cells. The fibres of the basilar membrane are estimated at 24,000 ; the fibres of the cochlear nerve at 14,000 . It will be understood that the counting of structures as minute as these yields results which cannot be more than approximately accurate. Helmholtz, assuming that each are of Corti indicates an analytical element, accounted for the apparent deficiency in their number by assuming that a tone of which the pitch fell between two arches set both in sympathetic vibration, the arch which was nearest in pitch to the tone vibrating the more strongly. In this way he anticipated an objection which has often been brought against his theory of a long series of resonators.

In opposition to Helmholtz's theory it is pointed out that when a violinist runs his finger up a bowed string, the pitch rises with perfect smoothness; it does not bump along from resonator to resonator. Especially in the case of very high tones given out by a siren, it is urged that at the rare intervals at which a resonator in the ear is tuned for the tone which the siren is emitting it should sound much louder than when the tone falls midway between two resonators. But the whole question of the nature of the response of the analytical elements is too obscure at present for the discussion of points so nice as this.

Many who think that Helmholtz's theory of resonators is based upon principles of physics and of physiology which must be regarded as the starting-points of any explanation of the analysis of sounds by the ear and the mind, hold that it goes too far in searching for a separate resonator for every distinguishable tone. The coohlea, as we have already said, does not offer anything like so extensive a choice as this, if regard be had to the tension or length of its elements, and not to their numbers. Those who accept it as an axiom that the cochlea contains a series of responding instruments-but a series far more limited in range than the gamut of our sound-perceptions-seek to discover in musical tones qualities which unite them in groups. Just as in the case of colour-sensations they recognize four (or six) elementary qualities which excite four (or six) pieces of responding apparatus, so also in the case of hearing they seek for a limited number of tone-qualities and 
a correspondingly limited number of elementary sensations. The ideal of those who take this view is an octave of qualities and of elementary sensations sounded in the middle of the scale when $x$ nerve-endings are stimulated, as the octave above when $2 x$ nerves respond, the octave below with $\frac{x}{2}$. Such a conception seems to guide thought round insurmountable barriers. There is, however, a risk of making too much of the periodic intervals, because they take so important a place in music. At one side of the gap which sound bridges between the individual and his environment is an elastic body shaking at any possible rate within the range of hearing. At the other side of the gap is the ear. If, having arranged several thousands of stones along the side of the road in order of size, I were to state, picking up No. 512, "This is the fundamental of which No. 1,024 is the octave," answer would be made to me: "It may be that the larger could be broken into halves, each as heavy as the smaller stone; but I recognize no difference between the stones in shape, colour, or hardness." A vibrating string divides into equal segments, each of which vibrates within the vibrations of the whole string, sounding the octave. We recognize a similarity in quality between tones and their octaves because we are accustomed to hear the octave, the most prominent of overtones, in all musical sounds. Hence, from association, it has become more difficult to distinguish a note from its octave than it is to distinguish it from its fifth ; but it does not follow that the effect of 1,024 vibrations upon the sensory cells more nearly resembles the effect of 512 than does that of 768. But at this point we are compelled to construct some hypothesis as to the way in which the vibrations affect the sensory cells. The protoplasm of the cells is not directly sensitive to them. We can account for the generation of impulses in the nerve connected with a particular cell, or group of cells, only on the supposition that a resonating mechanism which responds to vibrations of a certain frequency shakes the cell. Even then it seems necessary to suppose that there is an accessory mechanism which disturbs the cell protoplasm sufficiently to render the shake effective, probably the hairs rubbing against the tectorial membrane. Anatomical study gives us no confidence in the theory of the 
existence of several thousands of resonators tuned to as many notes of different pitch. It remains for the physicists to say whether or not we may picture one of these minute resonators as responding to a given note in 10 separate octaves, another in 9 ... another in only 1 . The physicists, on their part, may very properly ask the anatomists to point out the resonators, and even to reproduce them in models of dimensions which allow of experimental investigation.

It is generally agreed that the sensation of a chord is compounded of the sensations to which each of its constituent tones gives rise, and that our power of analysing the compound is a question of attention. A musician can direct his attention to either sensation at will. It is not equally certain that a person who has no knowledge of music can do the same. Familiarity with musical instruments gives us so exact a knowledge of the way in which compound tones are produced that it becomes a difficult matter to decide whether, when we say that we can pick out the $\mathrm{E}$ or the $\mathrm{G}$ of the common chord, it means that we can hear it as distinct from $\mathbf{C}$ and $\mathrm{C}^{\prime}$, or whether it means that, knowing the constitution of the chord, we think about the $\mathrm{E}$ or the $\mathrm{G}$ when we hear the compound tone, to the exclusion of its other constituents. Then, again, the several strings which we try to strike simultaneously do not actually " toe the line." Their vibrations are not in the same phase, even though the strings be in absolute tune. Discrepancy of phase may favour the singling out of the several constituents of the chord. There we touch upon a problem which we passed over in silence when attempting to give an idea of the nature of the pulsations which reach the ear. We then (p. 405) described the partial pulsations which are superimposed upon the main pulsation as if they necessarily started simultaneously with it. We assumed that the phase difference of the partials was zero. But it is clear that difforences of phase of its constituent tones may produce an almost infinite number of variations in the form of a compound "wave" of sound. Is the ear variously affected by different forms of wave? Does difference of phase result in difference of sensation? In broad terms, the answer to this question must be in the negative ; although it can be shown that in certain cases a change in phase of the several constituents of a compound 
tone, without any alteration in their number or their loudness, makes a change in its acoustic quality. Any attempt to correlate physical changes-the movements of air in the outer ear -with the effects which they may be supposed to have upon the organ of Corti must take into account this wide range of variation of wave-form. We have called attention to the difficulties which it introduces ; but have no hope of indicating the way in which they may be overcome.

Nothing connected with the physiology of the sense of hearing is more remarkable than its capacity for education. The cochlea of one human being is as extensive and as elaborate in structure as that of another, yet some men can make an infinitely more refined use of it as an analytical apparatus than can others. A native of the Torres Straits cannot distinguish as two separate notes sounds which are less than a semitone apart. Sir Michael Costa could distinguish sounds into the sixty-fourth parts of semitones. The cochlea of a cat is not less elaborate than that of a man, yet Man's mental life is based upon the analysis of auditory sensations. His supreme advance in the animal scale has depended upon the invention of language, by means of which he communicates and receives information, thus rendering experience eternal, notwithstanding the transience of the individuals who acquire and transmit it. An animal is born, finds out, dies. A man starts with the wisdom of the race beneath his feet.

Hearing has a nebulous origin in sensations of movement or displacement. The connection between the two special senses -the sense of orientation and the sense of hearing, properly so-called-remains always intimate. David danced before the Ark of the Lord. All people, savage and civilized, associate music with movement. High in the animal scale appears the sense-organ which enables its possessor to discriminate musical tones. By its use Man has developed with great rapidity-as secular time is reckoned-an intelligence which removes him from all other animals a planet's space. The sounding of his organ of Corti by pure tones and combinations of pure tones gives him extreme pleasure, although it in no way ministers to his intelligence. Yet there is in the enjoyment of music a quality of pleasure which makes it near akin to the satisfaction which we experience in exercising the intellect. 


\section{CHAPTER XV}

\section{SKIN-SENSATIONS}

THE senses, according to a time-honoured classification, are five in number-smell, sight, taste, hearing, and common sensation, or touch ; but such a classification of our sensations and of the organs which originate them is too crude for modern needs. Already we have shown that, whereas the nose and the tongue afford the same kind of information, the ear affords information of two, perhaps of three, different kinds. Within the realm of common sensation we pick out three special senses served by specialized sense-organs-touch, cold and heat-and, possibly, a fourth, served by non-specialized nerves, to which alone the epithet " common" properly applies.

The skin is supplied with nerves-naked fibrils-in the richest abundance. They are most easily demonstrated in the layer which covers the cornea, thanks to its transparency; in this, as shown in Fig. 41, having branched on the front of the fibrous tissue of which the cornea is composed, the nerves pass towards the surface, forming connections with every one of its cells, or, at any rate, with every cell of the more superficial of the three or four layers of which the epithelium is made up. Ramified nerve-twigs of this type do not, under ordinary conditions, convey any sensations to consciousness. So long as the skin-cells with which they are connected are healthy, the nerve-twigs establish for them connections with the central nervous system by which their nutrition is regulated ; but they carry no impulses to which we can direct attention. The movement of blinking is accompanied by no sensation until the edges of the eyelids come in contact. A pencil pressed against the lid evokes touch-sensations from the skin, but none from the cornea which underlies it. When 
a tiny beetle injures the surface of the cornea by scratching the epithelial cells with its horny wings and legs, the ruptured nerve-filaments convey to consciousness impulses, or, as we prefer to express it, an influence which is felt as pain. But even the pain caused by injury to the cornea is trifling as compared with that which originates in the under-sides of the lids, where not only is the epithelium supplied with branching nerve-twigs, but specialized organs of touch are present to localize the seat of injury. Everywhere the epithelium

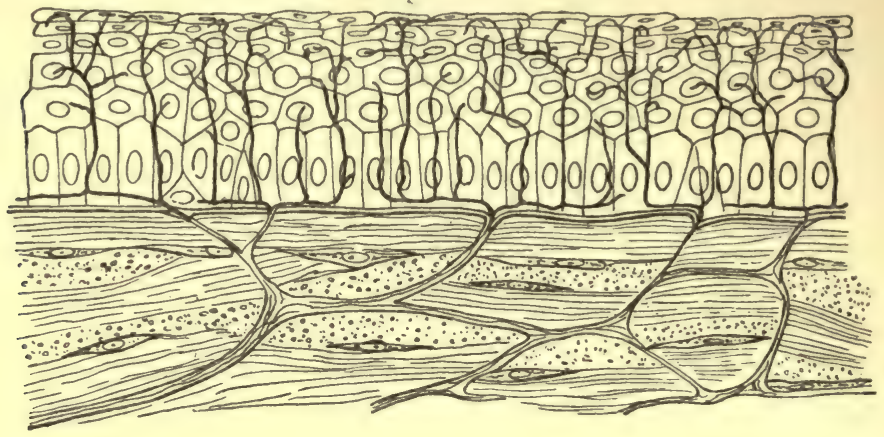

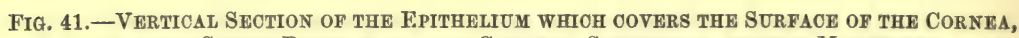
and of a Small Portion of the Corneal Substande, higly Magnified.

The black lines are naked nerve-flbres (stained with chloride of gold), which are distributed amongst the cells of the more superficial strata of the epithelium in very great abundance. The corneal substance is composed of sheets of transparent flbres with intervening cells. As the fibres of the several sheets cross one another at various angles, they are cut, some transversely, others in the direction of their length.

covering the surface of the body is so abundantly supplied that a successful staining of nerve-filaments induces one to think that every epithelial cell has its nervous affiliation. These are the nerves of common sensation, if we retain the term ; but sensation so common, so obscure, so little differentiated that we know no more about it than we know about the air which envelops our hands and faces on a warm, windless day. Yet the air, when it moves, gives rise to a dim, broad, generalized sensation, which may be focussed into definiteness by a sensitive nerve.

An observer who has devoted himself for many years to the investigation of skin-sensations, and especially of the "referred pains " which are due to diseases of the viscera, recently caused the large cutaneous nerve which supplies the thumb 
side of the forearm and hand to be cut in his own arm, in order that he might study carefully the revival of sensations. $\mathrm{He}$ found that he never lost his ability to recognize displacements of the tissues beneath the skin. Pacinian bodies and other end-organs of deep-lying nerves recorded pressure and tension caused by pushing or rubbing with a blunt instrument. Seven weeks after the injury he began to recognize stimuli that do harm-hot things, cold things, pricking with a pinalthough his power of localizing the spot injured was extremely vague. In seven weeks, that is to say, the protopathic nerves, which do not follow the same definite lines as the nerves of the special senses, but form open networks with many alternative paths, had re-established their skin connections. Only gradually and very slowly did critical sensations return-the ability to distinguish degrees of warmth, to recognize as separate two points of a pair of compasses, to feel a touch with cottonwool.

According to a theory set forth in this book (p. 312), pain is not a set of sensations, but a condition of the central nervous system which renders it unduly excitable, or excitable in a particular manner, to impulses which have the same local origin as the nerve-current which sets up the condition of pain. When a nerve of the skin has been cut, the epithelial ramifications are renewed before any specialized tactile or other sense-organs have regained their nervous connections. When the area which has regained its surface ramifications, but has not regained its sense-organs, is injured, no localization of pain results. Indeed, the obscure sensations which are then experienced if the skin be injured can hardly be deseribed as painful. The ramified nerves pour their agitation into the grey matter of the spinal cord ; but it is not the agitation per se which causes pain. It is the passage of impulses through the agitated area that gives to them, when they reach consciousness, not only a topographical meaning, but also a distressful feeling. Until the specialized organs of the skin have been restored to working order, there are no impulses to pass through the agitated grey matter, and therefore no feelings of pain. According to this view there are two systems of afferent nerves, the protopathic and the specialized or critical. The former is very widely and very abundantly distributed to the surface 
of the body, the lungs, the alimentary canal, and other viscera. It has no end-organs, no defined tracts in the central nervous system, no definite connections with the cortex of the great brain. The currents which it conducts, if they originate in the visceral part of this system, have no direct effect in consciousness ; but if they originate on the surface of the body, or in the alimentary canal at the lower end of the cosophagus, or in certain other situations, they co-operate with stimuli of heat, cold, or traction. The critical system works in a more definite way. Its impulses originate in sense-organs. Starting with a certain potential, they are transmitted by the discharge of a succession of linked neurones. When they reach the cortex their potential is sufficiently high to evoke consciousness. Their distribution in the cortex is as definite as their origin.

Specialized sense-organs are necessary for the origin of all sensations. Within the epithelium are certain cells which look as if they were specialized for sensory purposes. The deeper sheet, or derma, of the skin is abundantly provided with structures in which nerves end in the most elaborate and complicated ways (Fig. 42). They are found especially in the papillæ of connective tissue, which, set in rows, form the ridges that one can see at the finger-tips and in various other situations. All of these organs are made up of groups of epithelial cells which, displaced from the epidermis, have sunk into the derma, with the nerves connected with them. In their further development the nervous part of the apparatus is complicated by branching, the branches being thickened and usually flattened into ribbons, which lie on the external surfaces of the cells or between them. A more or less marked capsule is provided for the organ by condensation of connective tissue.

Anyone can convince himself that the skin is not uniformly sensitive. He may test it first for the minimal stimulus which excites a sensation of touch. With a hair of the head-it must not be a very fine one-cut across with scissors, and held between finger and thumb at the right distance from the cut end, the skin of the palm of the hand is prodded. Every here and there a spot is found which is insensitive to so slight a pressure. These spots are neither large nor very close together. If the hairless skin of the arm between the elbow and the 
armpit be investigated in the same way, much larger blank areas are met with-oval patches more than $\frac{1}{4}$ inch in diameter. When a hairy surface is tested, it is found that contact with a hair can always be felt; and when the hairs are shaved, the touch-spots are found to extend around or from the points at which hairs pierce the epidermis. Touchless areas lie between them. Hair-follicles receive tufts of nerve-filaments, and it appears that they are the chief organs of touch. "Touchcorpuscles," which are found in great numbers in the papillæ
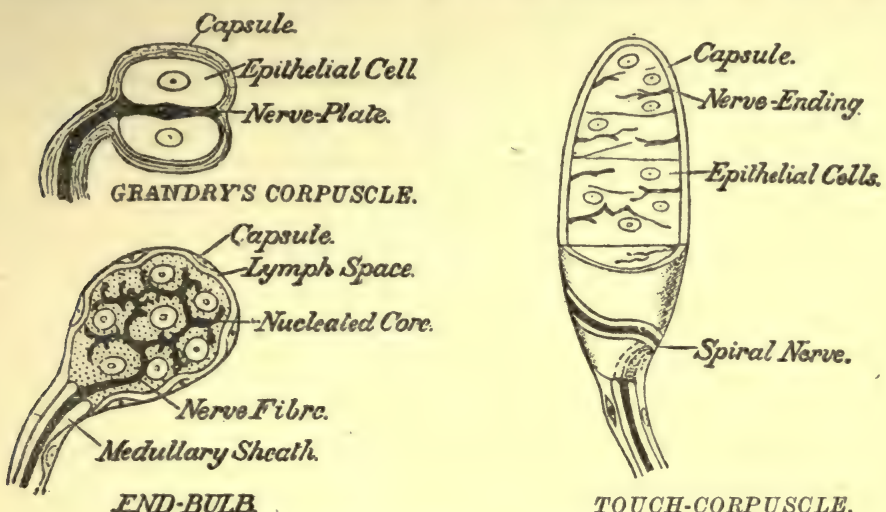

TOUCH-CORPUSCLE.

Fig. 42.-Sense-Organs susceptible to Pressure.

All are formed on essentially the same plan; a fibrous capsule invests a group of epithelial cells amongst which a nerve ramifles. The simplest form is known as a Grandry's corpuscle-a nerve ending in one or two plates between two or three epithelial cells. These organs are found in great numbers in the bills of aquatic birds. If a duck is watched whilst it is gobbling mud at the margin of a pond, it will be seen to have a remarkable capacity for discriminating between the shells of small snails, which it can crush, and stones, which it needs to drop from its bill. Its bill is also provided with small Pacinian corpuscles (Fig. 43). Touch-corpuscles, more elaborate in form than the one figured, are found in the papillæ of the skin of the fingers and elsewhere. They appear to be modifled hair-follicles. End-bulbs occur in the conjunctiva and elsewhere, and especially in the peritoneum. Together with Pacinian corpuscles, they are accountable for sensations connected with the distension of the stomach and intestines.

of the skin of the fingers and elsewhere, may probably be regarded as, genetically, hair-follicles which have not developed hairs.

If sensitiveness to pain is investigated by tapping very gently with a needle-or, better, by using a stiff horsehair fixed in a cleft stick, from which it projects about $\frac{1}{4}$ inch-it will be found that every here and there are spots which are exceedingly sensitive, whilst adjoining them are areas which are moderately sensitive, and between these areas small spots or stretches of 
skin which do not give the smarting sensation even though the horsehair be pushed until it doubles up.

Testing now for sensitiveness to cold with a cold blunt motal point, "cold-spots" can be mapped on the skin. If the metal

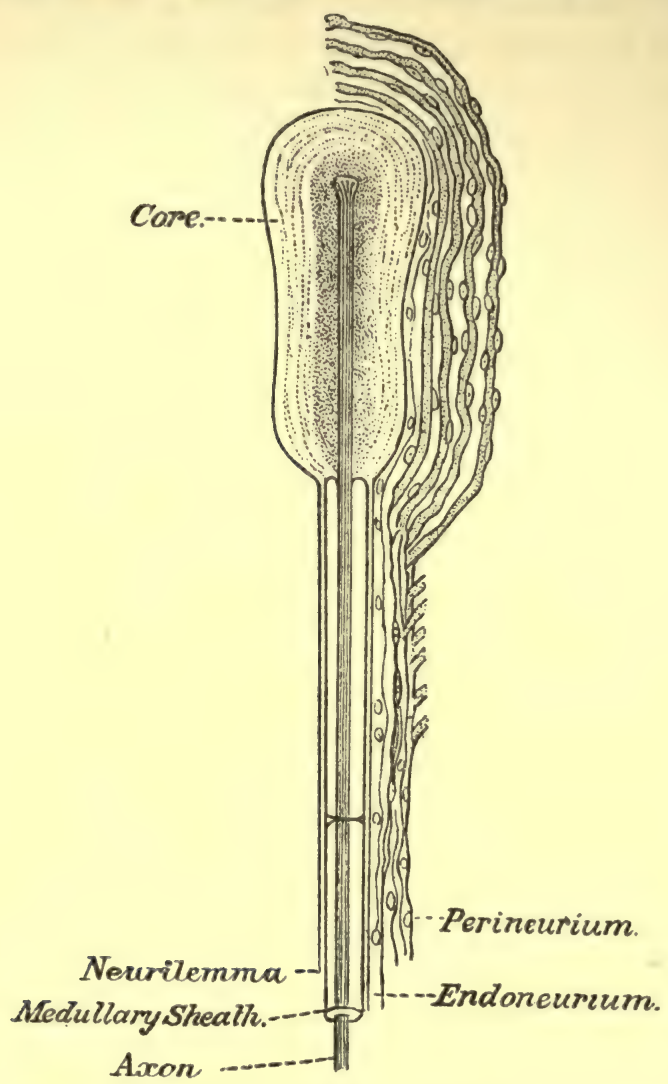

Fig. 43.-PACinian CORPUSCle.

These organs are especially numerous in the neighbourhood of tendons and ligaments. They are also present beneath the skin of the hands and feet. Their capsules are formed of a great number of concentric lamellæ of connective tissue, enclosing lymph-spaces. Within the capsule is a core of finely granular substance, which also shows a tendency to a lamellar disposition. The structure of these relatively large sense-organs is highly suggestive of sensitiveness to pressure, traction, or rubbing.

is warmed to about $50^{\circ}$ C., "heat-spots" are found. The different kinds of spot are very irregularly distributed. They may coincide, or overlap, or leave blank spaces. Their relative abundance varies. In some regions touch-spots, in others cold- 
spots, in others heat-spots, are more closely grouped. The tongue and the hand, and especially the tips of the fingers, are most sensitive to touch; but whereas the tongue is also exceedingly sensitive to warmth, the hands are relatively insensitive. Yet, speaking generally, parts especially sensitive to touch are little sensitive to temperature, and vice versa. Sensitiveness to cold is much more widespread than sensitiveness to heat. It is concentrated in the skin covering the abdominal viscera. A cold douche directed between the shoulders is doubtfully felt as cold. There is no doubt whatever about it when it strikes the skin over the stomach.

From these observations it appears that the skin contains three sets of organs sensitive respectively to touch, cold, and heat. Certain investigators hold that it also contains specific organs, or nerve-endings, sensitive to painful stimulants ; but in this case there is the obvious difficulty of distinguishing between pain and touch. At no spot can pure pain be evoked free from any consciousness of touch.

To a certain extent the combinations of epithelial cells and nerve-endings in the skin fulfil the negative requirement of sense-organs; each kind, whilst specially sensitive to its own specific stimulant, is insensitive to stimulants of other kinds. But mutual exclusion is not absolute in the case of cold and warmth. If a warmed metal point be applied to a cold spot, it produces a sensation of cold. Our feelings of warmth and cold are to a large degree comparative. Lukewarm water feels cold to hands just taken out of hot water ; moderately cold water appears luke-warm to hands that have been in contact with ice. The sensory apparatus for cold and heat soon adapts itself, or, in physiological language, it is soon fatigued. If after a prolonged bath at the body temperature a foot be plunged into very hot water and withdrawn quickly, the feeling which first ensues is one of cold. It is indistinguishable from the feeling provoked by dipping the foot into cold water. The sensation of cold subsequently gives place to one of painful warmth. This does not indicate that the heat-spots have been waked out of their lethargy by excessive stimulation. On the contrary, it is the cold-spots which, when they were first stimulated by the very hot water, answered "Cold," that now cry out "Hot"; for both cold-spots and heat-spots, 
when strongly stimulated, yield the same sensation. Indeed, it appears that the mind relies upon the simultaneous stimulation of adjacent heat-spots and cold-spots for the assurance that the thing with which the skin is in contact is really hot. If two metal points, one kept warm and the other cold, are applied simultaneously to two closely adjacent spots of skin, the resulting sensation is "hot." When the cold point is withdrawn, or replaced by a second warm point, the sensation sinks to "warm." 


\section{CHAPTER XVI}

\section{VOICE AND SPEECH}

A CUT carried horizontally backwards across the cartilage which projects forwards as Adam's apple, a quarter of an inch below its notch, would show that it is $\mathbf{V}$-shaped, the point of the $\mathbf{V}$ in front. Each limb of the $\mathbf{V}$ is a broad plate. In the mid-line is a gap, the rima glottidis, through which the windpipe communicates with the pharynx (Fig. 45). It is overhung by the stiff leaf-shaped epiglottis, the edge of which can be felt with the finger behind the tongue. ( $\gamma \lambda \omega \tau \tau$ is, the mouthpiece of a reed-pipe, is the term commonly used, for short, for the rima glottidis.) When air is being drawn into the lungs, the glottis is widely open. In speaking or singing it is almost closed. It is tightly shut whilst food is passing down the gullet.

The glottis is bounded, as to its anterior two-thirds, by two membranous folds, the vocal cords. In its posterior third it has a triangular cartilage, the arytenoid, on either side. A distinction is sometimes drawn between the anterior part, bounded by the vocal cords, and the whole glottis, the former being termed " rima vocalis "; but it is scarcely justified, for, although it is true that the anterior part is essentially the organ of voice, and its margins alone vibrate when high notes are sung, the anterior ends of the arytenoid cartilages also vibrate during the production of low notes. (The substance of these processes is not, properly speaking, cartilage; it resembles the epiglottis in containing a great abundance of elastic fibres.) And here we must warn the reader not to picture to himself a vocal "cord" as a kind of fiddle-string. It bears no resemblance to a cord, as we ordinarily understand the word; it is but a fold of mucous membrane, such as one might pinch up between finger and thumb from the inner side of the cheek. 
Its capacity for vibration depends upon the tenseness which is given to it by the pressure of the lymph with which it is distended, and vast numbers of exceedingly slender elastic fibres which traverse it.

The first cartilage below the thyroid-it may be felt with the finger - is termed "cricoid" ( $\kappa$ íkos, a ring), from its resemblance to a signet-ring. Narrow in front, its large signet

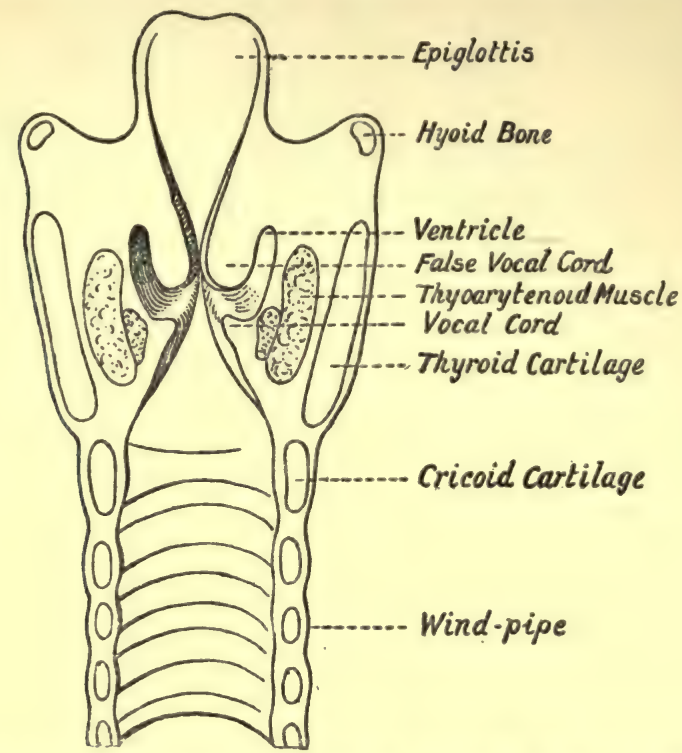

Fig. 44.-The ANterior half of the Larynx seen from BeHIND.

The drawing shows the folds of mucous membrane, the vocal cords, which stretch from the tips of the arytenoid cartilages to the recess behind the median portion of the thyroid cartilage. To the outer side of each vocal cord is seen the thyro-arytenoid muscle (cut across), consisting of a broad outer portion, chiefly concerned in closing the glottis during the act of swallowing, and a smaller internal portion, which regulates the length and the thickness of the segment of the cord allowed to vibrate.

projects upwards, within the $\mathbf{V}$ of the thyroid, behind, and on the top of the signet rest the two arytenoids. Each arytenoid is a triangular pyramid, its anterior, external, and upper angles prolonged into processes. It is united with the cricoid by a swivel joint, which allows its anterior process to swing inwards or outwards under the influence of two antagonistic muscles attached to its outer angle-the lateral and posterior cricoarytenoids. Another muscle attached only to the arytenoids draws them together. Still another muscle-or two muscles, 
for it is in two separate bands-unites the anterior process of the arytenoid with the back surface of the thyroid just on the outer side of the attachment into that cartilage of the vocal cord. The internal thyro-arytenoid muscle is a comparatively narrow band; the external thyro-arytenoid muscle is thick and broad.* By the simultaneous contraction of the encircling

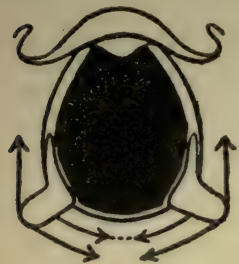

A

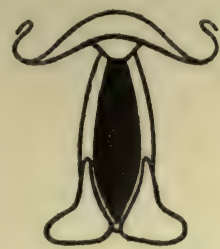

B

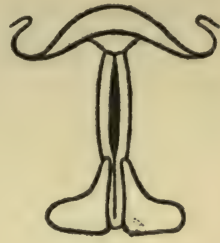

C

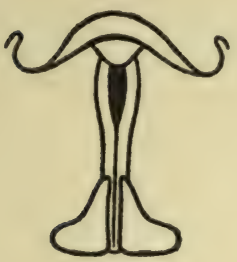

D

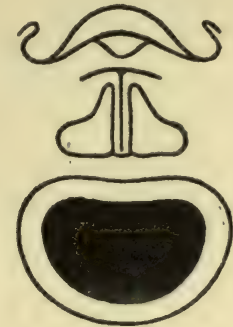

$\mathrm{E}$

Fig. 45.-THE APERTURE OF THE GLOTTIS SEEN FROM ABOVE.

The leaf-like structure in front of it is the epiglottis ; the two triangular structures at the back, the arytenoid cartilages; the white bands on either side, the vocal cords. A, The glottis is widely open during inspiration. Arrows show the lines of action of the muscles which rotate, and approximate, the cartilages. Attached to their outer angles, and pulling these angles forwards, the lateral crico-arytenoid muscles ; pulling them backwards and inwards, the posterior erico-arytenoid muscles. Drawing the cartilages together, the arytenoid muscles. B, The glottis during speaking in a deep chest-voice, or when a low note of the lower register is being sung. C, During the production of a high note of the lower register. D, During the production of a note of the head-register. E, During the act of swallowing ; the arytenoid cartilages are drawn towards the epiglottis; the aperture is folded into a $\mathbf{T}$; the pharynx (the tube behind the glottis) is distended.

muscles the larynx is closely squeezed together, the anterior portion of the slit forming a $\mathrm{T}$, with the transverse limb in front. This occurs only in swallowing. Under the co-operating contractions of the several muscles, the glottis assumes a variety of shapes. The external crico-arytenoids rotate the anterior angles of the arytenoid cartilages inwards (Fig. 45, A).

* A bullock's larynx is an admirable object of study. In almost all points of form and structure it is practically identical with the human larynx, and its large size makes it easy to dissect. 
If at the same time the arytenoid muscle draws the cartilages together, the glottis is reduced to a slit (Fig. 45, C). The posterior crico-arytenoid muscles rotate the cartilages outwards. If the arytenoid muscle is at the same time relaxed, the glottis gapes to its fullest extent (Fig. 45, A). The freer the opening, the less is the resistance to the blast of air, the gentler the vibrations of the cords, the lower the voice. The closer the slit, the greater is the resistance which the air in the windpipe has to overcome in passing through it, and consequently the more ample the vibrations into which it throws the vocal cords.

The vocal cords are the tongues of a reed-pipe, which, commencing in the chest at the point where the great bronchi join to form the windpipe, comprises the larynx, and, above the larynx, the complicated chambers of the throat, mouth, and nasal cavities, including the spaces within the bones of the head which open out of them. The pitch of the voice depends upon (1) the length of the vocal cords, and (2) their tension. The first factor is fixed for every individual. The voice is base, baritone, tenor, in a man ; contralto, mezzo-soprano, soprano, in a woman-in proportion as the cords are long, of medium length, or short. A man's vocal cords measure, on the average, 15 millimetres, a woman's 11 millimetres. When a boy is from twelve to fifteen years of age his vocal cords double in length, and the "breaking" of the voice occurs as he gives up trying to get high notes out of his longer cords, and allows them to produce manly tones of an octave lower.

The lower posterior angles of the thyroid cartilages articulate with the cricoid. If the four cartilages are freed from all soft tissues without disturbing the thyro-cricoid, or crico-arytenoid joints, and if, while the thyroid is held in one hand, a finger of the other is placed on the front of the cricoid, it will be found that as this is depressed the arytenoid cartilages which rest upon its signet are tilted upwards and forwards within the thyroid; as it is raised, they are tilted away from it. In life this movement is effected by a muscle-the crico-thyroid (Fig. 46)-attached to the front of the cricoid cartilage and to the under border of the lateral plate of the thyroid. This is the muscle of supreme importance in the production of the voice. The thyroid cartilage is slung in a fixed position by 
the hyoid bone (to be felt in the neck above it). The cricothyroid muscle, being unable to depress the thyroid, raises the front of the cricoid cartilage, tilts back the arytenoids, tightens the vocal cords. As the voice ascends the scale, the tension of the cords is progressively increased, and their vibrations rendered proportionately more rapid. The range of the human voice is about three and a half octaves; of individual voices about two octaves; if the shrill cry of a baby, which may reach the third $G$ above the middle $C$, or even higher $\left(E^{\prime \prime \prime \prime}\right.$ or $\left.\mathrm{F}^{\prime \prime \prime \prime}\right)$,

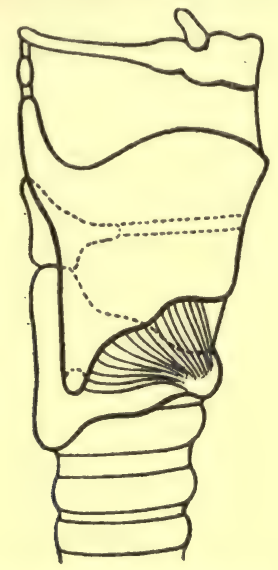

Fig. 46.-The Larynx From the Right Side.

From above downwards : the hyoid bone, thyro-hyoid membrane, thyroid cartilage, cricoid cartilage, trachea. The upper and posterior angle of the wing of the thyroid cartilage is suspended from the hyoid bone; its lower and posterior angle articulated with the cricoid cartilage. On the summit of the cricoid cartilage it articulates the arytenoid. Dotted lines indicate the position of the vocal cord. The crico-thyroid muscle, which raises the front of the cricoid, tilting the arytenoid cartilage backwards and tightening the vocal cord, extends, fan-like, from the front of the cricoid to the lower border of the wing of the thyroid.

be excluded. Exceptional voices have a range far greater than two octaves. Falsetto voice is produced by throwing half of the vocal cord out of vibration (the way in which this is accomplished is not clear), and at the same time raising the back of the tongue to the wall of the throat in such a manner as to cut off all the lower part of the upper resonating chamber, leaving it only the mouth and the cavities of the nose.

So far the mechanism of voice is easily understood. As the scale is ascended, the vocal cords are progressively tightened by the contraction of the crico-thyroid muscles. But an analysis 
of the feelings experienced during singing (and of the quality of the sounds produced) shows that by themselves these muscles are not able to make changes in the tension of the cords sufficient to account for the full range of the voice. Or, put in another way, the tension of the vocal cords is not altered to the extent which would be necessary if upon it alone depended a range of from two to three octaves. It is obvious that by some means the length or thickness, or both, of the portions of the cords vibrating is changed as the scale is ascended. If commencement be made on a low note, a point is reached, after a certain number of notes have been sung, at which a sudden change occurs. There is an alteration in the quality of sound, the more marked, the less well trained the singer. The singer experiences a feeling of relief. If a finger be placed on his crico-thyroid muscle, a relaxation of its anterior fibres can be detected. As he proceeds up the scale, these fibres again tighten. At a certain point there is again a change in the quality of voice, and in the feelings which accompany its production. The two points at which change occurs are said to divide the voice into three "registers "- the lower, or chest-register, the middle, and the upper, or head-register. A great effort is needed to hold either register above its natural range.

The physiology of the registers is a subject far too thorny for handling in this book. The larynx can be watched with the laryngoscope during the production of notes of different pitch, but observers are not in accord regarding the appearances which it presents, or their interpretation. The possibilities of changing the reed which vibrates, the vocal cord, otherwise than by increasing the direct pull upon it exerted by the cricothyroid muscle, appear to be as follows : (1) During the production of the lowest notes the elastic portion of the arytenoid cartilage may be included with the cord. It may be thrown out of vibration by its rotation inwards (under the action of the lateral crico-arytenoid muscle) until it is pressed against its fellow. (2) Certain portions of the cord may be damped by partial contractions of the internal thyro-arytenoid muscle. It has been frequently stated, although the statement is not accepted by all anatomists, that some of the fibres which take origin from the arytenoid cartilage end in the cord, 
instead of passing right through to the thyroid. It is supposed that by their contraction they throw the posterior portion of the cord-even, it is asserted, as much as its posterior twothirds in the higher head-notes-out of vibration. (3) It appears that the width (thickness) of the cord vibrating is also regulated by the contraction of the thyro-arytenoid muscle. Those who regard the diminution in the thickness and width of the vibrating fold of mucous membrane and underlying elastic tissue as the chief factor in the adaptation of the larynx for the middle register lay great stress upon the sense of relief from muscular effort which accompanies the transition. Less force is needed to tighten the thinner cord. They also call attention to the loss in volume of the voice when the lower register is left, and to its greater softness. The lower is spoken of as the thick register, the middle as thin, and the upper (on the hypothesis that part only of the cord vibrates) as the small register.

Singing reveals the possibilities of the larynx as a musical instrument. In speech the larynx plays a part, but the form of the syllabic sounds and the relative prominence of overtones in the vowels is of more importance than pitch. Flexibility of voice is dependent upon ability to increase or diminish at will the size of the resonating chambers of the throat. mouth, and nose, or the freedom of access to them. Conversation is carried on in the lower or chest-register. When a practised speaker mounts a platform, he spends the first few minutes in ascertaining the pitch of the hall-that is to say, the pitch of his voice to which the room resonates most freely. Having found the proper tone, he endeavours to maintain a uniform tension of his vocal cords, and therefore a uniform pitch. He relieves the monotony of speech by suitable variations of its overtones. Nothing is more uncomfortable to listen to than an oration delivered in cadences. The speaking voice should be full, round, and musical, and free from affectation-as guiltless of the intoning or preaching quality as it is of harshness or of vulgar flatness. A flexible voice is capable of producing, as occasion calls for them, tones of any and every quality. With the throat and mouth set for the syllable " haw," it is impossible to do justice to such words as "king" and "queen." The voice-tones of a superior person are as 
distasteful to the hearer as those of a vulgarian. Unpleasant also is a nasal twang, illogically so called, since it is due, not to the opening of the resonating chambers of the nose, but to the restriction of the entry of air into them. In this it is somewhat similar to the effect produced by a severe cold. Resonance in the nasal chambers produces a clear, ringing voice.

A little consideration of the varying qualities of different voices suffices to show how largely they depend on resonance. When vowel-sounds are analysed, it is found that the distinctive character of each of them is dependent upon the overtones which it contains. For every vowel the overtones are fixed, or very nearly so, no matter what may be the pitch of the note to which the vowel is sounded.

It is much to be regretted that the alphabet was settled before the physiology of speech was understood. Were it based upon reasonable principles, children would be spared the bewilderment which overtakes them when they endeavour to establish in their minds some kind of relation between the names of consonants and their effects upon the blast of air as it passes through throat and mouth, and between tongue and palate, teeth and lips. The vowels, had physiologists defined them, would have been real pure vowel-tones- $\overline{0 o}, o$, $a h, \overline{e e}$ - sounds which can be sustained for an indefinite time, and allowed to die away without deterioration in their quality. $A$ ( $e ́$ as pronounced in France) is doubtfully pure-it has a tendency to tail off in $\overline{e e} ; \bar{i}$ is frankly a diphthong, ai $(a h-\overline{e e})$. Try to hold a long final note on the syllable "nigh"! An international standard of vowel-sounds would have been fixed, by giving the vibrating periods of the tuning-forks for which in each several case the resonating chambers are shaped, and defining the relative accentuation of each overtone. Greatest boon of all, the irruption of the Essex dialect would have been dammed. It would not have been allowed to inundate London, or to submerge Australia, debasing our English tongue. In Cockney speech vowels degenerate down the line of greatest indolence. $A w$ becomes or, or $a r ; a$ becomes $i$. It requires a greater effort to pronounce a full $a$ than a flat $a$, a definite flat $a$ than $i$. And worse than a Cockney's unwillingness to take the trouble necessary for the production of dignified 
vowel-tones is his reluctance to make the effort required for the holding of any tone. In his mouth virile, self-reliant vowels are replaced by emasculated diphthongs, which collapse as they present themselves to the ear. It costs trouble to fix the mouth-chamber before a vowel is sounded and to hold it steady until it is finished. $A h$ slides down through $a i$ to $\overline{e e} ; i$ slips into $\overline{e e}$. "Cow" becomes kyow; "you," ye-u-ow; "cart," kyart. And just as the effort needed for the filling of the vowels is shirked, so also is grudged the expenditure of an accessory blast for their aspiration.

When a vowel is whispered, although the vocal cords do not vibrate, the blast passing through the resonating chambers produces the overtones characteristic of the vowel. Anyone who feels his own larynx while he sings, to the same note, the various vowels between $\overline{o O}$ and $\bar{e}-$ he may please himself as to the number of $a i$, $e u$, and $\breve{u}$ vowels he interposes between these two extremes-will recognize that it is pulled farther and farther upwards by the muscles which surround it. The cavity of the mouth is at the same time made shorter and broader for each succeeding vowel. Singing the several vowels before a piano, and at the same time striking various keys, it is felt in the mouth that the resonance of that chamber is reinforced by certain selected notes. Certain tuning-forks, when sounded in front of the mouth shaped for a vowel, ring out more loudly, because the mouth cavity resonates to their prime tones. The overtones of the vowels can be analysed in this way. Conversely, by sounding simultaneously an appropriate selection of tuning-forks, each with the right degree of force, the overtones of a vowel can be synthesised. Thus if whilst one tuning-fork is sounding $\mathrm{B}_{1} b$ ( $\mathrm{B} b$ above middle $\mathrm{C}$ ), two others be added giving $\mathrm{B}_{2} b$ (loud) and $\mathrm{F}_{3}$ (soft), the composite sound resembles the vowel $o$. If to these same three forks, with $\mathrm{F}_{3}$ sounding more strongly, $\mathrm{B}_{3} \mathrm{~b}$ and a loud $\mathrm{D}_{4}$ be added, the sound changes to $a h$.

The organ of voice is a combination of a reed-pipe with resonating chambers, the shape of which can be changed at will. The quality characteristic of a vowel is given to it by adding to the note produced in the larynx sounds due to the resonance of the throat and mouth. On the assumption (not allowed by all authorities) that, since the resonating chambers 
are not sound-producers, they can only add to the larynxtone, as "formants" of a vowel, its own harmonics-sounds which they have picked out of it-it follows that, if, when the prime is changed, the resonators were not adapted to the new note, they would be dumb. If this attitude in regard to the question be justified, there must be a certain amount of variation in the quality of a vowel as the scale is ascended. But a vowel is not a musical tone; it is a conventional sound. Its whole value depends upon its retaining, as nearly as may be, the same quality, whatever be the pitch of its prime tone. By adjusting the form of the throat and mouth, we can not only prevent one vowel from passing into another, but we can keep it so nearly true to itself as to convince the ear that its quality is unchanged: $\overline{O 0}$ remains $\overline{o o}$, and $a h a h$, although the form of the sound as produced on $\mathrm{C} \notin$ is different to its form when sung to $\mathrm{C}$.

Apart from the general distinction that low notes are taken more easily with vowels requiring a large mouth-cavity, and high notes with those providing a small one, there are certain very distinct relations between vowel-sounds and musical tones which need to be borne in mind in setting words to music. A singer changes a word when he feels that its vowel-tone does not allow him to give to the note to which it is set the fullest expression of which he is capable.

An account of the physiology of the production of consonants is to be found in most text-books of grammar. 


\section{N D E X}

ABSORPTION from alimentary canal, 129

Accelerator nerves of heart, 237

Accommodation of the eye for distance, 391

for light, 390

Acromegaly, 93

Addison's disease, 91

Adrenalin, action on the kidney, 209 formed in suprarenal capsule, 92

Air, quantity inspired, 173 quantity needed by individual, 191

Air-cells of lungs, 168

Albumin made by plants, 12

Alcohol, effect on nerve conduction, 301

Alimentary canal, morphology of, 98 nerves of, 104

Altitude, highest, attained by climbers, 187

Alveoli of lungs, their number, 169

Amides produced from proteins, 119

Amœba, irritability of its protoplasm, 10

Amyl nitrite, effect on vascular system, 237

Anæmia, treatment with iron, 67

Anæsthetics, influence on protoplasm, 11

Analysis by animals, 12

Angina pectoris, 237

Angler fish, its nerve-cells, 31

Animal machine and its driver, 354, 358

Animals, hunting versus hunted, 366

not reflex machines, 358

relative insensibility to the knife, 361

Antitoxins, formation by protoplasm, 20

Aorta, diameter of, 232

Aphasia, 352

Apnoa, condition of arrested respiration, 181

Appendicitis, increased frequency of, 101

Appetite, a safe guide, 114

Arteries, blood-pressure in, 234, 239 structure of wall of, 233

Artificial respiration, 183

Asphyxia, 182

Association zones in the cortex of the great brain, 348

Asthma, due to reflex contraction of small bronchi, 167

Astigmatism, correction by glasses, 393 due to modern print, 269
Attention, effect of, in heightening pain, 361

Bacteria, diminution of number in intestine on milk diet, 138

of alimentary canal, 135

of Bulgarian sour milk, 138

of the River Ganges, 141

in an infant's intestine, 136

their rôle in nature, 20

Balance-sheet of body, how drawn up, 149

Balloon, highest altitude attained in, 187

Basket-cells in nervous system, 324,340

Bat's squeak, number of vibrations, 418

Bats, flight not dependent on vision, 381

Beats in music, explanation of, 407

Beetle, muscle of, 261

Belladonna, physiological action, 109

Bile, composition, 117

function in regard to absorption of fat, 133

relation to digestion, 117

Bile-pigment, origin from hæmoglobin, $69,82,118$

Bioplasm, the essential substance of a living cell, 148

Birds, sense of hearing of, 410

Blind spot, how filled in, 395

Blisters, 41

Blood, amount ejected by heart, 219

circulation-time, 219

composition of, 59

gases of, amount, 190 tension, 61

lodged in abdominal veins, 234, 236

Blood-eorpuscles, cellular nature, 28

life-story, 62

number, 61

origin, 63,64

structure, 60

Blood-platelets, 74

Blood-poisoning, 57

Blushing, 243

Bowman's description of kidney, 200 dises in muscle, 259

Brain. Cf. Cerobellum, Cortex of cerebrum

blood-supply of, 352

Bread, digestion of, 120

Breathing, mechanism of, 171

Bruises, explanation of play of colours, 69

Bulgarian milk-germ, 138 
Capillary vessels, circulation of blood in, 232

migration of leucocytes from, 232

structure of their walls, 38

Carbohydrate foods, chemical composition, 147

Carbonic acid, carried by blood, 60

liberation in lungs, 61, 189

Carbonic oxide, compound with hæmoglobin, 187

Carnivora, absorption of fat from alimentary canal of, 133

Cartilage, growth, 28

Catalysis, 17

Cell theory, 26

Cells, constituent parts, 26,28

size, 30

specialization of function in, 35

Cells of Purkinje in the cerebellum, 303, 340

Cellulose, digestion of, 137

Cerebellum, cases of deficiency of, 341 connections with cerebro-spinal axis, 340

development of granules of, 299, 303

lobes, 338

minute anatomy, 339

phylogeny, 338

relation to tone of muscles, 342

Cerebral hemisphere, an outgrowth towards olfactory pit, 334

in animals with various sensory endowments, 349

Cerebro-spinal fluid, 50

Chemical activity of protoplasm, 12 messengers, 89,123

processes in plants, 15

Chemiotaxis of leucocytes, 56, 364

Children, brain in, 346

development of astigmatism in eyes of, 269

Chill, catching a, 242

Chloroform. Cf. Anæsthetics

Cholesterin, 118

Chromatolysis in nerve-cells, 320

Chrome-silver method of colouring nerve-tissue, 293

Chyme, food converted into, 126

Circulation of the blood, 218

Circulation-time, 219

Cirrhosis of liver, 42

Coagulation of blood, 69

Cochlea, anatomy, 413

Cockney dialect, the degradation of vowel-sounds, 439

Coke fire, poisonous fumes from, 186

Cold-spots in skin, 429

Collaterals of nerves, 297

Colon, length and disposition of, 101

Colour-blindness, 385
Colour vision, 385

Colours, reason for apparent fading in twilight, 378

Conductivity of protoplasm, 248

Consciousness, does not come within physiological investigation, 360

its part in animal life, 359

Control experiments, their value, 72

Convolutions of brain, 345

Cooking, effect upon digestibility of meat, 120

Corneal epithelium, sensitiveness of, 424

Corpus striatum of brain, 344

Cortex of cerebrum, discovery of excitability of, 344

fissures and convolutions, 345

functional areas, 352

myelination of its fibres, 345

sensory and association areas, 346

structure of, 347

variations in different animals, 349

Corti, organ of, its structure, 414

theories of function of, 416

Coughing, mechanism of, 180

Crayfish, tone of claw-muscle of, 273

Cretinism, 85, 90

Cricket, chirp of, 261

Crypts of Lieberkühn, 103

Curdling of milk, 75

Dancing, association of sound with movement, 422

Day's work, food required for, 151

Deafness due to sore throat, 412

Degeneration of nerves after section, 326

Depressor nerve of the heart, 237

Diabetes, excretion of more carbohydrate than contained in food, 143

Dialysis, explanation of the process, 40 , 128

Diaphragm, function in respiration, 171

Diastases, destructive ferments, 18

Diet, limits of possible variations in, 153

of labouring classes, 152

Digestibility of bread, meat, fish, etc., 120,125

Digestion, mechanism of, 96

vascular changes during, 235

waits on appetite, 114

Digitalis, action on heart and kidney, 209

Diphtheria, antitoxin of, 20

Diuretics, 209

Dog's sense of smell, 370

Dreams, theory of, 362

Dropsy, 42 
Drowning, resuscitation from, 183

Drugs, physiology of, 95

Ductless glands, 94

Dyspnoea, difficult respiration, 181

Ear, anatomy, 411

bones of, 412

differentiation into separate senseorgans, 410

in fishes, 410

phylogeny, 409

Eel's blood injected into mammal, 20

Effector, an organ which exhibits change in response to stimulation, 253

Egg-albumin destroyed by blood, 19

Electric organs, 288 phenomena of muscles, 279

Emotions, their relation to vaso-motor changes, 242

Energy, expended by body, 151 source of the body's, 152

of stimulus compared with energy of muscular response, 254

Engines, body compared with, 152, 256

Epiglottis during swallowing, 433

Equilibrium, maintenance of, in walking, 342

Erepsin, ferment of intestinal juice, 119

Errors of sensory judgment, 402

Excretion, 195

Eye, accommodation for distance, 391

adaptation for darkness, 390

blind spot, 394

optical defects of, 393

phylogeny, 334

refractive media, formation of image by, 391

Eyeball, abnormalities in shape of, 392

anatomy, 373

development, 374

muscles of, indefatigable, 269

Fat, absorption of, 131, 132

accumulation of, relation to foods consumed, 144

chemistry, 132

digestion, 133

laid down in connective tissues, 145 stored in liver, 145

Fatigue, causes of, 45,268

Fermentation, 16

Ferments, chemical nature, 18 classification, 16,18 physiological importance, 18

Fibrin of blood, its antecedents, 75

Fireflies, source of their light, 291

Fish, sense of smell of, 365 supposed to be frightened by noise, 410

Flatulence, cause of, 114, 125, 136
Foods, classification, 142

history of, after absorption, 142

relative value, $147,151,153,157$

residue after digestion and absorption, 194

Foramen ovale of heart, sometimes perforate, 218

Frigate-bird, turbinate bones of, 166

Frog, supposed to be found entombed in rock, 164

Functional interdependence of organs, 94

Functions transferred to other organs, 87

Gall-stones, cause of formation of, 118

Galvani's observation of contraction of a frog's muscles, 277

Ganges, purifying water of, 138

Ganglia of sympathetic chain, function, 325

Ganglion cells of retina, 376

$$
\text { spinal, } 299,333
$$

Gaseous tension, meaning of expression, 188

Gases of blood, their exchange in the lungs, 184

Gastric glands, structure, 123

juice, amount secreted, 114

composition, 114

digestive action, 115

Golatin as article of diet, 158

Giant cells, 65

Glands, vaso-motor nerves of, 109, 241

Glycogen, formula, 147

as muscle food, 148

stored in liver, 147

Goitre, cause of, 84 .

Granules, appearance of, in glands, 110 of cerebellum, development of, 299 , 303

Grey matter, formation of paths in, 356

Growth, a function of protoplasm, 24 a reaction to work, 47

Hæmatin, 68

Hæmatoidin, 68

Hæmochromogen, 68

Hæmoglobin, crystalline form, 66, 186 formula, 66

as oxygen carrier, 66,186

spectrum, 68,185

Hæmophilia, non-coagulability of blood, 76

Hallucinations, 362

Headache, a pain in the scalp, 106, 319 the brain's warning of fatigue, 269 from strain of eye-muscles, 268

Hearing, analysis of compound vibrations, 405

capacity dependent upon educa. tion, 422 
Hearing, Helmholtz's theory of analysis of sounds, 419

range of sensations, 418

sense of, 404

upper limit, 418

Heart, anatomy, 217

automatism of, 238

development, 218

murmurs, 229

muscular tissue, minute structure, 261

nerves regulating beat, 237, 239

sounds of, 228

valves, their mechanism, 226

work done by, 219, 223

Heat, production of, by muscles, 254, 256

Heat-spots in skin, 429

Helmholtz's theory of organ of Corti, 419

Hering's theory of colour-vision, 388

Hormones, meaning of term, 89, 124

of pancreas and liver, 127

of stomach, 123

Humours in ancient medical theory, 79

Hunter, experiment of grafting cock's spur in its comb, 47

Hydrochloric acid, part taken in digestion, 114

Hydrophobia, protective inoculation, 78

Hyperpnœa, excessive respiratory efforts, 182

Hypoblast, a layer of the embryo, 97

Illusions of movement, 335,384 of size and distance, 400

Immunity, acquisition of, 20

Impulse of the heart, 225 rate of passage in muscle, 280 in nerve, 278,280

theory of nerve conduction, 282

Inhibition, explanation of term, 311 of reflex actions, 311

Insects, efficiency of their muscles, 261

Instinct, due to brain-pattern, 359

Intelligence of animals, 359

Internal secretions, 83

Intestinal juice, digestive action, 119

Intestine, large, sacculation of its walls, 101

small, folds and glands of mucous membrane, 102

Intestines, movements of, 103

nerves of, 105

size and situation, 100

Iodine, importance of, to economy, 89

Iodothyrin, goitre due to deficiency of, 90

Iris, its function in regulating admission of light to eye, 394

Iron in food, 67

in hæmoglobin, 67

use of, in treatment of anæmia, 67

Irritability, a function of protoplasm, 10
Japanese, cultivation of sense of smell by, 370

Judgment of angles, 402

of distance and size, 401

of meaning of sensations, 396,399

Kidney, ancestral history, 195

elimination of indigo by, 207

of birds and reptiles, 200, 207

hydrostatic mechanism, 189

minute anatomy, 196

Kinæsthetic sensations, absence from dreams, 363

part played by, in voluntary actions, 354

representation in cortex of brain, 350, 352

Knee-jerk, 274

Labyrinth of ear, 413

Lactate of ammonia, relation to urea, 13

Lacteals, lymphatic vessels of alimentary canal, 43, 131

Lactic acid produced in muscle, 46,146

Larynx, closure during swallowing, 433 structure of, 430

Latent period of muscle after nervous impulse reaches it, 278

Laughter, respiratory mechanism of, 180

Locithin produced by metabolism of nerve-tissue, 118

Leech, ganglion cells of, 298

Leucocytes as protective agents, 52 death of, 54,57

migration of, 49

number in lymph and in blood, 49,61

origin of, 33,51

source of fibrin-ferment, $\mathbf{7 4}$

Leucocythæmia, excess of leucocytes in the blood, 215

Levers to which muscles are attached, 286

Light, emission of, by animals, 291

Lime, influence upon coagulation of blood, 75

curdling of milk, 75

Lithates, or urates, constituents of calculi, 213

Liver, destruction of red blood-corpuscles in, 83

form and structure of, 160

former theories of its functions, 129,163

manufactures urea and uric acid, 146,162

of well-fed sheep, 147

origin of, in vertebrate phylogeny,

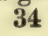


Liver stores food, especially glycogen, $46,145,147,161$

Locomotor ataxy, 341

Ludwig's view of mechanism of kidney, 200

Luminous glands, 291

Lung, exchange of gases in, 173, 184, 190 nerve-supply, 178 structure, 168

Lymph, amount of, in body, 37 composition, 49 relation to blood, 51

Lymph-spaces, 37, 43, 49

Lymphatic glands, structure of, 54

Lymphatic vessels, 43

Malapterurus, electric organs, 288 immense neurones of, 295

Manometer for measuring blood-pressure, description of, 238

Man's ancestry, 153

Massage of abdominal viscera, 101 of muscles, 48

Meal, the story of a, 120

Meat, diet consisting solely of, 157 digestion of, 121 extracts of, as articles of diet, 159

Megacaryocytes, 65

Memory, physiological explanation, 356

Metabolism, chemical change in living tissue, 12, 273

Methæmoglobin, 69

Microscope, its discovery, 26

Migration of birds, 359

Milk, call for secretion of, by a hormone, 94

chemical and physical constitution, 132

digestion of, 127

Milk diet, reduction of bacteria in alimentary canal on, 138

Mind, physiology of, 354

Mosquitoes, production of sound by, 261

Motile cells, 32

Mountain sickness, 187

Mountains, highest climbed, 187

Mucous membrane, use of term, 97

Murmurs, in chest, in diseases of lungs, 169

of heart, 229

Muscle, change in appearance under microscope during contraction, 263

chemistry of contraction, 266

contraction a phenomenon of osmosis, 258

electric phenomena of, 278

means of promoting growth of, 271

measurement of its power, 285

nature of impulse which leads to contraction of, 282
Muscle of heart, its minute structure, 224

of insects, its efficiency, 261

plain, its minute structure, 258

plasma, its coagulation, 266

rhythm of voluntary contraction, 279

theory of its structure as a mechanism liberating energy, 234, 255

tone of, 272

tracings taken of contracting, 278

voluntary, its minute structure, 259

wastes when its nerve is severed, 274

work done by, proportional to load, 286

Muscles, arrangement in regard to the bones which they move, 286

co-operation in lifting a weight, 287

Muscular energy, source of, 235

Muscularis mucosæ of alimentary canal, 103

Musculi papillares of heart, 227

Music, chords admissible in, 408

Indian, division of octave, 408

primitive, prevalence of minor chords, 408

Musical tones and overtones, 406

Myelination of nerves, order of, 345

Myxœdema, dependent on disease of thyroid gland, 85

Myxomycetes, fusion of cell bodies of, 27

Nasal chambers, air warmed in, 166

Negroes, their long heels, 285

Nerve, conduction in, theory of, 282

degeneration, 326

electrical phenomena, 279

indefatigable, 282

regeneration, 326

structure, 296

Nerves, depressor, 237

experiment of crossing, 327

fifth, 316

of heart, 239

of intestines, 426

protopathic and critical systems of, 425

secretory, of the salivary glands, 109

splanchnic, 236

superior laryngeal, 178

vagus, 104

vaso-motor, 239

Nerve-cells last throughout life, 148

limitations of their functions, 321

store of energy in, 320

transfer of impulses from cell to cell, 177, 300

their relation to muscle-fibres, 274 varying size of, 295,322

Nerve-centres, 176 
Nerve-force, improper use of expression, 281

Nerve-impulses, distribution in grey ,mattor, 305

reinforcement of, 320

resistance to, at synapses, 306

Nerve-nets, pericellular, 301, 319

Nervous system, neuronic and extraneuronic conduction, 310 phylogeny of, 332

Neuro-fibrillæ, 298

Neurone, origin of term, 293 transmission of current by, 328 various types of, 296,323

Night-blindness, 378

Nissl's bodies, source of nervous energy, 320

Nitric oxide, combination with hæmoglobin, 186

Nitrogenous equilibrium, 150 food, stimulating effect of, 157 waste, 210

Nceud vital of Flourens, 176

Normal diet, 151

Normal salt solution, 82

Nucleo-proteins, source of uric acid, 215

Odours, classification of, 366

Edema, or dropsy, 42

Olfactory membrane, structure, 366

Optic nerve, number of fibres, 378

Organ of Corti, structure, 415 theory of function, 417

Organs that have lost their prime functions, 87

Orientation, sense of, 335

Osmosis, 40, 128, 201 cause of muscular contraction, 235

Osteoblasts, bone-forming cells, 32

Osteoclasts, bone-eating cells, 65

Oxygen, amount required per diem, 166 carried by red blood-corpuscles, 66

Pain, influence of, upon action, 359 referred from viscera to surface of body, 316

relation to sensation, 313,425

theory of, 312,425

Pancreas, structure, 116

Pancreatic juice, constitution, 116 fat-splitting ferment of, 133

Papillæ of the tongue, various forms of, 97

Parathyroids, 86

Pepsin, digestive action, 115

Peptone prevents coagulation of blood, 77

Pericellular nerve-nets, 301

Perspiration, cools the surface of the body, 236

repressed during fever, 257
Peyer's patches of lymph-follicles in intestine, 53

Phagocytes, germ-eating leucocytes, 60 consumption of red blood-corpuscles by, 82

Phosphenes, developed by pressure on eyeball, 383

Phosphorescence, cause of, 291

Phrenology, 343

Pictures, suggestion of solidity in, 401

Pineal body, phylogeny, 334

Pituitary body, 93

Plants, anæsthetized by ether, 12, 24

their metabolism, 15

their respiration, 24

Pleura, lining membrane of chest, 172

Pleurisy, pain of, 313

Pleuritic fluid, absorption of, 223

Pneumonia, changes in lung during, 169

Portal system of bloodvessels, 80 regulator of vascular tone, 236

Power of muscles, 285

Precipitins formed in blood, 19

Proteins, absorption by alimentary canal, 145

chemical constitution, 6

dietetic value, 157

fate after absorption, 212

Protopathic nerves, 425

Protoplasm, arrangement in cells, 30 constitution, 7

Huxley's definition, 6

Pulse, cause of, 244

records of, 245

variations, 247

Purgatives, theory of action, 128

Purkinje cells of cerebellum, 303,340

shadows of retina] vessels, 375

Pus, origin of, from leucocytes, 57

Pyramids of cortex of great brain, 346

Rabbit's ear, vaso-motor changes in, 235

Receptor, an organ specially sensitive to stimulation, 253

Referred pains from viscera, 316

Reflex action, inhibition of, 311

of scratching, 330

vinegar experiment with frog, 307

Regeneration of nerves, 326

Renal-portal circulation, 199

Renewal of tissues, 148

Rennin, ferment of milk, 16

Resistance in nervous system, laws of, 177,307

Respiration, artificial, 179 effect on circulation, 221

a function of protoplasm, 23, 164

movements of, 171

nervous mechanism, 175,179

in tissues, 165, 193 
Respiratory centre in medulla oblongata, 176, 178, 182

Respiratory quotient, 174

Retina, structure, 374

Retinal pigment, relation to vision, 381

Rice ordeal, arrest of secretion of saliva, 112

Rigor mortis, 266

Rods and cones, respective functions in vision, 378

Rowing, value of, as exercise, 287

Saccharin, taste of, 367

Saline frog, respiration in, 193

Saliva, chemical constitution, 107 function of, 96, 107

Salivary glands, mechanism of secretion, 108

nerves of, 109, 236

Salts, absorption of, in alimentary canal, 128

Scientific method, definition of, 71

Scratch reflex, in dog, 330

Sea-sickness, 106

Secretin, hormone of pancreas and liver, 127

Secretion, accumulation of granules in cells, and their discharge, 110

a response to stimulation, 111

not a process of filtration, 110

Semicircular canals, their functions, 410 their positions in space, $\mathbf{3 3 5}$

Sensations, their apparent fusion, 356 many which escape attention, 318 , 355

neutralization of one by another, 356

Sense-organs, origin in vertebrata, 336

Sensory areas in cortex of the great brain, 348

Sensory nerves, their connection with cerebro-spinal axis, 304

Shell-fish, poisonous extract of, 41

Shivering due to loss of heat from skin, 257

Sight. $C f$. Vision

Skate, electric organs of, 289

Skilled movements, dependent upon kinæsthetic sensations, 357

Skin, experiment of cutting nerve, 424 variety of sensations from, 423

Sleep, condition of neurones in, 362

Sleeping sickness, 33

Smallpox, protection against, 78

Smell, disappearance of sense of, in later life, 370

dog's dependence upon sense of, 366 reason for mental associations with sensations of, 371

sensitiveness to mercaptan, 365

Smells, nice and nasty, 369
Smoking, mental effect of, 371

Sneezing on looking at bright light, 317

Sore-throat, cause of deafness, 412

Soul, Aristotle's definition, 32

Sound, mode of conduction, 404 rapidity of vibrations of, 406,418

Sounds of the heart, 228

periodic and aperiodic, 409

Spectacles, defects of eyeball which call for, 392

Speech, derangements of, due to disэase of the brain, 353

mechanism of, 437

Sphygmographs for recording pulse, 245

Spinal dog, reflex action in, 330

frog, reflex action in, 307

ganglia, development of cells, 299

Splanchnic nerves, regulation of bloodpressure by, 236

Spleen, destruction of blood-corpuscles in, 80

structure, 79

Squint, correction of double vision in, 397

Starch, formula, 15

Star-shapes due to puckering of crystalline lens, 393

Starvation, statistics of, 156

Stiffness of muscles, cause of, 45,271 ,

Stimuli to muscles and nerves, 248

Stokes, discovery of spectrum of blood, 68

Stomach, digestion in, 120

glands of, 123

referred pains from, 316

shape and size, 99

Stone in the bladder, its cause, 213

Subconscious self, 355

Sugars, digestion of, 120, 136 formulæ, 15

Sun, apparent size near horizon, 399

Suprarenal capsules, their structure and function, 91

Sweetbread as article of diet, 215

Sympathetic system of nerves, 243,325 diameter of fibres, 325

Synapses of nerve-cells, resistance interposed at, 306

Synaptases, constructive ferments, 18

Synthesis by plants, 15

Tapeworms, resist digestion in the intestines, 21

Taste, confusion with sense of smell, 364 localization on tongue, 367 sense of, in fishes, 365 sensitiveness to quinine, 369

Taste-bulbs, their structure, 368

Tattooing, removal of pigment by leuco. cytes, 55

Tea, its dietetic value, 122 
Teeth, 96

Tendon, the growth of, from cells, 28

Tension of gases in the lungs, 190

Tetanus, the vibratile contraction of muscle, 279

Thoracic duct, discharges lymph into veins, 43, 131

Thorax, negative pressure in, 222

Thorns on dendrites of nerve-cells, 300

Thyroid body or gland, forms an internal secretion, 86

relation to goitre, 85

structure of, 85

Tight-lacing, deformation of organs which it causes, 220

Tigroids, in nerve-cells, stores of energy, 320

Tissues, respiration in, 165, 193

Tone of muscles, 272

Tongue, as organ of taste, 367

Tonsils, function as guardians of the fauces, 53

structure, 52

Torpedo, electric organs of, 290

Touch, sensations of, 426

Toxins produced by microbes, 20

Urea, amount relatively to proteins consumed, 155

antecedents of, 146, 212

chemical formula, 211

secreted during period of starvation, 156

Uric acid, amount secreted daily, 213 artificial production of, 13 chemical formula, 13, 214 diathesis, its relation to diet, 140

due to metabolism of leucocytes, 53,216

form in which excreted, 207

made in the liver of birds, 13

Urticaria due to abnormal composition of lymph, 41

Vaccination, protective value of, 22

Valves of heart, their mechanism, 226
Vascular system, tone of, 236, 240

Vaso-constrictor nerves, 236

Vaso-dilator nerves, 236

Vegetables, dietetic value of, 139

digestion of, 125, 137

Vermiform appendix, 88

Villi of intestine, absorption of food by, 130

fat seen in, during active digestion, 134

Viscera, their insensitiveness to injury, 316,426

Vision, colour contrasts, 382

duration of images, 382

judgment of distance and size, 411 solidity, 401

stereoscopic, doctrine of corresponding points, 397

Visual purple, 381

Vital action, definition of expression, 205

Vivisection, 4

Vocal cords, structure, 431 how modified in singing, 435

Voice, breaking of, in boys, 434

falsetto, how produced, 435

range of human, $\mathbf{4 3 5}$

registers, 436

Vomiting, 105

Vowels, synthesis by tuning-forks, 439

Wandering cells, 33

Warmth, appreciation of, by skin, 429

Waste substances, classification, 194 how eliminated from body, 59

Waterfall, negative after-image of, 384

Water-weed, experiment proving that it respires, 24

Wear and tear of bioplasm, 145

Wisdom-tooth, tending to disappear, 96

Yawning, beneficial effect on circulation, 222

nervous mechanism of, 180

Young's theory of colour-vision, 385

Zymogen, 110

THE END 


\section{Dr. Hutchison's Works.}

\section{FOOD AND THE PRINCIPLES OF DIETETICS.}

By ROBERT HUTCHISON, M.D. EDIN., F.R.C.P., Assistant Physician to the London Hospital and to the Hospital for Siok Children, Great Ormond Street.

$\mathrm{xx}+582$ pages, with 3 Plates in Colour and 34 Illustrations in the text. Demy 8vo., red buckram, 16s. net.

\section{NEW AND REVISED EDITION.}

CHAPTER

\section{OUTLINE OF CONTENTS.}

I. The Nature, Nutritive Constituents, and Relative Values of Foods.

II. The Amount of Food required in Health.

III. On the Influence of Various Conditions upon the Amount of Food required.

IV. Animal Foods.

V. Jellies-Fish.

VI. Soups, Beef-Extracts, Beef-Juices, Beef-Tea, and Beef-Powders.

VII. Milk.

VIII. Foods derived from Milk.

IX. Oheese, Eggs, and Egg Substitutes.

X. Vegetable Foods.

XI. The Cereals: Wheat-Bread.

XII. Bread (continued)-Other Cereals.

XIII. The Pulses-Roots, and Tubers.

XIV. Vegetables-Fruits-Nuts-Fungi-Algæ and Lichens

$\mathrm{XV}$. Sugar, Spices, and Condiments.

XVI. Mineral Constituents of the Food.

XVII. Water and Mineral Waters.

XVIII. Tea, Coffee, and Cocoa.

XIX. Alcohol.

XX. Alcoholic Beverages : Spirits and Malt Liquors.

XXI. Alcoholic Beverages (continued): Wines.

XXII. The Cooking of Foods.

XXIII. The Digestion of Food in Health.

XXIV. The Principles of Feeding in Infancy and Childhood : Human Milk.

XXV. The Principles of Feeding in Infancy and Childhood (continued): Substitutes for Human Milk.

XXVI. The Principles of Feeding in Infancy and Childhood (continued): Other Substitutes for Hurnan Milk (Peptonized Milk, Condensed Milk, Proprietary Foods); Feeding of Older Children.

XXVII. The Principles of Feeding in Disease.

XXVIII. The Principles of Feeding in Disease (continued).

XXIX. Artificial and Predigested Foods and Artificial Feeding. 


\section{Dr. Hutchison's Works.}

\section{LECTURES ON DISEASES OF CHILDREN.}

Bx ROBERT HUTCHISON, M.D. EdIN., F.R.C.P., Physician to the London Hospital and Assistant Physician to the Hospital for Sick Children, Great Ormond Street.

Author of "Food and the Principles of Dietetics."

\section{THIRD IMPRESSION.}

xii +338 pages. With Illustrations. Crown 8vo., cloth, 8s. $6 \mathrm{~d}$. net (post free 8s. 10d.).

CHAPTER

CONTENTS.

I. The Clinical Examination of Sick Children.

II. The Artificial Feeding of Infants.

III. The Digestive Disorders of Infancy-Colic and Vomiting.

IV. The Digestive Disorders of Infancy (continued)-Diarrhoea.

V. The Wasting Diseases of Infancy-Marasmus and Congenital Syphilis.

VI. Tuberculosis in Childhood.

VII. Rickets.

VIII. Infantile Scurvy.

IX. The Dyspepsias of the Second Dentition.

$\mathrm{X}$. Rheumatism in Childhood.

XI. The Respiratory Diseases of Children.

XII. Some Functional Nervous Diseases of Childhood.

XIII. Some Functional Nervous Diseases of Childhood (continued).

XIV. The Paralyses of Childhood.

XV. Meningitis.

XVI. On Mental Deficiency in Childhood.

XVII. The Blood Disorders of Early Life.

XVIII. Some Common Symptoms of Disease in Children and their Diagnostic Significance.

XIX. Some Medical Aspects of Adenoid Vegetations in Infancy and Childhood.

\section{APPLIED PHYSIOLOGY.}

\section{A Handbook for Students of Medicine.}

By ROBERT HUTCHISON, M.D., F.R.C.P.

Crown 8vo., 7s. 6d. net (post free 7s. 10d.).

The author of a standard work on diet is not likely to err by being too theoretical. The principle of Dr. Hutchison's new book is to bring physiology from the laboratory to the bedside. "Physiology," he writes, "is studied in the laboratory, and clinical medicine in the wards, and too often one finds that the student is incapable of applying his scientific knowledge to his clinical work." 


\section{Mr. Edward Arnold's Books on Biology.}

\section{THE EVOLUTION THEORY.}

BY Dr. AUGUST WEISMANN,

Professor of Zoology in the University of Freiburg in Breisgau.

Translated, with the Author's co-operation,

BY J. ARTHUR THOMSON,

Regius Professor of Natural History in the University of Aberdeen,

$$
\text { AND }
$$

MARGARET THOMSON.

Two vols., xvi +416 and viii +396 pages, with over 130 Illustrations.

Royal 8vo., cloth, 32s. net (post free 32s. 7d.).

\section{ANIMAL BEHAVIOUR.}

By Professor C. LLOYD MORGAN, LL.D., F.R.S.,

Principal of University College, Bristol.

viii +344 pages, with 26 Illustrations. Crown 8 vo., cloth, 7s. 6 d. net

(post free 7s. 11d.).

\section{HABIT AND INSTINCT.}

By Professor C. LLOYD MORGaN.

viii +352 pages. Demy 8vo., cloth, $16 \mathrm{~s}$.

\section{A TEXT-BOOK OF ZOOLOGY.}

BY G. P. MUDGE, A.R.C.Sc. LoND., F.Z.S.,

Lecturer on Botany and Zoology at the London School of Medicine for Women (University of London) and at the Regent Street Polytechnic; and Demonstrator on Biology at the

London Hospital Medical College (University of London).

xvi +416 pages, with 2 Coloured Plates and numerous original Illustrations.

Crown 8vo., cloth, 7s. 6d.

\section{THE LIFE OF THE SALMON.}

With Reference more espectally to the Fish in Scotland.

BY W. L. CALDERWOOD, F.R.S.E.,

Inspector of Salmon Fisheries for Scotland.

Illustrated. Demy 8vo., 7s. 6d. net (post free 7s. 10d.).

\section{A CLASS-BOOK OF BOTANY.}

BY G. P. MUDGE, A.R.C.Sc. LoND., F.Z.S., AND

ARTHUR J. MASLEN, F.L.S.,

Lecturer on Botany at the Woolwich Polytechnic.

xvi +512 pages, with over 200 Illustrations. Crown 8vo., cloth, 7s. 6 d.

\section{HOUSE, GARDEN, AND FIELD.}

A Collection of Short Nature Studies.

Br L. C. MIALL, F.R.S., Professor of Biology in the University of Leeds, and Fullerian Professor of Physiology
in the Royal Institution.

With numerous Illustrations. viii +316 pages. Crown 8vo., cloth, $6 \mathrm{~s}$.

LONDON : EDWARD ARNOLD, 41 \& 43 MADDOX STREET, W. 


\section{Mr. Edward Arnold's Books on Psychology.}

\section{A TEXT-BOOK OF EXPERIMENTAL PSYCHOLOGY.}

BY C. S. MYERS,

Professor of Psychology at King's College, London University.

Crown 8vo., 7s. 6d, net (post free 7s. 11d.).

For some time past the lack of a text-book on Experimental Psychology has been keenly felt. The literature of the subject is now so scattered and so profuse that a student must have at his command a small library of books and periodicals if he wishes to pursue a course of independent reading. Accordingly, it is the object of the forthcoming work to give an account of the more important results that have been obtained in this field of research, as well as to describe the methods and principles of psychological experiment. To each chapter an account is appended of experiments which may be performed by the student in the laboratory, and illustrations are given of the apparatus employed therein.

\section{AN INTRODUCTION TO CHILD.STUDY.}

BY W. B. DRUMMOND, M.B., O.M., F.R.C.P.E., Medical Officer and Lecturer on Hygiene to the Edinburgh Proviucial Cummittee for the Training of Teachers.

Author of "The Child: His Nature and Nurture."

Crown 8vo., cloth, 6s. net (post free 6s. 4d.).

CONTENTS.

Preparation-Caution in Child-Study-Biology and Child-Study-The Methods of Child-Study-How to Study a Baby-Weights and Measures, some Facts of Growth-The Senses and the Nervous System-The Health of the ChildFatigue--The Instincts of Children-Instinct and Habit-The Interests of Children-Forms of Expression-Some Moral Characteristics-Religion and the Child-Peculiar and Exceptional Ohildren.

\section{THE CHILD'S IMIND: ITS GROWTH AND TRAINING.}

By W. E. URWICK, M.A.

Crown 8vo., cloth, 4s. 6d. net (post free 4s. 10d.).

CONTENTS.

The Bearing of Biology on Educational Theology-The Mechanism of LearningMind's Main Characteristics and Early Products-Lower Processes of Learning -Learning and the Growth of Ideas-Final Values of Learning-Growth of Ideas in Imaginative Process : Normal Types of Learning-Reasoning as Mind's Emergency Process-Growth of Immediate Values-Some Conclusions.

\section{PSYCHOLOGY FOR TEACHERS.}

By C. LLOYD MORGAN, LL.D., F.R.S., Principal of University College, Bristol.

New Edition, entirely rewritten. xii 307 pages. Crown 8vo., cloth, 4s. 6d.

For this edition, Professor Lloyd Morgan has entirely rewritten, and very considerably enlarged, his well-known work on this important subject. He has, in fact, practically made a new book of it.

LONDON : EDWARD ARNOLD, 41 \& 43 MADDOX STREET, W. 


\section{Mr. Edward Arnold's List of Technical \& Scientific Publications}

Extract from the LIVERPOOL POST of Dec, 4, 1907 :-

"During recent years Mr. Edward Arnold has placed in the hands of engineers and others interested in applied science a large number of volumes which, independently altogether of their intrinsic merits as scientific works, are very fine examples of the printers' and engravers' art, and from their appearance alone would be an ornament to any scientific student's library. Fortunately for the purchaser, the publisher has shown a wise discrimination in the technical books he has added to his list, with the result that the contents of the volumes are almost without exception as worthy of perusal and study as their appearance is attractive."

\section{The Dressing of Minerals.}

BY HENRY LOUIS, M.A.,

Professor of Mining and Lecturer on Surveyin, Armstrong College, Newcastle-on-Tyne.

With about 400 Illustrations. Royal 8vo., 3os. net.

The object of this book is to fill a gap in technological literature which exists between works on Mining and works on Metallurgy. On the intermediate processes, by which the minerals unearthed by the miner are prepared for the smelter and for their use in arts and manufactures, no English text-book has yet appeared. The present work should, therefore, be very welcome to students, as well as to miners and metallurgists.

\section{ARNOLD'S GEOLOGICAL SERIES.}

GENERAL EDITOR: DR. J. E. MARR, F.R.S.

The economic aspect of geology is yearly receiving more attention in our great educational centres, and the books of this series are designed in the first place for students of economic geology. It is believed, however, that they will be found useful to the -student of general geology, and also to surveyors and others who are concerned wiih the practical applications of the science.

\section{The Geology of Coal and Coal-Mining.}

By WALCOT GIBSON, D.Sc., F.G.S.

352 pages. With 45 Illustrations. Crown 8 vo., 7s. 6d. net (post free 7s. rod.).

Many years' professional experience among the coalfields of "this country and of South Africa have enabled the author to treat his subject in a thoroughly original manner. The book therefore contains a great amount of valuable information, as well as many criticisms and suggestions which have not hitherto appeared in any text-book on the subject.

IN PREPARATION.

\section{The Geology of Ore Deposits.}

By H. H. THOMAS AND D. A. MACALISTER, Of the Geological Survey.

Illustrated. Crown 8vo., 7s, 6d. net (post free 7 s. Iod.). 


\section{Electrical Traction.}

By ERNEST WILSON, Whit. Sch., M.I.E.E., Professor of Electrical Engineering in the Siemens Laboratory, King's College, London, AND FRANCIS LYDALL, B.A., B.Sc.

New Edition. Rewritten and Greatly Enlarged.

Two volumes, sold separately. Demy 8vo., cloth.

Vol. 1., with about 270 Illustrations and Index.

Vol. II., with about I 70 Illustrations and Index.

I 5s. net each volume (post free I5s. 5d.).

"We are most decidedly of the opinion that both of these volumes will prove of great value to engineers, and that the last volume, in view of the present great interest in the question of single-phase traction, is of the utmost importance, for in it for the first time is published a great amount of data with reference to which, hitherto, the manufacturing companies concerned have observed great secrecy."-The Times (Engineering Supplement).

\section{A Text-Book of Electrical Engineering.}

BY DR. ADOLF THOMÄLEN.

Translated by G. W. O. HOWE, M.Sc., Whiт. Sch., A.M.I.E.E., Lecturer in Electrical Engineering at the Central Technical College, South Kensington.

With 454 Illustrations. Royal 8vo., cloth, I5s. net (post free I 5s. 6d.).

This translation of the "Kurze Lehrbuch der Electrotechnik" is intended to fill the gap which appears to exist between the elementary text-books and the specialized works on various branches of electrical engineering.

\section{Alternating Currents.}

A Text-Book for Students of Engineering.

By C. G. LAMB, M.A., B.Sc.,

Clare College, Cambridge ; Associate Member of the Institution of Electrical Engineers; Associate of the City and Guilds of London Institute.

viii +325 pages. With upwards of 230 Illustrations. Demy 8 vo., cloth, Ios. 6d. net (post free Io:. I Id.).

\section{Electric and Magnetic Circuits.}

By ELLIS H. CRAPPER, M.I.E.E.,

Head of the Electrical Engineering Department in the University College, Sheffield.

viii +380 pages. Demy 8vo., cloth, Ios. 6d. net (post free Ios. IId.).

\section{Applied Electricity.}

A Text-Book of Electrical Engineering for "Second Year" Students.

BY J. PALEY YORKE,

Head of the Physics and Electrical Engineering Department at the London County Council School of Engineering and Navigation, Poplar.

xii +420 pages. Crown 8 vo., cloth, 7s. 6 d. 


\section{Hydraulics.}

By F. C. LEA, B.Sc., A.M.Inst.C.E.,

Senior Whitworth Scholar, A.R.C.S. ; Lecturer in Applied Mechanics and E.ngineering Design, City and Guilds of London Central Technical College, London.

With 367 Diagrams. Demy 8vo., 18s. net (post free I8s. 5d.).

This book is intended to supply the want felt by students and teachers alike for a text-book of Hydraulics to practically cover the syllabuses of London and other Universities, and of the Institution of Civil Engineers.

\section{Hydraulics.}

\section{BY RAYMOND BUSQUET,}

Professeur à l'École Industrielle de Lyon.

Authorized English Edition.

Translated by A. H. PEAKE, M.A.,

Demonstrator in Mechanism and Applied Mechanics in the University of Cambridge.

viii +3I2 pages. With 49 Illustrations. Demy 8vo, cloth, 7s. 6d. net (post free $7 \mathrm{~s}$. Iod.).

\section{The Balancing of Engines.}

By W. E. DALBY, M.A., B.Sc., M.Inst.C.E., M.I.M.E., Professor of Engineering, City and Guilds of London Central Technical College.

Second Edition, Revised and Enlarged.

xii +283 pages. With upwards of 180 Illustrations.

Demy 8vo., cloth, Ios. 6d. net (post free Ios. Iod.).

\section{Valves and Valve Gear Mechanisms.}

By W. E. DALBY, M.A., B.Sc., M.Inst.C.E., M.I.M.E., Professor of Engineering, City and Guilds of London Central Technical College.

xviii +366 pages. With upwards of 200 Illustrations.

Royal 8vo., cloth, 2Is. net (post free £I Is. 5d.).

\section{Power Gas Producers.}

\section{Their Design and Application.}

\section{By PHILIP W. ROBSON,}

Of the National Gas Engine Co., Ltd. ; sometime Vice-Principal of the Municipal Schoul of Technology, Manchester.

Demy 8vo., cloth, Ios. 6d. net (post free Ios. IId.).

The recent enormous increase in the use of gas power is largely due to the improvements in gas producers. This book, which is written by a well-known expert, goes thoroughly into the theory, design, and application of all kinds of plants, with chapters on working and general management. 


\section{The Strength and Elasticity of Structural Members.}

By R. J. WOODS, M.E., M.Inst.C.E., Formerly Fellow and Assistant Professor of Engineering, Royal Indian Engineering College, Cooper's Hill.

Second Edition, Revised.

xii +310 pages. With 292 Illustrations. Demy 8vo., cloth, ros. 6d. net (post free IOS. Iod.).

\section{Calculus for Engineers.}

By JOHN PERRY, M.E., D.Sc., F.R.S.,

Professor of Mechanics and Mathematics in the Royal College of Science, London; Vice-President of the Physical Society; Vice-President of the Institution of Electrical Engineers.

NINTH IMPRESSION.

viii +382 pages. With 106 Illustrations. Crown 8vo., cloth, 7s. 6d.

\section{Mathematical Drawing.}

Including the Graphic Solution of Equations.

BY G. M. MINCHIN, M.A., F.R.S.,

Formerly Professor of Applied Mathematics at the Royal Indian Engineering College, Cooper's Hill ;

AND JOHN BORTHWICK DALE, M.A., Assistant Professor of Mathematics at King's College, London.

Crown 8vo., cloth, 7s. 6d. net (post free 7s. Iod.).

\section{Five-Figure Tables of Mathematical Functions.}

Comprising Tables of Logarithms, Powers of Numbers, Trigonometric, Elliptic, and other Transcendental Functions.

BY JOHN BORTHWICK DALE, M.A.,

Assistant Professor of Mathematics at King's College, London.

vi +92 pages. Demy 8vo., cloth, 3s. 6d. net (post free 3s. Iod.).

This collection of Tables has been selected for use in the examinations of the University of London.

\section{Logarithmic and Trigonometric Tables (To}

Five Places of Decimals). By JOHN BORTHWICK DALE, M.A., Assistant Professor of Mathematics at King's College, London. Demy 8vo., cloth, 2s. net (post free 2s. 3d.).

\section{Traverse Tables. With an Introductory Chapter}

on Co-ordinate Surveying. By Henry LOUIS, M.A., Professor of Mining and Lecturer on Surveying, Armstrong College, Newcastle-on-Tyne ; and G. W. CAUNT, M.A.. Lecturer in Mathematics, Armstrong College, Newcastle-on-Tyne. xxviii +92 pages. Demy 8 vo., flexible cloth, rounded corners, 4s. 6d net (post free 4s. 9d.). 


\section{Organic Chemistry for Advanced Students.}

By JUliUs B. COHEN, PH.D., B.Sc.,

Professor of Organic Chemistry in the University of Leeds, and Associate of Owens College, Manchester.

Demy 8vo., cloth, 21s. net (post free 2is. 6d.).

The book is written for students who have already completed an elementary course of Organic Chemistry, and is intended largely to take the place of the advanced text-book. For it has long been the opinion of the author that, when the principles of classification and synthesis and the properties of fundamental groups have been acquired, the object of the teacher should be, not to multiply facts of a similar kind, but rather to present to the student a broad and general outline of the more important branches of the subject. This method of treatment, whilst it avoids the dictionary arrangement which the text-book requires, leaves the writer the free disposal of his materials, so that he can bring together related substances, irrespective of their nature, and deal thoroughly with important theoretical questions which are often inadequately treated in the text-book.

\section{The Chemical Synthesis of Vital Products and the Inter-relations between Organic Compounds.}

By RAPHAEL MELDOLA, F.R.S., V.P.C.S., F.I.C., etc.,

Professor of Chemistry in the City and Guilds of London Technical College, Finsbury.

Vol. I., xvi +338 pages. Super Royal 8vo., cloth, 2 Is. net

(post free 2 Is. 5d.).

The great achievements of modern Organic Chemistry in the domain of the synthesis or artificial production of compounds which are known to be formed as the result of the vital activities of plants and animals have not of late years been systematically recorded. The object of the present book is to set forth a statement, as complete as possible, of the existing state of knowledge in this most important branch of science.

\section{The Chemistry of the Diazo-Compounds.}

By JOHN CANNELL CAIN, D.Sc. (Manchester and Tübingen), Editor of the Publications of the Chemical Society.

176 pages. Demy 8vo., Ios. 6d. net (post free Ios. Iod.).

\section{Lectures on Theoreticaland Physical Chemistry.}

BY DR. J. H. VAN 'T HOFF,

Professor of Chemistry at the University of Berlin.

Translated by R. A. LEHFELDT, D.Sc.,

Professor of Physics at the Transvaal Technical Institute, Johannesburg.

In three volumes, demy 8vo., cloth, $28 \mathrm{~s}$. net (post free $28 \mathrm{~s} .7 \mathrm{~d}$.), or separately as follows :

PART I. CHEMICAL DYNAMICS. 254 pages, with 63 Illustrations. I2s. net (post free I2s. 5d.).

PART II. CHEMICAL STATICS. I 56 pages, with 33 Illustrations. 8s. 6d. net (post free 8s. Iod.).

PART III. RELATIONS BETWEEN PROPERTIES AND COMPOSITION. I43 pages, 7s. 6d. net (post free 7s. Iod.). 


\title{
Experimental Researches with the Electric Furnace.
}

BY HENRI MOISSAN, Membre de l'Institut; Professor of Chemistry at the Sorbonne. AUTHORIZED ENGLISH EDITION.

Translated by A. T. de MOUILPIED, M.Sc., Ph.D., Assistant Lecturer in Chemistry in the University of Liverponl.

xii +307 pages, with Illustrations. Demy 8 vo., cloth, Ios. $6 \mathrm{~d}$. net (post free Ios. Iod.).

"There is hardly a page of it which is not crowded with interest, and hardly a section which does not teem with suggestion; and if the coming of this English edition of the book has been so long delayed, we may still be thankful that it has come at last, and come in a form which it is a pleasure to handle and a delight to read,"-Electrical Review.

\section{Electrolytic Preparations.}

\section{Exercises for use in the Laboratory by Chemists and Electro-Chemists.}

BY DR. KARL ELBS,

Professor of Organic and Physical Chemistry at the University of Giessen.

Translated by R. S. HUTTON, M.Sc.,

Demonstrator and Lecturer on Electro-Chemistry at the University of Manchester.

xii + Ioo pages. Demy 8vo., cloth, 4s. 6d. net (post free 4s. Iod.).

The book contains a complete course of examples on the application of electrolysis to the preparation of both inorganic and organic substances. It will be found useful as filling a distinct gap in the textbook literature suitable for use in chemical laboratories, and should enable the chemist to make use of the many valuable and elegant methods of preparation which have been worked out during recent years, the advantages and ease of application of which he cannot appreciate without such a guide.

\section{Introduction to Metallurgical Chemistry for Technical Students.}

\author{
By J. H. Stansbie, B.Sc. (Lond.), F.I.C.,
}

Associate of Mason University College, and Lecturer in the Birmingham University Technical Schuol.

SECOND EDITION.

xii +252 pages. Crown 8vo., cloth, 4s. $6 \mathrm{~d}$.

\section{An Experimental Course of Chemistry for Agri-} cultural Students. By T. S. Dymond, F.I.C., Lately Principal Lecturer in the Agricultural Department, County Technical Laboratories, Chelmsford. New Impression. 192 pages, with 50 Illustrations. Crown 8vo., cloth, 2s. 6 d.

\section{A History of Chemistry.}

By DR. HUGO BAUER, Royal Technical Institute, Stuttgart.

Translated by R. V. STANFORD, B.Sc. (LOND.), Priestley Research Scholar in the University of Birmingham.

Crown 8vo., cloth, 3s. 6d. net. 


\title{
The Becquerel Rays and the Properties of Radium.
}

\author{
BY THE HON. R. J. STRUTT, F.R.S., \\ Fellow of Trinity College, Cambridge. \\ SEcond Edition, REvised AND ENLARgED. \\ viii +222 pages, with Diagrams. Demy 8vo., cloth, 8s. 6d. net \\ (post free 8s. Iod.).
}

"If only a few more books of this type were written, there might be some hope of a general appreciation of the methods, aims, and results of science, which would go far to promote its study. . . . A book for which no praise can be excessive." Athencum.

\section{Astronomical Discovery.}

BY HERBERT HALL TURNER, D.Sc., F.R.S.,

Savilian Professor of Astronomy in the University of Oxford.

xii +225 pages, with Plates and Diagrams. Demy 8vo., cloth, Ios. 6d. net (post free Ios. IId.).

\section{An Introduction to the Theory of Optics.}

By ARTHUR SCHUSTER, Ph.D., Sc.D., F.R.S.,

Recently Professor of Physics at the University of Manchester.

xvi +340 pages, with Illustrations. Demy 8 vo., cloth, I 5 s. net

(post free I 5s. 5d.).

"We know of no book written with a set purpose better adapted to serve the purpose for which it was written, nor any that the earnest student of optics will find more interesting and profitable. The work itself, without the confession of the preface, shows that Professor Schuster is a teacher, and every page bears evidence that he is a master of his subject. . . . We heartily recommend the book to our readers." Ophthalmic Review.

\section{Wood.}

A Manual of the Natural History and Industrial Applications of the Timbers of Commerce.

By G. S. BOULGER, F.L.S., F.G.S., A.S.I.,

Professor of Botany and Lecturer on Forestry in the City of London College, and formerly in the Royal Agricultural College.

NEw Edition. Revised and Enlarged and profusely illustrated.

Demy 8vo., I2s. 6d. net (post free I2s. I Id.).

"It is just the book that has long been wanted by land agents, foresters, and woodmen, and it should find a place in all technical school libraries."-Field.

\section{Manual of Alcoholic Fermentation and the Allied Industries.}

By CHARLES G. MATTHEWS, F.I.C., F.C.S., ETc.

xvi 295 pages, with 8 Plates and 40 Illustrations. Crown 8vo., cloth, 7s. 6d. net (post free 7s. Iod.).

" This is a book worthy of its author, and well worth perusing by every student. - The student, both old and young, as well as the practical brewer, will find this book gives him some very useful information."-Brewers' Guardian. 


\section{The Evolution Theory. By Dr. August Weis-}

MANN, Professor of Zoology in the University of Freiburg in Breisgau. Translated, with the Author's co-operation, by J. ARTHUR THOMSON, Regius Professor of Natural History in the University of Aberdeen ; and MARGARET THOMSON. Two vols., xvi +416 and viii +396 pages, with over I30 Illustrations. Royal 8vo., cloth, 32s. net.

" The subject has never been so fully and comprehensively expounded before ; and it is not necessary to subscribe to all the author's tenets in order to recognise the value and the absorbing interest of his exposition, with its prodigious wealth of illustration, its vast store of zoological knowledge, its ingenious interpretations and far-reaching theories. English readers have reason to be grateful to Professor and Mrs. Thomson for their admirable translation." - The Times.

\section{The Chances of Death and Other Studies in}

Evolution. By Karl Pfarson, M.A., F.R.S, Professor of Applied Mathematics in University College, London, and formerly Fellow of King's College, Cambridge. 2 vols., xii +388 and 460 pages, with numerous Illustrations. Demy 8 vo., cloth, 25s, net (post free 25s. 6d.).

\section{The Life of the Salmon.}

With reference more especially to the Fish in Scotland. By W. L. CALDERWOOD, F.R.S.E., Inspector of Salmon Fisheries for Scotland. Illustrated. Demy 8vo,, 7s. 6d, net.

"We have no hesitation whatever in advising all persons interested in the salmon, whether as fishermen, naturalists, or legislators, to add this book to their libraries." -Nature.

\section{Animal Behaviour. By Professor C. Llovd} MoRgan, LL.D., F.R.S., Principal of University College, Bristol. viii +344 pages, with 26 Illustrations. Crown 8vo., cloth, 7s. 6d. net (post free 7 s. IId.).

This important contribution to the fascinating subject of animal psychology covers the whole ground from the behaviour of cells up to that of the most highly developed animals.

\section{$B Y T H E S A M E$ AUTHOR.}

\section{Habit and Instinct. viii $+35^{2}$ pages, with Photo-} gravure Frontispiece. Demy 8vo., cloth, I6s.

Professor AlfRed RUSSEl WAllace:- "An admirable introduction to the study of a most important and fascinating branch of biology, now for the first time based upon a substantial foundation of carefully observed facts and logical induction from them."

\section{BY THE SAME AUTHOR.}

The Springs of Conduct. Cheaper Edition. viii +317 pages. Large crown 8vo., cloth, 3s. 6d. This volume deals with the Source and Limits of Knowledge, the Study of Nature, the Evolution of Scientific Knowledge, Body, and Mind, Choice, Feeling, and Conduct.

$B Y$ THE SAME AUTHOR.

Psychology for Teachers. New Edition, entirely rewritten. xii +308 pages. Crown 8 vo., cloth, 45. $6 \mathrm{~d}$.

An Introduction to Child Study. By Dr. W. B. DRUMMOND. Crown 8vo., cloth, 6s. net.

The Child's Mind: Its Growth and Training. By W. E. URWICK, University of Leeds. Crown 8vo., cloth, 4s. 6d. net. 
$x^{2} 7^{2}$ 



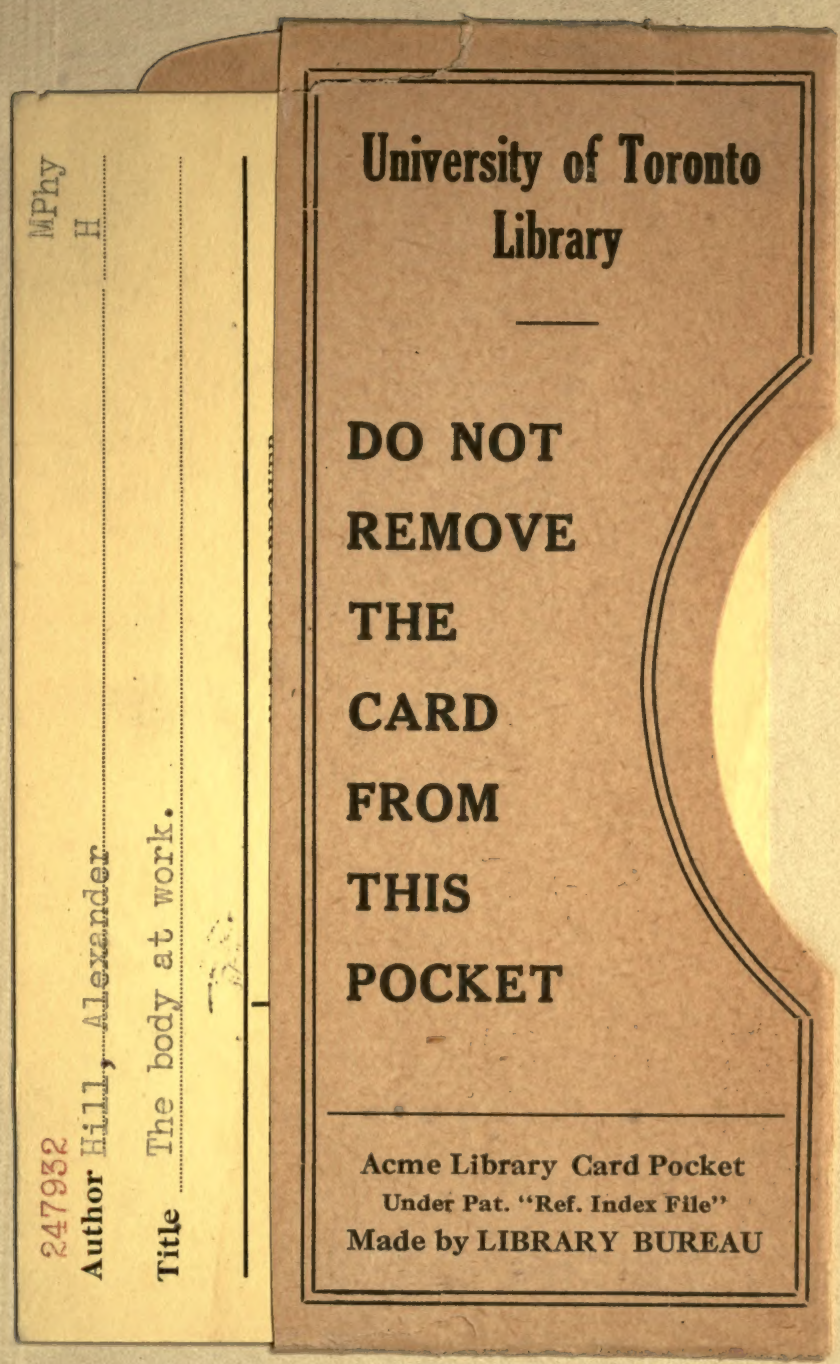


